\title{
Synthesis of Modified Biomolecules to Investigate Neuronal Processes
}

\author{
Dissertation
}

\author{
for the award of the degree \\ "Doctor rerum naturalium" \\ of the Georg-August University Göttingen \\ within the doctoral program Chemistry \\ of the Georg-August-University School of Science (GAUSS)
}

submitted by

\section{Patrick Menzel}

from Burgwedel

Göttingen 2020 



\section{Thesis Committee}

Prof. Dr. Ulf Diederichsen

Institute of Organic and Biomolecular Chemistry, Georg-August-University Göttingen

Prof. Dr. Claudia Steinem

Institute of Organic and Biomolecular Chemistry, Georg-August-University Göttingen

\section{Members of the Examination Board}

\section{Reviewer}

Prof. Dr. Ulf Diederichsen

Institute of Organic and Biomolecular Chemistry, Georg-August-University Göttingen Prof. Dr. Claudia Steinem

Institute of Organic and Biomolecular Chemistry, Georg-August-University Göttingen

\section{Additional Members of the Examination Board}

Prof. Dr. Reinhard Jahn

Institute of Neurobiology, Max Planck Institute for Biophysical Chemistry, Göttingen

Prof. Dr. Silvio Rizzoli

Department of Neuro- and Sensory Physiology, University Medical Center Göttingen

Dr. Stefan Glöggler

Center for Biostructural Imaging of Neurodegeneration (BIN), Georg-August-University

Göttingen

Dr. Holm Frauendorf

Institute of Organic and Biomolecular Chemistry, Georg-August-University Göttingen

Dr. Michael John

Institute of Organic and Biomolecular Chemistry, Georg-August-University Göttingen

Date of oral examination: December 22, 2020 

The work described in this thesis was carried out under the supervision and guidance of Prof. Dr. Ulf Diederichsen at the Institute of Organic and Biomolecular Chemistry of the GeorgAugust University Göttingen between January 2017 and November 2020.

This work was supported by the Cluster of Excellence and DFG Research Center Nanoscale Microscopy and Molecular Physiology of the Brain (CNMPB)

Parts of chapter 1.2.2 of this thesis have been published as:

B. E. Hubrich, P. M. Menzel, B. Kugler, U. Diederichsen, Peptide Nucleic Acids: Methods and Protocols, P.Nielsen (Ed.), $3^{\text {rd }}$ Edition, Springer Verlag, Wiesbaden, 2020.

\section{Declaration of Authorship}

Hereby, I declare that I prepared the doctoral thesis entitled "Synthesis of Modified Biomolecules to Investigate Neuronal Processes" on my own and with no other sources and aids than quoted.

Göttingen, November 09, 2020

Patrick Menzel 



\section{Contents}

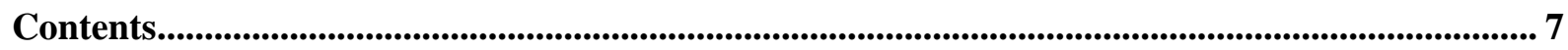

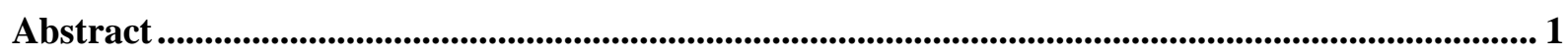

1 Synthesis of Chloride-Sensors to Investigate Neurotransmitter filling in Synaptic Vesicles.. 3

1.1 Introduction - Synaptic Vesicle .............................................................................................................. 3

1.1.1 Vesicular Neurotransmitter Transporters .................................................................... 4

1.1.2 The Vesicular Glutamate Transporters........................................................................ 5

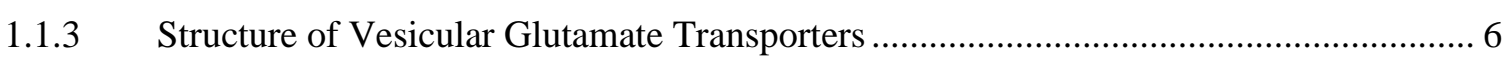

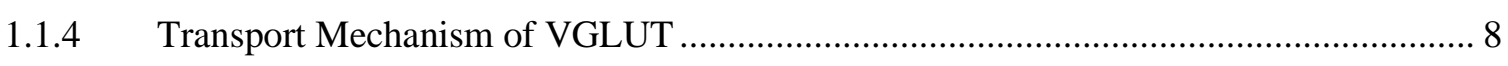

1.1.4.1 Mediation of Chloride Transport into Synaptic Vesicles ...................................... 9

1.1.4.2 Allosteric Activation of VGLUT by Luminal Chloride ....................................... 11

1.1.4.3 VGLUT Model for Ion Transport across Vesicular Membranes............................ 12

1.1.5 Development of Fluorescent Chloride Sensor Molecules ............................................ 14

1.1.5.1 Chloride Sensitive Protein Reporters ..................................................................... 15

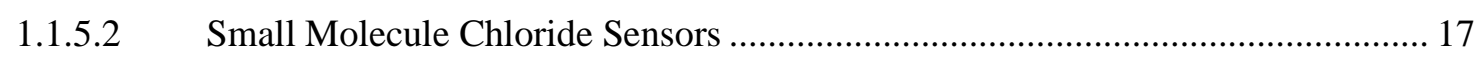

1.1.5.3 Second Generation Sensor - Development of Ratiometric Quantification ........... 19

1.1.5.4 Chloride Sensor conjugated to a DNA Scaffold.................................................... 20

1.2 Design and Synthesis of PNA-DNA based Chloride Sensors............................................... 25

1.2.1 Synthesis of PNA based Sensing Module ….................................................................. 26

1.2.2 Optimised Synthesis Strategy for PNA Oligomers (molecule part I) ............................ 27

1.2.2.1 Synthesis of BAC Modified Peptide-PNA Oligomer............................................. 30

1.2.3 Synthesis of Membrane Anchor Modified PNA Sensors (Molecule Part II) ................. 31

1.2.3.1 Synthesis of Cholesterol-Modified Peptide-PNA Oligomer .................................. 32

1.2.3.2 Synthesis of a Palmitoyl-Modified Peptide-PNA Oligomer .................................. 34

1.2.4 Introduction of Cleavage Sites in Modified PNA Sensors (molecule part III) .............. 35

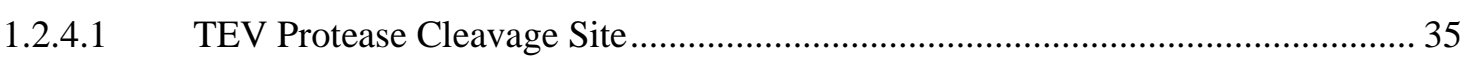

1.2.4.2 Factor Xa Protease Cleavage Site ..................................................................... 38

1.2.4.3 Cathepsin B Protease Cleavage Site ..................................................................... 40

1.2.4.4 Membrane Incorporation of Palmitoyl Modified PNA Sensors ............................. 41

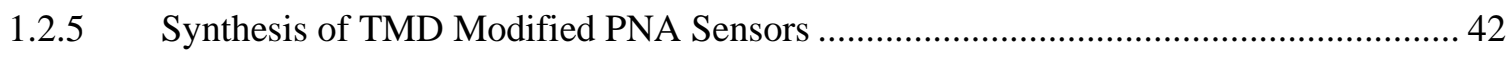

1.2.5.1 Membrane Incorporation and Distribution of TMD-Modified PNA Sensors ....... 44

1.2.5.2 Formation of the Chloride Sensitive PNA-DNA Complex .................................... 46

1.2.6 Synthesis of His-Tag Modified PNA Sensors ........................................................... 48

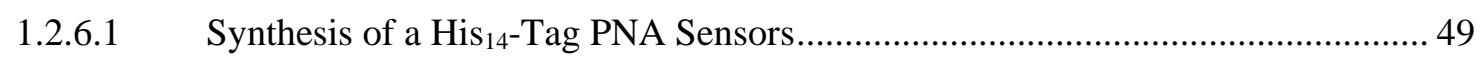

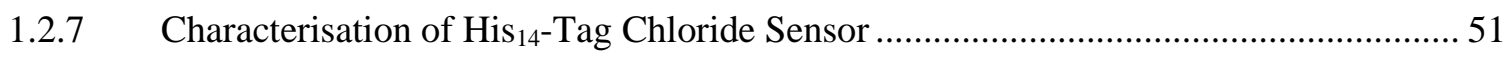

1.2.7.1 Formation studies of the His He $_{14}$-Tag Chloride Sensor............................................... 51 


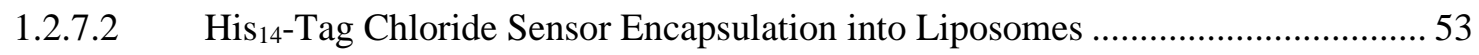

1.2.7.3 Purification and Chloride Measurements of Sensor Modified Liposomes .............. 55

1.2.7.4 Functional Assays of the His ${ }_{14}$-Tag Chloride Sensor ............................................. 57

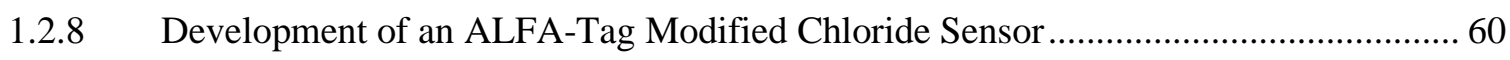

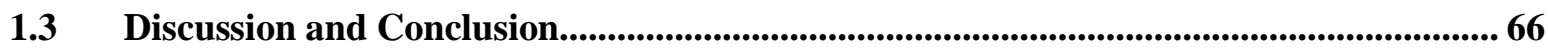

2 Investigation of Voltage-Gated Sodium Channels by modified Conotoxins ............................ 71

2.1 Structure and Function of Voltage-Gated Sodium Channels............................................ 71

2.1.1 Voltage-Gated Sodium Channel Isoforms.................................................................... 73

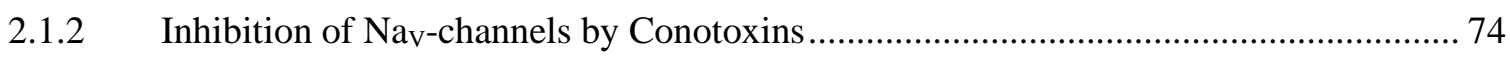

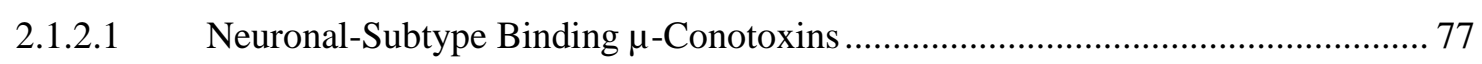

2.1.3 Oxidative Folding of Disulphide-Rich Peptides................................................................ 79

2.1.3.1 Regioselective Folding of Disulphide-Rich Peptides .............................................. 81

2.2 Design and Synthesis of Modified $\mu$-Conotoxin SIIIA ........................................................ 83

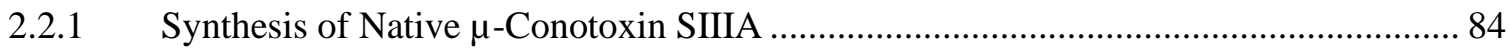

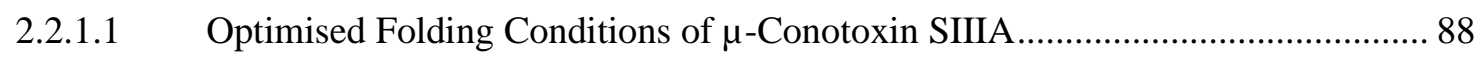

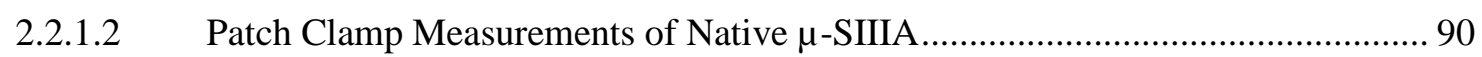

2.2.2 Synthesis of Labelled $\mu$-Conotoxin SIIIA ......................................................................... 94

2.2.3 New Synthesis Strategies for Labelling $\mu$-Conotoxin SIIIA ........................................... 97

2.2.3.1 Synthesis of Allocam Protected Alkyne Modified $\mu$-SIIIA ……............................ 97

2.2.3.2 Synthesis of tert-Butyl and MeOBzl Protected Alkyne Modified $\mu$-SIIIA........... 98

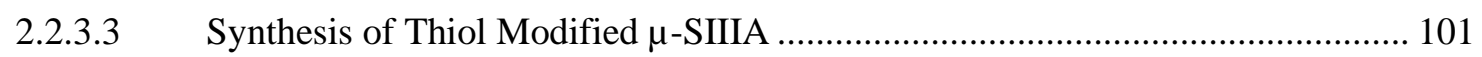

2.2.3.4 Synthesis of Amine Modified $\mu$-SIIIA .................................................................. 102

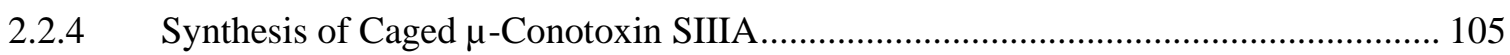

2.2.4.1 Characteristics of Photocleavable Protecting Groups............................................ 105

2.2.4.2 Caging Experiments of the $\mu$-Conotoxin SIIIA ..................................................... 106

2.2.4.3 Synthesis of Nvoc Protected Lysine .................................................................... 107

2.2.4.4 Synthesis of Nitrobenzyl Based Protected Aspartic Acid ................................... 108

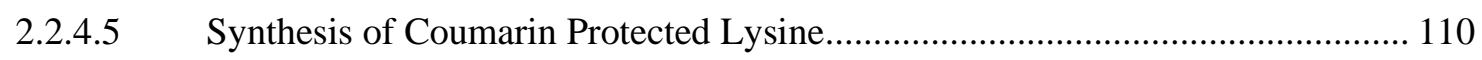

2.2.5 Synthesis of DEACM Caged $\mu$-Conotoxin SIIIA …...................................................... 112

2.2.5.1 Uncaging Experiments of DEACM Modified $\mu$-SIIIA ….................................... 113

2.2.5.2 Patch Clamp Measurements of DEACM Caged $\mu$-SIIIA ...................................... 114

2.2.5.3 Verification of the Uncaging Process by U-HPLC............................................. 118

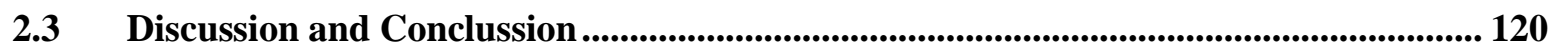

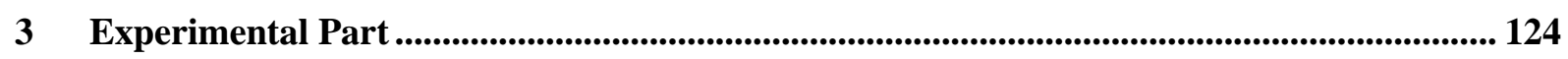

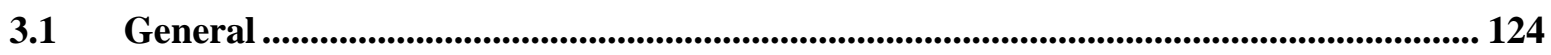

3.2 Chromatography .................................................................................................................. 125

3.3 Characterisation ............................................................................................................................... 126

3.4 Standard Operating Protocols (SOPs) ........................................................................................... 130 
3.4.1 SOPS for the Synthesis of Peptide-PNA based $\mathrm{Cl}^{-}$sensors........................................ 130

3.4.1.1 SOP1: Automated Solid-Phase Peptide Synthesis............................................... 130

3.4.1.2 SOP2: Automated SPPS for PNA Oligomers................................................... 131

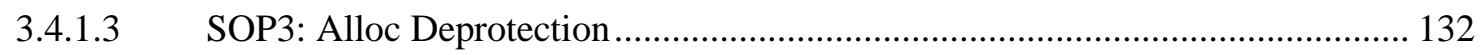

3.4.1.4 SOP4: Labelling of Peptides-PNA Oligomers with the BAC-Fluorophore ......... 132

3.4.1.5 SOP5: Labelling of Peptide-PNA Oligomers with the NBD Fluorophore ........... 132

3.4.1.6 SOP 6 Labelling of peptides with the AlexaFluor488 Fluorophore ..................... 132

3.4.1.7 SOP 7 Cleavage of Peptides and Peptide-PNA Oligomers from the Resin......... 133

3.4.2 SOPS for the Synthesis of Modified $\mu$-Conotoxin SIIIA ……....................................... 133

3.4.2.1 SOP8: Preloading Resin with RAMAGE Linker ................................................... 133

3.4.2.2 SOP9: Coupling of the First Amino Acid ............................................................... 133

3.4.2.3 SOP10: Determination of the Occupancy ............................................................ 134

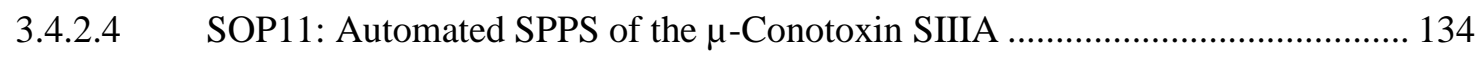

3.4.2.5 SOP 12: Cleavage of $\mu$-Conotoxin SIIIA Derivates from the Resin .................... 135

3.4.2.6 SOP 13: Synthesis of $\mu$-SIIIA: Formation of the First Disulphide Bridge .......... 135

3.4.2.7 SOP 14: Synthesis of $\mu$-SIIIA: Formation of the Second Disulphide Bridge ..... 136

3.4.2.8 SOP 15: Synthesis of Thiol or Amine Modified $\mu$-Conotoxin SIIIA................... 136

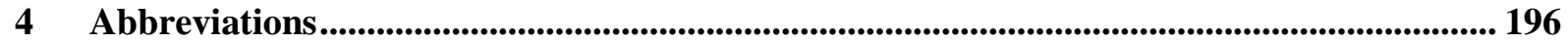

5 Bibliography............................................................................................................................... 201

6 List of Figures .............................................................................................................................................. 215

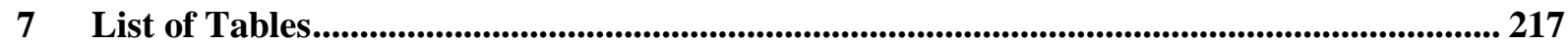

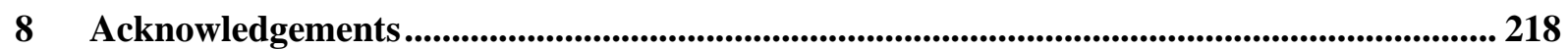

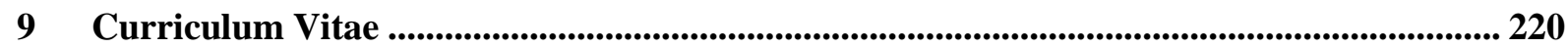





\section{Abstract}

Action potentials are an essential process for signal transmission in excitable cells. At chemical synapses, the action potential triggers the release of neurotransmitters from synaptic vesicles to transmit the signal to the target cells. After signal transmission, the neurotransmitters are refilled into synaptic vesicles. The uptake mechanism of the primary excitatory neurotransmitter glutamate is mediated by the vesicular glutamate transporter (VGLUT). ${ }^{[1,2]}$ However, the efficiency of glutamate transport by VGLUT is tightly regulated by the extravesicular and luminal $\mathrm{Cl}^{-}$concentrations. ${ }^{[3,4]}$ Despite many years of research, quantitative measurements of the $\mathrm{Cl}^{-}$conductance through VGLUT and the regulation of neurotransmitter uptake are still under discussion. For unravelling of the complex dependence between $\mathrm{Cl}^{-}$and glutamate transport, fluorescent $\mathrm{Cl}^{-}$sensors were developed, which allow measurements of the intravesicular $\mathrm{Cl}^{-}$concentration. The sensor was labelled with a $\mathrm{Cl}^{-}$sensitive $\mathrm{BAC}$ fluorophore and the insensitive AlexaFluor 647 dye. This combination of dyes allows a ratiometric measurement of the $\mathrm{Cl}^{-}$concentrations, independent of the experimental parameters. ${ }^{[5-7]}$ The BAC fluorophore, thereby, has the advantage of $\mathrm{Cl}^{-}$sensitivity within the physiological range, $\mathrm{pH}$ insensitivity as well as prolonged excitation and emission wavelengths compared to $\mathrm{Cl}^{-}$ sensitive protein reporters. The backbone of the sensor, which connects both fluorophores, is composed of a PNA-DNA scaffold (PNA = peptide nucleic acid), combining the sensing, normalising and stabilisation module. ${ }^{[5,6,8]}$ The PNA-DNA backbone was further modified with membrane anchors, for enhanced incorporation of the $\mathrm{Cl}^{-}$sensor into the luminal site of the liposome. Modification with a protease cleavage sequence or affinity tag allows the specific removal of extravesicular orientated sensors from the liposome. For VGLUT dependent measurements of $\mathrm{Cl}^{-}$concentration, liposomes were co-reconstituted with a bacterial proton pump $\left(\mathrm{TF}_{0} \mathrm{~F}_{1}\right)$ and VGLUT. Therefore, incorporation of the $\mathrm{Cl}^{-}$sensor allows direct quantification and kinetic measurement of $\mathrm{Cl}^{-}$in- and efflux through VGLUT in a controllable system. 
Apart from investigations of $\mathrm{Cl}^{-}$dependence during the neurotransmitter uptake of glutamate through VGLUT, an attempt of specific blockage of voltage-gated sodium (Nav) channels was studied in chapter two of this thesis. Nav channels are key players of the initiation and propagation of action potentials. Dysfunctions of the nine different isoforms are linked to diseases of the nervous system, heart rhythm and skeletal muscles contraction. ${ }^{\left[{ }^{[10]}\right.}$ However, especially malfunctions of the Nav1.2 channel subtype are associated with neurological disorders. ${ }^{[11]}$ For further understanding of the disease development and progression, improved visualisation of the Nav1.2 channels are essential. Therefore, new fluorescently labelled inhibitors with high affinity and specificity are required. Screening studies of neurotoxins from venomous cone snails revealed the $\mu$-conotoxin SIIIA, which blocks the Nav1.2 channel with nanomolar affinity and the skeletal Nav1.4 channel with submicromolar affinity. ${ }^{[12-14]}$ The synthesis and challenging folding of the $\mu$-conotoxin SIIIA could be improved by using a regioselective folding strategy with orthogonal thiol-protecting groups. After the successful formation of the native $\mu$-SIIIA, the molecule was labelled with an AlexaFluor 488 fluorophore enabling channel visualisation. For activity measurements with the $\mu$-conotoxin SIIIA, a caging approach was developed, which precisely allows Nav channel inhibition upon photocleavage of the caging group. 


\section{Synthesis of Chloride-Sensors to Investigate Neurotransmitter filling in Synaptic Vesicles}

\subsection{Introduction - Synaptic Vesicle}

The communication between neurons and their target cells occurs at the synapse and is regulated by action potentials. In a synapse, an incoming action potential results in the release of neurotransmitters from synaptic vesicles to transmit the signal to the target cell. Synaptic vesicles (SV) are lipid bilayer compartments with a small size $40 \mathrm{~nm}$ diameter, which are restricted to the uptake, storage and release of neurotransmitters. ${ }^{[1,2,15]}$

The neurotransmitter release process, induced by an action potential, is called exocytosis and proceeds in three distinct steps: docking, priming and fusion. In the docking step, the SV is recruited to the active zone (see figure 1.1). This zone is a specialised region close to the presynaptic membrane characterised by an accumulation of cytoskeleton-associated proteins. ${ }^{[1,16,17]}$ The docking process is mediated by the soluble $N$-ethylmaleimide-sensitivefactor attachment receptors (SNAREs) proteins, ensuring the fusion of synaptic vesicles with the presynaptic membrane. Next, the synaptic vesicles must be converted into a ready-to-fuse state. All docked and fused synaptic vesicles belong to the readily releasable pool (RRP), allowing immediate vesicle fusion upon electrical stimulation. ${ }^{[18-20]}$

The incoming action potential depolarises the synapse, resulting in the activation of voltagegated $\mathrm{Ca}^{2+}$ channels. The $\mathrm{Ca}^{2+}$ influx triggers the fusion of synaptic vesicles with the presynaptic nerve terminal, releasing neurotransmitters into the synaptic cleft. These neurotransmitters activate the postsynaptic receptors and therefore induce an ion influx in the postsynaptic membrane, which generates a new action potential. ${ }^{[1,21]}$ After fusion, the synaptic vesicles are endocytosed, recycled by clathrin-coated proteins and refilled with neurotransmitters for a new round of exocytosis. ${ }^{[1,20]}$ 


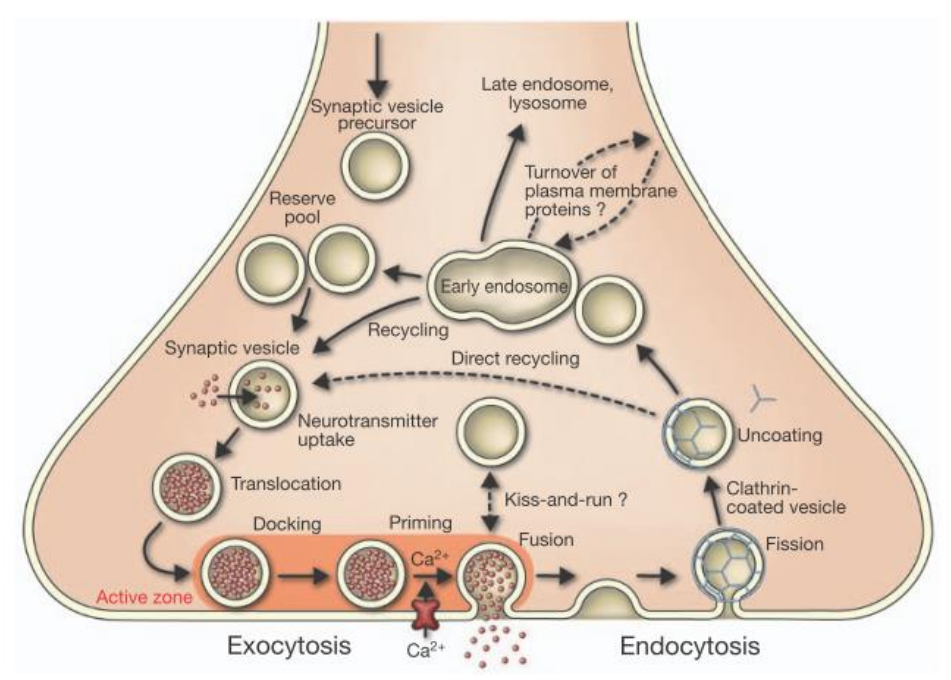

Figure 1.1: Cycling of Neurotransmitters and Synaptic vesicles. Neurotransmitter filled synaptic vesicles are transported to the active zone in the nerve terminal. After docking and priming, the neurotransmitters are released into the synaptic cleft, triggered by $\mathrm{Ca}^{2+}$ influx. The synaptic vesicles are recycled by endocytosis and refilled with neurotransmitters. Reprinted with permission from R. JAHN et al., Nature 2012, 490, 201-207.

Vesicular neurotransmitter transporters regulate the refilling of neurotransmitters in endocytosed synaptic vesicles. All vesicular transporters are secondary active transporters that require an electrochemical gradient $\left(\Delta \mu \mathrm{H}^{+}\right)$, which is generated by a vacuolar-type $\mathrm{H}^{+}$-ATPase (V-ATPase) in the membrane of the SVs. The V-ATPase hydrolyses cytosolic ATP and pumps the corresponding $\mathrm{H}^{+}$into the vesicle lumen, building a $\mathrm{pH}$ gradient $(\Delta \mathrm{pH})$ as well as a positive membrane potential $(\Delta \Psi)$ across the vesicle membrane. The $\mathrm{pH}$ gradient, as well as $\Delta \Psi$, contribute to the electrochemical gradient $\left(\Delta \mu \mathrm{H}^{+}\right)$which can be calculated by the equation: ${ }^{[22-24]}$

$$
\Delta \mu \mathrm{H}^{+}=\Delta \Psi+(2.3 R T / z F) \log \left(\left[\mathrm{H}^{+}\right]_{\text {in }} /\left[\mathrm{H}^{+}\right]_{\text {out }}\right)
$$

$R=$ gas constant,$T=$ temperature, $z=$ ion valence, $F=$ Faraday constant

\subsubsection{Vesicular Neurotransmitter Transporters}

So far, four different vesicular neurotransmitter transporters have been discovered and can be classified depending on the neurotransmitters they transport. ${ }^{[25]}$ The first identified group were vesicular monoamine transporters (VMATs), which transport monoamines like dopamine, serotonin, noradrenaline or adrenaline. VMATs exhibit a submicromolar affinity for their substrates due to the low cytosolic concentration of monoamines. ${ }^{[26,27]}$ Uptake of monoamine in SVs is driven by an antiport mechanism, exchanging two luminal $\mathrm{H}^{+}$for one positively charged monoamine. This transport mechanism mainly depends on the $\Delta \mathrm{pH}$ component of 
$\Delta \mu \mathrm{H}^{+}{ }^{[28,29]}$ The vesicular acetylcholine transporter (VAchT) is closely related to the VMATs, showing a similar transport mechanism by exchanging luminal protons for one positively charged acetylcholine. ${ }^{[28,30]}$

The third group is the vesicular inhibitory amino acid transporter (VIAAT or VGAT) responsible for the uptake of $\gamma$-aminobutyric acid (GABA) and glycine in the synaptic vesicle. The transport of non-charged GABA and glycine is believed to be mediated by an $\mathrm{H}^{+}$-antiport mechanism requiring both components of $\Delta \mu \mathrm{H}^{+}{ }^{[31,32]}$

The transport of the primary excitatory neurotransmitter glutamate is mediated by the vesicular glutamate transporter (VGLUT). The main driving force for the negatively charged glutamate uptake is the membrane potential $\Delta \Psi$ (see figure 1.2).$^{[33]}$ The transport of glutamate is highly complex and was extensively investigated over the last years. ${ }^{[34,35]}$

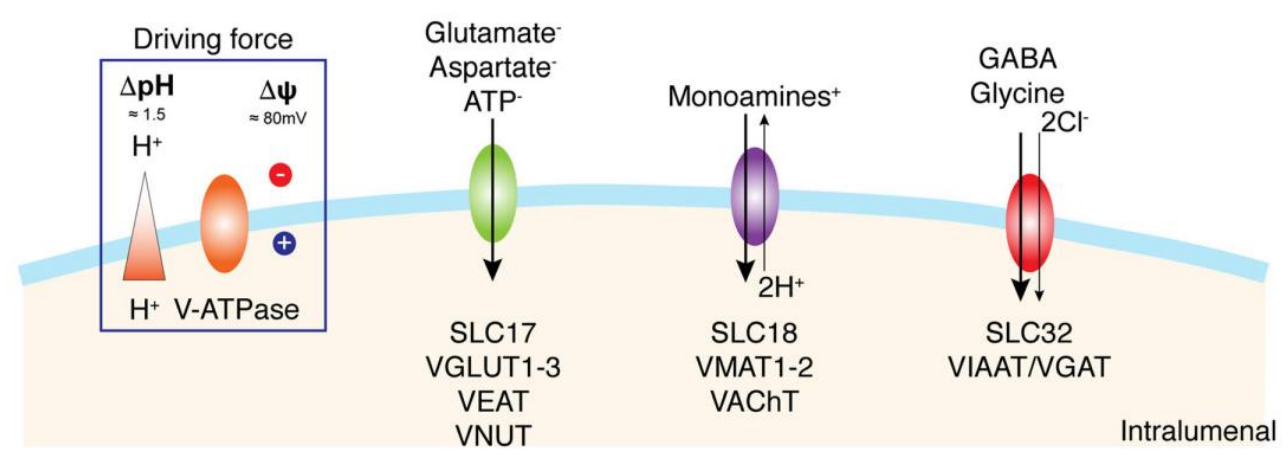

Figure 1.2: Proton-dependent neurotransmitter transport into vesicles. Refilling and storing of different neurotransmitters into synaptic vesicles, driven by the $\Delta \mu \mathrm{H}^{+}$electrochemical gradient generated by the V-ATPase. Reprinted with permission from N. PIETRANCOSTA et al., Mol. Neurobiol. 2020, 57, 3118-3142.

\subsubsection{The Vesicular Glutamate Transporters}

The requirement of VGLUT for brain function and their involvement in different neurological pathologies was demonstrated in various mice studies. ${ }^{[35-38]}$ Analysis of heterozygous VGLUT knock-out mice revealed not only issues in the sensory processing, learning as well as the memory, they also suffered from neuropathic pain and anxiety. ${ }^{[39]}$ Furthermore, psychiatric and neurological disorders like depression, Alzheimer's disease, epilepsy or schizophrenia can be traced back to an imbalance in glutamatergic neurotransmission. ${ }^{[40-43]}$ Most of these neurological diseases are not curable and the only available treatment option is the reduction of symptoms or delay of progression. Especially psychiatric medications are strictly controlled due to the variety of off-target effects. ${ }^{[44]}$ Consequently, a better understanding of the complex 
processes of glutamatergic transmission in synaptic vesicles is required to develop new psychoactive drugs with a higher specification and fewer side effects.

The VGLUTs are members of solute carrier transmembrane transporters (SLC17) and were first described as $\mathrm{Na}^{+}$-dependent inorganic phosphate transporter. The first experiments that showed a contribution of VGLUT in transmitting glutamate were performed in the 1980s with purified synaptic vesicles. ${ }^{[45]}$ Remarkably, VGLUT has a relatively low affinity for glutamate (1-2 mM) but an inability to transport structurally similar amino acids like glutamine or aspartate. ${ }^{[33,46]}$ Current estimations suggest a glutamate concentration between 60 and $120 \mathrm{mM}$ in synaptic vesicles. $^{[47]}$

So far, three different isoforms of the vesicular glutamate transporters (VGLUT1-3) have been described, which exhibit a high sequence homology in mammals. ${ }^{[35,48,49]}$ VGLUT1 is the most abundant isoform in the CNS and is expressed in the cerebral cortex, hippocampus and the amygdala, whereas VGLUT2 is primarily found in subcortical excitatory neurons. ${ }^{[36,50]}$ Compared to VMATs and VAchT, the expression of VGLUTs is limited to nerve endings where they constantly control the glutamate transport between plasma membrane, endosomes and synaptic vesicles. ${ }^{[51,52]}$

Surprisingly, VGLUT3 is expressed in non-glutamatergic neurons, disproving DALE's principle that neurons release only one type of neurotransmitters. These findings indicated that glutamate act as a co-transmitter in these cells. ${ }^{[50]}$ However, the consequences and mechanism of corelease for vesicle filling and neurotransmitter release are still under discussion. ${ }^{[53,54]}$

\subsubsection{Structure of Vesicular Glutamate Transporters}

For further insights into the mechanism of glutamate transport, structural informations of VGLUTs are required. Recently, LI et al. report the first cryo-electron microscopy structure of rat VGLUT2 at $3.8 \AA$ resolution, facilitated by an antigen-binding fragment (Fab) (see figure 1.3 A). ${ }^{[55]}$ Since VGLUT1 and VGLUT2 exhibit a sequence homology of $75 \%$, it is assumed that both transporters share structural similarities. ${ }^{[55]}$ Analysis of the cryo-EM structure revealed that VGLUT2 consists of 12 transmembrane helices, as predicted by modelling studies and hydropathy plots. ${ }^{[56]}$ The helices are connected by cytoplasmic loops and are organised into an $\mathrm{N}$ - and $\mathrm{C}$-domain (see figure $1.3 \mathrm{~B}$ and $1.3 \mathrm{C}$ ). ${ }^{[55]}$ 


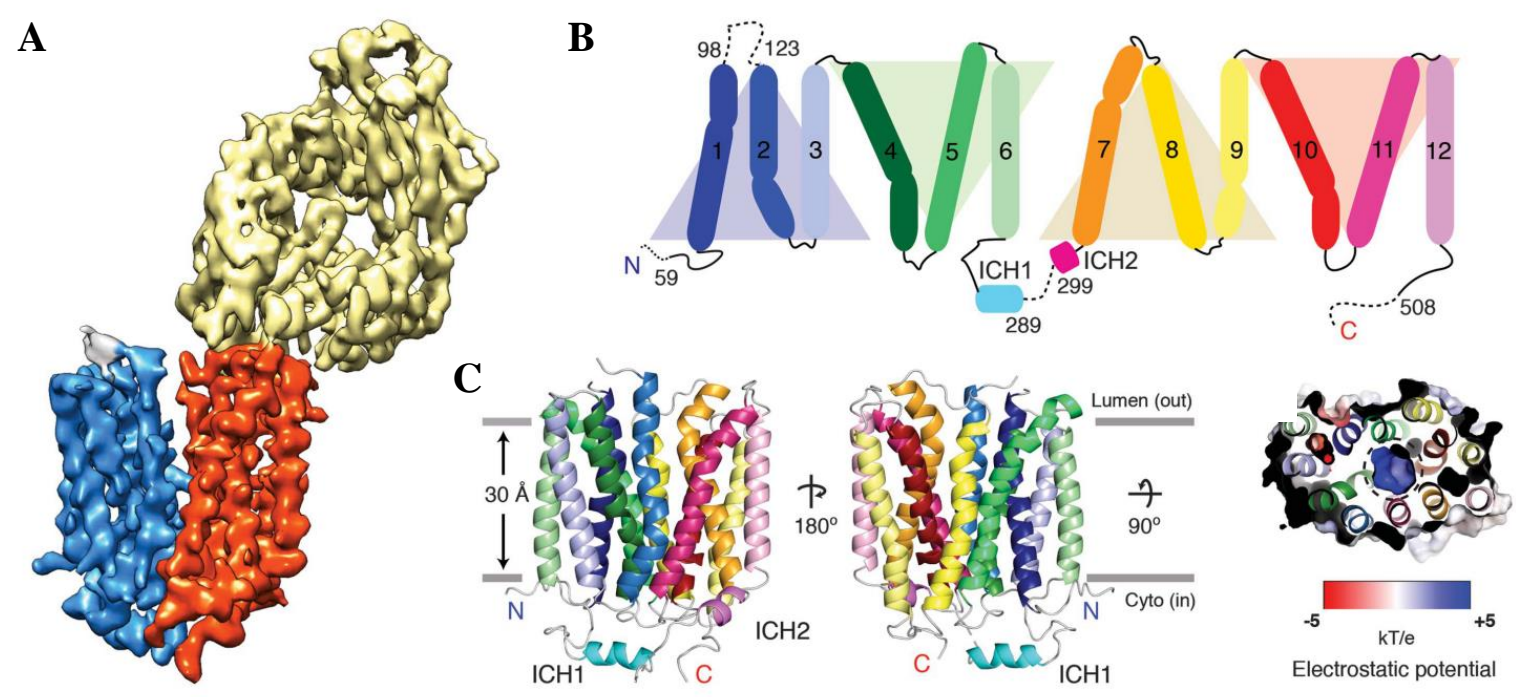

Figure 1.3: Structure of VGLUT. A) Cryo-EM structure of the VGLUT2. The N-domain is coloured in blue, the C-domain is presented in red and Fab is coloured in yellow. B) Schematic presentation of the transmembrane domains of VGLUT2. C) Structure of VGLUT2. The helices are highlighted in the colours according to the display in B. Reprinted with permission from F. LI et al., Science 2020, 368, 893-897.

The central cavity of VGLUT consists of positively charged residues ensuring the transport of the negatively charged glutamate. The glutamate-binding site is formed by the two domains consisting of the residues Arg88, H128, Glu191 (N-Domain) as well as Arg322, His487, Glu396, Cys321 (C-Domain) (Figure 1.4 A). Especially the Arg88 residue is highly conserved in transporters of the SLC17 family, forming a salt bridge with one carboxyl-group of the glutamate. The residue Glu191 and His128 are part of the N-Domain and protonation of the Glu191 residue liberates the adjacent Arg88, which subsequently can interact and transport glutamate. If no substrate is bound to the Glu191 residue, the channel is blocked by a charged pair formed with $\operatorname{Arg} 88$ (see figure $1.4 \mathrm{~B}$ and $1.4 \mathrm{C}$ ). ${ }^{[55]}$

The residue Arg322 faces Arg88 from the opposite side of the binding pocket and recognises the glutamate substrate's second carboxy group. The relevance of both arginine residues was illustrated in mutation studies, showing that R88A reduced the glutamate transport, whereas the mutation of R322A eliminates the transport. The residues of the C-Domain His487, Glu396 as well as C321 are in proximity to the Arg322 and exclusively conserved along VGLUT and not in the SLC17 family, indicating an essential role of the second carboxyl group in the binding process of glutamate (Figure $1.4 \mathrm{D}) .^{[55,57]}$ 

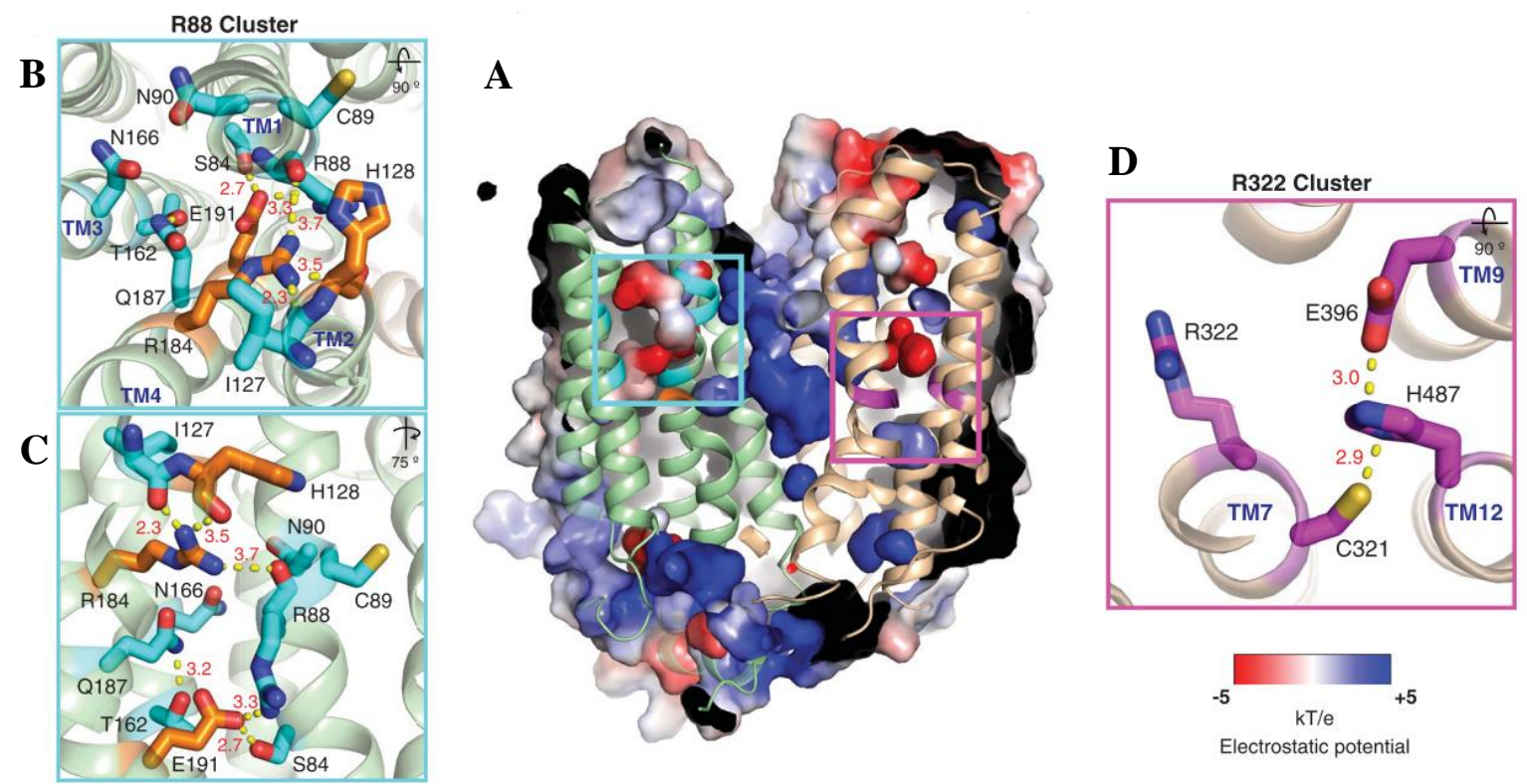

Figure 1.4: Functional residues of VGLUT. A) Electrostatic surface of VGLUT2. The central cavity consists of two domains. The $\mathrm{N}$-domain is highlighted in cyan and the $\mathrm{C}$-domain in pink. B) Top view of $\mathrm{N}$-domain, showing the essential residues and distances between the sidechains ( $\AA$ ). C) Side-view of the N-Domain. D) Top view of the C-domain. Reprinted with permission from F. Li et al., Science 2020, 368, 893-897.

\subsubsection{Transport Mechanism of VGLUT}

Over the last years, enormous efforts have been made to understand the transport mechanisms and the charge movements in the process of the neurotransmitter filling by VGLUT. The charge neutrality for each glutamate transport cycle is especially essential due to the small volume of synaptic vesicles. An imbalance of only one charge can result in a change of the membrane potential of $2 \mathrm{mV}$. Also, a single free proton in the lumen of synaptic vesicles leads to a decrease in the $\mathrm{pH}$ from 7.4 to $4 .{ }^{[3,58]}$ Consequently, the efficient loading of glutamate into synaptic vesicles requires different exit or entry pathways for ions to ensure a charge and $\mathrm{pH}$ balance.

The luminal $\mathrm{H}^{+}$is transported by the V-ATPase into the synaptic vesicle and allosterically activate VGLUT to transport glutamate. The main driving force for glutamate transport is the membrane potential $(\Delta \Psi)$, which was demonstrated by specific inhibition of the $\Delta \mathrm{pH}$ component. Measurements with the electroneutral cation antiporter nigericin, which exchanges luminal $\mathrm{H}^{+}$with extravesicular $\mathrm{K}^{+}$, showed no difference in the glutamate transport. ${ }^{[99,60]}$

During the transport, the negative charge of glutamate is balanced by the luminal $\mathrm{H}^{+}$, causing glutamic acid formation. ${ }^{[33,61]}$ After exhausting the buffering capacity of the synaptic vesicles, a cation- $\mathrm{H}^{+}$exchanger (NHE) catalyses the replacement of luminal $\mathrm{H}^{+}$for external $\mathrm{Na}^{+}$or $\mathrm{K}^{+}$ and therefore supports the glutamate uptake by decreasing $\Delta \mathrm{pH}$ and maintaining $\Delta \Psi{ }^{\left[{ }^{[62]}\right.}$ 
Remarkably, PREOBRASCHENSKI et al. and ERIKSEN et al. presented that VGLUT itself also has a $\mathrm{K}^{+} / \mathrm{H}^{+}$exchange activity, enabling a charge-neutral $\mathrm{H}^{+}$exit pathway to prevent an overacidification of the lumen by accumulating high glutamic acid concentrations. Consequently, their measurements with reconstituted liposomes showed a significant increase in the glutamate uptake in the presence of extravesicular $\mathrm{K}^{+} .^{[3,63]}$ Treatment with the VGLUT inhibitor Evans Blue reduces the glutamate uptake, indicating a blockage of the $\mathrm{K}^{+} / \mathrm{H}^{+}$exchange. ${ }^{[3]}$

Additionally, NAITO and UEDA reported that the efficiency of the glutamate uptake shows a biphasic dependency on the extravesicular $\mathrm{Cl}^{-}$concentration. A substantial increase in the glutamate uptake in synaptic vesicles was measured at low chloride concentrations (4-6 mM), whereas high extravesicular $\mathrm{Cl}^{-}$concentrations (> $20 \mathrm{mM}$ ) lead to an inhibitory effect (see figure 1.5). ${ }^{[33,50,64]}$ The inhibition of the glutamate uptake at high $\mathrm{Cl}^{-}$levels was explained by an increased influx of $\mathrm{Cl}^{-}$, causing an enhanced $\Delta \mathrm{pH}$ due to the generation of $\mathrm{HCl}$ and the dissipation of $\Delta \Psi$. The stimulating effect at low $\mathrm{Cl}^{-}$concentrations are attributed to an allosteric $\mathrm{Cl}^{-}$binding site on the cytoplasmic side of the transporter. ${ }^{[59,64-66]}$

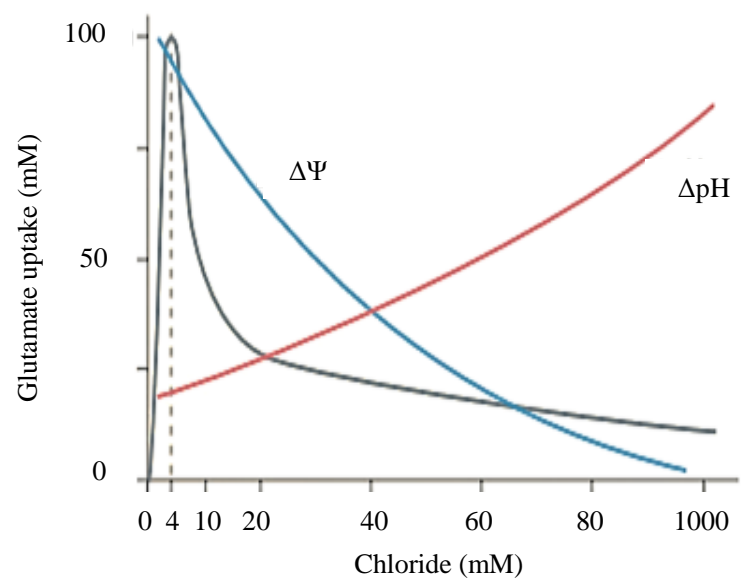

Figure 1.5: Cl- dependence of vesicular glutamate uptake. At low $\mathrm{Cl}^{-}$concentrations the glutamate uptake (black line) and $\Delta \Psi$ (blue line) are maximal whereas $\Delta \mathrm{pH}$ (red line) between the vesicular lumen and the cytoplasm is low. High extravesicular $\mathrm{Cl}^{-}$concentration increases the $\Delta \mathrm{pH}$ due to the formation of $\mathrm{HCl}$ and subsequently decreases $\Delta \Psi$ and the glutamate accumulation. Reprinted with permission from S. EL MESTIKAWY et al., Nat. Rev. Neurosci. 2011, 12, 204-216.

\subsubsection{Mediation of Chloride Transport into Synaptic Vesicles}

Since $\mathrm{Cl}^{-}$plays an essential role in the regulation of the glutamate transport, the question remained, whether $\mathrm{Cl}^{-}$conductance is mediated by chloride transporters, like ClC3 or by VGLUT itself. ${ }^{[4,67]} \mathrm{ClC} 3$ is a $\mathrm{Cl}^{-} / \mathrm{H}^{+}$exchanger with a 2:1 stoichiometry, which is localised in synaptic vesicles and along the endosomal pathway. ${ }^{[67,68]}$ In different studies, it was 
demonstrated, that $\mathrm{ClC} 3$ facilitates acidification of lysosomes and endosomes by an accumulation of luminal chloride. ${ }^{[69-72]}$ Also, the disruption of $\mathrm{ClC} 3$ in rodent models causes neurodegeneration represented by the disability of synaptic vesicle acidification. ${ }^{[67,73]}$ However, investigations of synaptic vesicles isolated from $\mathrm{ClC} 3$ knock-out mice revealed no difference in the biphasic $\mathrm{Cl}^{-}$dependence. The glutamate uptake was still increased at low $\mathrm{Cl}^{-}$ concentrations and inhibited at high $\mathrm{Cl}^{-}$concentrations. These results indicated that other proteins or transporters are involved in the chloride uptake in synaptic vesicles. ${ }^{[3,4]}$

Schenck et al. performed similar experiments with VGLUT1 knock-out mice, showing a strong reduction in the $\mathrm{Cl}^{-}$and glutamate-dependent acidification, identifying VGLUT as $\mathrm{Cl}^{-}$ transporter. ${ }^{[4]}$ These results were confirmed by measuring a $\mathrm{Cl}^{-}$induced acidification in liposomes co-reconstituted exclusively with VGLUT1 and the bacterial proton pump $\mathrm{TF}_{0} \mathrm{~F}_{1}$ ATPase (see figure 1.6 A). ${ }^{[3]}$ Furthermore, PREOBRASCHENSKI et al. fused purified synaptic vesicles with large liposomes, including the $\mathrm{TF}_{0} \mathrm{~F}_{1}$-ATPase to exclude differences in the behaviour of VGLUT in a native environment. The hybrid vesicles contained all endogenous proteins in the correct orientation. They showed chloride-dependent acidification and chloridestimulated glutamate uptake, indicating that $\mathrm{Cl}^{-}$conductance is mediated by VGLUT and not the $\mathrm{ClC} 3$ channel (see figure $1.6 \mathrm{~B}$ ). ${ }^{[3]} \mathrm{CHANG}$ et al. revealed by measuring endosome currents, that extravesicular $\mathrm{Cl}^{-}$directly competes with glutamate for permeation through VGLUT, independent of $\Delta \Psi$. Therefore, the inhibition of the glutamate uptake at high $\mathrm{Cl}^{-}$concentrations can be explained by a compeptitive binding and not exclusively by the dissipation of the driving force $\Delta \Psi \cdot{ }^{[34,74]}$
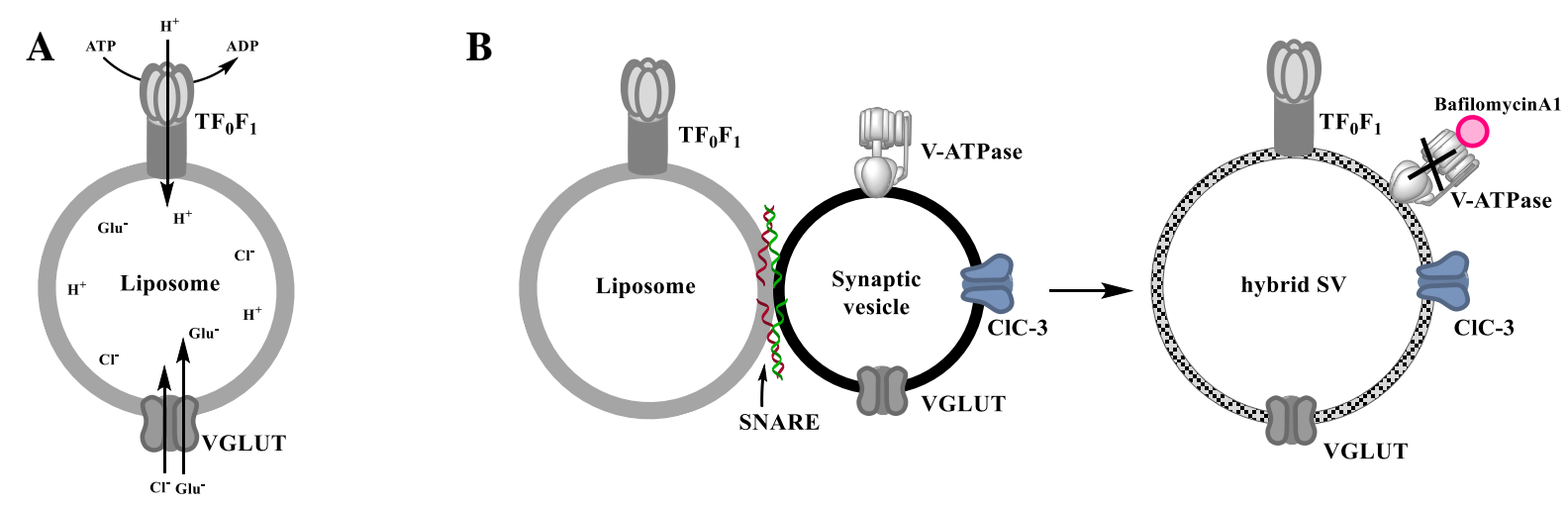

Figure 1.6: Proteoliposome model for $\mathrm{Cl}^{-}$transport mechanism. A) Reconstituted liposomes with $\mathrm{TF}_{0} \mathrm{~F}_{1}$ ATPase and VGLUT to measure $\mathrm{Cl}^{-}$transport through VGLUT during acidification. B) Formation of hybrid vesicles, by SNARE-mediated fusion of $\mathrm{TF}_{0} \mathrm{~F}_{1}$ liposomes and native synaptic vesicles. Interference with unfused synaptic vesicles was excluded by blocking the endogenous V-ATPase activity with bafilomycinA1 ${ }^{[3]}$. 


\subsubsection{Allosteric Activation of VGLUT by Luminal Chloride}

SCHENCK et al. could further discover that not only extravesicular $\mathrm{Cl}^{-}$influences the glutamate transport but also high intravesicular $\mathrm{Cl}^{-}$concentrations allosterically activate the glutamate uptake. Measurements in reconstituted liposomes, preloaded with $100 \mathrm{mM} \mathrm{KCl}$, showed a threefold increased glutamate uptake compared to the negative control. These results indicated one or several allosteric $\mathrm{Cl}^{-}$binding sides that are accessible from the luminal as well as the cytoplasmic site. ${ }^{[3,4,63]}$ These findings are in line with the assumption that synaptic vesicle are regenerated and filled with high levels of $\mathrm{Na}^{+}$and $\mathrm{Cl}^{-}(\sim 130 \mathrm{mM})$ during the process of endocytosis. ${ }^{[46]}$ The export of luminal $\mathrm{Cl}^{-}$into the cytoplasm can also drive the glutamate uptake exclusively, which was reported by MARTINEAU et al., performing live-cell imaging experiments of hippocampal neurons with a $\mathrm{pH}-$ and $\mathrm{Cl}^{-}$sensitive fluorophores. ${ }^{[47,63]}$ Additionally, CHANG et al. presented electrophysiological recordings of VGLUT-associated currents, independent of an $\mathrm{H}^{+}$pump, ion gradients or $\Delta \Psi$. Their measurements in endosomes also confirmed an allosteric activation of the glutamate transport by luminal $\mathrm{Cl}^{-} \cdot{ }^{[74]}$

For more in-depth insights into the relationship between allosteric activation and $\mathrm{Cl}^{-}$ permeation, CHANG et al. performed mutation studies, revealing that the luminal activation of the glutamate transport depends on the interaction of $\mathrm{Cl}^{-}$with a highly conserved arginine residue in transmembrane helix 4. Further, they presented that neutralisation of the arginine residue by interaction with luminal $\mathrm{Cl}^{-}$is sufficient to initiate the allosteric activation required for glutamate transport and $\mathrm{Cl}^{-}$conductance. ${ }^{[74,75]}$ The mutations studies, in which the arginine residue was replaced by an uncharged alanine, caused a permanent influx of glutamate and $\mathrm{Cl}^{-}$ even in the absence of luminal $\mathrm{Cl}^{-} .^{[74]}$

Based on these findings, EDWARDS and colleagues proposed a mechanism for the glutamate uptake within the synaptic vesicle cycle (see figure 1.7). After endocytosis, the high luminal $\mathrm{Cl}^{-}$concentration trapped in synaptic vesicles activate the glutamate transport by neutralising the positive charge of the Arg residue in TM 4. The efflux of luminal $\mathrm{Cl}^{-}$along its concentration gradient generates the membrane potential necessary for the glutamate uptake. The glutamate $/ \mathrm{Cl}^{-}$exchange mechanism allows an electroneutral filling without affecting the osmotic pressure. As the luminal $\mathrm{Cl}^{-}$concentration decreases, the glutamate uptake is driven by the membrane potential generated by the V-ATPase pumping $\mathrm{H}^{+}$in the vesicle lumen. The glutamate filling stops when the membrane potential dissipates and the luminal $\mathrm{pH}$ decreases, resulting in an inactivation of VGLUT. This process prevents the leakage of glutamate from filled vesicles. Finally, the filled synaptic vesicles release the glutamate in the synaptic cleft during the process of exocytosis. ${ }^{[47,55,74,75]}$ 


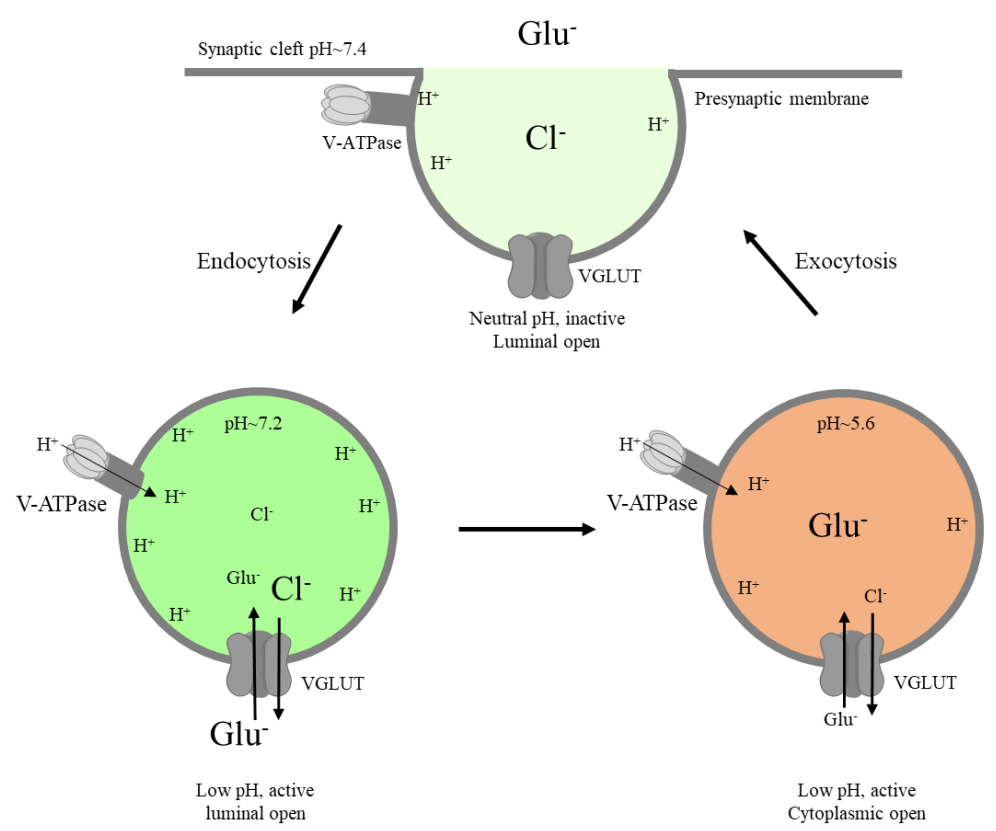

Figure 1.7: Proposed mechanism of glutamate uptake in the synaptic vesicle. Top: Glutamate is released into the synaptic cleft. Left: After endocytosis, the synaptic vesicles contain high luminal $\mathrm{Cl}^{-}$concentrations and their efflux promotes the glutamate uptake by VGLUT. The glutamate influx is also driven by the $\Delta \Psi$ generated by V-ATPase, pumping $\mathrm{H}^{+}$in the vesicle. The entry of glutamate causes a decrease in the luminal $\mathrm{pH}$. Right: In the late stage of the cycle, the acidified synaptic vesicles are filled with glutamate and the efflux of $\mathrm{Cl}^{-}$maintains $\Delta \Psi \cdot{ }^{[55,75]}$

\subsubsection{VGLUT Model for Ion Transport across Vesicular Membranes}

The fundamental role of VGLUT for glutamate uptake was discovered in the recent years, showing that VGLUT not only transports glutamate into the synaptic vesicles, it also controls the transport of $\mathrm{Cl}^{-}$and functions as $\mathrm{K}^{+} / \mathrm{H}^{+}$antiporter. These different binding modes allow VGLUT to control ionic- or charge changes tightly and to optimise the uptake of glutamate into synaptic vesicles. ${ }^{[3,74]}$ PREOBRASCHENSKI et al. developed a detailed model of the VGLUT transport mechanism, assuming that VGLUT consists of two anionic and at least one cationic binding site. VGLUT can also switch between two conformational states, in which it is either open to the cytoplasmic (state I) or luminal side (state II) (see figure 1.8). ${ }^{[3]}$ 


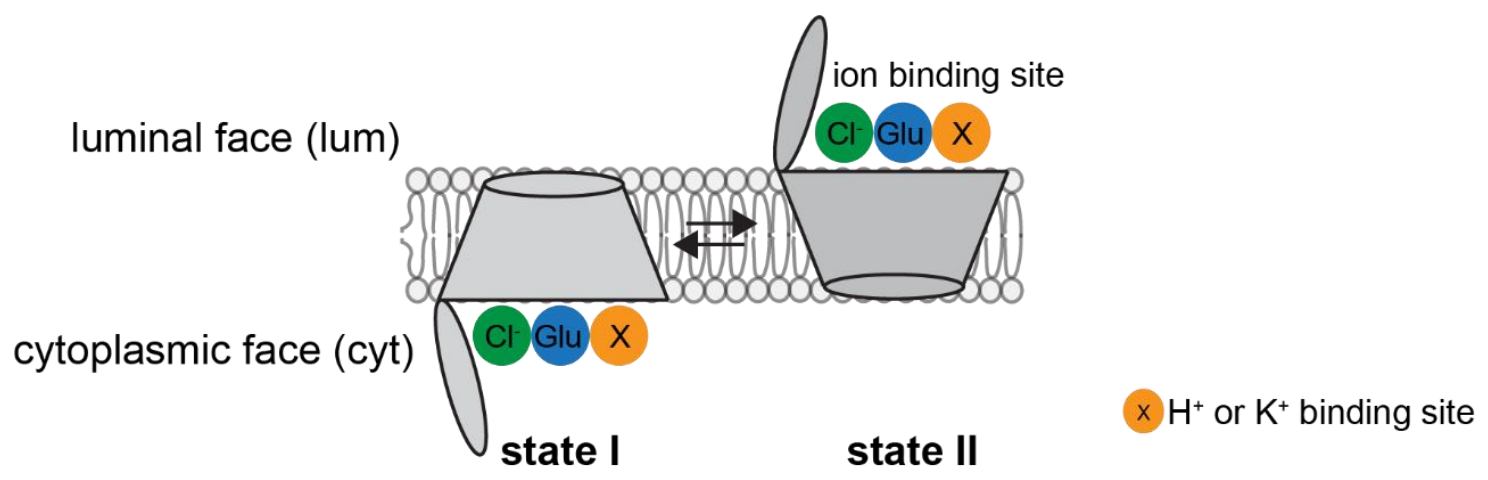

Figure 1.8: Model of the VGLUT transport mechanism. VGLUT has two anionic and one cationic binding site and can be open to either the cytoplasmic (state I) or luminal side (state II). ${ }^{[3]}$

When VGLUT is open to the cytoplasmic side, one of the anionic binding sites specifically binds $\mathrm{Cl}^{-}$with a millimolar affinity (see figure 1.9). The high millimolar $\mathrm{Cl}^{-}$concentration, which is present under physiological conditions, leads to a constantly occupied binding position. Thus, no net $\mathrm{Cl}^{-}$transport occurs independently of the conformational state of the transporter. When the transporter is open to the cytoplasmic side, glutamate can bind with a higher affinity to the second anionic binding site compared to $\mathrm{Cl}^{-}$. The binding of glutamate can already trigger a conformational change of VGLUT, opening the binding pocket to the luminal side, where glutamate dissociates. When VGLUT is open to the luminal side (state II), the glutamate binding site is occupied by $\mathrm{Cl}^{-}$, due to much higher luminal $\mathrm{Cl}^{-}$concentration compared to glutamate. Consequently, the conformational switch from state II to state I results in the efflux of $\mathrm{Cl}^{-}$from the synaptic vesicle. The conformational change of VGLUT in both directions is accelerated when the $\mathrm{Cl}^{-}$binding pocket is also occupied, indicating that $\mathrm{Cl}^{-}$ binding lowers the activation energy for the conformational switch. The glutamate uptake is maximal when chloride is present on the luminal as well as the cytoplasmic side. ${ }^{[3,74]}$

In the late stages of the synaptic vesicle cycle, when the luminal $\mathrm{Cl}^{-}$concentration is strongly reduced, the accumulation of glutamate in the vesicle causes an acidification of the lumen. For an efficient glutamate loading in the vesicle even at low intravesicular $\mathrm{pH}(\sim 5.5)$, an $\mathrm{H}^{+}$efflux pathway is required. This exit strategy is provided by the $\mathrm{K}^{+} / \mathrm{H}^{+}$antiporter function of VGLUT, which transports $\mathrm{H}^{+}$from the luminal to the cytoplasmic site, whereas $\mathrm{K}^{+}$is transported in the reverse direction. This $\mathrm{K}^{+} / \mathrm{H}^{+}$exchange mediates the glutamate uptake by maintaining $\Delta \Psi$ and decreasing $\Delta \mathrm{pH}$. This model shows that VGLUT exhibit $\mathrm{Cl}^{-}$and $\mathrm{H}^{+}$binding sites, which are not tightly coupled to the glutamate transport. This allows adjustments of the changing ionic concentrations during the transport mechanism resulting in an efficient glutamate loading. ${ }^{[3,44,62]}$ 


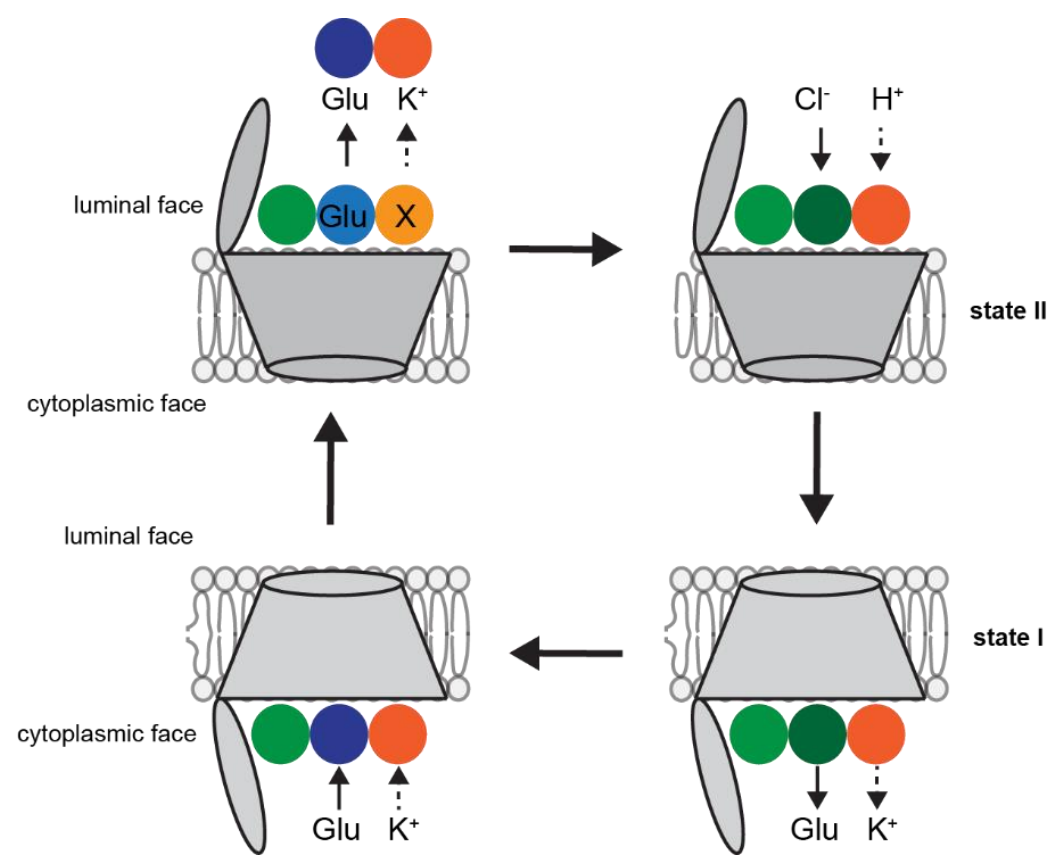

Figure 1.9: Elevated model of the VGLUT transport mechanism. When VGLUT is open to the cytoplasmic site (state I), it is loaded with glutamate, $\mathrm{Cl}^{-}$and $\mathrm{K}^{+}$. After the conformational change glutamate and $\mathrm{K}^{+}$are dissociated into the lumen (state II). Next, the glutamate binding site is loaded with $\mathrm{Cl}^{-}$and the cationic site binds an $\mathrm{H}^{+}$, which are transported to the cytoplasm. ${ }^{[3]}$

\subsubsection{Development of Fluorescent Chloride Sensor Molecules}

The physiological importance of $\mathrm{Cl}^{-}$is not only limited to the process of glutamate uptake into synaptic vesicles, but it is also involved in numerous cellular functions like regulation of cell volume and charge balance as well as stabilisation of the resting membrane potential. ${ }^{[76,77]}$ However, mutations in $\mathrm{Cl}^{-}$channels or transporters can lead to an imbalance in the chloride homeostasis, which is associated with the occurrence of several diseases like cystic fibrosis, lysosomal storage disease, osteoporosis or epilepsy. ${ }^{[78,79]}$ For further understanding of these disease progression, more profound insights into the $\mathrm{Cl}^{-}$channel activity are essential. Consequently, there is a great need for measuring intracellular $\mathrm{Cl}^{-}$concentrations to characterise channel activity and cellular processes further. This physiological studies can be addressed by using $\mathrm{Cl}^{-}$sensor molecules. ${ }^{[8]}$

The first measurements of intracellular chloride concentrations were presented by NEILD and THOMAS in 1974, by using a chloride-sensitive microelectrode to measure currents in giant neurons of the snail Helix aspersa. However, this method revealed major drawbacks regarding the restriction to large cells, the time-consuming preparation and the limited selectivity for chloride. ${ }^{[80]}$ Also, radioactive tracers showed only insufficient sensitivity and limited time resolution. ${ }^{[1]}$ Sensor molecules based on optical methods were developed, providing the 
advantage of spatially and temporally resolved measurements of the $\mathrm{Cl}^{-}$concentration. Over the last years, an enormous variety of fluorescent $\mathrm{Cl}^{-}$-sensors have been developed, which can be categorised into two groups: $\mathrm{Cl}^{-}$-sensitive fluorescent proteins and $\mathrm{Cl}^{-}$-sensitive small molecule dyes. $^{[8,82]}$

\subsubsection{Chloride Sensitive Protein Reporters}

Generally, protein-based sensors provide the advantage of subcellular targeting and cellular retention compared to small molecule sensors. One of the first $\mathrm{Cl}^{-}$sensitive fluorescent proteins was presented by WACHTER and REMINGTON showing that the fluorescence of the yellow fluorescent protein (YFP) is quenched in the presence of halide or nitrate ions $\left(K_{\mathrm{D}}=777 \mathrm{mM}\right) .{ }^{[83]}$ YFP is a homolog of the green fluorescent protein (GFP), containing the S65G, V68L, S72A and T203Y mutations. Recognising the opportunity to transform YFP into $\mathrm{a} \mathrm{Cl}^{-}$sensor for intracellular measurements, several mutation studies were performed to increase the $\mathrm{Cl}^{-}$affinity and brightness. Especially, the mutant YFP-H148Q-I152L revealed an improved $K_{\mathrm{D}}$ for $\mathrm{Cl}^{-}$from $777 \mathrm{mM}$ to $88 \mathrm{mM}$ as well as an accelerated response. ${ }^{[84,85]}$

However, the YFP sensors exhibit a pH-dependence under physiological conditions, which strongly affects the $\mathrm{Cl}^{-}$sensitivity (at pH $6.0 K_{\mathrm{D}}=32 \mathrm{mM}$, pH $7.5 K_{\mathrm{D}}=777 \mathrm{mM}$ ), limiting the in vitro or in vivo application. Furthermore, YFP provides only a single optical signal which is influenced by the local probe concentration, optical path length, light scattering, illumination intensity or photobleaching. ${ }^{[82]}$ Consequently, quantitative measurements of the $\mathrm{Cl}^{-}$ concentration with YFP are highly laborious and imprecise. To overcome these downsides, a ratiometric sensor was developed, combining YFP with a second $\mathrm{Cl}^{-}$insensitive fluorophore, enabling the normalisation of the YFP distribution. Based on the emission ratio of both fluorophores, the intracellular $\mathrm{Cl}^{-}$concentration and changes in the local environment can be precisely calculated. ${ }^{[8,82]}$

One of the first ratiometric YFP-based chloride sensors was CL-SENSOR, which consists of the previously described YFP mutant and the chloride-insensitive cyan fluorescent protein (CFP) connected by a flexible peptide linker (see figure 1.10). ${ }^{[86]}$ Due to the overlap between the CFP emission $\left(\lambda_{\mathrm{Em}}=485 \mathrm{~nm}\right)$ and YFP excitation spectrum, the fluorophores form a FöRSTER resonance energy transfer (FRET) pair. High $\mathrm{Cl}^{-}$concentrations quench the emission of the YFP fluorophore without affecting the $\mathrm{Cl}^{-}$insensitive CFP fluorophore. Recordings of the emission ratio of YFP to CFP provides an absolute measurement of the $\mathrm{Cl}$ - concentration theoretically. Compared to other ratiometric reporters, CL-SENSOR exhibit a high $\mathrm{Cl}^{-}$affinity $\left(K_{\mathrm{D}}=30 \mathrm{mM}\right)$ 
and can be applied to monitor $\mathrm{Cl}^{-}$concentrations under physiological conditions (3-60 mM). ${ }^{[86,87]}$ However, the CL-SENSOR exhibit the crucial disadvantage of $\mathrm{pH}$ sensitivity, which affects the $\mathrm{Cl}^{-}$affinity. The FRET readout is thereby distorted, resulting in imprecise chloride measurements. ${ }^{[86]}$
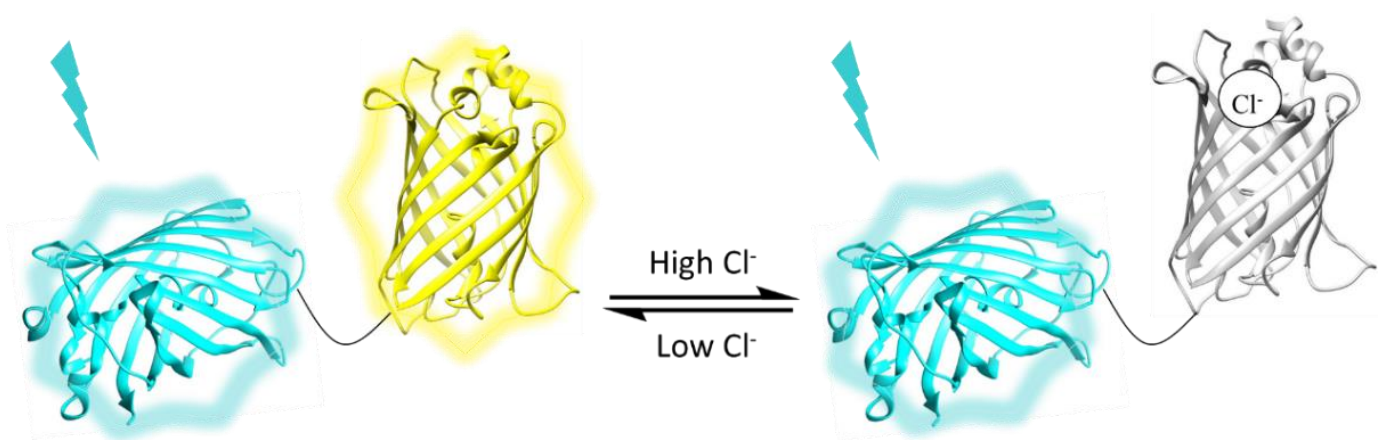

Figure 1.10: Schematic representation of CL-SENSOR. The $\mathrm{Cl}^{-}$insensitive $\mathrm{CFP}$ (cyan) and the $\mathrm{Cl}^{-}$sensitive YFP (yellow) mutant are forming a FRET pair. ${ }^{[86]}$

To overcome these limitations, AROSIO et al. developed a non-FRET based sensor, called ClopHensor, which allows simultaneous measurements of the intracellular $\mathrm{pH}$ and $\mathrm{Cl}^{-}$ concentration. ${ }^{[88]} \mathrm{A}$ specific halogen-binding site was introduced into the $\mathrm{pH}$-sensor protein $E^{2}$ GFP by a single substitution mutation T203Y. ${ }^{[89]}$ To perform ratiometric measurements, the $E^{2} \mathrm{GFP}$ is fused with the normalising red fluorescent protein (DsRed), which is insensitive to variations of $\mathrm{Cl}^{-}$as well as $\mathrm{H}^{+}$concentration (see figure 1.11). The $\mathrm{pH}$ and $\mathrm{Cl}^{-}$concentration can be calculated by measuring the ratio of green-to-cyan fluorescence of $\mathrm{E}^{2} \mathrm{GFP}$, because $\mathrm{Cl}^{-}$ quenches both cyan and green fluorescence, whereas $\mathrm{H}^{+}$exclusively quenches the green fluorescence of $\mathrm{E}^{2} \mathrm{GFP}{ }^{[88,90]}$
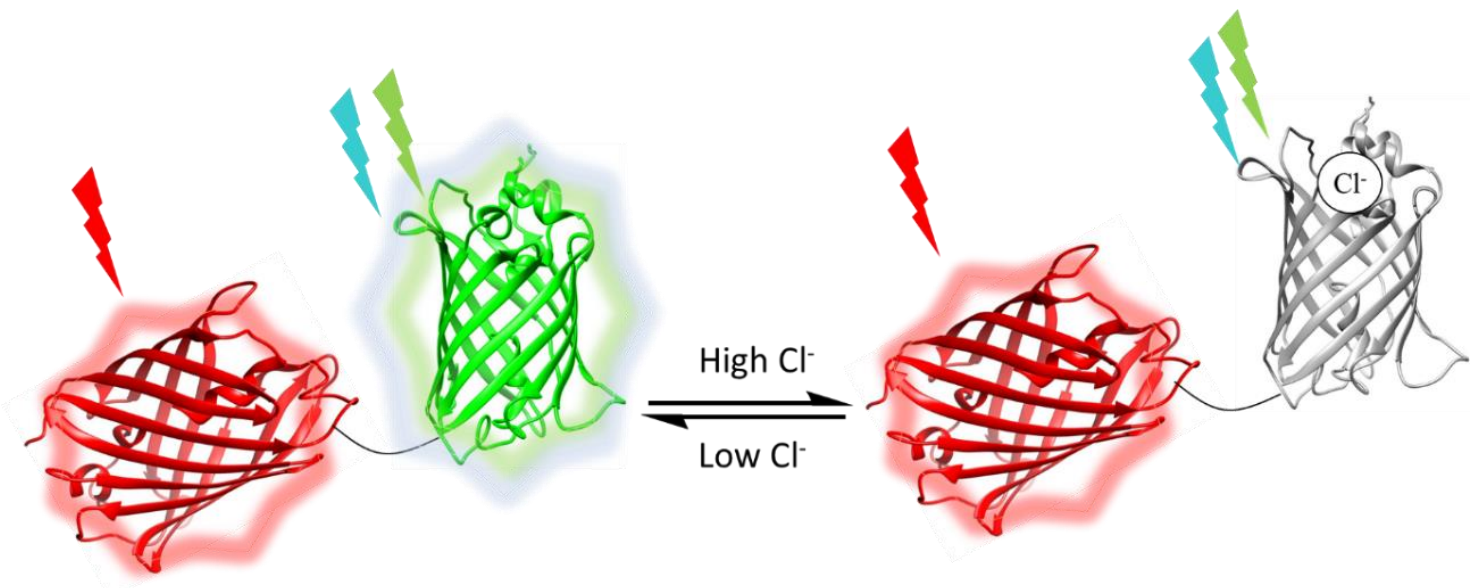

Figure 1.11: Schematic presentation of the CLOPHENSOR. The sensor consists of the $\mathrm{Cl}^{-}$and $\mathrm{pH}$-sensitive $\mathrm{E}^{2} \mathrm{GFP}$ and the normalising DsRed protein. Binding of $\mathrm{Cl}^{-}$to the $\mathrm{E}^{2} \mathrm{GFP}$ quenches the fluorescence. ${ }^{[88]}$ 
Besides all advantages, ClopHensor still exhibits some limitations regarding their complex analysis, low photostability, aggregation of the DsRed fluorophore and they are not applicable to all physiological chloride concentrations. ${ }^{[5,8]}$ Since all protein-based $\mathrm{Cl}^{-}$sensors do not fulfil the criteria required for precise intracellular $\mathrm{Cl}^{-}$measurements, $\mathrm{Cl}^{-}$sensitive small molecule dyes were extensively investigated. This group of $\mathrm{Cl}^{-}$sensors has the major advantage of $\mathrm{pH}$ insensitivity and $\mathrm{Cl}^{-}$sensitivity across the entire physiological range $(3-60 \mathrm{mM}) .{ }^{[82,91,92]}$

\subsubsection{Small Molecule Chloride Sensors}

The idea of measuring intracellular chloride concentration with small fluorescent molecules was inspired by the success of $\mathrm{pH}-$ and $\mathrm{Ca}^{2+}$ sensors, providing a sensitive and non-invasive method. ${ }^{[93,94]}$ For the design of fluorescent indicators used for specialised biological applications, the sensors must fulfil different criteria. The fluorescence of the $\mathrm{Cl}^{-}$sensitive indicator must be sensitive to changes in the physiological range of $\mathrm{Cl}^{-}(3-60 \mathrm{mM})$. Furthermore, they need to respond selectively to changes in the $\mathrm{Cl}^{-}$concentration, be nontoxic, insensitive to $\mathrm{pH}$-changes and cell impermeable. Also, high excitation and emission wavelengths ( $>400 \mathrm{~nm}$ ), as well as a high quantum yield of the sensor, are required to minimise phototoxicity and autofluorescence from UV excitation. ${ }^{[8,95,96]}$

Based on the mentioned criteria, VERKMAN and colleagues synthesised the first generation of chloride-sensitive dyes 6-methoxy- $N$-(3-sulfopropyl)quinolinium $\quad(S P Q, \quad 1), \quad N$ (ethoxycarbonylmethyl)-6-methoxyquninolinium bromide (MQAE, 2) and 6-methoxy- $N$ ethylquinolinium iodide (MEQ, 3) as well as the second generation 10,10'-bis[3carboxypropyl]-9,9’biacridinium dinitrate $(\mathrm{BAC}, \mathbf{4})$ (see figure 1.12 and table 1.1). ${ }^{[95,97-99]}$ 
A

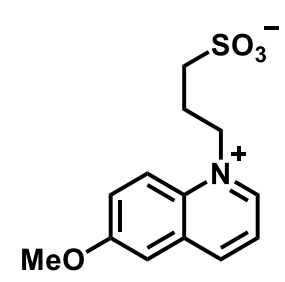

B

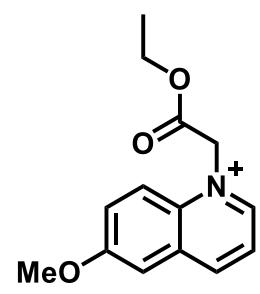

MQAE, 2
$\mathbf{C}$<smiles>CC[n+]1cccc2cc(OC)ccc21</smiles>

D<smiles>O=C(O)CCC[n+]1cc2[n+](CCCC(=O)O)c3ccccc3c-2c(-c2c3ccccc3[n+](CCCC(=O)O)c3ccccc23)c2ccccc21</smiles>

BAC, 4

Figure 1.12: Examples for the first-generation $\mathrm{Cl}^{-}$-sensitive small molecules. A) SPQ (1), B) MQAE (2), C) MEQ (3) and D) the second-generation $\mathrm{Cl}^{-}$sensor BAC (4). ${ }^{[7,95]}$

Table 1.1: Overview of the fluorescent $\mathrm{Cl}^{-}$-sensitive small molecule dyes. SPQ, MQAE, MEQ and BAC presenting their absorption and emission maxima, extinction coefficient as well as $K_{\mathrm{SV} .}{ }^{[7,95,98,99]}$

\begin{tabular}{cccccc}
\hline Name & Generation & Abs $[\mathbf{n m}]$ & $\mathbf{E m ~}[\mathbf{n m}]$ & $\boldsymbol{\varepsilon}\left[\mathbf{M}^{\mathbf{- 1}} \cdot \mathbf{c m}^{-\mathbf{1}}\right]$ & $\boldsymbol{K}_{\mathbf{S v}}\left[\mathbf{M}^{\mathbf{- 1}}\right]$ \\
\hline SPQ (1) & 1 & 344 & 443 & 3700 & 12 \\
MQAE (2) & 1 & 350 & 460 & 2800 & 25 \\
MEQ (3) & 1 & 344 & 442 & 3900 & 19 \\
BAC (4) & 2 & 434 & 505 & 12500 & 56
\end{tabular}

All these sensors undergo dynamic collisional quenching which occurs when the excited sensor molecule collides with $\mathrm{Cl}^{-}$and returns to the ground state through a non-radiative transition. The resulting decrease in the fluorescence intensity and lifetime is directly related to the chloride concentration, which can be calculated by the STERN-VOLLMER equation (1.2). Thereby, the STERN-VOLLMER quenching constant $\left(K_{\mathrm{SV}}\right)$ describes the $\mathrm{Cl}^{-}$concentration that quenches half of the fluorescence and is a crucial parameter to define the optimal working range for each dye. ${ }^{[82,91]}$

$$
\mathrm{F}([\mathrm{Cl}])=\frac{\mathrm{F}_{0}}{1+K_{\mathrm{SV}} \cdot[\mathrm{Cl}]}
$$

$\mathrm{F}_{0}=$ fluorescence in the absence of $\mathrm{Cl}^{-} ; \mathrm{F}=$ fluorescence in the presence of $\mathrm{Cl}^{-} ; \mathrm{K}_{\mathrm{SV}}=$ STERN-VOLLMER quenching constant.

The first generation sensors, SPQ (1), MQAQ (2) and MEQ (3) have been used for measurements of the $\mathrm{Cl}^{-}$concentrations in neurons, glia, epithelial cells or fibroblasts. ${ }^{[100-102]}$ 
However, the small molecule $\mathrm{Cl}^{-}$sensors revealed some critical limitations regarding photobleaching, cell impermeability, self-quenching, UV-excitation and especially the absence of ratiometry. Due to the lack of a second emission wavelength, the fluorescence readout depends on the sensor uptake, cellular distribution and optical thickness. Therefore, they cannot provide an accurate measurement of the chloride concentration. ${ }^{[8]}$

\subsubsection{Second Generation Sensor - Development of Ratiometric Quantification}

To overcome all these limitations, VERKMAN and colleagues developed a ratiometric sensor based on the second-generation $\mathrm{Cl}^{-}$sensitive dye BAC (4). ${ }^{[7]}$ Compared to the previously described quinolinium based sensors, the BAC fluorophore (4) exhibits significant advantages and fulfils all criteria of a fluorescent indicator for biological applications. Especially the increased chromophore size by the tricyclic acridine structure shifts the excitation and emission spectra of BAC to longer wavelengths, reducing phototoxicity. Furthermore, BAC (4) also revealed a high chloride sensitivity in the physiological range (3-60 mM), a pH insensitivity, intracellular stability and membrane impermeability. ${ }^{[7]}$ Similar to the quinolinium sensor, BAC (4) is also collisionally quenched by $\mathrm{Cl}^{-}$or other halides. Due to the low concentration of other halides in living systems, this influence can be neglected. ${ }^{[103-105]}$

For ratiometric measurements, the green fluorescent BAC fluorophore (4) was conjugated to the chloride insensitive red fluorescent tetramethylrhodamine (TMR), connected by an amino dextran linker (40 kD) (see figure 1.13). With the ratiometric BAC-TMR-dextran sensor (5), SONAWANE et al. measured the dependence between chloride accumulation and acidification in endosomes and Chinese hamster ovary cells. ${ }^{[7]}$ However, the BAC-TMR-construct (5) varies from batch-to-batch when used for intracellular measurements, as the stoichiometry of the BAC and TMR binding to the dextran scaffold cannot be controlled. This leads to inconsistent measurements due to the unequal loading densities of both fluorophores. Furthermore, direct conjugation of the BAC fluorophore to proteins, lead to a reduced fluorescence by $90 \%$, despite the introduction of long spacers. ${ }^{[8,106]}$ 


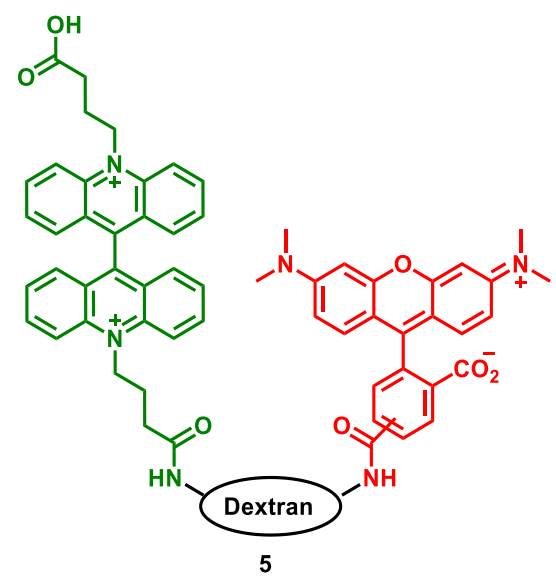

Figure 1.13: Structure of a ratiometric $\mathrm{Cl}^{-}$sensor (5). The chloride sensitive BAC and TMR fluorophores are conjugated to a dextran scaffold. ${ }^{[7]}$

\subsubsection{Chloride Sensor conjugated to a DNA Scaffold}

Interestingly, KRISHNAN and colleagues presented an approach, which combined two fluorophores in a precise 1:1 stoichiometry, enabling them to target organelles or cellular pathways. ${ }^{[107,108]}$ The fluorophores were conjugated to a DNA scaffold, which provides the advantage of a modular structure, making the scaffold targetable, programmable as well as biocompatible. The first application of a fluorophore labelled DNA nanodevice was a $\mathrm{pH}$ sensor, called I-switch, used to investigate cellular processes. The sensor was labelled at the 3' and 5 ' position with the fluorophores AlexaFluor 488 and AlexaFluor 647, which can form a FRET pair after conformational changes caused by protonation of the cytosine-rich region in the DNA scaffold (see figure 1.14). Due to a specific double-strand (ds) DNA sequence d(AT) ${ }_{4}$, the I-switch can target to the lumen of endocytic organelles in Drosophila haemocytes. ${ }^{[107-109]}$

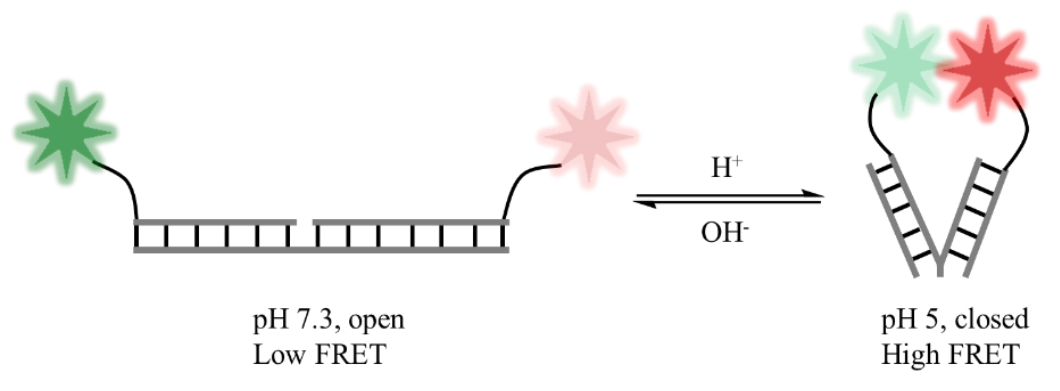

Figure 1.14: Structure of the I-switch sensor. The I-switch is present in the open state at high $\mathrm{pH}$ (low FRET) and in a closed state at low $\mathrm{pH}$ (high FRET). ${ }^{[107]}$ 
Inspired by the concept of the I-switch sensor and the synthesis of the $\mathrm{Cl}^{-}$sensitive $\mathrm{BAC}$ fluorophore (4), KRISHNAN and co-workers developed a ratiometric DNA nanodevice to measure intracellular $\mathrm{Cl}^{-}$concentrations in specific organelles. ${ }^{[7,107]}$ The BAC fluorophore (4) was conjugated to a $\mathrm{Cl}^{-}$insensitive normalising AlexaFluor 647 fluorophore, by linking both fluorophores to a single-stranded DNA sequence (BAC-ssDNA) in a 1:1 stoichiometry (see figure 1.15) ${ }^{[6,108]}$ Accordingly, the BAC fluorescence intensity $(\mathrm{G})$ is linearly reduced with increasing $\mathrm{Cl}^{-}$concentration, whereas the fluorescence intensity (R) of AlexaFluor 647 remains constant. Based on the ratio of the emission intensities $(\mathrm{R} / \mathrm{G})$, the $\mathrm{Cl}^{-}$concentration can be calculated. However, conjugation of BAC to the DNA scaffold significantly decreases the fluorescence intensity, chloride sensitivity $\left(K_{\mathrm{SV}}=33.6 \mathrm{M}^{-1}\right)$, as well as fluorescence lifetime (5.5 ns), compared to the free BAC (4) (see table 1.1). ${ }^{[6]}$

This reduction can be explained by a photoinduced electron transfer (PET) between guanine and BAC, quenching the fluorescence. The efficiency of PET-based quenching from guanine is firmly distance-dependent and reduces with increasing distance. Thus, guanine is placed ten nucleotides (nt) apart from BAC and the formation of BAC-double-stranded DNA prevented the development of globular structures that bring guanine and BAC close together. ${ }^{[6]}$ Surprisingly, the duplexation showed no significant increase in the fluorescence intensity or chloride sensitivity. This could be explained by intercalation of BAC into the dsDNA, which limits the accessibility of BAC to chloride. ${ }^{[6]}$

To investigate the effect of groove binding and intercalation of BAC into the ds-DNA, various ds-DNA scaffolds were synthesised, bearing BAC internally (BAC-dsDNAB0) or creating bulges of different sizes around $\mathrm{BAC}\left(\mathrm{BAC}-\mathrm{dsDNA}_{\mathrm{B} 1}\right)$. The bulges were designed to enhance the conformational flexibility of BAC or to place BAC away from the helical scaffold to enhance the accessibility for chloride. However, determination of the chloride sensitivity revealed for all constructs no significant increase in sensitivity compared to BAC-single stranded (ss) DNA (see table 1.2). These results indicate a strong interaction between the positively charged acridine core of BAC and the negatively charged DNA backbone, leading to increased PET-based quenching and intercalation due to the proximity. ${ }^{[6]}$ 
A

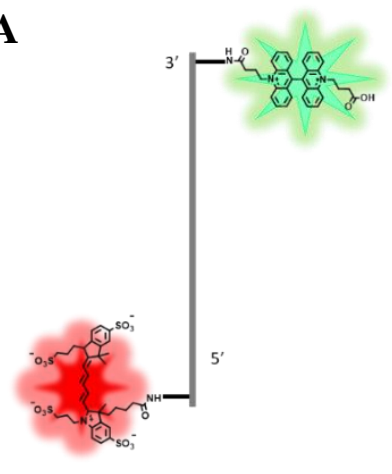

B

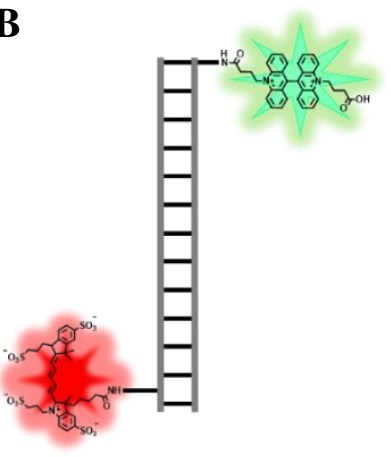

C

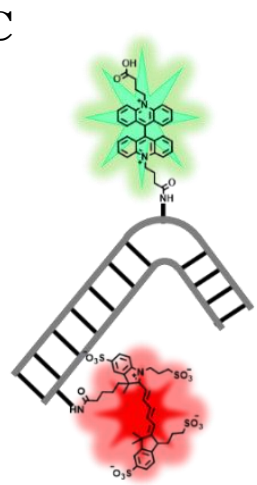

Figure 1.15: Schematic structure of various DNA based sensor. A) BAC-ssDNA B) BAC-dsDNA C) BACdsDNA with bulges. The BAC fluorophore is shown in green, whereas Alexa Fluor 647 is presented in red. ${ }^{[6]}$

To overcome the limitation of electrostatic interaction between BAC and the negatively charged phosphate backbone, the DNA scaffold was replaced by a peptide nucleic acid (PNA) oligomer. The PNA consists structurally of purine and pyrimidine bases, which are linked to a noncharged peptidic backbone and can form stable PNA/PNA as well as PNA/DNA strands. Measurements of a synthesised BAC-dsPNA oligomer revealed an increased chloride sensitivity, fluorescence lifetime $(9.2 \mathrm{~ns})$ as well as $\mathrm{Cl}^{-}$sensitivity $\left(K_{\mathrm{SV}}=71 \mathrm{M}^{-1}\right)$ which are comparable with the sensitivity of free BAC (4) (lifetime: $9.2 \mathrm{~ns}, K_{\mathrm{SV}}=56 \mathrm{M}^{-1}$ ). ${ }^{[6]}$

Nevertheless, the application of BAC-dsPNA for intracellular $\mathrm{Cl}^{-}$sensing is limited. Precise ratiometric measurements require a spacing of at least $40 \mathrm{nt}(10 \mathrm{~nm})$ between the sensing BAC as well as normalising AlexaFluor 647 fluorophores to preserves photophysical characteristics of both fluorophores and to exclude FRET effects. ${ }^{[110]}$ Since the synthesis of PNA oligomers is still limited to a length of $20 \mathrm{nt}$, a BAC-dsPNA nanodevice would lead to imprecise measurements. ${ }^{[111]}$ Furthermore, the specific targeting of the nanodevice sensors to pathways or organelles is based on dsDNA sequences. 
Table 1.2: Overview of the photophysical characteristics of different BAC-labelled constructs. STERNVOLLMER constant $\left(K_{\mathrm{SV}}\right)$, average lifetime $\left(\tau_{0 \text { Avg }}\right)$, fold change in fluorescence intensity $\left.24.4-90.9 \mathrm{mM}^{-} \mathrm{Cl}^{-}\right]$.

\begin{tabular}{lllc}
\hline Construct & $\boldsymbol{K}_{\mathbf{S v}}\left(\mathbf{M}^{-\mathbf{1}}\right)$ & $\boldsymbol{\tau}_{\mathbf{0} \text { Avg }}(\mathbf{n s})$ & Fold change $(\mathbf{R} / \mathbf{G})$ \\
\hline BAC (4) & $56.4 \pm 0.6$ & $9.15 \pm 0.01$ & 2.89 \\
BAC-ssDNA & $33.6 \pm 0.8$ & $5.47 \pm 0.25$ & 1.24 \\
BAC-dsDNA $_{\mathrm{B} 0}$ & $33.6 \pm 1.8$ & $5.16 \pm 0.19$ & 1.21 \\
BAC-dsDNA $_{\mathrm{B} 1}$ & $3.9 \pm 0.5$ & $2.68 \pm 0.11$ & 1.3 \\
BAC-dsDNA & $7.7 \pm 1.1$ & $3.57 \pm 0.26$ & 1.3 \\
BAC-ssPNA & $64.3 \pm 1.1$ & $7.35 \pm 0.06$ & 1.82 \\
BAC-dsPNA & $70.8 \pm 0.2$ & $7.80 \pm 0.08$ & 2.72 \\
CLENSOR & $58.6 \pm 0.3$ & $7.40 \pm 0.01$ & 1.82 \\
\hline
\end{tabular}

Consequently, to combine the benefits of the specific intracellular targeting of the ds-DNA and the improved chloride sensitivity of the PNA, the KRISHNAN group designed a nanodevice, called CLENSOR, consisting of three different modules. ${ }^{[6,92]}$ The sensing module is a 12-mer PNA sequence $(\mathrm{P})$ carrying the chloride-sensitive BAC fluorophore (4). The normalising module is a 38-mer DNA sequence (D2) conjugated with a chloride insensitive AlexaFluor 647 fluorophore and the targeting sequence, which is a 26-mer DNA sequence (D1) bearing a recognition sequence for endosomal targeting (see figure 1.16). The P and D1 module hybridise with the complementary DNA strand D2 to form a DNA-PNA hybrid duplex. The ds-DNA part of CLENSOR, consisting of D1 and D2, can also be linked with different recognition sequences to target specific organelles, for example, with an RNA aptamer to investigate human transferrin receptors. ${ }^{[5]}$ 
$\mathbf{A}$

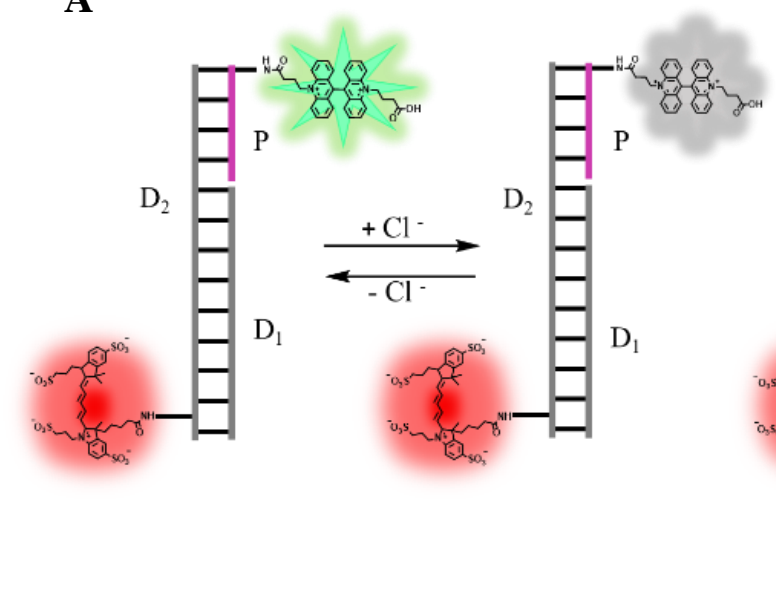

B

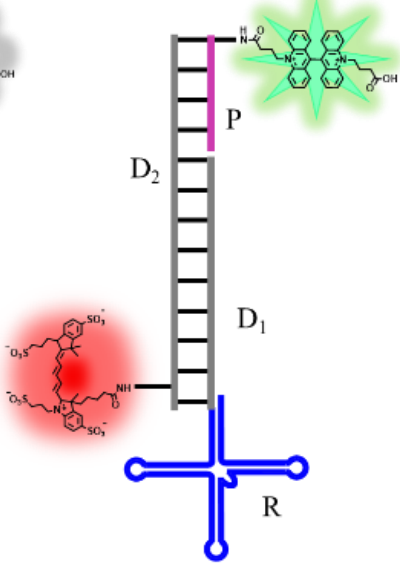

Figure 1.16: Schematic presentation of CLENSOR. A) Structure of the CLENSOR: the sensing module (P, pink) consists of PNA and BAC (4), the normalising DNA module (D2, grey) bears the AlexaFluor 647 and the targeting DNA module (D1, grey). In the presence of $\mathrm{Cl}^{-}, \mathrm{BAC}(4)$ is collisional quenched, whereas the AlexaFluor 647 is $\mathrm{Cl}^{-}$insensitive. B) Modified Clensor with an RNA aptamer structure ( $\mathrm{R}$, blue) to target the human transferrin receptor. ${ }^{[92]}$

Measurements with the CLENSOR molecule under physiological conditions showed a $33 \%$ improved fluorescence intensity compared to the BAC-dsDNA. Fluorescence lifetime and chloride titration experiments revealed a lifetime of $8.3 \mathrm{~ns}$ and a $K_{\mathrm{SV}}$ value of $58.6 \mathrm{M}^{-1}$, which is comparable with characteristics of free BAC (4), showing similar sensitivity. Subsequently, CLENSOR was applied to measure characteristics of $\mathrm{Cl}^{-}$concentrations along the endolysosomal pathway in Drosophila melanogaster. ${ }^{[5]}$

Compared to protein-based $\mathrm{Cl}^{-}$sensors, the CLENSOR molecule, provides the advantage of a high $\mathrm{Cl}^{-}$sensitivity in the physiological range, a rapid response $(<1 \mathrm{~ms})$ and insensitivity to physiological changes in $\mathrm{pH}$. Due to conjugation of the BAC and AlexaFluor 647 fluorophore to the PNA-DNA scaffold, the sensor is also ratiometric and can specifically target intracellular pathways or organelles. In addition, the modular structure of CLENSOR allows the introduction of modifications for special transport and storage in the desired compartment. ${ }^{[6,92]}$ 


\subsection{Design and Synthesis of PNA-DNA based Chloride Sensors}

Vesicular neurotransmitter transporters play an essential role in neurological processes, by filling neurotransmitters in synaptic vesicles. Especially, imbalance in the glutamatergic neurotransmission can be linked to various neurological diseases, which are currently not curable and the available treatment with psychiatric therapeutics can cause various off-target effects. ${ }^{[40-43]}$ Consequently, for the development of highly specific therapeutics, more in-depth insights into the glutamate transport mechanism are required. One decisive question is how different $\mathrm{Cl}^{-}$concentrations can influence the dynamics and mechanisms of glutamate uptake by VGLUT. For answering this question, a fluorescent $\mathrm{Cl}^{-}$sensor was developed, to measure the $\mathrm{Cl}^{-}$influx through VGLUT during the glutamate transport.

For kinetic measurements of the $\mathrm{Cl}^{-}$influx, the sensor was encapsulated into the lumen of the proteoliposomes. The $\mathrm{Cl}^{-}$sensor was thus modified with a hydrophobic membrane anchor to ensure that the sensor remains in the liposome during reconstitution. Due to the conjugation of the membrane anchor, the $\mathrm{Cl}^{-}$sensor incorporates to the luminal as well as extravesicular site of the proteoliposomes. However, the extravesicular orientated sensors disturb the measurements of the $\mathrm{Cl}^{-}$influx through VGLUT since the fluorescence signals from luminal and extravesicular sensors cannot be differentiated. To overcome this limitation, a $\mathrm{Cl}^{-}$sensor has to be synthesised, consisting of a membrane anchor and a cleavage sequence, which allows the removal of all outside orientated sensors of the reconstituted liposome. The $\mathrm{Cl}^{-}$sensitive fluorophore has to be conjugated to an insensitive fluorophore, to perform ratiometric measurements.

To fulfil these criteria, a new $\mathrm{Cl}^{-}$sensor was developed, based on the design of the CLENSOR molecule from KRISHNAN and colleagues. ${ }^{[5,6]}$ The BAC fluorophore (4) was selected as $\mathrm{Cl}^{-}$ sensitive fluorophore due to its sensitivity in the physiological range of $\mathrm{Cl}^{-}$from 3 to $>100 \mathrm{mM}$, $\mathrm{pH}$ insensitivity and prolonged excitation as well as emission wavelength. ${ }^{[7,92]}$ For the ratiometric design, the $\mathrm{BAC}$ fluorophore (4) was linked to a $\mathrm{Cl}^{-}$insensitive AlexaFluor 647 fluorophore in a 1:1 stoichiometry by a PNA-DNA scaffold, consisting of three modules. The sensing module is a 12-mer peptide nucleic acid (PNA) oligomer conjugated to the BAC fluorophore (4), whereas the normalising AlexaFluor 647 fluorophore is linked to a 38-mer DNA (D2) sequence. For spatial separation between sensing and normalising dye, a stabilising 26-mer DNA (D1) module is introduced, which hybridises with the PNA and the D2 module (see figure 1.17). To target the $\mathrm{Cl}^{-}$sensor in the lumen of the proteoliposomes, a peptidic linker sequence was attached to the PNA scaffold. This linker sequence allows, on the one hand, the 
conjugation of a palmitoyl or cholesterol anchor for membrane incorporation and on the other hand the introduction of a protease cleavage site. Consequently, treatment with a protease leads to a cleavage between the sensor and the membrane anchor, removing all extravesicular orientated sensors from the proteoliposomes. The intravesicular orientated sensors is not affected by the protease cleavage due to their membrane impermeability.

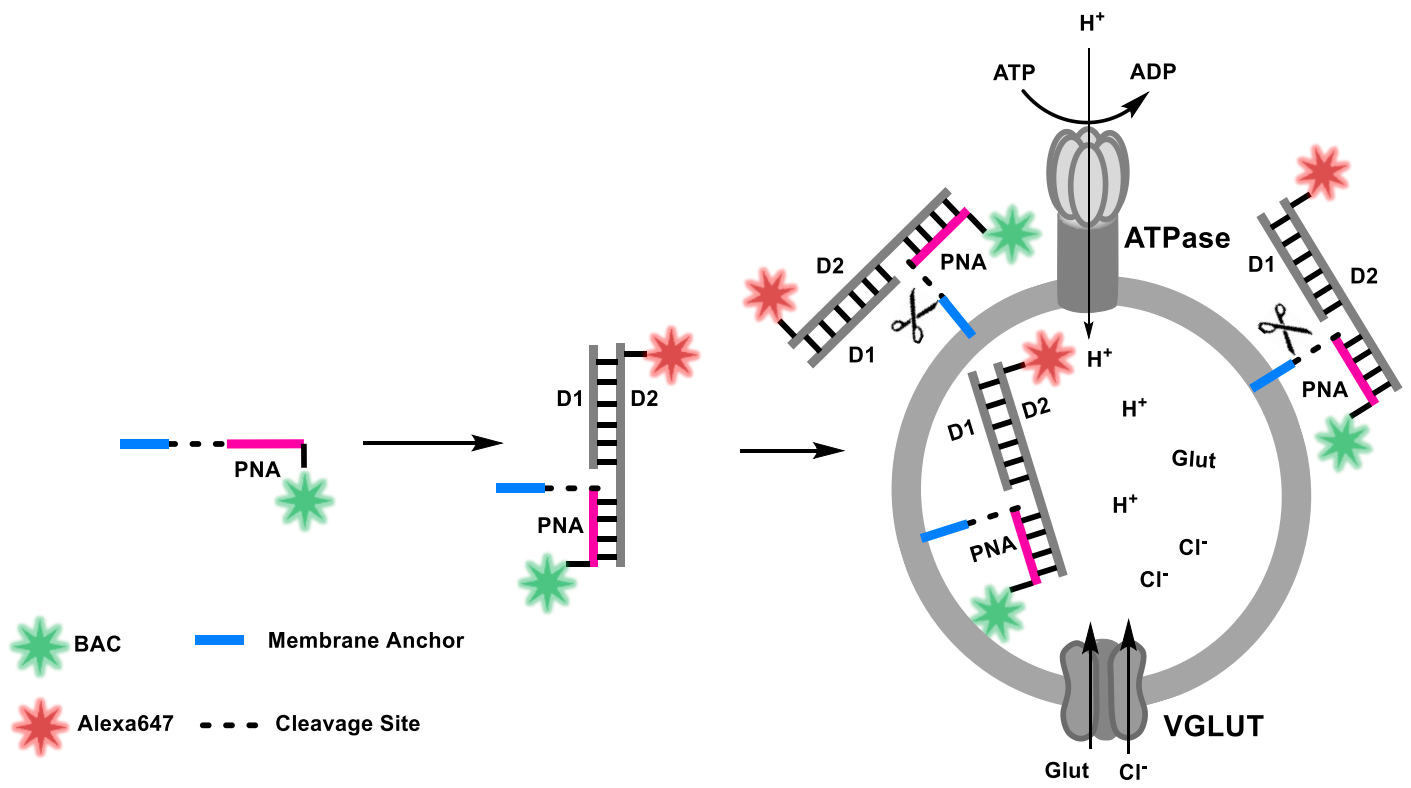

Figure 1.17: Structure and function of the $\mathrm{Cl}^{-}$sensors. The PNA strand is labelled with the $\mathrm{Cl}^{-}$sensitive $\mathrm{BAC}$ fluorophore (4), which is modified with a membrane anchor and a cleavage site. The D2 strand is the normalising module, containing a $\mathrm{Cl}^{-}$insensitive AlexaFluor 647 fluorophore. D1 is the stabilising module, which hybridises with D2. Incorporation of the $\mathrm{Cl}^{-}$sensor in proteoliposomes leads to luminal and extravesicular orientated sensors. All outside orientated sensors can be removed by addition of a protease, cleaving the linker sequence between the sensor and membrane anchor.

\subsubsection{Synthesis of PNA based Sensing Module}

In this chapter, the synthesis of a ratiometric PNA-DNA based $\mathrm{Cl}^{-}$sensors is described, which are modified with a membrane anchor and a protease cleavage sequence to measure intravesicular $\mathrm{Cl}^{-}$concentrations in proteoliposomes. Therefore, the protease recognition motive was conjugated to the peptidic backbone of the 12-mer PNA sensing module and the membrane anchor was linked to a side chain residue of the protease recognition motif at the $C$-terminal part of the peptide-PNA oligomer. The $\mathrm{Cl}^{-}$sensitive BAC fluorophore (4) was coupled to a lysine residue at the $N$-terminal part of the oligomer (see figure 1.18). Since the formation of PNA oligomers and their labelling with the BAC fluorophore (4) are challenging, an optimised synthesis strategy for PNA coupling as well as fluorophore labelling was introduced in the first step (part I). Following, different membrane anchors (part II) and 
protease recognition sequences (part III) were incorporated into the PNA sequence, to identify efficient liposome incorporation and cleavage conditions for the $\mathrm{Cl}^{-}$sensor.

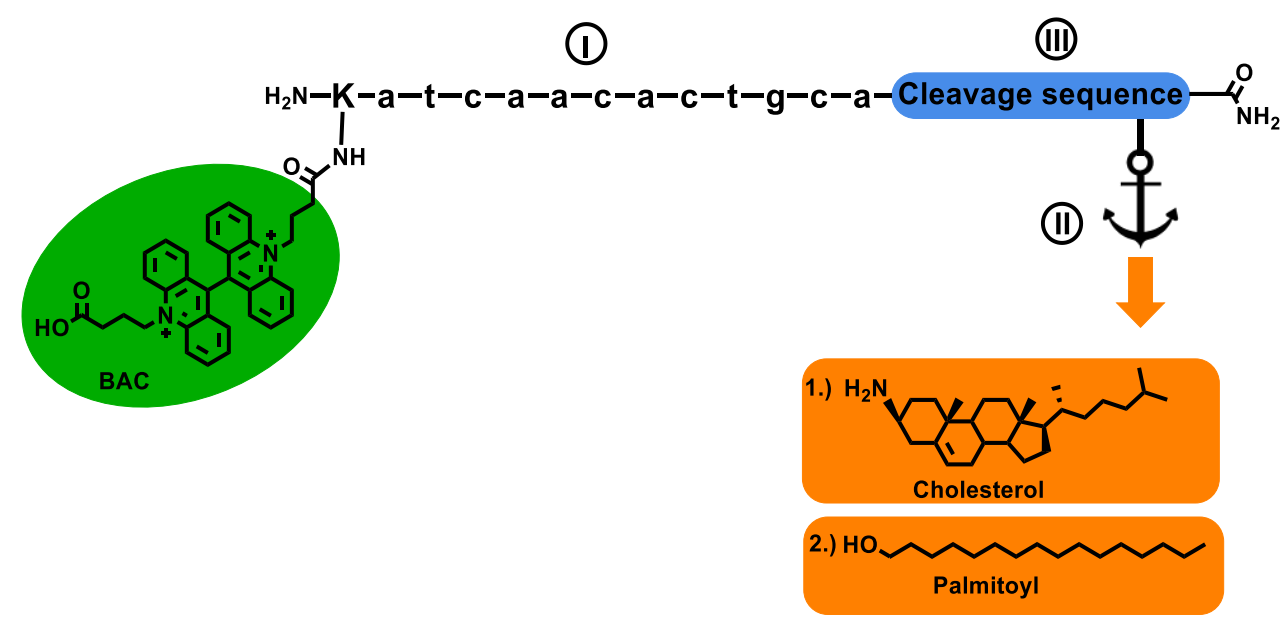

Figure 1.18: Target structure of $\mathrm{Cl}^{-}$sensitive PNA module. The structure consists of three parts: the PNA sequence and the $\mathrm{Cl}^{-}$sensitive BAC fluorophore (4) (green; I), the membrane anchor (orange; II) as well as a protease cleavage sequence (blue; III). The PNA residues are shown in lowercase letters. The $C$-terminus of the peptide-PNA oligomer is amidated.

\subsubsection{Optimised Synthesis Strategy for PNA Oligomers (molecule part I)}

PNA oligomers are artificially synthesised polymers, which were developed by NIELSEN, EGHOLM, BERG and BUCHARDT as DNA mimics. ${ }^{[12]}$ Over the last years, PNA strands were frequently used in diagnostic assays, as tools for molecular biology or as drug leads. ${ }^{[113-116]}$ In contrast to the DNA structure, the sugar-phosphate backbone is replaced in the PNA scaffold by a synthetic peptide backbone consisting of $N$-(2-aminoethyl)-glycine (aeg) units (see figure 1.19). The PNA monomers are linked by peptide bonds and the corresponding nucleobases are attached to the backbone by methylene carbonyl linkages. ${ }^{[117]}$ Due to the lack of electrostatic repulsion, PNA oligomers can form stronger interactions with the complementary DNA or RNA strands than the corresponding DNA/DNA or RNA/RNA strands. They are also stable over a wide temperature and $\mathrm{pH}$ range as well as resistant towards proteases or nucleases due to their unnatural backbone. ${ }^{[118,119]}$ 


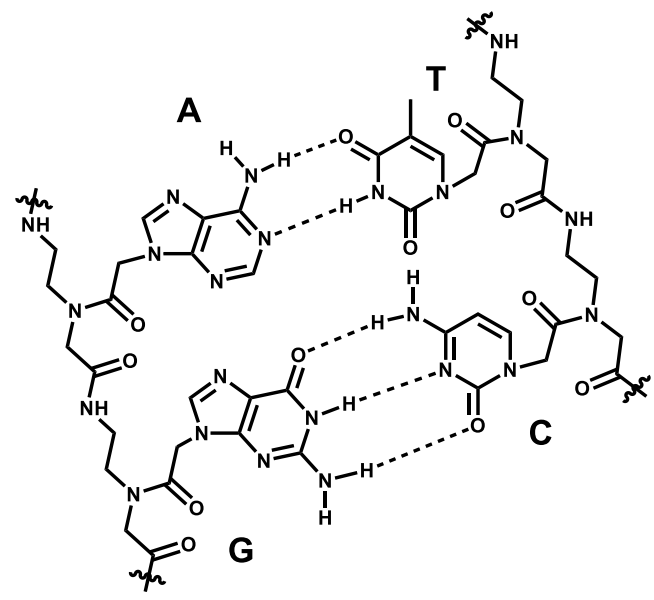

Figure 1.19: Structure of the aeg-PNA. Duplexation of PNA strands according to WATSON-CRICK base pairing.

In the first step, the PNA oligomer sequence of the sensing module was synthesised by solidphase peptide synthesis (SPPS). The simple structure of the unmodified PNA scaffold allows the optimisation of coupling and labelling conditions since the formation of PNA oligomers is limited by the low solubility of the monomers in DMF and their poor coupling efficiency. ${ }^{[11,119]}$ Previous PNA synthesis protocols from SAHA et al. or MIDDEL et al. reported a manual doublecoupling method for PNA monomers for $1 \mathrm{~h}$ at $\mathrm{rt}^{[5,120]}$ The deprotection of the $N$-terminal Fmoc-group was performed with $20 \%$ piperidine for two times $10 \mathrm{~min}$ at $\mathrm{rt}$. Due to the long coupling and deprotection times, the synthesis of the 12-mer PNA oligomer lasted three to four days. To reduce the time needed for PNA synthesis, an optimised coupling protocol for PNA monomers was developed, using automated SPPS on a peptide synthesiser.

For increasing the coupling efficiency and reduction of the reaction times, a microwave-assisted coupling method was developed using $N, N^{\prime}$-diisopropylcarbodiimide (DIC) as activator and ethyl cyanohydroxyiminoacetate (Oxyma) as activator base. Higher coupling temperatures of $50{ }^{\circ} \mathrm{C}$ lead thereby to a reduced coupling time of $25 \mathrm{~min}$. Further attempts to increase the coupling temperature to $75{ }^{\circ} \mathrm{C}$ lead to no product formation. The deprotection conditions were also enhanced by applying $2 \%$ piperazine at $50{ }^{\circ} \mathrm{C}$ for $5 \mathrm{~min}$. Furthermore, the formation of side products was reduced by blocking unreacted amino acids with acetic anhydride after the coupling process. Since acetic anhydride in the capping solution can acetylated nucleobases, the resin-bound peptide-PNA hybrids were washed with $5 \%$ DIPEA, which reverse the nucleoacetylation and reduces the formation of side-products. The optimised SPPS protocol for the synthesis of peptide-PNA oligomers lead to enhanced yields and purity as well as significantly reduced reaction times compared to the manually coupled PNA protocol. ${ }^{[121]}$ The amino acids were coupled by standard Fmoc-chemistry using DIC and Oxyma as coupling reagents and elevated reaction temperatures of $90{ }^{\circ} \mathrm{C}$ for $8 \mathrm{~min}$ at $30 \mathrm{~W}$. 
Based on these findings, the 12-mer PNA sequence, which was adopted from the CLENSOR molecule, was synthesised on a Rink Amide resin with low loading density, to prevent aggregation of the oligomers during synthesis (see figure $1.20 \mathrm{~A}$ ). ${ }^{[5]}$ As a first amino acid, an additional lysine residue was coupled to increase the solubility of the oligomer. For the introduction of the BAC fluorophore (4), an orthogonal Alloc-protected lysine residue was attached to the $N$-terminal part of the PNA sequence, which can be selectively deprotected by addition of Tetrakis(triphenylphosphine)palladium $(0)\left(\operatorname{Pd}\left(\mathrm{PPh}_{3}\right)_{4}\right)$. However, the free amine group of the lysine residue caused cleavage of the adjacent $N$-terminal Fmoc group, leading to unspecific binding of the BAC fluorophore (4) to the lysine and $N$-terminal amine group. To overcome this limitation, an additional Boc protected alanine residue was introduced to the $N$-terminal position. The Boc group is stable towards nucleophilic attack from the $\varepsilon$-amine of the lysine and can be removed under acidic conditions. After completion of the coupling process, a test cleavage confirmed the successful formation of the PNA oligomer (6) sequence by HPLC and mass spectrometry analysis (see figure 1.20 B). Consequently, the $\mathrm{Cl}^{-}$sensitive BAC fluorophore (4) was synthesised in the next step, to perform labelling experiments of the PNA oligomer.

A

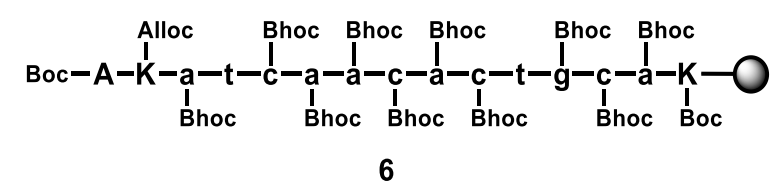

B

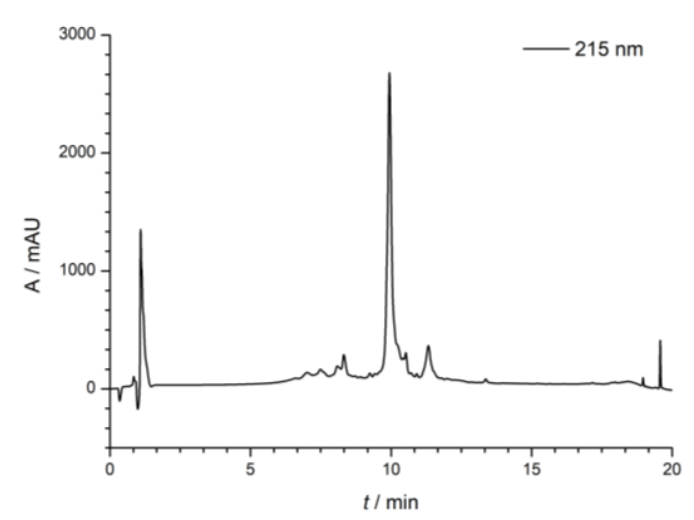

Figure 1.20: Synthesis and analysis of PNA oligomer. A) Structure of the PNA oligomer (6), bearing an Allocprotecting group for selective introduction of the BAC-fluorophore (4). B) U-HPLC chromatogram of the PNA oligomer (6) after test cleavage. U-HPLC gradient: 1-30\% MeCN in 15 min.

The BAC fluorophore (4) was synthesised over three steps, according to the literature procedure from SonAWANE et al. (see figure 1.21). ${ }^{[7]}$ First, the acridone (7) was heated under basic conditions in $\gamma$-butyrolactone (8), forming the 10-[carboxypropyl]-9(10H)-acridone (9), which was subsequently dimerised in a MCMURRY reaction by addition of zinc and $\mathrm{HCl}$ in ethanol. In 
the last step, the 10,10'-bis[3-caboxypropyl]-9,9'biacridylidene (10) was dissolved and heated in nitric acid (2 M) producing BAC (4) with an overall yield of $12 \%$ over three steps.

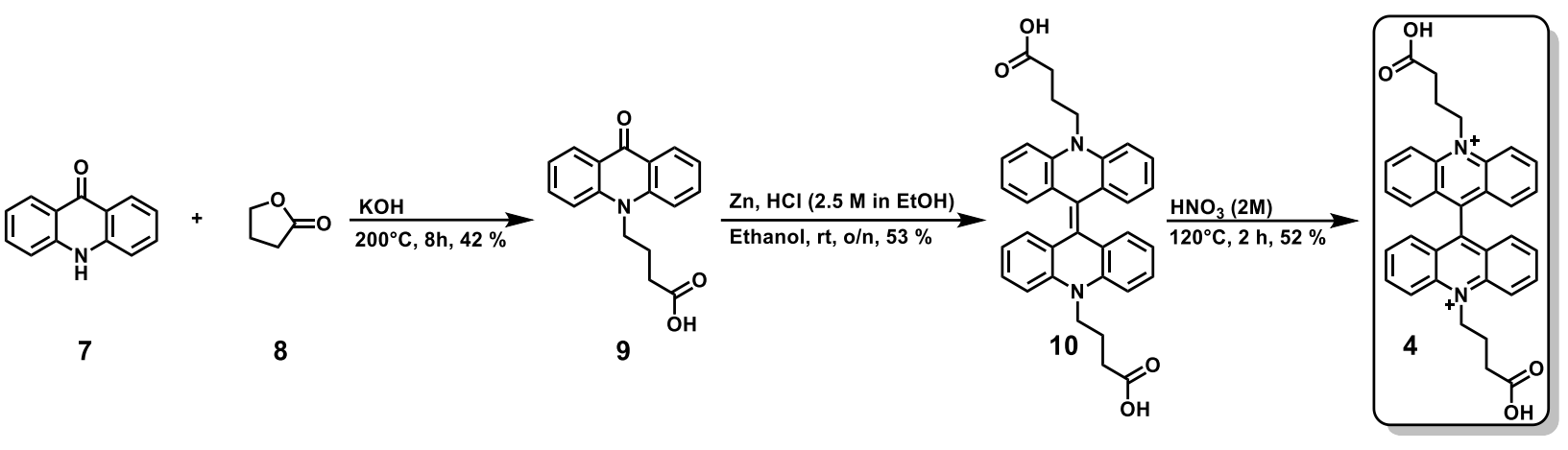

Figure 1.21: Synthesis of the $\mathrm{Cl}^{-}$sensitive BAC fluorophore (4). The synthesis was performed over three steps, starting from the acridone (7).

\subsubsection{Synthesis of BAC Modified Peptide-PNA Oligomer}

Before the BAC fluorophore (4) can be conjugated to the PNA oligomer, the Alloc protecting group of the $N$-terminal lysine residue was removed with $\mathrm{Pd}\left(\mathrm{PPh}_{3}\right)_{4}$ and phenylsilane under microwave irradiation for $5 \mathrm{~min}$ at $38^{\circ} \mathrm{C}$ and $15 \mathrm{~W} .{ }^{[122]}$ This method allows a complete cleavage of the Alloc-group with significantly reduced reaction times, compared to previous procedures. ${ }^{[123]}$ Following, the BAC fluorophore (4) was coupled to the free $\varepsilon$-amine group of the lysine side chain residue according to the protocol from SAHA et al. ${ }^{[6,106]}$ The BAC fluorophore (4) was activated by $N$-hydroxysuccinimide (NHS) as well as 1-ethyl-3-(3dimethylaminopropyl)carbodiimide (EDC) for $2 \mathrm{~h}$ at $\mathrm{rt}$, followed by incubation with the resinbound PNA oligomer (6) for $24 \mathrm{~h}$ at rt. After cleavage of the PNA sequence from the resin with trifluoroacetic acid (TFA), triisopropylsilane (TIS), $\mathrm{H}_{2} \mathrm{O}(95 / 2.5 / 2.5, v / v / v)$, the labelling process was analysed by LC-MS revealing no product formation.

In a second approach, a labelling method for fluorescent $\mathrm{Ca}^{2+}$-sensors to $\beta$-peptides was tested. For the activation of the BAC fluorophore (4), a mixture of ((7-azabenzotriazol-1yloxy)tripyrrolidinophosphonium hexafluorophosphate) (PyAOP), 1-hydroxy-7azabenzotriazole (HOAT), N,N-diisopropylethylamine (DIPEA) in DMF was used. The labelling reaction was performed under microwave irradiation at an elevated temperature of $70{ }^{\circ} \mathrm{C}$ for $3 \mathrm{~h}$ at $15 \mathrm{~W}$ (see figure $1.22 \mathrm{~A}$ ). ${ }^{[124]}$ Compared to the frequently used 1-(bis(dimethylamino)methylene)-1H-1,2,3-triazolo[4,5-b]pyridinium 3-oxide hexafluorophosphate (HATU), PyAOP provides the advantage of no deactivation of free amine groups by guanidinylation. ${ }^{[124,125]}$ After fluorophore labelling, the PNA oligomer and alle acid sensitive 
protecting groups were removed by addition of TFA/TIS/ $\mathrm{H}_{2} \mathrm{O}(95 / 2.5 / 2.5, v / v / v)$. The successful synthesis of the BAC labelled PNA oligomer (11) was confirmed by HPLC and mass spectrometry analysis showing a complete conversion of the starting material (see figure $1.22 \mathrm{~B}$ and $1.22 \mathrm{C})$.

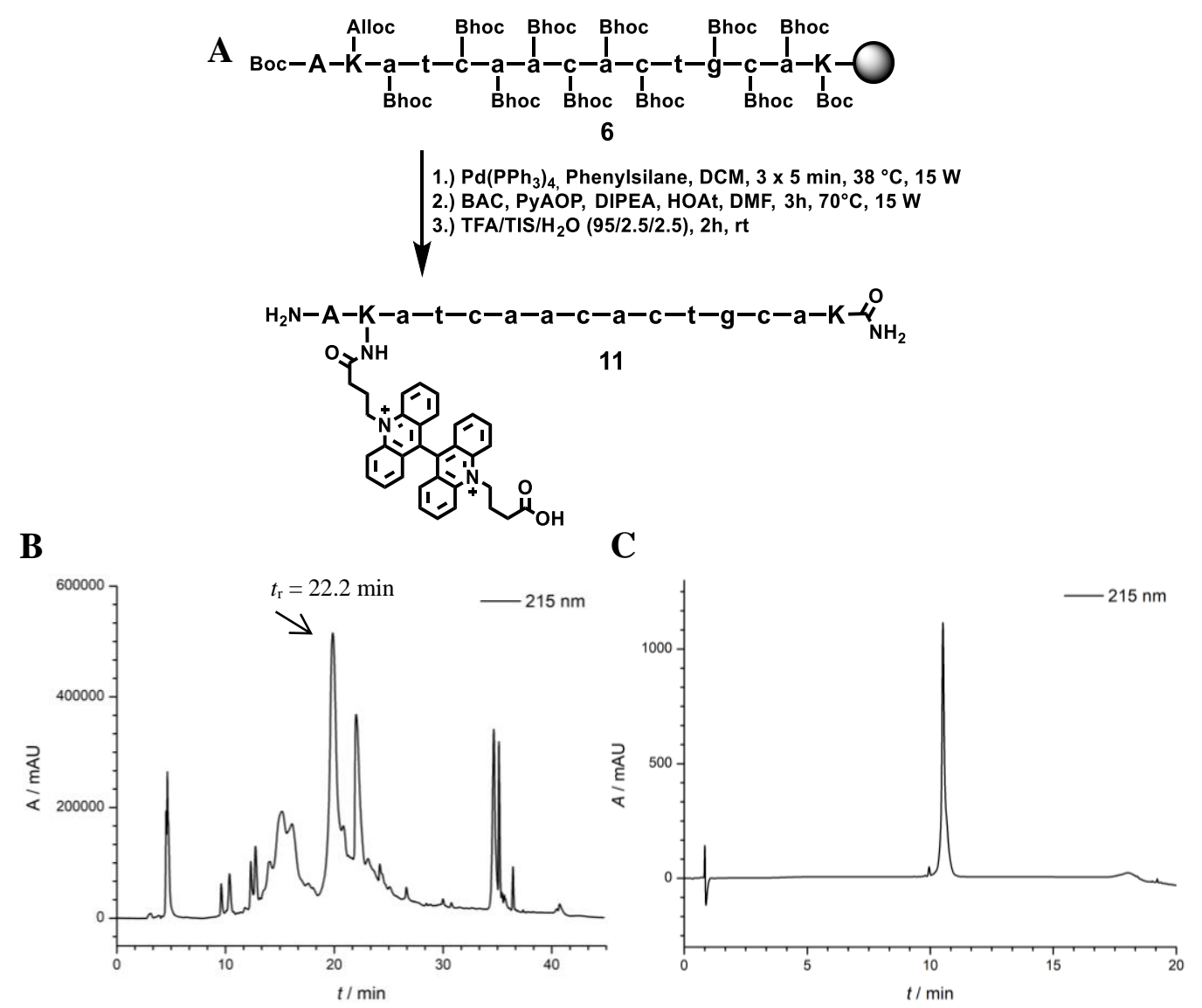

Figure 1.22: Synthesis and Analysis of BAC labelled PNA oligomer. A) Synthesis of BAC modified peptidePNA oligomer (11). B) HPLC chromatogram of the of PNA oligomer (11) after BAC labelling. The isolated product peak is highlighted with an arrow. HPLC gradient: 1-30 \% MeCN in 30 min. C) U-HPLC chromatogram of the purified PNA-BAC sensor (11). U-HPLC gradient: 1-30\% MeCN in 15 min.

\subsubsection{Synthesis of Membrane Anchor Modified PNA Sensors (Molecule Part II)}

After optimisation of the PNA coupling protocol and BAC labelling conditions, a membrane anchor had to be incorporated, for encapsulation of the sensor in the liposome. For identification of optimal anchoring conditions, a modified cholesterol derivate and a palmitoyl linker were selected, due to previous studies with modified $\beta$-peptides, showing a successful accumulation in membranes for both modifications. ${ }^{[126,127]}$ 


\subsubsection{Synthesis of Cholesterol-Modified Peptide-PNA Oligomer}

Starting with the incorporation of the cholesterol anchor, an amine-modified cholesterol derivate was synthesised, which can be linked to a free carboxyl group of the resin-bound PNA sequence. The synthesis of the amine-modified cholesterol derivate was performed over three steps according to the synthesis protocol from SuN et al. (see figure 1.23). ${ }^{[128]}$ In the first step, the hydroxyl group of cholesterol (12) was converted into a good leaving group by adding methanesulfonyl chloride forming the cholesteryl mesylate (13). Following, the cholesteryl mesylate (14) was treated with trimethylsilyl azide $\left(\mathrm{TMSN}_{3}\right)$ and boron trifluoride etherate $\left(\mathrm{BF}_{3} \cdot \mathrm{OEt}_{2}\right)$ to produce the azido-5-cholestene $(\mathbf{1 4})$. In the final step, the azide group was reduced by the addition of lithium aluminium hydride to form the amino-5-cholestene (15) with an overall yield of $18 \%$.

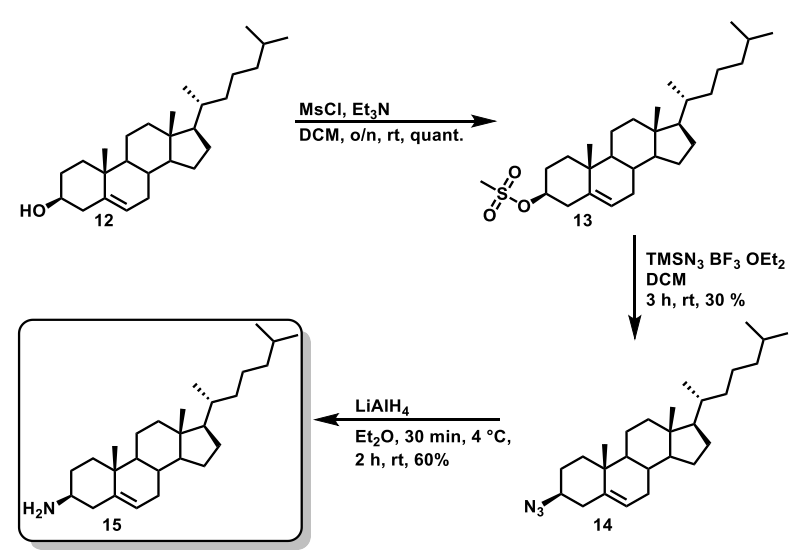

Figure 1.23: Synthesis of amino-5-cholestene (15). The reaction was performed over three steps, starting from cholesterol (12).

For the incorporation of the amine-modified cholesterol anchor (15), a PNA oligomer sequence (16) with an allyl protected glutamic acid residue at the $C$-terminal part was synthesised by the optimised PNA coupling protocol (see chapter 1.3.2). After synthesis of the oligomer, the allyl-group was cleaved with $\mathrm{Pd}\left(\mathrm{PPh}_{3}\right)_{4}$ and the amine-modified cholesterol anchor (15) was conjugated to the free carboxyl group by addition of the activators (1-cyano-2-ethoxy-2oxoethyliden-aminooxy)dimethylamino-morpholino-carbenium hexafluorophosphate (COMU) and DIPEA (see figure 1.24). The reaction process was analysed after $24 \mathrm{~h}$ by a test cleavage and LC-MS analysis, revealing no conversion of the deprotected PNA oligomer. Also different coupling reagents, like HOAt/HATU, DIC/Oxyma or DIC/HOAt and longer reaction times lead to no product formation. These findings can probably be explained by the steric structure of the cholesterol molecule and their tendency to aggregate. Different approaches from HÖGER, to directly incorporate cholesterol modified aspartic acid building block by SPPS, lead to no conversion of the starting material. ${ }^{[127]}$ 


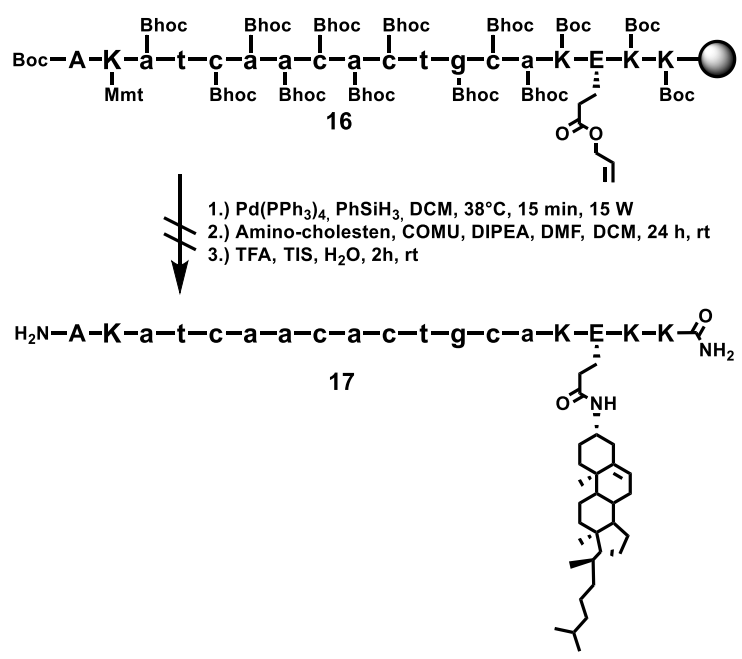

Figure 1.24: Synthesis approach of cholesterol modified peptide-PNA oligomers (17).

As a consequence, a strategy for cholesterol coupling in solution was developed, by synthesising the PNA sequence on the RAMAGE linker modified chlorotrityl resin (18) (see figure 1.25). ${ }^{[129]}$ This approach allows the cleavage of the fully protected peptide-PNA oligomer (19) from the resin with $10 \%$ acetic acid and the coupling of the sterically demanding cholesterol anchor to the PNA oligomer in solution. Nevertheless, cholesterol (15) coupling with COMU and DIPEA as well as HOAt/HATU lead to no product (17) formation, indicating that the bulky cholesterol structure is incompatible for coupling to PNA oligomers. Based on these findings, it was assumed that another membrane anchor with a reduced steric demand was required.

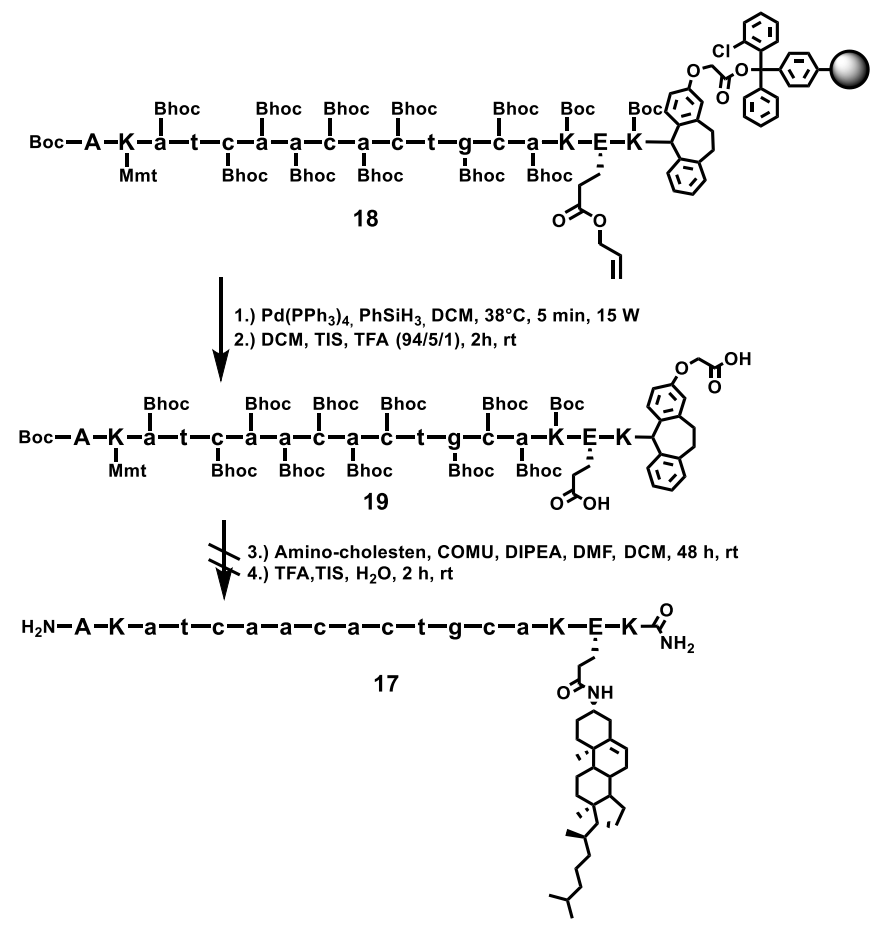

Figure 1.25: Synthesis approach of cholesterol modified peptide-PNA oligomer (17) in solution. 


\subsubsection{Synthesis of a Palmitoyl-Modified Peptide-PNA Oligomer}

Besides the cholesterol motif, palmitoyl anchors are frequently used for the incorporation of peptides in membranes, providing the advantage of a reduced steric structure and increased solubility. An effective strategy for the incorporation of palmitoyl anchors in peptide sequences is the synthesis of a palmitoyl modified aspartic acid residue by the formation of an ester bond, which can directly be used for SPPS. ${ }^{[130]}$

The synthesis of the palmitoyl modified aspartic acid building block for SPPS was performed in two steps according to the literature procedure (see figure 1.26). ${ }^{[130]}$ First, FmocAsp(palmitoyl)-O $\mathrm{O}^{t} \mathrm{Bu}(\mathbf{2 2})$ was obtained by esterification of the palmityl alcohol (21) and the protected Fmoc-Asp-O $\mathrm{O}^{t} \mathrm{Bu}$ (20) using 1-(2-mesitylensulfonyl)-3-nitro-1H-1,2,4-triazole (MSNT) as well as 1-methylimidazole (MeIm) as activators. In the second step, the $C$-terminal tert-butyl ester group was cleaved with TFA in DCM afforded Fmoc-Asp(palmitoyl)-OH (23) with an overall yield of $71 \%$.
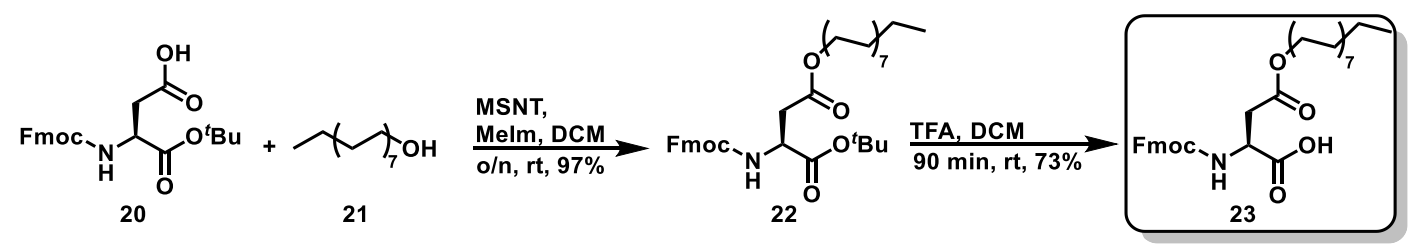

Figure 1.26: Synthesis of Fmoc-Asp(palmitoyl)-OH (23) over two steps.

Standard microwave coupling conditions achieved the incorporation of the palmitoyl-modified aspartic acid building block (23) into the $C$-terminal part of the PNA oligomer (24) at an elevated temperature of $90{ }^{\circ} \mathrm{C}$ (see figure $1.27 \mathrm{~A}$ ). To increase the solubility of the palmitoyl modified PNA oligomer (24), two additional lysine residues were introduced at the $C$-terminal part. After completion of the coupling process, a test cleavage was performed, confirming the successful synthesis of the PNA oligomer modified with a palmitoyl anchor. Following, the Alloc protecting group of the $N$-terminal lysine residue was removed with $\mathrm{Pd}\left(\mathrm{Ph}_{3}\right)_{4}$ and the BAC fluorophore (4) was coupled under microwave irradiation for $3 \mathrm{~h}$ at $70{ }^{\circ} \mathrm{C}$ and $15 \mathrm{~W}$. Verification of the labelling process by HPLC and mass spectrometry analysis, revealed the formation of the palmitoyl modified PNA-BAC sensor (25) (see figure 1.27 B and 1.27 C). 


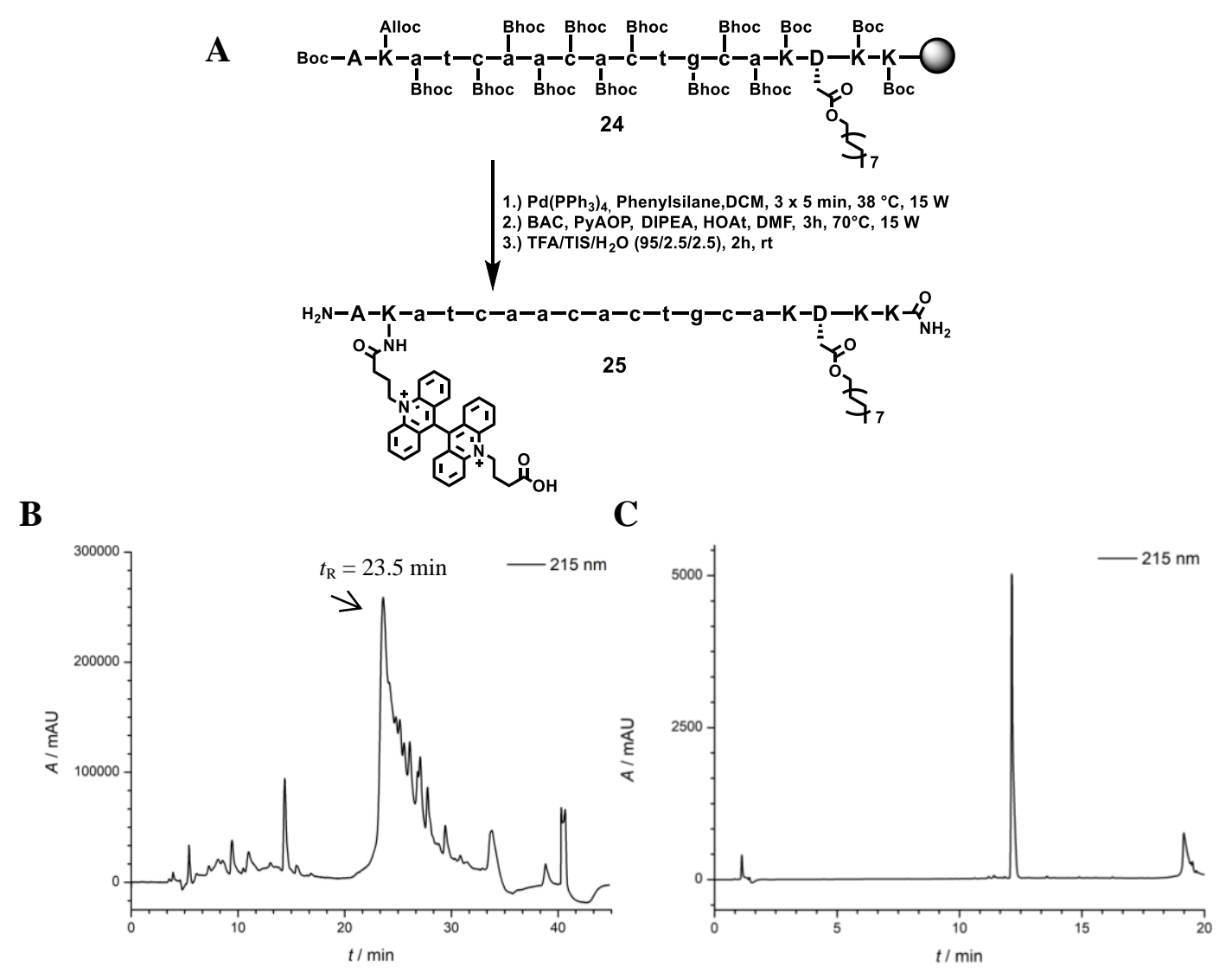

Figure 1.27: Synthesis and analysis of palmitoyl and BAC modified PNA oligomer. A) BAC labelling of the palmitoyl-modified PNA oligomer (25). B) HPLC chromatogram of palmitoyl-PNA oligomer (25) after BAC labelling. The isolated product peak is highlighted with an arrow. HPLC gradient: $10-60 \% \mathrm{MeCN}$ in $30 \mathrm{~min}$. C) U-HPLC chromatogram of the purified palmitoyl -PNA-BAC sensor (25). U-HPLC gradient: 1-30\% MeCN in $15 \mathrm{~min}$.

\subsubsection{Introduction of Cleavage Sites in Modified PNA Sensors (molecule part III)}

After successful synthesis and purification of the palmitoyl modified PNA-BAC sensor (25), the optimised synthesis strategy was applied for the introduction of a protease recognition motif (see figure 1.18). The cleavage sequence was incorporated between the palmitoyl modified aspartic acid and the PNA part, allowing the cleavage of extravesicular orientated sensors on the liposome. To ensure an efficient cleavage process, different proteases were tested.

\subsubsection{TEV Protease Cleavage Site}

Screening of different proteases and their cleavage conditions revealed the tobacco etch virus (TEV) protease, which is frequently used for the cleavage of fusion proteins or affinity tags. ${ }^{[131,132]}$ The TEV protease recognises the sequence Glu-Asn-Leu-Tyr-Phe-Gln-Gly/Ser and cuts specifically between the Gln and Gly/Ser residues. Additionally, the TEV protease 
demonstrated a high cleavage activity over a broad $\mathrm{pH}(4-9)$ and temperature range $\left(4{ }^{\circ} \mathrm{C}\right.$ $37^{\circ} \mathrm{C}$ ), without the formation of non-specific proteolysis side products. ${ }^{[133,134]}$

For investigations of the cleavage efficiency, a TEV recognition sequence was introduced between the palmitoyl anchor and the PNA part of the sensing module, to allow cleavage between the anchor and the sensor (see figure 1.28). To prevent blockage of the TEV protease by the highly flexible palmitoyl chain, a linker sequence was incorporated, separating the $C$ terminal palmitoyl chain from the TEV recognition sequence. The linker sequence consists of a repetitive glycine serine motive. The synthesis of the peptide-PNA sensor (26) with a palmitoyl anchor and the TEV recognition site was performed by optimised PNA coupling protocol (see chapter 1.3.2). After deprotection of the Alloc group, the BAC fluorophore (4) was coupled under microwave irradiation and the peptide-PNA oligomer was cleaved from the resin. HPLC purification of the crude oligomer yielded the palmitoyl modified peptide-PNA sensor (27) with a TEV recognition sequence in high purity.

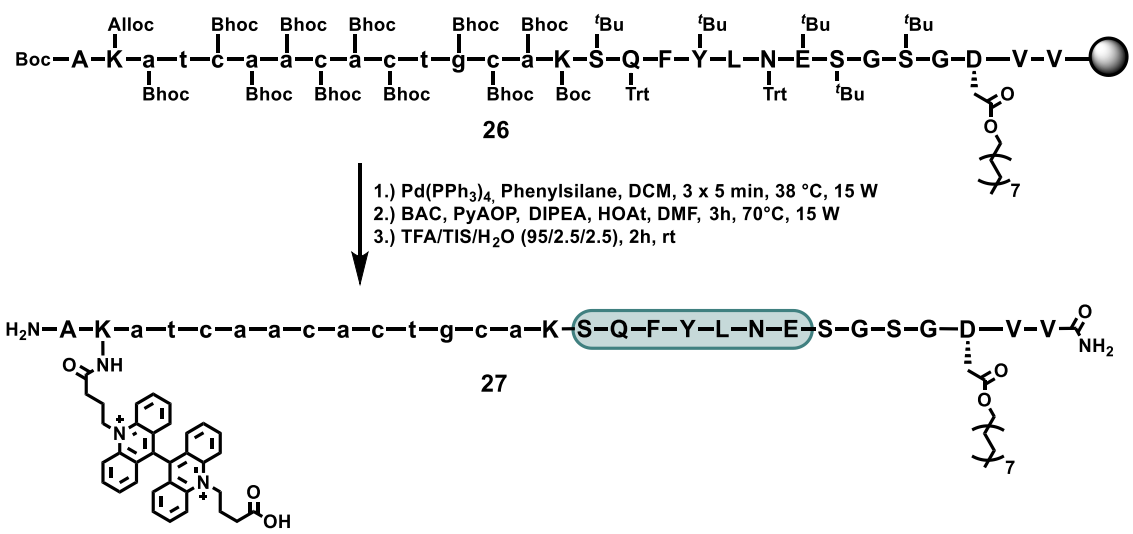

Figure 1.28: Synthesis of palmitoyl, TEV and BAC modified peptide-PNA oligomer (27). The protease cleavage site is highlighted in grey.

In the next step, the TEV protease-mediated cleavage of the synthesised PNA sensor (27) was investigated (see figure 1.29). The purified peptide-PNA sensor (27) was dissolved in the reaction buffer (50 mM Tris- $\mathrm{HCl}, 0.5 \mathrm{mM}$ EDTA, $1 \mathrm{mM}$ DTT, $\mathrm{pH} 8.0$ ), heated to $37^{\circ} \mathrm{C}$ and the TEV protease $(3000 \mathrm{U} / \mathrm{mg})$ was added in a ratio of 100:1 $(v / v)$. The cleavage process was monitored after incubation times of $6 \mathrm{~h}, 12 \mathrm{~h}$ and $24 \mathrm{~h}$ by U-HPLC, showing no conversion of the starting material (27). Also, increased concentrations of the TEV protease (50:1 or 20:1 $(v / v))$ and reduced incubation temperatures $\left(20^{\circ} \mathrm{C}, 4^{\circ} \mathrm{C}\right)$ lead to no cleavage of the peptidePNA sensor. 


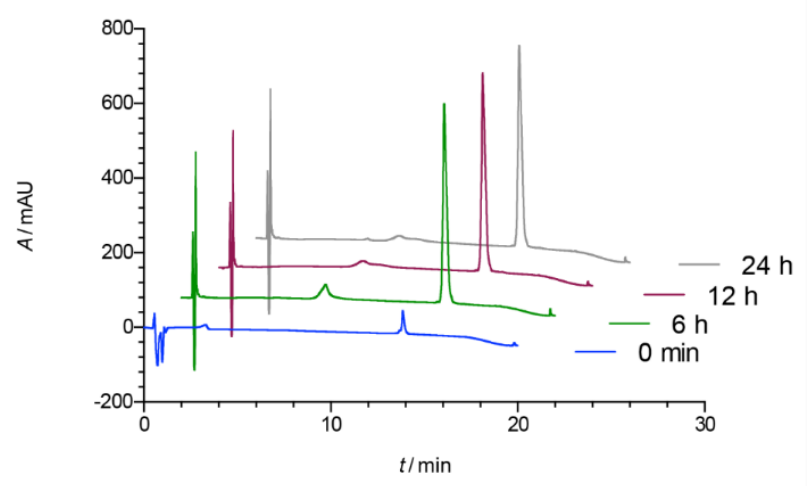

Figure 1.29: TEV-protease cleavage experiments of the palmitoyl modified PNA sensor (27). This experiment was analysed by U-HPLC after incubation times of $0 \mathrm{~min}, 6 \mathrm{~h}, 12 \mathrm{~h}$ and $24 \mathrm{~h}$ at $37^{\circ} \mathrm{C}$. U-HPLC gradient: $10-60 \%$ $\mathrm{MeCN}$ in $15 \mathrm{~min}$.

These findings indicate a blockage of the TEV protease activity, caused either by the PNA structure or by the palmitoyl anchor. Consequently, different test peptides were synthesised without the PNA structure (28) and with increased Gly-Ser linker length to reduce possible blockage from the palmitoyl linker (29) (see table 1.3). To exclude micelle formation of the peptide-PNA oligomers, which would limit the accessibility of the cleavage sequence, a peptide sequence (30) lacking the hydrophobic palmitoyl anchor was also synthesised (see figure $1.30 \mathrm{~A})$. For verification of the cleavage activity, a peptide (31) consisting exclusively of the recognition sequence and two additional lysine residues was tested as a positive control. After the synthesis of different test peptides, the cleavage process was investigated. The peptides were dissolved in the reaction buffer and the TEV protease was added $(100: 1, v / v)$ at $37{ }^{\circ} \mathrm{C}$. The cleavage process was analysed by U-HPLC after $1 \mathrm{~h}, 3 \mathrm{~h}, 5 \mathrm{~h}$ and $24 \mathrm{~h}$, showing no cleavage for any of the tested peptides. Also, testing of various protease concentrations (10:1 or $1000: 1, v / v)$ and reaction temperatures $\left(4^{\circ} \mathrm{C}, 20^{\circ} \mathrm{C}\right)$, revealed no conversion of the test peptides under these conditions. The activity of the TEV protease was verified with a control protein (provided by RASHI GoEL, Group of Prof. REINHARD JAHN), showing a complete cleavage after $2 \mathrm{~h}$ (see figure $1.30 \mathrm{~B}$ ). These results indicated that the TEV protease is unsuitable for the cleavage of short peptide sequences since these are probably unable to enter the TEV protease binding pocket. Thus, a different protease is required with higher catalytic activity, which has already been applied for the cleavage of small peptide structures. 
Table 1.3: Overview of the different test peptides with the TEV recognition sequence.

\begin{tabular}{|c|c|c|}
\hline Peptide & Sequence & Comment \\
\hline 28 & $\mathrm{H}_{2} \mathrm{~N}-\mathrm{A}-\mathrm{K}-\mathrm{S}-\mathbf{Q}-\mathbf{F}-\mathrm{Y}-\mathbf{L}-\mathrm{N}-\mathbf{E}-\mathrm{S}-\mathrm{G}-\mathrm{S}-\mathrm{G}-\mathrm{D}\left(\mathrm{C}_{16}\right)-\mathrm{V}-\mathrm{V}-\mathrm{CONH}_{2}$ & $\begin{array}{l}\text { Palmitoyl-anchor, linker and TEV- } \\
\text { recognition sequence }\end{array}$ \\
\hline 29 & $\begin{array}{l}\mathrm{H}_{2} \mathrm{~N}-\mathrm{A}-\mathrm{K}-\mathrm{S}-\mathbf{Q}-\mathbf{F}-\mathbf{Y}-\mathbf{L}-\mathbf{N}-\mathbf{E}-\mathrm{S}-\mathrm{G}-\mathrm{S}-\mathrm{G}-\mathrm{S}-\mathrm{G}-\mathrm{S}-\mathrm{G}-\mathrm{D}\left(\mathrm{C}_{16}\right)-\mathrm{V}- \\
\mathrm{V}-\mathrm{CONH}_{2}\end{array}$ & $\begin{array}{l}\text { Palmitoyl-anchor, increased linker } \\
\text { length and TEV-recognition sequence }\end{array}$ \\
\hline 30 & $\begin{array}{l}\mathrm{H}_{2} \mathrm{~N}-\mathrm{A}-\mathrm{K}-\mathrm{S}-\mathbf{Q}-\mathbf{F}-\mathbf{Y}-\mathbf{L}-\mathbf{N}-\mathbf{E}-\mathrm{S}-\mathrm{G}-\mathrm{S}-\mathrm{G}-\mathrm{S}-\mathrm{G}-\mathrm{S}-\mathrm{G}-\mathrm{D}-\mathrm{V}-\mathrm{V}- \\
\mathrm{CONH}_{2}\end{array}$ & $\begin{array}{l}\text { Increased linker length and TEV- } \\
\text { recognition sequence }\end{array}$ \\
\hline 31 & $\mathrm{H}_{2} \mathrm{~N}-\mathrm{K}-\mathrm{K}-\mathbf{G}-\mathbf{Q}-\mathbf{F}-\mathbf{Y}-\mathbf{L}-\mathbf{N}-\mathbf{E}-\mathrm{CONH}_{2}$ & TEV-recognition sequence \\
\hline
\end{tabular}

A

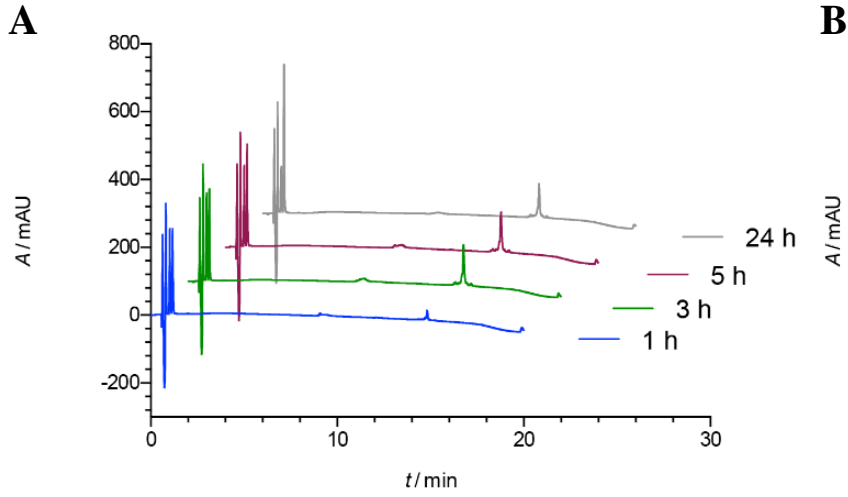

B

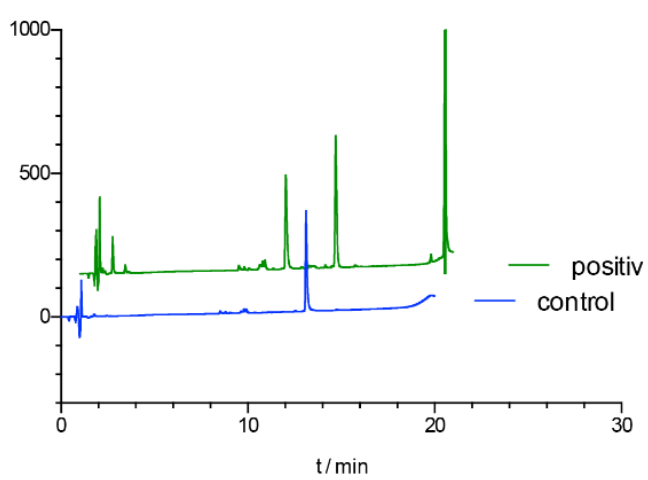

Figure 1.30: TEV-cleavage experiments of different test peptides. A) TEV-protease cleavage experiments of the palmitoyl-modified peptide sequence (29) with increased Gly-Ser linker length analysed by U-HPLC after incubation times of $1 \mathrm{~h}, 3 \mathrm{~h}, 5 \mathrm{~h}$ and $24 \mathrm{~h}$ at $37^{\circ} \mathrm{C}$. U-HPLC gradient: 10-60\% MeCN in $15 \mathrm{~min}$. B) TEV-protease cleavage experiments of a control protein by U-HPLC after incubation times of $2 \mathrm{~h}$ at $37{ }^{\circ} \mathrm{C}$. U-HPLC gradient: $10-60 \% \mathrm{MeCN}$ in $15 \mathrm{~min}$.

\subsubsection{Factor Xa Protease Cleavage Site}

Screening of proteases, which have been successfully used for the cleavage of small peptides, revealed the Factor Xa protease. This site-specific endoprotease cleaves the $C$-terminal peptide bond after the recognition motif Ile-Glu-Gly-Arg with high specificity. ${ }^{[135]}$ To investigate the cleavage conditions of the Factor Xa protease, a test peptide (32) with the recognition motif was synthesised (see figure 1.31 A). For the cleavage experiments, the peptide was dissolved in the reaction buffer, incubated at $37{ }^{\circ} \mathrm{C}$ and subsequently, the Factor Xa protease (20 U/mg) was added in a ratio of 50:1 ( $/ v / v)$. The cleavage process was analysed by U-HPLC after $5 \mathrm{~h}, 7 \mathrm{~h}$ and $12 \mathrm{~h}$, showing a complete conversion of the starting material (32) under these conditions after $12 \mathrm{~h}$ (see figure $1.31 \mathrm{~B}$ ). 


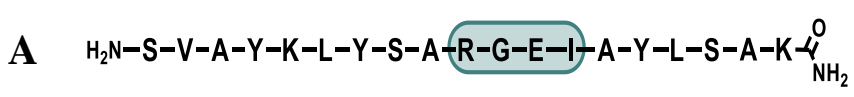

32

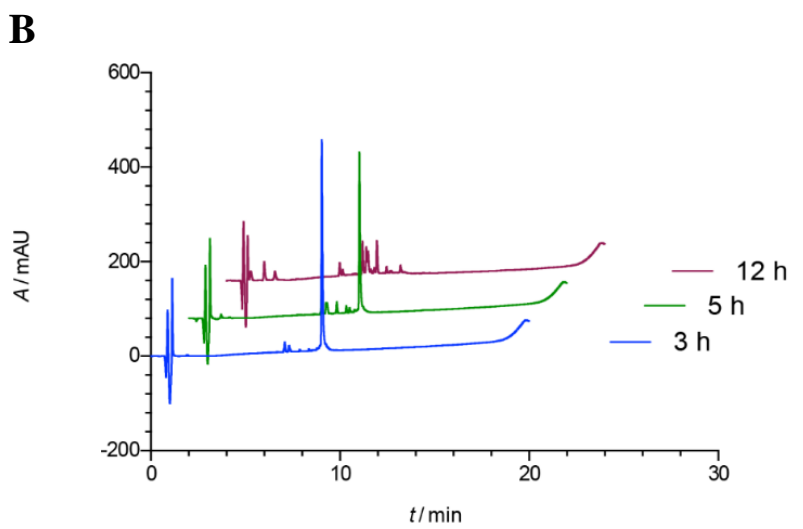

Figure 1.31: Structure and cleavage of Factor Xa test peptides. A) Sequence of the Factor Xa test peptide (32) with the recognition motif I-E-G-R. The protease cleavage site is highlighted in grey. B) Factor Xa-protease cleavage experiments of $\mathbf{3 2}$ analysed by U-HPLC after incubation times of $3 \mathrm{~h}, 5 \mathrm{~h}$ and $12 \mathrm{~h}$. U-HPLC gradient: $10-60 \% \mathrm{MeCN}$ in $15 \mathrm{~min}$.

Consequently, the Factor Xa cleavage sequence was incorporated into the palmitoyl-modified PNA sensor. The synthesis of the peptide-PNA oligomer (33) was performed as previously described by the optimised coupling conditions (see chapter 1.2.2). After labelling with the BAC fluorophore (4) and HPLC purification, the palmitoyl modified peptide-PNA sensor (34) with a Factor Xa recognition sequence was obtained in high purity (see figure 1.32 A). Next, the cleavage properties were investigated, by dissolving the peptide-PNA sensor (34) in protease reaction buffer with the Factor Xa protease $(50: 1, v / v)$ at $37^{\circ} \mathrm{C}$. However, analysis of the cleavage process revealed only the starting material (34) after $2 \mathrm{~h}, 4 \mathrm{~h}, 16 \mathrm{~h}$ and $48 \mathrm{~h}$ (see figure 1.32 B). Different cleavage conditions with higher protease concentration (25:1 or 10:1, $v / v)$ and reduced incubation temperatures $\left(23{ }^{\circ} \mathrm{C}, 4{ }^{\circ} \mathrm{C}\right)$ also revealed no cleavage under all conditions. Based on these findings, it was assumed that the PNA scaffold probably blocks the activity of the Factor Xa protease. Since the preliminary results from the test peptide showed a slow cleavage process, a protease with higher activity is required. 

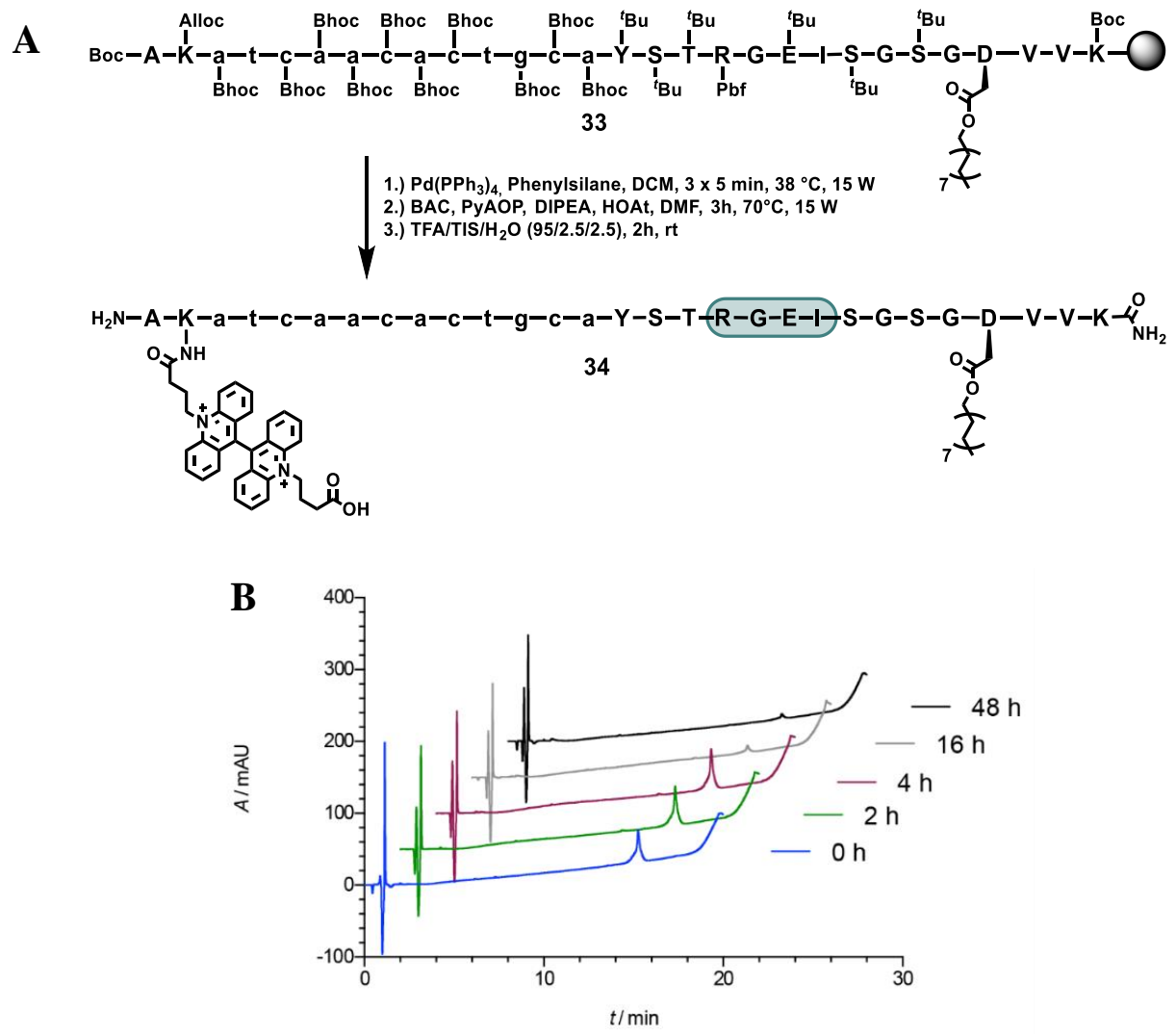

Figure 1.32: Synthesis and Factor Xa cleavage of palmitoyl-modified PNA sensors. A) Synthesis of palmitoyl modified peptide-PNA sensor (34) with a Factor Xa recognition sequence. The protease cleavage site is highlighted in grey. B) Factor Xa-protease cleavage experiments of the Factor Xa modified peptide-PNA sensor (34) analysed by U-HPLC after incubation times of $2 \mathrm{~h}, 4 \mathrm{~h}, 16 \mathrm{~h}$ and $48 \mathrm{~h}$ at $37^{\circ} \mathrm{C}$. HPLC gradient: 10-60\% MeCN in $15 \mathrm{~min}$.

\subsubsection{Cathepsin B Protease Cleavage Site}

In a third approach, the highly active lysosomal cathepsin B protease was tested, which is upregulated under pathological conditions like in metastatic tumours. ${ }^{[136]}$ Several cancer drugs are conjugated with cathepsin B cleavable linkers, allowing a controlled release of the drug in the targeted tissue to reduce off-target effects. ${ }^{[137,138]}$ Commonly used recognition motifs are the dipeptides valine-citrulline or phenylalanine-lysine, which are stable under physiological conditions but rapidly hydrolysed by addition of cathepsin B.

Consequently, a palmitoyl modified peptide-PNA sensor (35) with a cathepsin B recognition sequence was synthesised by the optimised coupling protocol and purified by HPLC (see figure $1.33 \mathrm{~A})$. For the cleavage experiments, the cathepsin B protease was activated by a mixture of DTT and EDTA for $15 \mathrm{~min}$ at $\mathrm{rt}$. The activated cathepsin B (10 U/mg) was then transferred to the dissolved peptide-PNA oligomer (36) and the mixture was incubated at $37{ }^{\circ} \mathrm{C}$. The cleavage efficiency was measured after $30 \mathrm{~min}, 60 \mathrm{~min}, 90 \mathrm{~min}$ and $20 \mathrm{~h}$ by U-HPLC as well as mass 
spectrometry analysis showing a complete conversion of the starting material after 30 min (see figure $1.33 \mathrm{~B})$.

A

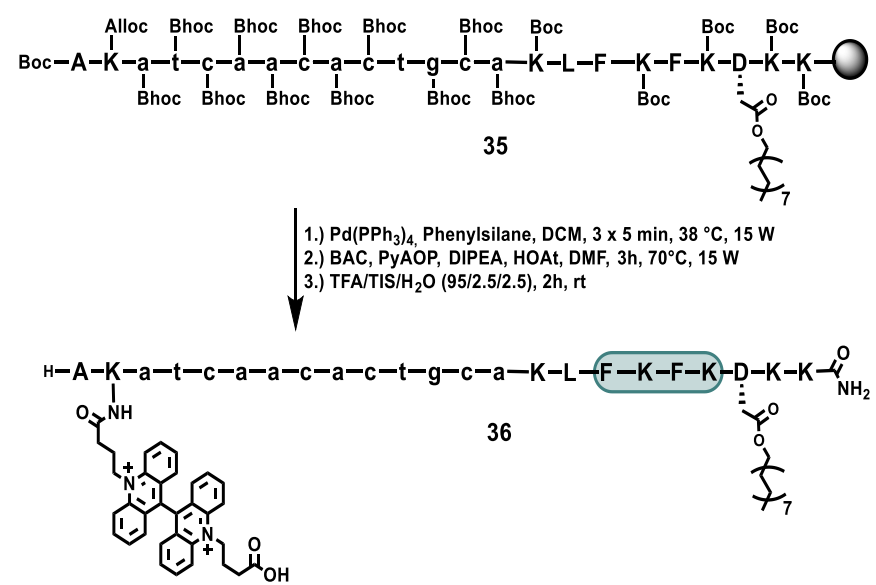

B

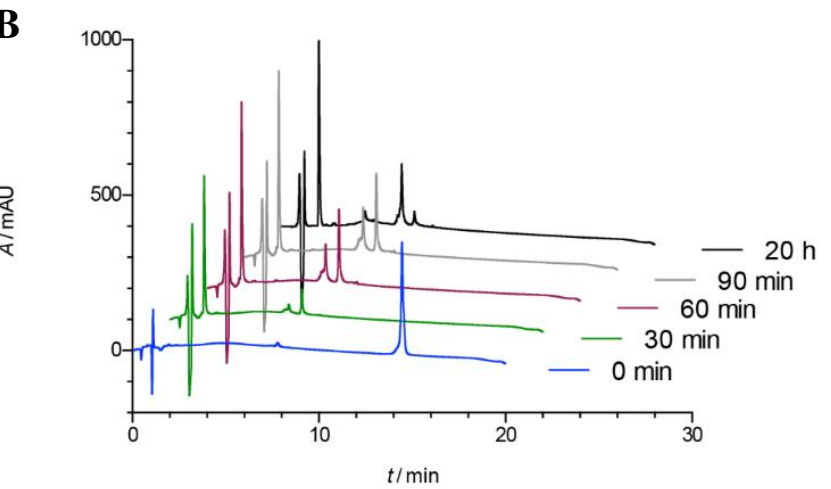

Figure 1.33: Synthesis and cathepsin B cleavage of palmitoyl-modified PNA sensors. A) Synthesis of palmitoyl modified peptide-PNA sensor (36) with a cathepsin B recognition sequence. The protease cleavage site is highlighted in grey. B) Cathepsin B protease cleavage experiments of the cathepsin B modified peptide-PNA sensor (36), analysed by U-HPLC after incubation times of $0 \mathrm{~min}, 30 \mathrm{~min}, 60 \mathrm{~min}, 90 \mathrm{~min}$ and $20 \mathrm{~h}$ at $37^{\circ} \mathrm{C}$. HPLC gradient: $10-60 \% \mathrm{MeCN}$ in 15 min.

\subsubsection{Membrane Incorporation of Palmitoyl Modified PNA Sensors}

After successful cleavage experiments with the cathepsin B protease, the membrane incorporation efficiency of the palmitoyl modified PNA sensor was determined by densitygradient centrifugation performed by RASHI GOEL. The peptide-PNA sensors (36) were therefore incorporated into liposomes (DOPC/DOPS/DOPE/cholesterol, 63/10/2/25) by manual extrusion and the proteoliposomes were overlaid with a Nycodenz gradient $(40 \%, 30 \%$ and $0 \%$ ) as well as a buffer layer on top. During centrifugation, the low-density proteoliposomes with incorporated peptide-PNA oligomers migrate to the top buffer fraction (see figure 1.34). Contrary, unbound free peptide-PNA sensors (36) accumulate in the lower fractions, due to their higher density. After centrifugation, the separation between the different Nycodenz fractions was analysed by fluorescence measurements. 


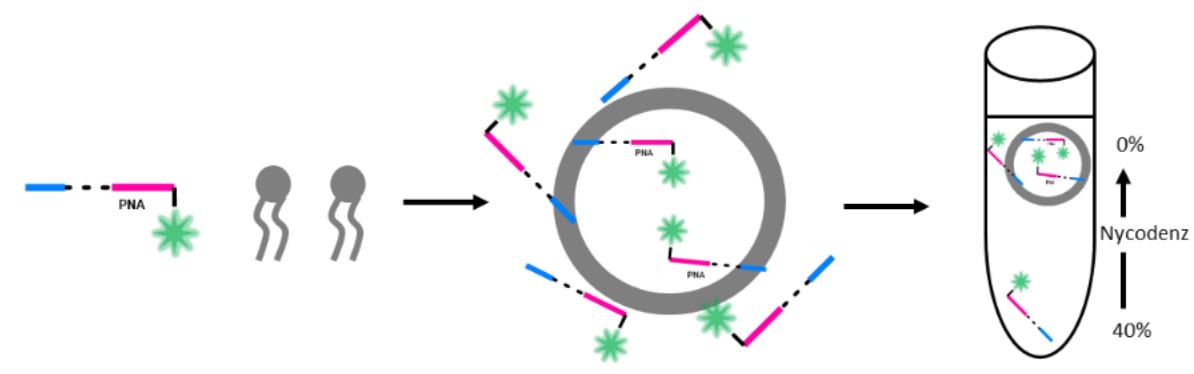

Figure 1.34: Separation of incorporated sensors by density-gradient centrifugation. Incorporation of palmitoyl modified peptide-PNA sensors into liposomes by manual extrusion. The efficiency of the incorporation process was analysed by density-gradient centrifugation.

Analysis of the co-flotation assay revealed for the palmitoyl modified PNA sensors (36) inefficient incorporation in liposomes in the top fractions compared to the Texas Red fluorophore, which was used as the positive control (see figure 1.35). The floating properties of the palmitoyl modified peptide-PNA sensor (36) without liposomes (free BAC) were analysed, to exclude accumulation of the sensor itself in the top fractions. Based on these findings, it was assumed that the hydrophobic effect of one palmitoyl moiety is not sufficient to anchor the hydrophilic PNA strand in the membrane. The formation of liposomes was further hampered, due to aggregation of the palmitoyl-modified sensors.

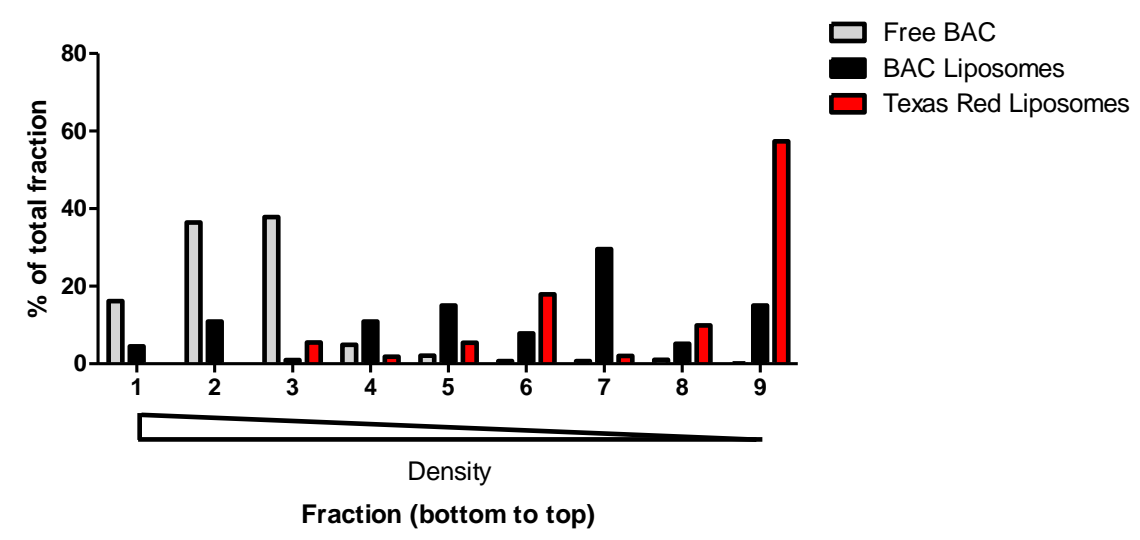

Figure 1.35: Incorporation of the palmitoyl-modified PNA sensor in liposomes. The relative BAC fluorescence intensity $\left(\lambda_{\mathrm{Ex}}=450 \mathrm{~nm}, \lambda_{\mathrm{Em}}=520 \mathrm{~nm}\right)$ was measured in the different fractions of the density gradient centrifugation. The fractions for the palmitoyl-PNA sensor with the liposomes are highlighted in black, liposomes incubated with Texas Red are shown in red and only sensor without liposomes is presented in grey. The experiment was performed by RASHI GOEL.

\subsubsection{Synthesis of TMD Modified PNA Sensors}

As the incorporation of the palmitoyl modified PNA sensors into the liposome was comparatively low, a more hydrophobic membrane anchor for improved incorporation had to be identified. Different studies of SNARE peptide model systems and spin labelled 
transmembrane peptides, revealed efficient incorporation of the transmembrane domain (TMD) modified peptides into liposomes. ${ }^{[139,140]}$ The transmembrane domains are a part of membranespanning protein domains, consisting predominantly of nonpolar amino acid residues. Screening of different transmembrane domains revealed the lysine-flanked model peptide (KALP), which is frequently used for anchoring of peptides into the membrane. ${ }^{[141]}$ The KALP TMD consists of an alternating leucine and alanine core as well as two lysine residues at the $N$ - and $C$-terminus. The amine group of the lysine side chains can interact with the polar lipid head groups and therefore enhances the solubility. Structurally, the KALP domain forms a stable $\alpha$-helical structure and is incorporated into the membrane in a transmembrane orientation. The TMD-modified PNA oligomer (37) was synthesised by the optimised PNA coupling protocol (see figure 1.36 A). To improve the coupling efficiency of the hydrophobic TMD domain, the amino acids were coupled with increased DIC $(0.1 \mathrm{M})$ and Oxyma $(0.2 \mathrm{M})$ concentration as well as prolonged reaction times $\left(10 \mathrm{~min}\right.$ at $\left.90^{\circ} \mathrm{C}\right)$. The recognition sequence for the cathepsin B protease and a short Gly-Ser linker sequence were placed between the TMD and the PNA sequence. After synthesis of the TMD-PNA oligomer (37), the Alloc protecting group was removed and the BAC fluorophore was conjugated under microwave irradiation. HPLC purification of the crude mixture yielded the TMD-PNA sensor (38) with a cathepsin B recognition motif, which was directly used for cleavage experiments. The cathepsin B protease was activated and transferred to the TMD modified PNA sensor. The cleavage process was monitored by U-HPLC after $30 \mathrm{~min}$ and $60 \mathrm{~min}$, revealing a complete conversion of the starting material (38) after $30 \mathrm{~min}$ (see figure $1.36 \mathrm{~B}$ ). These results were confirmed by mass spectrometry analysis. 

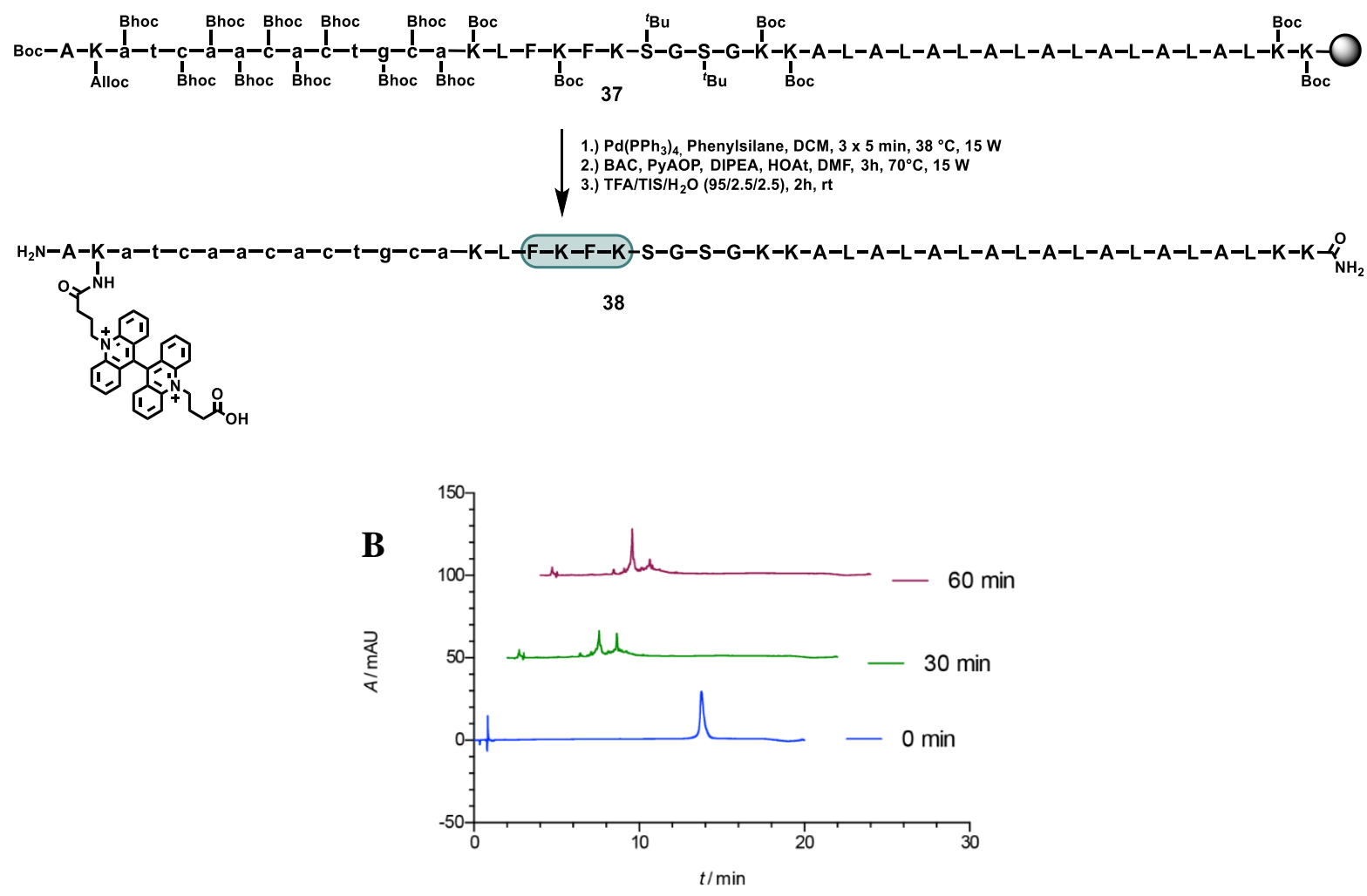

Figure 1.36: Synthesis and Cathepsin B cleavage of TMD-modified PNA sensors. A) Synthesised of a TMD modified peptide-PNA sensor (38) with a cathepsin B recognition sequence. The recognition sequence is highlighted in grey. B) Cathepsin B protease cleavage experiments of the cathepsin B modified TMD-PNA sensor (38) analysed by U-HPLC after incubation times of $0 \mathrm{~min}, 30 \mathrm{~min}, 60 \mathrm{~min}$ at $37{ }^{\circ} \mathrm{C}$. U-HPLC gradient: $10-60 \%$ $\mathrm{MeCN}$ in15 min.

\subsubsection{Membrane Incorporation and Distribution of TMD-Modified PNA Sensors}

Following the successful cathepsin B cleavage experiments, the incorporation efficiency of the TMD-modified PNA sensor (38) into liposome DOPC/DOPS/DOPE/cholesterol (63/10/2/25) by manual extrusion was analysed by density-gradient centrifugation (performed by RASHI GOEL). Measurements of the BAC fluorescence intensity in the different fractions revealed a high BAC fluorescence intensity in the top fraction of the density gradient (4). In contrast, marginal BAC signals were detected in the bottom fractions (1 and 2) (see figure 1.37). These results confirmed highly efficient incorporation of the BAC labelled TMD-PNA oligomer into the liposomes. The fluorophore Texas Red was again used as positive control and the floating properties of the TMD-PNA sensor without liposomes were analysed, revealing a low accumulation in the top fraction. 


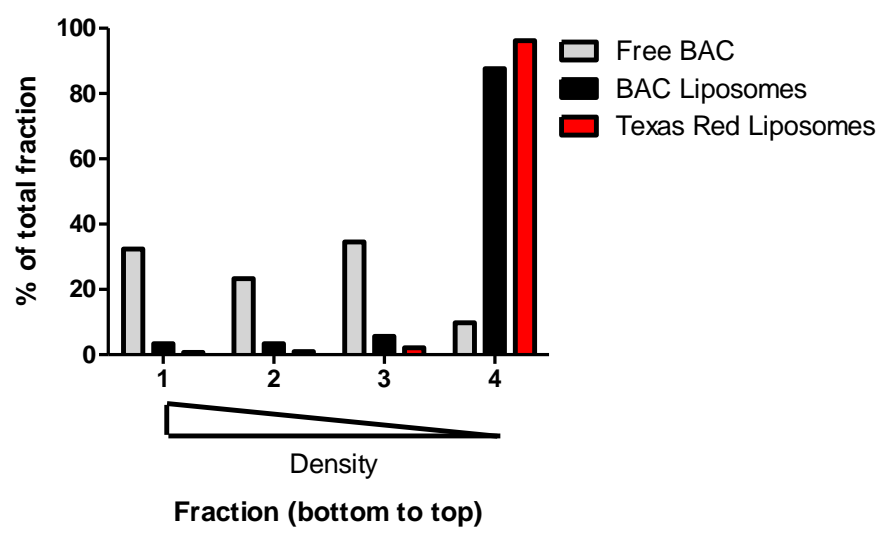

Figure 1.37: Incorporation assay of the TMD-modified PNA sensor in liposomes. The relative BAC fluorescence intensity $\left(\lambda_{\mathrm{Ex}}=450 \mathrm{~nm}, \lambda_{\mathrm{Em}}=520 \mathrm{~nm}\right.$ ) was measured in the different fractions of the density gradient centrifugation. The fractions for TMD-PNA sensor with the liposomes are highlighted in black, liposomes incubated with Texas Red are shown in red and only sensor without liposomes is presented in grey. The experiment was performed by RASHI GOEL.

After incorporation experiments of the TMD-PNA sensors (38) into liposomes, the incorporation efficiency between intra- and extravesicular orientated sensors was determined. The TMD part of the sensor was synthesised and labelled with the 7-nitro-2-1,3-benzoxadiazole (NBD)-fluorophore. The fluorescence of the NBD dye can be quenched by addition of sodium dithionite $\left(\mathrm{Na}_{2} \mathrm{~S}_{2} \mathrm{O}_{4}{ }^{2-}\right)$, which induces a reduction of the electron-withdrawing nitro group (39) to an electron-donating amine group (40) (see figure 1.38 A). The sodium dithionite is unable to cross the membrane due to the charges. Thus, only the NBD fluorescence of the extravesicular orientated sensors are quenched, whereas the intravesicular NBD fluorescence signals are not affected (see figure $1.38 \mathrm{~B}$ ).

A

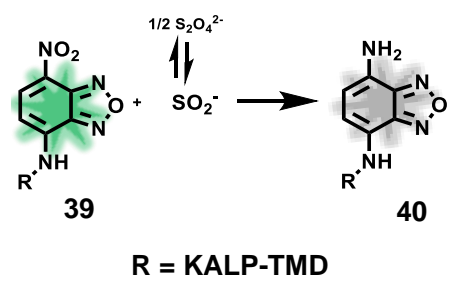

B

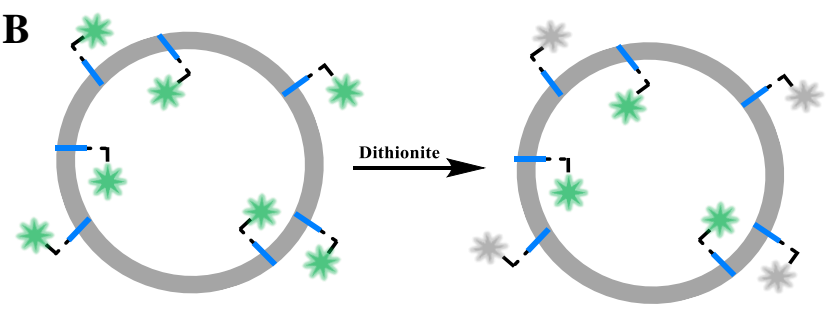

Figure 1.38: Quenching experiments in fluorophore labelled TMD peptides. A) Reduction of the 7-nitro-2,1,3benzoxoadiazol-4-yl (NBD) fluorophore (39) to the corresponding 7-amino-2,1,3-benzoxoadiazol-4-yl (40) by addition of sodium dithionite. B) The sodium dithionite quenches all extravesicular orientated NBD-labelled KALP-TMD oligomers, whereas intracellular sensors are not quenched.

The NBD-labelling of the TMD sequence was performed according to the literature procedure (see figure $1.39 \mathrm{~A}) .{ }^{[123]}$ In the next step, the Alloc protecting group was removed and the NBD$\mathrm{Cl}$ was coupled to the $\mathrm{N}$-terminal lysine residue with a mixture of DIPEA in DMF at $\mathrm{rt}$ for $24 \mathrm{~h}$. 
After cleavage from the resin and subsequent HPLC purification, the NBD-labelled TMD oligomer (42) was incorporated into liposomes by manual extrusion. The incorporation efficiency of the NBD labelled TMD oligomer was measured before and after treatment with sodium dithionite. The proteoliposomes were incubated with $50 \mathrm{mM} \mathrm{Na} 2 \mathrm{~S}_{2} \mathrm{O}_{4}$ for $5 \mathrm{~min}$ at $\mathrm{rt}$. Fluorescence measurements revealed an NBD fluorescence reduction of about $55 \%$ after quenching with sodium dithionite (see figure $1.39 \mathrm{~B}$ ). These results highlight the nearly equal intra- and extravesicular integration of the NBD labelled TMD oligomer (42).
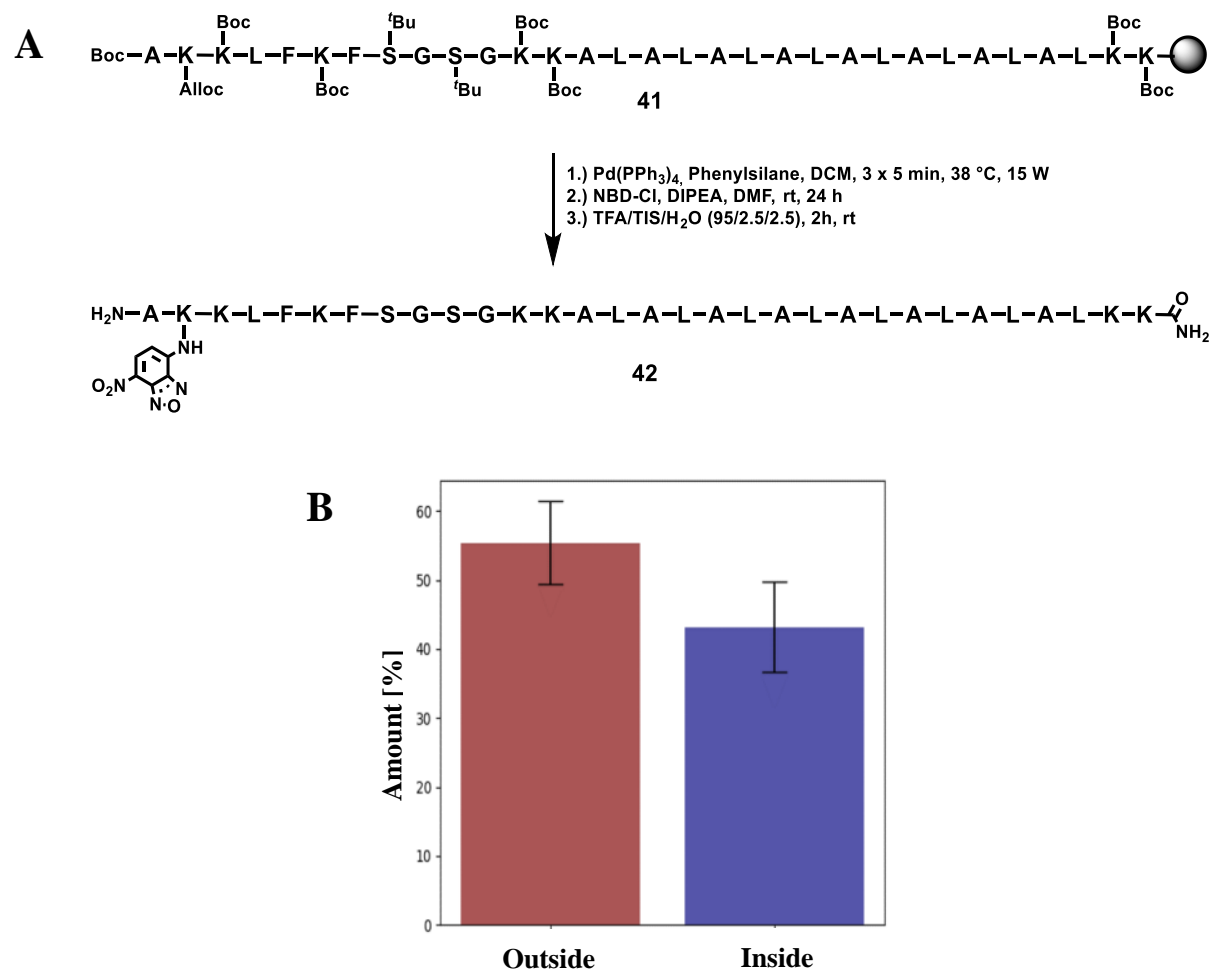

Figure 1.39: Synthesis and incorporation assay of NBD labelled TMD peptides. A) Synthesis of NBD modified TMD sequence (42). B) Fluorescence change after treatment of lipid vesicles containing NBD-labelled TMD oligomers with sodium dithionite. The experiment was performed by FREDERIKE MAAß.

\subsubsection{Formation of the Chloride Sensitive PNA-DNA Complex}

After successful incorporation of the TMD-PNA sensors (38) into liposomes, with complementary DNA oligomers (D1: stabilizing module, D2: normalizing module) was investigated next. The formation of the TMD modified PNA sensors (38) and the commercially available D1 and D2 strands were analysed by a gel mobility shift assay on a native polyacrylamide gel (see figure 1.40; see table 1.4). First, all three components were mixed in equimolar ratios and were annealed at $90{ }^{\circ} \mathrm{C}$ for $5 \mathrm{~min}$, decreasing the temperature every $15 \mathrm{~min}$ by $5{ }^{\circ} \mathrm{C}$. Analysis of the TMD- $\mathrm{Cl}^{-}$sensor formation revealed only a complexation of the D1-D2 oligomers (lane 4). Adaptation of the annealing temperature for the TMD-PNA strand with the 
preformed D1-D2 complex was tested, to optimize process conditions (lane 5-7). However, only for the annealing temperature of $35^{\circ} \mathrm{C}$, small amounts of the $\mathrm{TMD}-\mathrm{Cl}^{-}$sensor were observed (lane 7).

Further improvement of complex formation by increasing the concentration of TMD-PNA strand could not be achieved (data not shown). The BAC labelled PNA oligomer (11) without a TMD was used as a positive control, revealing the PNA-DNA complex formation and only small amounts of D1-D2 (lane 3).

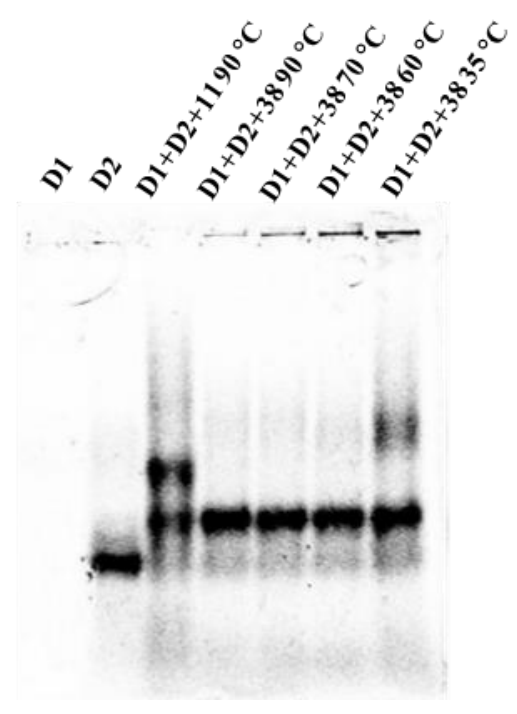

Figure 1.40: Gel mobility shift assay for visualisation of the $\mathrm{Cl}^{-}$sensor formation. The captured band detection results from the emission of the AlexaFluor 647 fluorophore. Lane 1: D1, Lane 2: D2, Lane 3: D1+D2+11, Lane 4: D1+D2+38 at $90{ }^{\circ} \mathrm{C}$, Lane 5: D1+D2+38 at $70{ }^{\circ} \mathrm{C}$, Lane 6: D1+D2+38 at $60{ }^{\circ} \mathrm{C}$, Lane 7: D1+D2+38 at $35{ }^{\circ} \mathrm{C}$. The experiment was performed by BENJAMIN IDE.

Table 1.4: Sequence of the peptide-PNA and DNA oligomers..

\begin{tabular}{|c|c|c|}
\hline Name & Sequence & Comment \\
\hline D1 & 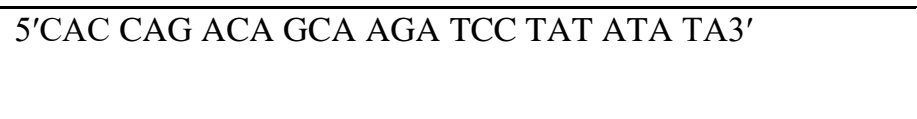 & $\begin{array}{l}\text { DNA strand: stabilizing module } \\
(\mathrm{M}=7996 \mathrm{~g} / \mathrm{mol})\end{array}$ \\
\hline D2 & $\begin{array}{l}\text { 5'TAT AT(AlexaFluor 647)A TAG GAT CTT GCT GTC TGG } \\
\text { TGT GCA GTG TTG AT3' }\end{array}$ & $\begin{array}{l}\text { DNA strand: normalizing module } \\
(\mathrm{M}=12571 \mathrm{~g} / \mathrm{mol})\end{array}$ \\
\hline 38 & $\begin{array}{l}\mathrm{H}_{2} \mathrm{~N}-\mathrm{A}-\mathrm{K}(\mathrm{BAC})-\mathrm{a}-\mathrm{t}-\mathrm{c}-\mathrm{a}-\mathrm{a}-\mathrm{c}-\mathrm{a}-\mathrm{c}-\mathrm{t}-\mathrm{g}-\mathrm{c}-\mathrm{a}-\mathrm{K}-\mathrm{L}-\mathrm{F}-\mathrm{K}-\mathrm{F}-\mathrm{K}-\mathrm{K}-\mathrm{L}-\mathrm{A}- \\
\text { L-A-L-A-L-A-L-A-L-A-L-A-L-A-K-K-CONH }\end{array}$ & $\begin{array}{l}\text { PNA strand: sensing module } \\
(\mathrm{M}=6580 \mathrm{~g} / \mathrm{mol})\end{array}$ \\
\hline 11 & $\mathrm{H}_{2} \mathrm{~N}-\mathrm{A}-\mathrm{K}(\mathrm{BAC})-\mathrm{a}-\mathrm{t}-\mathrm{c}-\mathrm{a}-\mathrm{a}-\mathrm{c}-\mathrm{a}-\mathrm{c}-\mathrm{t}-\mathrm{g}-\mathrm{c}-\mathrm{a}-\mathrm{K}-\mathrm{CONH}_{2}$ & $\begin{array}{l}\text { PNA strand: sensing module } \\
(\mathrm{M}=4057 \mathrm{~g} / \mathrm{mol})\end{array}$ \\
\hline
\end{tabular}


This inefficient $\mathrm{Cl}^{-}$sensor complex formation of the TMD-modified PNA oligomer (38) with the complementary DNA strands might be caused by the hydrophobic TMD, which avoid interactions with the charged DNA backbone. Also, the steric hindrance and rigidity of the $\alpha$-helical structure from the TMD can block the interaction with the DNA strands. Due to the incomplete complex formation, the $\mathrm{Cl}^{-}$sensor cannot be used for precise measurements. This might cause the batch-to-batch variability between the $\mathrm{Cl}^{-}$sensitive BAC fluorophore and the $\mathrm{Cl}^{-}$insensitive AlexaFluor 647 fluorophore. Based on these results, a new $\mathrm{Cl}^{-}$sensor with improved complex formation as well as efficient encapsulation and cleavage of the extravesicular orientated sensors was required.

\subsubsection{Synthesis of His-Tag Modified PNA Sensors}

Since the hydrophobic TMD hinders the interaction of PNA and complementary DNA, a new membrane anchoring system for the $\mathrm{Cl}^{-}$sensor was required. The demanded characteristic of reduced hydrophobicity and efficient removal of extravesicular orientated sensors could be found in affinity tags. VASIC et al. firstly presented this interesting approach of protein incorporation into liposomes, by introducing an affinity tag to the substrate and thereby increasing their encapsulation efficient during liposome formation. ${ }^{[142]}$ Therefore, a polyhistidine sequence can be introduced to the $N$ - or $C$-terminus of the target protein. The benefit of the polyhistidine sequence (His-tag) is the strong interaction with $\mathrm{Ni}^{2+}$, which can be incorporated as $\mathrm{Ni}^{2+}$-nitrilotriacetic acid (NTA) labelled phospholipids in liposomes (see figure 1.41). The addition of imidazole leads to efficient removal of the histidine- $\mathrm{Ni}^{2+}$ interaction, which is a crucial requirement for the elimination of the extravesicular orientated sensors. Thus a His-tag modified PNA sensor was synthesised for improving the sensor complex formation. The protease cleavage site is no longer required, as the extravesicular orientated sensors can be removed by imidazole. 

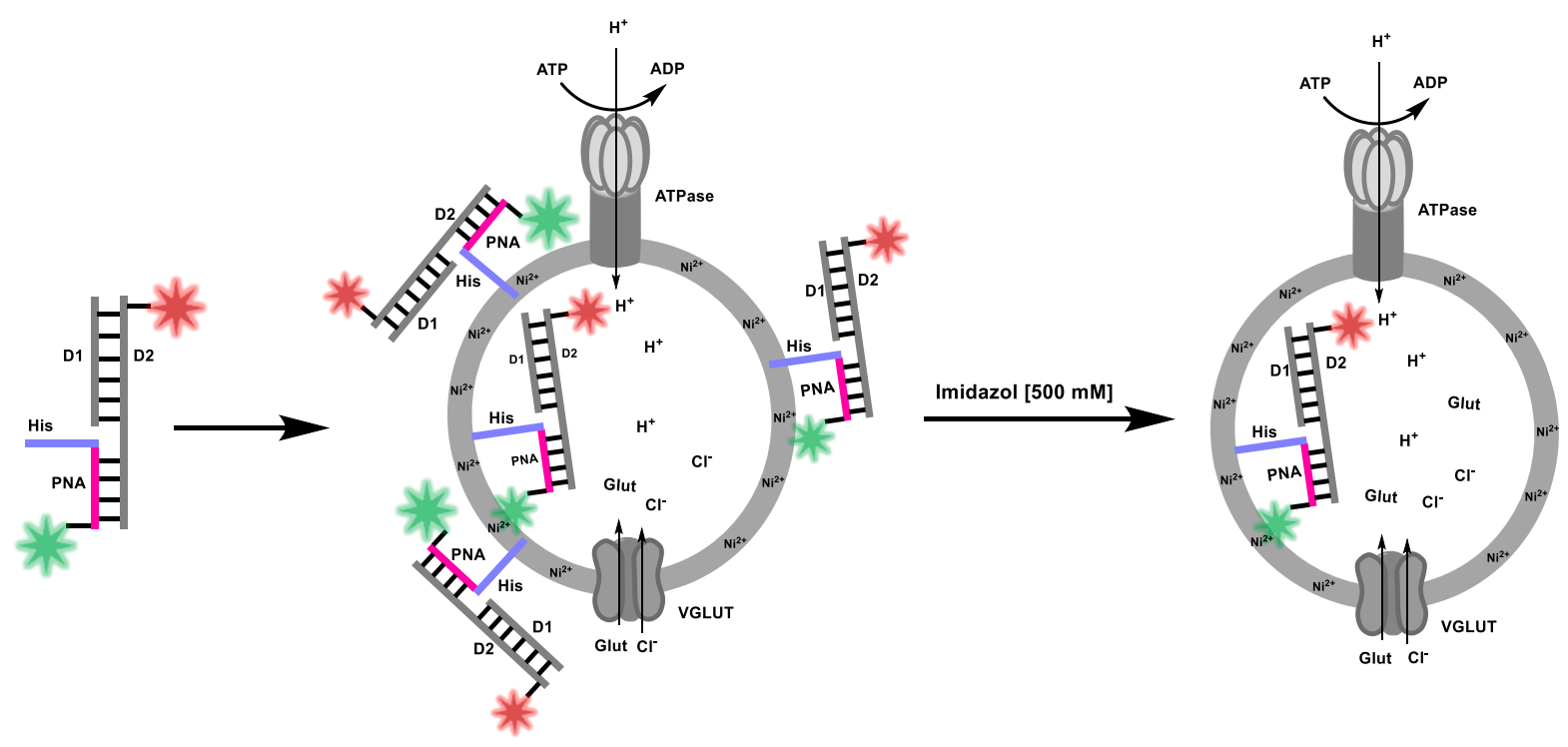

Figure 1.41: Strategy for a more effective $\mathrm{Cl}^{-}$-sensor encapsulation. For this approach, $\mathrm{Ni}^{2+}-\mathrm{NTA}$ labelled liposomes were used. The His-tagged $\mathrm{Cl}^{-}$-sensor was incorporated in $\mathrm{Ni}^{2+}-\mathrm{NTA}$ modified liposomes. After detergent removal, the $\mathrm{Cl}^{-}$-sensors were coupled to the intra-and extravesicular site of the membrane. Following, treatment with imidazole cleaves the extravesicular bound sensors.

\subsubsection{Synthesis of a His14-Tag PNA Sensors}

Polyhistidine tags are widely disseminated for protein purification and consist of six or more consecutive histidine residues, which guarantee a high selectivity due to the rare occurrence of oligohistidine segments in proteins. The tag can be introduced to the $C$ - or $N$-terminus of the target protein by polymerase chain reaction (PCR) or encoding the target protein in a vector. The synthesis of histidine sequences by SPPS is challenging due to their tendency to epimerise during the coupling step (see figure 1.42.). The free electron pair of the imidazole $N^{\pi}$ thereby deprotects the $\alpha$-carbon (43), forming an achiral ester enolate (44), which can be converted to the L- or D-isomer (44). ${ }^{[143]}$ Analysis of different histidine protecting groups revealed that coupling of histidine with the frequently used trityl (Trt) group at $50{ }^{\circ} \mathrm{C}$ for $10 \mathrm{~min}$ lead to an elevated formation of the D-isomer $(5.8 \%)$. However, Boc-protected histidine revealed a reduced D-isomer formation of $0.18 \%$ under the same conditions. The Fmoc-His(Boc)-OH also showed a low tendency to epimerize (1.2\%) at higher coupling temperatures of $90{ }^{\circ} \mathrm{C}$ for $2 \mathrm{~min}$ compared to Fmoc-His(Trt)-OH $(22 \%) .{ }^{[144]}$ This can be explained by the decreased basicity caused by the electron-withdrawing effect of the carbamate group. 


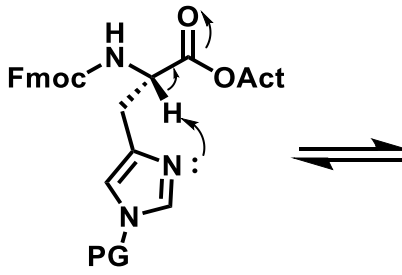

43

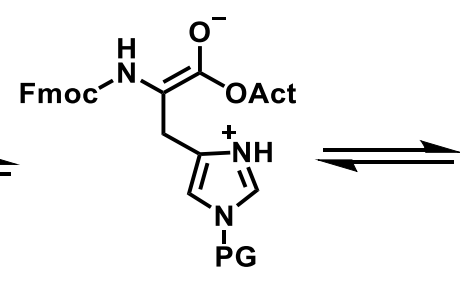

44

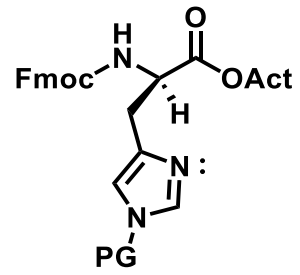

45

Figure 1.42: Epimerization of Fmoc-His(PG)-OH during SPPS activation.

Previous experiments from LATA et al. demonstrated that longer polyhistidine-tags (His 10 ) possess 10 -fold higher affinity compared to the frequently used His 6 -tag. ${ }^{[145-147]}$ Further studies from VASIC revealed a high encapsulation efficiency of proteins modified with a His 14 sequence in $\mathrm{Ni}^{2+}$ containing liposomes. ${ }^{[142]}$ The His 14 sequence consists of four polyhistidine segments with three or four residues each, which are connected by flexible glycine, serine or threonine linkers. The structure of the His 14 sequence allows the preferred interaction of two histidine residues $(\mathrm{i}$ and $\mathrm{i}+2 / \mathrm{i}$ and $\mathrm{i}+5)$ with the $\mathrm{Ni}^{2+}$.

To develop efficient SPPS conditions for the synthesis of the His 14 PNA sensor, the Bocprotected histidine residues were used and the coupling was performed at a reduced temperature of $50{ }^{\circ} \mathrm{C}$ to minimize epimerization (see figure $1.43 \mathrm{~A}$ ). ${ }^{[144]}$ Further increased coupling times of two times $25 \mathrm{~min}$ and higher activator concentrations (DIC: $0.1 \mathrm{M}$, Oxyma: $0.2 \mathrm{M}$ ) lead to an improved crude purity compared to initial coupling conditions $\left(10 \mathrm{~min}, 75^{\circ} \mathrm{C}\right)$. The PNA part of the oligomer was synthesised by the optimised coupling conditions. After completion of the coupling process, a test cleavage and LC-MS analysis were performed, revealing a successful synthesis of the His ${ }_{14}$-PNA oligomer (46). In the final step, the Alloc protection group of the $N$-terminal lysine residue was cleaved, and the BAC fluorophore (4) was introduced. HPLC purification and mass analysis revealed the formation of the BAC-labelled His ${ }_{14}$-PNA sensor (47) with high purity (see figure $1.42 \mathrm{~B}$ and $1.42 \mathrm{C}$ ). 
A

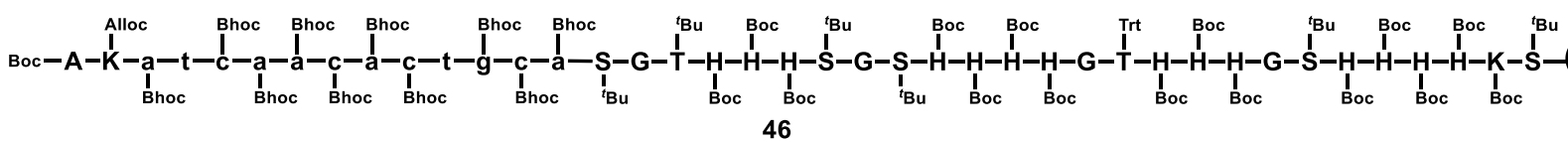

1.) $\mathrm{Pd}\left(\mathrm{PPh}_{3}\right)_{4}, \mathrm{Phenylsilane,} \mathrm{DCM}, 3 \times 5 \mathrm{~min}, 38^{\circ} \mathrm{C}, 15 \mathrm{~W}$ 2.) BAC, PYAOP, DIPEA, HOAt, DMF, $3 \mathrm{~h}, 70^{\circ} \mathrm{C}, 15 \mathrm{~W}$

3.) $\mathrm{TFA} / \mathrm{TIS} / \mathrm{H}_{2} \mathrm{O}(95 / 2.5 / 2.5), 2 \mathrm{~h}$, rt

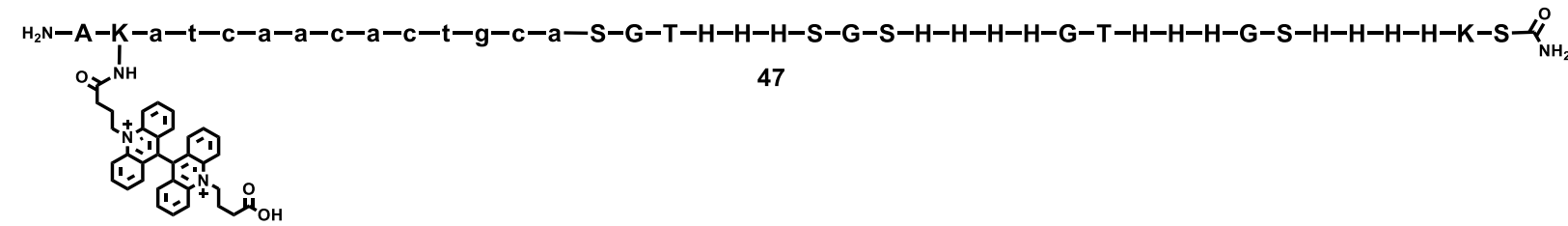

B

C
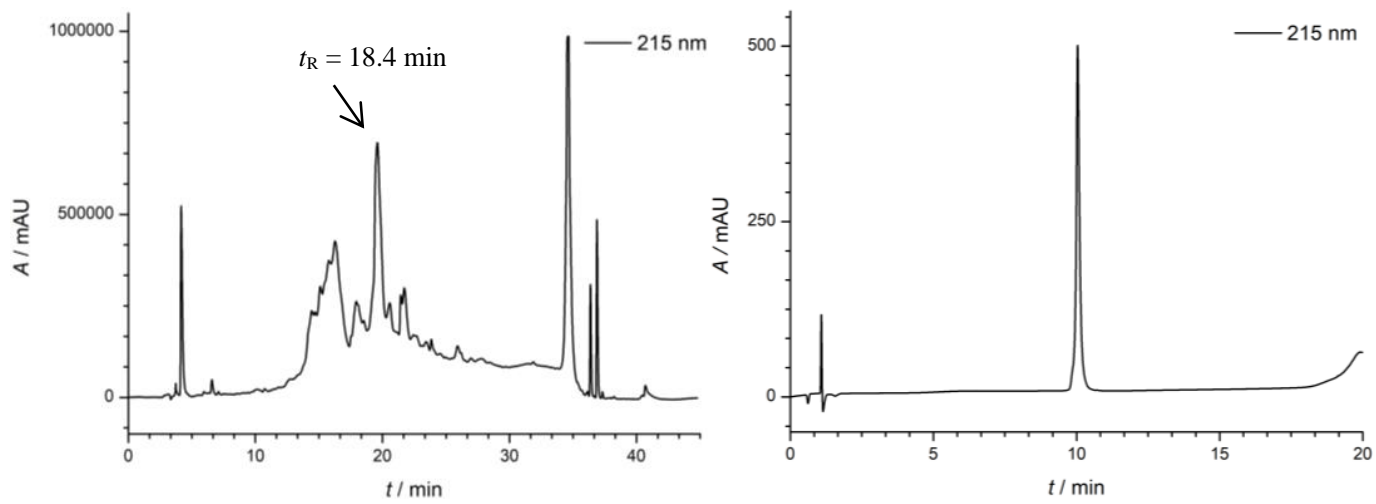

Figure 1.43: Synthesis and analysis of His 14 -PNA sensors. A) Synthesis of BAC-labelled His ${ }_{14}$-PNA oligomer (47). B) HPLC chromatogram of the crude His ${ }_{14}$-PNA oligomer (47) after BAC labelling. The isolated product peak is highlighted with an arrow. HPLC gradient: $1-40 \% \mathrm{MeCN}$ in $30 \mathrm{~min}$. C) U-HPLC chromatogram of the purified BAC-labelled-His ${ }_{14}$-PNA oligomer (47). HPLC gradient: 1-40\% MeCN in 15 min.

\subsubsection{Characterisation of His14-Tag Chloride Sensor}

\subsubsection{Formation studies of the His14-Tag Chloride Sensor}

The BAC-labelled His 14 -PNA oligomer (47) was characterised by JENNIFER STRUCK and RASHI GoEl (Group of PROF REINHARD JAHN). In the first step, the $\mathrm{Cl}^{-}$sensor formation of the BAClabelled His ${ }_{14}$-PNA oligomer (47) with the complementary DNA strands was analysed by a gel shift assay on a native polyacrylamide gel (PAGE) (see figure 1.44). Mixing of equimolar ratios of the PNA and DNA strands lead to the formation of the desired $\mathrm{Cl}^{-}$sensor, with a low amounts of free D1 and D2 complex (lane 4). To achieve a full complexation, an increased ratio of 1.25 of the His 14 -PNA oligomer (47) was used, showing the formation of the $\mathrm{His}_{14}-\mathrm{Cl}^{-}$sensor without free DNA complexes (lane 5). Further increase of the PNA sensor ratio to 1.5 did not achieve 
an improvement of the complex formation (lane 6). Consequently, the following experiments were performed in a molar ratio of 1:1:1.25.

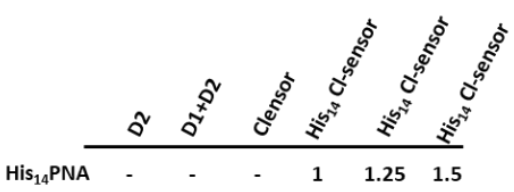

Figure 1.44: Gel mobility assay visualizing the His 14 Clensor formation. The captured band is indicated by the emission of the AlexaFluor 647 fluorophore. Lane 1: D2, Lane 2: D1+D2, Lane 3: D1+D2+11 (molar ratio: 1:1:1), Lane 4: D1+D2+47 (molar ratio: 1:1:1), Lane 5: D1+D2+47 (molar ratio: 1:1:1.25), Lane 6: D1+D2+47 (molar ratio: 1:1:1.5). The experiment was performed by JENNIFER STRUCK.

In the next step, the sensitivity of the $\mathrm{His}_{14}$ modified sensor towards $\mathrm{Cl}^{-}$was evaluated, by measuring the emission spectra of the BAC- and AlexaFluor 647 fluorophore in the physiological range of $\mathrm{Cl}^{-}(0-75 \mathrm{mM})$. The fluorescence intensity of the BAC fluorophore was reduced in a dose-dependent manner with increasing $\mathrm{Cl}^{-}$concentrations when measured at the wavelength of $505 \mathrm{~nm}(\mathrm{G})$ (see figure $1.45 \mathrm{~B}$ ). However, the fluorescence intensity of the AlexaFluor 647 fluorophore (R), measured at a wavelength of $670 \mathrm{~nm}$, remained constant (see figure $1.45 \mathrm{C}$ ). For quantification of the $\mathrm{Cl}^{-}$concentration, the STERN-VOLLMER quenching constant $\left(K_{\mathrm{SV}}\right)$ of the $\mathrm{His}_{14} \mathrm{Cl}^{-}$sensor was calculated. $\mathrm{A} \mathrm{Cl}^{-}$calibration profile was created, fitting the $\mathrm{R} / \mathrm{G}$ ratios dependent on the $\mathrm{Cl}^{-}$concentration, according to the STERN-VOLLMER equation (see figure $1.45 \mathrm{D}$ ). Based on the $\mathrm{Cl}^{-}$calibration profile, an apparent $K_{\mathrm{SV}}$ of $21.15 \pm 0.33 \mathrm{M}^{-1}$ for the His ${ }_{14} \mathrm{Cl}^{-}$sensor was calculated. 
A

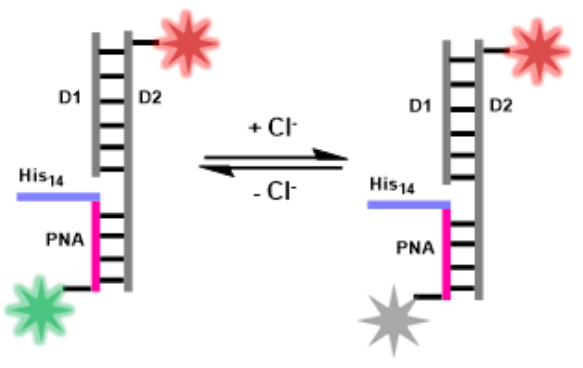

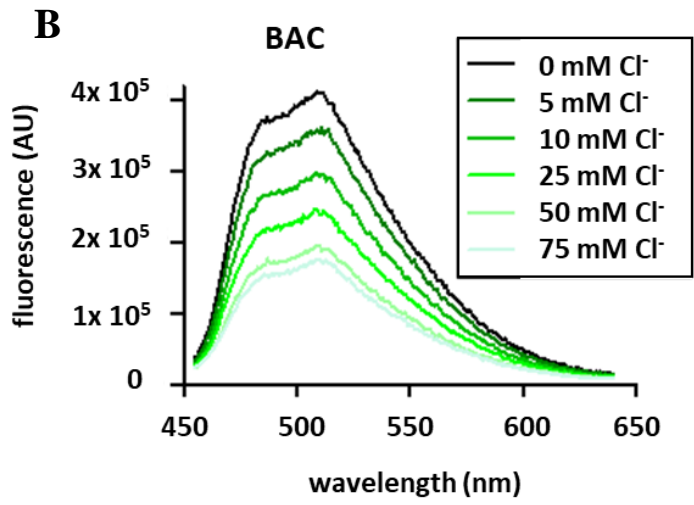

D

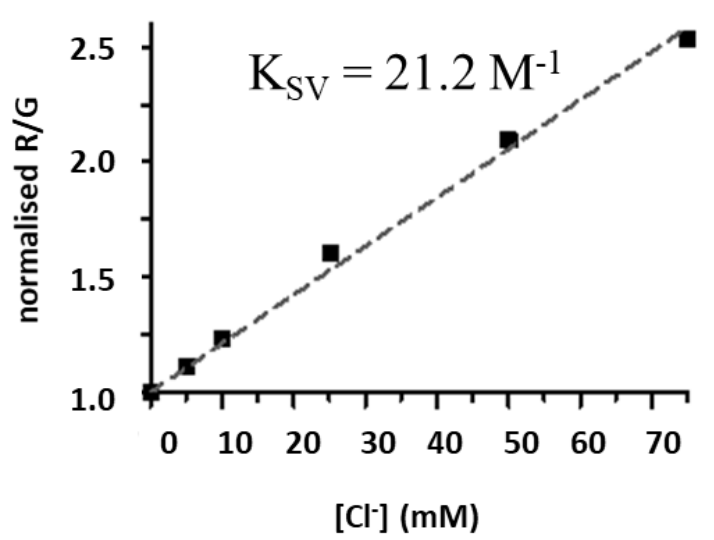

Figure 1.45: $\mathrm{Cl}^{-}$sensitivity measurements of the His ${ }_{14}-\mathrm{Cl}^{-}$sensor. A) Structure of the His ${ }_{14}$-Clensor, PNA sensing module (pink) contains the His 14 tag (blue) and the BAC fluorophore (green), D1 stabilizing module (grey) and D2 normalizing module (grey) containing the AlexaFluor 647 fluorophore (red). B) BAC fluorescence emission spectra at $\left[\mathrm{Cl}^{-}\right]$ranging from $0-75 \mathrm{mM}$ using $\lambda_{\mathrm{Ex}}=434 \mathrm{~nm}$. C) AlexaFluor 647 fluorescence emission spectra at $\left[\mathrm{Cl}^{-}\right]$ranging from $0-75 \mathrm{mM}$ using $\lambda_{\mathrm{Ex}}=650 \mathrm{~nm}$. D) Calibration profile of His $\mathrm{H}_{14}$-Clensor visualizing AlexaFluor 647 and BAC fluorescence intensity ratio $(\mathrm{R} / \mathrm{G})$ versus $\left[\mathrm{Cl}^{-}\right]$. The $\mathrm{R} / \mathrm{G}$ values were normalized to blank. The experiments were performed by JENNIFER STRUCK.

\subsubsection{His14-Tag Chloride Sensor Encapsulation into Liposomes}

After successful complex formation and measurements of the $\mathrm{Cl}^{-}$sensitivity, the encapsulation efficiency of the $\mathrm{His}_{14}-\mathrm{Cl}^{-}$sensor into liposomes and further their cleavage from the extravesicular site was evaluated in the following steps. For efficient removal of the extravesicular incorporated sensors, three different approaches were developed using imidazole to remove the His-tag, a DNase I to degrade the sensor or a combination of imidazole and the enzyme.

Before the cleavage experiments can be performed, the integrity of the $\mathrm{His}_{14}-\mathrm{Cl}^{-}$sensor towards imidazole and DNase I was verified by gel mobility assay, to exclude denaturation of the sensor 
by imidazole or blockage of the DNase activity by the PNA structure (see figure 1.46). The results of the gel mobility assay revealed no affection of the $\mathrm{His}_{14}-\mathrm{Cl}^{-}$sensor integrity after the treatment with $400 \mathrm{mM}$ imidazole, whereas treatment with DNase I $(1 \mathrm{U} / \mu \mathrm{L})$ resulted in the degradation of the sensor. Surprisingly, the combination of both compounds leads to incomplete degradation of the DNA strands, presumably caused by the reduced enzymatic activity of the DNase induced by imidazole. Interestingly, incubation of the BAC fluorophore with imidazole revealed a quenching effect for the BAC fluorescence, whereas the emission spectrum of the AlexaFluor 647 fluorophore remains unaffected (data not shown). Based on these findings, it is essential to incorporate a dialysis step in the encapsulation procedure to exclude falsification of the BAC fluorescence by imidazole.

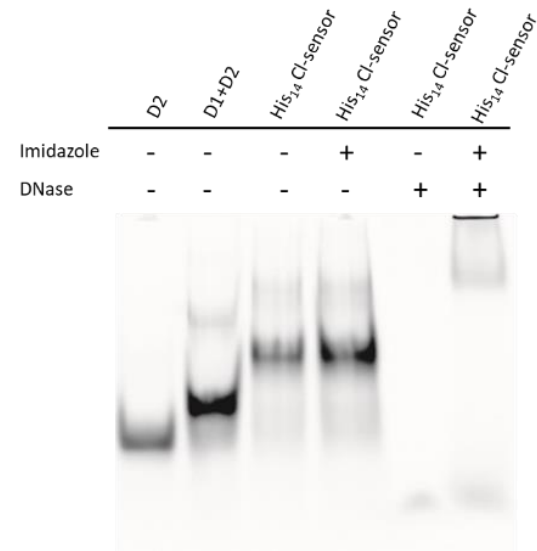

Figure 1.46: Integrity analysis of $\mathrm{His}_{14} \mathrm{Cl}^{-}$sensor by gel mobility shift assay. The sensor was incubated with imidazole, DNase I or with a combination. Lane 1: D2, Lane 2: D1 and D2, Lane 3: His ${ }_{14-\mathrm{Cl}^{-}}$sensor, Lane 4: His 14- $^{-}$ $\mathrm{Cl}^{-}$sensor treated with $400 \mathrm{mM}$ imidazole, Lane 5: $\mathrm{His}_{14}-\mathrm{Cl}^{-}$sensor treated with DNase I, Lane 6: $\mathrm{His}_{14}-\mathrm{Cl}^{-}$sensor treated with imidazole and DNase I. Experiment was performed by JENNIFER STRUCK.

After the assessment of $\mathrm{His}_{14}-\mathrm{Cl}^{-}$sensor integrity towards imidazole and DNase, the incorporation efficiency and the cleavage of extravesicular orientated sensors by imidazole or DNase were analysed. Therefore, liposomes (DOPC $63 \%$, DOPS $10 \%$, cholesterol $25 \%$ ) modified with DGS-NTA $\left(\mathrm{Ni}^{2+}\right)$ lipids $(2 \%)$ were prepared and the $\mathrm{His}_{14}-\mathrm{Cl}^{-}$sensor was incorporated by manual extrusion. The incorporation of the sensors into the liposomes was evaluated by density gradient centrifugation, measuring the fluorescence intensity of the top fractions. The AlexaFluor 647 emission intensity was used as a readout for the incorporation and cleavage efficiency, as the BAC fluorescence is quenched by imidazole.

The results from the co-flotation experiments revealed a 3.7 times higher $\mathrm{His}_{14}-\mathrm{Cl}^{-}$sensor incorporation in liposomes containing $2 \%$ DGS-NTA $\left(\mathrm{Ni}^{2+}\right)$ lipids, compared to liposomes without $\mathrm{Ni}^{2+}$ lipids (see figure $1.47 \mathrm{~A}$ ). Treatment of $\mathrm{Ni}^{2+}$ modified liposomes with imidazole 
resulted in a decrease of the AlexaFluor 647 fluorescence intensity by approximately $50 \%$, compared to $\mathrm{Ni}^{2+}$ liposome without imidazole. However, the fluorescence intensity for $\mathrm{Ni}^{2+}$ free liposome remained constant after imidazole addition. These results confirm successful incorporation of the $\mathrm{His}_{14}-\mathrm{Cl}^{-}$sensor in $\mathrm{Ni}^{2+}$ modified liposomes and the cleavage of extravesicular orientated sensors. The reduced fluorescence intensity of $50 \%$ after imidazole treatment indicates an equal intra- and extravesicular integration of the sensor, similar to previous investigations with the TMD based sensors (see chapter 1.3.5.1). Despite the successful encapsulation, the incorporation efficiency of the $\mathrm{His}_{14}-\mathrm{Cl}^{-}$sensor into the liposome was relatively low, probably caused by steric hindrance of the PNA-DNA scaffold or low accessibility of the $\mathrm{His}_{14}$ tag to the $\mathrm{Ni}^{2+}$-NTA lipids.

On the contrary, the results for DNase mediated cleavage of extravesicular orientated $\mathrm{His}_{14} \mathrm{Cl}^{-}$ sensor revealed a reduced fluorescence intensity of AlexaFluor 647, whereas the BAC emission intensity was increased (see figure $1.47 \mathrm{~B}$; BAC data not shown). This effect can probably be explained by the resistance of the PNA structure towards the DNase, which only degrades the D1 and D2 strands by cleaving their phosphate backbone. Considering the outcome of these experiments, imidazole was used as cleavage reagent for the extravesicular orientated sensor in further experiments.
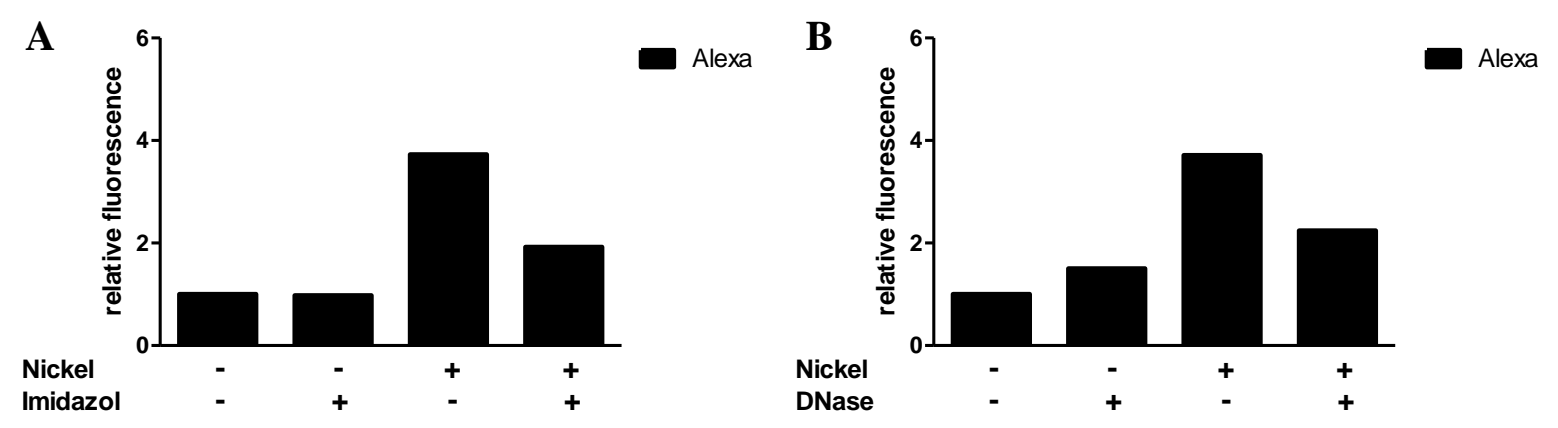

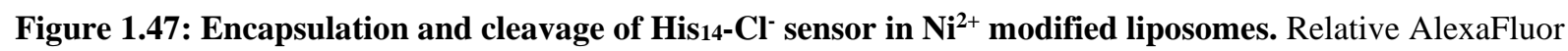
647 fluorescence intensity of the top fractions from density gradient centrifugation normalized to the control fluorescence intensity. A) Top fractions of density gradient centrifugation for liposomes with or without $\mathrm{Ni}^{2+}$ and imidazole. B) Top fractions of density gradient centrifugation for liposomes with or without $\mathrm{Ni}^{2+}$ and $\mathrm{DNase}$. The experiments were performed by JENNIFER STRUCK.

\subsubsection{Purification and Chloride Measurements of Sensor Modified Liposomes}

After successful encapsulation of the sensor into liposomes, the $\mathrm{His}_{14}-\mathrm{Cl}^{-}$sensor was separated from the cleaved free sensor by gel filtration chromatography. The reduced interaction with the stationary phase of the liposomes was taken as an advantage to separate the liposomes form 
free sensor (data not shown). The structural integrity of the liposome was subsequently verified by dynamic light scattering (DLS) measurements.

Next, the efficiency of the separation process was analysed. The fractions of the purified liposomes were therefore separated by density gradient centrifugation, revealing a high fluorescence intensity in the top fractions (8-10) and only minor amounts of the unbound sensor (fractions 1-5) (see figure 1.48 A). The purified liposomes were used to determine the chloride sensitivity of the encapsulated $\mathrm{His}_{14}-\mathrm{Cl}^{-}$sensors. Measurements of the AlexaFluor $647(\mathrm{R})$ and BAC $(G)$ ratio of intact liposomes with encapsulated sensor showed no response to increasing $\mathrm{Cl}^{-}$concentrations. Contrary, disruption of the liposomes with Triton X-100 caused a dosedependent increase of the $\mathrm{R} / \mathrm{G}$ ratio due to the release of the BAC fluorophore from the luminal liposome site (see figure 1.48 B). Calculations of the apparent STERN-VOLLMER quenching constant revealed for the intact liposomes a $K_{\mathrm{SV}}$ of $0.99 \mathrm{M}^{-1}$ and after disruption a quenching constant of $K_{\mathrm{Sv}}=13.58 \mathrm{M}^{-1}$. These results approved the successful encapsulation and efficient cleavage of the extravesicular orientated $\mathrm{His}_{14}-\mathrm{Cl}^{-}$sensors. The sensors still reveal a dosedependent response towards $\mathrm{Cl}^{-}$concentration after liposome reconstitution. The difference in $K_{\mathrm{SV}}$ compared to the free $\mathrm{His}_{14}-\mathrm{Cl}^{-}$sensor $\left(K_{\mathrm{SV}}=21.15 \mathrm{M}^{-1}\right)$ might be caused by the lipid environment changes in the buffer composition or by imidazole, which was not completely removed during the dialysis steps.

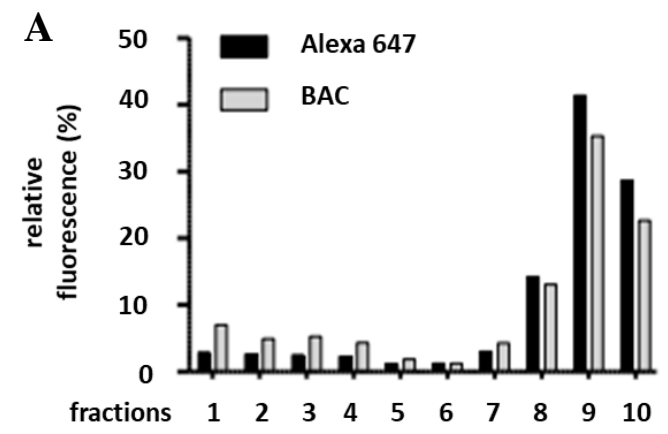

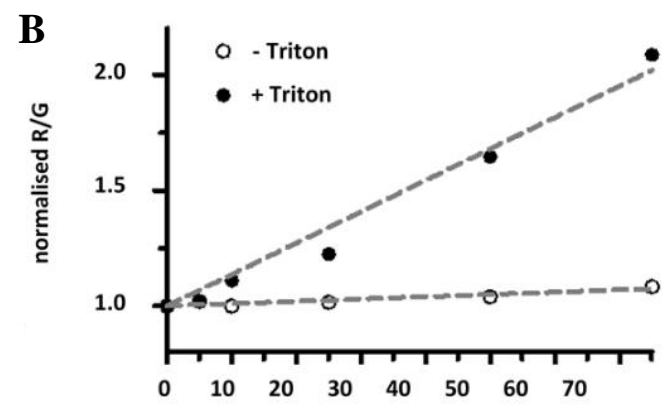

$[\mathrm{Cl}](\mathrm{mM})$

Figure 1.48: Purification and $\mathrm{Cl}^{-}$measurements of encapsulated His $\mathrm{H}_{14}$ sensors in liposomes. A) Density gradient chromatography profile of purified liposomes containing the His ${ }_{14}$-Clensor. The fractions were analysed and the relative fluorescence intensity was calculated based on the total fluorescence intensity of all fractions. B) Measurements of the $\mathrm{Cl}^{-}$calibration profile of liposomes containing the $\mathrm{His}_{14} \mathrm{Cl}^{-}$sensor either treated with or without Triton X-100. The emission spectra of BAC and AlexaFluor 647 were measured at $\mathrm{Cl}^{-}$concentrations ranging from 0-75 mM. The $\mathrm{R} / \mathrm{G}$ values at different $\mathrm{Cl}^{-}$concentrations were normalized to $0 \mathrm{mM} \mathrm{Cl}^{-}$, equation of linear fit $\mathrm{R} / \mathrm{G}=1+0.99 \mathrm{M}^{-1} \cdot\left[\mathrm{Cl}^{-}\right]$; plus Triton $\mathrm{X}-100 \mathrm{R} / \mathrm{G}=1+13.58 \mathrm{M}^{-1}\left[\mathrm{Cl}^{-}\right]$. The experiments were performed by JENNIFER STRUCK. 


\subsubsection{Functional Assays of the His14-Tag Chloride Sensor}

To measure the intravesicular $\mathrm{Cl}^{-}$concentrations in the liposomes, the $\mathrm{His}_{14}-\mathrm{Cl}^{-}$sensor has to be encapsulated in $\mathrm{Ni}^{2+}$ modified liposomes together with VGLUT1 and $\mathrm{TF}_{0} \mathrm{~F}_{1}$ (ATP synthase for thermophilic Bacillus PS3). In preliminary experiments, the activity of VGLUT and $\mathrm{TF}_{0} \mathrm{~F}_{1}$ in reconstituted $\mathrm{Ni}^{2+}$ modified liposomes was confirmed, showing ATP-induced acidification and influx of external $\mathrm{Cl}^{-}$as a counter ion (data not shown). Changes of the luminal $\mathrm{pH}$ were measured with $\mathrm{pH}$-sensitive dye Acridine Orange (AO). Furthermore, acidification experiments were performed in the presence of imidazole, which revealed no effect on the VGLUT1 and $\mathrm{TF}_{0} \mathrm{~F}_{1}$ activity.

Based on these data, $\mathrm{Cl}^{-}$concentrations were measured in proteoliposomes by the $\mathrm{His}_{14}-\mathrm{Cl}^{-}$ sensor. Liposomes with encapsulated $\mathrm{His}_{14}-\mathrm{Cl}^{-}$-sensor were formed by manual extrusion and VGLUT as well as $\mathrm{TF}_{0} \mathrm{~F}_{1}$ were reconstituted by detergent removal (see figure $1.49 \mathrm{~A}$ ). All extravesicular orientated sensors were removed by addition of imidazole and the liposomes were purified by gel filtration chromatography. The excess of imidazole was removed by dialysis, to prevent quenching of the BAC fluorophore. The purity of the liposomes was verified by density gradient centrifugation and the integrity of the liposomes was confirmed by DLS measurements (see figure $1.49 \mathrm{~B}$ ).

Further, the successful incorporation of VGLUT and $\mathrm{TF}_{0} \mathrm{~F}_{1}$ was proven by density gradient centrifugation analysed by SDS-PAGE or Western Blot (see figure $1.49 \mathrm{C}$ ). Next, the activity of VGLUT and $\mathrm{TF}_{0} \mathrm{~F}_{1}$ in the presence of $\mathrm{His}_{14}-\mathrm{Cl}^{-}$sensor was analysed by measuring the ATPmediated acidification with $30 \mathrm{mM}$ external $\mathrm{Cl}^{-}$. These results revealed strong acidification of the liposomes ( $\Delta \%$ of $61.14 \%$ ), similar to previous acidification assays, confirming the activity of VGLUT1 and $\mathrm{TF}_{0} \mathrm{~F}_{1}$ in the presence of the sensor (see figure 1.49 D). 
A

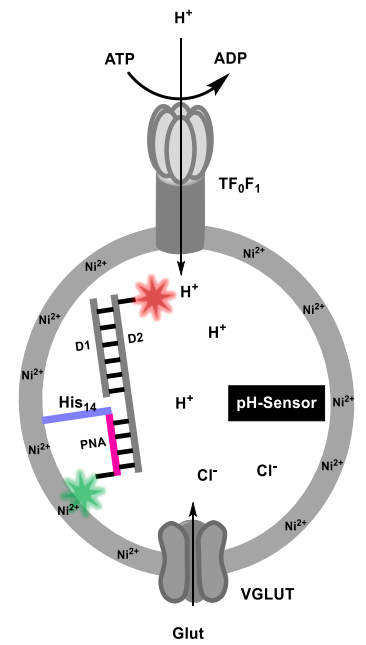

C

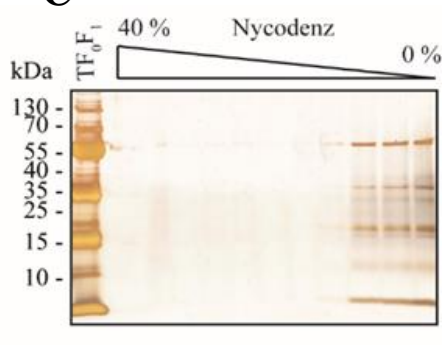

C

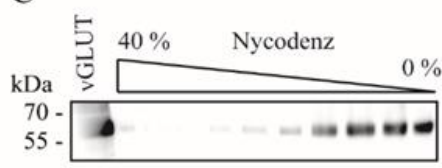

B

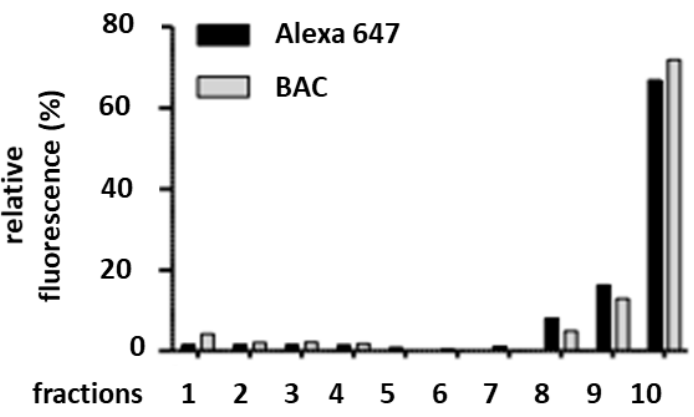

D

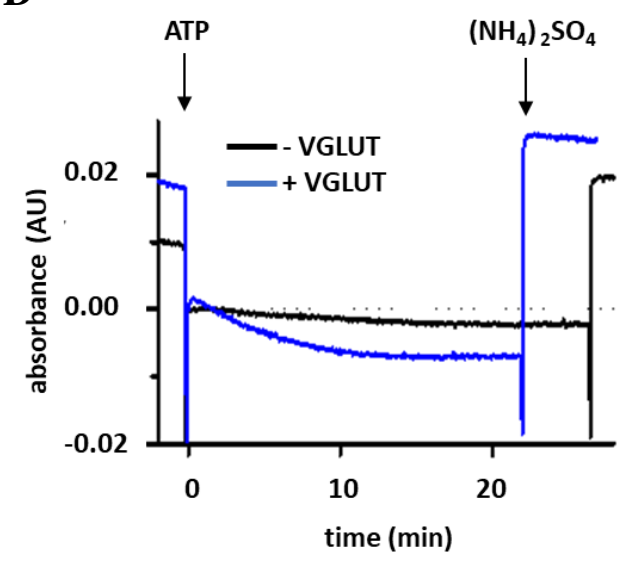

Figure 1.49: Activity measurements of encapsulated VGLUT, $\mathrm{TF}_{0} \mathrm{~F}_{1}$ and $\mathrm{His}_{14}-\mathrm{Cl}^{-}$sensors in liposomes. A) Structure of the liposomes containing VGLUT, $\mathrm{TF}_{0} \mathrm{~F}_{1}$, $\mathrm{His}_{14}-\mathrm{Cl}^{-}$sensor and the $\mathrm{pH}$-Sensor Acridine Orange. B) Density gradient chromatography profile (fraction 1: $40 \%$ Nycodenz, fraction 10: $0 \%$ Nycodenz) of purified liposomes after size exclusion chromatography. The fractions were analysed by the relative fluorescence intensity, which was calculated based on the total fluorescence intensity of all fractions. C) Top: Silver stained SDS PAGE after density gradient centrifugation, showing co-migration of $\mathrm{TF}_{0} \mathrm{~F}_{1}$ with liposomes in top-fractions. Down: Western Blot analysis after density gradient centrifugation, showing co-migration of VGLUT in top fractions. D) $\mathrm{Cl}^{-}$dependent acidification of proteoliposomes lacking or containing VGLUT. Experiments were performed with $2 \mathrm{mM}$ ATP and $30 \mathrm{mM} \mathrm{KCl}$. The experiment was performed by JENNIFER STRUCK.

Finally, the $\mathrm{Cl}^{-}$conductance in liposomes containing VGLUT, $\mathrm{TF}_{0} \mathrm{~F}_{1}$ and encapsulated $\mathrm{His}_{14}-\mathrm{Cl}^{-}$sensor were measured by monitoring the BAC fluorescence intensity. Surprisingly, stepwise addition of $\mathrm{Cl}^{-}(30 \mathrm{mM})$ lead to no drastic decrease in the BAC fluorescence in the millisecond range, as observed for the $\mathrm{Cl}^{-}$calibration curves (see figure 1.50). The measurements of the BAC fluorescence intensity were disturbed by long stabilisation times and bleaching of the fluorophore caused by prolonged irradiation periods. During the fluorescence recordings, it was not possible to measure the fluorescence intensity of BAC and AlexaFluor 647 simultaneously. Dilution effects or other artefacts, which are influencing the 
BAC fluorescence intensity were also not considered. Thus, further measurements of the $\mathrm{Cl}^{-}$ conductance will be performed with total internal reflection fluorescence (TIRF) microscopy or fluorescence cross-correlation spectroscopy (FCCS), which allows the simultaneous monitoring of the BAC and AlexaFluor 647 fluorescence.

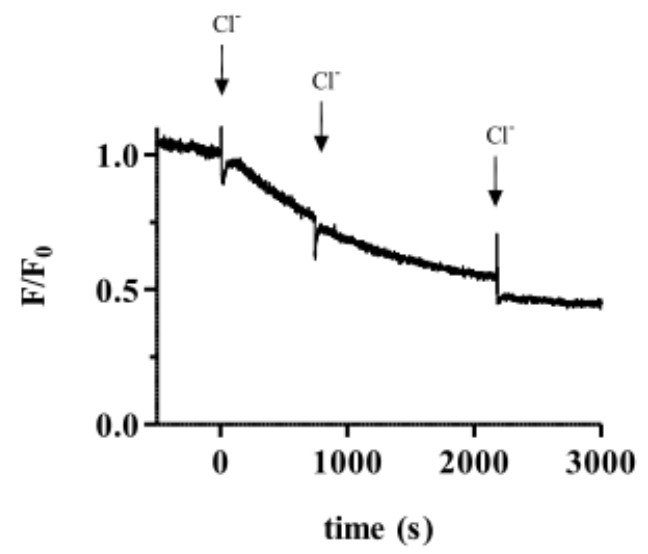

Figure 1.50: Time-dependent BAC fluorescence intensity of $\mathrm{His}_{14}-\mathrm{Cl}^{-}$-sensor. After signal stabilization, $30 \mathrm{mM} \mathrm{Cl}^{-}$was added each. The fluorescence intensity was normalized to the time point of signal stabilization. The experiment was performed by JENNIFER STRUCK. 


\subsubsection{Development of an ALFA-Tag Modified Chloride Sensor}

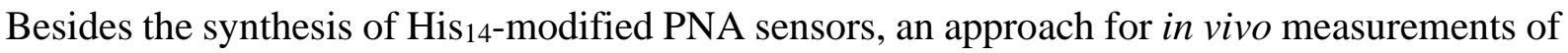
$\mathrm{Cl}^{-}$concentrations was developed. The idea of linking a sensor molecule to the luminal synaptotagmin 1 domain, described by MARTINEAU et al., was used for this strategy. ${ }^{[47]}$ Synaptotagmin is a transmembrane protein in synaptic vesicles, which is interacting with the SNARE complex, regulating the process of vesicle docking and fusion. Expression of the sensor in cultured hippocampal neurons leads to efficient incorporation in synaptic vesicles. However, MARTINEAU et al. used the CLOPHENSOR (see chapter 1.1.5.1), which produces imprecise measurements as it does not cover all physiological $\mathrm{Cl}^{-}$concentrations and the $\mathrm{Cl}^{-}$recordings require a complex $\mathrm{pH}$ correction factors. ${ }^{[5,88]}$

A bispecific monoclonal nanobody was engineered, which can simultaneously address the luminal synaptotagmin domain and binds specifically an epitope tag, which is conjugated to a $\mathrm{Cl}^{-}$sensor. This tag can be introduced into the PNA sensing module, allowing specific interaction with the nanobodies and incorporation of the PNA-DNA based $\mathrm{Cl}^{-}$sensor in synaptic vesicles during endocytosis. Since nanobodies are antibody fragments with reduced molecular weight (12-15 kDa compared to $150-160 \mathrm{kDa})$, the synaptic vesicle can incorporate a higher number of $\mathrm{Cl}^{-}$sensors. ${ }^{[148,149]}$

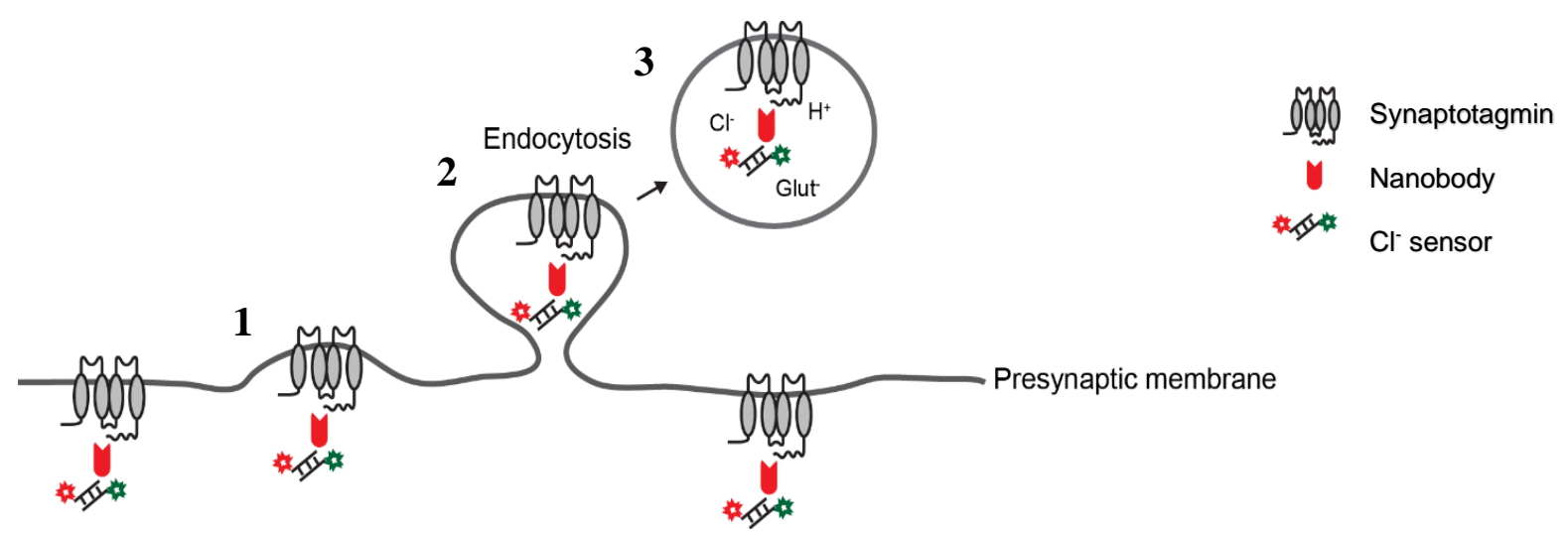

Figure 1.51: Strategy for $\mathrm{Cl}^{-}$-sensor incorporation in synaptic vesicles. Thereby, the luminal domain of synaptotagmin is addressed with a nanobody. Fusion with a second nanobody allows specific interaction with an epitope tag, which can be incorporated in PNA-DNA based $\mathrm{Cl}^{-}$sensors.

Screening of highly specific and potent epitope tags for protein purification or detection revealed the ALFA-tag, which is recognized by the ALFA nanobody (NbALFA) with 26 pM affinity. The ALFA-tag was already successful conjugated to fluorophores, enzymes and biomolecules, applicable for super-resolution microscopy, immunoprecipitations or detection of proteins in living cells. ${ }^{[150]}$ The sequence of the ALFA-tag (SRLEEELRRRLTE) was inspired by an artificial peptide, which is hydrophilic, uncharged under physiological $\mathrm{pH}$ and 
forms a stable $\alpha$-helical structure in solution. ${ }^{[151]}$ Furthermore, the ALFA tag sequence is compatible with protein functions and can be either incorporated at the $\mathrm{N}$ - or $\mathrm{C}$-terminus of a protein or between two domains. Other advantages of the ALFA tag are the low reactivity, as it lacks free amine or thiol groups, as well as reduced influence on protein conformation, as it is flanked by proline residues, which break secondary structures.

After engineering of a bispecific nanobody fairy, which targets, on the one hand, the luminal synaptotagmin domain and on the other hand the ALFA tag sequence, first incorporation experiments were performed (see figure 1.52). Therefore, an ALFA-tag modified GFP fluorophore and the nanobody fairy were incubated in primary cultured hippocampal neurons, showing a successful encapsulation after $10 \mathrm{~min}$ (experiment performed by RASHI GOEL). For removal of all extravesicular orientated nanobodies, a TEV protease cleavage site was introduced into the nanobody sequence to measure the intravesicular $\mathrm{Cl}^{-}$concentration exclusively. Despite so far unsuccessful experiments using the TEV protease, no hindrance was expected for this approach since previous studies revealed only limited TEV activity for the cleavage of small peptides.

$N$-Terminus

$C$-Terminus

\begin{tabular}{|l|l|l|l|}
\hline Nanobody Syt1 & TEV & 3 x FLAG & Nanobody ALFA tag \\
\hline
\end{tabular}

Figure 1.52: Structure of the nanobody fairy. The construct consists of a recognition unit against the luminal synaptotagmin domain and the ALFA tag sequence. The nanobodies are connected by a TEV recognition sequence to remove extracellular orientated sensors and a FLAG linker to increase the flexibility of both nanobodies.

Based on the successful encapsulation experiments of the GFP fluorophore, the ALFA tag sequence was introduced in the PNA sensing module of the $\mathrm{Cl}^{-}$sensor, to ensure incorporation in synaptic vesicle and measurements of the $\mathrm{Cl}^{-}$concentration. The ALFA tag recognition sequence was incorporated in the $C$-terminal part of the sensing module and the ALFA-PNA oligomer (48) was synthesised by the previously described optimised PNA coupling conditions (see chapter 1.2.2). To increase spatial separation, a short Gly-Ser linker, as well as proline residues, were introduced between the ALFA tag and the PNA oligomer (see figure 1.53 A). For the coupling of the Arg residue in the ALFA tag sequence, reduced reaction temperatures of $75{ }^{\circ} \mathrm{C}$ and enhanced reaction times $(10 \mathrm{~min})$ were applied, to prevent $\delta$-lactam formation during the coupling process. After cleavage of the Alloc protection group, the BAC fluorophore (4) was coupled under microwave irradiation and the crude oligomer was purified by HPLC (see figure $1.53 \mathrm{~B}$ ). U-HPLC and mass spectrometry confirmed the successful formation of the ALFA-tag PNA sensor (49) (see figure 1.53 C). 


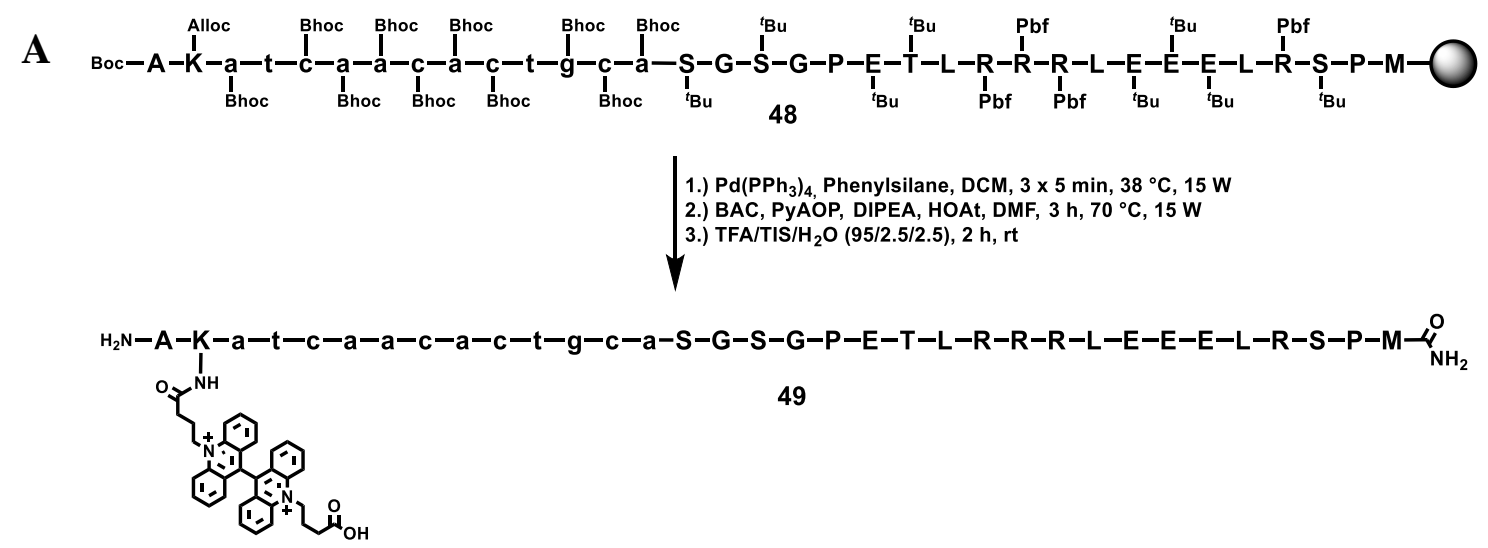

B

C
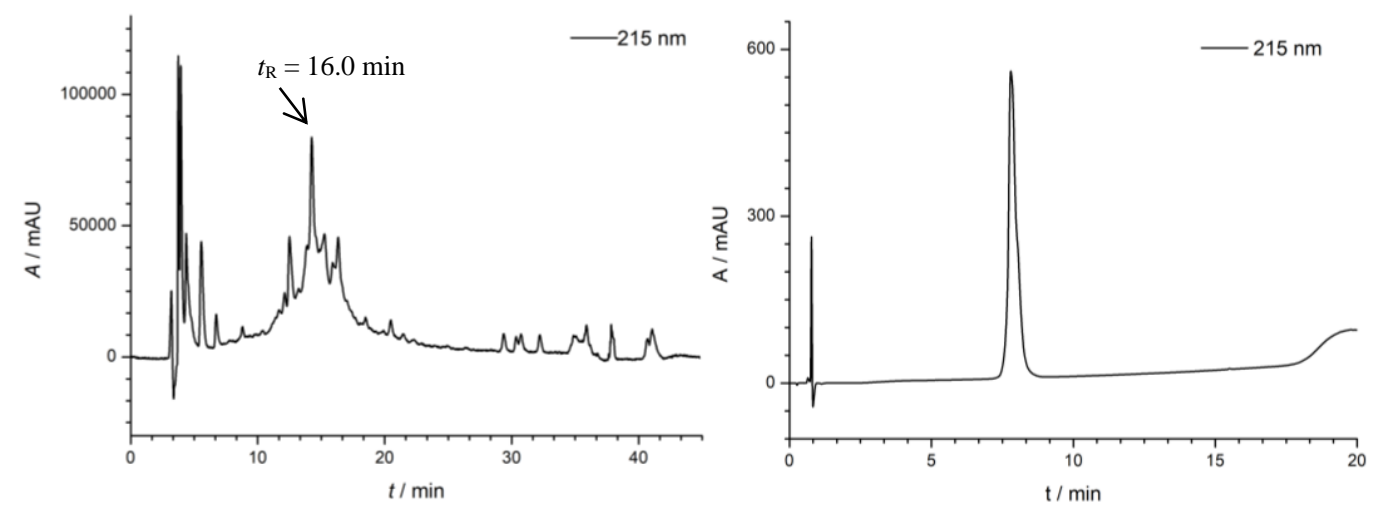

Figure 1.53: Synthesis and analysis of ALFA-tag modified PNA sensor. A) Synthesis of the ALFA-tag modified PNA sensor (49) for Cl- measurements in synaptic vesicles. B) HPLC chromatogram of the of ALFAPNA oligomer (49) after BAC labelling. The isolated product peak is highlighted with an arrow. HPLC gradient: $1-30 \% \mathrm{MeCN}$ in $45 \mathrm{~min}$. C) U-HPLC chromatogram of the purified ALFA-PNA-BAC sensor (49). U-HPLC gradient: $1-30 \% \mathrm{MeCN}$ in $20 \mathrm{~min}$.

Following, the synthesised ALFA-PNA sensor (49) was hybridized with the complementary DNA strands, by annealing all three components for $5 \mathrm{~min}$ at $90^{\circ} \mathrm{C}$. The successful formation of the ALFA-tag modified $\mathrm{Cl}^{-}$sensor was confirmed by gel mobility shift assay (data not shown) and the sensor was directly incubated with the ALFA nanobody at rt for $15 \mathrm{~min}$. The complex formation of the nanobody and the $\mathrm{Cl}^{-}$sensor was analysed by gel mobility shift assay visualized by silver staining, revealing only bands for the nanobody and the ALFA-Cl--sensor, however no complexation (see figure 1.54). 


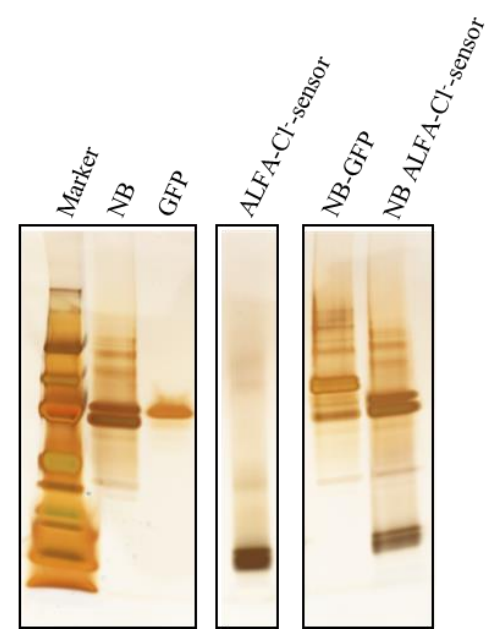

Figure 1.54: Gel mobility shift assay of the ALFA-Cl-sensor formation with the ALFA-nanobody. For visualisation, silver staining was used. Lane 1: Marker, Lane 2: ALFA nanobody, Lane 3: GFP as the positive control, Lane 4: ALFA- $\mathrm{Cl}^{-}$-sensor, Lane 5: ALFA nanobody and GFP, Lane 6: ALFA-Cl-sensor and ALFA nanobody. The experiment was performed by RASHI GOEL.

These findings indicated a blockage of the ALFA nanobody recognition process, probably caused by the bulky PNA-DNA structure, which hinders the accessibility of the ALFA-tag sequence. The complex formation of a simplified ALFA-tag peptide, lacking the PNA structure, with the nanobody was investigated. The ALFA-tag peptide consists of the recognition sequence and a fluorophore to visualise complexation with the nanobody and possible uptakes in synaptic vesicles. For facilitation of the binding process, an increased Gly-Ser linker sequence was introduced between the fluorophore and the ALFA recognition side (see figure 1.55). The peptide sequence (50) was synthesised by SPPS and the Arg residues were coupled at a reduced temperature of $75^{\circ} \mathrm{C}$. In the next step, the Alloc protecting group of the $\mathrm{N}$-terminal Lys residue was cleaved and an NHS-activated AlexaFluor 488 fluorophore was conjugated to the resin-bound peptide with a mixture of DIPEA and DMF at rt for $24 \mathrm{~h}$. After labelling, the peptide was cleaved from the resin with TFA, TIS, $\mathrm{H}_{2} \mathrm{O}(95 / 2.5 / 2.5, v / v / v)$ and the crude peptide (51) was purified by HPLC (data not shown). The fluorophore AlexaFluor 488 was selected, due to its stability, small size and high quantum yield. 


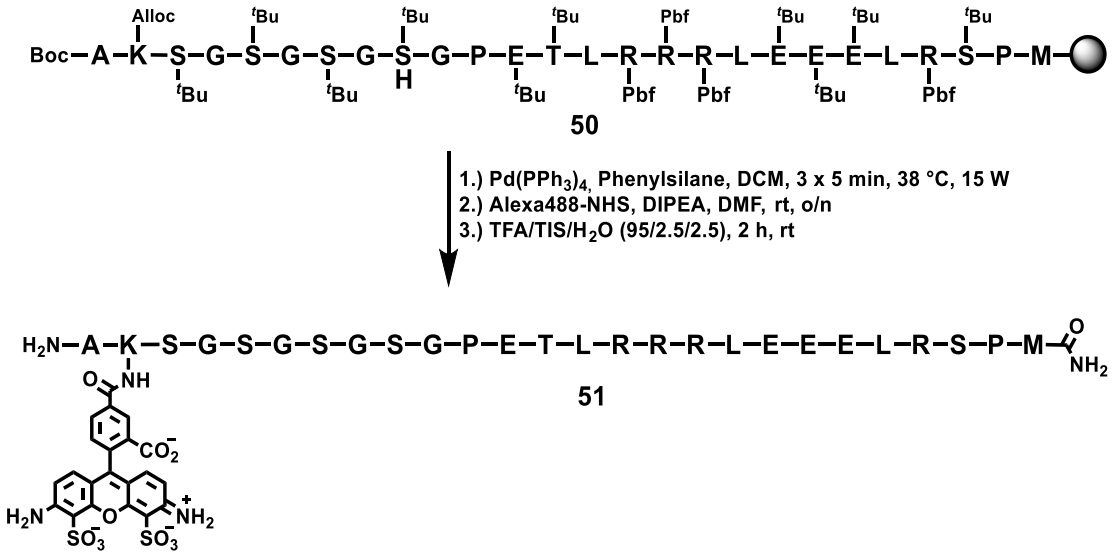

Figure 1.55: Synthesis of AlexaFluor 488 labelled ALFA-tag peptides (51).

The fluorophore labelled ALFA peptide (51) was used for complexation studies with the ALFA nanobody, which were analysed by gel mobility shift assay and visualised by the fluorescence emission of the AlexaFluor 488 fluorophore. The complexation experiments revealed for the AlexaFluor 488 labelled ALFA peptide (51) and the nanobody a band at approximately $40 \mathrm{kDa}$ as expected for the complex (see figure 1.56). However, similar bands were detected for the labelled ALFA peptide (51) itself, indicating the formation of aggregates and no complexation with the ALFA nanobody. This effect might be explained by the structure of the ALFA-tag sequence, which has a high tendency to oligomerise, caused by the charged arginine and glutamic acid residues. When conjugated to proteins, the tendency to oligomerise is reduced due to the small size of the ALFA-tag sequence compared to protein structures.

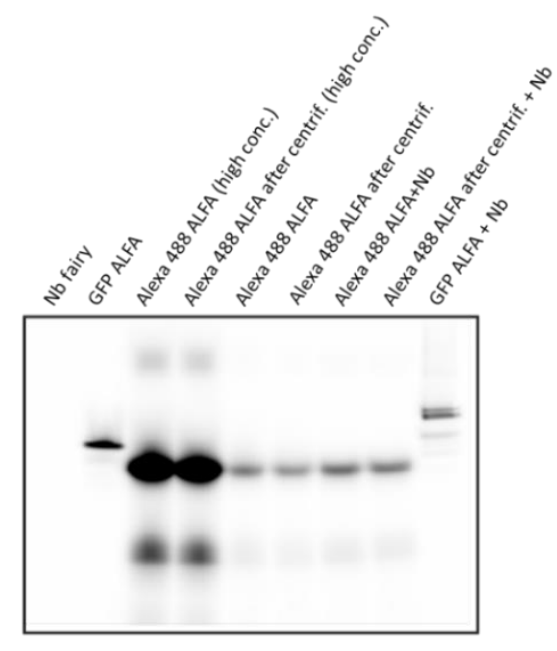

Figure 1.56: Gel mobility shift assay visualizing the AFLA-peptide nanobody formation. Lane 1: Nanobody, Lane 2: ALFA modified GFP (as positive control), Lane 3: AlexaFluor488-labelled peptide (high conc.), Lane 4: AlexaFluor488-labelled peptide after centrifugation to disrupt secondary structures (high conc.), Lane 5: AlexaFluor488-labelled peptide, Lane 6: AlexaFluor488-labelled peptide after centrifugation, Lane 7: AlexaFluor488-labelled peptide and the nanobody, Lane 8: AlexaFluor488-labelled peptide and nanobody after centrifugation, Lane 9: ALFA-GFP and nanobody. The experiment was performed by RASHI GOEL. 
Since the interaction between the ALFA tag modified peptides (51) and the nanobody are hindered, the ALFA-tag approach was replaced by HaloTag, which is frequently used for visualisation of the cellular process by super-resolution microscopy. The HaloTag is a self-labelling protein, which forms a covalent bond with reactive chloralkane linker, conjugated to fluorophores or sensors. ${ }^{[152,153]}$ Consequently, the HaloTag protein was fused with the nanobody against the luminal synaptotagmin domain, which allows the incorporation of chloralkane-modified PNA-DNA sensors into synaptic vesicles. Further incorporation experiments with the Halo fusion protein and modified PNA-DNA sensors are still pending. 


\subsection{Discussion and Conclusion}

The transmission of signals is managed by a complex network of neurons, which send signals along axons and thereby releasing neurotransmitters into the synaptic cleft. After signal transmission, the neurotransmitters are recycled and refilled into synaptic vesicles. ${ }^{[1,2]}$ Mainly, the reuptake process of the excitatory neurotransmitter glutamate was studied over the last years, as several neurological diseases can be linked to glutamate imbalance. ${ }^{[40,41]}$ The transport of glutamate into synaptic vesicles by VGLUT is proton gradient dependent. This gradient is generated by the V-ATPase. However, the efficiency of glutamate transport by VGLUT is tightly regulated by the extravesicular and luminal $\mathrm{Cl}^{-}$concentrations. ${ }^{[3,4,74]}$ For more in-depth insights into the $\mathrm{Cl}^{-}$dependence, a fluorescent $\mathrm{Cl}^{-}$sensor was developed to pursue the kinetics of the $\mathrm{Cl}^{-}$influx through VGLUT during the glutamate transport.

To complete this task, a ratiometric PNA-DNA based $\mathrm{Cl}^{-}$sensor was developed, which consists of three modules: a PNA sensing module (PNA) conjugated to the $\mathrm{Cl}^{-}$sensitive BAC fluorophore, a normalizing DNA module bearing the $\mathrm{Cl}^{-}$insensitive AlexaFluor 647 fluorophore and a stabilizing DNA module (see figure 1.57). For encapsulation of the $\mathrm{Cl}^{-}$-sensor into the lumen of the proteoliposomes, the PNA sensing module was modified with a hydrophobic membrane anchor and protease cleavage sequence. The incorporation of the cleavage motif allows the specific removal of extravesicular orientated sensors on the liposome by addition of a protease.

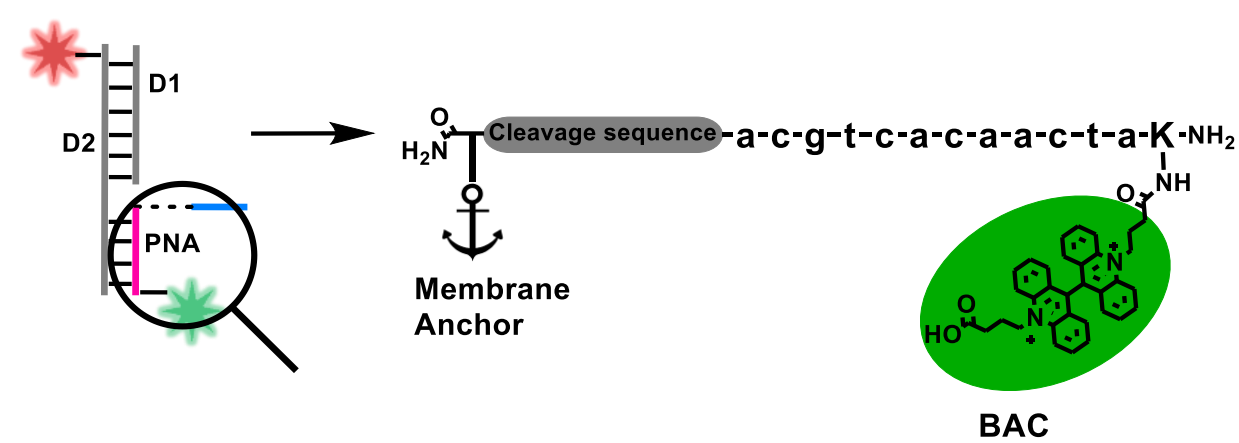

Figure 1.57: Structure of the PNA-DNA based $\mathrm{Cl}^{-}$sensor. The sensor consists of the normalizing and stabilizing DNA strands as well as the $\mathrm{Cl}^{-}$sensitive PNA module. Furthermore, the PNA strand is modified by a membrane anchor and a cleavage sequence, to encapsulate sensors in the liposome.

Peptide-PNA sensors, equipped with a palmitoyl membrane anchor and different protease recognition sequences, were synthesised by an optimised PNA coupling strategy. This method allows automated SPPS of the PNA monomers with significantly reduced reaction times $(2 \mathrm{x}$ 25 min at $\left.50{ }^{\circ} \mathrm{C}\right)$ compared to manually coupling protocols $(2 \times 1 \mathrm{~h}, \mathrm{rt})^{[5,120,121]}$ For further 
improvements of the synthesis process, the reaction temperature could be increased to $65{ }^{\circ} \mathrm{C}$ and therefore, the coupling times could be reduced to $15 \mathrm{~min}$. These new coupling conditions would allow the synthesis of a 12-mer PNA oligomer in almost half of the time. PAfter synthesis of the different peptide-PNA sensors, the cleavage process was analysed. Oligomers with a TEV or Factor Xa protease recognition sequence revealed no cleavage under different digestion conditions. In contrast, peptide-PNA oligomers with a cathepsin B recognition motif were completely cleaved after $30 \mathrm{~min}$. Despite efficient cleavage, cathepsin B protease revealed a reduced cleavage specificity compared to the TEV or Factor Xa protease. The cathepsin B protease cleaves the recognition motif Phe-Lys preferentially. Still, it is also able to cleave the sequence Phe-Arg or Gly-Phe-Leu-Gly. Therefore, removal of extravesicular sensors by cathepsin $\mathrm{B}$ could also affect the activity of VGLUT or the $\mathrm{TF}_{0} \mathrm{~F}_{1}$, since both proteins exhibit cathepsin B cleavage motifs. The cathepsin B protease is still applicable for in vitro measurements, however, removal of the extravesicular sensors has to take place before the proteins are incorporated into the liposomes. For further improvements of the cleavage process, a protease with higher specificity is required, which is also applicable for the cleavage of small peptide structures. The protease cleavage sequence could also be replaced by disulphide- or $\mathrm{pH}$-sensitive linker systems, which have been used for the synthesis of antibody-drug conjugates. $^{[154-156]}$

The membrane incorporation of palmitoyl modified PNA sensors was analysed by density gradient centrifugation. However, the results demonstrated inefficient incorporation of the sensor, presumably the hydrophobic effect of one palmitoyl moiety is not sufficient to anchor the PNA strand in the membrane.

The palmitoyl anchor was replaced by a hydrophobic transmembrane domain (TMD), which was introduced into the PNA sequence by an optimised SPPS coupling protocol. Further investigations of the TMD-modified PNA sensor revealed a fast cleavage by cathepsin B and efficient incorporation into the liposomes, showing an equal intra- and extravesicular distribution (see figure 1.58). However, annealing of the TMD-PNA sensor with the complementary DNA strands revealed no formation of the $\mathrm{Cl}^{-}$sensor, probably caused by the hydrophobic TMD, which hinders the interaction with the charged DNA backbone. To overcome this limitation, the distance between the TMD and PNA scaffold could be increased, by the introduction of a highly flexible PEG linker, which is frequently used for the application of drugs and biomolecules. ${ }^{[157]}$ The introduction of the PEG-linker would also increase the water solubility of the TMD-PNA sensor and therefore enhance the interaction with the DNA strands. 


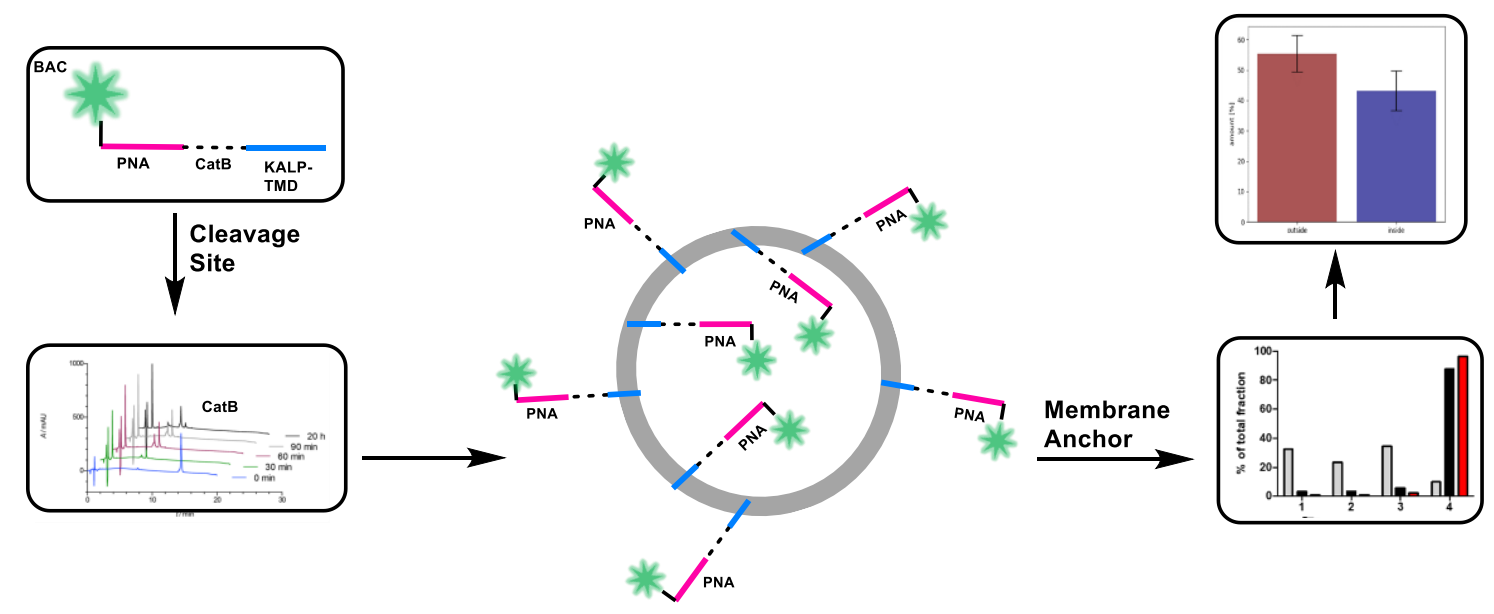

Figure 1.58: Overview of the results of the TMD and Cathepsin B modified PNA sensor. After synthesis of the sensor, the cleavage by the cathepsin B protease (left) and incorporation experiments (right) were performed.

Based on these findings, the TMD anchor was replaced by a hydrophilic polyhistidine sequence, which can specifically interact with $\mathrm{Ni}^{2+}$-NTA labelled phospholipids in liposomes. A protease cleavage sequence was no longer required since the addition of imidazole leads to efficient removal of extravesicular orientated sensors. Previous incorporation experiments revealed a higher encapsulation efficiency for more extended polyhistidine tags compared to the frequently used His6-tag. ${ }^{[142,145]}$ Therefore, a His ${ }_{14}$-PNA sensor was synthesised by optimised SPPS conditions. Further characterisations of the His ${ }_{14}$-PNA oligomer presented a successful complexation with the complementary DNA strands and a $\mathrm{Cl}^{-}$sensitivity of the $\mathrm{BAC}$ fluorophore $\left(K_{\mathrm{SV}}=21.15 \pm 0.33 \mathrm{M}^{-1}\right)$ in the physiological range of $\mathrm{Cl}^{-}$(see figure 1.59).

Following, the incorporation efficiency and the removal of extravesicular orientated $\mathrm{His}_{14}-\mathrm{Cl}^{-}$ sensor was verified. The results from the co-flotation experiments revealed a 3.7 times higher His $14-\mathrm{Cl}^{-}$sensor incorporation in liposomes modified with $\mathrm{Ni}^{2+}$ lipids and efficient removal of all outside orientated sensors by treatment with imidazole. However, the incorporation efficiency of the $\mathrm{His}_{14}-\mathrm{Cl}^{-}$sensor into liposomes was relatively low, probably caused by steric demand. In future experiments, different $\mathrm{Ni}^{2+}$ lipid or $\mathrm{Cl}^{-}$sensor concentrations have to be investigated, to identify optimal conditions to encapsulated a maximum number of sensors in the liposomes. Another improvement of incorporation might be achieved by replacing the His 14 sequence by a His 6 -tag, as reduced steric demand increase the incorporation amount. Preliminary experiments with a His6-tag modified PNA sensor presented already a successful $\mathrm{Cl}^{-}$sensor formation and further incorporation studies are under investigation.

After the encapsulation studies, the $\mathrm{His}_{14}-\mathrm{Cl}^{-}$sensor was incorporated into $\mathrm{Ni}^{2+}$ modified liposomes together with VGLUT and an ATPase $\left(\mathrm{TF}_{0} \mathrm{~F}_{1}\right)$, to measure the kinetics of $\mathrm{Cl}^{-}$influx 
through VGLUT in liposomes. First experiments revealed the successful incorporation of VGLUT and $\mathrm{TF}_{0} \mathrm{~F}_{1}$ in $\mathrm{Ni}^{2+}$ modified liposomes and their activity in the presence of encapsulated $\mathrm{His}_{14}-\mathrm{Cl}^{-}$sensor. Surprisingly, recordings of the $\mathrm{Cl}^{-}$conductance in liposomes containing VGLUT, $\mathrm{TF}_{0} \mathrm{~F}_{1}$ and encapsulated $\mathrm{His}_{14}-\mathrm{Cl}^{-}$sensor revealed no decrease in the $\mathrm{BAC}$ fluorescence intensity after addition of $\mathrm{Cl}^{-}$. However, the measurements were hampered by bleaching of the BAC fluorophore, caused by long irradiation times. Further experiments will be performed by TIRF or FCCS measurements, which allows the simultaneous monitoring of the AlexaFluor 647 and BAC fluorescence intensity. The AlexaFluor 647 emission intensity can be used to normalise the emission intensity of BAC.

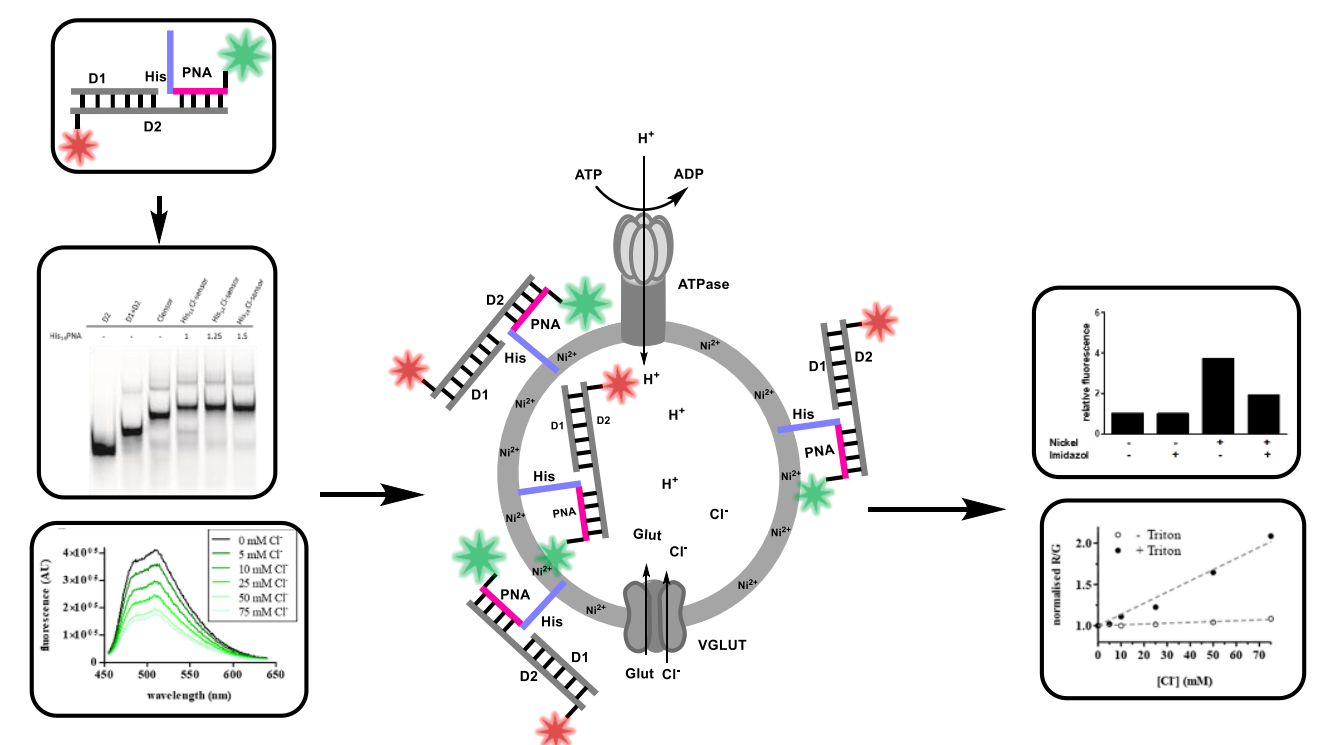

Figure 1.59: Overview of the results of the His 14 -PNA sensor. First, the $\mathrm{His}_{14}$-sensor was formed and the $\mathrm{Cl}^{-}$ sensitivity was measured (left). Afterwards, the incorporation and cleavage efficiency of the $\mathrm{Cl}^{-}$sensor were analysed (right).

Additionally, one approach for in vivo measurements of the $\mathrm{Cl}^{-}$concentration was developed. An ALFA tag nanobody was used to target the luminal synaptotagmin domain in synaptic vesicles. Simultaneously, the nanobody binds to a short ALFA-tag sequence, which consists of 15 amino acids. ${ }^{[150]}$ This ALFA sequence was introduced into the PNA sensing module of the $\mathrm{Cl}^{-}$sensor by SPPS. However, incubation of the ALFA-tag modified $\mathrm{Cl}^{-}$sensor and the nanobody lead to no complexation, presumably caused by the bulky PNA-DNA structure or oligomerisation of the ALFA-tag sequence. The ALFA-tag was replaced by a HaloTag protein, which can specifically interact with a chloralkane modified PNA-DNA sensor. The simple structure of the chloralkane linker might lead to improved interaction with the HaloTag and a more efficient complex formation. The chloralkane linker was introduced to the 3' end of the D1 strand instead of the PNA module, which might improve the accessibility of the linker 
towards the HaloTag protein. Complexation and encapsulation studies of the Halo fusion protein and the sensor is under current investigation.

Combining these results, different PNA-DNA based $\mathrm{Cl}^{-}$-sensors were synthesised with an optimised coupling strategy. This approach allows the successful incorporation of various membrane anchoring systems into the PNA sequence. Especially the introduction of the His 14-tag presented an elegant approach for efficient encapsulation in liposomes and controlled removal of all extravesicular orientated sensors by incubation with imidazole. Noteworthy, the strategy of liposome encapsulation of the His ${ }_{14}$-PNA-DNA sensors is not only limited to $\mathrm{Cl}^{-}$ sensing and presents, therefore a useful tool for measurements of ion concentrations. This will provide new opportunities to study transmembrane proteins and their transport mechanisms. One exciting application of the sensor is the profound examination of SNARE-mediated membrane fusion processes. 


\section{Investigation of Voltage-Gated Sodium Channels by modified Conotoxins}

\subsection{Structure and Function of Voltage-Gated Sodium Channels}

Voltage-gated sodium channels (VGSC or Nav channels) are integral membrane proteins that initiate electrical signalling in excitable cells and control a variety of physiological processes. The activity of $\mathrm{Na}_{\mathrm{v}}$ channels is tightly controlled by changes of the membrane potential, which causes a channel opening and therefore an influx of $\mathrm{Na}^{+}$along their electrochemical gradient. ${ }^{[158,159]}$ The Nav channels were first discovered by HoDGKIN and HuXLEY in 1952, by analysing action potentials of giant squid axons with voltage-clamp measurements. Their work revealed that signals in nerves are initiated by voltage-dependent activation of $\mathrm{Na}^{+}$influx. ${ }^{[160,161]}$ After inactivation (1-2 ms) of the Nav channels, ionic balance is reestablished by potassium channels, initiating a $\mathrm{K}^{+}$efflux. ${ }^{[159]}$

The first basic structural investigations of VGSCs were reported by BENESKI, CATTERALL and HARTSHORNE in the 1980 s, using azidonitrobenzoyl mono[ $\left.{ }^{125} \mathrm{I}\right]$ iodo, a photoactive derivate of the scorpion toxin, to identify subunits of rat brain sodium channels. ${ }^{[162,163]}$ More precise structural information and visualization of different functional states were revealed with recently published X-ray crystal structures of the bacterial Nav channels from the marine bacterium Magnetoccus sp. (strain MC-1). ${ }^{[164]}$ Although the crystal structure of the mammalian Nav channels is still unknown, homology models were developed based on the sequences of human Nav channels and bacterial crystallographic data. These models provided further insights into the functionality of these channels on a molecular level. ${ }^{[165,166]}$

The human $\mathrm{Nav}$ channels consist of a large pore-forming $\alpha$-subunit of $260 \mathrm{kDa}$, associated with auxiliary $\beta 1$ - and $\beta 2$-subunit of $30-40 \mathrm{kDa}$. The $\alpha$-subunit is a single-chain polypeptide with a length of 2000 amino acids, organized in four homologous domains, each containing the six transmembrane segments S1-S6 (see figure 2.1 A). ${ }^{[9,167,168]}$ The S1-S4 transmembrane segments form the voltage-sensing domain, in which the conserved S4 segment acts as the channel's voltage sensor, consists of positively charged arginine or lysine residues in every third position. ${ }^{[9,168]}$ The voltage sensors from each domain are organized around the channel pore, which is formed by the S5-S6 segments of all domains as well as the extracellular connecting pore loops. (see figure $2.1 \mathrm{~B}$ and $2.1 \mathrm{C}$ ). ${ }^{[169,170]}$ Upon depolarization, the S4 segment moves forward to the extracellular surface, inducing a conformational change which allows the opening of the channel and a $\mathrm{Na}^{+}$influx. During the depolarization process, the inactivation 
gate, formed by the short intracellular loop between S3 and S4, folds into the channel pore resulting in an inactivation of the channel and a refolding of the S4 segment to the resting position. ${ }^{[171]}$

The selectivity of the channel is ensured by a filter that distinguishes between $\mathrm{Na}^{+}$and other competing ions which differ in charge and radii. The negative charge of the channel attracts only cations, interacting with the narrowest part of the pore, which just fits for a $\mathrm{Na}^{+}$ion conjugated with one water molecule. ${ }^{[172]}$ The narrowest part of the pore is formed by a salt bridge between a lysine and glutamate or aspartate residue. Destruction of the salt bridge by replacing the lysine with an alanine residue destroys the selectivity of the channel and makes it permeable towards $\mathrm{K}^{+}, \mathrm{Ca}^{2+}$ and $\mathrm{Ba}^{2+} \cdot[9,173]$

$\mathbf{A}$

A. Domain I(DI)

Domain II (DII)

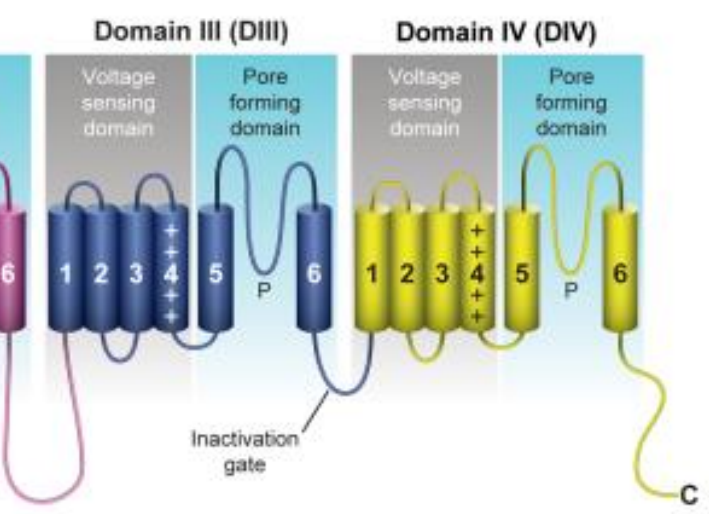

B

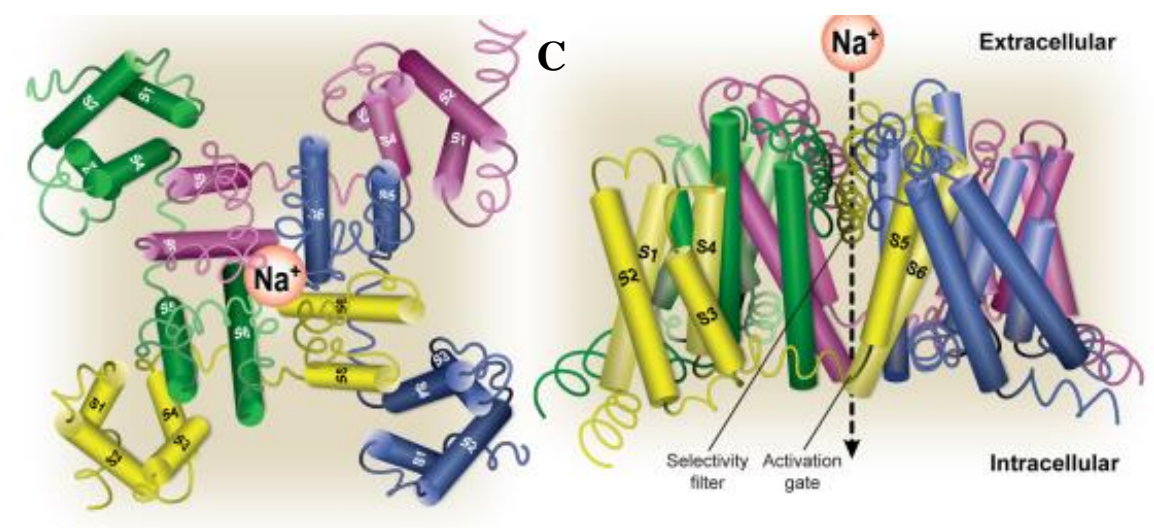

Figure 2.1: Structure of Nav channels. A) Transmembrane folding diagram of the $\alpha$-subunit of the human voltage-gated sodium channel. The channel consists of four homologous domains with six transmembrane segments each. The plus signs in the S4 segment represents the positively charged amino acids in the voltagesensing domain. B) View of the open-channel pore, from a crystal structure of bacterial Nav channel. C) Sideview of Nav channel in the open state. Reprinted with permission from M. DE LeRA RUIZ et al., J. Med. Chem. 2015, 58, 7093-7118. 


\subsubsection{Voltage-Gated Sodium Channel Isoforms}

Currently, nine different isoforms of the Nav channels (1.1-1.9) have been described with similar functionality and a high sequence homology over $50 \%$ in the transmembrane segments and extracellular loop regions. The Nav 1.1, 1.2, 1.3 and 1.6 are expressed in the central nervous system (CNS), whereas Nav 1.7-1.9 is restricted to the peripheral nervous system (PNS). The Nav1.4 and 1.5 channels occur predominantly in skeletal and cardiac muscles (see figure 2.2). ${ }^{[174]}$ Mutations or dysfunctions in the different Nav channels cause various diseases like chronic pain, neuromuscular disorders, cardiovascular diseases or psychiatric disorders. ${ }^{[10,175-}$ 177]

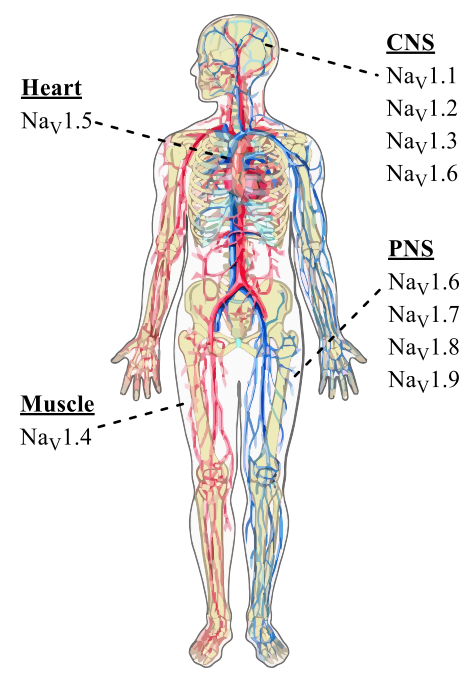

Figure 2.2: Tissue expression of the nine different voltage-gated sodium channel subtypes. ${ }^{[9]}$

Especially, dysfunction of the neuronal Nav1.2 channel, which is expressed in the axon initial segment and dendrites, is linked to psychiatric diseases like epilepsy, autism, Asperger syndrome or febrile seizures. ${ }^{[11,178,179]}$ Therefore, Nav1.2 might be a suitable target structure to address new treatment options for these diseases. The current used Nav inhibitors, like phenytoin carbamazepine or lamotrigine, can cause various off-target effects, due to subtype unspecific Nav channel binding. ${ }^{[180,181]}$ Consequently, there is a great interest in the development of specific inhibitors for the Nav1.2 channel to gain deeper insights into their molecular mechanisms as well as their localization and activity during different stages of the diseases. These findings would contribute to the development of new effective therapeutics with a higher specification and fewer side effects.

Different approaches already tried to determine the density and localization of the Nav1.2 channels with fluorophore-labelled antibodies for the $\alpha$-subunit of the channel. ${ }^{[182-184]}$ However, the usage of antibodies for immunostaining of Nav channels has significant drawbacks. The 
bulky size of the antibody distorts the precise localisation of Nav channels by enlarging the distance of the fluorophore to the channel. Furthermore, the labelled antibodies address intracellular epitopes, requiring an uptake into the neurons. Therefore, the cells have to be fixated and permeabilised, which makes them only applicable for in vitro studies. ${ }^{[185]}$ Also, several fluorophores can bind to one antibody and several antibodies can bind to one channel, making precise immunofluorescence measurement unfeasible. ${ }^{[182]}$ Therefore, new inhibitors have to be identified, which fulfil the following criteria: the inhibitor requires a high affinity and specificity for the Nav1.2 channels, it has to be compatible with fluorophore labelling strategies and it must bind to the extracellular site of the channel in a precise 1:1 stoichiometry.

\subsubsection{Inhibition of Nav-channels by Conotoxins}

Over the last years, different high-affinity inhibitors of the Nav channels have been described, which mostly are produced by venomous animals or poisonous plants. ${ }^{[9]}$ Depending on their structure, the inhibitors can be categorized into two main groups of peptidic- or non-peptidic toxins. In the group of non-peptidic inhibitors, especially the alkaloid neurotoxins tetrodotoxin (TTX) (52) was extensively studied (see figure $2.3 \mathrm{~A}$ ). The toxin is produced by the pufferfish, showing a nanomolar affinity for the Nav1.1-1.4, Nav1.6 and Nav1.7. Therefore, their application as a tool for channel investigations as well as their therapeutic potential is limited due to the lack of selectivity among different Nav subtypes. ${ }^{[186,187]}$ Also, other members of the group of non-peptidic toxins, like Batrachotoxin (53), revealed a high affinity for Nav channels but no subtype specificity (see figure $2.3 \mathrm{~B}$ ). ${ }^{[9,188]}$

A
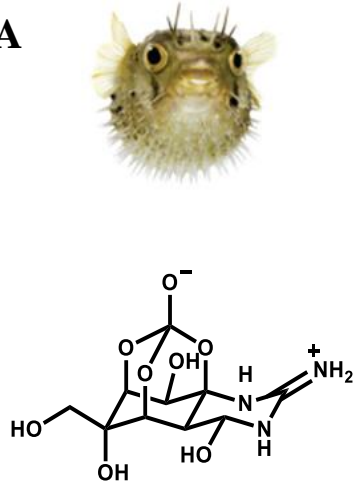

Tetrodotoxin, 52

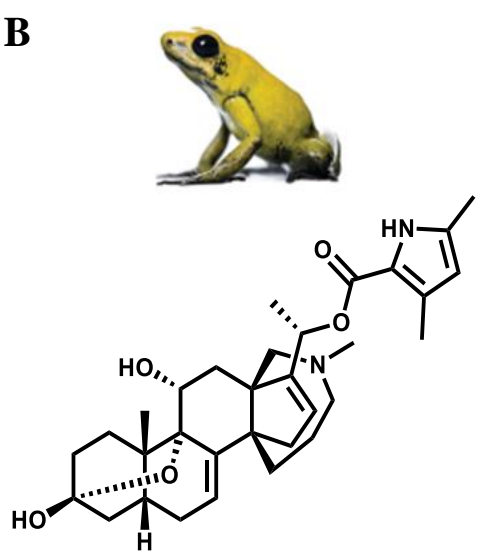

Batrachotoxin, 53

Figure 2.3: Non-peptidic toxins inhibiting the Nav channels. A) Tetrodotoxin (52) extracted from pufferfish. B) Batrachotoxin (53) isolated from the skin secretion of the Phyllobates frog. Reprinted with permission from $\mathrm{M}$. De LeRA RuIZ et al., J. Med. Chem. 2015, 58, 7093-7118. 
Hence, the search for highly selective Nav inhibitors was focussing on the group of venom peptides produced by spiders, scorpions, molluscs or marine cone snails. Although spiders, scorpions and sea anemones species produce up to 10 million bioactive peptides from which only $0.01 \%$ have been characterized, the highest potency, as well as subtype-selectivity for the Nav1.2 channel, was found in peptides isolated from the marine cone snail (see figure 2.4). ${ }^{[9,189,190]}$

A

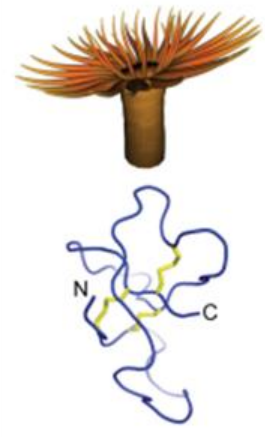

Anthopleurin B
B

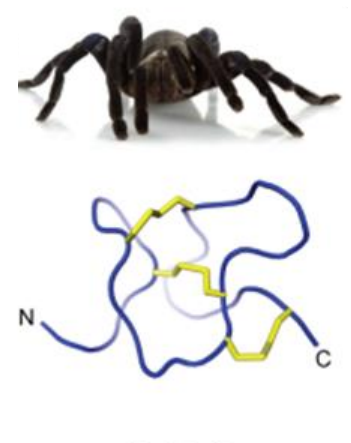

ProTX-II

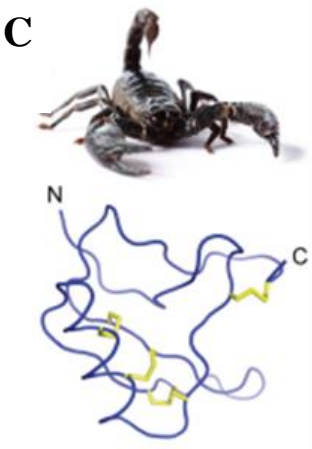

OD1
D
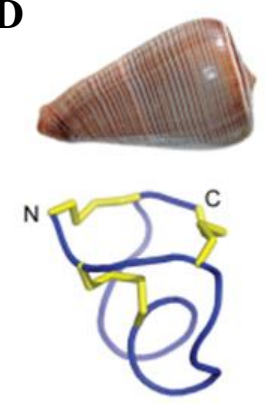

$\mu$-Conotoxin KIIIA

Figure 2.4: Peptidic toxins that inhibit Nav channels. A) Anthopleurin B isolated from the sea anemones. B) ProTX-II isolated from tarantulas. C) OD1 extracted from the scorpion. D) $\mu$-Conotoxin KIIIA extracted from cone snails. Reprinted with permission from M. DE LERA RUIZ et al., J. Med. Chem. 2015, 58, 7093-7118.

Cone snails are distributed throughout tropical and subtropical waters, where they are hunting fish, worms or molluscs. Since the cone snails are slow-moving, they use a venomous harpoon to inject their toxins to capture faster-moving preys. ${ }^{[191,192]}$ First investigations of the cone snail venoms were done in the 1960s due to several human deaths, caused by cone snail stings. In the following years, many researchers tried to unravel the pharmacological effects of the cone snail toxins. Their studies showed that the venom consists of a mixture of at least 100-200 neuroactive peptides, so-called conotoxins. These toxins cause an almost immediate paralysis of the prey by blocking ion channels, G-protein coupled receptors or neurotransmitter transporters. ${ }^{[193,194]}$ Structurally, conotoxins are small peptides with a length of 10-40 amino acid and well-defined secondary structures, which are stabilized by multiple conserved disulphide bonds. Additional post-translational modifications (PTMs) increase the stability towards degradation by proteases. ${ }^{[194,195]}$ Today, around 750 different species of the cone snails have been discovered, producing up to 70.000 neuroactive peptides from which only $0.1 \%$ have been characterized pharmacologically. ${ }^{[194,196]}$ The so far revealed conotoxins can be organized into superfamilies based on similarities of the $N$-terminal precursor sequences (see figure 2.5). Further, the superfamilies can be classified into families based on their cysteine framework, conotoxin family and target receptor, according to the International Union of Basic 
and Clinical Pharmacology (IUPHAR) nomenclature. ${ }^{[197]}$ The conotoxin nomenclature can be explained exemplary for the $\alpha$-conotoxin PnIA: $\alpha$ indicates the targeting of the nicotinic acetylcholine receptor, Pn signifies the origin Connus pennaceus, I determines the framework one and A denotes the order of discovery. ${ }^{[192,198,199]}$

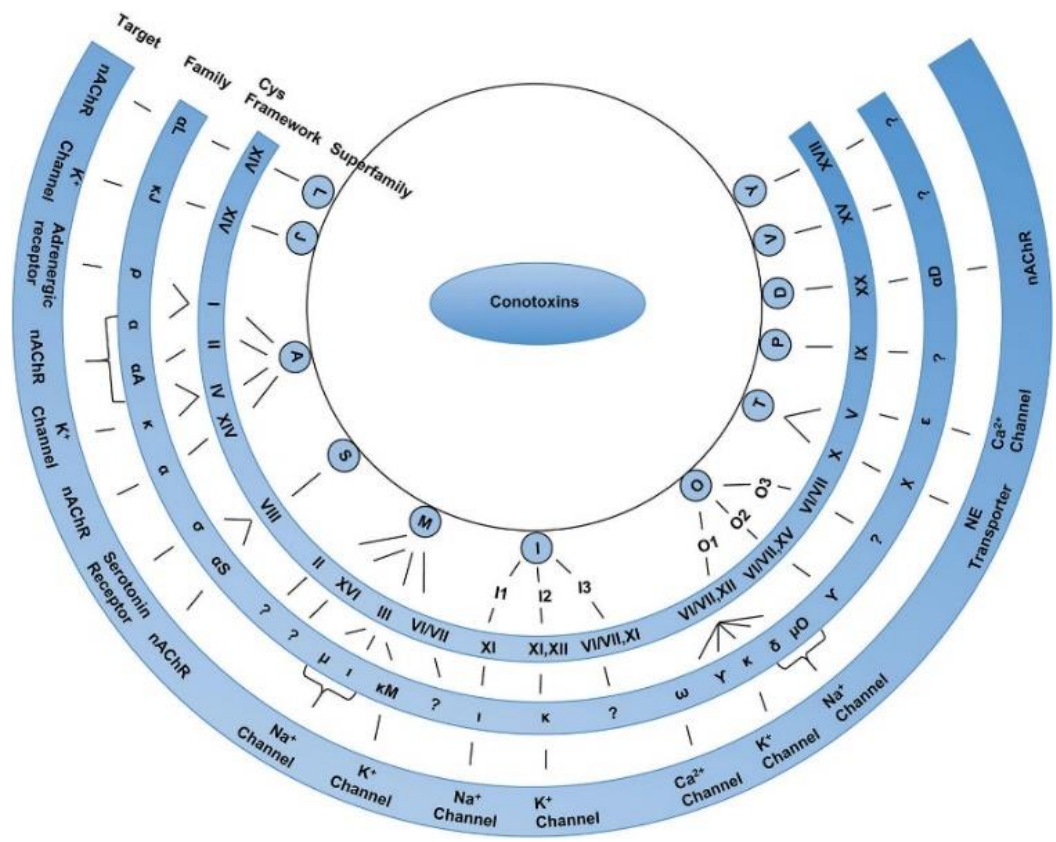

Figure 2.5: Classification of conotoxins. Conotoxins are categorized into superfamilies based on their conserved signal sequence homology. The different organisation in families depends on their cysteine frameworks and target structures. Reprinted with permission from K. B. AKONDI et al., Chem. Rev. 2014, 114, 5815-5847.

Due to the large number of neuroactive peptides produced by cone snails, the conotoxins exhibit a tremendous potential as lead molecules for the development of new therapeutics as well as tools for channel and receptor examination under physiological and pathological conditions. ${ }^{[194,195]}$ The $\omega$-conotoxin MVIIA (ziconotide) from the cone snail Conus magnus, was already approved by the Food and Drug Administration (FDA) in 2004 and is clinically used as an analgesic for the treatment of chronic pain. Ziconotide acts on the voltage-gated $\mathrm{Ca}^{2+}$ channel, by blocking neurotransmitter and substance $\mathrm{P}$ release, which is associated with inflammatory processes and pain. ${ }^{[200]}$ Compared to morphine, ziconotide is 1000 times more potent and causes less tolerance and addiction. However, ziconotide must be administered directly into the spinal fluid because of the lack of efficacy when ingested orally or intravenously. Based on this limitation ziconotide is used exclusively as an alternative therapy for chronic pain or opiate-resistant patients. ${ }^{[200,201]}$ 


\subsubsection{Neuronal-Subtype Binding $\mu$-Conotoxins}

Analysis of the characterized conotoxin families revealed four families $\left(\mu-, \mu \mathrm{O}-, \delta_{-}, \mathrm{i}_{-}\right)$which are explicitly affecting the functions of Nav channels. The $\mu$-conotoxins block the sodium conductance by direct blockage of the extracellular pore, whereas the $\mu \mathrm{O}$-conotoxins inhibit the sodium current by interfering with the voltage-sensors in domain $2 .{ }^{[202,203]}$ On the contrary $\delta$-and i-conotoxins disturbs the process of channel inactivation by modifying the voltage sensor domain D4. ${ }^{[204]}$

Among the conotoxins targeting $\mathrm{Nav}$ channels, the $\mu$-conotoxins are the most numerous and best-characterized class of conotoxins. They consist of 16-26 amino acids and their threedimensional structure is stabilized by three conserved disulphide bridges arranged in a class III framework (Cys1-Cys4, Cys2-Cys5, Cys3-Cys6). ${ }^{[205]}$ The net charge of the $\mu$-conotoxins $(+2$ to +7 ) allows them to electrostatically bind and block the negatively charged channel pore of the Nav channels (see table 2.1). Although the binding site of $\mu$-conotoxins overlap with the TTX binding site, the $\mu$-conotoxin interacts with more residues of the Nav-channel, resulting in the subtype selectivity. ${ }^{[203]}$

The first isolated and characterized $\mu$-conotoxin was the $\mu$-GIIIA from the venom of Conus geographus, consisting of 22 amino acids. Structure-activity studies of the $\mu$-GIIIA revealed that all three disulphide bridges, as well as the positively charged arginine residue in the second intercysteine loop, are essential for the blockage of the Nav channel. ${ }^{[206,207]}$ Furthermore, measurements with $\mu$-GIIIA indicated for the first time a Nav channel subtype selectivity, by showing specificity for the skeletal muscle channel Nav1.4 $\left(\mathrm{IC}_{50}=0.019 \mu \mathrm{M}\right)$ over the neuronal subtypes $\left(\mathrm{IC}_{50}=0.2-18 \mu \mathrm{M}\right)$. Measurements of the related $\mu$-conotoxins PIIIA and SmIIIA also revealed a preference for the muscular Nav1.4 channel. ${ }^{[207]}$

Shortly after the identification of $\mu$-GIIIA, $\mu$-PIIIA and $\mu$-SmIIIA, the first inhibitors of the neuronal Nav channels were identified by BULAJ et al., presenting the $\mu$-conotoxin SIIIA from Conus striatus and KIIIA from Conus kinoshitai. ${ }^{[12]}$ Characterisation of these toxins revealed for the $\mu$-SIIIA $\left(\mathrm{IC}_{50}=0.05 \mu \mathrm{M}\right)$ as well as $\mu$-KIIIA $\left(\mathrm{IC}_{50}=0.005 \mu \mathrm{M}\right)$ a low nanomolar affinity for the Nav1.2 channel. Beside their inhibition of the Nav1.2 channel, both conotoxins block the Nav1.4 as well as Nav1.6 channel in submicromolar concentration. ${ }^{[12,208,209]}$ Structurally, the $\mu$-SIIIA and $\mu$-KIIIA exhibit a strong sequence homology, with $\mu$-SIIIA having four additional residues in the $N$-terminal part. Further investigations of $\mu$-SIIIA analogues also revealed an analgesic potency by blocking action potentials in mice sciatic nerves, leading to a reduction of inflammatory pain. ${ }^{[14,210]}$ These results underline the therapeutic potential of $\mu$ - 
SIIIA as a potent inhibitor of the mammalian Nav1.2 channels. Furthermore, the four additional residues, which do not influence the overall activity of the $\mu$-SIIIA, makes it a powerful tool for research studies by introducing modifications. ${ }^{[210]}$

Table 2.1: $\boldsymbol{\mu}$-Conotoxin sequences and characteristics. Summary of the sequence, net charge, favoured $\mathrm{Nav}$ channels of selected $\mu$-conotoxins. The disulphide framework is observed between Cys1-Cys4, Cys2-Cys5, Cys3Cys6. $\mathrm{Z}=$ pyroglutamate and $\mathrm{O}=$ hydroxyproline. Table adapted from ${ }^{[14]}$.

\begin{tabular}{llll}
\hline $\boldsymbol{\mu}$-Conotoxin & Sequence & Net charge & Nav targets \\
\hline \multirow{2}{*}{ GIIIA } & RD-CCTOOKKCKDRQCKO-QRCCA & +6 & $1.4>1.1>1.6 .>1.2$ \\
\multirow{2}{*}{ PIIIA } & ZRLCCGFOKSCRSRQCKOH-RCC & +6 & $1.4>1.6=1.1>1.2>1.3$ \\
SmIIIA & ZR-CCNGRRGCSSRWCRDHSRCC & +5 & $1.4>1.2>1.1>1.3>1.6$ \\
SIIIA & ZN-CCNG--GCSSKWCRDHARCC & +2 & $1.2>1.4>1.6>1.1$ \\
KIIIA & --- CCN----CSSKWCRDHSRCC & +3 & $1.2>1.4>1.6>1.7$ \\
\hline
\end{tabular}

Structurally, the $\mu$-SIIIA consists of 20 amino acids with a net charge of +2 and three disulphide bridges, which are buried in the core of the peptide. The $N$-terminus is cyclized by the formation of pyroglutamate, which increases the stability against enzymatic degradation by exopeptidases. ${ }^{[211]}$ The $C$-terminal is posttranslationally modified by amidation, which affects the isoelectric point and enhances the binding to the negative surface of the Nav channel. Furthermore, the amidation protects the $\mu$-SIIIA against degradation by carboxypeptidase. ${ }^{[14,207]}$ Investigations of the 3D-structure by NMR studies revealed a compact $\alpha$-helix (residues 11-16) compared to the muscle subtype binding $\mu$-conotoxins which form distorted helices (see figure 2.6 A). ${ }^{[208]}$ Further structure-activity studies showed that the residues in the helical structure are most important for the channel interaction. Especially, replacement of the residues Lys11, Trp12, Arg14, His16 and Arg18 caused a significantly reduced binding affinity to the channel (see figure 2.6 B). ${ }^{[13,14]}$ However, for all mutations, a preference for the neuronal Nav1.2, compared to the Nav1.4 channel was still measurable. Interestingly, the mutation H16A leads to a disruption in the $\alpha$-helical motif, causing a significant decrease in the binding affinity for neuronal as well as muscle subtypes. ${ }^{[13,14]}$ 
A

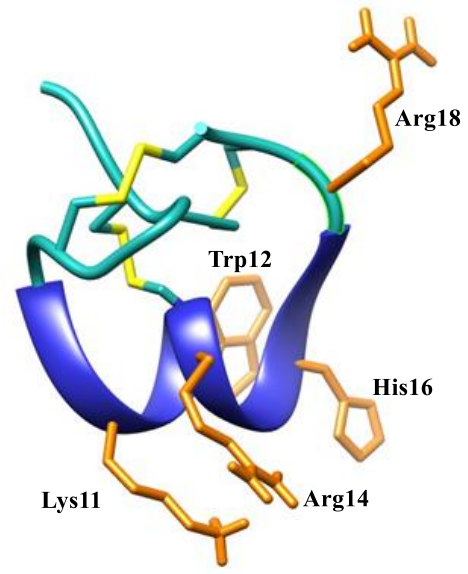

B

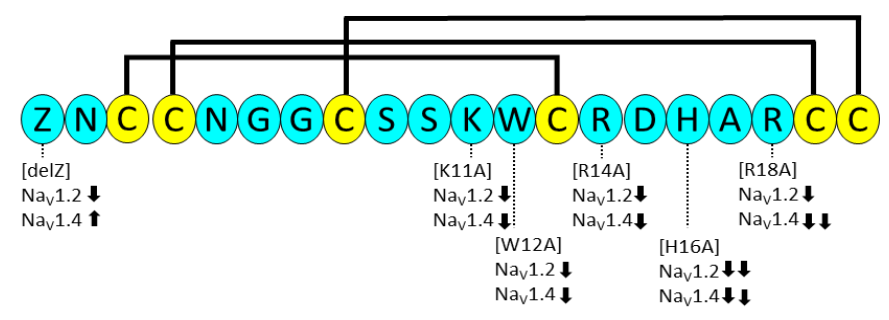

Figure 2.6: Structure and sequence of $\mu$-conotoxin SIIIA. A) Three-dimensional structure of $\mu$-SIIIA. The disulphide bridges are highlighted in yellow, the $\alpha$-helix in blue and the crucial residues for channel interaction are shown in orange (BMRB ID code: SMS20025). The graphic was created with USCF Chimera package. ${ }^{[212] .}$ B) Results of amino acid replacement on $\mathrm{Nav} 1.2$ and Nav1.4 subtype selectivity. ( $\uparrow$ ) indicates improved potency against $\mathrm{Na}_{\mathrm{V}} 1.2$ or $\mathrm{Na}_{\mathrm{V}} 1.4$ subtypes, whereas $(\downarrow)$ indicates a decreased potency. ${ }^{[207]}$

To improve the therapeutic potential of $\mu$-SIIIA, different synthetic analogues were developed to increase the stability under cellular conditions as well as specificity and affinity to the Nav1.2 channel. Therefore, the disulphide bonds were replaced by diselenide bridges, which revealed greater thermodynamic stability under reductive conditions and enhanced oxidative folding but decreased binding affinity to the Nav1.2 channel. ${ }^{[213,214]}$ Furthermore, stabilisation of the $\mu$-SIIIA structure by introduction of a lactam bridge causes a decrease in the affinity for Nav1.2 compared to native $\mu$-SIIIA. ${ }^{[215]}$ Interestingly, studies from GREEN et al. revealed that replacing the non-essential Asn, pGlu or Gly residues, by non-peptidic backbone spacers lead to a higher analgesic effect in mice models. ${ }^{[207]}$ These results indicate the potential of the non-essential positions for the introduction of modifications, without affecting the overall affinity and selectivity.

\subsubsection{Oxidative Folding of Disulphide-Rich Peptides}

One major drawback includes the limited availability of the $\mu$-conotoxin SIIIA for the research as well as a clinical application when extracted from the cone snail venom. ${ }^{[194]}$ Therefore, different approaches to produce sufficient amounts of $\mu$-SIIIA have been developed. On the one hand, conotoxins can be produced by recombinant expression in heterologous expression systems like $E$. coli or yeast. ${ }^{[216]}$ This method provides rapid production of $\mu$-SIIIA. However, purification of the conotoxin, as well as the incorporation of unnatural amino acids and different posttranslational modifications, are still challenging in recombinant expression systems. ${ }^{[194]}$ On 
the other hand, chemical synthesis provides more control over the introduction of modifications such as backbone cyclization or disulphide replacement to increase the stability of $\mu$-SIIIA against proteases. Therefore, the chemical synthesis of the conotoxin can be performed by solidphase peptide synthesis (SPPS), producing the linear precursor peptide. The folding of the conotoxin is induced by oxidation of the cysteine residues, which can be achieved by either oxidative folding or orthogonal protecting group strategy, leading to the formation of disulphide bridges. ${ }^{[194,217]}$

The oxidative folding strategy provides the advantage of only one cysteine protecting group for SPPS and one purification step (see figure 2.7). The conformation of the conotoxin depends mainly on the folding information encoded in the sequence and the number of disulphide bridges and isomers, which can be calculated by the equation $(2 n) ! /\left(2^{n} n !\right)$ with $n$ being the number of disulphide bonds. ${ }^{[194]}$ Accordingly, the $\mu$-SIIIA consists of three disulphide bridges and can theoretically form 15 isomers excluding topological isomers. However, only one isomer corresponds to the bioactive conformation, indicating the relevance of the folding process. ${ }^{[192]}$
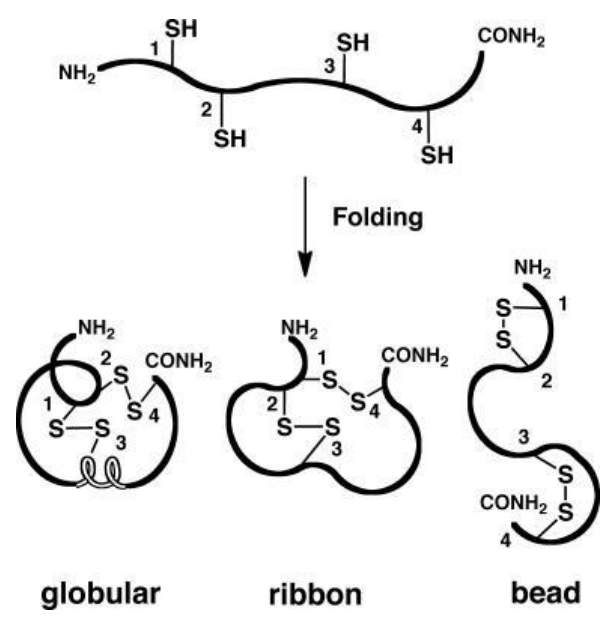

Figure 2.7: Formation possibilities in a peptide containing two-disulphide bonds. Reprinted with permission from K. B. AKONDI et al., Chem. Rev. 2014, 114, 5815-5847.

The successful formation of the native $\mu$-SIIIA by oxidative folding was achieved by the addition of reduced and oxidized glutathione (GSH/GSSG) in the basic buffer ( $\mathrm{pH}$ 7.5-8.5) and high dilution to prevent dimer or oligomer formations. ${ }^{[12,210,218]}$ Furthermore, the folding of the $\mu$-SIIIA can also be induced in ionic liquids (1-ethyl-3-methylimidazolium acetate), providing higher yields and accelerated reaction times. ${ }^{[219]}$

However, the limitations of the oxidative folding strategies were reported by RADZEY, showing that introduction of non-natural amino acids for fluorophore labelling, drastically disturb the 
folding process. ${ }^{[220]}$ A variety of different folding conditions, including GSH/GSSG in a Tris/HCl buffer or ionic liquids, were analysed, leading to multiple disulphide isomers, nonnative structures or low yields. ${ }^{[210,213]}$ To overcome these limitations, a regioselective oxidation strategy was developed, allowing a controlled formation of the disulphide bonds.

\subsubsection{Regioselective Folding of Disulphide-Rich Peptides}

The regioselective synthesis approach is based on orthogonal cysteine-protecting groups, which ensure a selective deprotection and a stepwise formation of the disulphide bonds. This strategy forms the desired disulphide connectivity and reduces the number of isomers during the folding process to a few topological isomers (see figure 2.8). ${ }^{[221,222]}$ For a controlled step-by-step formation of multiple disulphide bonds, cleavage and oxidation conditions are required, which prevent breaking and reshuffling of already formed disulphide bridges. Therefore, alkaline conditions, thiols or nucleophiles have to be avoided during all deprotection and disulphide formation steps.

Oxidative Folding

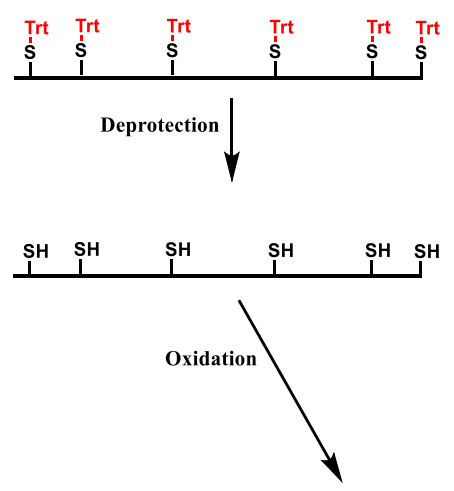

Regioselective Folding

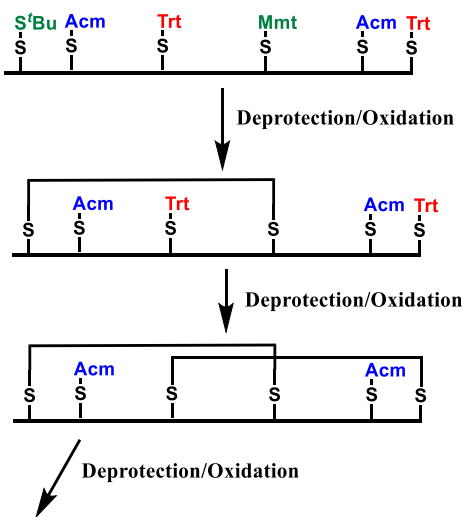

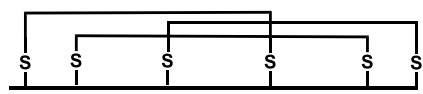

Figure 2.8: Comparison of oxidative one-step folding and regioselective synthesis strategy.

For the synthesis of conotoxins with multiple disulphide bridges, different approaches were reported using the following protecting groups: tert-butylthio $\left(\mathrm{S}^{t} \mathrm{Bu}\right)$, trityl (Trt), acetamidomethyl (ACM), tert-butyl $\left({ }^{t} \mathrm{Bu}\right)$ and methoxybenzyl (MeOBzl) (see table 2.2). ${ }^{[23,224]}$ However, the order of cleavage and deprotection, as well as reaction conditions, strongly affects the success and yields of the synthesis. For example, the $S^{t} \mathrm{Bu}$ group is cleaved reductively; therefore, it must be removed in the first folding step, to prevent a reduction of already preformed disulphide bridges. The Trt group is a frequently used thiol protecting group because it is labile towards TFA and is removed in the cleavage process of the peptide from the resin. 
Furthermore, the Trt-group is often combined with the orthogonal Acm group, which is stable against TFA and can be removed by iodine or mercury salts. However, the Acm cleavage conditions can cause oxidation of sensitive side chains like methionine, histidine, tryptophan or tyrosine residues. ${ }^{[194]}$ Commonly protecting groups for the formation of the $3^{\text {rd }}$ disulphide bridge are the $t \mathrm{Bu}$ and $\mathrm{MeOBzl}$ group. The $t \mathrm{Bu}$ group can be removed in a DMSO/TFA mixture at room temperature whereas MeOBzl is cleaved in DMSO/TFA at $60{ }^{\circ} \mathrm{C} .{ }^{[225]}$

Table 2.2: Overview of frequently used thiol protecting groups for SPPS. The structure, stability and standard cleavage conditions for the different protecting groups are shown. ${ }^{[194,223]}$

\begin{tabular}{|c|c|c|c|}
\hline Protecting group & Structure & Stabile & Cleavage \\
\hline Triphenylmethyl (Trt) & & base, nucleophiles & $\begin{array}{l}\text { diluted TFA }(30 \%), \mathrm{I}_{2}, \mathrm{Ag}^{+}, \mathrm{Hg}^{2+} \text {, } \\
\mathrm{Ti}^{3+}, \mathrm{RSCl}\end{array}$ \\
\hline Acetoamidomethyl (Acm) & & TFA, HF $\left(0{ }^{\circ} \mathrm{C}\right)$, base & $\begin{array}{l}\mathrm{I}_{2}, \mathrm{Hg}^{2+}, \mathrm{Ag}^{+}, \mathrm{Ti}^{3+}, \mathrm{RSCl}, \mathrm{Ph}_{2} \mathrm{SO} / \\
\mathrm{MeSiCl}_{3}\end{array}$ \\
\hline Tert-Butylthio $\left(\mathrm{S}^{t} \mathrm{Bu}\right)$ & & TFA, base, $\mathrm{RSCl}$ & $\mathrm{RSH}, \mathrm{Bu}_{3} \mathrm{P},\left(\mathrm{HOOCCH}_{2} \mathrm{CH}_{2}\right)_{3} \mathrm{P}$ \\
\hline Tert-Butyl $\left({ }^{t} \mathrm{Bu}\right)$ & & $\begin{array}{l}\text { TFA, HF }\left(0{ }^{\circ} \mathrm{C}\right) \text {, base, } \\
\operatorname{Ag}(\mathrm{I}), \mathrm{I}_{2}\end{array}$ & $\begin{array}{l}\mathrm{HF}\left(20^{\circ} \mathrm{C}\right), \mathrm{Hg}^{2+}, \mathrm{RSCl}, \mathrm{Ph}_{2} \mathrm{SO} / \\
\mathrm{MeSiCl}_{3}\end{array}$ \\
\hline 4-Methylbenzyl (MeBzl) & & TFA, HF, base & $\begin{array}{l}\mathrm{HF} \quad\left(0{ }^{\circ} \mathrm{C}\right), \quad \mathrm{Ti}^{3+}, \quad \mathrm{RSCl}, \quad \mathrm{Ph}_{2} \mathrm{SO} / \\
\mathrm{MeSiCl}_{3}\end{array}$ \\
\hline Monomethoxytrityl (Mmt) & & base & $\begin{array}{l}\text { diluted TFA }(0.5 \%), \mathrm{Hg}^{2+}, \mathrm{Ag}^{+}, \mathrm{I}_{2} \text {, } \\
\mathrm{Ti}^{3+}\end{array}$ \\
\hline
\end{tabular}




\subsection{Design and Synthesis of Modified $\mu$-Conotoxin SIIIA}

Voltage-gated sodium channels are involved in all physiological processes by controlling the influx of $\mathrm{Na}^{+}$across the membrane. ${ }^{[11,178,179]}$ Especially mutations and dysfunctions in the neuronal Nav1.2 channel are associated with various neurological diseases. ${ }^{[11,178,179]}$ However, for the development of new specific therapeutics, a deeper understanding of the molecular mechanisms and the expression levels of these channels are fundamental. A potent inhibitor of the Nav1.2 channel is required, which can be conjugated to a fluorescent dye, to visualize the localization and distribution of these channels by fluorescence microscopy.

For identification of potent inhibitors of the Nav1.2 channels, a screening approach was performed. Thereby, the $\mu$-conotoxin SIIIA and KIIIA, which were isolated from the venomous cone snails, were identified to preferentially block the Nav1.2 channel with low nanomolar affinity and further the skeletal Nav1.4 channel with submicromolar affinity. ${ }^{[12,207]}$ Comparison of the sequence of both conotoxins revealed additional pyroglutamate and asparagine residues at the $N$-terminus as well as two glycine residues in the first loop for the $\mu$-SIIIA. Since these residues are non-essential for the channel interaction, they are suitable positions for a chemical substitution of a fluorophore, without affecting the binding affinity of the conotoxin. ${ }^{[210,220]}$ Consequently, the $\mu$-SIIIA was selected as a lead structure for fluorophore labelling and channel investigations.

Commonly used labelling strategies, addressing amine or thiol groups in the protein of interest, cannot be applied for the $\mu$-SIIIA. ${ }^{[226-228]}$ On the one hand, labelling of a newly introduced amine group would lead to unspecific labelling of the native lysine residue in the $\alpha$-helix, which is essential for the conotoxin channel interaction. ${ }^{[207]}$ On the other hand, the introduction of another thiol group by a cysteine would result in misfolding of the $\mu$-SIIIA, by the formation of a non-native disulphide bond. To overcome these limitations, the $\mu$-SIIIA can be labelled by a biorthogonal copper-catalysed azide-alkyne cycloaddition (CuAAC). Therefore, an alkyne linker is introduced into the sequence of $\mu$-SIIIA, which can be conjugated to an azide modified fluorophore.

In previous studies from RADZEY, different positions in the $\mu$-SIIIA sequence were modified with a fluorophore and analysed regarding their binding affinity towards Nav1.2 in patch-clamp measurements. ${ }^{[220]}$ The $\mu$-SIIIA derivative, which was labelled at the Gly6 position, revealed the highest blocking activity compared to $C$ - or $N$-terminal labelled derivates. ${ }^{[220]}$ However, accurate determination of the Nav1.2 channel distribution by fluorescence microscopy and kinetic measurements of the labelled $\mu$-SIIIA were not performed. The measurements from 
RADZEY also revealed that the AlexaFluor 647 fluorophore exhibit a tendency of sticking to membranes and the charges of the fluorophore disturb the channel interaction. ${ }^{[220]}$ The fluorophore was therefore replaced with the AlexaFluor 488 fluorophore, which was attached to an alkyne linker in the position of the Gly6 of the modified $\mu$-SIIIA (see figure 2.9). ${ }^{[229]}$
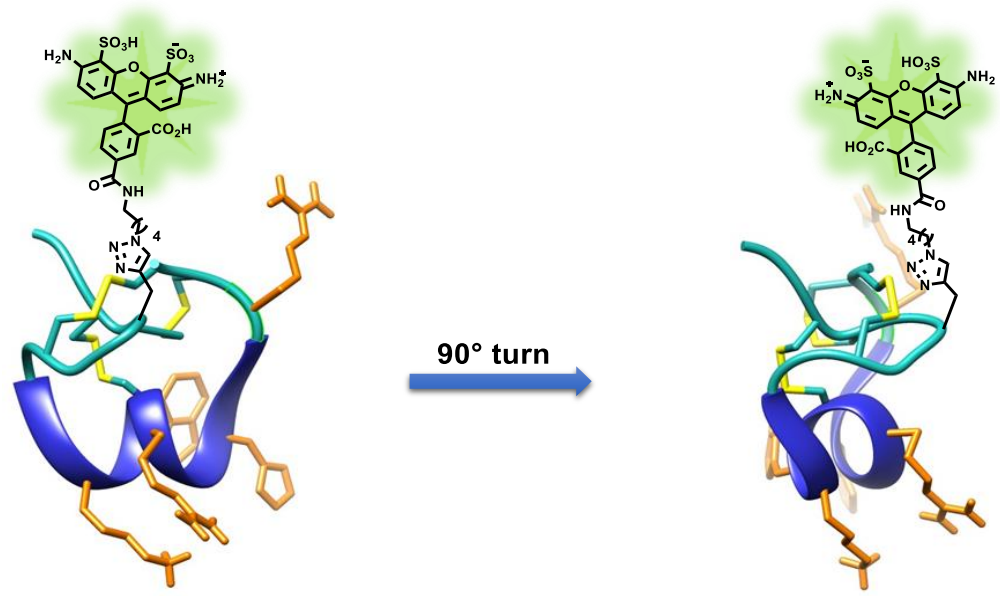

Figure 2.9: 3D Structure of the $\mu$-SIIIA modified with AlexaFluor 488. The fluorophore was attached to an alkyne linker in the position of the Gly6. The fluorophore is highlighted in green, the disulphide bridges in yellow and main residues for the channel interaction are presented in orange (BMRB ID code: SMS20025). The graphic was created with USCF Chimera package. ${ }^{[212]}$

\subsubsection{Synthesis of Native $\mu$-Conotoxin SIIIA}

In the following chapter, the synthesis of native and fluorophore labelled $\mu$-conotoxins SIIIA are described to target Nav channels (see figure 2.10). Previous results from RADZEY revealed the formation of multiple disulphide isomers when using the oxidative folding strategy for the synthesis of an alkyne modified $\mu$-SIIIA derivative. ${ }^{[220]}$ A regioselective folding strategy for the synthesis of an alkyne-modified $\mu$-SIIIA was developed, based on the work from RADZEY.${ }^{[179]}$ This synthesis strategy ensures the correct folding of three disulphide bridges and induces the native topology by a stepwise deprotection of orthogonal cysteine protecting groups. After folding of the disulphide bridges, the $\mu$-SIIIA can be labelled with a fluorophore by $\mathrm{CuAAC}$ reaction. ${ }^{[220]}$

First, the unmodified $\mu$-SIIIA was synthesised to verify whether the regioselective folding strategy forms the native conformation of the toxin. Therefore, the synthesised $\mu$-SIIIA was characterised by patch-clamp measurements and the blocking activity was compared with literature data for the native $\mu$-SIIIA. ${ }^{[13,202,222]}$ The simple structure of the unmodified $\mu$-SIIIA 
was used to optimize reaction and folding conditions, which can be later applied for the synthesis fluorophore labelled $\mu$-SIIIA derivatives.

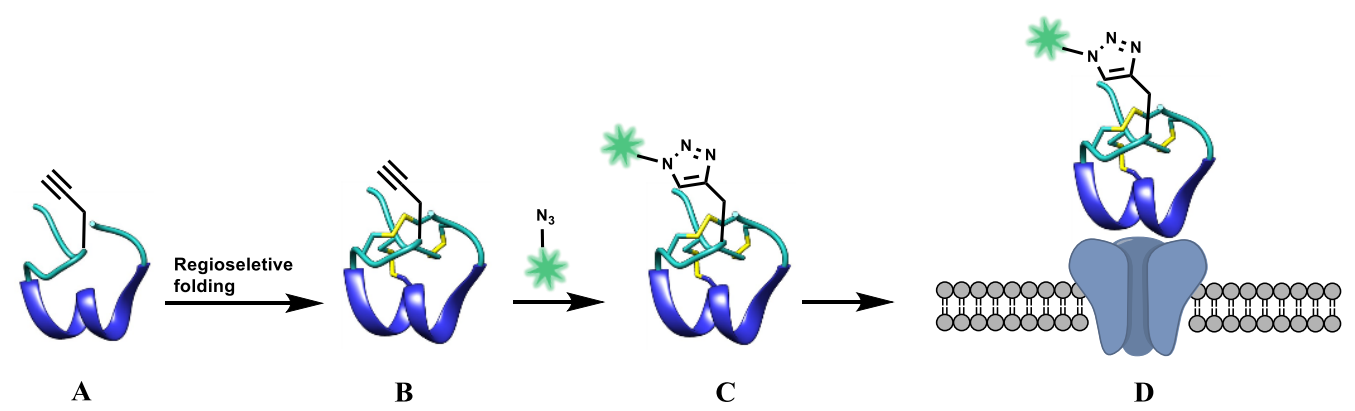

Figure 2.10: Schematic overview of the $\mu$-SIIIA synthesis into a fluorescent labelled toxin. In the first step, a linear peptide was synthesised, containing an alkyne linker for biorthogonal labelling (A). After the formation of the disulphide bridges (B), the fluorophore is attached to the folded peptide (C), allowing visualisation of the $\mathrm{Na}_{\mathrm{V}}$ channel distribution (D).

The linear precursor sequence of the unmodified $\mu$-SIIIA was synthesised on a 2-chlorotrityl chloride resin, using Fmoc-based solid-phase peptide synthesis (SPPS) on a peptide synthesizer. The chlorotrityl resin was modified with a RAMAGE-linker to introduce a $C$-terminal amide group to the peptide sequence. ${ }^{[129]}$ To avoid peptide aggregation during SPPS, a resin with low loading $(<0.35 \mathrm{mmol} / \mathrm{g})$ density was used. The peptides can be cleaved from the resin under mild conditions with $10 \%$ acetic acid, producing the protected peptide in solution (see figure 2.11). Disulphide bonds can be built in high dilution, which prevents dimer or oligomer formation caused by intermolecular disulphide bridges. ${ }^{[194]}$ Coupling of the Fmoc-protected amino acids was performed under microwave irradiation at $50{ }^{\circ} \mathrm{C}$ for $10 \mathrm{~min}$, due to the sensitivity of the chlorotrityl resin towards elevated temperatures. ${ }^{[231]}$ Activation of the amino acids was achieved with DIC as activator and Oxyma as activator base.

A controlled folding process of the three disulphide bonds is essential for the synthesis of the $\mu$-SIIIA since studies of the disulphide isomers of the $\mu$-conotoxin PIIIA revealed either no or a reduced (30-50\%) channel blockage activity compared to the native toxin. ${ }^{[230]}$ The formation of the first disulphide bridge is crucial and the yields can be enhanced by activation of the first cysteine residue. ${ }^{[220]}$ The resin-bound peptide (54) was treated with $20 \%$ 2-mercaptoethanol in DMF to remove the $\mathrm{S}^{t} \mathrm{Bu}$ protecting group, forming the free thiol group at Cys3 (see figure 2.11). Following, the thiol group was activated by introduction of an electron-withdrawing 2-thiopyridine (SPyr) substituent, which promotes the formation of a disulphide bond in the following step. The protected peptide (55) was cleaved from the resin with $10 \%$ acetic acid $(\mathrm{AcOH})$ and the monomethoxytrityl (Mmt) protecting group at Cys13 was removed with $0.5 \%$ 
TFA in DCM under high dilution. The first disulphide bridge (56) was formed under basic conditions in a mixture of triethylamine (10\%) and DCM, causing deprotonation of the free thiol group which can attack, as a nucleophile, the activated Cys(SPyr).

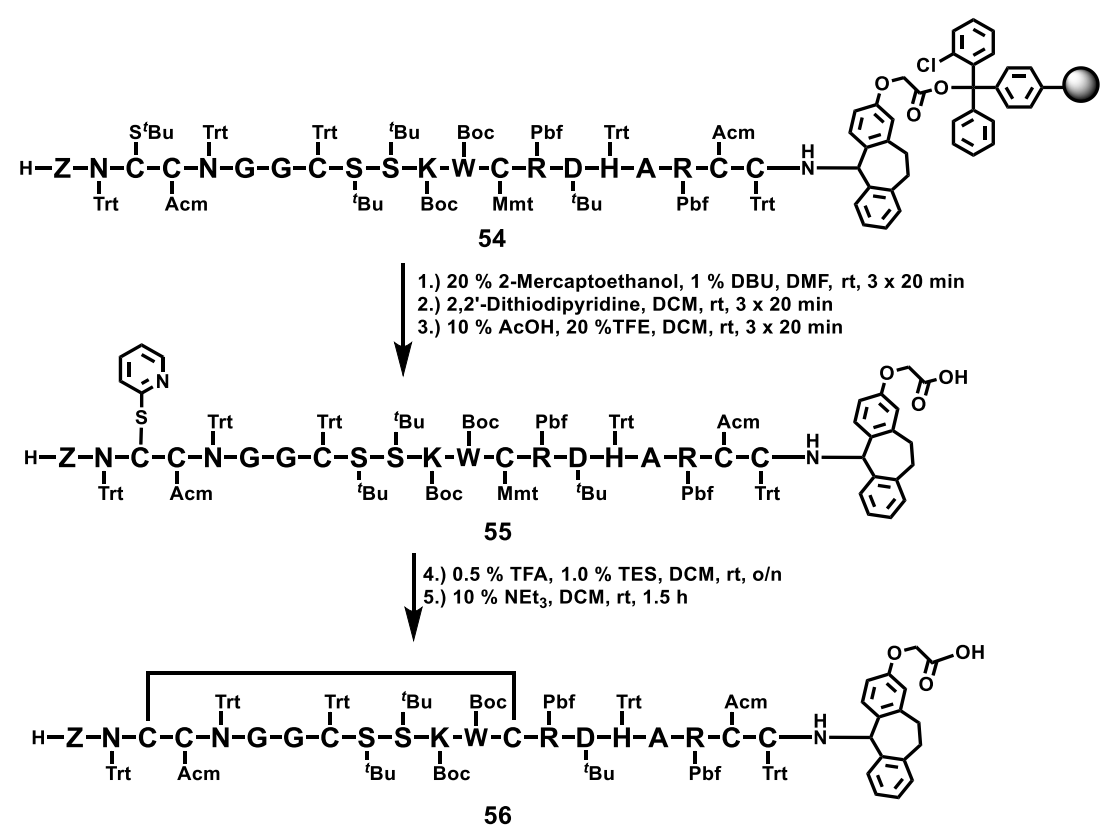

Figure 2.11: Formation of the first disulphide bridge of the native $\mu$-SIIIA. After deprotection of the $S^{t} \mathrm{Bu}$ group, the free thiol group was activated by $2,2^{\prime}$-dithiodipyridine. Afterwards, the peptide (54) was cleaved from the resin and the Mmt protecting group was removed, forming the first disulphide bridge (56) under basic conditions. $\mathrm{Z}$ represents pyroglutamate.

After the formation of the first disulphide bridge (56), all acid-sensitive protecting groups were removed with a mixture of TFA, TIS and $\mathrm{H}_{2} \mathrm{O}$, leading to the crude $\mu$-SIIIA (57) with two Acm protected cysteine residues (see figure 2.12). The oxidation of the second disulphide bridge was induced by DMSO, which can be applied over a broad $\mathrm{pH}$ range from 1 to 8 . Especially acidic conditions accelerate the oxidation rates and prevent reshuffling of already built disulphide bridges. $^{[232]}$ However, low $\mathrm{pH}(<3)$ or high DMSO concentrations $(>50 \%)$ can oxidize sensitive side-chain residues like histidine or tryptophan. ${ }^{[194]}$ The formation of the second disulphide bridge was performed under mild oxidizing conditions with $20 \%$ DMSO in acetate buffer at pH 6. The crude peptide (58) was purified by HPLC yielding one major product fraction. 


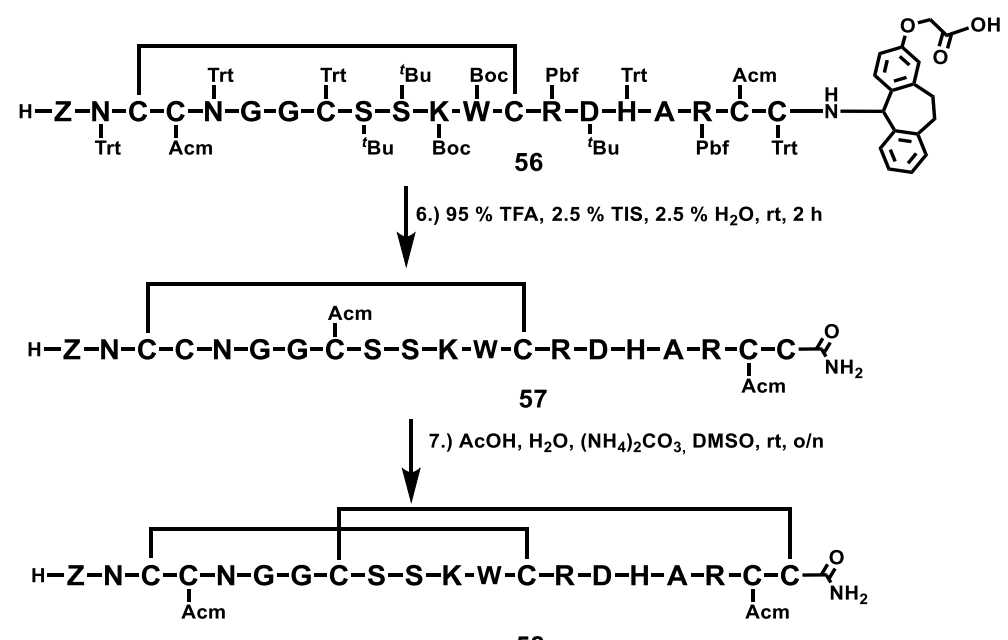

58

Figure 2.12: Formation of the second disulphide bridge of the native $\boldsymbol{\mu}$-SIIIA. After cleavage of all acid protecting groups, the second disulphide bridge (58) was formed in acetate buffer with $20 \%$ DMSO at pH 6 . The $C$-terminus is amidated.

Finally, the two Acm protecting groups were oxidatively cleaved by addition of iodine, which simultaneously induced the formation of the third disulphide bridge. Iodine-mediated cleavage can also cause oxidation of the sensitive residues histidine or tryptophan in the $\mu$-SIIIA sequence and longer reaction times $(>4 \mathrm{~h}$ ) can lead to a reshuffling of already formed disulphide bridges. ${ }^{[233]}$ Consequently, the purified $\mu$-SIIIA with two disulphide bonds was dissolved in a mixture of $\mathrm{AcOH} / \mathrm{H}_{2} \mathrm{O}$, whereby the scavenger Ac-Trp-OMe was added to prevent oxidative side reactions. ${ }^{[233]}$ After the addition of iodine, the reaction mixture was stirred for $1 \mathrm{~h}$ and the addition of ascorbic acid quenched the excess of iodine. HPLC purification and mass spectrometry analysis of the crude reaction mixture showed the formation of four main product species (59), which have the same mass but show different retention times on the HPLC-column (isomer 59.1.1-59.1.4, see figure 2.13). Due to the regioselective synthesis strategy, the different product fractions are most likely topological isomers, which form the same disulphide linkage with different conformations. Detailed structural analysis of these isomers by NMR spectroscopy was not carried out so far, due to the small amounts of the four isolated isomers. 


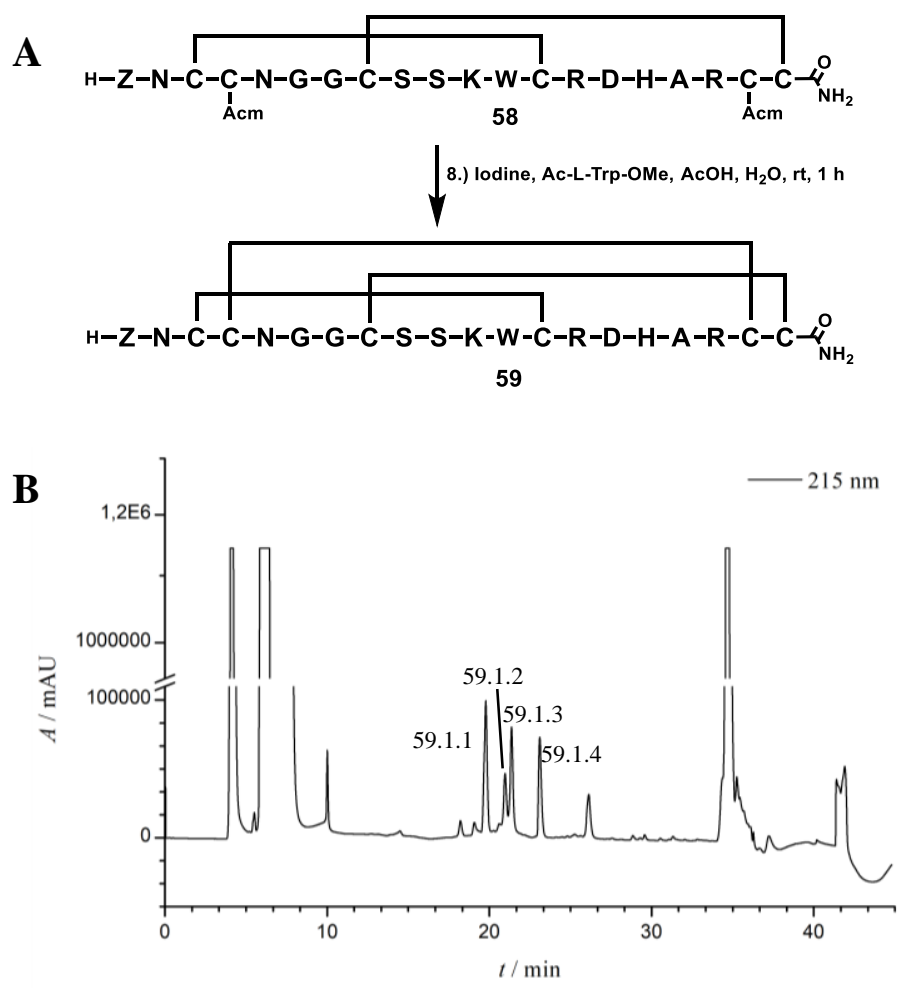

Figure 2.13: Formation of the third disulphide bridge of the $\boldsymbol{\mu}$-SIIIA. A) Cleavage of the Acm protecting groups of the $\mu$-SIIIA (58) by addition of iodine in $\mathrm{AcOH} / \mathrm{H}_{2} \mathrm{O}$. B) HPLC chromatogram of the crude $\mu$-SIIIA (59) with three disulphide bridges. The four product peaks (59.1.1-59.1.4) are highlighted with numbers. HPLC gradient: $1-40 \% \mathrm{MeCN}$ in $30 \mathrm{~min}$.

\subsubsection{Optimised Folding Conditions of $\mu$-Conotoxin SIIIA}

The observed formation of topological isomers by the regioselective approach was already reported for the synthesis of the enterotoxin and the conotoxin PnID B. Patch-clamp measurements of these topological isomers revealed a significant reduction of the biological activity compared to the native conformation. ${ }^{[234,235]}$ ESPIRITU explained these differences of activity by the formation of distinct contortions or knots within the structure of the isomers. ${ }^{[234]}$ Further analysis of the enterotoxin folding process by the three disulphide bridges (Cys1-Cys4, Cys2-Cys5, Cys3-Cys6) revealed a dependence between the order of disulphide bond formation and the proportion of topological isomers. The overall conformation is thereby strongly influenced by the formation of the last disulphide bond. ${ }^{[236]}$ Thus, an optimised synthesis strategy for the $\mu$-SIIIA is required to not only reduce the number of topological isomers but also to increase the yields for the native conformation to create a cost-effective product.

Based on these findings, an optimised folding strategy for the $\mu$-SIIIA was developed by analysing the 3D structure of $\mu$-SIIIA. The analysis revealed two disulphide bonds (A: Cys3-Cys13, B: Cys4-Cys19) buried in the core of the peptide and one disulphide bridge facing 
the surface (C: Cys8-Cys20) (see figure 2.14 A). The synthesis strategy based on the work from RADZEY forms firstly one core disulphide bridge followed by the surface disulphide bridge. The last bridge is formed in the core between the Cys4-Cys19. This procedure results in four topological isomers potentially caused by the more variable core orientation of the cysteine residues. Changing the order of disulphide formation by rearranging the position of the protecting groups can affect the number of topological isomers. By firstly forming the interior disulphide bonds Cys3-Cys13 and Cys4-Cys19 of the $\mu$-conotoxin SIIIA, the globular structure is preformed (see figure $2.14 \mathrm{~B}$ ). In the last step, the disulphide bond on the surface is built between the Cys8 and Cys20 without affecting the overall conformation.

A

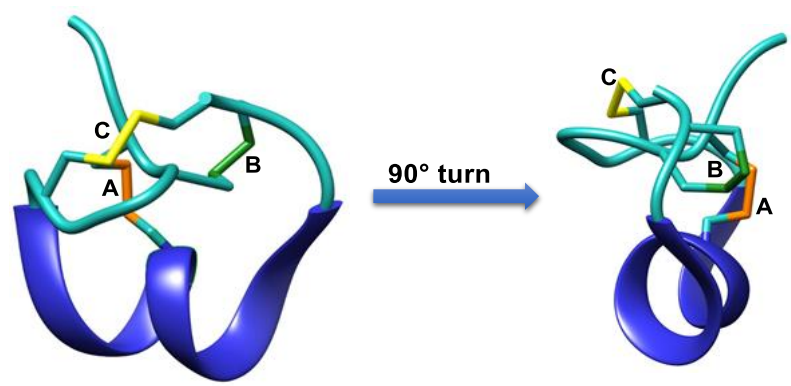

B

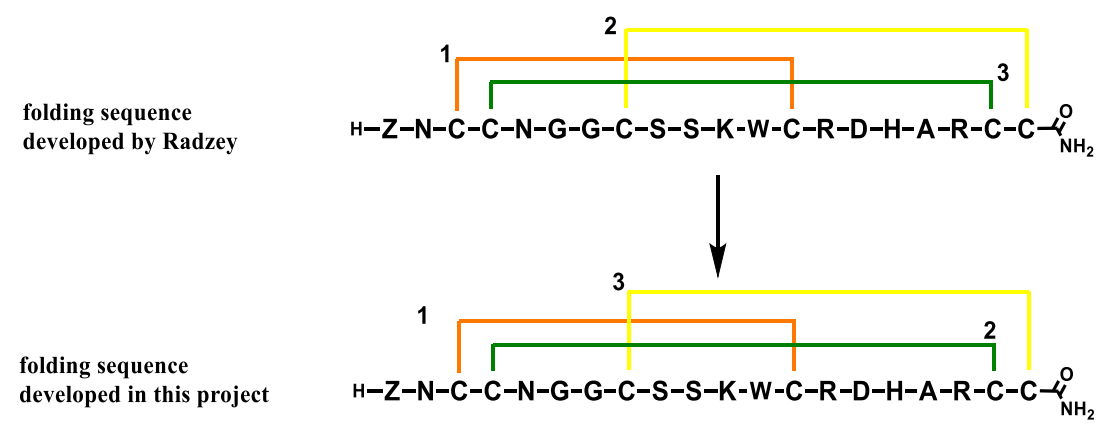

Figure 2.14: Folding strategy of disulphide bonds. A) 3D Structure of the $\mu$-SIIIA. The disulphide bridge C8C20 is highlighted in yellow, Cys3-Cys13 in orange and Cys4-Cys19 in green (BMRB ID code: SMS20025). The graphic was created with USCF Chimera package. $\left.{ }^{[212]} \mathbf{B}\right)$ Comparison of the folding strategy for the synthesis of the $\mu$-SIIIA developed by Radzey and in this work.

Based on these assumptions, a new folding pattern for the synthesis of the native $\mu$-conotoxin SIIIA (60) by an interchange of the cysteine protecting groups to Cys3-St Bu, Cys13-Mmt, Cys4,19-Trt, Cys8,20-Acm was established (see figure 2.15 A). Similar to the previously described method, first the $\mathrm{S}^{t} \mathrm{Bu}$, as well as Mmt group, were cleaved and the Cys3-Cys13 disulphide bridge was built (see chapter 2.2.1). After removal of all acid-sensitive protecting groups, the second core disulphide bridge was formed, and the crude peptide (61) was purified by HPLC obtaining one isomer. Finally, the Acm groups were cleaved with iodine for $1 \mathrm{~h}$, leading to the formation of only two instead of four topological isomers (59.2.1 and 59.2.2), which were separated by HPLC (see figure $2.15 \mathrm{~B}$ and $2.15 \mathrm{C}$ ). These results highlight the 
importance of the order of disulphide bond formation as the presumable number of non-active topological isomers could be reduced from three to one.

A

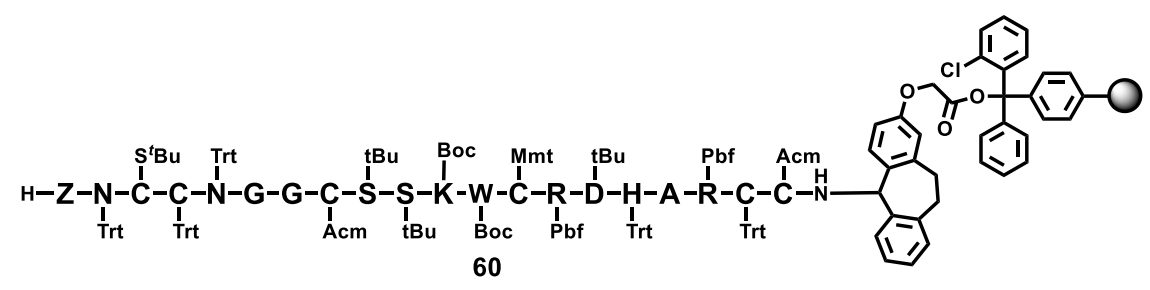

60

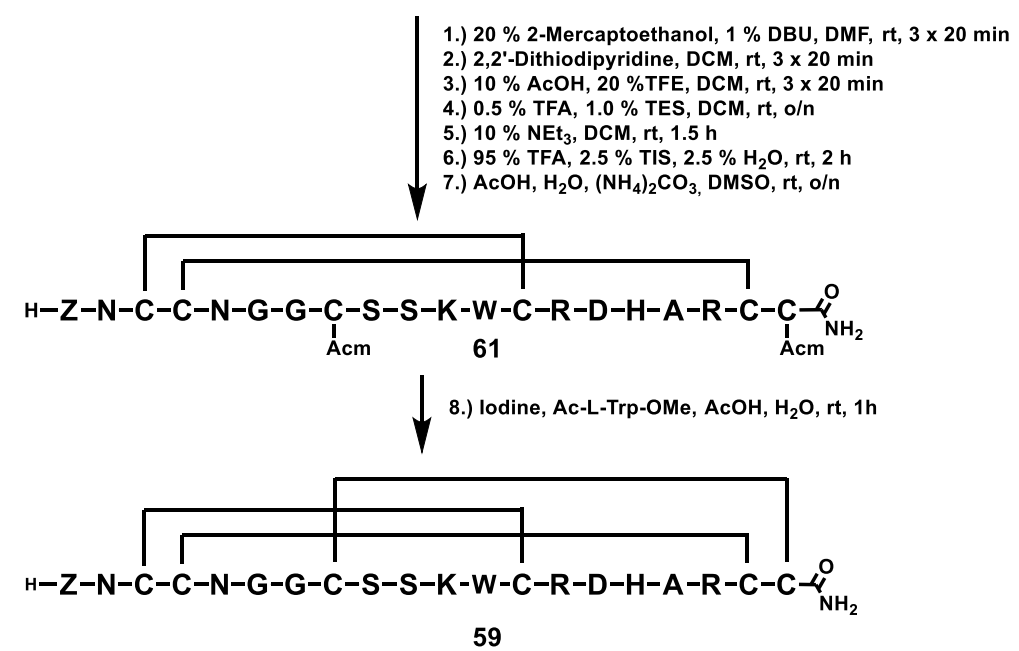

B

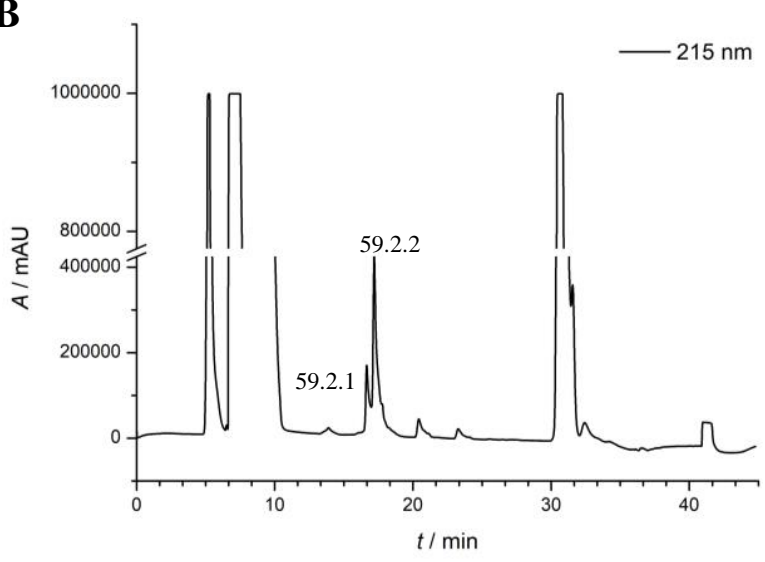

C

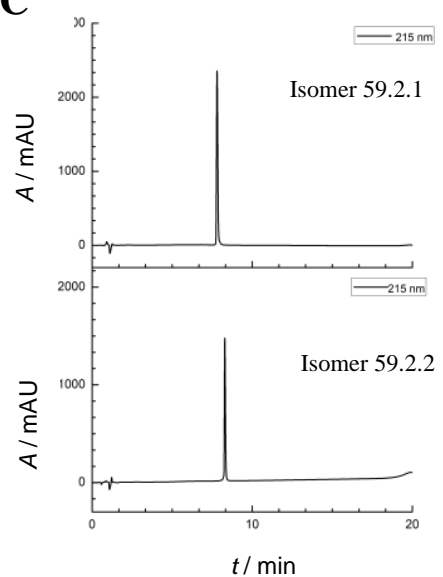

Figure 2.15: Synthesis and purification of native $\mu$-SIIIA. A) Synthesis of the native $\mu$-SIIIA (59). Compared to the synthesis approach from RADZEY, the Acm protected cysteine residues are placed in the positions Cys8 and Cys20 (60). B) HPLC chromatogram of crude native $\mu$-SIIIA (59). The improved folding strategy received the isomers 59.2.1 and 59.2.2. HPLC gradient: $1-40 \% \mathrm{MeCN}$ in $30 \mathrm{~min}$. C) U-HPLC chromatogram of the purified isomers of the $\mu$-SIIIA. HPLC gradient: $1-40 \% \mathrm{MeCN}$ in $15 \mathrm{~min}$.

\subsubsection{Patch Clamp Measurements of Native $\mu$-SIIIA}

Whole-cell patch-clamp experiments carried out measurements of the $\mathrm{NaV}$ channel blocking activity by the topological isomers 59.2.1 and 59.2.2. Previous measurements of the 
$\mu$-conotoxin SIIIA (59) revealed a micromolar potency for Nav1.2 channels as well as a blockage of the Nav1.4 channels with a submicromolar potency. ${ }^{[12,13,202]}$ These electrophysiological characteristics can be used as a reference for the verification of the native $\mu$-SIIIA isomer.

Since expression and patch-clamp measurements of the Nav1.2 channel in cells are challenging, the blocking experiments of both isomers were performed by measuring currents through the Nav1.4 channel. Furthermore, the interaction of the $\mu$-SIIIA and the Nav1.4 channel was intensively investigated, providing detailed information about the toxin-channel interaction as well as the association and dissociation process. These data support the characterisation of newly synthesised isomers and modified $\mu$-SIIIA derivates. ${ }^{[202]}$

Patch-clamp experiments of the isomer 59.2.1 and 59.2.2 of the $\mu$-SIIIA were performed by VIVIEN KISTMACHER (Group of Prof. ENRICO LEIPOLD, University Lübeck). The inhibition studies were conducted with HEK 293 cells, which were transfected with rat Nav1.4-channel expression plasmids. The measurements of the sodium currents were performed $24-48 \mathrm{~h}$ after transfection and the $\mu$-SIIIA isomers were locally applied with a glass pipette. The channel availability was monitored every $3 \mathrm{sec}$ with a pulse, consisting of hyperpolarization to $-80 \mathrm{mV}$ and depolarization to $60 \mathrm{mV}$.

Measurements of isomer 59.2.1 of the $\mu$-SIIIA at a concentration of $1 \mu \mathrm{M}$ revealed an $88 \%$ inhibition of the Nav1.4 current reaching a steady-state phase after $350 \mathrm{sec}$ (see figure $2.16 \mathrm{~A}$ ). A 10-fold increase of the isomer 59.2.1 concentrations resulted in an increase of current inhibition up to $90 \%$ after $80 \mathrm{sec}$. Saturating concentration of $100 \mu \mathrm{M} \mu$-SIIIA leads to a maximal blocking activity of $93 \%$, reaching a steady-state phase after $25 \mathrm{sec}$. Analysis of the dissociation process revealed an almost irreversible binding, showing a sustained blocking activity of $80 \%$ after a washout time of $20 \min \left(\tau_{\mathrm{off}}=6816 \pm 85 \mathrm{sec}\right)$. These results revealed a strong interaction between isomer 59.2.1 and the Nav1.4 channel but no complete blockage of the sodium currents, similar to previous experiments with the native $\mu$-SIIIA. ${ }^{[202]}$

To compare the efficiency of the binding process of the isomer 59.2.1 with the native $\mu$-SIIIA, the dissociation constant $\left(K_{\mathrm{D}}\right)$ has to be calculated. The $K_{\mathrm{D}}$ value can be derived from the association rate $\left(k_{\mathrm{on}}\right)$, which are dependent on the toxin concentration, and the independent dissociation rate $\left(k_{\text {off }}\right)$. The $k_{\text {off }}$ value $\left(1.5 \cdot 10^{-4} \mathrm{~s}^{-1}\right)$ was recorded in the blocking experiments, whereas $k_{\text {on }}$ cannot directly be measured and has to be calculated from the experimentally observed association rate $\left(k_{\mathrm{obs}}\right)$. The $k_{\mathrm{obs}}$ rate is the sum of $k_{\mathrm{on}}$ and $k_{\text {off }}$ depending on the analyte 
concentration, which describes the association and dissociation of the toxins from the channel during the experiment (see equation 2.1).

$$
k_{\text {obs }}=k_{\text {on }} \cdot\left[\mathrm{c}_{\mu-\text { SIIIA }}\right]+k_{\text {off }}
$$

The $k_{\mathrm{obs}}$ were plotted against the corresponding concentration of isomer 59.2.1 and the $k_{\text {on }}$ rate $\left(5.4 \cdot 10^{-3} \mu \mathrm{M}^{-1} \mathrm{~s}^{-1}\right.$ ) was calculated from the slope of the linear regression (see figure 2.16 B). Based on the $k_{\text {on }}$ and $k_{\text {off }}$ kinetics, a $K_{\mathrm{D}}\left(K_{\mathrm{D}}=k_{\text {off }} / k_{\text {on }}\right)$ value of $28 \pm 5 \mathrm{nM}$ was calculated for the isomer 59.2.1. Comparable $K_{\mathrm{D}}$ values were measured for the native $\mu$-SIIIA by YAO et al. in Xenopus oocytes. ${ }^{[208,237]}$ Based on the slow dissociation process and the $K_{\mathrm{D}}$ value, the isomer 59.2.1 were identified to form the native conformation of $\mu$-SIIIA.

A
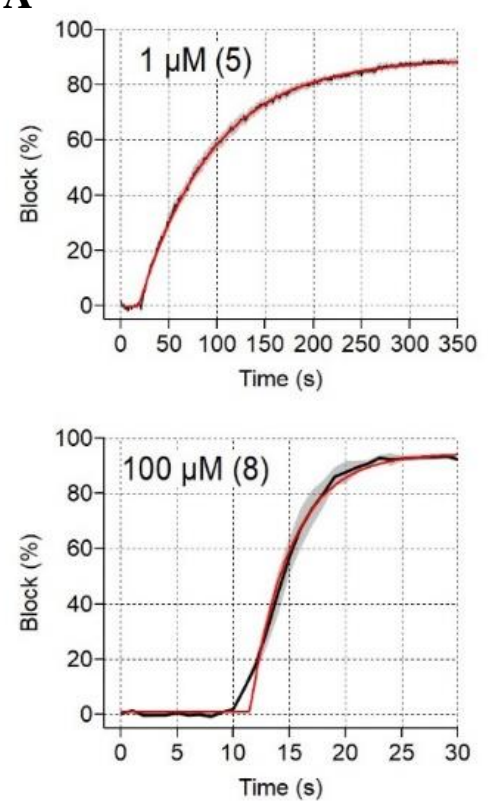
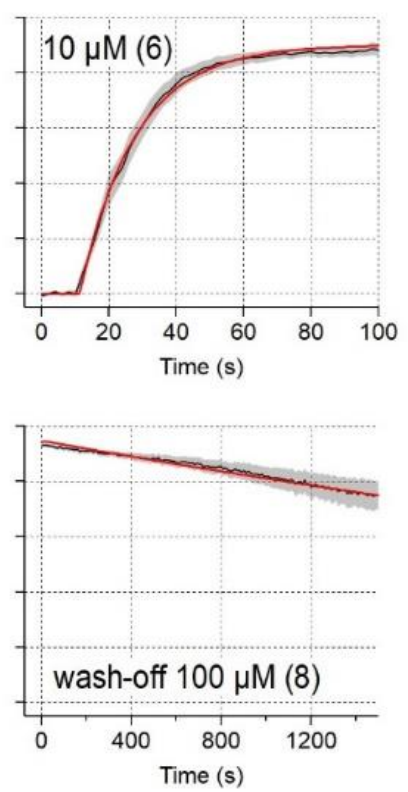

B

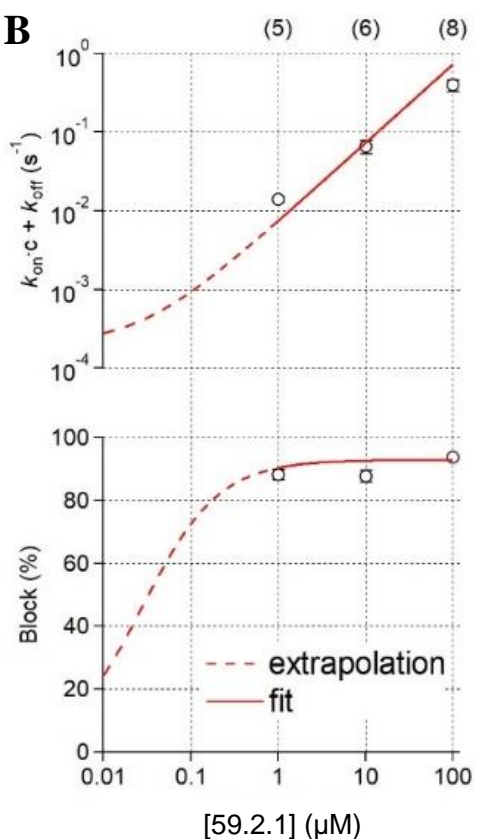

Figure 2.16: Patch-clamp measurements of isomer 2.1. A) Blocking and wash-off kinetics of the isomer 59.2.1 of $\mu$-SIIIA at $1 \mu \mathrm{M}, 10 \mu \mathrm{M}$ and $100 \mu \mathrm{M}$ concentration. The data points are highlighted in black. The SEM is indicated by grey shading and the red lines are single-exponential fits. The number of experimental replicates is shown in parentheses. B) Dose-response curve shows the association and dissociation kinetics (top) and percentage of sodium current inhibition as a function of the $\mu$-SIIIA concentration (bottom). Straight lines are data fits; dashed lines are extrapolations of data fits. The experiment was performed by VIVIEN KISTMACHER.

The patch-clamp measurements of isomer 59.2.2 of $\mu$-SIIIA $(10 \mu \mathrm{M})$ showed a reduced blocking activity of $58 \%$ at a steady-state level after $80 \mathrm{sec}$, compared to a blocking activity of $90 \%$ for isomer 59.2.1 (see figure 2.17 A). Also, a 10-times higher concentration caused only a maximal inhibition of $87 \%$ of the sodium currents after $25 \mathrm{sec}$. Measurements of the dissociation kinetics showed a fast wash-off from the Nav1.4 channel and a full recovery of the current after $400 \mathrm{sec}$ washout time $\left(\tau_{\mathrm{off}}=124 \pm 19 \mathrm{sec}\right)$. Calculation of the $K_{\mathrm{D}}$ value of 
isomer 59.2.2 yielded 140 times higher $K_{\mathrm{D}}$ value $(5.1 \pm 1.2 \mu \mathrm{M})$ compared to isomer 59.2.1 (see figure $2.17 \mathrm{~B}$ ). The fast dissociation process and the increased $K_{\mathrm{D}}$ value indicate a reduced binding affinity between the channel and the toxin probably caused by a non-native conformation of the isomer 59.2.2. Since the toxins were synthesised by a regioselective synthesis strategy, the isomer 59.2.2 is probably forming a topological isomer, with correct disulphide linkage but non-native overall conformation.

A
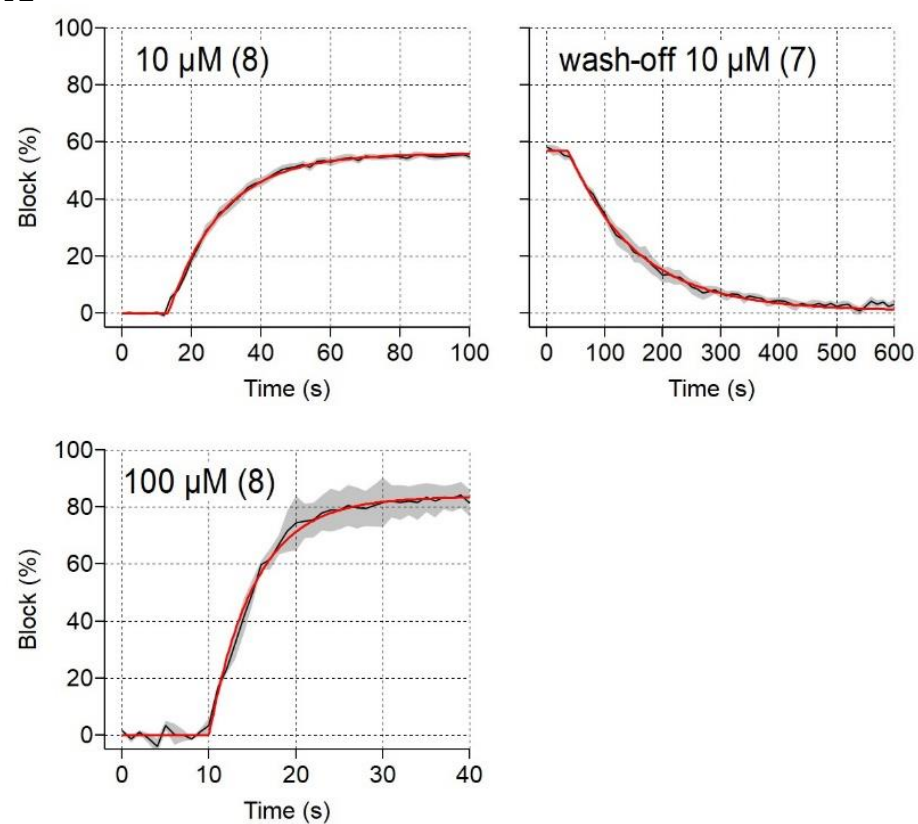

B

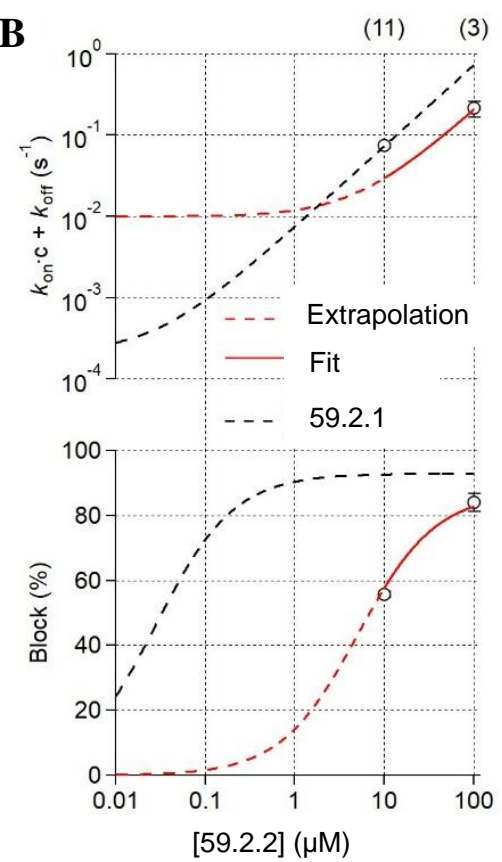

Figure 2.17: Patch-clamp measurements of isomer 59.2.2. A) Blocking and wash-off kinetics of isomer 59.2.2 of $\mu$-SIIIA at $10 \mu \mathrm{M}$ and $100 \mu \mathrm{M}$ concentration. The data points are highlighted in black. The SEM is indicated by grey shading and the red lines are single-exponential fits. The number of experimental replicates is shown in parentheses. B) Dose-response curve shows the association and dissociation kinetics (top) and percentage of sodium current inhibition as a function of the $\mu$-SIIIA concentration (bottom). Straight lines are data fits; dashed lines are extrapolations of data fits. The experiment was performed by VIVIEN KISTMACHER. 


\subsubsection{Synthesis of Labelled $\mu$-Conotoxin SIIIA}

After the successful synthesis and identification of the native $\mu$-SIIIA (59), the optimised folding strategy was applied for the development of fluorophore-labelled $\mu$-SIIIA. A propargylglycine residue was incorporated at the position of Gly6 via SPPS, allowing the coupling of the fluorophore by copper(I)-catalysed alkyne-azide cycloaddition (CuAAC). The synthesis of the linear peptide (62) and the formation of the first and second disulphide bridge was performed analogously to the synthesis of the native $\mu$-SIIIA (59) (see chapter 2.2.1.1). After HPLC purification, the $\mu$-SIIIA (63) with two disulphide bonds and the Acm protecting groups at Cys8 and Cys20 was isolated. Compared to the synthesis protocol of the native $\mu$-SIIIA (59), optimised reaction conditions are required for the cleavage of the Acm groups, as the alkyne linker is sensitive towards iodine. However, other cleavage conditions of the Acm groups were already examined by RADZEY, revealing that mercury(II) acetate or silver(I) trifluoroacetate lead to a reduction of the disulphide bridges. ${ }^{[220]}$ Thus, the iodine mediated cleavage process was analysed by LC-MS to identify whether Acm deprotection or iodination of the alkyne group occurred first. The results showed an efficient cleavage of the Acm groups after $10 \mathrm{~min}$ and first traces of iodated side product after $20 \mathrm{~min}$. The formation of the third disulphide bridge was induced with a mixture of iodine in $\mathrm{AcOH} / \mathrm{H}_{2} \mathrm{O}$ and the reaction was stopped after $15 \mathrm{~min}$ (see figure 2.18 A). HPLC and mass spectrometry analysis of the reaction process revealed the formation of the desired alkyne-modified $\mu$-SIIIA (64) as main product (fraction 1, see figure $2.18 \mathrm{~B}$ ) and only small amounts of the starting material (fraction 2) as well as iodated side product (fraction 3). The purified alkyne-modified $\mu$-SIIIA (64) was used for fluorophore labelling studies.

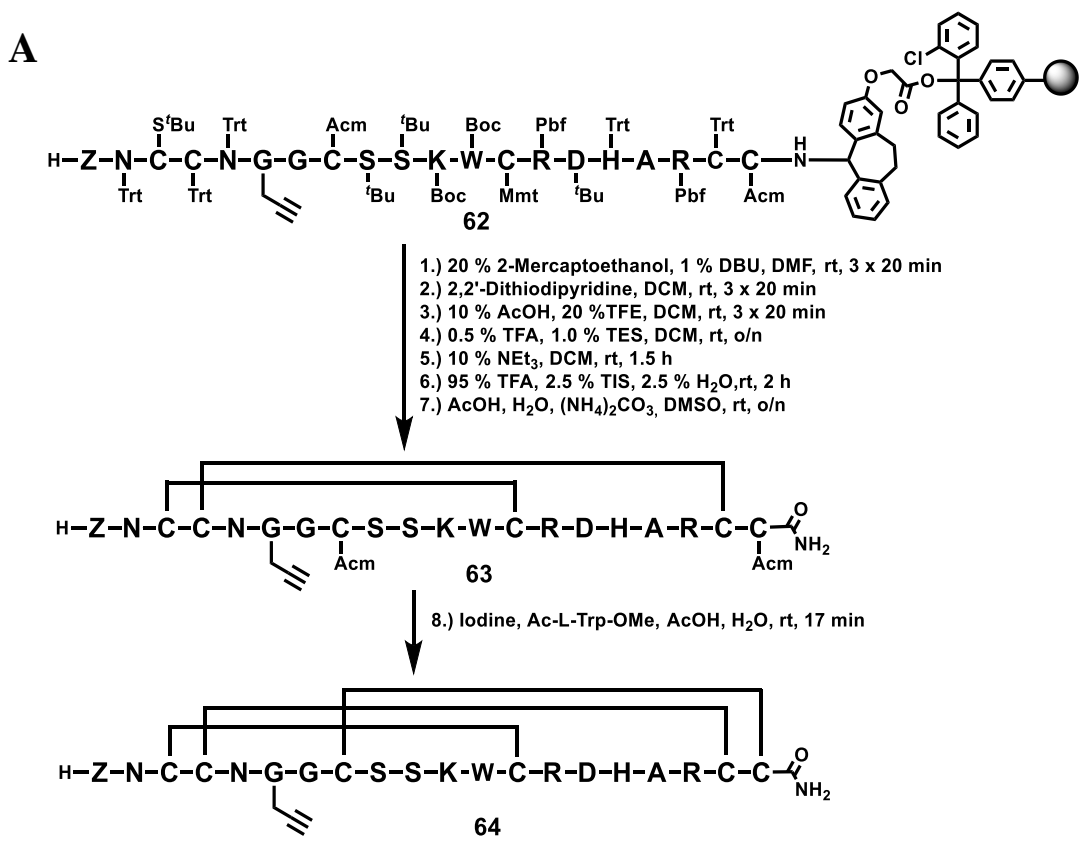




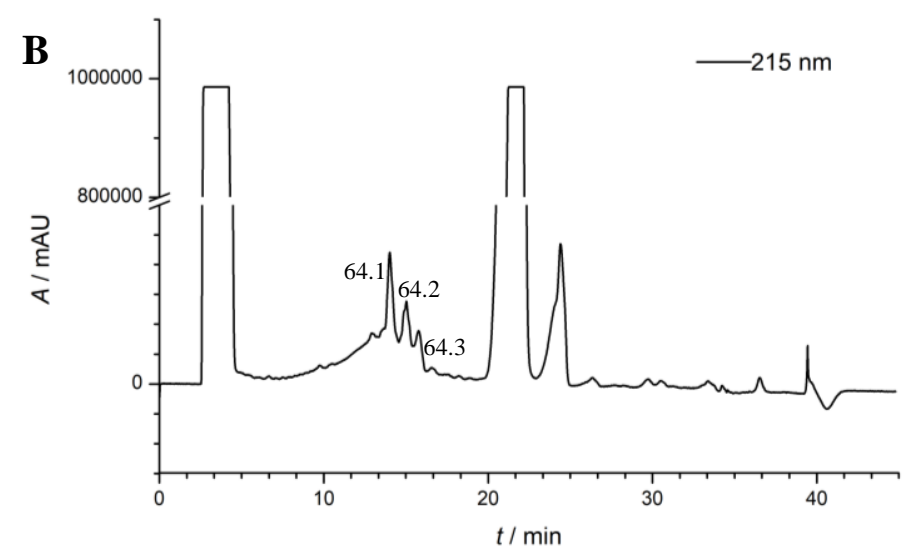

Figure 2.18: Synthesis and purification of alkyne modified $\boldsymbol{\mu}$-SIIIA. A) Synthesis of propargylglycine modified $\mu$-SIIIA (64) by the optimised synthesis strategy. B) HPLC chromatogram of crude alkyne-modified $\mu$-SIIIA (64). The product fraction (1) was separated from the starting material (2) and the diiodated side product (3). HPLC gradient: $1-40 \% \mathrm{MeCN}$ in $30 \mathrm{~min}$.

For imaging, the channel distribution, the alkyne modified $\mu$-SIIIA (64) was labelled with the AlexaFluor 488 fluorophore as it exhibits a small size, high stability and quantum yield as well as reduced membrane interaction. ${ }^{[238]}$ The labelling reaction was performed by a biorthogonal $\mathrm{CuAAC}$ reaction. Compared to other labelling strategies, the $\mathrm{CuAAC}$ reaction exhibits a higher specificity as the reaction only takes place at the alkyne linker. The CuAAC also shows a high tolerance towards functional groups, can be performed under mild reaction conditions and yields an irreversible bond. ${ }^{[239]}$ However, CuAAC reaction has some restrictions regarding its prolonged reaction times for peptides as substrates as well as the formation of reactive oxygen species, which can cause oxidation of sensitive side-chain residues. ${ }^{[240,241]}$ As a consequence, the reaction has to be performed under exclusion of oxygen and in the presence of stabilising ligands like tris(3-hydroxypropyltriazolylmethyl)amine (THPTA). The addition of stabilizing agents preserves the catalytic active copper(I) species and thus, enhances the reaction process significantly. ${ }^{[239]}$

The fluorophore labelling of the $\mu$-SIIIA was first performed according to a protocol from EMPTING et al. ${ }^{[242]}$ The azide modified AlexaFluor 488 fluorophore and alkyne modified $\mu$-SIIIA (64) were treated with copper(II) sulphate pentahydrate, THPTA and sodium ascorbate as reducing agent in degassed water. DIPEA was added to increase the formation of the active copper acetylide complex and accelerate the reaction time. ${ }^{[242]}$ However, analysis of the reaction progress by LC-MS revealed no conversion of the starting material after $48 \mathrm{~h}$.

Consequently, a recently reported method from RINK and THOMAS was tested. ${ }^{[241]}$ They presented an efficient method for fluorophore and tag labelling of coiled-coil peptides by CuAAC. ${ }^{[241]}$ The alkyne-modified $\mu$-SIIIA (64) was mixed with AlexaFluor 488 azide 
fluorophore and $\mathrm{CuSO}_{4} / \mathrm{THPTA}$ /sodium ascorbate as the catalytic system. The reaction was performed in degassed tert-butanol/PBS under inert gas atmosphere and was incubated at $35{ }^{\circ} \mathrm{C}$ for $48 \mathrm{~h}$ (see figure $2.19 \mathrm{~A}$ ). Analysis of the labelling reaction by LC-MS revealed the conversion of the starting material and successful formation of AlexaFluor 488 labelled $\mu$ SIIIA (65). After HPLC purification $33 \mathrm{nmol}$ of the desired product were isolated (see figure 2.19 B). To conclude, the labelled $\mu$-SIIIA (65) was successfully synthesised by a regioselective approach and conjugated to a fluorophore by CuAAC. The affinity and selectivity of the labelled $\mu$-SIIIA (65) is currently investigated by patch-clamp experiments and further imaging experiments will be performed in near future.

A
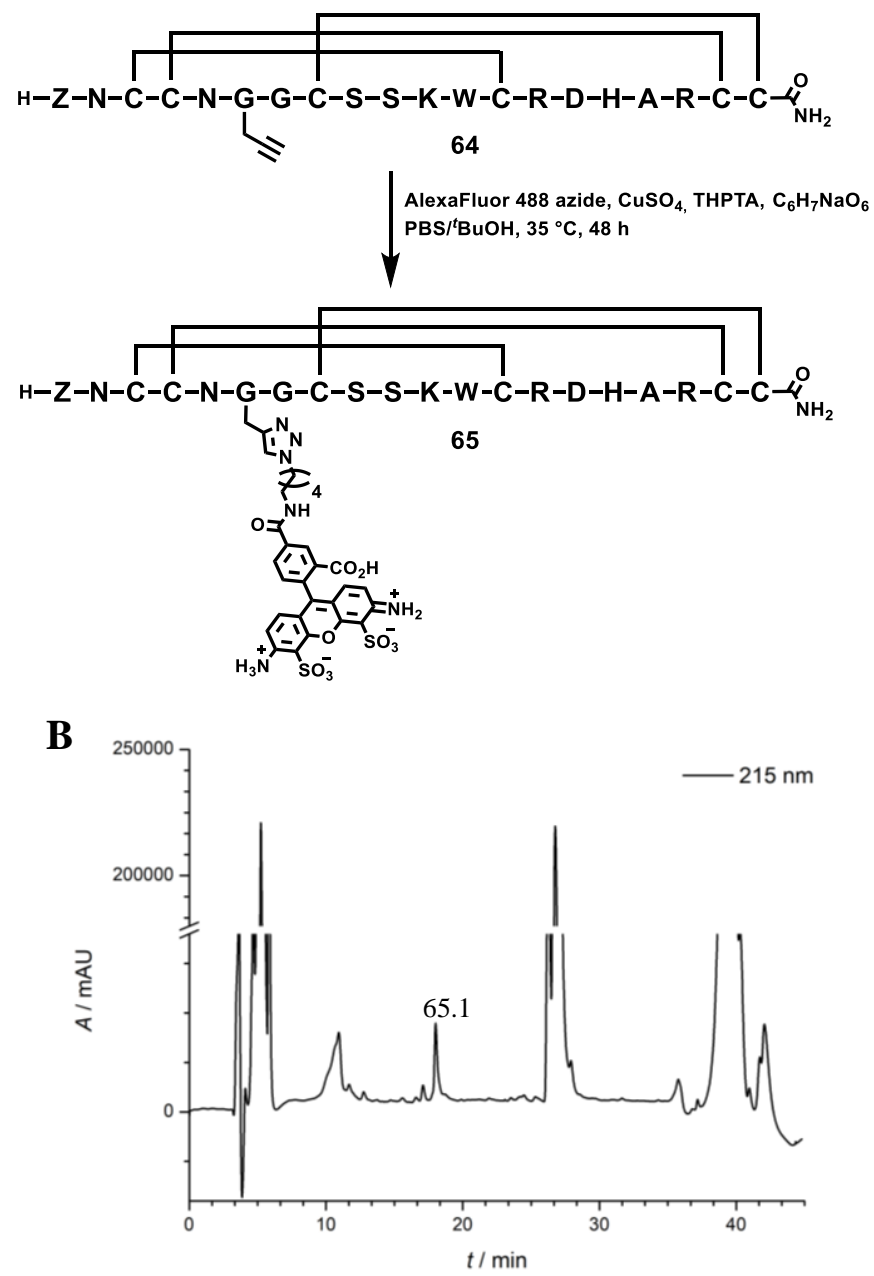

Figure 2.19: Synthesis and purification of AlexaFluor 488 labelled $\mu$-SIIIA. A) Synthesis of the AlexaFluor 488 labelled $\mu$-SIIIA (65) by CuAAC. B) HPLC chromatogram of the crude $\mu$-SIIIA (65) after fluorophore labelling. The desired product (65.1) was received with high purity. HPLC gradient: 1-40\% MeCN in $30 \mathrm{~min}$. 


\subsubsection{New Synthesis Strategies for Labelling $\mu$-Conotoxin SIIIA}

Despite the successful synthesis of the fluorophore labelled $\mu$-SIIIA (65), low yields and the complicated synthesis route limit its application for Nav channel investigations. Especially the deprotection of the Acm group with iodine and therefore the formation of the third disulphide bridge proceeded insufficiently. The iodine also caused side reactions with the sensitive alkyne linker, which restricts the accessibility of the alkyne for the CuAAC reaction. To overcome these synthetic limitations, various folding strategies were investigated, including new thiol protecting groups as well as a rearrangement of the utilised protecting groups.

\subsubsection{Synthesis of Allocam Protected Alkyne Modified $\mu$-SIIIA}

The first approach was inspired by the work of KONDASINGHE et al., who reported the synthesis and application of a new thiol protecting group allyloxycarbonyl aminomethyl (Allocam). ${ }^{\text {[243] }}$ The Allocam group can be removed by $\mathrm{Pd}(0), \mathrm{I}_{2}, \mathrm{~N}$-chlorosuccinimide or high concentrations of TFA whereas it is stable at low TFA concentrations $(<30 \%)$ which are required for Trt or Mmt cleavage. ${ }^{[243]}$ Furthermore, Allocam is compatible with the $\mathrm{S}^{t} \mathrm{Bu}$ group, showing no removal or oxidation after addition of 2-mercaptoethanol and dithiodipyridine. ${ }^{[244]}$ Based on these results, a synthesis strategy for $\mu$-SIIIA (66), including the $S^{t} \mathrm{Bu}, \mathrm{Mmt}$, Trt and Allocam group, was developed (see figure 2.20). After deprotection of the $S^{t} \mathrm{Bu} / \mathrm{Mmt}$ groups and creation of the first disulphide bridge, the Trt-groups, as well as all acid sensitive protecting groups, were cleaved with a mixture of DCM, TFA, TIS and $\mathrm{H}_{2} \mathrm{O}(65 / 30 / 2.5 / 2.5, v / v / v / v)$. HPLC purification of the crude peptide yielded two isomers with two disulphide bridges and the remaining Allocam protecting groups (67.1-67.2). The formation of a second isomer indicates a disturbance of the folding process caused by the Allocam groups. In the last step the Allocam groups were removed with $\mathrm{Pd}(\mathrm{OAc})_{2}, 5 \% \mathrm{AcOH}, 3 \% \mathrm{NMM}$ in DMSO for $48 \mathrm{~h}$. However, analysis of the reaction progress by LC-MS revealed only the starting material and no product formation (64). 


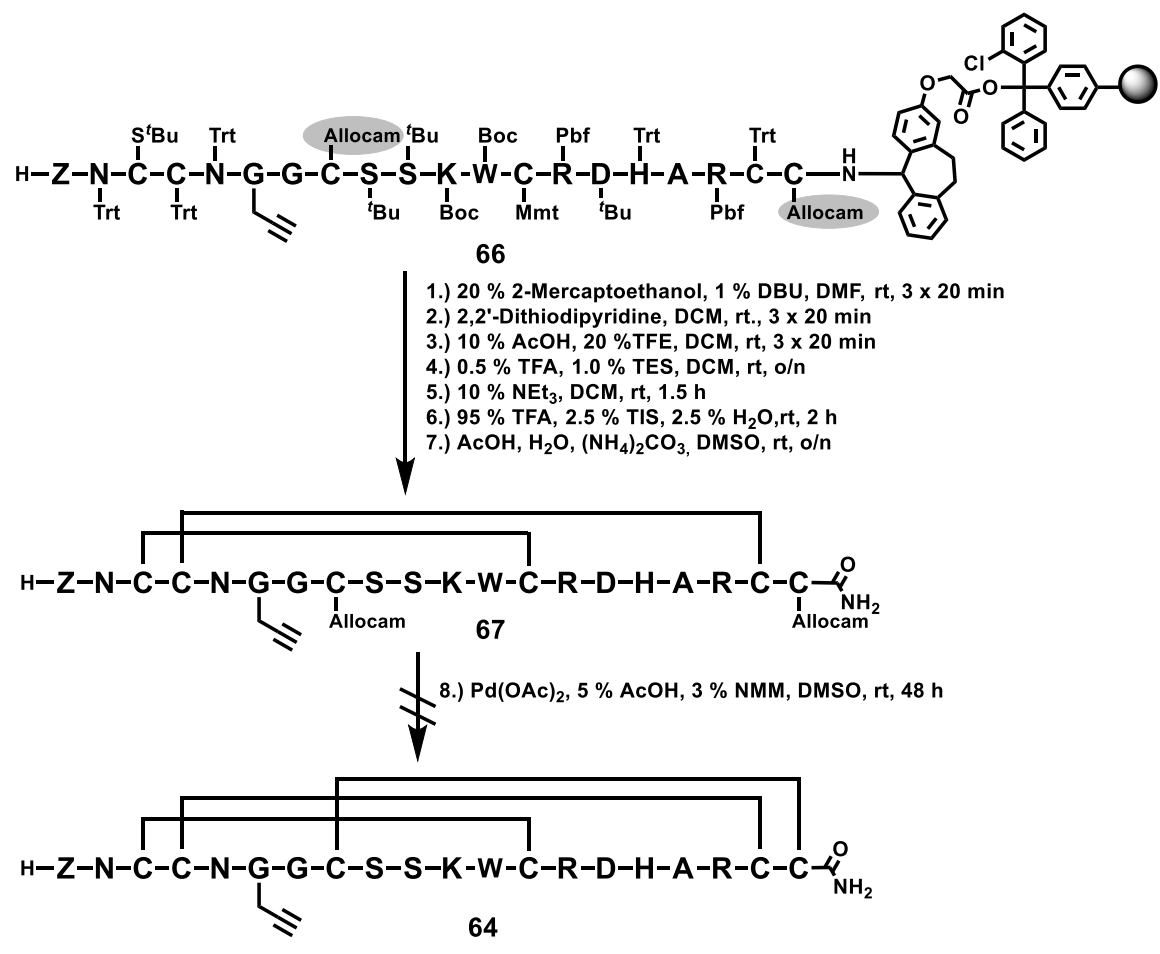

Figure 2.20: Synthesis strategy for the Allocam-protected alkyne-modified $\mu$-SIIIA.

\subsubsection{Synthesis of tert-Butyl and MeOBzl Protected Alkyne Modified $\mu$-SIIIA}

In a second approach, the Acm protecting group was replaced with the tert-butyl group as described in the work of WU et al., presenting a regioselective synthesis of peptides containing four disulphide bonds. ${ }^{[245]}$ The tert-butyl group is orthogonal to the $\mathrm{S}^{t} \mathrm{Bu}, \mathrm{Mmt}$ and Trt groups and can be cleaved with strong acids. Following, the tert-butyl group protected modified alkyne $\mu$-SIIIA (68) was synthesised and the first, as well as second disulphide bridge (69), was formed as described previously (see chapter 2.2.1). The removal of the tert-butyl group and formation of the last disulphide bridge was performed in a mixture of TFA, methyl trichlorosilane and diphenylsulphoxide at $4{ }^{\circ} \mathrm{C}$ (see figure 2.21). After a reaction time of $30 \mathrm{~min}, 2 \mathrm{~h}$ and $12 \mathrm{~h}$, the process was analysed by LC-MS revealing no product formation (64). Different cleavage conditions with $5 \%$ DMSO in TFA at rt were tested, showing removal of the tert-butyl groups after $2 \mathrm{~h}$. However, the isolated product showed a mass difference $\Delta \mathrm{m} / \mathrm{z}$ of +16 , indicating a hydroxylation of the sensitive Trp or His residue. Due to the essential function of both residues for the channel interaction, this approach was not pursued further. 


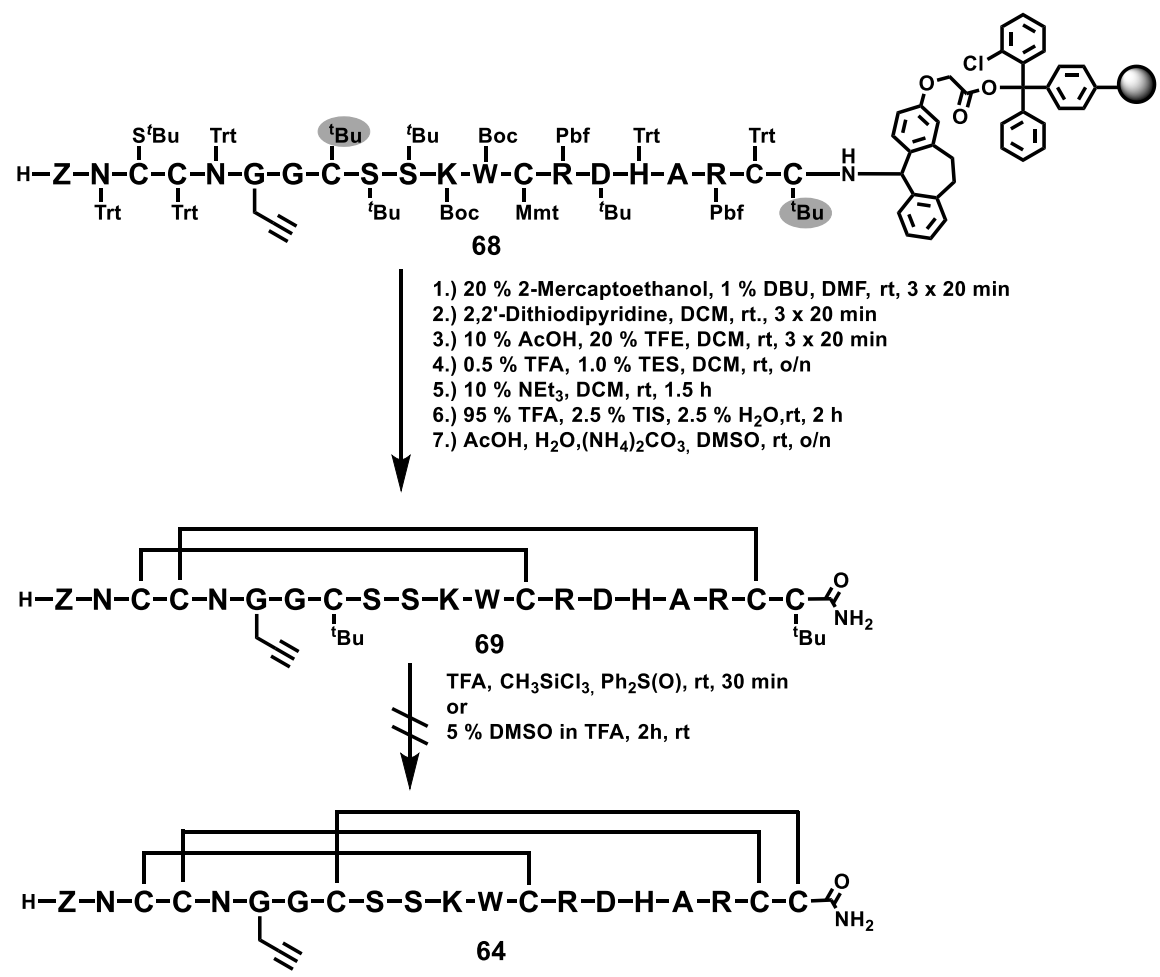

Figure 2.21: Synthesis strategy of tert-butyl-protected and alkyne modified $\mu$-SIIIA.

In a third approach, the Acm groups were substituted by MeOBzl protecting groups, which have been successfully used for the synthesis of fluorescent labelled zinc finger peptides. ${ }^{[246]}$ The MeOBzl group is stable under TFA-cleavage conditions and can be deprotected with trifluoromethanesulfonic acid (TFMSA). Thus, MeOBzl protected Cys8 and Cys20 were incorporated into an alkyne-modified $\mu$-SIIIA (70) and the first, as well as second disulphide bridge, were formed (see figure 2.22). Purification of the crude peptide by HPLC yielded two isomers (70.1-70.2). In the last step, the remaining MeOBzl groups were cleaved in a mixture of $10 \%$ TFMSA, $88 \%$ TFA and $2 \%$ Thioansiole. The cleavage progress was analysed after $2 \mathrm{~h}, 4 \mathrm{~h}$ and $6 \mathrm{~h}$ by LC-MS, revealing only traces of the desired product (64). An improved product formation was detected by using $98 \%$ TFA and $2 \%$ TIS for $12 \mathrm{~h}$ at $37{ }^{\circ} \mathrm{C}$. [247] Unfortunately, this method caused oxidation of sensitive side-chain residues as well. 


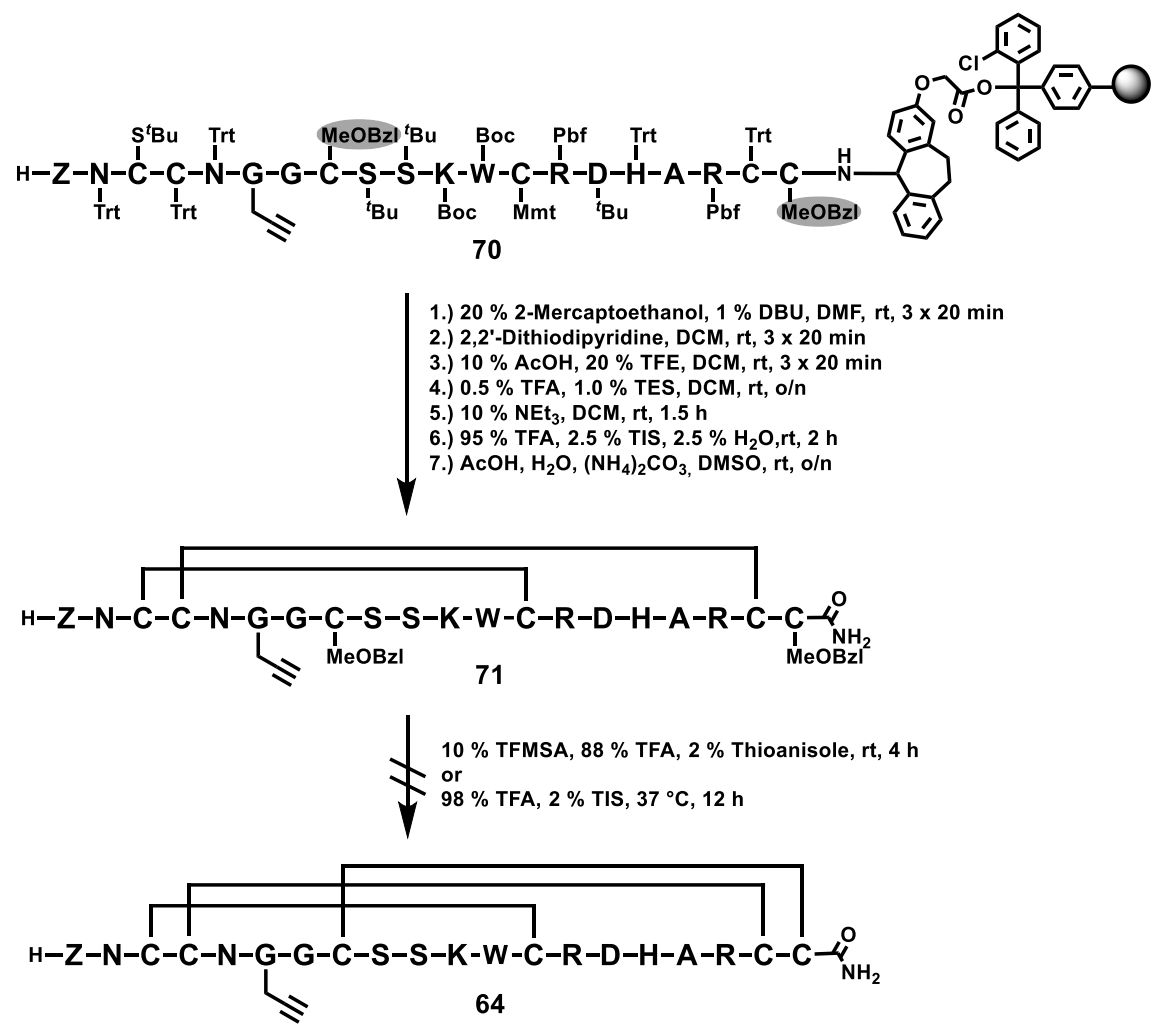

Figure 2.22: Synthesis strategy of MeOBzl-protected and alkyne modified $\mu$-SIIIA.

Summarizing, all investigated protecting groups for replacing the Acm groups revealed limitations. Investigation of the Allocam modified $\mu$-SIIIA showed no cleavage of the protecting groups by addition of $\mathrm{Pd}(\mathrm{OAc})_{2}$ in DMSO. Introduction of the Allocam groups disturbed the folding process of the $\mu$-SIIIA, causing two topological isomers (67.1-67.2) after the formation of the second disulphide bridge, which reduced the overall yield. Analysis of the deprotection experiments for tert-butyl and MeOBzl modified $\mu$-SIIIA displayed either no cleavage of the protecting groups or oxidation of the sensitive side chain residues. These results indicate that cleavage conditions for tert-butyl or MeOBzl protecting group are incompatible for peptides containing sensitive Trp or His residues. ${ }^{[248,249]}$ The introduction of the bulky $\mathrm{MeOBzl}$ group also interferes with the folding process, causing the formation of two topological isomers (70.1-70.2), similar to the Allocam protected $\mu$-SIIIA.

Therefore, new synthesis strategies were developed, in which the alkyne linker for CuAAC mediated fluorophore labelling was replaced by either a lysine or cysteine residue. These residues can be labelled with NHS ester or maleimide activated fluorophores, providing the advantage of reduced sensitivity compared to the alkyne linker. These labelling approaches are compatible with the $\mathrm{S}^{t} \mathrm{Bu}, \mathrm{Mmt}$, Trt and Acm protecting groups, which have successfully been used for the synthesis of $\mu$-SIIIA, without oxidizing sensitive side chain residues. 


\subsubsection{Synthesis of Thiol Modified $\mu$-SIIIA}

A frequently applied fluorophore labelling strategy for peptides and proteins is the thiolmaleimide conjugation. This reaction requires a free thiol group which can selectively react with a maleimide activated fluorophore in the presence of a base. ${ }^{[250]}$ To achieve controlled labelling of the $\mu$-SIIIA at a specific position, an additional cysteine residue, which is not involved in the formation of the disulphide bonds, has to be introduced into the sequence. The newly introduced cysteine residue will be cleaved after the formation of the third disulphide bond. A new synthetic route for the formation of the three disulphide bridges was developed using the orthogonal $\mathrm{StBu}, \mathrm{Mmt}$ and Trt protecting groups exclusively. The additional cysteine residue was protected with an Acm group and was introduced in the position of the Gly6 (72).

In the first step, the $\mathrm{S}^{t} \mathrm{Bu}$ protecting groups of $\mathrm{Cys} 3$ and $\mathrm{Cys} 13$ were removed with 2-mercaptoethanol in DMF and the peptide was cleaved from the resin with $10 \%$ acetic acid (see figure 2.23). The first disulphide bridge was formed between the Cys3 and Cys13 in DCM with triethylamine (10\%). Following, the two Mmt groups were cleaved with $0.5 \%$ TFA and the second disulphide bridge (73) was formed in acetate buffer (pH 6, 20 \% DMSO). The Trt-, as well as all acid-sensitive protecting groups, were cleaved in a mixture of TFA/TIS/ $\mathrm{H}_{2} \mathrm{O}$ and the last disulphide bridge was formed in acetate buffer ( $\mathrm{pH} \mathrm{6,20 \%} \mathrm{DMSO).} \mathrm{After} \mathrm{HPLC}$ purification, two topological isomers of the Acm protected $\mu$-SIIIA (74.1-74.2) with three disulphide bonds were obtained in high yields. Due to lack of an alkyne moiety, the Acm cleavage with iodine in $\mathrm{AcOH} / \mathrm{H}_{2} \mathrm{O}$ was performed with prolonged reaction times of $1 \mathrm{~h}$. However, LC-MS analysis of the crude peptide revealed the formation of an intermolecular disulphide bridge conjugating two $\mu$-SIIIA molecules (75). Cleavage of the intermolecular disulphide bridge by addition of reducing agents would also cause a breakage of the three intramolecular disulphide bonds, leading to an unfolding of the toxin and loss of activity. These limitations required a different approach for fluorophore labelling. 


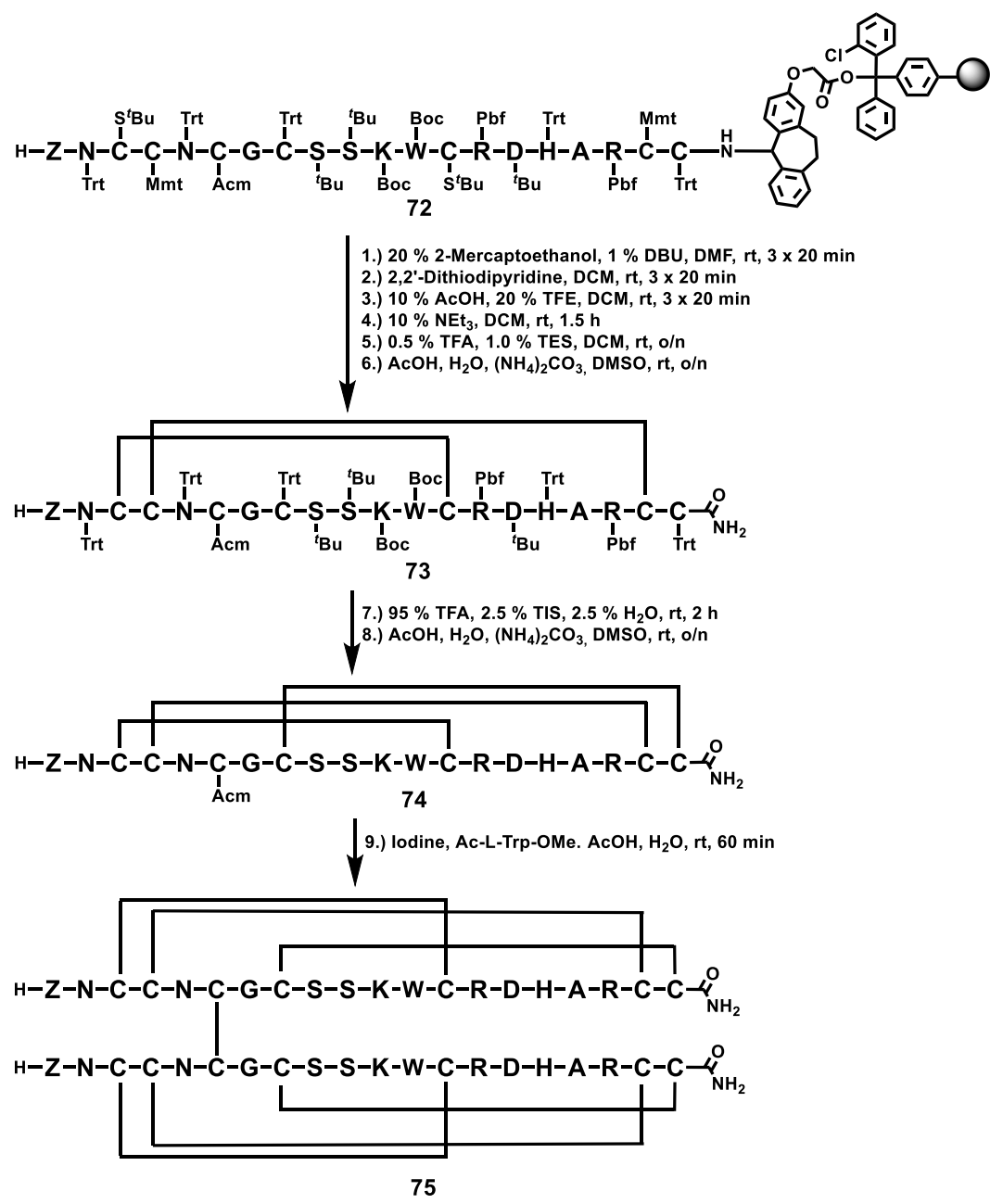

Figure 2.23: Synthesis approach for Cys(Acm)-modified $\mu$-SIIIA.

\subsubsection{Synthesis of Amine Modified $\mu$-SIIIA}

Since the introduction of an additional cysteine residue for fluorophore labelling causes the formation of intermolecular disulphide bridges, the Cys6 was replaced by an additional lysine residue. The $\varepsilon$-amino group of the lysine side chain can be conjugated with an NHS activated fluorophore, forming a stable amide bond with high yields. ${ }^{[226,228,251]}$ The successful labelling with NHS-activated fluorophores was recently reported for Hspla, a short cysteine-rich peptide, which selectively inhibits Nav1.7 channels. ${ }^{[252]}$ However, fluorophore labelling of the folded $\mu$-SIIIA could also cause unspecific labelling of the Lys11, which is essential for the channel interaction. Thus, the introduction of the fluorophore in the position of the Lys11 would drastically decrease the affinity towards $\mathrm{Na}_{\mathrm{v}}$ channel. To overcome these limitations, an Allocprotected Lys residue was placed in the position of Gly6, whereby the Alloc protecting group is orthogonal to the $\mathrm{S}^{t} \mathrm{Bu}, \mathrm{Mmt}$ as well as Trt groups and can be removed with $\mathrm{Pd}\left(\mathrm{PPh}_{3}\right)_{4}$. The initial cleavage of the Alloc-protecting group prevents the labelling of the still protected Lys11. 
Consequently, a linear Lys(Alloc)-modified $\mu$-SIIIA (76), bearing the $\mathrm{S}^{t} \mathrm{Bu}$, Mmt and Trt protecting groups, was synthesised. After the formation of the first and second disulphide bridge (77) by deprotecting the $\mathrm{S}^{t} \mathrm{Bu}$ and $\mathrm{Mmt}$ groups (see chapter 2.2.3.3), the Alloc group was cleaved with $\mathrm{Pd}\left(\mathrm{PPh}_{3}\right)_{4}$ and phenylsilane under an argon atmosphere (see figure $2.24 \mathrm{~A}$ ). In the next step, an NHS activated fluorophore was coupled to the free amine group of the Lys6, producing the fluorophore-labelled, protected $\mu$-SIIIA with two disulphide bonds (78). For the first labelling experiments, tetramethylrhodamine (TMR) was used as a fluorophore, as it can easily be activated with $N$-hydroxysuccinimid, producing activated TMR with high yields. In the last step, the Trt, as well as all acid-sensitive protecting groups, were cleaved and the third disulphide bridge (79) was formed in acetate buffer. The reaction mixture was purified by HPLC, yielding two topological isomers (79.1-79.2) of the TMR labelled $\mu$-SIIIA with significantly higher yields compared to the CuAAC labelling strategy (see figure 2.24 B). Further examination of the TMR labelled $\mu$-SIIIA isomers (79.1-79.2), regarding their affinity and selectivity for $\mathrm{Nav}$ channels, have to be performed by patch-clamp experiments and fluorescence microscopy. 
A

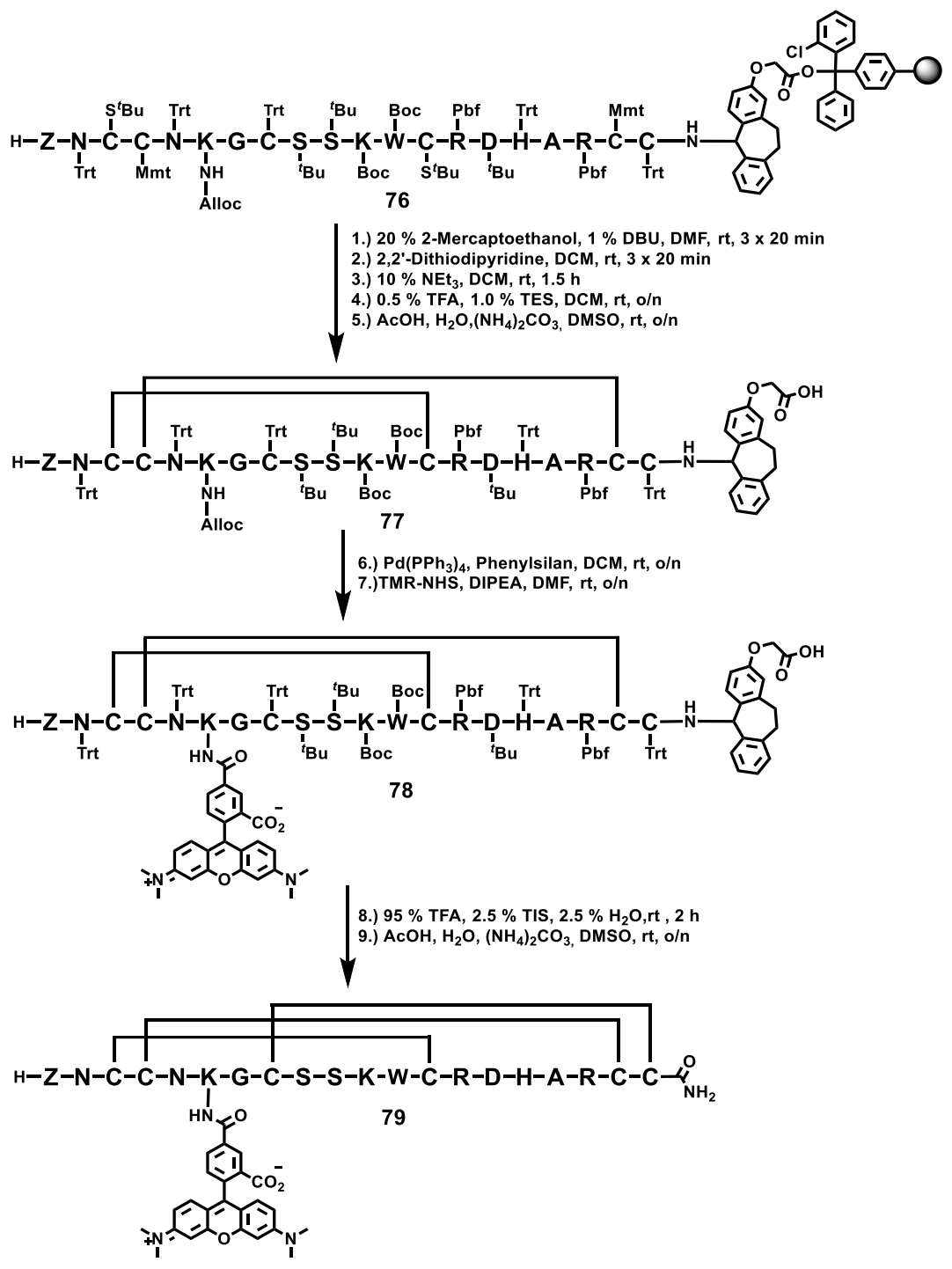

B

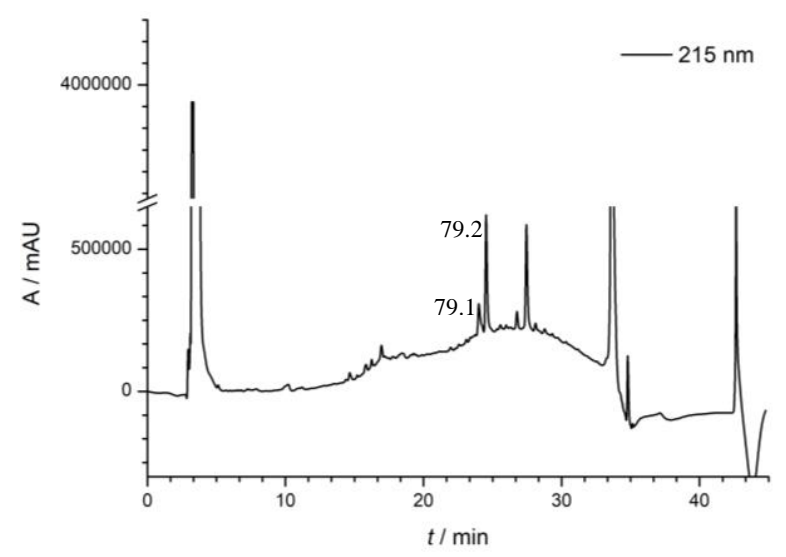

Figure 2.24: Synthesis and purification of TMR labelled $\boldsymbol{\mu}$-SIIIA. A) Synthesis approach for TMR modified $\mu$-SIIIA (79). B) HPLC chromatogram of TMR modified $\mu$-SIIIA (79), yielding two topological isomers (79.179.2). HPLC gradient: $1-40 \% \mathrm{MeCN}$ in $30 \mathrm{~min}$. 


\subsubsection{Synthesis of Caged $\mu$-Conotoxin SIIIA}

Besides the visualisation of the Nav1.2 channel distribution with fluorophore labelled $\mu$-SIIIA, there is a great interest in controlling the activity of the Nav channels on a cellular level in specific neuronal compartments. This specific control could provide more in-depth insight into channel mechanisms and how dysfunctions of the Nav1.2 are linked to neurological diseases. For controlled measurements of the channel activity, a highly specific inhibitor is required, which is modified with a caging group, reducing the channel blocking the activity of the inhibitor. A spatially and temporally controlled cleavage of the caging group switches the inhibitor from an inactive to an active state, allowing regulation of the Nav channel activity.

For controlled blockage experiments of the Nav1.2 channel, the $\mu$-conotoxin SIIIA was selected as an inhibitor and was modified with a photocleavable protecting group (PPG). The PPG is conjugated to an amino acid residue, which is crucial for interaction with the Nav and therefore prevents the binding of the caged $\mu$-SIIIA to the channel (see figure 2.25). A controlled blockage of the channel activity can be induced by irradiation of the caged $\mu$-SIIIA, causing cleavage of the PPG, which regenerates the blocking activity of the $\mu$-SIIIA.

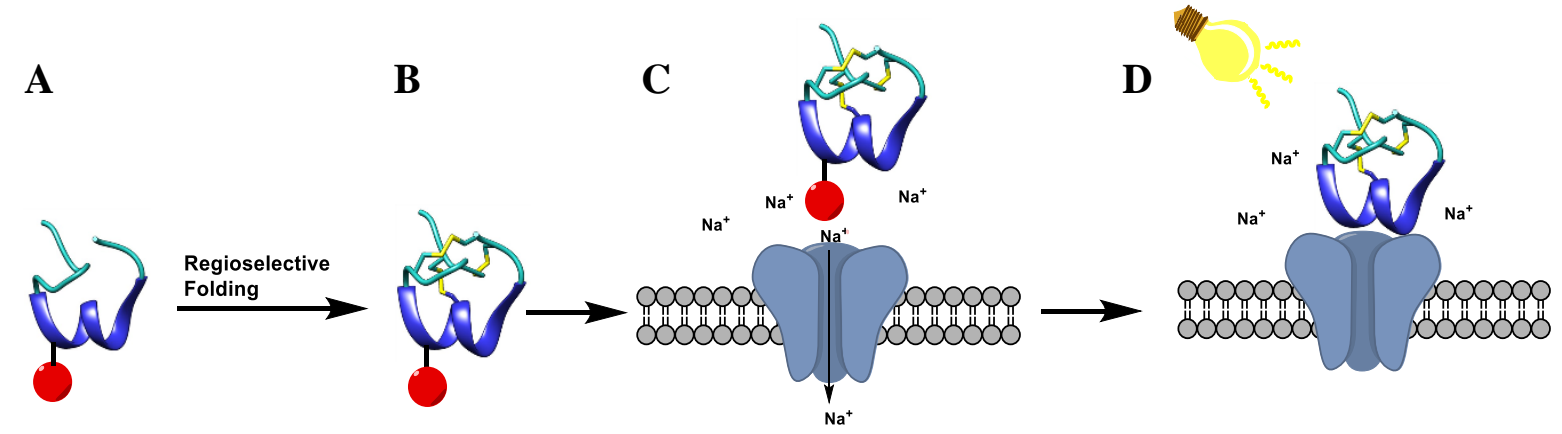

Figure 2.25: Overview of the synthesis and application of caged neurotoxins. The linear $\mu$-SIIIA bearing a PPG was synthesised (A) and folded to its 3D structure (B). The caged $\mu$-SIIIA provides a tool for channel investigations and for controlling the action potential propagation. The caged conotoxin can be switched from an inactive state to $(\mathbf{C})$ an active state by $\mathrm{UV}$-irradiation. Only the active state of the conotoxin can inhibit the $\mathrm{Na}_{\mathrm{V}}$ channel activity (D).

\subsubsection{Characteristics of Photocleavable Protecting Groups}

Over the last years, PPG became a powerful tool to study dynamic intracellular processes or pathways in biological systems. The PPG can temporarily mask one of the functional groups in the caged molecule, which is responsible for the bioactivity. Thereby, the cleavage of the PPG by UV irradiation triggers the activation of the caged molecule. Photo-induced cleavage allows a precise spatial and temporal control over the release of the compound under mild conditions, 
without the addition of other chemicals or invasive methods. ${ }^{[253-255]}$ For in vitro and in vivo application, the PPGs require a strong absorption at $\lambda>340 \mathrm{~nm}$ as well as rapid photo-cleavage kinetics to prevent phototoxicity caused by prolonged illumination. Additional requirements of the PPG strategy are the stability towards cellular conditions and biocompatibility of the cleavage products. ${ }^{[256]}$

\subsubsection{Caging Experiments of the $\mu$-Conotoxin SIIIA}

For controlled blocking experiments of the Nav1.2 channel activity, the $\mu$-SIIIA was selected as the target molecule. Therefore, a PPG is conjugated to one of the essential residues for channel blocking to ensure no interaction between the PPG modified $\mu$-SIIA and the channel. As previously described, several structure analysis studies of the $\mu$-SIIIA have been performed, revealing a crucial role of the residues in the $\alpha$-helix Lys11, Trp12, Arg14, Asp15, His 16 and Arg18 for the interaction with the channel. From these residues, especially the Lys 11 is an attractive position for covalent linkage of modifications, as the lysine $\varepsilon$-amine is nucleophilic. Further substitutions of the Asp15 with positively charged or aromatic residues significantly decreases the affinity towards Nav1.2. ${ }^{[13]}$ Conjugation of a bulky PPG could effectively decrease the channel blocking, making Asp15 an exciting position for the introduction of a caging group (see figure 2.26). Based on the structural analysis of the $\mu$-SIIIA, lysine and aspartic acid were selected as target residue for conjugation and incorporation of a PPG into the $\mu$-SIIIA sequence by SPPS.

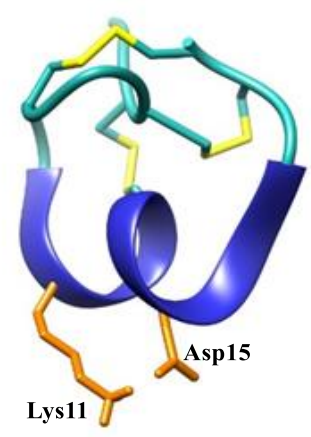

Figure 2.26: 3D Structure of the $\boldsymbol{\mu}$-conotoxin SIIIA. The residues Lys 11 and Asp 15 were selected for the introduction of a caging group and are highlighted in orange (BMRB ID code: SMS20025). The graphic was created with USCF Chimera package. ${ }^{[212]}$ 


\subsubsection{Synthesis of Nvoc Protected Lysine}

The most frequently used photocleavable protecting groups are the $o$-nitrobenzyl, due to their simple structure and synthesis. They can also be attached to different functional groups in the target molecule and a variety of substitutions patterns for the nitrobenzyl moiety have been reported. ${ }^{[257]}$ Depending on these substitutions, the photochemical properties of the caging groups, like absorption spectrum, quantum yield and cleavage efficiency, can be adjusted. ${ }^{[257]}$ Significantly, the 6-nitroveratryloxycarbonyl (Nvoc) group is used for in vitro or in vivo measurements because of their two electron-donating methoxy groups, which increase the absorbance to longer wavelengths ( $\lambda>350 \mathrm{~nm}$ compared to $265 \mathrm{~nm}$ for $o$-nitrobenzyl). ${ }^{[256-258]}$ Photo-induced cleavage of the Nvoc group though leads to the formation of cytotoxic nitrosoaldehyde side products, limiting the application of the Nvoc group. ${ }^{[257]}$ Nevertheless, the straightforward synthesis of the Nvoc protecting group allows the investigation of cleavage conditions for caged peptides.

A lysine building block, modified with the Nvoc group for SPPS, was synthesised over two steps (see figure 2.27). ${ }^{[259]}$ First, the 4-nitrophenyl chloroformate (80) reacted with 4,5-dimethoxy-2-nitrobenzyl alcohol (81) to form the activated 6-nitrovertaryl-4'-nitrophenyl carbonate (82). In the second step, the activated carbonate was converted with Fmoc-Lys-OH in a substitution reaction to form the target compound Fmoc-Lys(Nvoc)-OH (83) with an overall yield of $60 \%$.

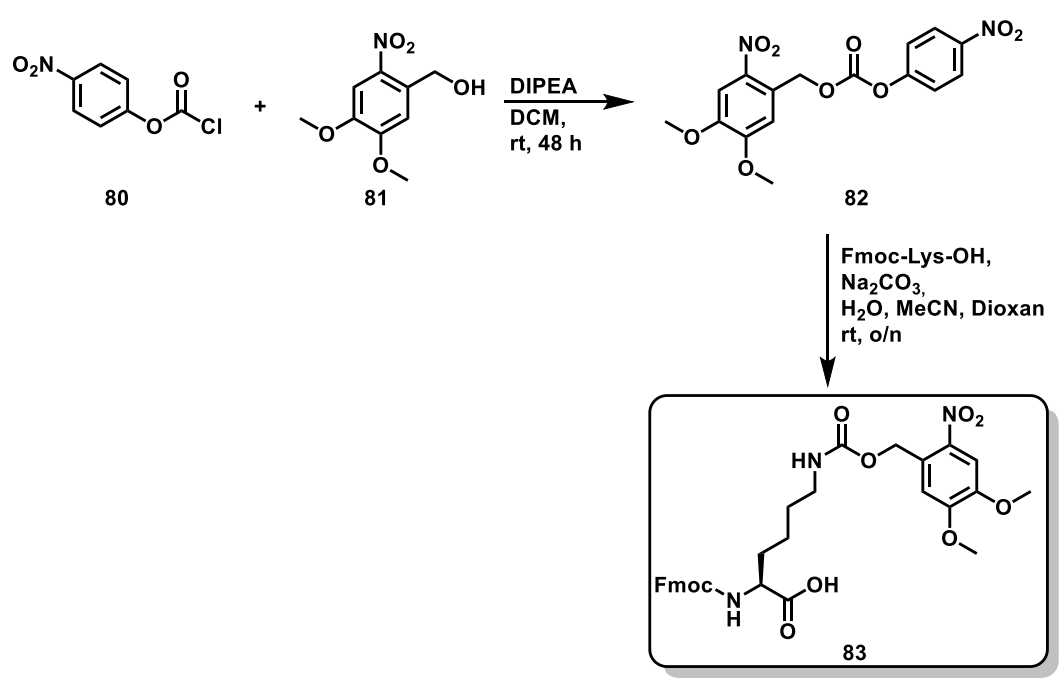

Figure 2.27: Synthesis of the caged Fmoc-Lys(Nvoc)-OH building block for SPPS over two steps.

After synthesis of Fmoc-Lys(Nvoc)-OH, the building block was incorporated into a short test peptide by SPPS to examine the coupling conditions as well as the photocleavage properties. The sequence of the test peptide construct consists of two serine residues, which increase the 
solubility, as well as hydrophobic residues to prevent side reactions with the cleaved nitrosoaldehyde by-products. Additionally, a phenylalanine residue was incorporated, to estimate the peptide concentrations for the uncaging experiments. The peptide was synthesised automatically by SPPS on a Rink amide resin (see figure $2.28 \mathrm{~A}$ ). Fmoc-Lys(Nvoc)-OH was coupled at a reduced temperature of $50{ }^{\circ} \mathrm{C}$ and an extended reaction time of $10 \mathrm{~min}$ at $15 \mathrm{~W}$. After HPLC purification of the desired product, the photocleavage of the caged peptide (84) was investigated. Therefore, the peptide was dissolved in MilliQ, transferred into a quartz cuvette and irradiated with a mercury UV lamp at $650 \mathrm{~W}$ and 300-400 nm. ${ }^{[260]}$ The uncaging progress was analysed after exposure times of $1 \mathrm{~min}, 3 \mathrm{~min}, 5 \mathrm{~min}, 10 \mathrm{~min}$ and $15 \mathrm{~min}$ by U-HPLC, revealing incomplete uncaging progress even after $15 \mathrm{~min}$ (see figure $2.28 \mathrm{~B}$ ). These long cleavage times limit the in vivo applications, due to photodamage as well as photo-induced destruction of already formed disulphide bonds. ${ }^{[261]}$

A

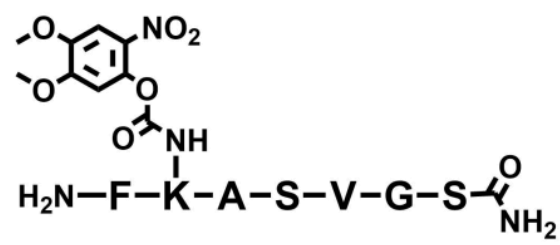

84

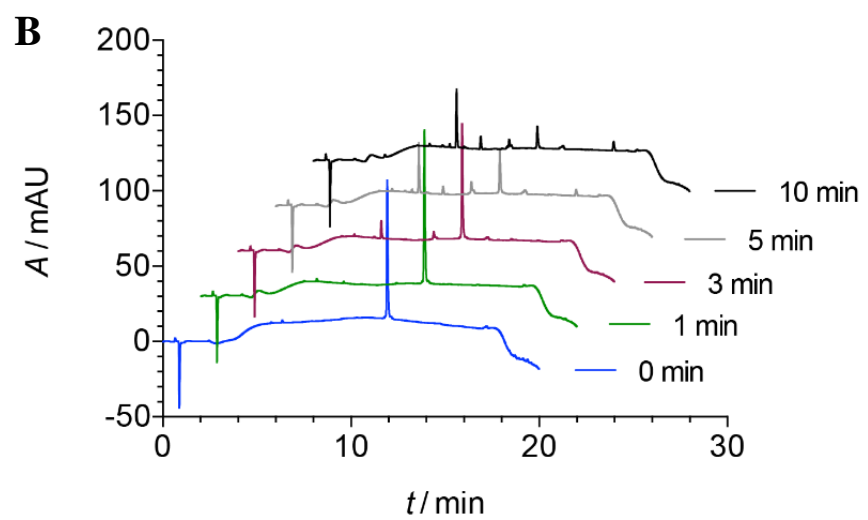

Figure 2.28: Structure and uncaging experiments of Nvoc modified peptides. A) Synthesis of Nvoc caged test peptide (84) to investigate cleavage conditions. B) Photocleavage experiment of the Nvoc caged peptide (84), analysed by U-HPLC after irradiation for $0 \mathrm{~min}, 1 \mathrm{~min}, 3 \mathrm{~min}, 5 \mathrm{~min}$ and $10 \mathrm{~min}$. HPLC gradient: 1-40\% MeCN in $15 \mathrm{~min}$.

\subsubsection{Synthesis of Nitrobenzyl Based Protected Aspartic Acid}

Based on the insufficient cleavage process of the Nvoc-protecting group, a PPG with higher cleavage efficiency is required, to reduce the irradiation times. Analysis of several nitrobenzylbased PPG revealed the 3-(4,5-dimthoxy-2-nitrophenyl)-2-butyl (DMNPB) as a suitable protecting group since it can be conjugated to glutamic or aspartic acid. ${ }^{[262]}$ Measurements of the DMNPB protecting group revealed a higher quantum yield and an increased cleavage process at $354 \mathrm{~nm}$, compared to the Nvoc protecting group. Additionally, the DMNPB group can be applied in biological systems as it forms the less reactive and non-toxic acetophenone 
side product. ${ }^{[263,264]}$ The Asp15 residue of the $\mu$-SIIIA was selected for an introduction of the DMNPB group as it is essential for the conotoxin channel interaction.

The synthesis of a DMNPB protected aspartic acid building block for SPPS was performed according to SPECHT et al. in five steps (see figure 2.29). ${ }^{[263]}$ First, the 3,4-dimethoxyphenyl acetone (85) was selectively methylated by methyl iodide and sodium hydride leading to 3(3,4-dimethoxyphenyl)-butan-2-one (86). Next, the nitro group was incorporated at the ortho position of the aromatic ring using acetic acid and nitric acid yielding 3-(3,4-dimethoxy-2nitrophenyl)-butan-2-one (87). Reduction of the ketone by addition of sodium borohydride produces the alcohol 3-(4,5-dimethoxyphenyl)-butan-2-ol (88). Esterification of the alcohol (88) and the unprotected side chain of Fmoc-Asp- $\mathrm{O}^{t} \mathrm{Bu}$ was achieved by activation with dimethylaminopyridine (DMAP) and 1-ethyl-3-(3-dimethylaminopropyl)carbodiimide (EDC), yielding Fmoc-Asp(DMNPB)-O'Bu (89). In the final step, the tert-butyl group was removed by TFA leading to the Fmoc-Asp(DMNPB)-OH (90) with an overall yield of $34 \%$.

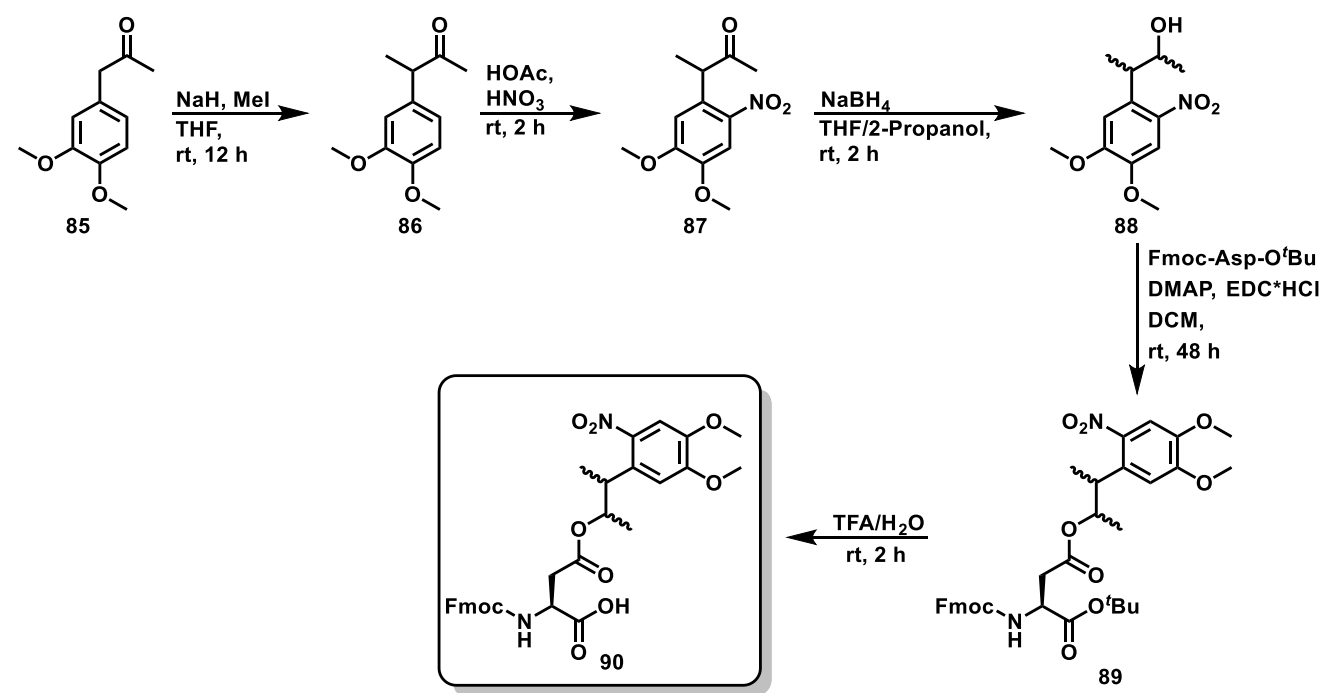

Figure 2.29: Synthesis of the caged Fmoc-Asp(DMNPB)-OH building block for SPPS.

To analyse the cleavage properties of the DMNPB protecting group compared to Nvoc group, the DMNPB protected aspartic acid (90) was incorporated into the test peptide sequence (91) (see figure $2.30 \mathrm{~A}$ ). The peptide was synthesised by SPPS and the Fmoc-Asp(DMNPB)-OH (90) was coupled at $50{ }^{\circ} \mathrm{C}$ for $10 \mathrm{~min}$ at $15 \mathrm{~W}$. After HPLC purification, the cleavage process of the DMNPB caged peptide (91) was investigated after irradiation times of $1 \mathrm{~min}, 3 \mathrm{~min}$, $5 \mathrm{~min}, 10 \mathrm{~min}, 15 \mathrm{~min}$ and $20 \mathrm{~min}$. Analysis of the HPLC chromatograms revealed an incomplete cleavage of the DMNPB group after $10 \mathrm{~min}$ as well as side product fractions (see figure $2.30 \mathrm{~B}$ ). Thus, a new PPG structure lacking a nitrobenzyl scaffold might lead to higher photocleavage rates and consequently, a complete uncaging process. 
A

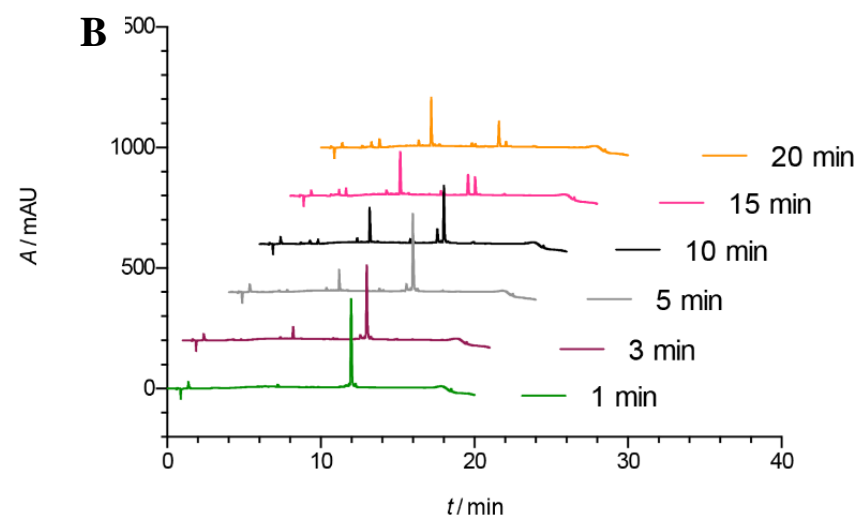

Figure 2.30: Structure and uncaging experiments of DMNPB modified peptides. A) Synthesis of DMNPB caged test peptide (91). B) Photocleavage experiment of the DMNPB caged peptide (91), analysed by HPLC after irradiation for $1 \mathrm{~min}, 3 \mathrm{~min}, 5 \mathrm{~min}, 10 \mathrm{~min}, 15 \mathrm{~min}$ and $20 \mathrm{~min}$. HPLC gradient: 1-40\% MeCN in $15 \mathrm{~min}$.

\subsubsection{Synthesis of Coumarin Protected Lysine}

After a screening of several PPG structures with different cleavage properties, the coumarin based PPGs were identified, revealing a challenging synthesis but a fast photocleavage compared to the nitrobenzyl derivatives. They exhibit a high absorption coefficient at wavelengths above $350 \mathrm{~nm}$, visible light sensitivity and biocompatibility. ${ }^{[255]}$ The coumarin groups also provided different substitution possibilities to adjust the absorption wavelengths $\left(\lambda_{\max }=310-490 \mathrm{~nm}\right) .{ }^{[257]}$ Especially, the (7-(diethylamino)coumarin-4-yl)methyl (DEACM) group conjugated to cAMP, ATP or GABA have been extensively used for studying cellular processes, showing an efficient photocleavage, high quantum yields and intense absorptivity at $>300 \mathrm{~nm}^{[265-267]}$

Since the DEACM group can be conjugated to free amine groups, the DEACM protected lysine (95) was synthesised over three steps, according to the method from SCHÖNLEBER et al. and KoTZUR et al. (see figure 2.31). ${ }^{[268,269]}$ In the first step, the methyl group of 7-diethylamino-4methyl-coumarin (92) was oxidized by selenium dioxide and reduced to the corresponding alcohol (93) by $\mathrm{NaBH}_{4}$. The isolated 7-diethylamino-4-hydroxymethyl-coumarin (93) was activated by 4-nitrophenyl chloroformate (80), DMAP and $\mathrm{NEt}_{3}$ to form the activated carbonate (94). In the final step, the carbonate was reacted with Fmoc-Lys-OH to yield the desired FmocLys(DEACM)-OH (95) under basic conditions with an overall yield of $14 \%$. 

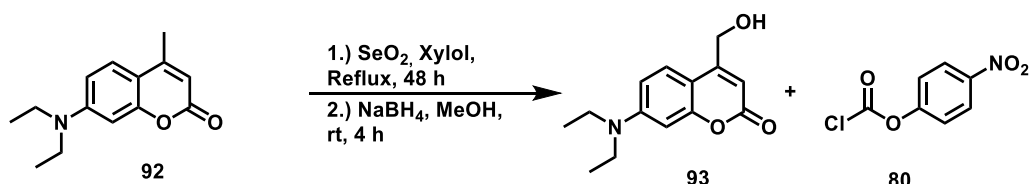

93

80

$\mid \begin{aligned} & \text { DMAP, NEt } \\ & \text { DCM } \\ & \text { rt, o/n }\end{aligned}$
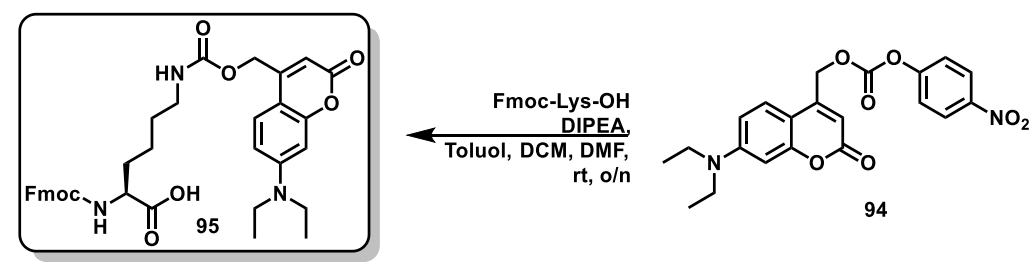

Figure 2.31: Three-step synthesis of Fmoc-Lys(DEACM)-OH building block for SPPS.

Next, the photocleavage process of the DEACM protected Lys was analysed, by incorporating the building block in the previously described test peptide sequence by SPPS (see figure $2.32 \mathrm{~A})$. The coupling of the Fmoc-Lys(DEACM)-OH (95) was performed two-times for $10 \mathrm{~min}$ at $50{ }^{\circ} \mathrm{C}$ and $15 \mathrm{~W}$. After HPLC purification, the DEACM protected peptide (96) was dissolved in MilliQ and irradiated by a handheld laser (200 mW, $405 \mathrm{~nm})$ for $30 \mathrm{sec}, 1 \mathrm{~min}$, $2 \mathrm{~min}, 3 \mathrm{~min}$ as well as $5 \mathrm{~min}$ (see figure $2.32 \mathrm{~B}$ ). The cleavage progress was monitored by HPLC, revealing a complete deprotection of DEACM group after $3 \mathrm{~min}$. The formation of the uncaged peptide was confirmed by mass spectrometry. Compared to the nitrobenzyl based Nvoc and DMNPB caging groups, the DEACM group revealed a fast photocleavage without the formation of side products. Therefore, the DEACM group was incorporated in the $\mu$-SIIIA sequence, to perform controlled blocking experiments of the Nav channels.
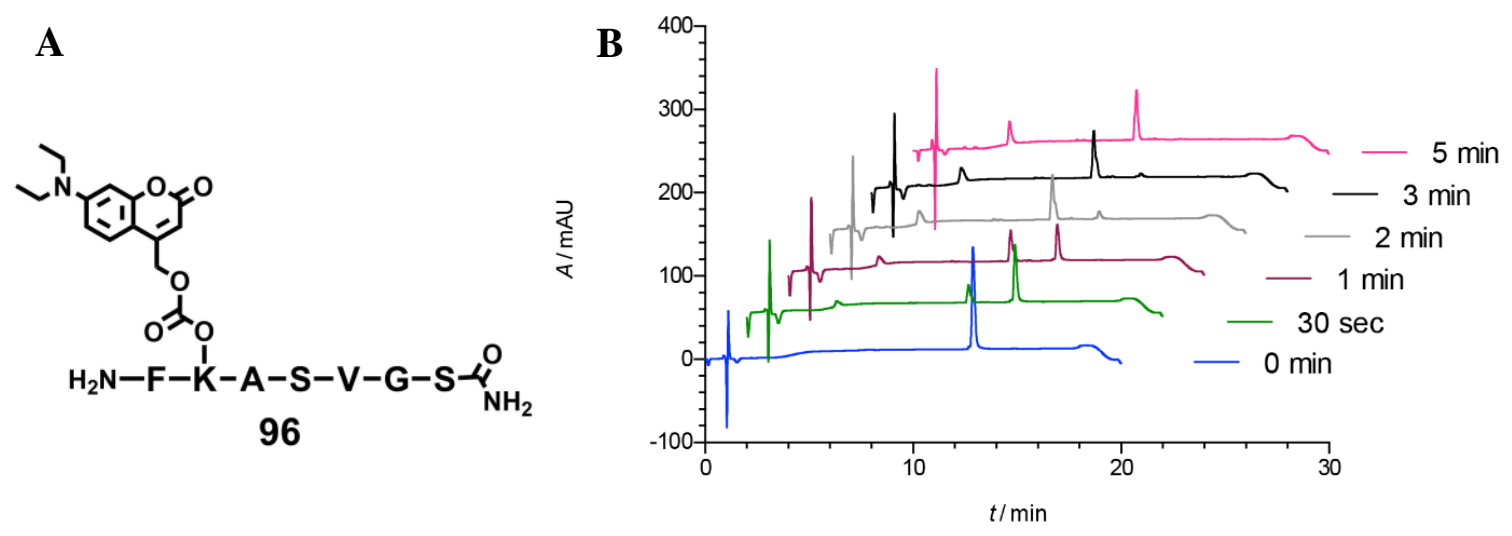

Figure 2.32: Structure and uncaging experiments of DEACM modified peptides. A) Synthesis of DEACM caged test peptide (96). B) Photocleavage experiment of the DEACM caged peptide (96), analysed by HPLC after irradiation for $0 \mathrm{~min}, 30 \mathrm{sec}, 1 \mathrm{~min}, 2 \mathrm{~min}, 3 \mathrm{~min}$ and $5 \mathrm{~min}$. HPLC gradient: 1-40\% MeCN in $15 \mathrm{~min}$. 


\subsubsection{Synthesis of DEACM Caged $\mu$-Conotoxin SIIIA}

After the successful synthesis and preliminary uncaging experiments, the Fmoc-Lys(DEACM)$\mathrm{OH}$ (95) building block was integrated into the $\mu$-SIIIA sequence at the position of the Lys11. Since the sensitive alkyne-linker is missing in this sequence, the cysteine protecting groups $\mathrm{S}^{t} \mathrm{Bu}, \mathrm{Mmt}$, Trt and Acm were selected, for the regioselective formation of the three disulphide bonds. The synthesis of the linear peptide (97) was performed by SPPS on a RAMAGE-Linker modified chlorotrityl-resin (see chapter 2.2.1.1). The first disulphide bridge was formed after cleavage of the $\mathrm{S}^{t} \mathrm{Bu}$ and $\mathrm{Mmt}$ group under basic conditions (see figure $2.33 \mathrm{~A}$ ). Subsequently, the Trt and all acid-sensitive protecting groups were cleaved in a mixture of TFA, TIS, $\mathrm{H}_{2} \mathrm{O}$ and the second disulphide bridge was formed in acetate buffer in $20 \% \mathrm{DMSO}$ at $\mathrm{pH}$ 6. HPLC purification of the crude peptide yielded one major product (98) with two disulphide bonds and the DEACM as well as Acm protection groups still attached. In the last step, the Acm groups were cleaved and the third disulphide bond, on the surface of the $\mu$-SIIIA structure, was formed after the addition of iodine. Shortening of the reaction time to $20 \mathrm{~min}$ prevented possible side reaction of iodine with the DEACM group. HPLC and mass spectrometry analysis revealed a full conversion of the starting material and the formation of four topological isomers (99.199.4) of the DEACM protected $\mu$-SIIIA (99) (see figure 2.33 B). Interestingly, the incorporation of the bulky DEACM group disturbs the folding process of the conotoxins, causing two additional topological isomers compared to the folding of the native $\mu$-SIIIA (59). 
A

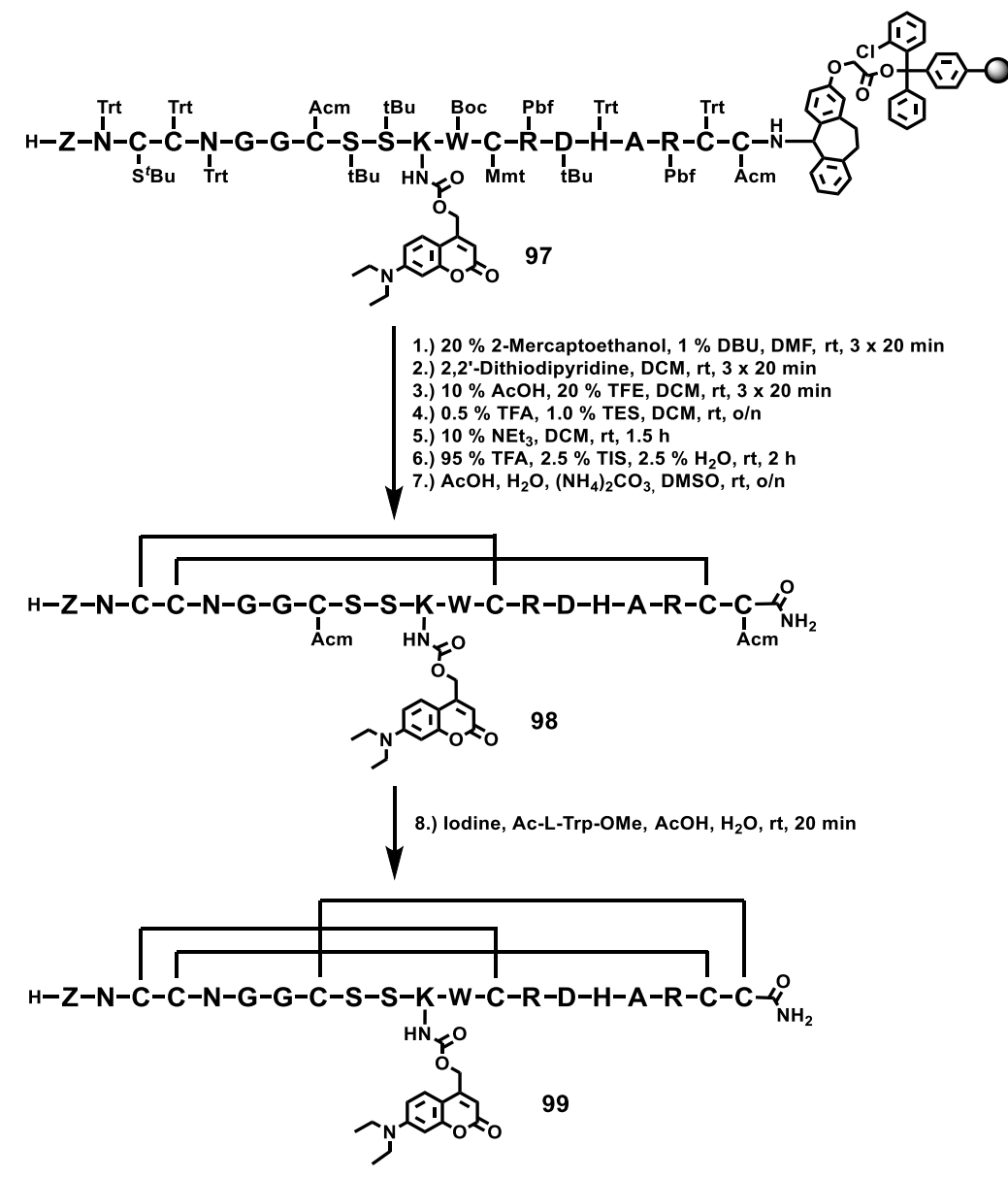

B

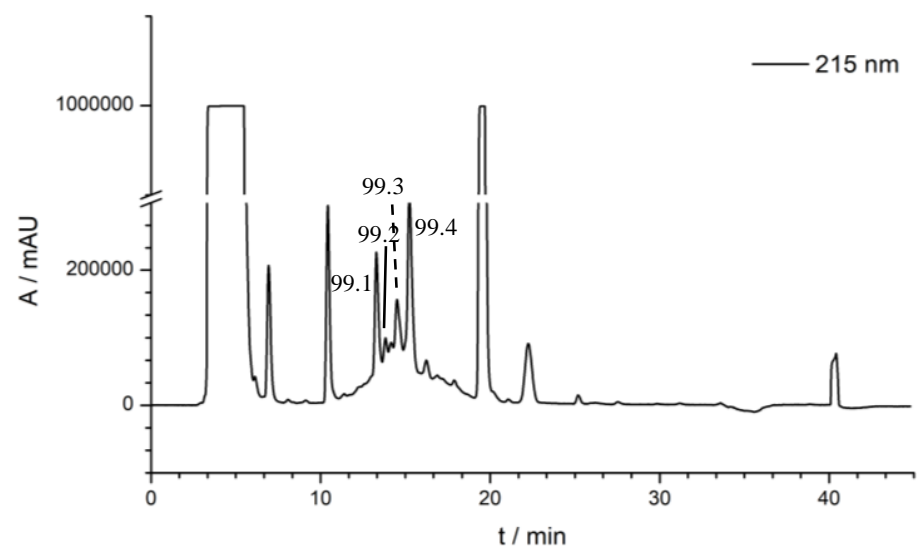

Figure 2.33: Synthesis and purification of the DECAM caged $\mu$-SIIIA. A) Synthesis of DEACM caged $\mu$-SIIIA (99). B) HPLC chromatogram of DEACM modified $\mu$-SIIIA (99), yielding four topological isomers (99.1-99.4). HPLC gradient: $1-40 \% \mathrm{MeCN}$ in 30 min.

\subsubsection{Uncaging Experiments of DEACM Modified $\mu$-SIIIA}

Since irradiation of conotoxins or disulphide rich peptides can destroy formed disulphide bonds, the photocleavage process of the DEACM modified $\mu$-SIIIA (99) was evaluated. ${ }^{[261]}$ Therefore, isomer 99.1 of the DEACM protected $\mu$-SIIIA was diluted in MilliQ to a concentration of $40 \mu \mathrm{M}$ and irradiated by a laser $(200 \mathrm{~mW}, 405 \mathrm{~nm}$ ) for $30 \mathrm{sec}, 1 \mathrm{~min}, 2 \mathrm{~min}, 3 \mathrm{~min}$ and $5 \mathrm{~min}$ 
(see figure 2.34). The deprotection process was monitored by HPLC and analysed by mass spectrometry, showing a reduction of the starting material (retention time of $13 \mathrm{~min}$ ) as well as the formation of the uncaged native $\mu$-SIIIA (59), which elutes after $8 \mathrm{~min}$. The HPLC results revealed an almost complete deprotection after an irradiation time of $3 \mathrm{~min}$, without the formation of side products or destruction of disulphide bridges. Detailed structural analysis of the conformation of the isomers by NMR spectroscopy was not carried out, due to the small amounts. Characterisation experiments of the different isomers (99.1-99.4) were analysed in patch-clamp measurements.

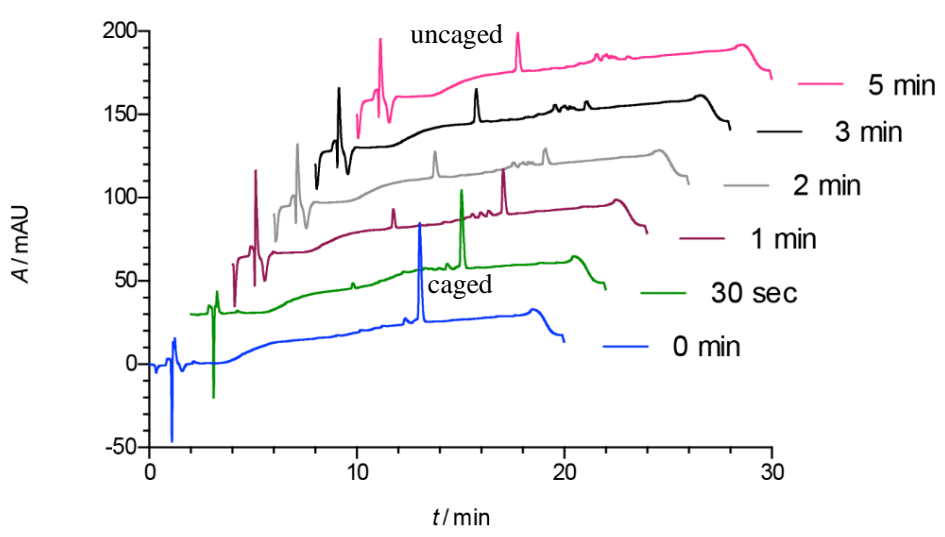

Figure 2.34: Photocleavage experiments of DEACM caged $\boldsymbol{\mu}$-SIIIA. This experiment was analysed by HPLC after irradiation for $30 \mathrm{sec}, 1 \mathrm{~min}, 2 \mathrm{~min}, 3 \mathrm{~min}$ and $5 \mathrm{~min}$. The DEACM caged $\mu$-SIIIA (99.1) revealed a retention time of 13 min, whereas the uncaged $\mu$-SIIIA (59) elutes after 8 min. HPLC gradient: 1-40\% MeCN in 15 min.

\subsubsection{Patch Clamp Measurements of DEACM Caged $\mu$-SIIIA}

To perform controlled inhibition studies of the Nav channel, the blocking activity and affinity of the DEACM protected $\mu$-SIIIA (99) after irradiation has to be investigated. Patch-clamp experiments of the irradiated isomers (99.1-99.4) were performed and analysed by V. KISTMACHER and E. LEIPOLD (University Lübeck). Similar to the inhibition studies of the native $\mu$-SIIIA isomers (59.2.1-59.2.2), the measurements were performed in HEK 293 cells transfected with rat Nav1.4-channel expression plasmid (see chapter 2.2.1.2). Measurement on the Nav1.4 channel facilitates the characterisation of the caged $\mu$-SIIIA isomers (99.1-99.4), due to the comparative data from the native $\mu$-SIIIA (59). After identification of the bioactive isomer and efficient cleavage conditions, further measurements have to be performed on the Nav1.2 channel.

Before the measurements were carried out, the DEACM caged $\mu$-SIIIA isomers (99.1-99.4) were diluted to a concentration of $10 \mu \mathrm{M}$ and were irradiated with a $40 \mathrm{~mW}$ LED light source 
(CooLED PE300) at $400 \pm 25 \mathrm{~nm}$. Since the LED light source has a reduced power compared to the handheld laser $(200 \mathrm{~mW})$, which was used in the preliminary uncaging experiments (see chapter 2.2.5.1), various irradiation periods were tested. After irradiation, the $\mu$-SIIIA isomers were used for whole-cell voltage-clamp experiment to measure the Nav1.4 currents of the HEK 293 cells.

Patch-clamp measurements of the isomer 99.1 of the DEACM protected $\mu$-SIIIA revealed for the non-irradiated sample no blockage of the Nav1.4 current (see figure $2.35 \mathrm{~A}$ ). This confirms that blockage of the $\varepsilon$-amino group of the Lys side chain by conjugation of a PPG, disperse the interaction of the $\mu$-SIIIA and the Nav-channel. Recordings of the first uncaging experiment showed a maximal blocking activity of $38 \%$ after an irradiation time of $5 \mathrm{~min}$. These results confirmed a successful reactivation of the caged $\mu$-SIIIA after irradiation. However, the reduced blocking activity of the isomer 99.1 compared to the native $\mu$-SIIIA indicate an incomplete uncaging process. Therefore, the exposure time was extended to $20 \mathrm{~min}, 35 \mathrm{~min}$ and $60 \mathrm{~min}$, showing an increased channel inhibition with longer irradiation times. A maximum of the blocking activity of $80 \%$ was reached after $35 \mathrm{~min}$, whereas longer exposure times of $60 \mathrm{~min}$ lead to no significant increase in the channel blocking. Recordings of the dissociation process for the isomer 99.1 after an irradiation time of $35 \mathrm{~min}$, revealed a slow dissociation $\left(\tau_{\text {Diss }}=\right.$ $1690 \pm 183 \mathrm{~s}$ ) of the uncaged $\mu$-SIIIA from the channel, similar to the isomer 59.2.1 of the native $\mu$-SIIIA ( $\tau_{\text {Diss }}=3640 \pm 163 \mathrm{~s}$ ). Based on the similar dissociation kinetics of the isomers 99.1 and the isomer 59.2.1 of the native $\mu$-SIIIA, the efficiency of the uncaging process was calculated from the blocking activity, using the native $\mu$-SIIIA data as a reference (see figure $2.35 \mathrm{~B})$. The effective concentration of reactivated $\mu$-SIIIA $(10 \mu \mathrm{M})$ after an exposure time of $35 \mathrm{~min}$ corresponded to $300 \mathrm{~nm}$ of the native $\mu$-SIIIA, representing a cleavage efficiency of only $3 \%$. Despite the leaky channel blocking, the slow dissociation of the uncaged toxin from the channel, indicate that isomer $\mathbf{9 9 . 1}$ forms a native conformation and the reduced blocking activity is caused by an incomplete uncaging process. 
A
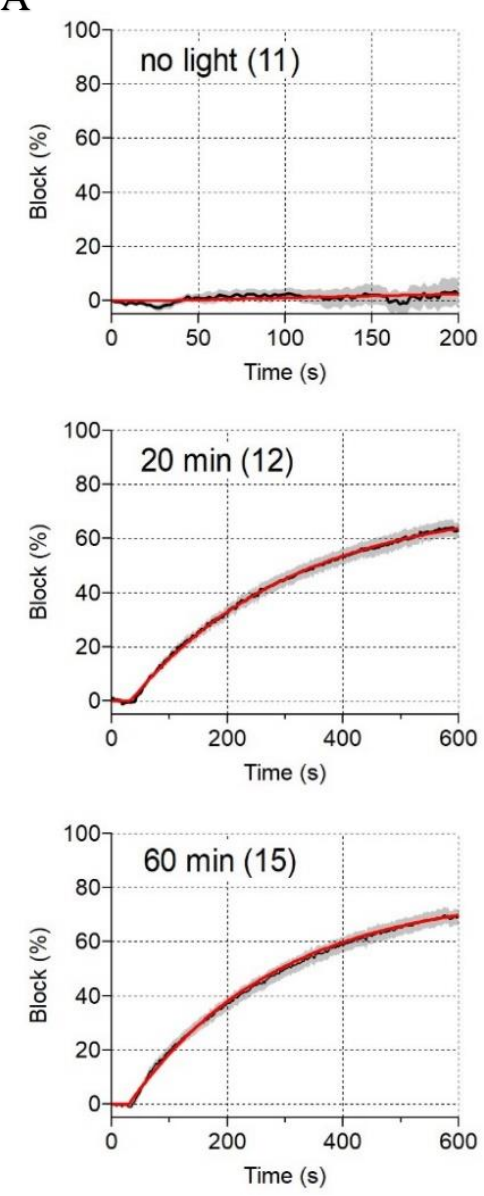
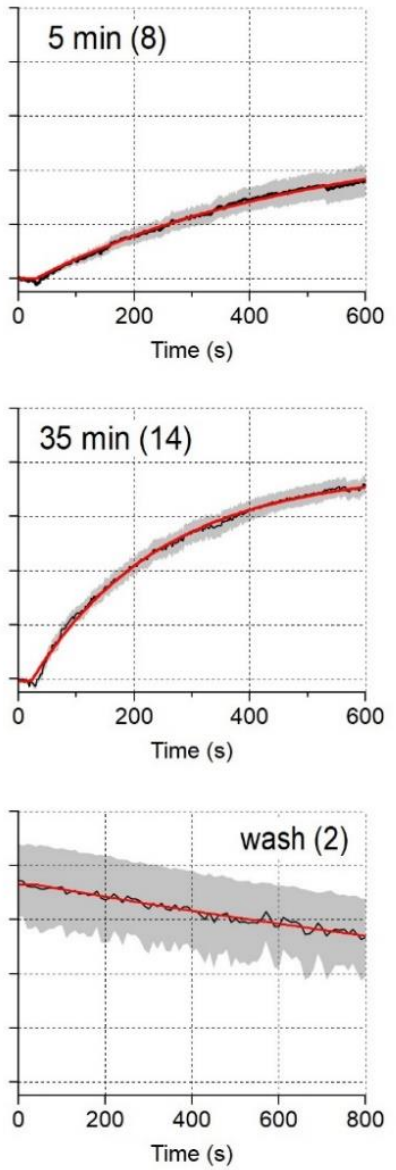

B
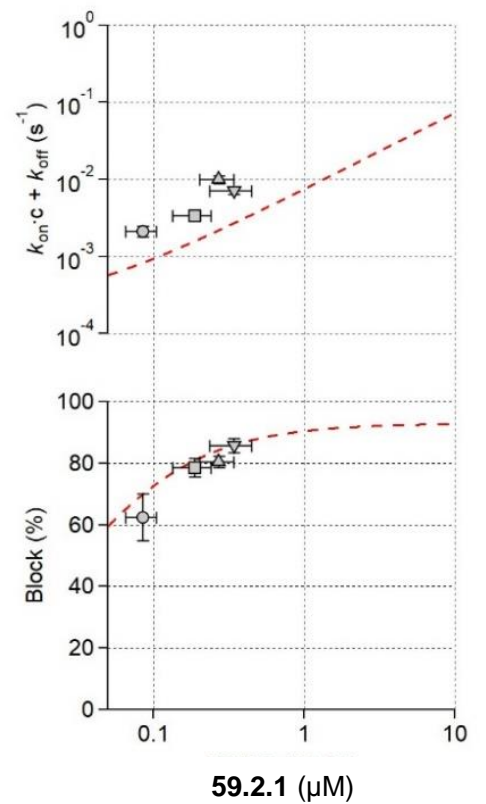

Figure 2.35: Patch-clamp experiments of isomer 4.1. A) Blocking and wash-off kinetics of isomer 99.1 of DEACM-protected $\mu$-SIIIA $(10 \mu \mathrm{M})$ after irradiation for $0 \mathrm{~min}, 5 \mathrm{~min}, 20 \mathrm{~min}, 35 \mathrm{~min}$ and $60 \mathrm{~min}$. The data points are highlighted in black. The SEM is indicated by grey shading and the red lines are single-exponential fits. The number of experimental replicates is shown in parentheses. B) Dose-response curve shows the association and dissociation kinetics and percentage of sodium current inhibition as a function of the native $\mu$-SIIIA (59.2.1) concentration (red dashed lines). Data points from $10 \mu \mathrm{M}$ DEACM-caged $\mu$-SIIIA after irradiation are highlighted in grey. The experiment was performed by VIVIEN KISTMACHER.

The results of the uncaging studies showed for isomer 99.1 the highest blocking activity after an irradiation time of $35 \mathrm{~min}$. Thus, the isomer 99.2 was irradiated for $35 \mathrm{~min}$ and the blocking activity was measured (see figure $2.36 \mathrm{~A}$ ). Recordings of the sodium current revealed a marginally reduced blocking activity of $60 \%$ compared to the isomer 99.1. Measurements of dissociation kinetics showed a slow wash-off from the channel ( $\tau_{\text {Diss }}=2520 \pm 855 \mathrm{~s}$ ), remaining a blocking activity of $50 \%$ after a washout time of $17 \mathrm{~min}$, similar to the isomer 99.1 of the native $\mu$-SIIIA. Consequently, the effective concentration of the uncaged isomer $99.2(10 \mu \mathrm{M})$ was estimated after irradiation, revealing a cleavage efficiency of only $0.6 \%$ (see figure 2.36 B). Based on the slow dissociation kinetics, it can be assumed that the isomer $\mathbf{9 9 . 2}$ also forms the native conformation or a topological isomer with a closely related structure. 
A

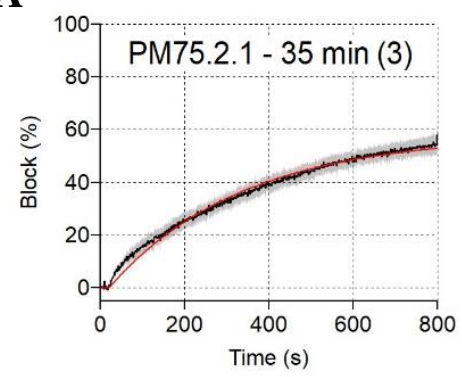

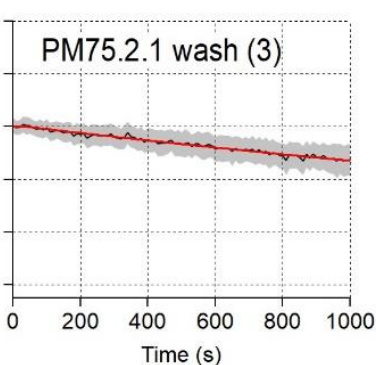

B

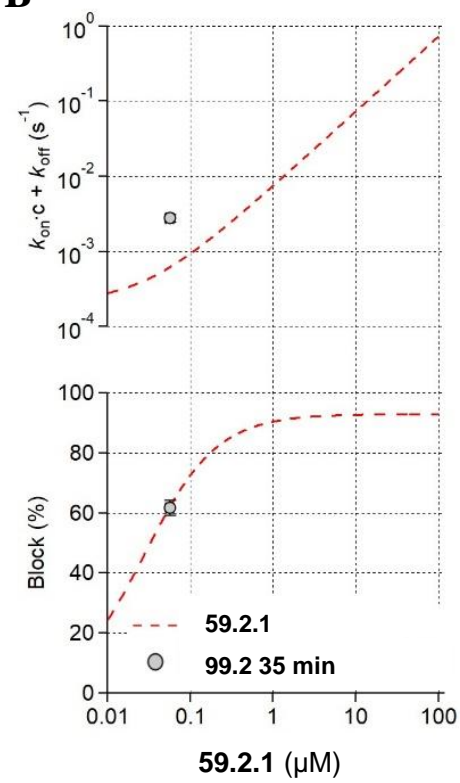

Figure 2.35: Patch-clamp experiments of isomer 4.2. A) Blocking and wash-off kinetics of isomer 99.2 of DEACM-protected $\mu$-SIIIA $(10 \mu \mathrm{M})$ after irradiation for $35 \mathrm{~min}$. B) Dose-response curve shows the association and dissociation kinetics and percentage of sodium current inhibition as a function of the native $\mu$-SIIIA (59.2.1) concentration (red dashed lines). Data points from $10 \mu \mathrm{M}$ of the second isomer after 35 min irradiation are highlighted in grey. The experiment was performed by VIVIEN KISTMACHER.

Patch-clamp measurements of isomer 99.3 of the DEACM protected $\mu$-SIIIA $(10 \mu \mathrm{M})$ showed a reduced blocking activity of about $16 \%$ after an exposure time of $35 \mathrm{~min}$ compared to the isomer 99.1 and 99.2 (see figure 2.37 A). Additionally, the dissociation kinetics revealed a fast wash-off from the Nav1.4 channel and an almost full recovery of the sodium current after 5 min $\left(\tau_{\text {Diss }}=214 \pm 36 \mathrm{~s}\right.$ ), compared to the isomer 99.1 and 99.2 (see figure 2.37 B). These results suggest that the isomer $\mathbf{9 9 . 3}$ forms a non-native conformation with a reduced affinity for the Nav1.4 similar to isomer 59.2.2 of the native $\mu$-SIIIA. The patch-clamp measurements of isomer 99.4 have to be further investigated. 
A

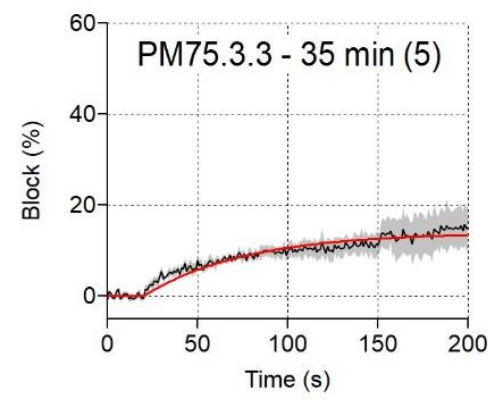

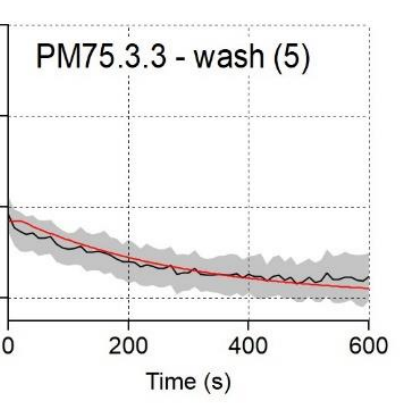

B

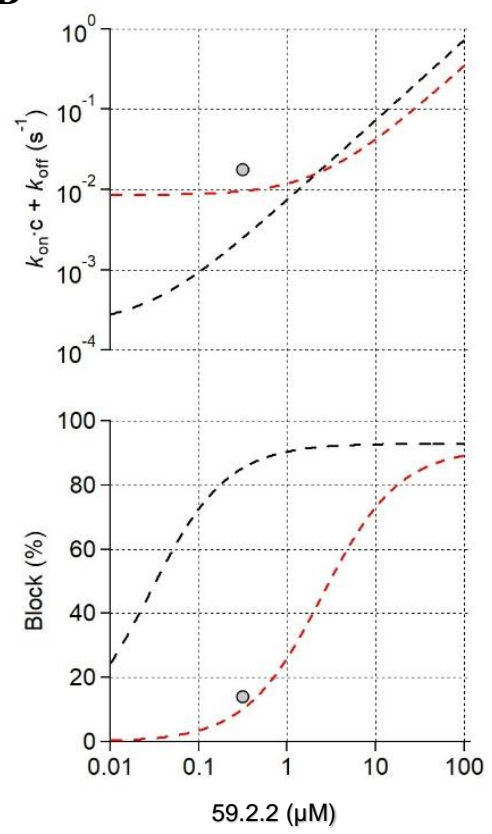

Figure 2.36: Patch-clamp experiments of isomer 4.3. A) Blocking and wash-off kinetics of isomer 99.3 of DEACM-protected $\mu$-SIIIA $(10 \mu \mathrm{M})$ after irradiation for $35 \mathrm{~min}$. B) Dose-response curve shows the association and dissociation kinetics and percentage of sodium current inhibition as a function of the native $\mu$-SIIIA (59.2.2) concentration (red dashed lines) and non-native $\mu$-SIIIA (black dashed lines). Data points from $10 \mu \mathrm{M}$ of the second isomer after $35 \mathrm{~min}$ irradiation are highlighted in grey. The experiment was performed by VIVIEN KISTMACHER.

\subsubsection{Verification of the Uncaging Process by U-HPLC}

Since isomer 99.1 and 99.2 revealed slow dissociation kinetics, it can be assumed that both isomers form the native conformation. However, the significantly reduced blocking activity, compared to the native $\mu$-SIIIA, indicates an incomplete uncaging process of the DEACM group and therefore limited inhibition of the sodium currents. To verify the efficiency of the cleavage process for both isomers, the toxins were analysed by U-HPLC and mass spectrometry after irradiation of $35 \mathrm{~min}$ with the $40 \mathrm{~mW}$ LED light source (CooLED PE300) at $400 \pm 25 \mathrm{~nm}$.

The U-HPLC chromatogram of the isomer 99.1 revealed the formation of the uncaged $\mu$-SIIIA (59) with a retention time of $6.2 \mathrm{~min}$, but also significant amounts of the DEACM caged $\mu$-SIIIA (99) (15.7 min) (see figure 2.38 A). Beside the caged and uncaged $\mu$-SIIIA, several side product fractions were detected, probably caused by the long exposure times. This prolonged UV irradiation can induce photolysis of the disulphide bonds as well as heat-related denaturation of the conformation. Investigation of the isomer 99.2 after irradiation for $35 \mathrm{~min}$, showed only minor amounts of the uncaged $\mu$-SIIIA (59) and a reduced uncaging efficiency compared to the isomer 99.1 (see figure 2.38 B). These results are consistent with the data from 
the patch-clamp measurements, in which a reduced blocking activity was measured for isomer 99.2. Furthermore, the caged $\mu$-SIIIA (99), as well as side products, were detected with substantial higher concentrations. Based on the U-HPLC chromatograms, the relative peak area of the uncaged $\mu$-SIIIA isomers were calculated and related to the caged $\mu$-SIIIA as well as side product peaks. The calculations revealed a cleavage efficiency for the isomer $\mathbf{9 9 . 1}$ of $20 \%$ and $4 \%$ for the 99.2, showing minimal higher deprotection rates as for the patch-clamp measurements.

A

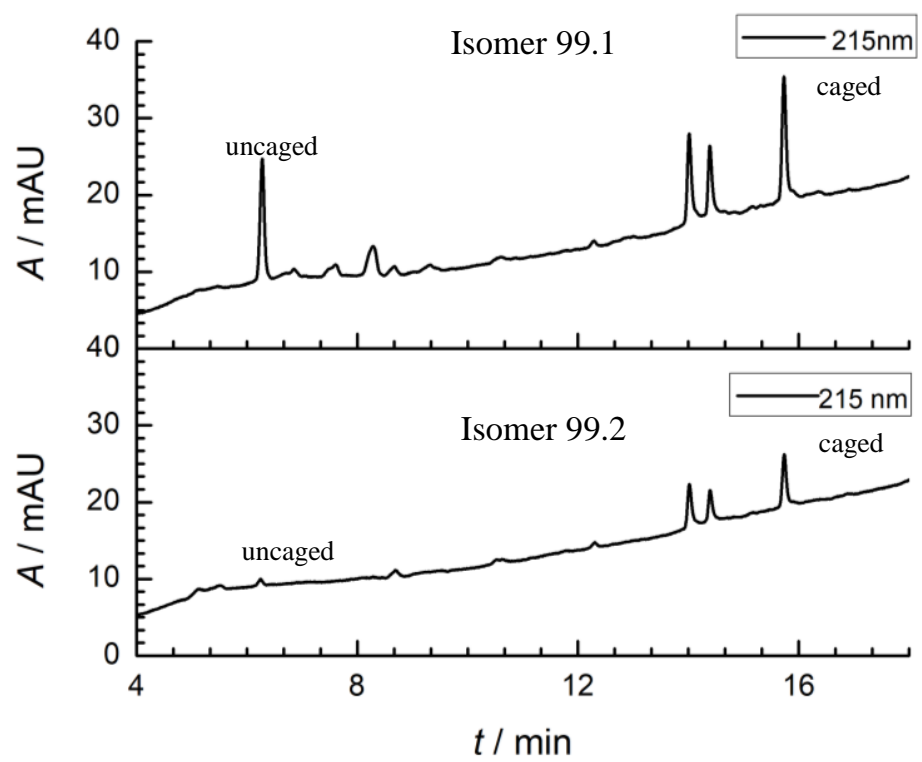

Figure 2.37: HPLC analysis of irradiated isomer 99.1 and 99.2. A) U-HPLC chromatogram of isomer 99.1 of the DEACM-protected $\mu$-SIIIA after irradiation for $35 \mathrm{~min}$. The uncaged $\mu$-SIIIA (59) was detected after $6.2 \mathrm{~min}$ and the caged $\mu$-SIIIA (99) elutes after $15.7 \mathrm{~min}$. HPLC gradient: $1-40 \% \mathrm{MeCN}$ in $15 \mathrm{~min}$. B) U-HPLC chromatogram of isomer $\mathbf{9 9 . 2}$ after irradiation for $35 \mathrm{~min}$. The uncaged $\mu$-SIIIA (59) was detected after $6.1 \mathrm{~min}$ and the caged $\mu$-SIIIA (99) elutes after 15.6 min. HPLC gradient: 1-40\% MeCN in 15 min.

In summary, analysis of the U-HPLC chromatograms for the isomers 99.1 and $\mathbf{9 9 . 2}$ after an exposure time of $35 \mathrm{~min}$, showed an incomplete cleavage of the DEACM group and the formation of several side products, causing the reduced blocking activity. The long irradiation times with the LED light source are likely to destroy the disulphide connectivity or conformation of the conotoxin. Therefore, patch-clamp measurements of isomer 99.1 and 99.2 should be performed again with optimised cleavage conditions, using low substrate concentrations and a quartz cuvette with a large surface area as well as a diameter $\leq 1 \mathrm{~mm}$ for optimal exposure conditions. The LED light source should also be replaced by the handheld laser, which was used in previous uncaging studies (see chapter 2.2.5.1), showing full cleavage after $3 \mathrm{~min}$, without the formation of side products. 


\subsection{Discussion and Conclussion}

Voltage-gated sodium channels are involved in the initiation and propagation of action potentials and control a variety of different physiological processes. ${ }^{[166,171]}$ From the nine different Nav channel isoforms, especially dysfunctions and mutations in the Nav1.2 subtype are linked to different neurological diseases. ${ }^{[171]}$ For the development of specific therapeutics, more profound insights into the molecular mechanisms and expression levels of the Nav1.2 channels are required. Fluorescent labelled neurotoxins were used to address Nav channels distribution and activity. Compared to labelled antibodies, the neurotoxins revealed higher selectivity for the different channel subtypes and a 1:1 binding stoichiometry. ${ }^{[182]}$ In screening studies, the disulphide-rich $\mu$-conotoxin SIIIA was identified, which inhibits the Nav1.2 channel with low nanomolar affinity and the skeletal Nav1.4 channel with submicromolar affinity. ${ }^{[12,13,209]}$ By contrast with $\mu$-conotoxins, the $\mu$-SIIIA (59) exhibit additional residues, which are non-essential for the channel interaction and therefore are suitable positions for fluorophore introduction. ${ }^{[14,207]}$

For the synthesis of $\mu$-SIIIA derivatives, a regioselective synthesis strategy was developed to ensure the correct disulphide connectivity and native conformation. An optimisation of the folding strategy, by rearranging the order of disulphide bridges, lead to the formation of only two topological isomers (59.2.1-59.2.2), instead of four (see figure 2.39). The isomers were separated by HPLC and analysed by patch-clamp measurements, revealing for isomer 59.2.1 a high blocking activity (93\%) as well as slow dissociation kinetics, indicating that the isomer 59.2.1 forms the conformation of the native $\mu$-SIIIA. The results for the isomer 59.2.2 revealed a reduced channel blocking activity and a fast dissociation process. Based on these results, it was assumed that isomer 59.2.2 forms a topological isomer with a non-native structure. Further structural analysis of 59.2.2 could help to investigate whether the reduced blocking activity is either based on different topological structure or disulphide connectivity caused by a reshuffling of the disulphide bonds. For clarification of the disulphide connectivity, NMR spectroscopy or MS/MS analysis can be performed. For the MS/MS measurements, the peptides get partially reduced and alkylated by addition of iodoacetamide. Following, a different combination between 2-, 4- and 6- alkylated peptides were obtained, which can be analysed by MALDI MS/MS. ${ }^{[230]}$ More structural in-depth analysis was not carried out as only a limited amount of material was synthesised. 


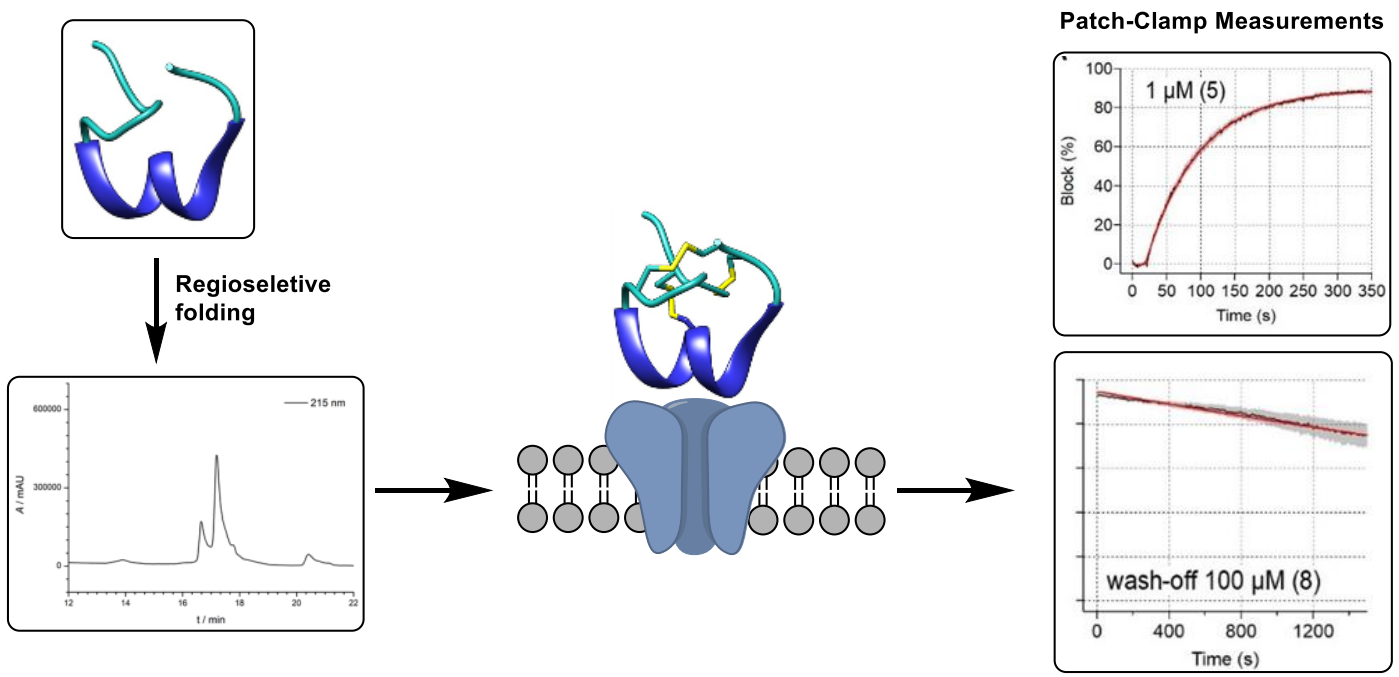

Figure 2.38: Overview of the results of the native $\mu$-SIIIA. After synthesis and purification of the native $\mu$-SIIIA, patch-clamp measurements were performed.

After the successful formation of the native $\mu$-conotoxin SIIIA (59), the regioselective folding strategy was applied for the synthesis of a fluorophore labelled $\mu$-SIIIA derivative. The commonly used NHS-fluorophore labelling method, described by HoNE et al. or GONZALES et al., revealed limitations for our approach, as the introduction of NHS-fluorophore would lead to a site unspecific binding to all nucleophilic lysine residues and $N$-terminal amine group in the folded peptide. For the specific introduction of the fluorophore, an alkyne linker was introduced into the position of the Gly6, which is non-essential for channel interaction. Following, the three disulphide bridges were formed and the alkyne-modified $\mu$-SIIIA was labelled with an AlexaFluor 488 fluorophore via copper(I)-catalysed azide-alkyne cycloaddition (CuAAC) reaction, producing one isomer (see figure 2.40). The affinity and selectivity of the labelled $\mu$-SIIIA (65) for the Nav1.2 will be analysed by patch-clamp measurements, followed by fluorescence microscopy experiments. ${ }^{[227,270,271]}$
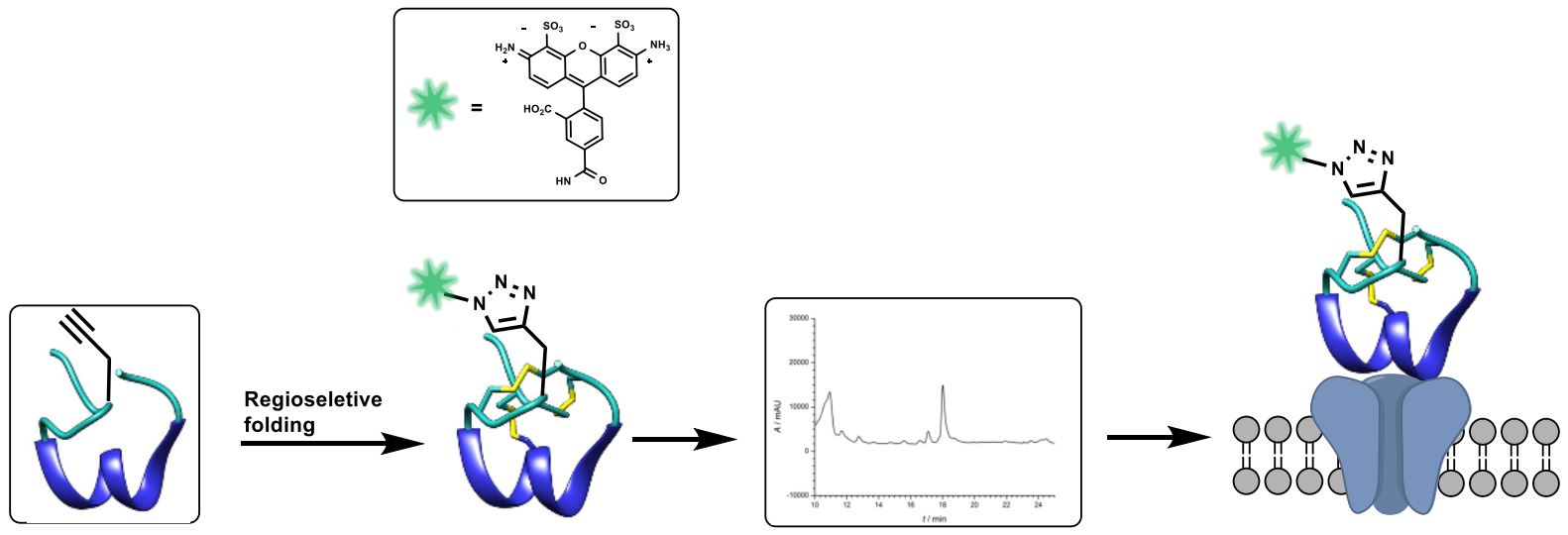

Figure 2.39: Overview of the results of the AlexaFluor 488 labelled $\mu$-SIIIA. After folding of the alkynemodified $\mu$-SIIIA, the AlexaFluor 448 fluorophore was introduced and purified by HPLC. 
Despite the successful labelling of the $\mu$-SIIIA, different folding strategies and cysteine protecting groups were investigated to facilitate the folding protocol and to increase the yields. Therefore, the Acm protecting group, which requires harsh cleavage conditions, was substituted with the Allocam group, tert-butyl or MeOBzl group. All tested protecting groups were either not cleavable or caused oxidation of sensitive side chain residues in the $\mu$-SIIIA sequence. To overcome these limitations, various labelling strategies were analysed to eliminate the reactive alkyne linker for CuAAC. In a first approach, an additional Cys residue was introduced at the position of the Gly6, which can be labelled with maleimide activated fluorophores. The newly introduced cysteine residue was not involved in the formation of disulphide bridges and was deprotected after the formation of the last disulphide bridge. Unfortunately, deprotection of the cysteine lead to the formation of intermolecular disulphide bonds. In a second approach, an Alloc-protected lysine residue was introduced in the position of Gly6, which can be selectively deprotected and labelled with an NHS-activated TMR fluorophore. At the same time, all other nucleophilic groups were still protected. After the formation of the third disulphide bridge, two isomers (79.1-79.2) of the TMR-labelled $\mu$-SIIIA were isolated with significantly higher yields compared to the CuAAC labelling strategy. Patch-clamp measurements and fluorescence microscopy will be performed for further characterisations of the isomers. Theses results will provide deeper insights into the folding process and how the introduction of the bulky TMR groups influences the overall conformation, compared to the AlexaFluor 488 labelled $\mu$-SIIIA For addressing the scientific challenge of channel activity monitoring, a $\mu$-SIIIA derivative modified with a photocleavable protecting group (PPG) was synthesised. This labelling strategy allows the controlled blockage of the Nav channel activity. The introduction of the PPG effectively prevented the interaction of the $\mu$-SIIIA derivatives with the Nav channel. A controlled blockage of the channel activity was achieved by UV irradiation, as the PPG is cleaved from the $\mu$-SIIIA enabling the binding and therefore blocking of the channel. Investigations of different PPGs displayed the DEACM group as PPG with the highest photocleavage rates compared to the Nvoc or DMNPB group. The DEACM group was incorporated into the $\mu$-SIIIA sequence by conjugation to the $\varepsilon$-amino group of the lysine residue. After successful synthesis and purification of caged $\mu$-SIIIA, four different isomers were isolated and analysed by patch-clamp measurements. Recordings of two caged isomers (99.1-99.2) revealed no blockage of the channel currents. As expected, irradiation by aED light source lead to an increased channel inhibition, reaching a maximum of blocking activity of $70 \%$ after an exposure time of $35 \mathrm{~min}$. Measurements of the dissociation kinetics revealed a slow wash-off from the channel, similar to the native $\mu$-SIIIA. These findings highlight the 
potent blocking of the Nav channel by the two isomers after the uncaging process. Nevertheless, a reduced blocking activity could be recorded caused by an incomplete uncaging process. These findings were confirmed by U-HPLC, showing an incomplete cleavage of the DEACM group and the formation of several side product fractions. For further investigations of the caged $\mu$ SIIIA, a more powerful UV light is indispensable to achieve complete cleavage of the $\mu$-SIIIA as shown in preliminary experiments.

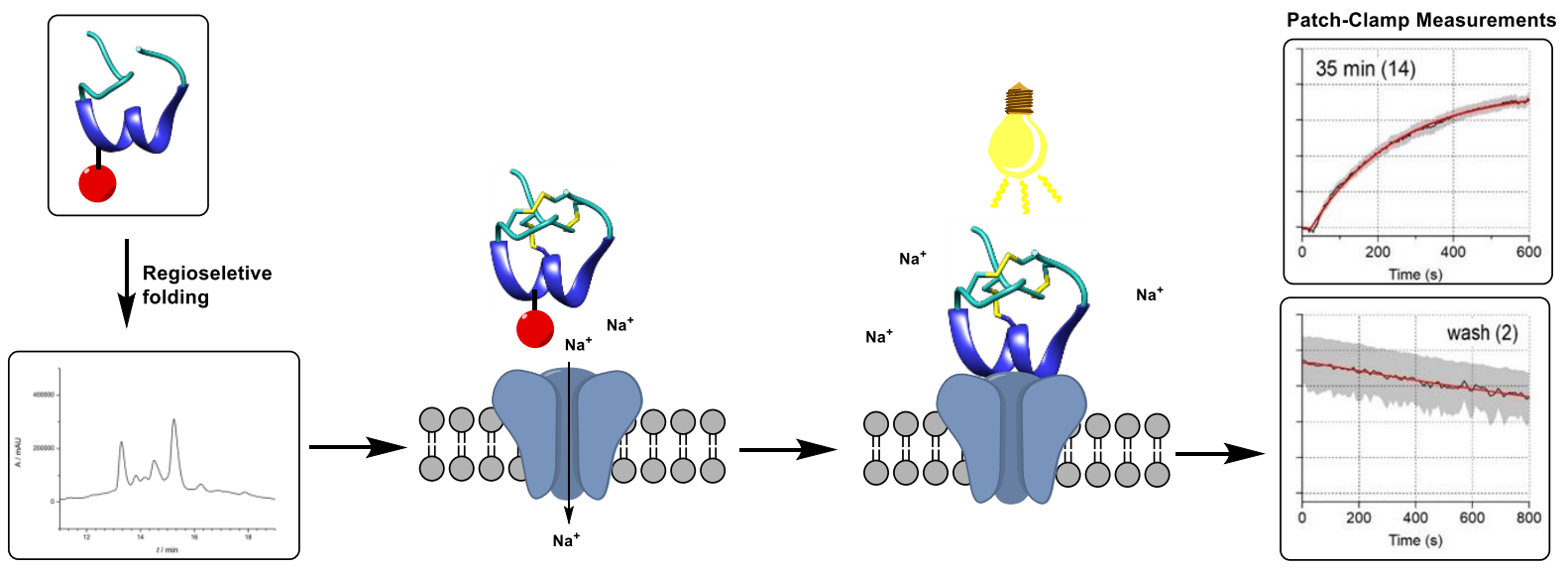

Figure 2.40: Overview of the results of the PPG modified $\mu$-SIIIA. After the introduction of the DEACM group, uncaging and patch-clamp experiments were performed.

Summarizing these results, different $\mu$-conotoxin SIIIA derivates were synthesised with an optimised regioselective folding strategy, which allows the controlled formation of three disulphide bridges and the introduction of different modifications in the $\mu$-SIIIA sequence. Compared to previously reported toxin labelling strategies, the incorporation of the fluorophore by biorthogonal CuAAC reaction enables the specific introduction in the folded $\mu$-SIIIA without the formation of side products. ${ }^{[227,270,271]}$ Furthermore, the uncaging experiments presented for the first time, the successful synthesis and reactivation of a PPG modified conotoxin to control Nav channel activity. Notably, the folding strategy as well as labelling approach are not $\mu$-SIIIA specific and can therefore be transferred to other toxins consisting of three disulphide bonds. This opens new possibilities for channel characterization and consequently, further investigations of channel dysfunction based disorders. 


\section{Experimental Part}

\subsection{General}

\section{Solvents}

Dry solvents were purchased from SIGMA-ALDRICH (Taufkirchen, Germany) and ACROS ORGANICS (Geel, Belgium). Technical solvents were distilled before use. Solvents of analytical and HPLC grade were purchased from FLUKA (Taufkirchen, Germany), VWR INTERNATIONAL (Fontenay-sous-Bois, France), ACROS ORgANICS (Geel, Belgium) and SigMA-Aldrich (Taufkirchen, Germany). Solvents for the NMR measurements were supplied by DEUTERIO (Kastellaun, Germany). Ultra-pure water was obtained by purifying demineralized water with the purification device arium mini lab water system by SARTORIUS (Göttingen, Germany).

\section{Reagents}

All reagents were purchased in the highest grade available and were used as supplied. Amino acid derivatives, resins and coupling reagents for solid-phase peptide synthesis were obtained from NovABIOCHEM (Darmstadt, Germany), BACHEM (Bubendorf, Switzerland), GL BIOCHEM (Shanghai, China), IRIS BIOTECH (Marktredwitz, Germany), BLD PhARMATECH LTD. (Shanghai, China) as well as VWR (Darmstadt, Germany). Other chemicals were purchased from MercK (Darmstadt, Germany), FISHER SCIENTIFIC (Nidderau, Germany), AlFA AESAR (Karlsruhe, Germany), BACHEM (Bubendorf, Switzerland), TCI (Eschborn, Germany), VWR (Darmstadt, Germany), NovaBiochem (Darmstadt, Germany), Acros Organics (Geel, Belgium) and SIGMA-ALDRICH (Taufkirchen, Germany). DNA Oligomers were purchased from IBA (Göttingen, Germany). PNA Oligomers were obtained from ASM Research Chemicals (Hannover, Germany).

\section{Reactions}

Air as well as water-sensitive reactions were carried out under argon (> 99.996\%) atmosphere using standard SCHLENK-technique in dry solvents. The glass apparatus was heated under reduced pressure and flushed with argon. The solution was added through a septum and solids in a counter stream of argon. 


\section{Freeze-drying}

Freeze-drying of peptides or synthesised building blocks was performed by an Alpha-2-4-LD plus benchtop freeze-dryer from CHRIST (Osterode am Harz, Germany) connected to a Vacuubrand chemistry hybrid pump RC5 high vacuum pump (Wertheim, Germany). Aqueous samples with minor amounts of acetonitrile collected from the HPLC device were frozen with liquid nitrogen and dried under reduced pressure $(<1 \mathrm{mbar})$. Small amounts $(<2 \mathrm{~mL})$ were freeze-dried using an evacuable $R V C$ 2-18 CD plus vacuum centrifuge from CHRIST.

\subsection{Chromatography}

\section{Thin-layer chromatography (TLC)}

Analytical TLC was performed on TLC $60 \mathrm{~F}_{254}$ silica gel coated aluminium sheets from MERCK (Darmstadt, Germany). The substances were detected via fluorescence quenching (254 nm, $366 \mathrm{~nm})$ or by the following TLC stains: ninhydrin solution $(1.5 \mathrm{~g}$ ninhydrin, $3 \mathrm{~mL}$ acetic acid in $100 \mathrm{~mL} n$-butanol) or potassium permanganate $\left(1.5 \mathrm{~g} \mathrm{KMnO}_{4}, 10 \mathrm{~g} \mathrm{~K}_{2} \mathrm{CO}_{3}\right.$ and $1.25 \mathrm{~mL}$ $10 \% \mathrm{NaOH}$ in $200 \mathrm{~mL}$ water)

\section{Flash column chromatography}

Flash column chromatography was performed using silica gel $60 \mathrm{~F}_{254}$ (particle size: 40-63 $\mu \mathrm{m}$ ) purchased from MERCK (Darmstadt, Germany). Columns were packed with wet silica gel (50100 fold weight excess) and the sample was loaded as a concentrated solution or onto silica gel. The column was run at an overpressure of 0.5-1.0 bar. Elution of the product fractions was identified by TLC.

\section{High-Performance Liquid Chromatography (HPLC)}

Analytical or semi-preparative reversed-phase HPLC purifications were performed with a system from JASCO (Tokyo, Japan), consisting of two pumps PU-2020Plus, a 3-line degasser DG2080-53 and a diode array detector MD-2010Plus. Substances were eluted with a linear gradient MilliQ + $0.1 \%$ TFA to MeCN + $0.1 \%$ TFA. The following columns from MACHEREYNAGEL (Düren, Germany) were used for purification:

Analytical: MN Nucleodor 100-5-C18, $250 \mathrm{~nm}$ x $4.6 \mathrm{~mm}, 5 \mu \mathrm{m}$, flow rate: $1 \mathrm{~mL} / \mathrm{min}$. 
Semipreparative: MN Nucleodor 100-5-C18, $250 \mathrm{~mm}$ x $10 \mathrm{~mm}, 5 \mu \mathrm{m}$, flow rate: $3 \mathrm{~mL} / \mathrm{min}$.

Preparative: MN Nucleodor 100-5-C18, $250 \mathrm{~mm}$ x $21 \mathrm{~mm}, 5 \mu \mathrm{m}$, flow rate: $10 \mathrm{~mL} / \mathrm{min}$.

UV-detection was measured at $215 \mathrm{~nm}, 254 \mathrm{~nm}, 280 \mathrm{~nm}$ for non-labelled peptides. For peptides labelled with BAC, NBD, AlexaFluor 488 or TMR the UV-absorbances was recorded at $368 \mathrm{~nm}, 464 \mathrm{~nm}, 488 \mathrm{~nm}$, or $540 \mathrm{~nm}$ Before usage, the samples were dissolved in a mixture of MilliQ and MeCN, followed by filtration through a Chromafil RC-45/15 MS filter from MACHEREY-NAGEL.

\section{Ultra-High-Performance Liquid Chromatography (U-HPLC)}

An UltiMate 3000 from THERMO-FISCHER SCIENTIFIC (two pumps LPG-3400, autosampler WPS-3000SL, column compartment TCC-3000SD, Detector DAD-3000, interface Chromeleon) were used for analyses of uncaging-, enzyme cleavage experiments as well as peptide synthesis. Substances were eluted with MilliQ (0.085 \% TFA) and MeCN (0.1 \%TFA). The UV-absorption was detected with a photodiode arrays in a range of 200 to $450 \mathrm{~nm}$.

Analytical: ACE Exel ${ }^{\mathrm{TM}} 2 \mathrm{C} 18,100 \mathrm{~nm} \times 2.1 \mathrm{~mm}, 2 \mu \mathrm{m}$, flow rate: $0.3 \mathrm{ml} / \mathrm{min}$.

Prior analysis, the samples were dissolved in MilliQ and MeCN, followed by filtration.

\subsection{Characterisation}

\section{Nuclear magnetic resonance spectroscopy (NMR)}

${ }^{1} \mathrm{H}-\mathrm{NMR}$ spectra were recorded at $300 \mathrm{MHz}$ on a VNMRS-300 or a Mercury-VX 300 spectrometer from VARIAN (Palo Alto, USA). ${ }^{13} \mathrm{C}$-NMR spectra were measured at $126 \mathrm{MHz}$ on an Inova-500 from VARIAN (Palo Alto, USA). The sample temperature was $35^{\circ} \mathrm{C}$ for DMSO$d_{6}$ and $\mathrm{CD}_{3} \mathrm{OD}$, whereas samples in $\mathrm{CDCl}_{3}$ were measured at $27^{\circ} \mathrm{C}$. The residual proton signals of the solvents served as an internal standard (DMSO- $d_{6}: \delta=2.50 \mathrm{ppm}$ for ${ }^{1} \mathrm{H}-\mathrm{NMR}, \delta=$ $39.5 \mathrm{ppm}$ for ${ }^{13} \mathrm{C}-\mathrm{NMR}$; $\mathrm{CDCl}_{3}: \delta=7.26 \mathrm{ppm}$ for ${ }^{1} \mathrm{H}-\mathrm{NMR}, \delta=77.16 \mathrm{ppm}$ for ${ }^{13} \mathrm{C}-\mathrm{NMR}$; $\mathrm{CD}_{3} \mathrm{OD}: \delta=3.31$ and 4.78 ppm for ${ }^{1} \mathrm{H}-\mathrm{NMR}, \delta=49.15$ and 54.00 ppm for $\left.{ }^{13} \mathrm{C}-\mathrm{NMR}\right)$. Chemical shifts $\delta$ are given in parts per million (ppm) and the coupling constants $J$ were given in Hertz $(\mathrm{Hz})$. The following abbreviations for the multiplicities were used: $\mathrm{s}$ (singlet), $\mathrm{d}$ (doublet), $\mathrm{t}$ (triplet), q (quartet), $\mathrm{m}$ (multiplet) and br (broad). The coupling constant ${ }^{\mathrm{n}} J_{\mathrm{X}, \mathrm{Y}}$ is indicated in Hertz $(\mathrm{Hz})(\mathrm{n}=$ number of bonds between the coupling nuclei; $\mathrm{X}, \mathrm{Y}=$ coupling nuclei). 


\section{Mass Spectrometry}

Electrospray ionization (ESI) mass spectra and high-resolution ESI (HR-MS) spectra were recorded at a maXis or MicroTOF spectrometer by BRUKER DALTONIK GMBH (Bremen, Germany). The data were analysed with Compass Data analysis software (version 4.0) by Bruker. The samples were dissolved in MilliQ/MeOH (1:1), MeOH or DCM. The values are given in $\mathrm{m} / \mathrm{z}$ ratio, along with the relative intensity of the peak.

\section{Liquid Chromatography Mass Spectrometry}

Analytical LC-MS measurements were performed on an UltiMate 3000 system Scientific (two pumps LPG-3400, autosampler WPS-3000SL, column compartment TCC-3000SD, Detector DAD-3000, interface Chromeleon) from THERMO FISHER SCIENTIFIC GMBH and an ACE Exel $^{\mathrm{TM}} 2$ column $(\mathrm{C} 18,100 \mathrm{~nm} \times 2.1 \mathrm{~mm}, 2 \mu \mathrm{m})$ at $50{ }^{\circ} \mathrm{C}$ with a flow rate of $0.45 \mathrm{~mL} / \mathrm{min}$. Substances were eluted with MilliQ (0.1\% FA) and $\mathrm{MeCN}(0.1 \% \mathrm{FA})$ in $15 \mathrm{~min}$, coupled to an LTQ XL ion trap spectrometer from THERMO FISHER SCIENTIFIC GMBH. The UV-absorption was detected with a photodiode arrays in a range of 200 to $450 \mathrm{~nm}$.

\section{$\mathrm{UV} / \mathrm{V}$ is Spectroscopy}

UV spectra for determination of resin loading efficiency and peptide concentration was measured with a V-550 spectrometer from JASCO (Tokyo, Japan) or the THERMO SCIENTIFIC nanodrop ND-2000c spectrophotometer. Peptide concentrations were calculated by using LAMBERT-BEER law:

$$
\mathrm{c}=\frac{\mathrm{A}}{\varepsilon \cdot \mathrm{d}}
$$

$\mathrm{c}=$ concentration $(\mathrm{mmol} / \mathrm{l}), \mathrm{A}=\operatorname{absorption}(\mathrm{nm}), \varepsilon=$ molar extinction coefficient in $\left(\mathrm{cm}^{-1}\right.$ $\left.\cdot \mathrm{mmol}^{-1}\right), \mathrm{d}=$ pathlength (in $\mathrm{cm}$ )

The absorption was estimated either at $260 \mathrm{~nm}$ for peptide-PNA oligomers or at $280 \mathrm{~nm}$ for peptides containing tryptophan. The molecular absorption coefficients were calculated by summation of the single coefficients at a set wavelength. For fluorophore marked molecules, the absorption was measured for the maximum of the fluorophores (see table 4.1). 
Table 3.1: The extinction coefficients used for concentration estimations. Depicted for tryptophan, phenylalanine and the used fluorophores as well as PNA monomers to estimate the overall $\varepsilon$ peptide-PNA hybrids.

\begin{tabular}{lll}
\hline & $\lambda_{\text {abs }}[\mathbf{n m}]$ & $\varepsilon\left[\mathbf{M}^{\left.\mathbf{- 1} \cdot \mathbf{c m}^{-\mathbf{1}}\right]}\right.$ \\
\hline Trp & 280 & 5500 \\
Phe & 280 & 1490 \\
Phe & 280 & 200 \\
NBD & 466 & 22000 \\
AlexaFluor 488 & 495 & 73000 \\
AlexaFluor 647 & 650 & 270000 \\
S-S & 280 & 125 \\
TMR & 540 & 91000 \\
\hline PNA-a & 260 & 13700 \\
PNA-c & 260 & 6600 \\
PNA-g & 260 & 11700 \\
PNA-t & 260 & 8600 \\
\hline
\end{tabular}

\section{BCA Assay}

The concentration of DEACM-modified $\mu$-conotoxin SIIIA was determined by BCA assay. Therefore, the following reagents were used: micro-BCA assay reagent $\mathrm{A}\left(645 \mathrm{mM} \mathrm{Na} \mathrm{CO}_{3}\right.$, $70 \mathrm{mM}$ NaK Tatrat tetrahydrate, $\mathrm{pH}$ 11.25), micro-BCA assay reagent $\mathrm{B}$ (116 mM Bicinchoninic acid), micro-BCA assay reagent C (160 mM copper(II)sulphate pentahydrate), micro-BCA assay solution (reagent $\mathrm{A} / \mathrm{B} / \mathrm{C}(49: 49: 2, v / v / v)$.

All samples $(100 \mu \mathrm{L})$ were mixed with the micro-BCA assay solution $(100 \mu \mathrm{L})$ and were incubated at $60{ }^{\circ} \mathrm{C}$ for $15 \mathrm{~min}$. Following, the samples were cooled to room temperature and the absorbance at $562 \mathrm{~nm}$ was measured on a nanodrop ND-2000c spectrophotometer from THERMO SCIENTIFIC. Unknown concentrations were calculated by standard curve.

\section{Uncaging studies}

Photocleavage experiments of the Nvoc and DMNPB protecting group were performed using a mercury UV lamp from NEWPORT (Darmstadt, Germany) equipped with a mirror filter (280 $460 \mathrm{~nm}$ ) at $650 \mathrm{~W}$. Uncaging studies of DEACM protected peptides were carried out with 
Streamline Laser from OsElA INC. (Lachine, Canada) at $405 \mathrm{~nm}, 100 \mathrm{~mW}$ and $70 \mathrm{~mA}$. For irradiation, the caged peptide $(40 \mu \mathrm{M})$ was transferred to a $Q S$ Micro Fluorescence Cell quartz cuvette (115F-QS, light path: 10 × $2 \mathrm{~mm}$, volume: $400 \mu \mathrm{L}$, outer dimensions: $12.5 \times 12.5 \mathrm{x}$ $40 \mathrm{~mm}$ ) from Hellma GmBH \& Co. KG (Müllheim, Germany). The cuvette was centred $\sim 5 \mathrm{~cm}$ in front of the light source and aliquots of $15 \mu \mathrm{L}$ were taken after $30 \mathrm{sec}, 1 \mathrm{~min}, 2 \mathrm{~min}, 3 \mathrm{~min}$, $5 \mathrm{~min}$ and $10 \mathrm{~min}$. After irradiation, the samples were analysed by U-HPLC or LC-MS

\section{Protease Cleavage Studies}

Digestion experiments with TEV, Factor Xa (FXA) or cathepsin B protease were carried out under the following conditions. The peptides and peptide-PNA oligomers were dissolved in reaction buffer (TEV: $50 \mathrm{mM}$ Tris-HCl, $0.5 \mathrm{mM}$ EDTA and $1 \mathrm{mM}$ DTT, pH 8.0; FXa: $20 \mathrm{mM}$ Tris- $\mathrm{HCl}, 50 \mathrm{mM} \mathrm{NaCl}, 1 \mathrm{mM} \mathrm{CaCl}$, cathepsin B: $25 \mathrm{mM}$ sodium acetate, $1 \mathrm{mM}$ EDTA, pH 5.0) to a concentration of 50-500 $\mu \mathrm{M}$. Afterwards, the protease (TEV: $3.000 \mathrm{U} / \mathrm{mg}$, FXa: $20 \mathrm{U} / \mathrm{mg}$, cathepsin B: $10 \mathrm{U} / \mathrm{mg}$ ) was added and the reaction mixture was incubated at $37{ }^{\circ} \mathrm{C}$. For cathepsin B treatment, the protease was activated prior usage with $30 \mathrm{mM}$ DTT and $15 \mathrm{mM}$ EDTA for $15 \mathrm{~min}$ at $\mathrm{rt}$. Aliquots of $15 \mu \mathrm{L}$ were taken at various time points in between $30 \mathrm{~min}$ and $48 \mathrm{~h}$. After incubation, the protease cleavage process was analysed by U-HPLC or LC-MS.

\section{Programs}

NMR data were analysed by MestReNova (version 10) and graphs were plotted with OriginPro (version $8.5 \mathrm{G}$ ). Chemical structures and calculations of molecular masses were performed by ChemBioDraw (version 16). Structures of the $\mu$-conotoxin SIIIA were created by USCF Chimera package (version 1.11.2). 


\subsection{Standard Operating Protocols (SOPs)}

\subsubsection{SOPS for the Synthesis of Peptide-PNA based $\mathrm{Cl}^{-}$sensors}

\subsubsection{SOP1: Automated Solid-Phase Peptide Synthesis}

For the synthesis of different peptide sequences, a Liberty Blue peptide synthesizer from CEM (Matthews, North Carolina, USA) was used. For the synthesis, the following protected amino acids were prepared as 0.2 M solution in DMF: Fmoc-Ala-OH, Fmoc-Arg(Pbf)-OH, FmocAsn(Trt)-OH, Fmoc-Asp( $\left.\mathrm{O}^{t} \mathrm{Bu}\right)-\mathrm{OH}$, Fmoc-Gln(Trt)-OH, Fmoc-Glu( $\left.\mathrm{O}^{t} \mathrm{Bu}\right)-\mathrm{OH}$, Fmoc-Gly$\mathrm{OH}$, Fmoc-His(Boc)-OH, Fmoc-Ile-OH, Fmoc-Leu-OH, Fmoc-Lys(Boc)-OH, Fmoc-Met-OH, Fmoc-Phe-OH, Fmoc-Pro-OH, Fmoc-Ser $\left(\mathrm{O}^{t} \mathrm{Bu}\right)-\mathrm{OH}$, Fmoc-Thr $\left(\mathrm{O}^{t} \mathrm{Bu}\right)-\mathrm{OH}$, Fmoc-Tyr $\left(\mathrm{O}^{t} \mathrm{Bu}\right)-$ $\mathrm{OH}$, Fmoc-Val-OH, Fmoc-Lys(Alloc)-OH and Boc-Ala-OH.

Depending on the synthesis scale, different coupling conditions were performed as well as different stock solutions for the amino acids, deprotection solution, activator and activator base were prepared (see table 4.2). In the following, the peptide synthesis is shown exemplarily for a synthesis scale of $0.10 \mathrm{mmol}$. For the deprotection of the Fmoc group, $20 \%$ piperidine in $\operatorname{DMF}(v / v)$ was used. The amino acids were activated by DIC (0.50 M in DMF) as Activator and Oxyma (1.00 M in DMF) as Activator Base.

The non-preloaded resin (1.0 eq loading density $<0.32 \mathrm{mmol} / \mathrm{g})$ was placed in the reaction vessel of the peptide synthesizer and was swollen for $30 \mathrm{~min}$ in DMF. First, the $N$-terminal Fmoc protecting group was removed by addition of piperidine (20\% in DMF, $v / v)$ under microwave irradiation $\left(1: 75^{\circ} \mathrm{C}, 90 \mathrm{~W}, 15 \mathrm{~s} ; 2: 90^{\circ} \mathrm{C}, 20 \mathrm{~W}, 50 \mathrm{~s}\right)$. To achieve a complete cleavage of the Fmoc-group, the deprotection step was repeated once. After washing of the resin with DMF $(5 \times 4 \mathrm{~mL})$ the amino acid $(0.20 \mathrm{M})$, DIC $(0.50 \mathrm{M})$ as well as Oxyma $(1.00 \mathrm{M})$ were transferred to the reaction vessel. The coupling reaction was performed under microwave irradiation $\left(1: 75^{\circ} \mathrm{C}, 170 \mathrm{~W}, 15 \mathrm{~s} ; 2: 90{ }^{\circ} \mathrm{C}, 30 \mathrm{~W}, 110 \mathrm{~s}\right)$ and was repeated once. For the coupling of Fmoc-Arg(Pbf)-OH $\left(75^{\circ} \mathrm{C}, 30 \mathrm{~W}, 300 \mathrm{~s}\right)$ and Fmoc-His(Boc)-OH $\left(50{ }^{\circ} \mathrm{C}, 30 \mathrm{~W}\right.$, $1500 \mathrm{~min}$ ) specialised coupling cycles were used to suppress the $\gamma$-lactam formation for arginine and epimerization of histidine.

After the peptide synthesis was completed, the resin was transferred into a BD Syringe with PE-frit and was washed with DMF (6 x $4 \mathrm{~mL})$ as well as DCM (6 x $4 \mathrm{~mL})$. Following, the resin was dried under reduced pressure. 
Table 3.2: Overview of coupling and deprotection conditions depending on the synthesis scale.

\begin{tabular}{|c|c|c|c|c|}
\hline & $0.01 \mathrm{mmol}$ & $0.025 \mathrm{mmol}$ & $0.05 \mathrm{mmol}$ & $0.1 \mathrm{mmol}$ \\
\hline Deprotection & $2 \%$ Piperazine & $20 \%$ Piperidine & $20 \%$ Piperidine & $20 \%$ Piperidine \\
\hline Amino acids & $0.08 \mathrm{M}$ & $0.2 \mathrm{M}$ & $0.2 \mathrm{M}$ & $0.2 \mathrm{M}$ \\
\hline Activator & $0.05 \mathrm{M}$ DIC & $0.13 \mathrm{M} \mathrm{DIC}$ & $0.25 \mathrm{M} \mathrm{DIC}$ & $0.5 \mathrm{M} \mathrm{DIC}$ \\
\hline Activator Base & 0.1 M Oxyma & 0.25 M Oxyma & 0.5 M Oxyma & 1.0 M Oxyma \\
\hline Deprotection 1.: & $75^{\circ} \mathrm{C}, 155 \mathrm{~W}, 15 \mathrm{~s}$ & $75^{\circ} \mathrm{C}, 155 \mathrm{~W}, 15 \mathrm{~s}$ & $75^{\circ} \mathrm{C}, 210 \mathrm{~W}, 15 \mathrm{~s}$ & $75^{\circ} \mathrm{C}, 260 \mathrm{~W}, 15 \mathrm{~s}$ \\
\hline \multirow{3}{*}{ Coupling } & $90^{\circ} \mathrm{C}, 30 \mathrm{~W}, 165 \mathrm{~s}$ & $90^{\circ} \mathrm{C}, 30 \mathrm{~W}, 50 \mathrm{~s}$ & $90^{\circ} \mathrm{C}, 30 \mathrm{~W}, 50 \mathrm{~s}$ & $90^{\circ} \mathrm{C}, 60 \mathrm{~W}, 50 \mathrm{~s}$ \\
\hline & $75^{\circ} \mathrm{C}, 170 \mathrm{~W}, 15 \mathrm{~s}$ & $75^{\circ} \mathrm{C}, 170 \mathrm{~W}, 15 \mathrm{~s}$ & $75^{\circ} \mathrm{C}, 170 \mathrm{~W}, 15 \mathrm{~s}$ & $75^{\circ} \mathrm{C}, 170 \mathrm{~W}, 15 \mathrm{~s}$ \\
\hline & $90^{\circ} \mathrm{C}, 30 \mathrm{~W}, 465 \mathrm{sec}$ & $90^{\circ} \mathrm{C}, 30 \mathrm{~W}, 225 \mathrm{~s}$ & $90^{\circ} \mathrm{C}, 30 \mathrm{~W}, 110 \mathrm{~s}$ & $90^{\circ} \mathrm{C}, 30 \mathrm{~W}, 110 \mathrm{~s}$ \\
\hline
\end{tabular}

\subsubsection{SOP2: Automated SPPS for PNA Oligomers}

The synthesis of different peptide-PNA sequences was performed with a Liberty Blue peptide synthesizer from CEM (Matthews, North Carolina, USA) in a $0.01 \mathrm{mmol}$ scale. For the synthesis the following protected aeg-PNA monomers were prepared as $0.08 \mathrm{M}$ solution in DMF: Fmoc-a(Bhoc)-aeg-OH, Fmoc-c(Bhoc)-aeg-OH, Fmoc-g(Bhoc)-aeg-OH and Fmoc$\mathrm{t}(\mathrm{Bhoc})-\mathrm{aeg}-\mathrm{OH}$. The Fmoc-group was removed with piperazine in DMF (2\%) and the PNA monomers $(0.08 \mathrm{M})$ were activated with DIC $(0.05 \mathrm{M})$ as activator as well as Oxyma $(0.10 \mathrm{M})$ as activator base.

The resin containing the peptide sequence was placed in the reaction vessel and was swollen in DMF for $30 \mathrm{~min}$. First the Fmoc-group was removed by addition of the deprotection solution $(3 \mathrm{~mL})$ under microwave irradiation $(50 \mathrm{C}, 25 \mathrm{~W}, 300 \mathrm{~s})$. In order to achieve a complete cleavage of the Fmoc-group, the deprotection step was repeated once. After washing of the resin with DMF (4 x $4 \mathrm{~mL}$ ) the aeg-PNA monomer (0.08 M), DIC (0.05 M) as well as Oxyma $(0.10 \mathrm{M})$ were transferred to the reaction vessel. The coupling was performed under microwave irradiation $\left(50{ }^{\circ} \mathrm{C}, 25 \mathrm{~W}, 1500 \mathrm{~s}\right)$ and was repeated once. Following, the resin was washed with DMF $(4 \times 4 \mathrm{~mL})$ and free amine groups were capped with a solution of DMF/DIPEA/Ac $2 \mathrm{O}$ $(2.79 / 0.15 / 0.6, v / v / v)$ for 5 min at rt. Following, the resin was washed with $5 \%$ DIPEA in DMF and DMF (4 $\mathrm{x} 4 \mathrm{~mL})$. After the synthesis was completed, the resin was transferred into a BD syringe with a polyethylene (PE) frit and was washed with DMF (6 $\times 2 \mathrm{~mL})$ as well as DCM $(6 \times 2 \mathrm{~mL})$. Afterwards the resin was dried under reduced pressure. 


\subsubsection{SOP3: Alloc Deprotection}

According to the procedure of WILSON et al. the peptide-bound resin $(5.00 \mu \mathrm{mol})$ was incubate with DCM $(300 \mu \mathrm{l})$ and phenylsilane $(10.8 \mu \mathrm{L}, 75.0 \mu \mathrm{mol}, 15.0 \mathrm{eq}, \rho=0.88 \mathrm{~g} / \mathrm{mL})$ for 1 min. ${ }^{[122]}$ Afterwards, $\mathrm{Pd}\left(\mathrm{PPh}_{3}\right)_{4}(2.84 \mathrm{mg}, 2.50 \mu \mathrm{mol}, 0.50 \mathrm{eq})$ was added and the cleavage reaction was performed under microwave irradiation $\left(5 \mathrm{~min}, 38^{\circ} \mathrm{C}, 15 \mathrm{~W}\right)$. After repeating the deprotection step twice the resin was washed with DMF $(6 \times 2 \mathrm{~mL})$ as well as DCM $(6 \times 2 \mathrm{~mL})$ and was dried under reduced pressure.

\subsubsection{SOP4: Labelling of Peptides-PNA Oligomers with the BAC-Fluorophore}

Labelling of the BAC fluorophore to the resin-bound peptide-PNA oligomer was performed in $5.00 \mu \mathrm{mol}$ scale. Therefore, BAC fluorophore (13.3 mg, $25.0 \mu \mathrm{mol}, 5.00 \mathrm{eq})$ (4), PyAOP (26.1 mg, $50.0 \mu \mathrm{mol}, 10.0 \mathrm{eq})$, HOAt $(3.40 \mathrm{mg}, 25.0 \mu \mathrm{mol}, 5.00 \mathrm{eq})$ as well as DIPEA $(8.73 \mu \mathrm{L}, 50.0 \mu \mathrm{mol}, 10.0 \mathrm{eq})$ were dissolved in DMF $(400 \mu \mathrm{L})$ and added to the resin. The labelling reaction was performed under microwave irradiation for $3 \mathrm{~h}$ at $70{ }^{\circ} \mathrm{C}$ and $15 \mathrm{~W}$. Afterwards the resin was washed with DMF $(6 \times 2 \mathrm{~mL})$ as well as DCM $(6 \times 2 \mathrm{~mL})$ and was dried under reduced pressure.

\subsubsection{SOP5: Labelling of Peptide-PNA Oligomers with the NBD Fluorophore}

Labelling of the NBD fluorophore to the resin-bound peptide-PNA hybrid was performed in $5.00 \mu \mathrm{mol}$ scale under argon atmosphere. Therefore, NBD-Cl (10.0 mg, $50.0 \mu \mathrm{mol}, 10.0 \mathrm{eq})$ as well as DIPEA $(17.4 \mu \mathrm{L}, 100 \mu \mathrm{mol}, 20.0 \mathrm{eq})$ were dissolved in DMF (400 $\mu \mathrm{L})$ and added to the resin. The labelling reaction was performed for $12 \mathrm{~h}$ at rt. Afterwards the resin was washed with $\mathrm{DMF}(6 \times 2 \mathrm{~mL})$ as well as DCM $(6 \times 2 \mathrm{~mL})$ and was dried under reduced pressure.

\subsubsection{SOP 6 Labelling of peptides with the AlexaFluor488 Fluorophore}

Labelling of the AlexaFluor488 fluorophore to the resin-bound peptide was performed in $5.00 \mu \mathrm{mol}$ scale under argon atmosphere. Therefore, AlexaFluor488-NHS ester $(10.0 \mathrm{mg}$, $50.0 \mu \mathrm{mol}, 10.0 \mathrm{eq})$ as well as DIPEA $(17.4 \mu \mathrm{L}, 100 \mu \mathrm{mol}, 20.0 \mathrm{eq})$ were dissolved in DMF $(400 \mu \mathrm{L})$ and added to the resin. The labelling reaction was performed for $12 \mathrm{~h}$ at rt. Afterwards the resin was washed with DMF $(6 \times 2 \mathrm{~mL})$ as well as DCM $(6 \times 2 \mathrm{~mL})$ and was dried under reduced pressure. 


\subsubsection{SOP 7 Cleavage of Peptides and Peptide-PNA Oligomers from the Resin}

The cleavage of the peptide from the resin was performed in a BD syringe with a PE frit. The resin was shaken at room temperature for $2 \mathrm{~h}$ in TFA/TIS/ $\mathrm{H}_{2} \mathrm{O}(95 / 2.5 / 2.5, v / v / v)$. After cleavage the solution was concentrated under a nitrogen stream and the crude peptide was precipitated from $-20{ }^{\circ} \mathrm{C}$ methyl tert-butyl ether. The crude peptide was isolated by centrifugation $\left(9000 \mathrm{rpm},-10^{\circ} \mathrm{C}, 10 \mathrm{~min}\right)$, washed two times with ether and dried under reduced pressure.

\subsubsection{SOPS for the Synthesis of Modified $\mu$-Conotoxin SIIIA}

\subsubsection{SOP8: Preloading Resin with RAMAgE Linker}

2-Chlorotrityl chloride resin (385 mg, $0.58 \mathrm{mmol}, 100-200 \mathrm{mesh}, 1.50 \mathrm{mmol} / \mathrm{g}$ ) was swollen in an argon flushed flask in dry DCM (4 mL) for $30 \mathrm{~min}$. RAMAGE Linker (147 mg, $290 \mu \mathrm{mol}$, 0.50 eq), DIPEA (237 $\mu \mathrm{L}, 1.39 \mathrm{mmol}, 2.40 \mathrm{eq})$ were dissolved in DCM (4 mL), DMF (0.5 mL) and added to the resin. The mixture was stirred for $30 \mathrm{~min}$ and afterwards the resin was washed with DCM/MeOH/DIPEA (17:2:1, v/v/v), 3 x $5 \mathrm{~mL})$, DCM $(3 \times 5 \mathrm{~mL})$, DMF ( 3 x $5 \mathrm{~mL})$, DCM $(3 \times 5 \mathrm{~mL})$ and dried under reduced pressure.

\subsubsection{SOP9: Coupling of the First Amino Acid}

For 2-chlorotrityl chloride resin the first amino acid was coupled with manual SPPS. The resin was swollen in a BD discardit syringe with PE frit in DMF (5 mL) for $30 \mathrm{~min}$. The Fmoc-group was removed with $20 \%$ piperidine in DMF ( $3 \mathrm{~mL}$ ) under microwave assisted conditions (30 s, $\left.50{ }^{\circ} \mathrm{C}, 25 \mathrm{~W}\right)$ and was washed with $\mathrm{DMF}(3 \times 3 \mathrm{~mL})$. To receive a complete deprotection of the Fmoc-group the cleavage with $20 \%$ Piperidine in DMF $(3 \mathrm{~mL})$ was repeated $\left(3 \mathrm{~min}, 50{ }^{\circ} \mathrm{C}\right.$, $25 \mathrm{~W})$. After the resin was washed with DMF $(3 \times 5 \mathrm{~mL})$, DCM $(3 \times 5 \mathrm{~mL})$ and DMF $(3 \mathrm{x}$ $5 \mathrm{~mL}$ ) the first amino acid (5.00 eq) was coupled with DIC (4.90 eq) as well as HOBt (5.00 eq) in DMF $(3 \mathrm{~mL})$ under microwave assisted conditions $\left(10 \mathrm{~min}, 50^{\circ} \mathrm{C}, 25 \mathrm{~W}\right)$. To receive a complete loading of the first amino acid the coupling step was repeated once. Following, the resin was washed with DMF (3 x $5 \mathrm{~mL}), \mathrm{DCM}(3 \times 5 \mathrm{~mL}), \mathrm{DMF}(3 \times 5 \mathrm{~mL})$ and DCM $(3 \times$ $5 \mathrm{~mL}$ ) and dried under reduced pressure. 


\subsubsection{SOP10: Determination of the Occupancy}

The resin loading was measured using UV absorption recordings. A small amount of the resin $(5-10 \mathrm{mg})$ was placed in a graduated flask $(10 \mathrm{~mL})$ and DBU (2\% in NMP, $4.0 \mathrm{~mL})$ were added. After gentle shaking for $1 \mathrm{~h} \mathrm{MeCN}$ was added $(6 \mathrm{~mL})$. Afterwards the mixture was diluted with $\mathrm{MeCN}(1 / 12.5)$ and transferred in a $10.0 \mathrm{~mm}$ cuvette. The absorption was measured at $304 \mathrm{~nm}\left(\varepsilon_{304}=7624 \mathrm{~L} \cdot \mathrm{mol}^{-1} \cdot \mathrm{cm}^{-1}\right)$ and corrected by the reference. The Fmoc loading was calculated by the following equation:

$$
\rho_{\text {resin }}\left[\frac{\mathrm{mmol}}{\mathrm{g}}\right]=\frac{1}{\mathrm{~m}_{\text {resin }}[\mathrm{g}]} \cdot\left(\frac{\mathrm{A} \cdot \mathrm{V}[\mathrm{L}] \cdot f}{\varepsilon\left[\mathrm{L} \mathrm{mol}^{-1} \mathrm{~cm}^{-1}\right] \cdot \mathrm{d}[\mathrm{cm}]} \cdot 10^{6}\right)
$$

Thereby, $\rho_{\text {resin }}$ is the loading density of resin, $\mathrm{m}_{\text {resin }}$ is the mass of the analysed resin, $\mathrm{A}=$ absorption, $\mathrm{V}$ is the volume of the graduated flask $(0.01 \mathrm{~L}), f$ the thinning factor $(f=12.5), \varepsilon$ the absorption coefficient of the dibenzofulvene and $d$ the pathlength of the cuvette $(10.0 \mathrm{~mm})$.

\subsubsection{SOP11: Automated SPPS of the $\mu$-Conotoxin SIIIA}

Automated synthesis of the modified $\mu$-Conotoxin SIIIA derivates were performed with a Liberty Blue as well as Liberty Prime peptide synthesizer connected to a Discover microwave unit by CEM (Matthews, North Carolina, USA). The protected amino acids were used as a 0.20 M solution in DMF, depending on the synthesis scale. The Fmoc-group was deprotected with piperidine in DMF $(20 \%+0.10 \mathrm{M} \mathrm{HOBt})$. The amino acids were activated with DIC $(0.50 \mathrm{M})$ as activator and Oxyma $(1.00 \mathrm{M})$ as the activator base.

Prior usage the resin was swollen for $30 \mathrm{~min}$ in DMF. In the first step the Fmoc-group was removed by addition of the deprotection solution $(3 \mathrm{~mL})$ under microwave irradiation $\left(1: 75^{\circ} \mathrm{C}\right.$, $90 \mathrm{~W}, 15 \mathrm{~s} ; 2: 90 \mathrm{C}, 20 \mathrm{~W}, 50 \mathrm{~s})$. To get a complete removal of the Fmoc-group the deprotection step was repeated once. After several washing steps $(4 \times 4 \mathrm{~mL})$ the amino acids $(0.20 \mathrm{M})$, DIC $(0.50 \mathrm{M})$ as well as Oxyma $(1.00 \mathrm{M})$ were transferred to the reaction vessel and the coupling was performed under microwave irradiation $\left(1: 75^{\circ} \mathrm{C}, 170 \mathrm{~W}, 15 \mathrm{~s} ; 2: 90^{\circ} \mathrm{C}, 30 \mathrm{~W}, 110 \mathrm{~s}\right)$. To prevent the formation of truncated sequences the coupling step was repeated. For the coupling of Fmoc-Arg(Pbf)-OH modified coupling conditions were used $\left(75^{\circ} \mathrm{C}, 30 \mathrm{~W}, 300 \mathrm{~s}\right)$ to prevent $\gamma$-lactam formation. Also Fmoc-His(Boc)-OH were coupled under mild conditions $\left(50{ }^{\circ} \mathrm{C}\right.$, $30 \mathrm{~W}, 1500 \mathrm{~min}$ ) to reduce epimerization.

After the synthesis was completed, the resin was transferred into a BD syringe with a polyethylene (PE) frit and was washed with DMF and DCM. Afterwards the resin was dried under reduced pressure. 


\subsubsection{SOP 12: Cleavage of $\mu$-Conotoxin SIIIA Derivates from the Resin}

Test-cleavage of the conotoxin derivates or caged test peptides were performed in a BD syringe with a PE frit. Therefore, the resin was shaken at $\mathrm{rt}$ for $2 \mathrm{~h}$ in a cleavage solution $\mathrm{A}$ for conotoxin derivates (TFA/ $\mathrm{H}_{2} \mathrm{O} / 1,2$-ethanedithiol/phenol/thioanisole, $82.5 / 5 / 2.5 / 5 / 5, \quad v / v / v / v / v$ ) or in cleavage solution B for caged test peptides (TFA/TIS/ $\mathrm{H}_{2} \mathrm{O}, 95 / 2.5 / 2.5, v / v / v$ ). After cleavage the solution was concentrated under a nitrogen stream and the crude peptide was precipitated from $-20^{\circ} \mathrm{C}$ methyl tert-butyl ether. The crude peptide was isolated by centrifugation (9000 rpm, $10 \mathrm{~min},-10^{\circ} \mathrm{C}$ ) and dried under reduced pressure.

\subsubsection{SOP 13: Synthesis of $\mu$-SIIIA: Formation of the First Disulphide Bridge}

The $\mu$-conotoxin SIIIA was synthesised in a scale of $0.10 \mathrm{mmol}$. Prior usage the resin was swollen in DMF for $30 \mathrm{~min}$.

1.) The $S^{t} \mathrm{Bu}$ protecting group was removed using $20 \% 2$-mercaptoethanol and $1 \% \mathrm{DBU}$ in DMF (12 ml, $3 \times 4 \mathrm{~mL}, 20 \mathrm{~min}$ each) followed by washing with DMF (3 x $5 \mathrm{~mL})$, DCM (3 x $5 \mathrm{~mL}), \operatorname{DMF}(3 \times 5 \mathrm{~mL}), \operatorname{DCM}(3 \times 5 \mathrm{~mL})$.

2.) Activation of the free thiol by using $2,2^{\prime}$-dipyridyl disulphide $(270 \mathrm{mg}, 0.61 \mathrm{mmol})$ in $\operatorname{DCM}(12 \mathrm{~mL}, 3 \times 4 \mathrm{~mL}, 20 \mathrm{~min}$ each) followed by washing with DCM $(3 \times 5 \mathrm{ml}), \mathrm{DMF}$ $(3 \times 5 \mathrm{~mL}), \mathrm{DCM}(3 \times 5 \mathrm{ml})$.

3.) Cleavage of the peptide from the resin was performed using $10 \% \mathrm{AcOH}, 20 \% \mathrm{TFE}$ in DCM (12 mL, 3 x $4 \mathrm{~mL}, 20 \mathrm{~min}$ each).

4.) Under argon atmosphere the cleavage mixture was added to a solution of $0.2 \%$ TFA, $0.4 \%$ TES in DCM. The reaction was stirred for $4 \mathrm{~h}$ at $\mathrm{rt}$.

5.) Under argon atmosphere this solution was added dropwise over $30 \mathrm{~min}$ to a solution of $\mathrm{NEt}_{3} / \mathrm{DCM}(10: 90, v / v)$ and was stirred for $2 \mathrm{~h}$. The mixture was concentrated and the peptide was precipitated from $-20{ }^{\circ} \mathrm{C}$ cold methyl tert-butyl ether $(25 \mathrm{~mL})$, isolated by centrifugation (9000 rpm, $10 \mathrm{~min},-10 \mathrm{C}$ ), washed two times with $-20{ }^{\circ} \mathrm{C}$ cold methyl tert-butyl ether and dried under reduced pressure.

6.) For the deprotection of all acid-labile protecting groups $\mathrm{TFA} / \mathrm{H}_{2} \mathrm{O} / \mathrm{TIS}$ (95:2.5:2.5, $v / v / v)$ was added to the peptide and agitate for $2 \mathrm{~h}$ at $\mathrm{rt}$. The cleavage mixture was concentrated under a nitrogen stream and the crude peptide was precipitated from $-20{ }^{\circ} \mathrm{C}$ cold ether, isolated by centrifugation ( $9000 \mathrm{rpm}, 10 \mathrm{~min},-10^{\circ} \mathrm{C}$ ) and dried under reduced pressure. 


\subsubsection{SOP 14: Synthesis of $\mu$-SIIIA: Formation of the Second Disulphide Bridge}

According to the procedure of TAM et al. the crude peptide $(0.10 \mathrm{mmol})$ was added to a solution of $5 \%$ acetic acid in water $\left(50 \mathrm{~mL}\right.$, pH 6 , adjust with $\left.\left(\mathrm{NH}_{4}\right)_{2} \mathrm{CO}_{3}\right) .{ }^{[232]} \mathrm{DMSO}(12.5 \mathrm{~mL})$ was added to the reaction mixture and it was stirred for $4 \mathrm{~h}$ at $\mathrm{rt}$. Afterwards the solvent was removed under reduced pressure. The crude peptide was dissolved in TFA $(2 \mathrm{~mL})$ and precipitated with $-20{ }^{\circ} \mathrm{C}$ cold ether, isolated by centrifugation $\left(9000 \mathrm{rpm}, 10 \mathrm{~min},-10{ }^{\circ} \mathrm{C}\right.$ ) and dried under reduced pressure. The resulting colourless solid was purified by semipreparative HPLC.

\subsubsection{SOP 15: Synthesis of Thiol or Amine Modified $\mu$-Conotoxin SIIIA}

The thiol or amine modified $\mu$-conotoxin SIIIA was synthesised in a scale of $0.10 \mathrm{mmol}$. Due to the incorporation of the $S^{t} \mathrm{Bu}, \mathrm{Mmt}$ and Trt groups a modified regioselective folding strategy compared to SOP13 and SOP14 was required. Prior usage the resin was swollen in DMF for 30 min. protected with the

1.) The $\mathrm{S}^{t} \mathrm{Bu}$ protecting groups were removed using $20 \% 2$-mercaptoethanol and $1 \% \mathrm{DBU}$ in DMF (12 ml, $3 \times 4 \mathrm{~mL}, 20 \mathrm{~min}$ each) followed by washing with DMF (3 x $5 \mathrm{~mL}$ ), DCM ( 3 x $5 \mathrm{~mL})$, DMF ( $3 \times 5 \mathrm{~mL})$, DCM ( $3 \times 5 \mathrm{~mL})$.

2.) Activation of the free thiol by using 2,2'-dipyridyl disulphide (540 mg, $1.22 \mathrm{mmol}$ ) in DCM (12 mL, $3 \times 4 \mathrm{~mL}, 20 \mathrm{~min}$ each) followed by washing with DCM ( $3 \times 5 \mathrm{ml})$, DMF $(3 \times 5 \mathrm{~mL}), \mathrm{DCM}(3 \times 5 \mathrm{ml})$.

3.) Cleavage of the peptide from the resin was performed using $10 \% \mathrm{AcOH}, 20 \% \mathrm{TFE}$ in DCM (12 mL, 3 x 4 mL, 20 min each).

4.) For the formation of the first disulphide bridge, the cleavage mixture was transferred to a basic solution of $\mathrm{NEt}_{3} / \mathrm{DCM}(10: 90, v / v)$ under argon atmosphere and the mixture was stirred for $2 \mathrm{~h}$. Following, the solvent was removed under reduced pressure and the peptide was precipitated from cold methyl tert-butyl ether $(25 \mathrm{~mL})$, isolated by centrifugation (9000 rpm, $10 \mathrm{~min},-10 \mathrm{~min}$ ) and dried under reduced pressure.

5.) Cleavage of the Mmt protecting groups were performed with TFA/TES/DCM $(0.2: 0.4: 100, v / v / v)$ for $2 \mathrm{~h}$. Next, the reaction mixture was dried under reduced pressure.

6.) The crude peptide was added to solution of $5 \%$ acetic acid in water $(50 \mathrm{~mL}, \mathrm{pH} 6$, adjust with $\left.\left(\mathrm{NH}_{4}\right)_{2} \mathrm{CO}_{3}\right)$ and DMSO $(12.5 \mathrm{~mL})$. After stirring for $4 \mathrm{~h}$, the reaction mixture was concentrated under reduced pressure.

7.) For the deprotection of all acid-labile protecting groups TFA/ $\mathrm{H}_{2} \mathrm{O} / \mathrm{TIS}$ (95:2.5:2.5, $v / v / v$ ) was added to the peptide and agitate for $2 \mathrm{~h}$ at $\mathrm{rt}$. The cleavage mixture was 
concentrated under a nitrogen stream and the crude peptide was precipitated from $-20{ }^{\circ} \mathrm{C}$ cold ether, isolated by centrifugation $\left(9000 \mathrm{rpm}, 10 \mathrm{~min},-10^{\circ} \mathrm{C}\right.$ ), washed two times with cold ether and dried under reduced pressure.

8.) For the formation of the third disulphide bridge, the peptide was dissolved in a solution of $5 \%$ acetic acid in water $\left(50 \mathrm{~mL}, \mathrm{pH} 6\right.$, adjust with $\left.\left(\mathrm{NH}_{4}\right)_{2} \mathrm{CO}_{3}\right)$ and DMSO $(12.5 \mathrm{~mL})$. Following the mixture was stirred for $4 \mathrm{~h}$ and was concentrated under reduced pressure. The crude peptide was dissolved in TFA $(2 \mathrm{~mL})$ and precipitated with cold ether, isolated by centrifugation $\left(9000 \mathrm{rpm}, 10 \mathrm{~min},-10{ }^{\circ} \mathrm{C}\right)$ and dried under reduced pressure. The resulting colourless solid was purified by semipreparative HPLC. 


\section{Synthesis Peptide-PNA based $\mathrm{Cl}^{-}$sensors}

Synthesis of 4-(9-oxoacridin-10(9H)-yl)butanoic acid (9)

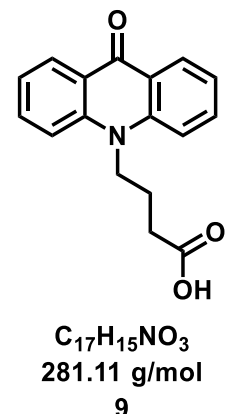

The synthesis was performed according to a protocol modified by LITT et al. ${ }^{[272]}$ A solution of acridone $(5.00 \mathrm{~g}, 25.6 \mathrm{mmol}, 1.00 \mathrm{eq})(7)$ and $\mathrm{KOH}(1.80 \mathrm{~g}, 32.0 \mathrm{mmol}, 1.30 \mathrm{eq})$ in ethanol $(80 \mathrm{~mL})$ was stirred at reflux for $2 \mathrm{~h}$. Afterwards the solvent was removed under reduced pressure. The residue was dissolved in butyrolactone $(39.1 \mathrm{~mL}, 512 \mathrm{mmol}, 20.0 \mathrm{eq}$, $\delta=1.13 \mathrm{~g} / \mathrm{mL})(\mathbf{8})$ and was stirred at reflux for $24 \mathrm{~h}$. The reaction mixture was allowed to cool to room temperature, filtered and the solvent was removed under reduced pressure. The resulting brown oil was purified by flash column chromatography (EtOAc/ $n$-pentane/NEt 3 1:1:0.01 $\rightarrow$ EtOAc/MeOH/AcOH 1:0.1:0.01, v/v/v) to yield the product 9 (2.99 g, $10.6 \mathrm{mmol}$, $42 \%$ ) as yellow solid.

${ }^{1} \mathbf{H}-\mathrm{NMR}\left(300 \mathrm{MHz}, \mathrm{DMSO}-d_{6}\right) ; \delta[\mathrm{ppm}]=1.94-2.08\left(\mathrm{~m}, 2 \mathrm{H}, \mathrm{CH}_{2}-\mathrm{CH}_{2}-\mathrm{CH}_{2}\right), 2.57$ (t, $\left.{ }^{3} J_{\mathrm{H}, \mathrm{H}}=6.8 \mathrm{~Hz}, 2 \mathrm{H}, \mathrm{CH}_{2}-\mathrm{COOH}\right), 4.43-4.54\left(\mathrm{~m}, 2 \mathrm{H}, \mathrm{CH}_{2}-\mathrm{CH}_{2}-\mathrm{CH}_{2}\right), 7.30-7.37(\mathrm{~m}, 2 \mathrm{H}$, C $\left.H_{\text {Arom. }}\right), 7.81-7.88\left(\mathrm{~m}, 2 \mathrm{H}, \mathrm{C} H_{\text {Arom. }}\right), 7.96\left(\mathrm{~d},{ }^{3} J_{\mathrm{H}, \mathrm{H}}=8.8 \mathrm{~Hz}, 2 \mathrm{H}, \mathrm{C} H_{\text {Arom. }}\right), 8.36\left(\mathrm{dd},{ }^{3} J_{\mathrm{H}, \mathrm{H}}=\right.$ $8.0 \mathrm{~Hz},{ }^{4} \mathrm{~J}_{\mathrm{H}, \mathrm{H}}=1.7 \mathrm{~Hz}, 2 \mathrm{H}, \mathrm{C} H_{\text {Arom. }}$.

${ }^{13}$ C-NMR $\left(126 \mathrm{MHz}, \mathrm{DMSO}-d_{6}\right) ; \delta[\mathrm{ppm}]=22.4\left(\mathrm{CH}_{2}-\mathrm{CH}_{2}-\mathrm{CH}_{2}\right), 30.9\left(\mathrm{CH}_{2}-\mathrm{COOH}\right), 45.3$ $\left(\mathrm{N}-\mathrm{CH}_{2}-\mathrm{CH}_{2}\right), 116.3\left(2 \mathrm{C}, 2 \times C_{\mathrm{Ar}}\right), 121.7\left(2 \mathrm{C}, 2 \times C_{\mathrm{Ar}}\right), 122.0\left(2 \mathrm{C}, 2 \times C_{\mathrm{Ar}}\right), 127.2(2 \mathrm{C}, 2 \mathrm{x}$ $\left.C_{\text {Ar. }}\right), 134.7$ (2C, 2 x $\left.C \mathrm{H}_{\text {Ar. }}\right), 141.9$ (2, 2 x $\left.C \mathrm{H}_{\text {Ar. }}\right), 174.9(C \mathrm{O}),. 176.9(C \mathrm{OOH})$.

MS (ESI): $\mathbf{m} / \mathbf{z}(\%)=282.1(12)[\mathrm{M}+\mathrm{H}]^{+}, 304.1(13)[\mathrm{M}+\mathrm{Na}]^{+}$.

HRMS (ESI): calc. for: $\mathrm{C}_{17} \mathrm{H}_{16} \mathrm{NO}_{3}$ : 282.1125, found.: 282.1124 [M+H] $]^{+}$, calc. for: $\mathrm{C}_{17} \mathrm{H}_{15} \mathrm{NO}_{3} \mathrm{Na}$ : 304.0944, found: $304.0939[\mathrm{M}+\mathrm{Na}]^{+}$. 


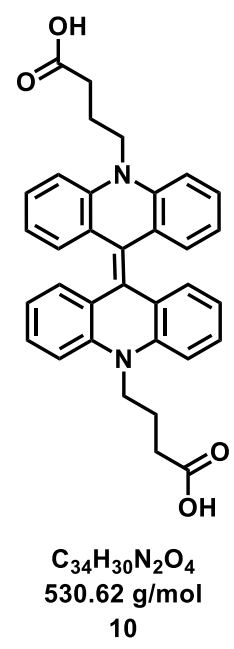

The synthesis was performed according to a protocol modified by MASSON et al. ${ }^{[273]}$ 4-(9oxoacridin-10(9H)-yl)butanoic acid (1.63 g, $5.79 \mathrm{mmol}, 1.00 \mathrm{eq})(9)$ and zinc powder (3.82 g, $58.0 \mathrm{mmol}, 10.0 \mathrm{eq})$ were dissolved in anhydrous ethanol $(20 \mathrm{ml})$ under argon atmosphere at $0{ }^{\circ} \mathrm{C}$. Subsequently, hydrogen chloride ethanolic solution $(2.50 \mathrm{M}, 47.0 \mathrm{~mL}, 115 \mathrm{mmol}$, $19.9 \mathrm{mmol}$ ) was added over $30 \mathrm{~min}$ and the solution was stirred at $\mathrm{rt}$ for $12 \mathrm{~h}$. After addition of water $(100 \mathrm{~mL})$, the mixture was filtered and recrystallized from hot ethanol. The product $\mathbf{1 0}$ (1.63 g, $3.07 \mathrm{mmol}, 53 \%)$ was obtained as a yellow solid.

${ }^{1} \mathbf{H}-\mathbf{N M R}\left(300 \mathrm{MHz}, \mathrm{DMSO}-d_{6}\right) ; \delta[\mathrm{ppm}]=1.96-2.12$ (m, 4H, 2x CH $\mathrm{CH}_{2}-\mathrm{CH}_{2}-\mathrm{CH}_{2}$ ), $2.40-2.48$ (m, 4H, $\left.2 \times \mathrm{CH}_{2}-\mathrm{COOH}\right), 4.04-4.21$ (m, 4H, $\left.2 \times \mathrm{CH}_{2}-\mathrm{CH}_{2}-\mathrm{CH}_{2}\right), 6.57-6.78$ (m, 8H, CH $H_{\text {Arom. }}$ ), $7.02-7.26\left(\mathrm{~m}, 4 \mathrm{H}, \mathrm{C} H_{\text {Arom. }}\right), 7.27-7.33\left(\mathrm{~d},{ }^{3} J_{\mathrm{H}, \mathrm{H}}=8.4 \mathrm{~Hz}, 4 \mathrm{H}, \mathrm{C} H_{\text {Arom. }}\right)$.

${ }^{13} \mathbf{C}-\mathrm{NMR}\left(126 \mathrm{MHz}, \mathrm{DMSO}-d_{6}\right) ; \delta[\mathrm{ppm}]=21.7\left(2 \mathrm{C}, 2 \times \mathrm{CH}_{2}-\mathrm{CH}_{2}-\mathrm{CH}_{2}\right), 31.1\left(2 \mathrm{C}, 2 \times \mathrm{CH}_{2}-\right.$ $\mathrm{COOH}), 44.7$ (2C, N-CH $\left.2-\mathrm{CH}_{2}\right), 114.3$ (4C, 4 x $\mathrm{CH}_{\mathrm{Ar}}$ ), 120.1 (4C, 4 x $\mathrm{CH}_{\mathrm{Ar}}$ ), 123.5 (2C, $C=C$ ), 124.4 (4C, 4 x $C \mathrm{H}_{\text {Ar. }}$ ), 127.7 (4C, 4 x $C \mathrm{H}_{\text {Ar. }}$ ), 127.8 (4, 4 x $\left.C \mathrm{H}_{\mathrm{Ar} .}\right), 143.8$ (4C, 4 x $\left.C \mathrm{H}_{\text {Ar. }}\right), 175.0$ $(2 \mathrm{C}, 2 \times \mathrm{COOH})$.

MS (ESI): $\mathbf{m} / \mathbf{z}(\%)=530.2(3)[\mathrm{M}+\mathrm{H}]^{+}, 265.1(100)[\mathrm{M}+2 \mathrm{H}]^{2+}$.

HRMS (ESI): calc. for: $\mathrm{C}_{34} \mathrm{H}_{31} \mathrm{~N}_{2} \mathrm{O}_{4}: 530.2200$, found.: $530.2188[\mathrm{M}+\mathrm{H}]^{+}$, calc. for: $\mathrm{C}_{34} \mathrm{H}_{32} \mathrm{~N}_{2} \mathrm{O}_{4}: 265.1095$, found.: $265.1099[\mathrm{M}+2 \mathrm{H}]^{2+}$. 
Synthesis 10,10'-bis(3-carboxypropyl)-[9,9'-biacridine]-10,10'-diium (4)

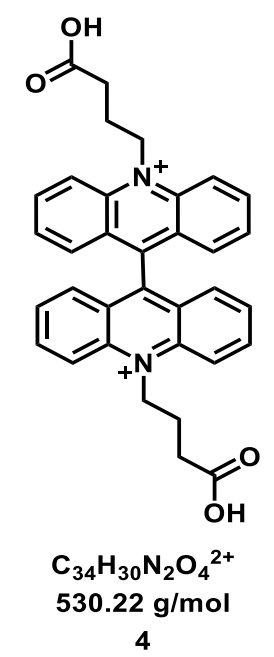

The synthesis was performed according to a protocol modified by MASSON et al. ${ }^{[273]} 4,4$ '(10H,10' H-[9,9'-biacridinylidene]-10,10'-diyl)dibutyric acid (1.63 g, $3.07 \mathrm{mmol}, 1.00 \mathrm{eq})$ (10) was suspended in nitric acid $(2.00 \mathrm{M}, 150 \mathrm{~mL})$ and was stirred at reflux for $2 \mathrm{~h}$ at $120{ }^{\circ} \mathrm{C}$. The reaction mixture was allowed to cool to rt, filtered and recrystallized from diluted nitric acid. The product $4(0.84 \mathrm{~g}, 1.58 \mathrm{mmol}, 52 \%)$ was obtained as a yellow solid.

${ }^{1} \mathbf{H}-\mathrm{NMR}\left(300 \mathrm{MHz}, \mathrm{CD}_{3} \mathrm{OD}\right) ; \delta[\mathrm{ppm}]=2.59-2.82\left(\mathrm{~m}, 4 \mathrm{H}, 2 \mathrm{x} \mathrm{CH}_{2}-\mathrm{CH}_{2}-\mathrm{CH}_{2}\right), 2.90-3.03$ (m, 4H, $\left.2 \times \mathrm{CH}_{2}-\mathrm{COOH}\right), 5.57-5.86\left(\mathrm{~m}, 4 \mathrm{H}, 2\right.$ x $\left.\mathrm{CH}_{2}-\mathrm{CH}_{2}-\mathrm{CH}_{2}\right), 7.68-7.84$ (m, 8H, $\mathrm{CH}_{\text {Arom. }}$ ), $8.36-8.66\left(\mathrm{~m}, 4 \mathrm{H}, \mathrm{C} H_{\text {Arom }}\right), 9.18\left(\mathrm{~d},{ }^{3} J_{\mathrm{H}, \mathrm{H}}=9.4 \mathrm{~Hz}, 4 \mathrm{H}, \mathrm{C} H_{\text {Arom. }}\right)$.

${ }^{13} \mathrm{C}-\mathrm{NMR}\left(126 \mathrm{MHz}, \mathrm{DMSO}-d_{6}\right) ; \delta[\mathrm{ppm}]=24.5\left(2 \mathrm{C}, 2 \times \mathrm{CH}_{2}-\mathrm{CH}_{2}-\mathrm{C}\right), 30.7\left(2 \mathrm{C}, 2 \times \mathrm{CH}_{2}-\right.$ $\mathrm{CH}_{2}-\mathrm{C}$ ), 50.9 (2C, $\left.2 \times \mathrm{N}-\mathrm{CH}_{2}-\mathrm{CH}_{2}\right), 119.7$ (4C, 4 x $\left.C_{\text {Arom. }}\right), 127.2$ (4C, 4 x $C_{\text {Arom. }}$ ), 129.3 (4C, 4 x $C_{\text {Arom. }}$ ), 129.7 (4C, 4 x $\left.C_{\text {Arom. }}\right), 139.7$ (4C, 4 x $\left.C_{\text {Arom. }}\right) 141.6$ (4C, 4 x $\left.C_{\text {Arom. }}\right), 152.0$ (2C, $\left.2 \times C_{\text {Arom.. }}\right), 174.8(2 \mathrm{C}, 2 \times C \mathrm{O})$

MS (ESI): $\mathbf{m} / \mathbf{z}(\%)=529.2(28)[\mathrm{M}+\mathrm{H}]^{+}$.

HRMS (ESI): calc. for: $\mathrm{C}_{34} \mathrm{H}_{29} \mathrm{~N}_{2} \mathrm{O}_{4}: 529.2122$, found.: $529.2112[\mathrm{M}+\mathrm{H}]^{+}$, calc. for: $\mathrm{C}_{34} \mathrm{H}_{28} \mathrm{~N}_{2} \mathrm{O}_{4} \mathrm{Na}$ : 551.1941, found.: $551.1923[\mathrm{M}+\mathrm{Na}]^{+}$. 


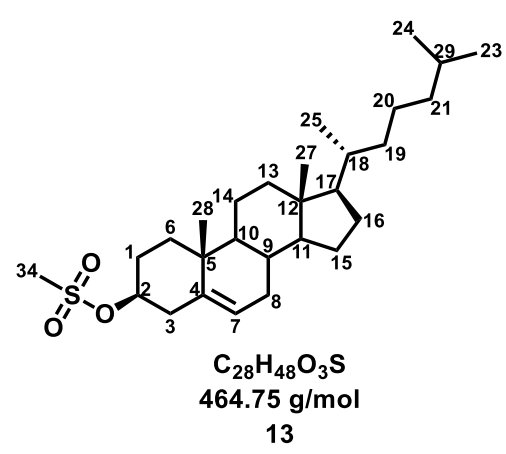

The synthesis was performed according to a protocol modified by SuN et al. ${ }^{[130]}$ Cholesterol $(8.00 \mathrm{~g}, 20.7 \mathrm{mmol}, 1.00 \mathrm{eq})$ (11) was dissolved in anhydrous DCM (110 mL) and triethylamine $(4.30 \mathrm{~mL}, 31.0 \mathrm{mmol}, 1.50 \mathrm{eq}, \delta=0.73 \mathrm{~g} / \mathrm{mol})$ under argon atmosphere at $0{ }^{\circ} \mathrm{C}$. Subsequently, methanesulfonyl chloride $(2.48 \mathrm{~mL}, 32.1 \mathrm{mmol}, 1.60 \mathrm{eq}, \delta=1.48 \mathrm{~g} / \mathrm{mol})$ was added dropwise and the reaction mixture was stirred for $12 \mathrm{~h}$ at rt. Afterwards, the solvent was removed under reduced pressure. The residue was dissolved in DCM $(10 \mathrm{~mL})$ and recrystallized from methanol. The product 13 (10.2 g, $21.7 \mathrm{mmol}$, quant.) was obtained as a colourless solid. ${ }^{1} \mathbf{H}-\mathbf{N M R}\left(300 \mathrm{MHz}, \mathrm{CDCl}_{3}\right) ; \delta[\mathrm{ppm}]=0.69\left(\mathrm{~s}, 3 \mathrm{H}, 27-\mathrm{CH}_{3}\right), 0.88\left(\mathrm{dd},{ }^{3} J_{\mathrm{H}, \mathrm{H}}=6.6 \mathrm{~Hz},{ }^{4} J_{\mathrm{H}, \mathrm{H}}=\right.$ $\left.1.3 \mathrm{~Hz}, 6 \mathrm{H}, 23-\mathrm{CH}_{3}, 24-\mathrm{CH}_{3}\right), 0.93\left(\mathrm{~d},{ }^{3} \mathrm{~J}_{\mathrm{H}, \mathrm{H}}=6.5 \mathrm{~Hz}, 3 \mathrm{H}, 25-\mathrm{CH}_{3}\right), 0.98-2.64(\mathrm{~m}, 31 \mathrm{H}, 1-$ $\mathrm{CH}_{2}, 3-\mathrm{CH}_{2}, 6-\mathrm{CH}_{2}, 8-\mathrm{CH}_{2}, 9-\mathrm{CH}, 10 \mathrm{CH}, 11-\mathrm{CH}, 13-\mathrm{CH}_{2}, 14-\mathrm{CH}_{2}, 15-\mathrm{CH}_{2}, 16-\mathrm{CH}_{2}, 17-\mathrm{CH}$, 18- $\left.\mathrm{CH}, 19-\mathrm{CH}_{2}, 20-\mathrm{CH}_{2}, 21-\mathrm{CH}_{2}, 22-\mathrm{CH}, 28-\mathrm{CH}_{3}\right), 3.02$ (s, 3H, 34- $\left.\mathrm{CH}_{3}\right), 4.43-4.65(\mathrm{~m}, 1 \mathrm{H}$, 2-CH), $5.40-5.48(\mathrm{~m}, 1 \mathrm{H}, 7-\mathrm{CH})$.

MS (ESI): $\mathbf{m} / \mathbf{z}(\%)=487.3(6)[\mathrm{M}+\mathrm{Na}]^{+}, 951.7(3)[2 \mathrm{M}+\mathrm{Na}]^{+}$.

HRMS (ESI): calc. for: $\mathrm{C}_{28} \mathrm{H}_{48} \mathrm{O}_{3} \mathrm{Na}: 487.3216$, found.: $487.3230[\mathrm{M}+\mathrm{Na}]^{+}$, calc. for: $\mathrm{C}_{28} \mathrm{H}_{48} \mathrm{O}_{3} \mathrm{NH}_{4}: 482.3662$, found.: $482.3668\left[\mathrm{M}+\mathrm{NH}_{4}\right]^{+}$. 
Synthesis of 3ß-Azido-5-cholestene (14)

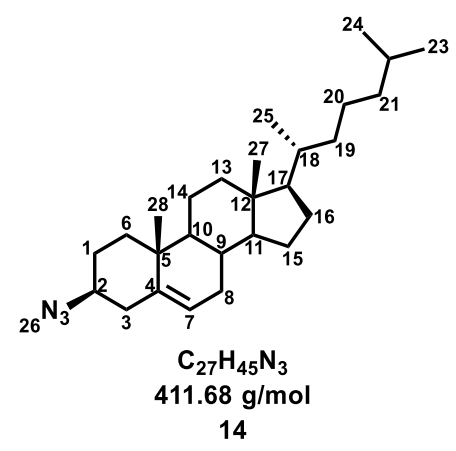

The synthesis was performed according to a protocol modified by SUN et al. ${ }^{[130]}$ Cholest-5-en$3 \beta$-yl mesylate $(4.00 \mathrm{~g}, 8.61 \mathrm{mmol}, 1.00 \mathrm{eq})(\mathbf{1 3})$ was dissolved in anhydrous DCM (40 mL) under argon atmosphere at $0{ }^{\circ} \mathrm{C}$. Trimethylsilyl azide $(1.25 \mathrm{~mL}, 9.47 \mathrm{mmol}, 1.10 \mathrm{eq}$, $\delta=0.87 \mathrm{~g} / \mathrm{mL})$ as well as boron trifluoride etherate $(2.18 \mathrm{~mL}, 17.2 \mathrm{mmol}, 2.00 \mathrm{eq}$, $\delta=1.12 \mathrm{~g} / \mathrm{mL}$ ) were added dropwise and the solution was stirred for $24 \mathrm{~h}$ at $\mathrm{rt}$. The reaction mixture was transferred into aqueous $\mathrm{NaOH}(2.00 \mathrm{M}, 50 \mathrm{~mL})$ and the aqueous phase was extracted with DCM $(100 \mathrm{~mL})$. The organic layer was washed with brine $(100 \mathrm{~mL})$ and dried over $\mathrm{Na}_{2} \mathrm{SO}_{4}$. The solvent was removed under reduced pressure and the resulting colourless oil was purified by flash column chromatography (DCM/MeOH 98:2,v/v). The product $14(1.04 \mathrm{~g}$, $2.53 \mathrm{mmol}, 30 \%$ ) was obtained as a colourless oil.

${ }^{1} \mathbf{H}-\mathbf{N M R}\left(300 \mathrm{MHz}, \mathrm{CDCl}_{3}\right) ; \delta[\mathrm{ppm}]=0.70\left(\mathrm{~s}, 3 \mathrm{H}, 27-\mathrm{CH}_{3}\right), 0.88\left(\mathrm{dd},{ }^{3} J_{\mathrm{H}, \mathrm{H}}=6.6 \mathrm{~Hz}\right.$, $\left.{ }^{4} J_{\mathrm{H}, \mathrm{H}}=1.4 \mathrm{~Hz}, 6 \mathrm{H}, 23-\mathrm{CH}_{3}, 24-\mathrm{CH}_{3}\right), 0.93\left(\mathrm{~d},{ }^{3} J_{\mathrm{H}, \mathrm{H}}=6.5 \mathrm{~Hz}, 3 \mathrm{H}, 25-\mathrm{CH}_{3}\right), 0.98-2.64(\mathrm{~m}$, $31 \mathrm{H}, 1-\mathrm{CH}_{2}, 3-\mathrm{CH}_{2}, 6-\mathrm{CH}_{2}, 8-\mathrm{CH}_{2}, 9-\mathrm{CH}, 10 \mathrm{CH}, 11-\mathrm{CH}, 13-\mathrm{CH}_{2}, 14-\mathrm{CH}_{2}, 15-\mathrm{CH}_{2}, 16-\mathrm{CH}_{2}$, 17- $\mathrm{CH}, 18-\mathrm{CH}, 19-\mathrm{CH}_{2}, 20-\mathrm{CH}_{2}, 21-\mathrm{CH}_{2}, 22-\mathrm{CH}, 28-\mathrm{CH}_{3}$ ), $3.15-3.30$ (s, 1H, 2-CH), $5.33-$ $5.49(\mathrm{~m}, 1 \mathrm{H}, 7-\mathrm{CH})$.

MS (EI): $\mathbf{m} / \mathbf{z}=411.4[\mathrm{M}]^{+}$. 


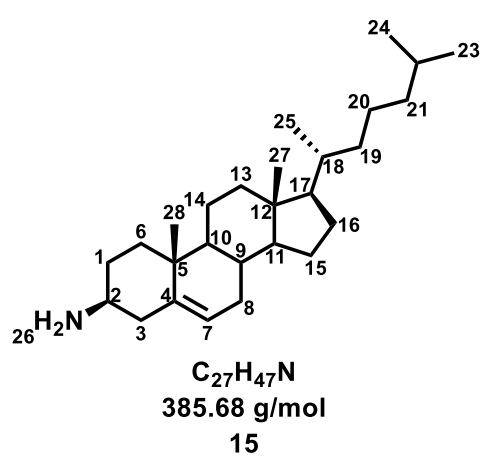

The synthesis was performed according to a protocol modified by Sun et al ${ }^{[130]} 3 \beta$-Azido-5cholestene (1.04 g, $2.53 \mathrm{mmol}, 1.00 \mathrm{eq})$ (14) was dissolved in anhydrous $\mathrm{Et}_{2} \mathrm{O}$ (12 ml) under argon atmosphere at $4{ }^{\circ} \mathrm{C}$ and $\mathrm{LiAlH}_{4}(0.11 \mathrm{~g}, 3.10 \mathrm{mmol}, 1.22 \mathrm{eq})$ was added slowly. The solution was stirred at $4{ }^{\circ} \mathrm{C}$ for $30 \mathrm{~min}$ and at $\mathrm{rt}$ for $2 \mathrm{~h}$. After quenching the excess of hydride with water, the organic layer was separated, and the aqueous phase was extracted with EtOAc $(3 \times 50 \mathrm{~mL})$. The organic layer was washed with brine $(100 \mathrm{~mL})$ and dried over $\mathrm{Na}_{2} \mathrm{SO}_{4}$. The solvent was removed under reduced pressure and the resulting colourless oil was purified by flash column chromatography ( $n$-pentane/EtOAc 2:1, v/v). The product $15(0.58 \mathrm{~g}, 1.52 \mathrm{mmol}$, $60 \%$ ) was obtained as a colourless solid.

${ }^{\mathbf{1}} \mathbf{H}-\mathbf{N M R}\left(300 \mathrm{MHz}, \mathrm{CDCl}_{3}\right) ; \delta[\mathrm{ppm}]=0.69\left(\mathrm{~s}, 3 \mathrm{H}, 27-\mathrm{CH}_{3}\right), 0.85-0.91\left(\mathrm{~m}, 6 \mathrm{H}, 23-\mathrm{CH}_{3}, 24-\right.$ $\left.\mathrm{CH}_{3}\right), 0.93\left(\mathrm{~d},{ }^{3} \mathrm{~J}_{\mathrm{H}, \mathrm{H}}=6.4 \mathrm{~Hz}, 3 \mathrm{H}, 25-\mathrm{CH}_{3}\right), 0.98-2.64\left(\mathrm{~m}, 31 \mathrm{H}, 1-\mathrm{CH}_{2}, 3-\mathrm{CH}_{2}, 6-\mathrm{CH}_{2}, 8-\mathrm{CH}_{2}\right.$, 9- $\mathrm{CH}, 10 \mathrm{CH}, 11-\mathrm{CH}, 13-\mathrm{CH}_{2}, 14-\mathrm{CH}_{2}, 15-\mathrm{CH}_{2}, 16-\mathrm{CH}_{2}, 17-\mathrm{CH}, 18-\mathrm{CH}, 19-\mathrm{CH}_{2}, 20-\mathrm{CH}_{2}, 21-$ $\left.\mathrm{CH}_{2}, 22-\mathrm{CH}, 28-\mathrm{CH}_{3}\right), 3.10-3.30$ (s, $\left.1 \mathrm{H}, 2-\mathrm{CH}\right), 5.30-5.49$ (m, 1H, 7-CH).

${ }^{13}$ C-NMR (126 MHz, DMSO- $\left.d_{6}\right) ; \delta[\mathrm{ppm}]=11.9(C), 18.7(C), 19.3(C), 21.2(C), 22.6(C)$, $22.8(C), 23.8(C), 24.3(C), 28.0(2 \mathrm{C}), 28.2(C), 31.8(2 \mathrm{C}), 35.8(C), 36.2(C) .37 .6(C), 38.2$ (C), $39.5(C), 39.7(C), 42.3(C), 50.1(C), 56.1(C), 56.7(C), 61.2(C), 122.5(C), 140.1(C)$ MS (ESI): $\mathbf{m} / \mathbf{z}(\%)=386.4(72)[\mathrm{M}+\mathrm{H}]^{+}$.

HRMS (ESI): calc. for: $\mathrm{C}_{27} \mathrm{H}_{47} \mathrm{~N}: 386.3781$, found.: $368.3773[\mathrm{M}+\mathrm{H}]^{+}$. 


\section{Synthesis of Fmoc-Asp(palmitoyl)-OH (23)}

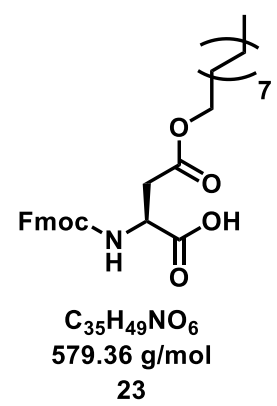

The synthesis was performed according to a protocol modified by LINNING et al. ${ }^{[130]}$. FmocAsp-OtBu (2.00 g, $4.86 \mathrm{mmol}, 1.00 \mathrm{eq})(\mathbf{2 0})$ was dissolved in anhydrous DCM (100 mL) under argon atmosphere at $\mathrm{rt}$ and MSNT (1.43 g, $5.35 \mathrm{mmol}, 1.10 \mathrm{eq})$ as well as 1-methylimidazole $(0.39 \mathrm{~mL}, 4.86 \mathrm{mmol}, 1.00 \mathrm{eq}, \rho=1.03 \mathrm{~g} / \mathrm{mL})$ were added. After stirring for $10 \mathrm{~min}$, hexadecanol (1.30 g, $5.35 \mathrm{mmol}, 1.10 \mathrm{eq})$ (21) was added and the solution was stirred overnight at rt. Following, $2 \%$ aqueous citric acid was added to the reaction mixture. The organic layer was washed with brine $(100 \mathrm{~mL})$ and dried over $\mathrm{Na}_{2} \mathrm{SO}_{4}$. The solvent was removed under reduced pressure and the resulting colourless oil was purified by flash column chromatography (Pen/EtOAc 2:1). The obtained Fmoc-Asp(palmitoyl)-O ${ }^{t} \mathrm{Bu}(2.99 \mathrm{~g}, 4.71 \mathrm{mmol}, 97$ \%) (22) was dissolved in TFA/DCM $(20 / 30, v / v)$ and stirred for $3 \mathrm{~h}$ at rt. The reaction mixture was diluted with DCM and washed with $\mathrm{H}_{2} \mathrm{O}(3 \times 50 \mathrm{~mL})$ as well as brine $(1 \times 50 \mathrm{~mL})$. After drying over $\mathrm{Na}_{2} \mathrm{SO}_{4}$ and removal of the solvent under reduced pressure, the residue was purified by flash column chromatography ( $n$-pentane/EtOAc/AcOH 1:1:0.01, $v / v / v$ ). The product 23 (2.06 g, $3.55 \mathrm{mmol}, 73 \%$ ) was obtained as a colourless solid.

${ }^{\mathbf{1}} \mathbf{H}-\mathbf{N M R}\left(300 \mathrm{MHz}, \mathrm{DMSO}-d_{6}\right) ; \delta[\mathrm{ppm}]=0.78-0.90\left(\mathrm{~m}, 3 \mathrm{H}, \mathrm{CH}_{3}\right), 1.15-1.30(\mathrm{~m}, 26 \mathrm{H}, 13$ x $\left.\mathrm{CH}_{2}\right), 1.49-1.53\left(\mathrm{~m}, 2 \mathrm{H}, \mathrm{O}-\mathrm{CH}_{2}-\mathrm{CH}_{2}\right), 2.58-2.90\left(\mathrm{~m}, 2 \mathrm{H}, \beta-\mathrm{CH}_{2}\right), 3.96-4.07(\mathrm{~m}, 2 \mathrm{H}$, $\left.\mathrm{CH}_{2}\right), 4.18-4.31\left(\mathrm{~m}, 3 \mathrm{H}\right.$, Fmoc-CH, Fmoc-CH $\mathrm{CH}_{2}, 4.35-4.41(\mathrm{~m}, 1 \mathrm{H}, \alpha-\mathrm{CH}), 7.32\left(\mathrm{t},{ }^{3} J_{\mathrm{H}, \mathrm{H}}=\right.$ $\left.7.4 \mathrm{~Hz}, 1 \mathrm{H}, \mathrm{Fmoc}-\mathrm{CH}_{\mathrm{Ar}}\right), 7.41\left(\mathrm{t},{ }^{3} J_{\mathrm{H}, \mathrm{H}}=7.4 \mathrm{~Hz}, 2 \mathrm{H}, \mathrm{Fmoc}-\mathrm{CH}_{\mathrm{Ar}}\right), 7.66-7.76(\mathrm{~m}, 3 \mathrm{H}, \mathrm{NH}$, Fmoc-CH $\left.H_{\mathrm{Ar}}\right), 7.89\left(\mathrm{~d},{ }^{3} J_{\mathrm{H}, \mathrm{H}}=7.5 \mathrm{~Hz}, 2 \mathrm{H}\right.$, Fmoc-CH $\left.H_{\mathrm{Ar}}\right)$

${ }^{13}$ C-NMR $\left(126 \mathrm{MHz}, \mathrm{CDCl}_{3}\right) ; \delta[\mathrm{ppm}]=14.2\left(\mathrm{CH}_{3}\right), 22.7\left(\mathrm{CH}_{2}-\mathrm{CH}_{3}\right), 25.9\left(\mathrm{CH}_{2}\right), 28.5\left(\mathrm{CH}_{2}\right)$, $29.3\left(\mathrm{CH}_{2}\right), 29.4\left(\mathrm{CH}_{2}\right), 29.5\left(\mathrm{CH}_{2}\right), 29.6\left(\mathrm{CH}_{2}\right), 29.7\left(2 \mathrm{C}, 2 \mathrm{x} \mathrm{CH}_{2}\right), 29.8\left(4 \mathrm{C}, 4 \times \mathrm{CH}_{2}\right), 32.0$ $\left(\mathrm{CH}_{2}\right), 36.4\left(\mathrm{CH}_{2}\right), 47.1$ (Fmoc- $\left.\mathrm{CH}\right), 50.2(\alpha-\mathrm{CH}), 65.5\left(\mathrm{O}-\mathrm{CH}_{2}\right), 67.5\left(\mathrm{Fmoc}-\mathrm{CH}_{2}\right), 120.0(2 \mathrm{C}$, $2 \times$ Fmoc- $\left.C_{\text {Ar. }}\right), 125.1$ ( $2 \mathrm{C}, 2$ x Fmoc- $\left.C_{\text {Ar. }}\right), 127.1$ (2C, 2 x Fmoc- $\left.C_{\text {Ar. }}\right), 127.8$ (2C, 2 x Fmoc- 
$\left.C_{\text {Ar. }}\right), 141.3\left(2 \mathrm{C}\right.$, Fmoc- $\left.C_{\text {Ar. }}\right), 143.6$ (Fmoc- $\left.C_{\text {Ar. }}\right), 143.8\left(\right.$ Fmoc- $\left.C_{\text {Ar. }}\right) 156.2(\mathrm{NH}-C=\mathrm{O}), 171.3$ $\left(\mathrm{CH}_{2}-\mathrm{CO}\right), 175.7\left(\mathrm{CO}_{2} \mathrm{H}\right)$.

MS (ESI): $\mathbf{m} / \mathbf{z}(\%)=580.4(32)[\mathrm{M}+\mathrm{H}]^{+}, 602.4(100)[\mathrm{M}+\mathrm{Na}]^{+}$.

HRMS (ESI): calc. for: $\mathrm{C}_{35} \mathrm{H}_{50} \mathrm{NO}_{6}: 580.3633$, found.: 580.3635 [M+H] ${ }^{+}$, calc. for: $\mathrm{C}_{35} \mathrm{H}_{49} \mathrm{NO}_{6} \mathrm{Na}: 602.3452$, found.: $602.3453[\mathrm{M}+\mathrm{Na}]^{+}$. 
Synthesis of a BAC-labelled PNA Oligomer (11)

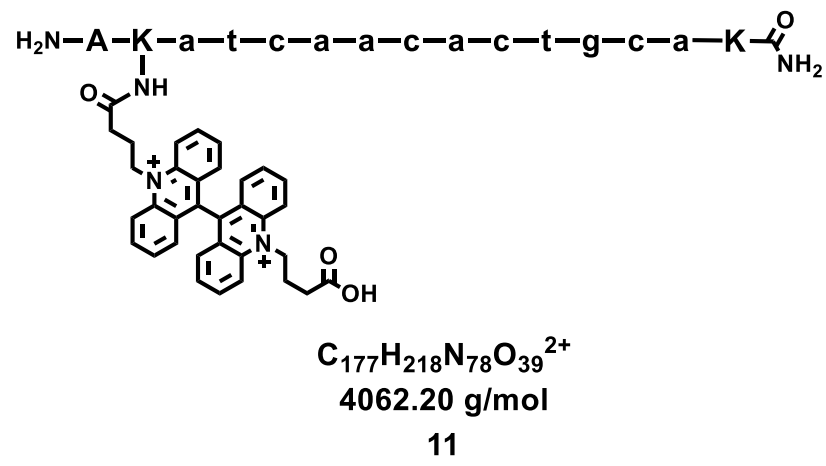

The synthesis of the peptide-PNA oligomer was performed on a Rink Amide MBHA resin $(10.0 \mu \mathrm{mol}, 0.17 \mathrm{mmol} / \mathrm{g})$ by automated SPPS according to SOP1 and SOP2. After deprotection of the Alloc group following SOP3 the lysine side chain was labelled with the BAC-fluorophore (4) as described in SOP4. The cleavage of the peptide-PNA oligomer from the resin was carried out as outlined in SOP7. The crude product was purified by semipreparative HPLC, yielding the peptide-PNA sensor $11(0.98 \mathrm{mg}, 0.24 \mu \mathrm{mol})$ as a yellow solid.

HPLC: $(250 \cdot 10 \mathrm{~mm}, 5 \mu \mathrm{m}, \mathrm{C} 18$, Gradient $=1-30 \% \mathrm{~B}$ in $30 \mathrm{~min}, \lambda[\mathrm{nm}]: 215,260,368)$ : $t_{\mathrm{R}}=22.2 \mathrm{~min}$.

MS (ESI): m/z $(\%)=1354.2(4)[\mathrm{M}+3 \mathrm{H}]^{3+}, 1015.9(33)[\mathrm{M}+4 \mathrm{H}]^{4+}, 813.0(88)[\mathrm{M}+5 \mathrm{H}]^{5+}$, $677.6(100)[\mathrm{M}+6 \mathrm{H}]^{6+}, 581.0(58)[\mathrm{M}+7 \mathrm{H}]^{7+}$.

HRMS (ESI): calc. for: $\mathrm{C}_{177} \mathrm{H}_{219} \mathrm{~N}_{78} \mathrm{O}_{39}: 1354.2533$, found.: $1354.2539[\mathrm{M}+3 \mathrm{H}]^{3+}$, calc. for: $\mathrm{C}_{177} \mathrm{H}_{220} \mathrm{~N}_{78} \mathrm{O}_{39}: 1015.9418$, found.: $1015.9432[\mathrm{M}+4 \mathrm{H}]^{4+}$, calc. for: $\mathrm{C}_{177} \mathrm{H}_{221} \mathrm{~N}_{78} \mathrm{O}_{39}: 812.9549$, found.: $812.9562[\mathrm{M}+5 \mathrm{H}]^{5+}$, calc. for: $\mathrm{C}_{177} \mathrm{H}_{222} \mathrm{~N}_{78} \mathrm{O}_{39}$ : 677.6312, found.: $677.6303[\mathrm{M}+6 \mathrm{H}]^{6+}$, calc. for: $\mathrm{C}_{177} \mathrm{H}_{223} \mathrm{~N}_{78} \mathrm{O}_{39}$ : 580.9698, found.: $580.9699[\mathrm{M}+7 \mathrm{H}]^{7+}$. 


\section{Synthesis of a Palmitoyl Modified Peptide-PNA Sensor (25)}

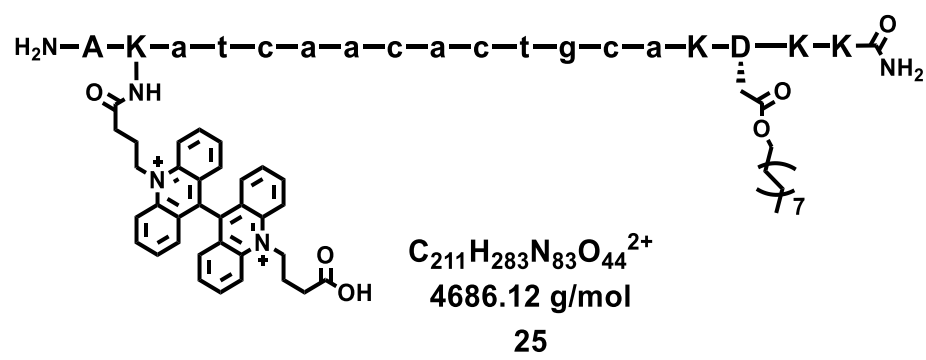

The synthesis of the peptide-PNA oligomer was performed on a Rink Amide MBHA resin $(10.0 \mu \mathrm{mol}, 0.17 \mathrm{mmol} / \mathrm{g})$ by automated SPPS according to SOP1 and SOP2. After deprotection of the Alloc group following SOP3 the lysine side chain was labelled with the BAC-fluorophore (4) as described in SOP4. The cleavage of the peptide-PNA oligomer from the resin was carried out as outlined in SOP7. The crude product was purified by semipreparative HPLC, yielding the peptide-PNA sensor $25(0.30 \mathrm{mg}, 0.85 \mu \mathrm{mol})$ as a yellow solid. HPLC: $(250 \cdot 10 \mathrm{~mm}, 5 \mu \mathrm{m}, \mathrm{C} 18$, Gradient $=10-60 \%$ B in $30 \mathrm{~min}, \lambda[\mathrm{nm}]: 215,260,368)$ : $t_{\mathrm{R}}=23.5 \mathrm{~min}$.

MS (ESI): $\mathbf{~ m / z ~}(\%)=1171.8(10)[\mathrm{M}+4 \mathrm{H}]^{4+}, 937.7(38)[\mathrm{M}+5 \mathrm{H}]^{5+}, 781.5(75)[\mathrm{M}+6 \mathrm{H}]^{6+}$.

HRMS (ESI): calc. for: $\mathrm{C}_{211} \mathrm{H}_{285} \mathrm{~N}_{83} \mathrm{O}_{44}: 1171.8162$, found.: $1171.8198[\mathrm{M}+4 \mathrm{H}]^{4+}$, calc. for: $\mathrm{C}_{211} \mathrm{H}_{286} \mathrm{~N}_{83} \mathrm{O}_{44}$ : 937.6544 , found.: $937.6566[\mathrm{M}+5 \mathrm{H}]^{5+}$, calc. for: $\mathrm{C}_{211} \mathrm{H}_{287} \mathrm{~N}_{83} \mathrm{O}_{44}: 781.5465$, found.: $781.5486[\mathrm{M}+6 \mathrm{H}]^{6+}$, calc. for: $\mathrm{C}_{211} \mathrm{H}_{288} \mathrm{~N}_{83} \mathrm{O}_{44}: 670.0409$, found.: $670.0420[\mathrm{M}+7 \mathrm{H}]^{7+}$, calc. for: $\mathrm{C}_{211} \mathrm{H}_{289} \mathrm{~N}_{83} \mathrm{O}_{44}: 586.4117$, found.: $586.4128[\mathrm{M}+8 \mathrm{H}]^{8+}$. 
Synthesis of a Palmitoyl Modified Peptide-PNA Sensor with a TEV Recognition Sequence (27)

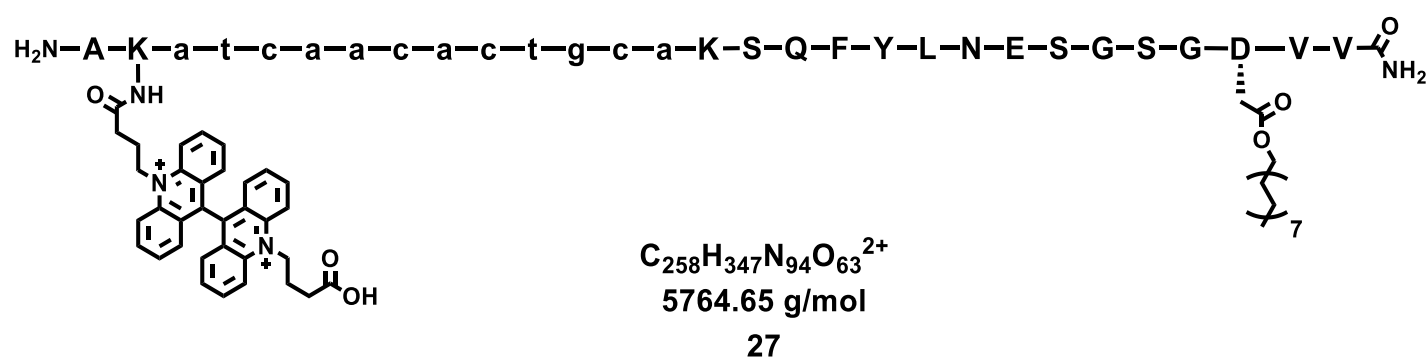

The synthesis of the peptide-PNA oligomer was performed on a Rink Amide MBHA resin $(10.0 \mu \mathrm{mol}, \quad 0.17 \mathrm{mmol} / \mathrm{g})$ by automated SPPS according to SOP1 and SOP2. After deprotection of the Alloc group following SOP3 the lysine side chain was labelled with the BAC-fluorophore (4) as described in SOP4. The cleavage of the peptide-PNA oligomer from the resin was carried out as outlined in SOP7. The crude product was purified by semipreparative HPLC, yielding the peptide-PNA sensor $27(0.25 \mathrm{mg}, 0.04 \mu \mathrm{mol})$ as a yellow solid. HPLC: $(250 \cdot 10 \mathrm{~mm}, 5 \mu \mathrm{m}, \mathrm{C} 18$, Gradient $=10-60 \%$ B in $30 \mathrm{~min}, \lambda[\mathrm{nm}]: 215,260,368)$ : $t_{\mathrm{R}}=17.8 \mathrm{~min}$.

MS (ESI): $\mathbf{m} / \mathbf{z}(\%)=962.0(32)[\mathrm{M}+5 \mathrm{H}]^{5+}$.

HRMS (ESI): calc. for: $\mathrm{C}_{258} \mathrm{H}_{347} \mathrm{~N}_{94} \mathrm{O}_{63}: 1154.5382$, found.: $1154.5382[\mathrm{M}+4 \mathrm{H}]^{4+}$, calc. for: $\mathrm{C}_{258} \mathrm{H}_{348} \mathrm{~N}_{94} \mathrm{O}_{63}: 962.2830$, found.: $962.2832[\mathrm{M}+5 \mathrm{H}]^{5+}$. 
Synthesis of a Palmitoyl Modified Test Peptide with a TEV Recognition Sequence (28)

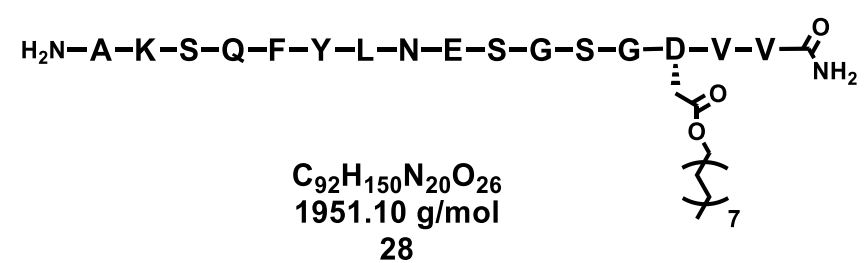

The synthesis of the peptide was performed on a Rink Amide MBHA resin $(25.0 \mu \mathrm{mol}$, $0.17 \mathrm{mmol} / \mathrm{g}$ ) by automated SPPS according to SOP1. The cleavage of the peptide sequence from the resin was carried out as outlined in SOP7. The crude product was purified by semipreparative HPLC, yielding the peptide $\mathbf{2 8}(2.42 \mathrm{mg}, 1.24 \mu \mathrm{mol})$ as a colourless solid.

HPLC: $(250 \cdot 10 \mathrm{~mm}, 5 \mu \mathrm{m}, \mathrm{C} 18$, Gradient $=10-60 \%$ B in $30 \mathrm{~min}, \lambda[\mathrm{nm}]: 215,254,280)$ : $t_{\mathrm{R}}=17.4 \mathrm{~min}$.

MS (ESI): $\mathbf{m} / \mathbf{z}(\%)=977.0(52)[\mathrm{M}+2 \mathrm{H}]^{2+}$.

HRMS (ESI): calc. for: $\mathrm{C}_{92} \mathrm{H}_{152} \mathrm{~N}_{20} \mathrm{O}_{26}: 976.5588$, found.: $976.5576[\mathrm{M}+2 \mathrm{H}]^{2+}$. 
Synthesis of a Palmitoyl Modified Test Peptide with a TEV Recognition Sequence and Increased Linker Sequence (29)

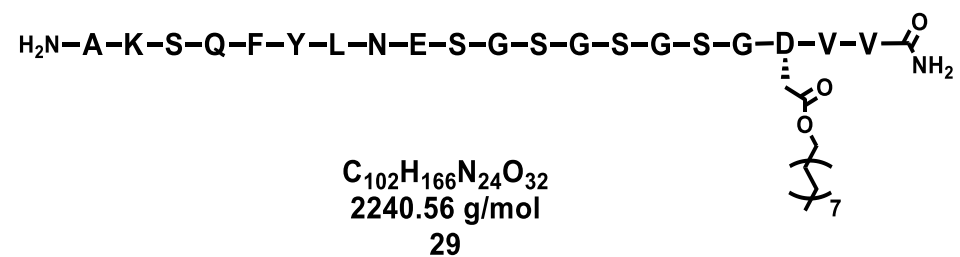

The synthesis of the peptide was performed on a Rink Amide MBHA resin $(25.0 \mu \mathrm{mol}$, $0.17 \mathrm{mmol} / \mathrm{g}$ ) by automated SPPS according to SOP1. The cleavage of the peptide sequence from the resin was carried out as outlined in SOP7. The crude product was purified by semipreparative HPLC, yielding the peptide $29(1.86 \mathrm{mg}, 0.83 \mu \mathrm{mol})$ as a colourless solid.

HPLC: $(250 \cdot 10 \mathrm{~mm}, 5 \mu \mathrm{m}, \mathrm{C} 18$, Gradient $=10-60 \%$ B in $30 \mathrm{~min}, \lambda[\mathrm{nm}]: 215,254,280)$ : $t_{\mathrm{R}}=15.9 \mathrm{~min}$.

MS (ESI): $\mathbf{m} / \mathbf{z}(\%)=1121.1(100)[\mathrm{M}+2 \mathrm{H}]^{2+}, 747.7(21)[\mathrm{M}+3 \mathrm{H}]^{3+}$.

HRMS (ESI): calc. for: $\mathrm{C}_{102} \mathrm{H}_{168} \mathrm{~N}_{24} \mathrm{O}_{32}: 1121.1138$, found.: $1121.1144[\mathrm{M}+2 \mathrm{H}]^{2+}$, calc. for: $\mathrm{C}_{102} \mathrm{H}_{169} \mathrm{~N}_{24} \mathrm{O}_{32}: 747.7449$, found.: $747.7439[\mathrm{M}+3 \mathrm{H}]^{3+}$. 
Synthesis of a Test Peptide with a TEV Recognition Sequence and Increased Linker Sequence (30)

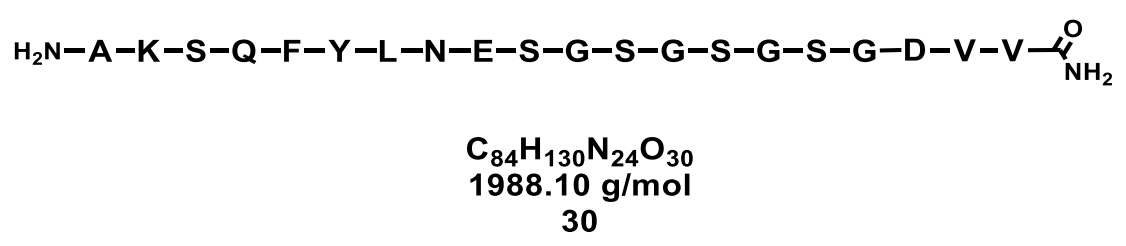

The synthesis of the peptide was performed on a Rink Amide MBHA r

esin $(25.0 \mu \mathrm{mol}, 0.17 \mathrm{mmol} / \mathrm{g})$ by automated SPPS according to SOP1. The cleavage of the peptide sequence from the resin was carried out as outlined in SOP7. The crude product was purified by semi-preparative HPLC, yielding the peptide $30(3.76 \mathrm{mg}, 1.89 \mu \mathrm{mol})$ as a yellow solid.

HPLC: $(250 \cdot 10 \mathrm{~mm}, 5 \mu \mathrm{m}, \mathrm{C} 18$, Gradient $=5-60 \% \mathrm{~B}$ in $30 \mathrm{~min}, \lambda[\mathrm{nm}]: 215,254,280)$ : $t_{\mathrm{R}}=16.9 \mathrm{~min}$.

MS (ESI): $\mathbf{m} / \mathbf{z}(\%)=995.0(100)[\mathrm{M}+2 \mathrm{H}]^{2+}, 663.3(78)[\mathrm{M}+3 \mathrm{H}]^{3+}$.

HRMS (ESI): calc. for: $\mathrm{C}_{84} \mathrm{H}_{132} \mathrm{~N}_{24} \mathrm{O}_{32}$ : 994.9730 , found.: $994.9729[\mathrm{M}+2 \mathrm{H}]^{2+}$, calc. for: $\mathrm{C}_{84} \mathrm{H}_{133} \mathrm{~N}_{24} \mathrm{O}_{32}: 663.6511$, found.: $663.6506[\mathrm{M}+3 \mathrm{H}]^{3+}$. 
Synthesis of a Test Peptide with a TEV Recognition Sequence (31)

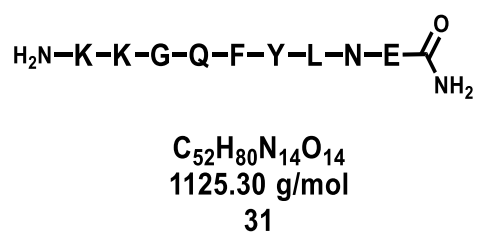

The synthesis of the peptide was performed on a Rink Amide MBHA resin $(25.0 \mu \mathrm{mol}$, $0.17 \mathrm{mmol} / \mathrm{g}$ ) by automated SPPS according to SOP1. The cleavage of the peptide sequence from the resin was carried out as outlined in SOP7. The crude product was purified by semipreparative HPLC, yielding the peptide $31(4.20 \mathrm{mg}, 3.73 \mu \mathrm{mol})$ as a colourless solid.

HPLC: $(250 \cdot 10 \mathrm{~mm}, 5 \mu \mathrm{m}, \mathrm{C} 18$, Gradient $=10-60 \%$ B in $30 \mathrm{~min}, \lambda[\mathrm{nm}]: 215,254,280)$ : $t_{\mathrm{R}}=12.6 \mathrm{~min}$.

MS (ESI): $\mathbf{m} / \mathbf{z}(\%)=1125.6(12)[\mathrm{M}+\mathrm{H}]^{+}, 563.3(100)[\mathrm{M}+2 \mathrm{H}]^{2+}$.

HRMS (ESI): calc. for: $\mathrm{C}_{52} \mathrm{H}_{81} \mathrm{~N}_{14} \mathrm{O}_{14}: 1125.6051$, found.: $1125.6027[\mathrm{M}+\mathrm{H}]^{+}$, calc. for: $\mathrm{C}_{52} \mathrm{H}_{82} \mathrm{~N}_{14} \mathrm{O}_{14}: 563.3062$, found.: $563.3063[\mathrm{M}+2 \mathrm{H}]^{2+}$, calc. for: $\mathrm{C}_{52} \mathrm{H}_{81} \mathrm{~N}_{14} \mathrm{O}_{14} \mathrm{Na}: 1147.5871$, found.: $1147.5852[\mathrm{M}+\mathrm{Na}]^{+}$. 
Synthesis of a Test Peptide with a Factor Xa Recognition Sequence (32)

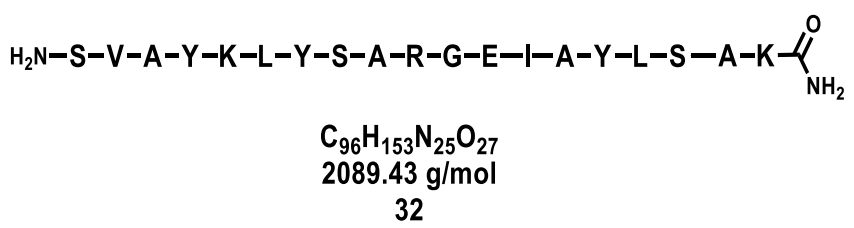

The synthesis of the peptide was performed on a Rink Amide MBHA resin $(25.0 \mu \mathrm{mol}$, $0.17 \mathrm{mmol} / \mathrm{g}$ ) by automated SPPS according to SOP1. The cleavage of the peptide sequence from the resin was carried out as outlined in SOP7. The crude product was purified by semipreparative HPLC, yielding the peptide $32(2.93 \mathrm{mg}, 1.40 \mu \mathrm{mol})$ as a colourless solid.

HPLC: $\left(250 \cdot 10 \mathrm{~mm}, 5 \mu \mathrm{m}, \mathrm{C}_{18}\right.$, Gradient $=10-60 \% \mathrm{~B}$ in $\left.30 \mathrm{~min}, \lambda[\mathrm{nm}]: 215,254,280\right)$ : $t_{\mathrm{R}}=17.8 \mathrm{~min}$.

MS (ESI): $\mathbf{m} / \mathbf{z}(\%)=1045.6(7)[\mathrm{M}+2 \mathrm{H}]^{2+}, 697.4(100)[\mathrm{M}+3 \mathrm{H}]^{3+}, 523.3(6)[\mathrm{M}+3 \mathrm{H}]^{3+}$.

HRMS (ESI): calc. for: $\mathrm{C}_{96} \mathrm{H}_{155} \mathrm{~N}_{25} \mathrm{O}_{27}: 1045.5771$, found.: $1045.5757[\mathrm{M}+2 \mathrm{H}]^{2+}$, calc. for: $\mathrm{C}_{96} \mathrm{H}_{156} \mathrm{~N}_{25} \mathrm{O}_{27}$ : 697.3872, found.: $697.3870[\mathrm{M}+3 \mathrm{H}]^{3+}$, calc. for: $\mathrm{C}_{96} \mathrm{H}_{157} \mathrm{~N}_{25} \mathrm{O}_{27}$ : 523.2922, found.: $523.2911[\mathrm{M}+4 \mathrm{H}]^{4+}$. 
Synthesis of a Palmitoyl Modified Peptide-PNA Sensor with a Factor Xa Recognition Sequence (34)

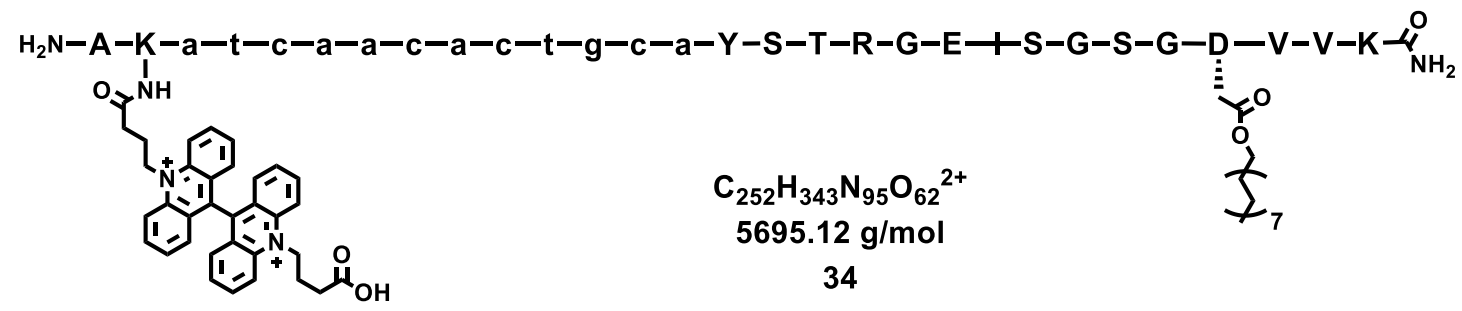

The synthesis of the peptide-PNA oligomer was performed on a Rink Amide MBHA resin $(10.0 \mu \mathrm{mol}, 0.17 \mathrm{mmol} / \mathrm{g})$ by automated SPPS according to SOP1 and SOP2. After deprotection of the Alloc group following SOP3 the lysine side chain was labelled with the BAC-fluorophore (4) as described in SOP4. The cleavage of the peptide-PNA oligomer from the resin was carried out as outlined in SOP7. The crude product was purified by semipreparative HPLC, yielding the peptide-PNA sensor $34(0.28 \mathrm{mg}, 0.05 \mu \mathrm{mol})$ as a yellow solid.

HPLC: $(250 \cdot 10 \mathrm{~mm}, 5 \mu \mathrm{m}, \mathrm{C} 18$, Gradient $=10-60 \%$ B in $30 \mathrm{~min}, \lambda[\mathrm{nm}]: 215,260,368)$ : $t_{\mathrm{R}}=25.6 \mathrm{~min}$.

MS (ESI): $\mathbf{m} / \mathbf{z}(\%)=1424.2(6)[\mathrm{M}+4 \mathrm{H}]^{4+}, 1139.5(19)[\mathrm{M}+5 \mathrm{H}]^{5+}, 949.8(38)[\mathrm{M}+6 \mathrm{H}]^{6+}$, $814.2(44)[\mathrm{M}+7 \mathrm{H}]^{7+}, 712.6(42)[\mathrm{M}+8 \mathrm{H}]^{8+}, 633.5(19)[\mathrm{M}+9 \mathrm{H}]^{9+}$.

HRMS (ESI): calc. for: $\mathrm{C}_{252} \mathrm{H}_{345} \mathrm{~N}_{95} \mathrm{O}_{62}: 1424.1710$, found.: $1424.1699[\mathrm{M}+4 \mathrm{H}]^{4+}$, calc. for: $\mathrm{C}_{252} \mathrm{H}_{346} \mathrm{~N}_{95} \mathrm{O}_{62}: 1139.5382$, found.: $1139.5374[\mathrm{M}+5 \mathrm{H}]^{5+}$, calc. for: $\mathrm{C}_{252} \mathrm{H}_{347} \mathrm{~N}_{95} \mathrm{O}_{62}$ : 949.7831 , found.: $949.7827[\mathrm{M}+6 \mathrm{H}]^{6+}$, calc. for: $\mathrm{C}_{252} \mathrm{H}_{348} \mathrm{~N}_{95} \mathrm{O}_{62}: 814.2437$, found.: $814.2426[\mathrm{M}+7 \mathrm{H}]^{7+}$, calc. for: $\mathrm{C}_{252} \mathrm{H}_{349} \mathrm{~N}_{95} \mathrm{O}_{62}: 712.5891$, found.: $712.5866[\mathrm{M}+8 \mathrm{H}]^{8+}$, calc. for: $\mathrm{C}_{252} \mathrm{H}_{350} \mathrm{~N}_{95} \mathrm{O}_{62}: 633.5245$, found.: $633.5230[\mathrm{M}+9 \mathrm{H}]^{9+}$. 


\section{Synthesis of Palmitoyl Modified Peptide-PNA Sensor with a Cathepsin B Recognition} Sequence (35)

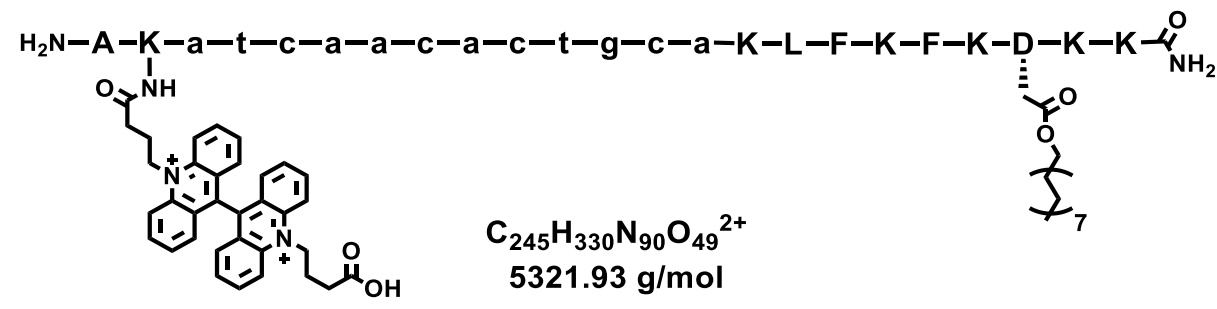

35

The synthesis of the peptide-PNA oligomer was performed on a Rink Amide MBHA resin $(10.0 \mu \mathrm{mol}, 0.17 \mathrm{mmol} / \mathrm{g})$ by automated SPPS according to SOP1 and SOP2. After deprotection of the Alloc group following SOP3 the lysine side chain was labelled with the BAC-fluorophore (4) as described in SOP4. The cleavage of the peptide-PNA oligomer from the resin was carried out as outlined in SOP7. The crude product was purified by semipreparative HPLC, yielding the peptide-PNA sensor $35(0.91 \mathrm{mg}, 0.17 \mu \mathrm{mol})$ as a yellow solid.

HPLC: $\left(250 \cdot 10 \mathrm{~mm}, 5 \mu \mathrm{m}, \mathrm{C}_{18}\right.$, Gradient $=10-60 \%$ B in $\left.30 \mathrm{~min}, \lambda[\mathrm{nm}]: 215,260,368\right)$ : $t_{\mathrm{R}}=18.1 \mathrm{~min}$.

MS (ESI): m/z $(\%)=1330.6(8)[\mathrm{M}+4 \mathrm{H}]^{4+}, 1064.9(29)[\mathrm{M}+5 \mathrm{H}]^{5+}, 887.6(61)[\mathrm{M}+6 \mathrm{H}]^{6+}$, $760.9(100)[\mathrm{M}+8 \mathrm{H}]^{8+}, 665.9(98)[\mathrm{M}+\mathrm{H}], 592.1(78)[\mathrm{M}+9 \mathrm{H}]^{9+}$.

HRMS (ESI): calc. for: $\mathrm{C}_{245} \mathrm{H}_{334} \mathrm{~N}_{90} \mathrm{O}_{49}: 1330.6611$, found.: $1330.6591[\mathrm{M}+4 \mathrm{H}]^{4+}$, calc. for: $\mathrm{C}_{245} \mathrm{H}_{335} \mathrm{~N}_{90} \mathrm{O}_{49}$ : 1064.7303, found.: $1064.7277[\mathrm{M}+5 \mathrm{H}]^{5+}$, calc. for: $\mathrm{C}_{245} \mathrm{H}_{336} \mathrm{~N}_{90} \mathrm{O}_{49}: 887.6105$, found.: $887.6093[\mathrm{M}+6 \mathrm{H}]^{6+}$, calc. for: $\mathrm{C}_{245} \mathrm{H}_{337} \mathrm{~N}_{90} \mathrm{O}_{49}: 760.9529$, found.: $760.9490[\mathrm{M}+7 \mathrm{H}]^{7+}$, calc. for: $\mathrm{C}_{245} \mathrm{H}_{338} \mathrm{~N}_{90} \mathrm{O}_{49}: 665.9597$, found.: $665.9574[\mathrm{M}+8 \mathrm{H}]^{8+}$, calc. for: $\mathrm{C}_{245} \mathrm{H}_{339} \mathrm{~N}_{90} \mathrm{O}_{49}: 592.0761$, found.: $592.0751[\mathrm{M}+9 \mathrm{H}]^{9+}$. 
Synthesis of Palmitoyl Modified Peptide-PNA Sensor with a Cathepsin B Recognition Sequence (38)

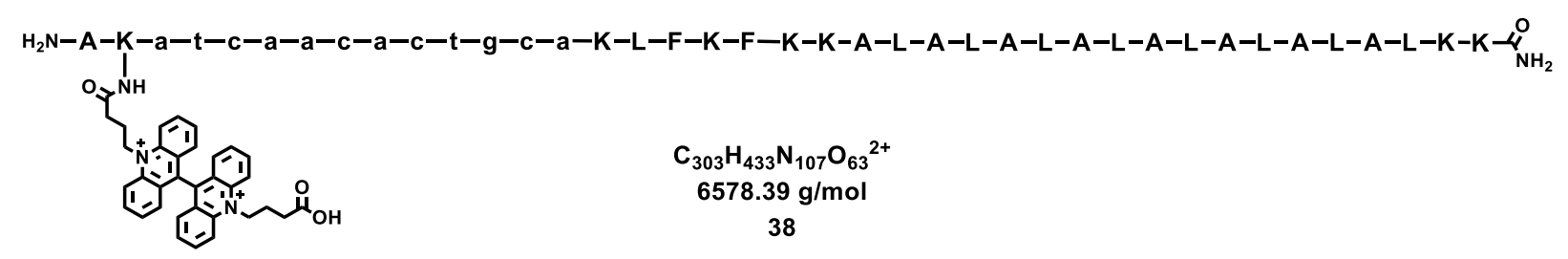

The synthesis of the peptide-PNA oligomer was performed on a Rink Amide MBHA resin $(10.0 \mu \mathrm{mol}, 0.17 \mathrm{mmol} / \mathrm{g})$ by automated SPPS according to SOP1 and SOP2. After deprotection of the Alloc group following SOP3 the lysine side chain was labelled with the BAC-fluorophore (4) as described in SOP4. The cleavage of the peptide-PNA oligomer from the resin was carried out as outlined in SOP7. The crude product was purified by semipreparative HPLC, yielding the peptide-PNA sensor $38(0.64 \mathrm{mg}, 0.10 \mu \mathrm{mol})$ as a yellow solid.

HPLC: $(250 \cdot 10 \mathrm{~mm}, 5 \mu \mathrm{m}, \mathrm{C} 18$, Gradient $=30-90 \%$ B in $30 \mathrm{~min}, \lambda[\mathrm{nm}]: 215,260,368)$ : $t_{\mathrm{R}}=11.5 \mathrm{~min}$.

MS (ESI): m/z $(\%)=1317.3(6)[\mathrm{M}+5 \mathrm{H}]^{5+}, 1097.9(25)[\mathrm{M}+6 \mathrm{H}]^{6+}, 823.8(69)[\mathrm{M}+7 \mathrm{H}]^{7+}$, $732.3(100)[\mathrm{M}+8 \mathrm{H}]^{8+}, 659.1(75)[\mathrm{M}+9 \mathrm{H}]^{9+}$.

HRMS (ESI): calc. for: $\mathrm{C}_{303} \mathrm{H}_{438} \mathrm{~N}_{107} \mathrm{O}_{63}: 1317.2883$, found.: $1317.2898[\mathrm{M}+5 \mathrm{H}]^{5+}$, calc. for: $\mathrm{C}_{303} \mathrm{H}_{439} \mathrm{~N}_{107} \mathrm{O}_{63}: 1097.9081$, found.: $1097.9088[\mathrm{M}+6 \mathrm{H}]^{6+}$, calc. for: $\mathrm{C}_{303} \mathrm{H}_{440} \mathrm{~N}_{107} \mathrm{O}_{63}: 823.6829$, found.: $823.6836[\mathrm{M}+7 \mathrm{H}]^{7+}$, calc. for: $\mathrm{C}_{303} \mathrm{H}_{441} \mathrm{~N}_{107} \mathrm{O}_{63}: 732.2745$, found.: $732.2708[\mathrm{M}+8 \mathrm{H}]^{8+}$, calc. for: $\mathrm{C}_{303} \mathrm{H}_{442} \mathrm{~N}_{107} \mathrm{O}_{63}: 659.1478$, found.: $659.1470[\mathrm{M}+9 \mathrm{H}]^{9+}$, calc. for: $\mathrm{C}_{303} \mathrm{H}_{443} \mathrm{~N}_{107} \mathrm{O}_{63}: 599.3168$, found.: $599.3173[\mathrm{M}+10 \mathrm{H}]^{10+}$. 


\section{Synthesis of TMD Modified peptide Labelled with NBD (42)}

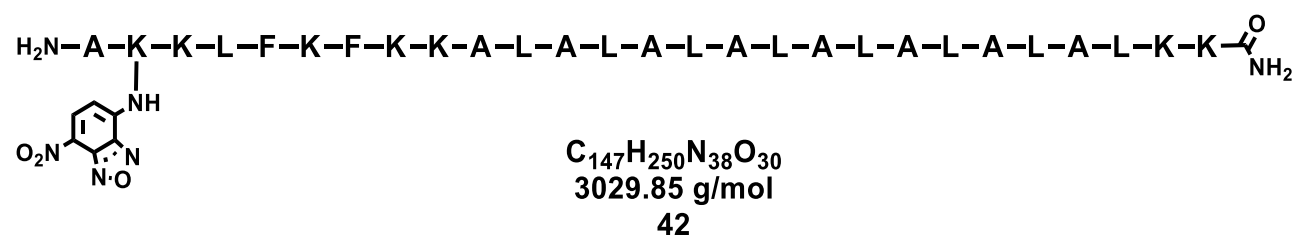

The synthesis of the peptide was performed on a Rink Amide MBHA resin $(10.0 \mu$ mol, $0.17 \mathrm{mmol} / \mathrm{g}$ ) by automated SPPS according to SOP1. After deprotection of the Alloc group following SOP3 the lysine side chain was labelled with the NBD-fluorophore as described in SOP5. The cleavage of the labelled peptide from the resin was carried out as outlined in SOP7. The crude product was purified by semi-preparative HPLC, yielding the peptide-PNA oligomer $42(0.95 \mathrm{mg}, 0.31 \mu \mathrm{mol})$ as a yellow solid.

HPLC: $(250 \cdot 10 \mathrm{~mm}, 5 \mu \mathrm{m}, \mathrm{C} 18$, Gradient $=30-70 \%$ B in $30 \mathrm{~min}, \lambda[\mathrm{nm}]: 215,260,467)$ : $t_{\mathrm{R}}=16.6 \mathrm{~min}$.

MS (ESI): $\mathbf{m} / \mathbf{z}(\%)=1010.6(11)[\mathrm{M}+3 \mathrm{H}]^{3+}, 758.2(51)[\mathrm{M}+4 \mathrm{H}]^{4+}, 606.8(100)[\mathrm{M}+5 \mathrm{H}]^{5+}$. HRMS (ESI): calc. for: $\mathrm{C}_{147} \mathrm{H}_{252} \mathrm{~N}_{38} \mathrm{O}_{30}: 1515.4690$, found.: $1515.4688[\mathrm{M}+2 \mathrm{H}]^{2+}$, calc. for: $\mathrm{C}_{147} \mathrm{H}_{253} \mathrm{~N}_{38} \mathrm{O}_{30}$ : 1010.6484, found.: $1010.6494[\mathrm{M}+3 \mathrm{H}]^{3+}$, calc. for: $\mathrm{C}_{147} \mathrm{H}_{254} \mathrm{~N}_{38} \mathrm{O}_{30}$ : 758.2381 , found.: $758.2386[\mathrm{M}+4 \mathrm{H}]^{4+}$, calc. for: $\mathrm{C}_{147} \mathrm{H}_{255} \mathrm{~N}_{38} \mathrm{O}_{30}: 606.7920$, found.: $606.7921[\mathrm{M}+5 \mathrm{H}]^{5+}$, calc. for: $\mathrm{C}_{147} \mathrm{H}_{256} \mathrm{~N}_{38} \mathrm{O}_{30}: 505.8278$, found.: $505.8300[\mathrm{M}+6 \mathrm{H}]^{6+}$. 
Synthesis of His14 Modified Peptide-PNA sensor (47)

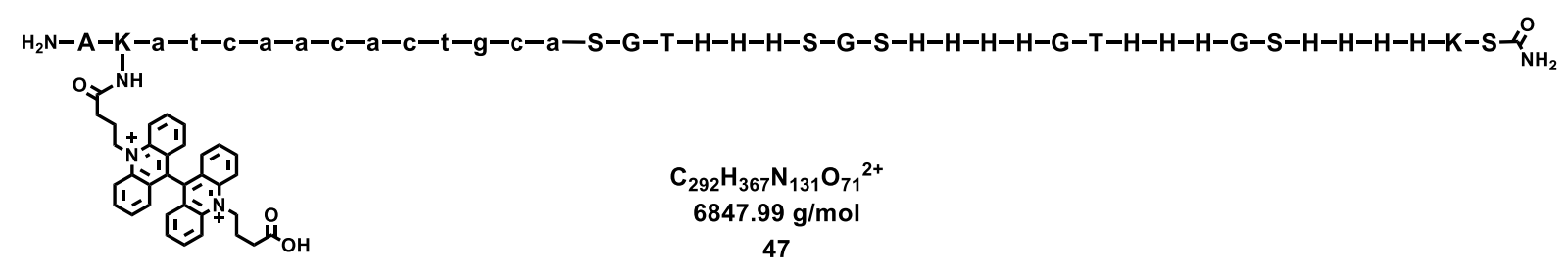

The synthesis of the peptide-PNA oligomer was performed on a Rink Amide MBHA resin $(10.0 \mu \mathrm{mol}, 0.17 \mathrm{mmol} / \mathrm{g})$ by automated SPPS according to SOP1 and SOP2. After deprotection of the Alloc group following SOP3 the lysine side chain was labelled with the BAC-fluorophore (4) as described in SOP4. The cleavage of the peptide-PNA oligomer from the resin was carried out as outlined in SOP7. The crude product was purified by semipreparative HPLC, yielding the peptide-PNA sensor $47(0.53 \mathrm{mg}, 0.08 \mu \mathrm{mol})$ as a yellow solid.

HPLC: $(250 \cdot 10 \mathrm{~mm}, 5 \mu \mathrm{m}, \mathrm{C} 18$, Gradient $=5-35 \%$ B in $30 \mathrm{~min}, \lambda[\mathrm{nm}]: 215,260,368)$ : $t_{\mathrm{R}}=18.4 \mathrm{~min}$.

MS (ESI): m/z $(\%)=1370.2(2)[\mathrm{M}+5 \mathrm{H}]^{5+}, 1141.8(10)[\mathrm{M}+6 \mathrm{H}]^{6+}, 979.0(10)[\mathrm{M}+7 \mathrm{H}]^{7+}$, $856.7(11)[\mathrm{M}+8 \mathrm{H}]^{8+}, 685.5(31)[\mathrm{M}+10 \mathrm{H}]^{10+}, 623.4(40)[\mathrm{M}+11 \mathrm{H}]^{11+}, 571.5(44)$ $[\mathrm{M}+12 \mathrm{H}]^{12+}$,

HRMS (ESI): calc. for: $\mathrm{C}_{292} \mathrm{H}_{370} \mathrm{~N}_{131} \mathrm{O}_{71}: 1369.9884$, found.: $1369.9902[\mathrm{M}+5 \mathrm{H}]^{5+}$, calc. for: $\mathrm{C}_{292} \mathrm{H}_{371} \mathrm{~N}_{131} \mathrm{O}_{71}: 1141.8248$, found.: $1141.8258[\mathrm{M}+6 \mathrm{H}]^{6+}$, calc. for: $\mathrm{C}_{292} \mathrm{H}_{372} \mathrm{~N}_{131} \mathrm{O}_{71}: 978.8509$, found.: $978.8471[\mathrm{M}+7 \mathrm{H}]^{7+}$, calc. for: $\mathrm{C}_{292} \mathrm{H}_{373} \mathrm{~N}_{131} \mathrm{O}_{71}: 856.6205$, found.: $856.6208[\mathrm{M}+8 \mathrm{H}]^{8+}$, calc. for: $\mathrm{C}_{292} \mathrm{H}_{374} \mathrm{~N}_{131} \mathrm{O}_{71}: 761.5523$, found.: $761.5533[\mathrm{M}+9 \mathrm{H}]^{9+}$, calc. for: $\mathrm{C}_{292} \mathrm{H}_{375} \mathrm{~N}_{131} \mathrm{O}_{71}: 685.4978$, found.: $685.4961[\mathrm{M}+10 \mathrm{H}]^{10+}$, calc. for: $\mathrm{C}_{292} \mathrm{H}_{376} \mathrm{~N}_{131} \mathrm{O}_{71}: 623.2714$, found.: $623.2707[\mathrm{M}+11 \mathrm{H}]^{11+}$, calc. for: $\mathrm{C}_{292} \mathrm{H}_{377} \mathrm{~N}_{131} \mathrm{O}_{71}: 571.4161$, found.: $571.4164[\mathrm{M}+12 \mathrm{H}]^{12+}$. 
Synthesis of ALFA-tag Modified Peptide-PNA sensor (49)

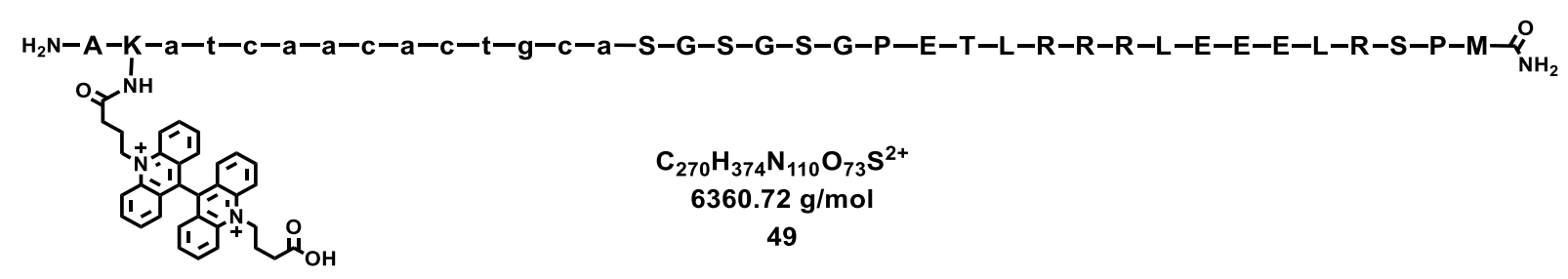

The synthesis of the peptide-PNA oligomer was performed on a Rink Amide MBHA resin $(10.0 \mu \mathrm{mol}, 0.17 \mathrm{mmol} / \mathrm{g})$ by automated SPPS according to SOP1 and SOP2. After deprotection of the Alloc group following SOP3 the lysine side chain was labelled with the BAC-fluorophore (4) as described in SOP4. The cleavage of the peptide-PNA oligomer from the resin was carried out as outlined in SOP7. The crude product was purified by semipreparative HPLC, yielding the peptide-PNA sensor $49(0.37 \mathrm{mg}, 0.06 \mu \mathrm{mol})$ as a yellow solid.

HPLC: $\left(250 \cdot 10 \mathrm{~mm}, 5 \mu \mathrm{m}, \mathrm{C}_{18}\right.$, Gradient $=10-60 \%$ B in $\left.30 \mathrm{~min}, \lambda[\mathrm{nm}]: 215,254,280\right)$ :

$t_{R}=16.0 \mathrm{~min}$.

MS (ESI): $\mathbf{m} / \mathbf{z}(\%)=1060.6(3)[\mathrm{M}+5 \mathrm{H}]^{5+}, 795.0(8)[\mathrm{M}+7 \mathrm{H}]^{7+}$.

HRMS (ESI): calc. for: $\mathrm{C}_{270} \mathrm{H}_{378} \mathrm{~N}_{110} \mathrm{O}_{73} \mathrm{~S}: 1272.5788$, found.: $1272.5761[\mathrm{M}+4 \mathrm{H}]^{4+}$, calc. for: $\mathrm{C}_{270} \mathrm{H}_{379} \mathrm{~N}_{110} \mathrm{O}_{73} \mathrm{~S}: 1060.6502$, found.: $1060.6520[\mathrm{M}+5 \mathrm{H}]^{5+}$, calc. for: $\mathrm{C}_{270} \mathrm{H}_{380} \mathrm{~N}_{110} \mathrm{O}_{73} \mathrm{~S}$ : 909.2726 , found.: $909.2699[\mathrm{M}+6 \mathrm{H}]^{6+}$, calc. for: $\mathrm{C}_{270} \mathrm{H}_{381} \mathrm{~N}_{110} \mathrm{O}_{73} \mathrm{~S}: 795.7395$, found.: $795.7419[\mathrm{M}+7 \mathrm{H}]^{7+}$, calc. for: $\mathrm{C}_{270} \mathrm{H}_{382} \mathrm{~N}_{110} \mathrm{O}_{73} \mathrm{~S}$ : 707.4359, found.: $707.4376[\mathrm{M}+8 \mathrm{H}]^{8+}$, calc. for: $\mathrm{C}_{270} \mathrm{H}_{383} \mathrm{~N}_{110} \mathrm{O}_{73} \mathrm{~S}$ : 636.7930 , found.: $636.7938[\mathrm{M}+9 \mathrm{H}]^{9+}$. 
Synthesis of an ALFA-tag Modified Peptide labelled with the AlexaFluor488 (51)

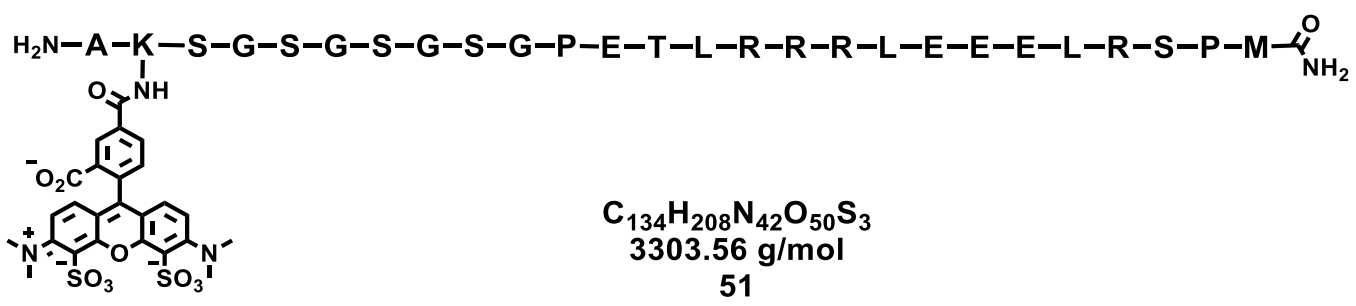

The synthesis of the peptide-PNA oligomer was performed on a Rink Amide MBHA resin $(10.0 \mu \mathrm{mol}, 0.17 \mathrm{mmol} / \mathrm{g})$ by automated SPPS according to SOP1 and SOP2. After deprotection of the Alloc group following SOP3 the lysine side chain was labelled with the BAC-fluorophore (4) as described in SOP4. The cleavage of the peptide-PNA oligomer from the resin was carried out as outlined in SOP7. The crude product was purified by semipreparative HPLC, yielding the peptide-PNA sensor $\mathbf{5 1}(0.64 \mathrm{mg}, 0.19 \mu \mathrm{mol})$ as a yellow solid.

HPLC: $\left(250 \cdot 10 \mathrm{~mm}, 5 \mu \mathrm{m}, \mathrm{C}_{18}\right.$, Gradient $=10-60 \%$ B in $\left.30 \mathrm{~min}, \lambda[\mathrm{nm}]: 215,254,280\right)$ : $\mathrm{t}_{\mathrm{R}}=15.3 \mathrm{~min}$.

MS (ESI): $\mathbf{m} / \mathbf{z}(\%)=1652.6(2)[\mathrm{M}+2 \mathrm{H}]^{2+}, 1102.1(6)[\mathrm{M}+6 \mathrm{H}]^{6+}$.

HRMS (ESI): calc. for: $\mathrm{C}_{134} \mathrm{H}_{210} \mathrm{~N}_{42} \mathrm{O}_{50} \mathrm{~S}_{3}: 1652.2180$, found.: $1652.2150[\mathrm{M}+2 \mathrm{H}]^{2+}$, calc. for: $\mathrm{C}_{134} \mathrm{H}_{211} \mathrm{~N}_{42} \mathrm{O}_{50} \mathrm{~S}_{3}: 1101.8144$, found.: $1101.8125[\mathrm{M}+3 \mathrm{H}]^{3+}$, calc. for: $\mathrm{C}_{134} \mathrm{H}_{212} \mathrm{~N}_{42} \mathrm{O}_{50} \mathrm{~S}_{3}: 826.6126$, found.: $826.6109[\mathrm{M}+4 \mathrm{H}]^{4+}$. 


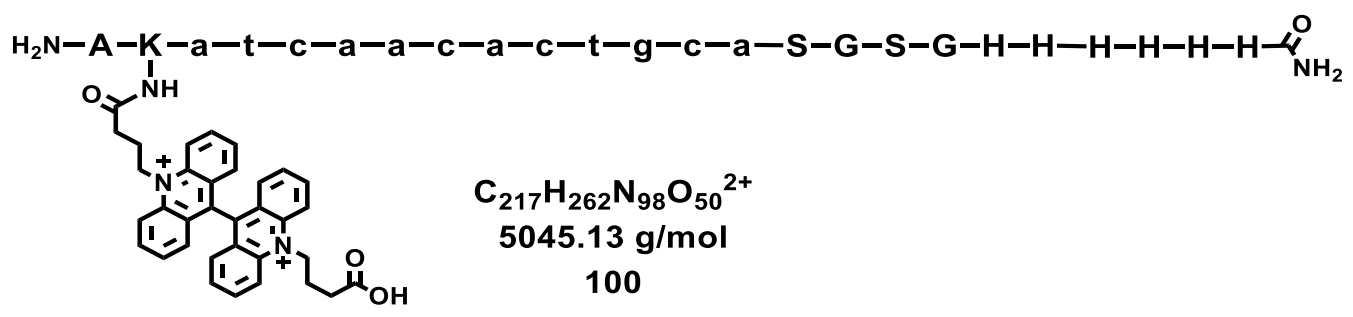

The synthesis of the peptide-PNA oligomer was performed on a Rink Amide MBHA Resin $(10.0 \mu \mathrm{mol}, 0.17 \mathrm{mmol} / \mathrm{g})$ by automated SPPS according to SOP1 and SOP2. After deprotection of the Alloc group following SOP3 the lysine side chain was labelled with the BAC-fluorophore (4) as described in SOP4. The cleavage of the peptide-PNA oligomer from the resin was carried out as outlined in SOP7. The crude product was purified by semipreparative HPLC, yielding the peptide-PNA sensor $100(0.53 \mathrm{mg}, 0.08 \mu \mathrm{mol})$ as a yellow solid.

HPLC: $\left(250 \cdot 10 \mathrm{~mm}, 5 \mu \mathrm{m}, \mathrm{C}_{18}\right.$, Gradient $=5-35 \% \mathrm{~B}$ in $\left.30 \mathrm{~min}, \lambda[\mathrm{nm}]: 215,260,368\right)$ : $t_{\mathrm{R}}=16.5 \mathrm{~min}$.

MS (ESI): $\mathbf{m} / \mathbf{z}(\%)=1009.6(2)[\mathrm{M}+5 \mathrm{H}]^{5+}, 841.3(6)[\mathrm{M}+6 \mathrm{H}]^{6+}, 721.3(7)[\mathrm{M}+7 \mathrm{H}]^{7+}, 631.3$

(10) $[\mathrm{M}+8 \mathrm{H}]^{8+}, 561.3(13)[\mathrm{M}+9 \mathrm{H}]^{9+}$.

HRMS (ESI): calc. for: $\mathrm{C}_{217} \mathrm{H}_{262} \mathrm{~N}_{98} \mathrm{O}_{50}: 1009.4277$, found.: $1009.4270[\mathrm{M}+5 \mathrm{H}]^{5+}$, calc. for: $\mathrm{C}_{217} \mathrm{H}_{263} \mathrm{~N}_{98} \mathrm{O}_{50}: 841.3577$, found.: $841.3565[\mathrm{M}+6 \mathrm{H}]^{6+}$, calc. for: $\mathrm{C}_{217} \mathrm{H}_{264} \mathrm{~N}_{98} \mathrm{O}_{50}: 721.3076$, found.: $721.3061[\mathrm{M}+7 \mathrm{H}]^{7+}$, calc. for: $\mathrm{C}_{217} \mathrm{H}_{265} \mathrm{~N}_{98} \mathrm{O}_{50}: 631.2701$, found.: $631.2684[\mathrm{M}+8 \mathrm{H}]^{8+}$, calc. for: $\mathrm{C}_{217} \mathrm{H}_{262} \mathrm{~N}_{98} \mathrm{O}_{50}: 561.2409$, found.: $561.2403[\mathrm{M}+9 \mathrm{H}]^{9+}$. 


\section{Synthesis of modified $\mu$-Conotoxin SIIIA}

\section{Synthesis of 4,5-Diemthoxy-2-nitrobenzyl(4-nitrophenyl)carbonate (82)}

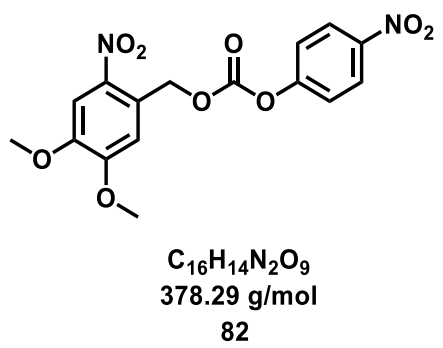

The synthesis was performed according to a protocol modified by FoMINA et al. ${ }^{[259]}$ 4,5-Dimethoxy-2-nitrobenzyl alcohol (2.50 g, $11.7 \mathrm{mmol}, 1.00$ eq.) (81) and 4-nitrophenyl chloroformate $(4.70 \mathrm{~g}, 23.5 \mathrm{mmol}, 2.00 \mathrm{eq})(\mathbf{8 0})$ were dissolved in anhydrous DCM (40 mL) under argon atmosphere. Subsequently, DIPEA (4.18 mL, $23.5 \mathrm{mmol}, 2.00 \mathrm{eq})$ was added and the reaction mixture was stirred for $12 \mathrm{~h}$ at $\mathrm{rt}$ under exclusion of light. The solvent was removed under reduced pressure and the residue was recrystallized from EtOH. After drying under reduced pressure, the product 82 (3.75 g, $9.93 \mathrm{mmol}, 85 \%)$ was obtained as yellow solid.

${ }^{1} \mathbf{H}-\mathrm{NMR}\left(300 \mathrm{MHz}, \mathrm{DMSO}-d_{6}\right) ; \delta[\mathrm{ppm}]=3.89$ (s, 3H, O-CH ), 3.92 (s, 3H, O-CH $), 5.61$ $\left(\mathrm{s}, 2 \mathrm{H}, \mathrm{CH}_{2}\right), 7.26\left(\mathrm{~s}, 1 \mathrm{H}, \mathrm{C} H_{\text {Ar. }}\right), 7.59\left(\mathrm{~d},{ }^{3} \mathrm{~J}_{\mathrm{H}, \mathrm{H}}=9.3 \mathrm{~Hz}, 2 \mathrm{H}, \mathrm{C} H_{\text {Ar. }}\right), 7.74\left(\mathrm{~s}, 1 \mathrm{H}, \mathrm{CH}_{\text {Ar. }}\right), 8.33$ $\left(\mathrm{d},{ }^{3} J_{\mathrm{H}, \mathrm{H}}=9.2 \mathrm{~Hz}, 2 \mathrm{H}, \mathrm{C} H_{\mathrm{Ar} .}\right)$.

${ }^{13}$ C-NMR $\left(126 \mathrm{MHz}, \mathrm{DMSO}-d_{6}\right) ; \delta[\mathrm{ppm}]=56.1\left(\mathrm{OCH}_{3}\right), 56.2\left(\mathrm{OCH}_{3}\right), 66.1\left(\mathrm{CH}_{2}\right), 108.2$ $\left(C_{\mathrm{Ar}}\right), 111.9\left(C_{\mathrm{Ar}}\right), 122.3$ ( 2 x $\left.C_{\mathrm{Ar}}\right), 124.1\left(C_{\mathrm{Ar}}\right), 125.2\left(2 \times C_{\mathrm{Ar}}\right), 139.8\left(C_{\mathrm{Ar}}\right), 145.1\left(C_{\mathrm{Ar}}\right)$, $148.1\left(C_{\mathrm{Ar}}\right), 151.3(C=\mathrm{O}), 152.9\left(C_{\mathrm{Ar}}\right), 154.9\left(C_{\mathrm{Ar}}\right)$.

MS (ESI): $\mathrm{m} / \mathrm{z}(\%)=401.1(100)[\mathrm{M}+\mathrm{Na}]^{+}, 779.2(46)[2 \mathrm{M}+\mathrm{Na}]^{+}$.

HRMS (ESI): calc. for $\mathrm{C}_{16} \mathrm{H}_{14} \mathrm{~N}_{2} \mathrm{O}_{9} \mathrm{Na}\left([\mathrm{M}+\mathrm{Na}]^{+}\right)$: 401.0592, found: 401.0593; calc. for $\mathrm{C}_{16} \mathrm{H}_{14} \mathrm{~N}_{2} \mathrm{O}_{9} \mathrm{~K}\left([\mathrm{M}+\mathrm{K}]^{+}\right)$: 417.0331, found: 417.0329 . 


\section{Synthesis of Fmoc-Lys(Nvoc)-OH (83)}

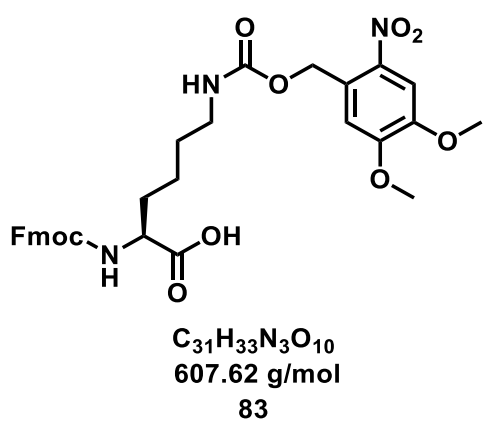

The synthesis was performed according to a protocol modified by DE GARCIA LUX et al. ${ }^{[274]}$ Fmoc-Lys-OH (1.10 g, $1.06 \mathrm{mmol}, 1.50 \mathrm{eq})$ and $\mathrm{Na}_{2} \mathrm{CO}_{3}(0.78 \mathrm{~g}, 2.70 \mathrm{mmol}, 1.00 \mathrm{eq})$ were dissolved in a mixture of dioxane, acetonitrile and $\mathrm{H}_{2} \mathrm{O}(36 \mathrm{~mL}, 1: 1: 1, v / v / v)$. 4,5-Diemthoxy2-nitrobenzyl(4-nitrophenyl)carbonate (1.02 g, $2.70 \mathrm{mmol}, 1.00 \mathrm{eq})(\mathbf{8 2})$ was added and the reaction mixture was stirred for $12 \mathrm{~h}$ at $\mathrm{rt}$ under exclusion of light. Afterwards, the solvent was removed under reduced pressure and crude product was purified by flash column chromatography (DCM/MeOH/AcOH 10:0.02:0.01, v/v/v). The product 83 (1.17 g, $1.94 \mathrm{mmol}$, $71 \%$ ) was obtained as a yellow solid.

${ }^{\mathbf{1}} \mathbf{H}-\mathbf{N M R}\left(300 \mathrm{MHz}, \mathrm{DMSO}-d_{6}\right) ; \delta[\mathrm{ppm}]=1.28-1.51\left(\mathrm{~m}, 4 \mathrm{H}, \delta-\mathrm{CH}_{2}, \gamma-\mathrm{CH}_{2}\right), 1.55-1.75$ $\left(\mathrm{m}, 2 \mathrm{H}, \beta-\mathrm{CH}_{2}\right), 2.96-3.08\left(\mathrm{~m}, 2 \mathrm{H} \varepsilon-\mathrm{CH}_{2}\right), 3.86$ (s, 3H, O-CH $), 3.88$ (s, 3H, O-CH3), $3.90-$ $3.96(\mathrm{~m}, 1 \mathrm{H}, \mathrm{Fmoc}-\mathrm{CH}), 4.17-4.32$ (m, 3H, Fmoc-CH,$\alpha-\mathrm{CH}), 5.32$ (s, 2H, Nvoc-CH $\left.\mathrm{CH}_{2}\right), 7.17$ (s, $1 \mathrm{H}$, Nvoc-C $\left.H_{\text {Ar. }}\right), 7.32\left(\mathrm{t},{ }^{3} J_{\mathrm{H}, \mathrm{H}}=7.4 \mathrm{~Hz}, 2 \mathrm{H}, \mathrm{Fmoc}-\mathrm{C} H_{\text {Ar. }}\right), 7.37-7.49$ (m, 3H, Fmoc-CH $H_{\text {Ar. }}$, Nvoc-CH $\left.H_{\text {Ar. }}\right), 7.61\left(\mathrm{~d},{ }^{3} J_{\mathrm{H}, \mathrm{H}}=8.0 \mathrm{~Hz}, 1 \mathrm{H}, \mathrm{NH}\right), 7.67-7.75\left(\mathrm{~m}, 2 \mathrm{H}, \mathrm{Fmoc}-\mathrm{C} H_{\mathrm{Ar}}\right), 7.88\left(\mathrm{~d},{ }^{3} J_{\mathrm{H}, \mathrm{H}}\right.$ $\left.=7.5 \mathrm{~Hz}, 2 \mathrm{H}, \mathrm{Fmoc}-\mathrm{CH}_{\text {Ar. }}\right)$.

${ }^{13}$ C-NMR $\left(126 \mathrm{MHz}, \mathrm{DMSO}-d_{6}\right) ; \delta[\mathrm{ppm}]=22.8\left(\gamma-\mathrm{CH}_{2}\right), 28.8\left(\delta-C \mathrm{H}_{2}\right), 30.3\left(\beta-C \mathrm{H}_{2}\right), 40.1$ $\left(\varepsilon-\mathrm{CH}_{2}\right), 46.6(\mathrm{Fmoc}-\mathrm{CH}) 53.7(\alpha-\mathrm{CH}), 56.0\left(2 \mathrm{C}, \mathrm{OCH}_{3}\right), 65.5\left(\mathrm{CH}_{2}\right), 67.0\left(\mathrm{Fmoc}-\mathrm{CH}_{2}\right), 107.5$ $\left(C_{\text {Ar. }}\right), 108.2$ ( $\left.C_{\text {Ar. }}\right), 120.0$ (2C, 2 x $\left.C_{\text {Ar. }}\right), 125.2$ (2C, 2 x $\left.C_{\text {Ar. }}\right), 127.0\left(2 \mathrm{C}, 2\right.$ x $\left.C_{\text {Ar. }}\right), 127.6(2 \mathrm{C}, 2$ x $\left.C_{\text {Ar. }}\right), 133.4$ ( $\left.C_{\text {Ar. }}\right), 139.3$ ( $\left.C_{\text {Ar. }}\right), 140.7$ ( 2 C, 2 x $\left.C_{\text {Ar. }}\right), 143.8$ ( 2 C, 2 x $\left.C_{\text {Ar. }}\right), 147.5$ ( $\left.C_{\text {Ar. }}\right), 153.4$ $\left(C_{\mathrm{Ar}}\right), 155.1(\mathrm{CO}), 156,1(\mathrm{CO}), 173.9\left(\mathrm{CO}_{2} \mathrm{H}\right)$

MS (ESI): m/z (\%) = $606.2(100)[\mathrm{M}-\mathrm{H}]^{-}$.

HRMS (ESI): calc. for $\mathrm{C}_{31} \mathrm{H}_{33} \mathrm{~N}_{3} \mathrm{O}_{10} \mathrm{Na}\left([\mathrm{M}+\mathrm{Na}]^{+}\right)$: 630.2058, found: 630.2023; calc. for $\mathrm{C}_{31} \mathrm{H}_{32} \mathrm{~N}_{3} \mathrm{O}_{10}\left([\mathrm{M}-\mathrm{H}]^{-}\right)$: 606.2093, found: 606.2090 . 


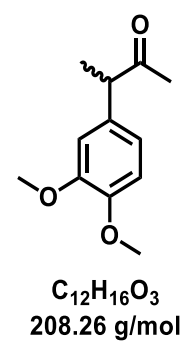

86

The synthesis was performed according to a protocol modified by SPECHT et al. ${ }^{[263]}$ 1-(3,4-Dimethoxyphenyl)propan-2-one (1.30 mL, $7.50 \mathrm{mmol}, 1.00 \mathrm{eq}, \delta=1.12 \mathrm{~g} / \mathrm{mL})(\mathbf{8 5})$ and sodium hydride (60\% in paraffin oil, $0.30 \mathrm{~g}, 7.50 \mathrm{mmol}, 1.00 \mathrm{eq})$ were dissolved in anhydrous THF (30 min) under argon atmosphere. After stirring at $\mathrm{rt}$ for $30 \mathrm{~min}$, the mixture was cooled to $0{ }^{\circ} \mathrm{C}$ and methyl iodide $(0.56 \mathrm{~mL}, 9.00 \mathrm{mmol}, 1.20 \mathrm{eq}, \delta=2.28 \mathrm{~g} / \mathrm{mL})$ was added. The reaction mixture was stirred for $6 \mathrm{~h}$ at $\mathrm{rt}$ and was quenched by addition of sat. $\mathrm{NaHCO}_{3}$ solution $(100 \mathrm{~mL})$. Following, the aqueous layer was extracted with EtOAc $(3 \times 50 \mathrm{~mL})$ and the combined layers were dried over $\mathrm{Na}_{2} \mathrm{SO}_{4}$. The solvent was removed under reduced pressure and the product 86 (1.61 g, quant.). was obtained as colourless oil.

${ }^{1} \mathbf{H}-\mathbf{N M R}\left(300 \mathrm{MHz}, \mathrm{DMSO}-d_{6}\right) ; \delta[\mathrm{ppm}]=1.26\left(\mathrm{~d},{ }^{3} J_{\mathrm{H}, \mathrm{H}}=7.0 \mathrm{~Hz}, 3 \mathrm{H}, \mathrm{CH}-\mathrm{CH}_{3}\right), 2.00(\mathrm{~s}, 3 \mathrm{H}$, $\left.\mathrm{CH}_{3}\right), 3.73$ (s, 3H, O-CH3), 3.75 (s, 3H, O-CH3), $3.74-3.83(\mathrm{~m}, 1 \mathrm{H}, \mathrm{CH}), 6.69-6.83$ (m, 2H, $\left.\mathrm{C} H_{\text {Ar. }}\right), 6.90\left(\mathrm{~d},{ }^{3} J_{\mathrm{H}, \mathrm{H}}=7.0 \mathrm{~Hz}, 1 \mathrm{H}, \mathrm{C} H_{\mathrm{Ar}}\right)$.

${ }^{13} \mathbf{C}-\mathbf{N M R}\left(126 \mathrm{MHz}, \mathrm{DMSO}-d_{6}\right) ; \delta[\mathrm{ppm}]=16.9\left(\mathrm{CH}-\mathrm{CH}_{3}\right), 27.9\left(\mathrm{CH}_{3}\right), 51.7(\mathrm{CH}), 55.4(2 \mathrm{C}$, 2 x O- $\left.C \mathrm{H}_{3}\right), 111.5\left(C_{\text {Ar. }}\right), 112.1\left(C_{\text {Ar. }}\right), 119.5\left(C_{\text {Ar. }}\right), 133.0\left(C_{\text {Ar. }}\right), 147.6\left(C_{\text {Arr }}\right), 148.7\left(C_{\text {Ar. }}\right), 207.9$ $(\mathrm{CO})$

MS (ESI): m/z (\%) = $209.1(100)[\mathrm{M}+\mathrm{H}]^{+}, 231.1(79)[\mathrm{M}+\mathrm{Na}]^{+}$.

HRMS (ESI): calc. for $\mathrm{C}_{12} \mathrm{H}_{17} \mathrm{O}_{3}\left([\mathrm{M}+\mathrm{H}]^{+}\right)$: 209.1172, found: 209.1179;

calc. for $\mathrm{C}_{12} \mathrm{H}_{16} \mathrm{O}_{3} \mathrm{Na}\left([\mathrm{M}+\mathrm{Na}]^{+}\right)$: 231.0992, found: 231.0994 . 


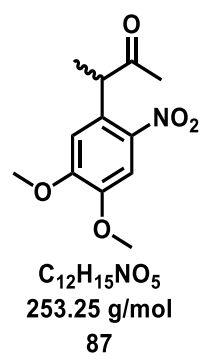

The synthesis was performed according to a protocol modified by SCHIRMACHER. ${ }^{[275]}$ 3-(3,4-Dimethoxyphenyl)butan-2-one (1.56 g, $7.50 \mathrm{mmol}, 1.00 \mathrm{eq})$ (86) was dissolved in acetic acid $(15 \mathrm{~mL})$, cooled to $0{ }^{\circ} \mathrm{C}$ and nitric acid $(1.50 \mathrm{~mL}, 36.0 \mathrm{mmol}, 4.80$ eq.) was added dropwise. After stirring for $60 \mathrm{~min}$ at $0{ }^{\circ} \mathrm{C}$, saturated $\mathrm{NaHCO}_{3}$ solution was added and the aqueous layer was extracted with EtOAc $(3 \times 50 \mathrm{~mL})$. The combined organic layers were washed with brine $(3 \times 50 \mathrm{~mL})$ and were dried over $\mathrm{Na}_{2} \mathrm{SO}_{4}$. The solvent was removed under reduced pressure and the product $87(1.83 \mathrm{~g}, 7.21 \mathrm{mmol}, 96 \%)$ was obtained as brown oil.

${ }^{1} \mathbf{H}-\mathbf{N M R}\left(300 \mathrm{MHz}, \mathrm{DMSO}-d_{6}\right) ; \delta[\mathrm{ppm}]=1.41\left(\mathrm{~d},{ }^{3} J_{\mathrm{H}, \mathrm{H}}=7.1 \mathrm{~Hz}, 3 \mathrm{H}, \mathrm{CH}-\mathrm{CH}_{3}\right), 2.10(\mathrm{~s}, 3 \mathrm{H}$, $\left.\mathrm{CH}_{3}\right), 3.86\left(\mathrm{~s}, 3 \mathrm{H}, \mathrm{O}-\mathrm{CH}_{3}\right), 3.90\left(\mathrm{~s}, 3 \mathrm{H}, \mathrm{O}-\mathrm{CH}_{3}\right), 3.97-4.09(\mathrm{~m}, 1 \mathrm{H}, \mathrm{CH}), 6.92\left(\mathrm{~s}, 1 \mathrm{H}, \mathrm{CH}_{\mathrm{Ar}}\right)$, 7.61 (s, $\left.1 \mathrm{H}, \mathrm{C} H_{\mathrm{Ar}}\right)$.

${ }^{13}$ C-NMR $\left(126 \mathrm{MHz}, \mathrm{DMSO}-d_{6}\right) ; \delta[\mathrm{ppm}]=16.0\left(\mathrm{CH}_{3}\right), 28.1\left(\mathrm{CH}_{3}\right), 48.6(\mathrm{CH}), 56.0(2 \mathrm{x}$ O- $\left.C \mathrm{H}_{3}\right), 108.1$ ( $\left.C_{\text {Ar. }}\right), 112.0\left(C_{\text {Ar. }}\right), 129.9$ ( $\left.C_{\text {Ar. }}\right), 140.5$ ( $\left.C_{\text {Ar. }}\right) 147.1\left(C_{\text {Ar. }}\right), 152.7\left(C_{\text {Ar. }}\right), 206.3$ $(C \mathrm{O})$

MS (ESI): $\mathrm{m} / \mathrm{z}(\%)=254.1(28)[\mathrm{M}+\mathrm{H}]^{+}, 276.1(100)[\mathrm{M}+\mathrm{Na}]^{+}$.

HRMS (ESI): calc. for $\mathrm{C}_{12} \mathrm{H}_{16} \mathrm{NO}_{5}\left([\mathrm{M}+\mathrm{H}]^{+}\right): 254.1023$, found: 254.1026, calc. for $\mathrm{C}_{12} \mathrm{H}_{15} \mathrm{NO}_{5} \mathrm{Na}\left([\mathrm{M}+\mathrm{Na}]^{+}\right)$: 276.0842, found: 276.0844 . 
Synthesis of 3-(4,5-Dimethoxy-2-nitrophenyl)butan-2-ol (88)

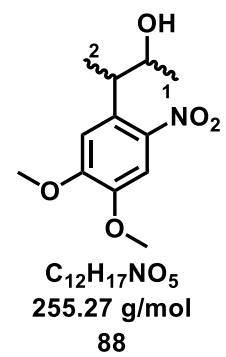

The synthesis was performed according to a protocol modified by SCHIRMACHER. ${ }^{\text {[275] }}$ 3-(4,5-dimethoxy-2-nitrophenyl)butan-2-one (1.14 g, $4.51 \mathrm{mmol}, 1.00 \mathrm{eq})$ (87) was dissolved in THF/2-propanol (3.3 mL, $2 \mathrm{~mL}, v / v)$ and sodium borohydride (0.43 g, $11.3 \mathrm{mmol}, 2.50 \mathrm{eq})$ was added. The reaction mixture was stirred for $2 \mathrm{~h}$ at $\mathrm{rt}$ and the excess of sodium borohydride was quenched with $\mathrm{HCl}(1 \mathrm{M})$ at $0{ }^{\circ} \mathrm{C}$. Following, the aqueous layer was extracted with EtOAc $(50 \mathrm{~mL})$. The combined organic layers were washed with saturated brine $(3 \times 50 \mathrm{~mL})$ and were dried over $\mathrm{Na}_{2} \mathrm{SO}_{4}$. The solvent was removed under reduced pressure and the product $\mathbf{8 8}$ (0.97 g, $3.83 \mathrm{mmol}, 85 \%$, erythro/threo (1:2)) was obtained as colourless solid.

${ }^{1}$ H-NMR $\left(300 \mathrm{MHz}, \mathrm{DMSO}-d_{6}\right) ; \delta[\mathrm{ppm}]=0.88\left(\mathrm{~d},{ }^{3} J_{\mathrm{H}, \mathrm{H}}=6.2 \mathrm{~Hz}, 1 \mathrm{H}, 1-\mathrm{CH}_{3}\right.$ (erythro)), 1.04 $\left(\mathrm{d},{ }^{3} J_{\mathrm{H}, \mathrm{H}}=6.2 \mathrm{~Hz}, 2 \mathrm{H}, 1-\mathrm{CH}_{3}(\right.$ threo $\left.)\right), 1.23\left(\mathrm{~d},{ }^{3} J_{\mathrm{H}, \mathrm{H}}=7.0 \mathrm{~Hz}, 2 \mathrm{H}, 2-\mathrm{CH}_{3}\right.$ (threo) $), 1.28\left(\mathrm{~d},{ }^{3} J_{\mathrm{H}, \mathrm{H}}\right.$ $=6.9 \mathrm{~Hz}, 1 \mathrm{H}, 2-\mathrm{CH}_{3}$ (erythro)), $3.11\left(\mathrm{q},{ }^{3} J_{\mathrm{H}, \mathrm{H}}=6.8 \mathrm{~Hz},{ }^{4} J_{\mathrm{H}, \mathrm{H}}=0.3 \mathrm{H}, \mathrm{CH}-\mathrm{CH}_{3}\right.$ (erythro)), 3.22 $\left(\mathrm{q},{ }^{3} \mathrm{~J}_{\mathrm{H}, \mathrm{H}}=7.0 \mathrm{~Hz},{ }^{4} \mathrm{~J}_{\mathrm{H}, \mathrm{H}}=0.7 \mathrm{H}, \mathrm{CH}-\mathrm{CH}_{3}\right.$ (threo), $3.81\left(\mathrm{~s}, 3 \mathrm{H}, \mathrm{OCH}_{3}\right), 3.86\left(\mathrm{~s}, 3 \mathrm{H}, \mathrm{OCH}_{3}\right), 3.87$

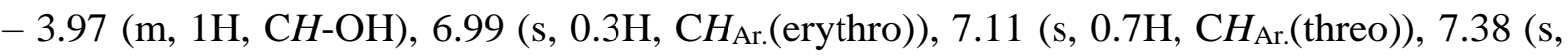
$0.7 \mathrm{H}, \mathrm{C} H_{\text {Ar. }}($ threo) $), 7.39$ (s, $0.3 \mathrm{H}, \mathrm{CH}_{\text {Ar. }}$ (erythro).

${ }^{13} \mathrm{C}-\mathrm{NMR}\left(126 \mathrm{MHz}, \mathrm{DMSO}-d_{6}\right) ; \delta[\mathrm{ppm}]=16.0\left(\mathrm{CH}_{3}\right), 22.1\left(\mathrm{CH}_{3}\right), 48.6(\mathrm{CH}), 56.0(2 \mathrm{C}, \mathrm{O}-$ $\left.C \mathrm{H}_{3}\right), 72.3(C \mathrm{H}), 108.1\left(C_{\mathrm{Ar}}\right), 112.0\left(C_{\mathrm{Ar}}\right), 129.9\left(C_{\mathrm{Ar} .}\right), 140.5\left(C_{\mathrm{Ar} .}\right), 147.1\left(C_{\mathrm{Ar}}\right), 152.7\left(C_{\mathrm{Ar} .}\right)$. MS (ESI): $\mathrm{m} / \mathrm{z}(\%)=278.1(100)[\mathrm{M}+\mathrm{H}]^{+}, 533.2(10)[2 \mathrm{M}+\mathrm{Na}]^{+}$.

HRMS (ESI): calc. for $\mathrm{C}_{12} \mathrm{H}_{17} \mathrm{NO}_{3} \mathrm{Na}\left([\mathrm{M}+\mathrm{Na}]^{+}\right)$: 278.0999, found: 278.1003 . 


\section{Synthesis of Fmoc-Asp(DMNPB)-O ${ }^{t} \mathrm{Bu}(89)$}

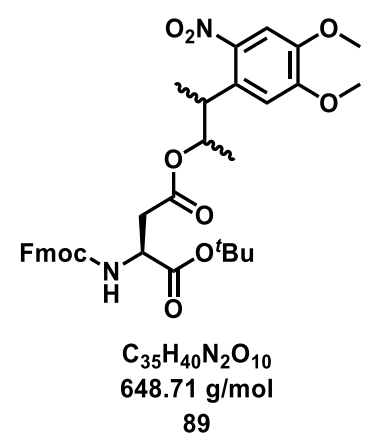

The synthesis was performed according to a protocol modified by SCHIRMACHER. ${ }^{[275]}$ 3-(4,5-Dimethoxy-2-nitrophenyl)butan-2-ol (0.97 g, $3.81 \mathrm{mmol}, 1.00 \mathrm{eq})$ (88) was dissolved in anhydrous DCM $(30 \mathrm{~mL})$ under argon atmosphere. Following, Fmoc-Asp-O ${ }^{t} \mathrm{Bu}(1.57 \mathrm{~g}$, $3.81 \mathrm{mmol}, 1.00 \mathrm{eq}), \mathrm{EDC} \cdot \mathrm{HCl}(0.80 \mathrm{~g}, 4.19 \mathrm{mmol}, 1.10 \mathrm{eq})$ as well as DMAP $(0.23 \mathrm{~g}$, $1.91 \mathrm{mmol}, 0.50 \mathrm{eq}$ ) were added and the mixture was stirred at rt for $24 \mathrm{~h}$. Afterwards, sat. $\mathrm{NaHCO}_{3}$ solution $(100 \mathrm{~mL})$ was added and the aqueous layer was extracted with EtOAc $(3 \mathrm{x}$ $50 \mathrm{~mL})$. The combined organic layers were washed with saturated brine $(3 \times 50 \mathrm{~mL})$ and were dried over $\mathrm{Na}_{2} \mathrm{SO}_{4}$. The solvent was removed under reduced pressure and crude product was purified using flash column chromatography ( $n$-pentane/EtOAc 1:0.2, $v / v)$. The product 89 (1.16 g, $1.79 \mathrm{mmol}, 47 \%)$ was obtained as a colourless solid.

${ }^{1} \mathbf{H}-\mathbf{N M R}\left(300 \mathrm{MHz}, \mathrm{DMSO}-d_{6}\right) ; \delta[\mathrm{ppm}]=0.96-1.15\left(\mathrm{~m}, 3 \mathrm{H}, \mathrm{O}-\mathrm{CH}-\mathrm{CH}_{3}\right), 1.27\left(\mathrm{~d},{ }^{3} J_{\mathrm{H}, \mathrm{H}}=\right.$ $\left.7.0 \mathrm{~Hz}, 3 \mathrm{H}, \mathrm{CH}_{3}\right), 1.91$ (s, 9H, 3 x CH$), 3.48-3.57$ (m, 2H, $\left.\beta-\mathrm{CH}_{2}\right), 3.77-3.91(\mathrm{~m}, 7 \mathrm{H}, 2 \mathrm{x}$ $\left.\mathrm{O}-\mathrm{CH}_{3}, \mathrm{CH}\right), 4.16-4.44$ (m, 4H, $\alpha-\mathrm{CH}$, Fmoc-CH, Fmoc-CH $\mathrm{CH}_{2}, 5.05-5.15$ (m, 1H, CH), 7.06 $-7.10\left(\mathrm{~m}, 1 \mathrm{H}, \mathrm{C} H_{\mathrm{Ar}}\right), 7.31\left(\mathrm{t},{ }^{3} J_{\mathrm{H}, \mathrm{H}}=8.3 \mathrm{~Hz}, 2 \mathrm{H}, \mathrm{Fmoc}-\mathrm{C} H_{\mathrm{Ar}}\right), 7.37-7.48$ (m, 3H, Fmoc$\left.\mathrm{C}_{\text {Ar. }}, \mathrm{CH}_{\mathrm{Ar}}\right), 7.63-7.72\left(\mathrm{~m}, 3 \mathrm{H}, \mathrm{Fmoc}-\mathrm{CH}_{\mathrm{Ar}}, \mathrm{N} H\right), 7.89\left(\mathrm{~d},{ }^{3} J_{\mathrm{H}, \mathrm{H}}=7.5 \mathrm{~Hz}, 2 \mathrm{H}, \mathrm{Fmoc}-\mathrm{C} H_{\mathrm{Ar}}\right)$. ${ }^{13}$ C-NMR $\left(126 \mathrm{MHz}, \mathrm{DMSO}-d_{6}\right) ; \delta[\mathrm{ppm}]=17.3\left(\mathrm{CH}_{3}\right), 17.9\left(\mathrm{CH}_{3}\right), 27.4\left(3 \mathrm{C}, \mathrm{OC}\left(\mathrm{CH}_{3}\right)_{3}\right)$, $36.0\left(\beta-\mathrm{CH}_{2}\right), 37.7\left(\mathrm{CH}-\mathrm{CH}_{3}\right), 46.5(\mathrm{Fmoc}-\mathrm{CH}), 50.9(\alpha-\mathrm{CH}), 55.9\left(\mathrm{O}-\mathrm{CH}_{3}\right), 56.1\left(\mathrm{O}-\mathrm{CH}_{3}\right)$,

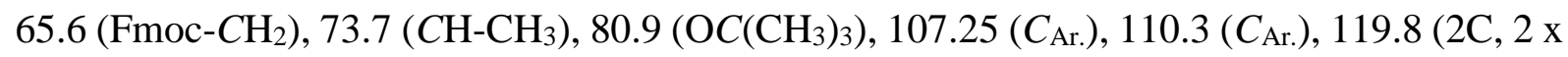
$\left.C_{\text {Ar. }}\right), 124.9$ (2C, 2 x $\left.C_{\text {Ar. }}\right), 126.8$ (2C, 2 x $\left.C_{\text {Ar. }}\right), 127.4\left(2 \mathrm{C}, 2\right.$ x $\left.C_{\text {Ar. }}\right), 130.6\left(C_{\text {Ar. }}\right), 140.5(2 \mathrm{C}, 2$ x $\left.C_{\text {Ar. }}\right), 142.5$ ( $\left.C_{\text {Ar. }}\right), 143.5$ (2C, 2 x $\left.C_{\text {Ar. }}\right), 146.8$ ( $\left.C_{\text {Ar. }}\right), 151.9\left(C_{\text {Ar. }}\right), 155.5$ (Fmoc- $\left.C O\right), 168.8$ $\left(\mathrm{CO}_{2}{ }^{t} \mathrm{Bu}\right), 169.9(\delta$-CO)

MS (ESI): $\mathrm{m} / \mathrm{z}(\%)=671.3(100)[\mathrm{M}+\mathrm{Na}]^{+}, 1319.5(60)[2 \mathrm{M}+\mathrm{Na}]^{+}$. 
HRMS (ESI): calc. for $\mathrm{C}_{35} \mathrm{H}_{41} \mathrm{~N}_{2} \mathrm{O}_{10}\left([\mathrm{M}+\mathrm{H}]^{+}\right): 649.2756$, found: 649.2742 calc. for $\mathrm{C}_{35} \mathrm{H}_{40} \mathrm{~N}_{2} \mathrm{O}_{10} \mathrm{Na}\left([\mathrm{M}+\mathrm{Na}]^{+}\right): 671.2575$, found: 671.2561 , calc. for $\mathrm{C}_{35} \mathrm{H}_{40} \mathrm{~N}_{2} \mathrm{O}_{10} \mathrm{~K}\left([\mathrm{M}+\mathrm{K}]^{+}\right)$: 687.2315, found: 687.2306. 


\section{Synthesis of Fmoc-Asp(DMNPB)-OH (90)}

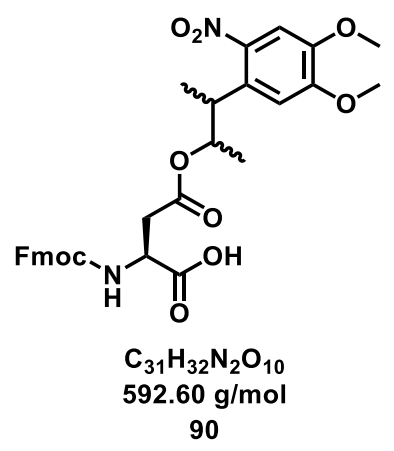

The synthesis was performed according to a protocol modified by SCHIRMACHER et al. ${ }^{[275]}$ Fmoc-Asp(DMNPB)-O ${ }^{t} \mathrm{Bu}(1.16 \mathrm{~g}, 1.79 \mathrm{mmol}, 1.00 \mathrm{eq})$ (89) was dissolved in TFA/DCM $(35 \mathrm{~mL}, 35 \mathrm{~mL}, v / v)$ and was stirred for $2 \mathrm{~h}$ at $\mathrm{rt}^{\left[{ }^{[263]}\right.}$ Following, the solvent was removed in a nitrogen stream and dried under reduced pressure. The crude product was purified by flash column chromatography ( $n$-pentane/EtOAc/AcOH 1:0.2:0.01, $v / v / v)$. The product $90(0.94 \mathrm{~g}$, $1.58 \mathrm{mmol}, 89 \%$ ) was obtained as a colourless solid.

${ }^{1} \mathbf{H}-\mathbf{N M R}\left(300 \mathrm{MHz}, \mathrm{DMSO}-d_{6}\right) ; \delta[\mathrm{ppm}]=0.96-1.10(\mathrm{~m}, 3 \mathrm{H}, \mathrm{O}-\mathrm{CH}-\mathrm{CH}), 1.27\left(\mathrm{~d},{ }^{3} J_{\mathrm{H}, \mathrm{H}}=\right.$ $\left.7.0 \mathrm{~Hz}, 3 \mathrm{H}, \mathrm{CH}_{3}\right), 2.53-2.61\left(\mathrm{~m}, 2 \mathrm{H}, \beta-\mathrm{CH}_{2}\right), 3.76-3.91\left(\mathrm{~m}, 7 \mathrm{H}, \mathrm{CH}, 2 \times \mathrm{O}-\mathrm{CH}_{3}\right), 4.15-$ 4.35 (m, 4H, Fmoc-CH, Fmoc-CH2, $\alpha-C H), 5.05-5.15$ (m. 1H, O-CH), 7.08 (d, ${ }^{4} J_{\mathrm{H}, \mathrm{H}}=4.5 \mathrm{~Hz}$, $\left.1 \mathrm{H}, \mathrm{C} H_{\text {Ar. }}\right), 7.28-7.35$ (m, 2H, Fmoc-CHAr. $), 7.37-7.48$ (m, 3H, Fmoc-CH $\left.H_{\text {Ar. }}, \mathrm{CH}_{\text {Ar. }}\right), 7.60-$ 7.75(m, 3H, Fmoc-C $\left.H_{\text {Ar. }}, \mathrm{N} H\right), 7.89\left(\mathrm{~d},{ }^{3} \mathrm{~J}_{\mathrm{H}, \mathrm{H}}=7.5 \mathrm{~Hz}, 2 \mathrm{H}\right.$, Fmoc-C $\left.H_{\text {Ar. }}\right)$.

${ }^{13}$ C-NMR $\left(126 \mathrm{MHz}, \mathrm{DMSO}-d_{6}\right) ; \delta[\mathrm{ppm}]=14.0\left(\mathrm{CH}_{3}\right), 17.3\left(\mathrm{CH}_{3}\right), 36.0\left(\mathrm{CH}_{2}\right), 37.7(\mathrm{CH})$, 46.6 (Fmoc-CH), 55.8 (2 x OCH3), $60.0(\alpha-\mathrm{CH}), 65.5\left(\right.$ Fmoc- $\left.\mathrm{CH}_{2}\right), 73.4(\mathrm{CH}), 107.3\left(\mathrm{CH}_{\text {Arom. }}\right)$, 110.4 ( $\left.C_{\text {Ar. }}\right), 119.8\left(2 \mathrm{C}, 2 \times C_{\text {Ar. }}\right), 124.9$ (2C, 2 x $\left.C_{\text {Ar. }}\right), 126.8\left(2 \mathrm{C}, 2 \times C_{\text {Ar.. }}\right), 127.4\left(2 \mathrm{C}, 2 \times C_{\text {Ar. }}\right)$, 130.6 ( $\left.C_{\text {Arom. }}\right), 140.4$ (2C, 2 x $\left.C_{\text {Ar. }}\right), 142.51$ ( $\left.C_{\text {Ar. }}\right), 143.5$ (2C, 2 x $\left.C_{\text {Ar. }}\right), 146.7\left(C_{\text {Ar. }}\right), 149.4\left(C_{\text {Ar. }}\right)$, 151.9 (Fmoc-CO), $168.8(\delta-\mathrm{CO}), 169.3\left(\mathrm{CO}_{2} \mathrm{H}\right)$

MS (ESI): $\mathrm{m} / \mathrm{z}(\%)=615.2(100)[\mathrm{M}+\mathrm{Na}]^{+}, 1207.4(26)[2 \mathrm{M}+\mathrm{Na}]^{+}$.

HRMS (ESI): calc. for $\mathrm{C}_{31} \mathrm{H}_{32} \mathrm{~N}_{2} \mathrm{O}_{10} \mathrm{Na}\left([\mathrm{M}+\mathrm{Na}]^{+}\right)$: 615.1949, found: 615.1948, calc. for $\mathrm{C}_{31} \mathrm{H}_{31} \mathrm{~N}_{2} \mathrm{O}_{10}\left([\mathrm{M}-\mathrm{H}]^{-}\right)$: 591.1984, found: 591.1977. 


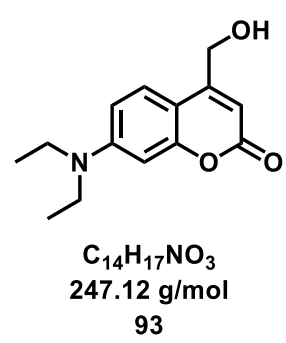

The synthesis was performed according to a protocol modified by ZHANG et al. ${ }^{[276]}$ A solution of 7-dimethylamino-4-methylcoumarin (4.21 g, $18.2 \mathrm{mmol}, 1.00 \mathrm{eq}$.) (92) and selenium dioxide $(2.10 \mathrm{~g}, 18.9 \mathrm{mmol}, 1.10 \mathrm{eq})$ in $p$-xylene $(350 \mathrm{~mL})$ was stirred at reflux for $48 \mathrm{~h}$. Following, the reaction mixture was filtered and the solvent was removed under reduced pressure. The residue was dissolved in methanol $(350 \mathrm{~mL})$ and sodium borohydride $(0.68 \mathrm{~g}$, $18.2 \mathrm{mmol}, 1.00 \mathrm{eq}$ ) was added slowly added to the solution. The mixture was stirred at $\mathrm{rt}$ for $4 \mathrm{~h}$ and was neutralized with by addition of $\mathrm{HCl}(1 \mathrm{M})$. The aqueous layer was extracted with $\operatorname{DCM}(3 \times 100 \mathrm{~mL})$ and the combined organic layers were washed with brine $(3 \times 50 \mathrm{~mL})$. After drying over $\mathrm{Na}_{2} \mathrm{SO}_{4}$, the solvent was removed under reduced pressure and the crude product was purified by flash column chromatography (DCM/acetone 1:0.04 $\rightarrow$ 1:0.2,v/v). The product $93(2.02 \mathrm{~g}, 8.17 \mathrm{mmol}, 44 \%)$ was obtained as an orange solid.

${ }^{1} \mathbf{H}-\mathbf{N M R}\left(300 \mathrm{MHz}, \mathrm{DMSO}-d_{6}\right) ; \delta[\mathrm{ppm}]=1.12\left(\mathrm{t},{ }^{3} J_{\mathrm{H}, \mathrm{H}}=7.0 \mathrm{~Hz}, 6 \mathrm{H}, 2 \times \mathrm{CH}_{3}\right), 3.42\left(\mathrm{q},{ }^{3} J_{\mathrm{H}, \mathrm{H}}\right.$ $\left.=7.3 \mathrm{~Hz}, 4 \mathrm{H}, 2 \times \mathrm{CH}_{2}\right), 4.65\left(\mathrm{~d},{ }^{4} \mathrm{~J}=1.5 \mathrm{~Hz}, 2 \mathrm{H}, \mathrm{CH}_{2}-\mathrm{OH}\right), 5.54(\mathrm{~s}, 1 \mathrm{H}, \mathrm{OH}), 6.05-6.09(\mathrm{~m}$, $1 \mathrm{H}, \mathrm{CH}), 6.52\left(\mathrm{~d},{ }^{4} J_{\mathrm{H}, \mathrm{H}}=2.5 \mathrm{~Hz}, 1 \mathrm{H}, \mathrm{CH} H_{\mathrm{Ar}}\right), 6.67\left(\mathrm{dd},{ }^{3} J_{\mathrm{H}, \mathrm{H}}=9.0 \mathrm{~Hz},{ }^{4} J_{\mathrm{H}, \mathrm{H}}=2.6 \mathrm{~Hz}, 1 \mathrm{H}\right.$, $\left.\mathrm{C} H_{\text {Ar. }}\right), 7.43\left(\mathrm{~d},{ }^{3} J_{\mathrm{H}, \mathrm{H}}=9.0 \mathrm{~Hz}, 1 \mathrm{H}, \mathrm{C} H_{\mathrm{Ar}}\right)$.

${ }^{13}$ C-NMR (126 MHz, DMSO- $\left.d_{6}\right) ; \delta[\mathrm{ppm}]=12.3\left(2 \mathrm{C}, 2 \times \mathrm{CH}_{3}\right), 44.0\left(2 \mathrm{C}, 2 \times \mathrm{CH}_{2}\right), 59.1\left(\mathrm{CH}_{2-}\right.$ OH $), 96.8\left(C_{\text {Ar. }}\right), 103.9\left(C_{\text {Ar. }}\right), 105.7\left(C_{\text {Ar. }}\right), 108.6(C H-C O), 125.1\left(C_{\text {Ar. }}\right), 150.2\left(C-\mathrm{CH}_{2}\right), 155.6$ $\left(C_{\mathrm{Ar}}\right), 156.9\left(C_{\mathrm{Ar}}\right), 161.2(C=\mathrm{O})$.

MS (ESI): m/z $(\%)=248.1(86)[\mathrm{M}+\mathrm{H}]^{+}, 270.1(40)[\mathrm{M}+\mathrm{Na}]^{+}, 495.3(11)[2 \mathrm{M}+\mathrm{H}]^{+}, 517.3$ (100) $[2 \mathrm{M}+\mathrm{Na}]^{+}, 764.4(40)[3 \mathrm{M}+\mathrm{Na}]^{+}$.

HRMS (ESI): calc. for $\mathrm{C}_{14} \mathrm{H}_{18} \mathrm{NO}_{3}\left([\mathrm{M}+\mathrm{H}]^{+}\right)$: 248.1281, found: 248.1285; calc. for $\mathrm{C}_{14} \mathrm{H}_{17} \mathrm{NO}_{3} \mathrm{Na}\left([\mathrm{M}+\mathrm{Na}]^{+}\right)$: 270.1101, found: 270.1102 . 


\section{Synthesis of (7-(Diethylamino)-2-oxo-2H-chromen-4-yl)methyl (4-nitrophenyl) carbonate (94)}

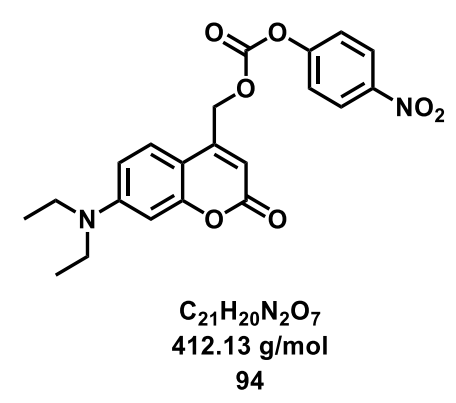

The synthesis was performed according to a protocol modified by KoTZUR et al. ${ }^{[269]}$ 7-(Diethylamino)-4-(hydroxymethyl)-2H-chromen-2-one (0.87 g, $3.52 \mathrm{mmol}, 1.00 \mathrm{eq})$ (93) was dissolved in DCM $(25 \mathrm{~mL})$ and cooled to $0{ }^{\circ} \mathrm{C}$. Afterwards $p$-nitrophenyl chloroformate (1.49 g, $7.39 \mathrm{mmol}, 2.10 \mathrm{eq})$ (80), dimethylaminopyridine (0.43 g, $3.52 \mathrm{mmol}, 1.00 \mathrm{eq})$ as well as DIPEA $(1.31 \mathrm{~mL}, 7.50 \mathrm{mmol}, 1.50 \mathrm{eq}, \delta=0.74 \mathrm{~g} / \mathrm{mL})$ were added and the reaction mixture was stirred for $12 \mathrm{~h}$ at $\mathrm{rt}$ under light exclusion. After removal of the solvent under reduced pressure, the residue was dissolved in EtOAc $(100 \mathrm{~mL})$ and washed with $1 \mathrm{M} \mathrm{H}_{3} \mathrm{PO}_{4}(3 \mathrm{x}$ $50 \mathrm{~mL}$ ). The organic layer was collected and dried over $\mathrm{Na}_{2} \mathrm{SO} 4$. The crude product was purified by flash column chromatography (DCM/acetone 1:0.04 $\rightarrow$ DCM/acetone 1:0.2, v/v) and the product 94 (0.85 g, $2.06 \mathrm{mmol}, 59 \%)$ was obtained as an orange solid.

${ }^{1} \mathbf{H}-\mathbf{N M R}\left(300 \mathrm{MHz}, \mathrm{DMSO}-d_{6}\right) ; \delta[\mathrm{ppm}]=1.13\left(\mathrm{t},{ }^{3} \mathrm{~J}_{\mathrm{H}, \mathrm{H}}=7.0 \mathrm{~Hz}, 6 \mathrm{H}, 2 \times \mathrm{CH}_{3}\right), 3.45\left(\mathrm{q},{ }^{3} J_{\mathrm{H}, \mathrm{H}}\right.$ $\left.=7.0 \mathrm{~Hz}, 4 \mathrm{H}, 2 \times \mathrm{CH}_{2}\right), 5.50\left(\mathrm{~s}, 2 \mathrm{H}, \mathrm{CH}_{2}-\mathrm{OH}\right), 6.12\left(\mathrm{~s}, 1 \mathrm{H}, \mathrm{CH}_{\mathrm{Ar}}\right), 6.57\left(\mathrm{~d},{ }^{4} J_{\mathrm{H}, \mathrm{H}}=2.5 \mathrm{~Hz}, 1 \mathrm{H}\right.$, $\left.\mathrm{C} H_{\mathrm{Ar}}\right), 6.73\left(\mathrm{dd},{ }^{3} J_{\mathrm{H}, \mathrm{H}}=9.1 \mathrm{~Hz},{ }^{4} J_{\mathrm{H}, \mathrm{H}}=2.7 \mathrm{~Hz}, 1 \mathrm{H}, \mathrm{C} H_{\mathrm{Ar}}\right), 7.54\left(\mathrm{~d},{ }^{3} J_{\mathrm{H}, \mathrm{H}}=9.0 \mathrm{~Hz}, 1 \mathrm{H}, \mathrm{C} H_{\mathrm{Ar}}\right)$, $7.60-7.67\left(\mathrm{~m}, 2 \mathrm{H}, \mathrm{C} H_{\text {Ar. }}\right), 8.31-8.39$ (m, 2H, $\left.\mathrm{CH}_{\text {Ar. }}\right)$.

${ }^{13}$ C-NMR (126 MHz, DMSO- $\left.d_{6}\right) ; \delta[\mathrm{ppm}]=12.3\left(2 \mathrm{C}, 2 \times \mathrm{CH}_{3}\right), 44.0\left(2 \mathrm{C}, 2 \times \mathrm{CH}_{2}\right), 65.9\left(\mathrm{CH}_{2}-\right.$ O), 96.9 ( $\left.C_{\text {Ar. }}\right), 105.5$ ( $\left.C_{\text {Ar. }}\right), 105.9$ ( $\left.C_{\text {Ar. }}\right), 108.8$ (CH-CO), $122.6\left(2 \mathrm{C}, 2\right.$ x $\left.C_{\text {Ar. }}\right), 125.4$ (2C. 2 x $\left.C_{\mathrm{Ar}}\right), 126.2$ ( $\left.C_{\mathrm{Ar}}\right), 145.3\left(C_{\mathrm{Ar}}\right), 148.9\left(C-\mathrm{CH}_{2}\right), 150.2\left(C_{\mathrm{Ar}}\right), 151.6(\mathrm{O}-C=\mathrm{O}), 155.11\left(C_{\mathrm{Ar}}\right)$, $155.85\left(C_{\text {Ar. }}\right), 160.5(C=\mathrm{O})$.

MS (ESI): $\mathrm{m} / \mathrm{z}(\%)=413.1(100)[\mathrm{M}+\mathrm{H}]^{+}, 847.3(40)[2 \mathrm{M}+\mathrm{Na}]^{+}$.

HRMS (ESI): calc. for $\mathrm{C}_{21} \mathrm{H}_{21} \mathrm{~N}_{2} \mathrm{O}_{7}\left([\mathrm{M}+\mathrm{H}]^{+}\right)$: 413.1343, found: 413.1334;

calc. for $\mathrm{C}_{21} \mathrm{H}_{21} \mathrm{~N}_{2} \mathrm{O}_{7} \mathrm{Na}\left([\mathrm{M}+\mathrm{Na}]^{+}\right)$: 435.1163, found: 435.1152 . 


\section{Synthesis of Fmoc-Lys(DEACM)-OH (95)}

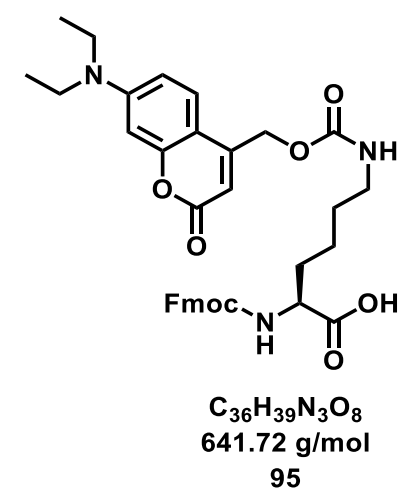

The synthesis was performed according to a protocol modified by SCHIRMACHER et al. ${ }^{[269]}$ (7-(Diethylamino)-2-oxo-2H-chromen-4-yl)methyl (4-nitrophenyl) carbonate (0.56 g, $1.36 \mathrm{mmol}, 1.00 \mathrm{eq})(\mathbf{9 4})$ was dissolved in a mixture of DCM/DMF $(15 \mathrm{~mL}, 15 \mathrm{~mL}, v / v)$ and was transferred to a solution of Fmoc-Lys-OH $(0.50 \mathrm{~g}, 1.36 \mathrm{mmol}, 1.00 \mathrm{eq})$ in in toluene $(40 \mathrm{~mL})$. The reaction mixture was cooled to $0{ }^{\circ} \mathrm{C}$ and DIPEA $(0.24 \mathrm{~mL}, 1.36 \mathrm{mmol}, 1.00 \mathrm{eq}$, $\delta=0.74 \mathrm{~g} / \mathrm{mL}$ ) was added. The solution was stirred for $24 \mathrm{~h}$ at $\mathrm{rt}$ under exclusion of light. Afterwards, the solvent was removed under reduced pressure and crude product was purified by flash column chromatography (DCM/MeOH/AcOH 1:0.05:0.01, v/v/v). The product 95 $(0.47 \mathrm{~g}, 0.73 \mathrm{mmol}, 54 \%)$ was obtained as a yellow solid.

${ }^{\mathbf{1}} \mathbf{H}-\mathbf{N M R}\left(300 \mathrm{MHz}, \mathrm{DMSO}-d_{6}\right) ; \delta[\mathrm{ppm}]=1.11\left(\mathrm{t},{ }^{3} \mathrm{~J}_{\mathrm{H}, \mathrm{H}}=7.00 \mathrm{~Hz}, 6 \mathrm{H}, 2 \times \mathrm{CH}_{3}\right), 1.28-1.79$ $\left(\mathrm{m}, 6 \mathrm{H}, \beta-\mathrm{CH}_{2}, \gamma-\mathrm{CH}_{2}, \delta-\mathrm{CH}_{2}\right), 3.00-3.03\left(\mathrm{~m}, 2 \mathrm{H}, \varepsilon-\mathrm{CH}_{2}\right), 3.42\left(\mathrm{q},{ }^{3} J_{\mathrm{H}, \mathrm{H}}=7.00 \mathrm{~Hz}, 4 \mathrm{H}, 2 \mathrm{x}\right.$ $\mathrm{CH}_{2}-\mathrm{CH}_{3}$ ), 3.86-3.96 (m, 1H, $\left.\alpha-\mathrm{CH}\right), 4.17-4.36$ (m, 3H, Fmoc- $\mathrm{CH}_{2}, \mathrm{Fmoc}-\mathrm{CH}$ ), 5.20 (s, 2H, O$\left.\mathrm{CH}_{2}\right), 5.97\left(\mathrm{~s}, 1 \mathrm{H}, \mathrm{C} H_{\text {Arom }}\right), 6.53\left(\mathrm{~d},{ }^{4} J_{\mathrm{H}, \mathrm{H}}=2.50 \mathrm{~Hz}, 1 \mathrm{H}, \mathrm{C} H_{\text {Arom }}\right), 6.67\left(\mathrm{dd},{ }^{3} J_{\mathrm{H}, \mathrm{H}}=9.10 \mathrm{~Hz}\right.$, ${ }^{4} J_{\mathrm{H}, \mathrm{H}}=2.50 \mathrm{~Hz}, 1 \mathrm{H}, \mathrm{C} H_{\text {Arom. }}$ ), $7.29-7.33\left(\mathrm{~m}, 2 \mathrm{H}, \mathrm{C} H_{\text {Arom. }}\right), 7.38-7.46$ (m, 3H, $\mathrm{CH}_{\text {Arom. }}$ ), 7.49$7.58\left(\mathrm{~m}, 2 \mathrm{H}, \mathrm{C} H_{\text {Arom. }}, \mathrm{NH}\right), 7.88\left(\mathrm{~d},{ }^{3} J_{\mathrm{H}, \mathrm{H}}=7.60 \mathrm{~Hz}, 2 \mathrm{H}, \mathrm{C} H_{\text {Arom }}\right), 7.89\left(\mathrm{~d},{ }^{3} J_{\mathrm{H}, \mathrm{H}}=7.60 \mathrm{~Hz}, 2 \mathrm{H}\right.$, ( $\left.H_{\text {Arom. }}\right)$.

${ }^{13}$ C-NMR $\left(126 \mathrm{MHz}, \mathrm{DMSO}-d_{6}\right) ; \delta[\mathrm{ppm}]=12.8\left(2 \times \mathrm{CH}_{3}\right), 23.3\left(\gamma-\mathrm{CH}_{2}\right), 29.4\left(\delta-\mathrm{CH}_{2}\right), 31.0$ $\left(\beta-\mathrm{CH}_{2}\right), 44.4$ (3C, $\left.2 \times \mathrm{CH}_{2}, \varepsilon-\mathrm{CH}_{2}\right), 47.1$ (Fmoc- $\left.\mathrm{CH}\right), 54.4(\alpha-\mathrm{CH}), 61.3\left(\mathrm{CH}_{2}-\mathrm{O}\right), 66.0(\mathrm{Fmoc}-$ $\left.C \mathrm{H}_{2}\right), 97.3$ ( $\left.C_{\text {Ar. }}\right), 104.9$ ( $\left.C_{\text {Ar. }}\right), 105.7$ ( $\left.C_{\text {Ar. }}\right), 109.2$ (C-CO), $120.0\left(C_{\text {Ar. }}\right), 120.5$ (2C, 2 x $\left.C_{\text {Ar. }}\right)$, 125.7 (2C, 2 x $\left.C_{\mathrm{Ar}}\right), 127.5$ (2C, 2 x $\left.C_{\mathrm{Ar}}\right), 128.1$ (2C, 2 x $\left.C_{\mathrm{Ar}}\right), 141.15$ (2C, 2 x $\left.C_{\mathrm{Ar}}\right), 144.3(2 \mathrm{C}$, $\left.2 \times C_{\text {Ar. }}\right), 150.9\left(C_{\text {Ar. }}\right), 152.4\left(C_{\text {Ar. }}\right), 155.9\left(C_{\text {Ar. }}\right), 156.2(\mathrm{O}-C \mathrm{O}), 156.5(C \mathrm{O}-\mathrm{NH}), 161.2(C=\mathrm{O})$, $174.4(\mathrm{COOH})$.

MS (ESI): $\mathbf{m} / \mathbf{z}(\%)=642.3(14)[\mathrm{M}+\mathrm{H}]^{+}, 664.3(100)[\mathrm{M}+\mathrm{Na}]^{+}, 1305.5(11)[2 \mathrm{M}+\mathrm{Na}]^{+}$. 
HRMS (ESI): calc. for: $\mathrm{C}_{36} \mathrm{H}_{40} \mathrm{~N}_{3} \mathrm{O}_{8}\left([\mathrm{M}+\mathrm{H}]^{+}\right): 642.2810$, found.: 642.2808, calc.: for $\mathrm{C}_{36} \mathrm{H}_{39} \mathrm{~N}_{3} \mathrm{O}_{8} \mathrm{Na}\left([\mathrm{M}+\mathrm{Na}]^{+}\right)$: 664.2629 , found.: 664.2624 . 
Synthesis of a Nvoc-protected test peptide (84)

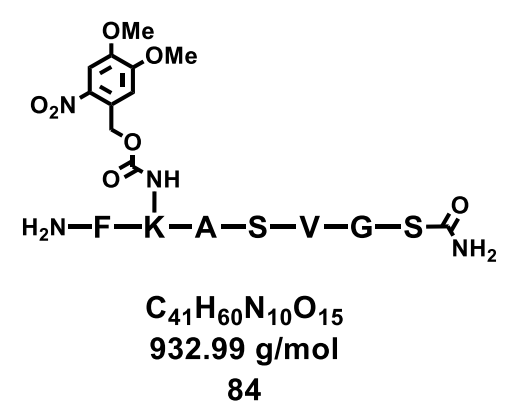

The peptide was synthesised on a Rink Amide MBHA resin $(25.0 \mu \mathrm{mol}, 0.76 \mathrm{mmol} / \mathrm{g})$. Fmoc$\operatorname{Ser}\left({ }^{t} \mathrm{Bu}\right)-\mathrm{OH}$ was coupled as first amino acid according to SOP9. The peptide was synthesised with automated SPPS following SOP11. The cleavage of the peptide from the resin was performed as described in SOP12 (cleavage mixture B). The crude peptide was purified by semipreparative HPLC to yield the product $84(2.43 \mathrm{mg}, 2.57 \mu \mathrm{mol})$ as a yellow solid.

HPLC: $(250 \cdot 10 \mathrm{~mm}, 5 \mu \mathrm{m}, \mathrm{C} 18$, Gradient $=0-50 \%$ B in $30 \mathrm{~min}, \lambda[\mathrm{nm}]: 215,254,280)$ : $t_{\mathrm{R}}=26.1 \mathrm{~min}$.

MS (ESI): $\mathbf{m} / \mathbf{z}(\%)=467.2(50)[\mathrm{M}+2 \mathrm{H}]^{2+}, 933.5(53)[\mathrm{M}+\mathrm{H}]^{+}$.

HRMS (ESI): calc. for: $\mathrm{C}_{41} \mathrm{H}_{61} \mathrm{~N}_{10} \mathrm{O}_{15}: 933.4312$ found: $933.4310[\mathrm{M}+\mathrm{H}]^{+}$, calc. for: $\mathrm{C}_{41} \mathrm{H}_{62} \mathrm{~N}_{10} \mathrm{O}_{15}: 467.2193$, found: $467.2185[\mathrm{M}+2 \mathrm{H}]^{2+}$. 
Synthesis of a DMNPB-protected test peptide (91)

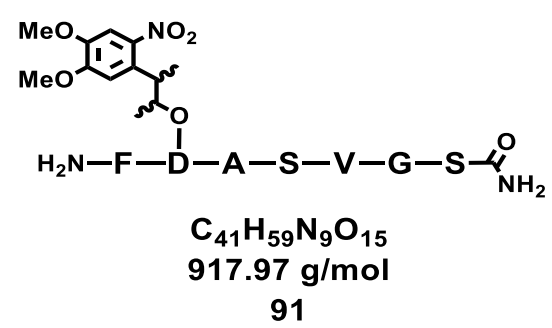

The peptide was synthesised on a Rink Amide MBHA resin $(25.0 \mu \mathrm{mol}, 0.76 \mathrm{mmol} / \mathrm{g})$. Fmoc$\operatorname{Ser}\left({ }^{t} \mathrm{Bu}\right)-\mathrm{OH}$ was coupled as first amino acid according to SOP9. The peptide was synthesised with automated SPPS following SOP11. The cleavage of the peptide from the resin was performed as described in SOP12 (cleavage mixture B). The crude peptide was purified by semipreparative HPLC to yield the product $91(1.83 \mathrm{mg}, 1.90 \mu \mathrm{mol})$ as a yellow solid

HPLC: $(250 \cdot 10 \mathrm{~mm}, 5 \mu \mathrm{m}, \mathrm{C} 18$, Gradient $=0-50 \% \mathrm{~B}$ in $30 \mathrm{~min}, \lambda[\mathrm{nm}]: 215,254,280)$ : $t_{\mathrm{R}}=27.7 \mathrm{~min}$.

MS (ESI): $\mathbf{m} / \mathbf{z}(\%)=483.3(50)[\mathrm{M}+2 \mathrm{H}]^{2+}, 918.5(74)[\mathrm{M}+\mathrm{H}]^{+}$.

HRMS (ESI): calc. for: $\mathrm{C}_{41} \mathrm{H}_{60} \mathrm{~N}_{9} \mathrm{O}_{15}$ : 918.4203 found: $918.4210[\mathrm{M}+\mathrm{H}]^{+}$, calc. for: $\mathrm{C}_{41} \mathrm{H}_{60} \mathrm{~N}_{9} \mathrm{O}_{15}: 459.7138$, found: $459.7138[\mathrm{M}+2 \mathrm{H}]^{2+}$. 
Synthesis of a DEACM-protected test peptide (96)

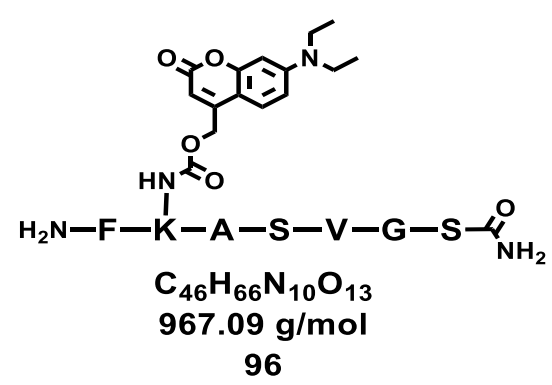

The peptide was synthesised on a Rink Amide MBHA resin $(25.0 \mu \mathrm{mol}, 0.76 \mathrm{mmol} / \mathrm{g})$. Fmoc$\operatorname{Ser}\left({ }^{t} \mathrm{Bu}\right)-\mathrm{OH}$ was coupled as first amino acid according to SOP9. The peptide was synthesised with automated SPPS following SOP11. The cleavage of the peptide from the resin was performed as described in SOP12 (cleavage mixture B). The crude peptide was purified by semipreparative HPLC to yield the product $96(2.02 \mathrm{mg}, 2.06 \mu \mathrm{mol})$ as a yellow solid

HPLC: $(250 \cdot 10 \mathrm{~mm}, 5 \mu \mathrm{m}, \mathrm{C} 18$, Gradient $=1-40 \% \mathrm{~B}$ in $30 \mathrm{~min}, \lambda[\mathrm{nm}]: 215,254,280)$ : $t_{\mathrm{R}}=20.9 \mathrm{~min}$.

MS (ESI): $\mathbf{m} / \mathbf{z}(\%)=967.5(100)[\mathrm{M}+\mathrm{H}]^{+}, 989.5(35)[\mathrm{M}+\mathrm{Na}]^{+}$.

HRMS (ESI): calc. for: $\mathrm{C}_{46} \mathrm{H}_{67} \mathrm{~N}_{10} \mathrm{O}_{13}: 967.4884$, found.: $967.4881[\mathrm{M}+\mathrm{H}]^{+}$, calc. for: $\mathrm{C}_{46} \mathrm{H}_{66} \mathrm{~N}_{10} \mathrm{O}_{13} \mathrm{Na}$ : 989.4703, found.: $989.4702[\mathrm{M}+\mathrm{Na}]^{+}$. 


\section{Synthesis of Native $\mu$-Conotoxin SIIIA by the Folding Strategy from RADZEY ${ }^{[220]}$}

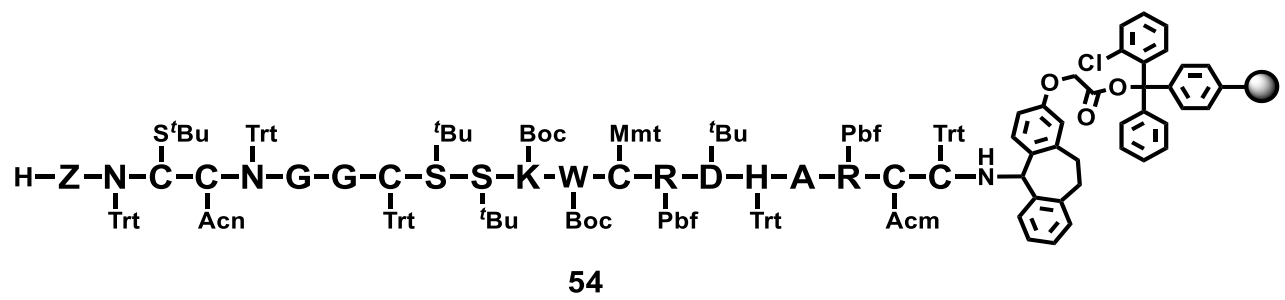

2-Chlorotrityl chloride resin $(0.70 \mathrm{~g}, 0.70 \mathrm{mmol}, 1-1.50 \mathrm{mmol} / \mathrm{g})$ was preloaded with the Ramage linker according to SOP8 and the occupancy $(0.28 \mathrm{mmol} / \mathrm{g})$ was determined following SOP10. The first amino acid Fmoc-Cys(Trt)-OH was coupled to the resin (238 mg, $0.10 \mathrm{mmol})$ as described in SOP9 and the peptide sequence was synthesised by automated SPPS following SOP11. The test cleavage of the native $\mu$-SIIIA (101) was carried out with cleavage mixture A as outlined in SOP12, yielding the Acm and $\mathrm{S}^{t} \mathrm{Bu}$ protected peptide.

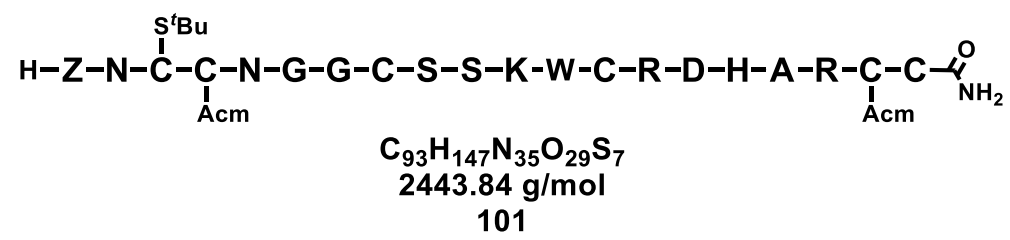

MS (ESI): $\mathbf{m} / \mathbf{z}(\%)=1222.5(18)[\mathrm{M}+2 \mathrm{H}]^{2+}, 815.3(100)[\mathrm{M}+3 \mathrm{H}]^{3+}, 611.7(50)[\mathrm{M}+4 \mathrm{H}]^{4+}$.

HRMS (ESI): calc. for: $\mathrm{C}_{93} \mathrm{H}_{149} \mathrm{~N}_{35} \mathrm{O}_{29} \mathrm{~S}_{7}: 122.4664$, found.: $1222.4666[\mathrm{M}+2 \mathrm{H}]^{2+}$, calc. for: $\mathrm{C}_{93} \mathrm{H}_{150} \mathrm{~N}_{35} \mathrm{O}_{29} \mathrm{~S}_{7}: 815.3134$, found.: $815.3130[\mathrm{M}+3 \mathrm{H}]^{3+}$, calc. for: $\mathrm{C}_{93} \mathrm{H}_{151} \mathrm{~N}_{35} \mathrm{O}_{29} \mathrm{~S}_{7}: 611.7368$, found.: $611.7363[\mathrm{M}+4 \mathrm{H}]^{4+}$. 
Synthesis of Native $\mu$-Conotoxin SIIIA: Formation of the First and Second Disulphide Bridge

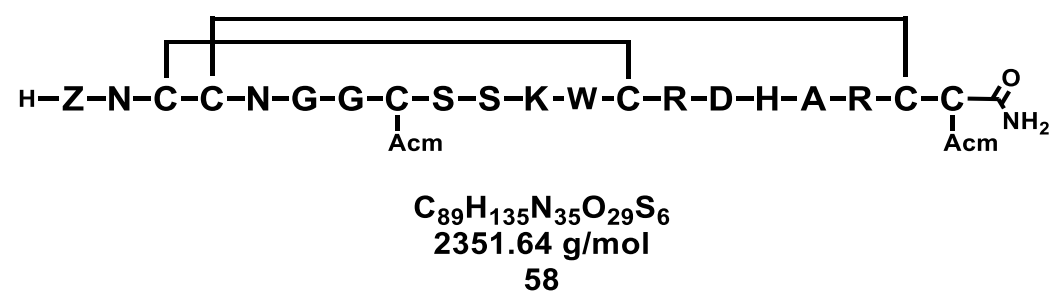

The formation of the first and second disulphide bridge was performed as described in SOP13 and SOP14. The crude peptide was purified by semipreparative HPLC and the product $\mathbf{5 8}$ (4.36 mg, $1.95 \mu \mathrm{mol}$ ) was obtained as a colourless solid.

HPLC: $(250 \cdot 10 \mathrm{~mm}, 5 \mu \mathrm{m}, \mathrm{C} 18$, Gradient $=1-40 \% \mathrm{~B}$ in $30 \mathrm{~min}, \lambda[\mathrm{nm}]: 215,254,280)$ : $t_{\mathrm{R}}=20.2 \mathrm{~min}$.

MS (ESI): $\mathbf{m} / \mathbf{z}(\%)=1176.4(22)[\mathrm{M}+2 \mathrm{H}]^{2+}, 784.6(100)[\mathrm{M}+3 \mathrm{H}]^{3+}, 588.7(11)[\mathrm{M}+4 \mathrm{H}]^{4+}$.

HRMS (ESI): calc. for: $\mathrm{C}_{89} \mathrm{H}_{137} \mathrm{~N}_{35} \mathrm{O}_{29} \mathrm{~S}_{6}: 1176.4330$, found.: $1176.4341[\mathrm{M}+2 \mathrm{H}]^{2+}$, calc. for: $\mathrm{C}_{89} \mathrm{H}_{138} \mathrm{~N}_{35} \mathrm{O}_{29} \mathrm{~S}_{6}: 784.6244$, found.: $784.6245[\mathrm{M}+3 \mathrm{H}]^{3+}$, calc. for: $\mathrm{C}_{89} \mathrm{H}_{139} \mathrm{~N}_{35} \mathrm{O}_{29} \mathrm{~S}_{6}: 588.7201$, found.: $588.7199[\mathrm{M}+4 \mathrm{H}]^{4+}$.

Synthesis of native $\mu$-conotoxin SIIIA: formation of the third disulphide bridge

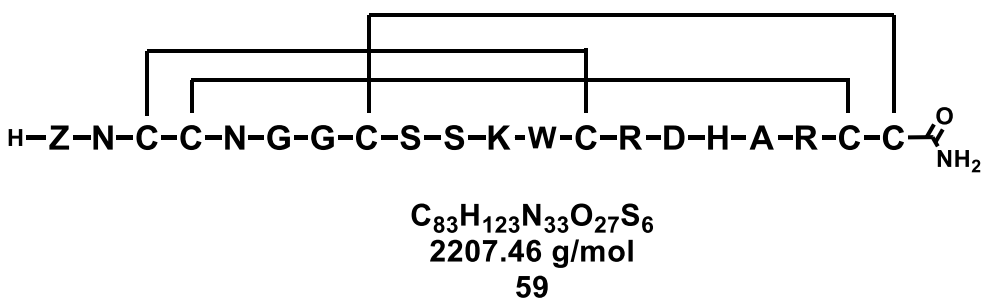

For cleavage of the Acm protecting groups and formation of the third disulphide bridge, $\mathbf{5 8}$ (4.36 mg, $1.95 \mu \mathrm{mol}, 1.00 \mathrm{eq})$ and Ac-L-Trp-OMe (5.13 mg, $19.5 \mu \mathrm{mol}, 10 \mathrm{eq})$ were dissolved in $\mathrm{AcOH} / \mathrm{H}_{2} \mathrm{O}(0.92 / 0.46, v / v)$. Following, a solution of iodine (12.3 mg, $\left.97.5 \mu \mathrm{mol}, 50.0 \mathrm{eq}\right)$, dissolved in acetic acid $(0.95 \mathrm{~mL})$, was added and the reaction mixture was stirred for $60 \mathrm{~min}$ at $\mathrm{rt}$. The reaction was quenched by addition of L-ascorbic acid (100 $\mathrm{mg}$ in $\left.0.75 \mathrm{~mL} \mathrm{H}_{2} \mathrm{O}\right)$ and 
the crude peptide was directly purified by semipreparative HPLC. Four isomers of the product 59 were obtained as a colourless solid.

1.) Isomer 59.1.1 (0.09 mg, $0.04 \mu \mathrm{mol})$

HPLC: $(250 \cdot 10 \mathrm{~mm}, 5 \mu \mathrm{m}, \mathrm{C} 18$, Gradient $=1-40 \%$ B in $30 \mathrm{~min}, \lambda[\mathrm{nm}]: 215,254,280)$ : $t_{\mathrm{R}}=19.8 \mathrm{~min}$.

MS (ESI): $\mathbf{m} / \mathbf{z}(\%)=1103.9(11)[\mathrm{M}+2 \mathrm{H}]^{2+}, 736.6(56)[\mathrm{M}+3 \mathrm{H}]^{3+}$.

HRMS (ESI): calc. for: $\mathrm{C}_{83} \mathrm{H}_{125} \mathrm{~N}_{33} \mathrm{O}_{27} \mathrm{~S}_{6}: 1103.8868$, found.: $1103.8868[\mathrm{M}+2 \mathrm{H}]^{2+}$, calc. for: $\mathrm{C}_{83} \mathrm{H}_{126} \mathrm{~N}_{33} \mathrm{O}_{27} \mathrm{~S}_{6}: 736.2603$, found.: $736.2596[\mathrm{M}+3 \mathrm{H}]^{3+}$, calc. for: $\mathrm{C}_{83} \mathrm{H}_{127} \mathrm{~N}_{33} \mathrm{O}_{27} \mathrm{~S}_{6}: 552.4470$, found.: $552.4464[\mathrm{M}+4 \mathrm{H}]^{4+}$.

2.) Isomer 59.1.2 $(0.04 \mathrm{mg}, 0.02 \mu \mathrm{mol})$

HPLC: $(250 \cdot 10 \mathrm{~mm}, 5 \mu \mathrm{m}, \mathrm{C} 18$, Gradient $=1-40 \%$ B in $30 \mathrm{~min}, \lambda[\mathrm{nm}]: 215,254,280)$ : $t_{\mathrm{R}}=20.9 \mathrm{~min}$.

MS (ESI): $\mathbf{m} / \mathbf{z}(\%)=1104.4(15)[\mathrm{M}+2 \mathrm{H}]^{2+}, 736.6(79)[\mathrm{M}+3 \mathrm{H}]^{3+}$.

HRMS (ESI): calc. for: $\mathrm{C}_{83} \mathrm{H}_{125} \mathrm{~N}_{33} \mathrm{O}_{27} \mathrm{~S}_{6}: 1103.8868$, found.: $1103.8872[\mathrm{M}+2 \mathrm{H}]^{2+}$, calc. for: $\mathrm{C}_{83} \mathrm{H}_{126} \mathrm{~N}_{33} \mathrm{O}_{27} \mathrm{~S}_{6}: 736.2603$, found.: $736.2597[\mathrm{M}+3 \mathrm{H}]^{3+}$, calc. for: $\mathrm{C}_{83} \mathrm{H}_{127} \mathrm{~N}_{33} \mathrm{O}_{27} \mathrm{~S}_{6}: 552.4470$, found.: $552.4462[\mathrm{M}+4 \mathrm{H}]^{4+}$.

3.) Isomer $\mathbf{5 9 . 1 . 3}(0.08 \mathrm{mg}, 0.04 \mu \mathrm{mol})$

HPLC: $(250 \cdot 10 \mathrm{~mm}, 5 \mu \mathrm{m}, \mathrm{C} 18$, Gradient $=1-40 \% \mathrm{~B}$ in $30 \mathrm{~min}, \lambda[\mathrm{nm}]: 215,254,280)$ : $t_{\mathrm{R}}=21.5 \mathrm{~min}$.

MS (ESI): m/z $(\%)=1104.4(15)[\mathrm{M}+2 \mathrm{H}]^{2+}, 736.6(53)[\mathrm{M}+3 \mathrm{H}]^{3+}$.

HRMS (ESI): calc. for: $\mathrm{C}_{83} \mathrm{H}_{125} \mathrm{~N}_{33} \mathrm{O}_{27} \mathrm{~S}_{6}: 1103.8868$, found.: $1103.8863[\mathrm{M}+2 \mathrm{H}]^{2+}$, calc. for: $\mathrm{C}_{83} \mathrm{H}_{126} \mathrm{~N}_{33} \mathrm{O}_{27} \mathrm{~S}_{6}: 736.2603$, found.: $736.2593[\mathrm{M}+3 \mathrm{H}]^{3+}$.

4.) Isomer 59.1.4 (0.12 mg, $0.05 \mu \mathrm{mol})$

HPLC: $(250 \cdot 10 \mathrm{~mm}, 5 \mu \mathrm{m}, \mathrm{C} 18$, Gradient $=1-40 \%$ B in $30 \mathrm{~min}, \lambda[\mathrm{nm}]: 215,254,280)$ : $t_{\mathrm{R}}=23.2 \mathrm{~min}$.

MS (ESI): $\mathbf{m} / \mathbf{z}(\%)=1104.4(6)[\mathrm{M}+2 \mathrm{H}]^{2+}, 736.6(21)[\mathrm{M}+3 \mathrm{H}]^{3+}$.

HRMS (ESI): calc. for: $\mathrm{C}_{83} \mathrm{H}_{125} \mathrm{~N}_{33} \mathrm{O}_{27} \mathrm{~S}_{6}: 1103.8868$, found.: $1103.8866[\mathrm{M}+2 \mathrm{H}]^{2+}$, calc. for: $\mathrm{C}_{83} \mathrm{H}_{126} \mathrm{~N}_{33} \mathrm{O}_{27} \mathrm{~S}_{6}: 736.2603$, found.: $736.2595[\mathrm{M}+3 \mathrm{H}]^{3+}$. 
Synthesis of Native $\mu$-Conotoxin SIIIA by the Optimised Folding Strategy

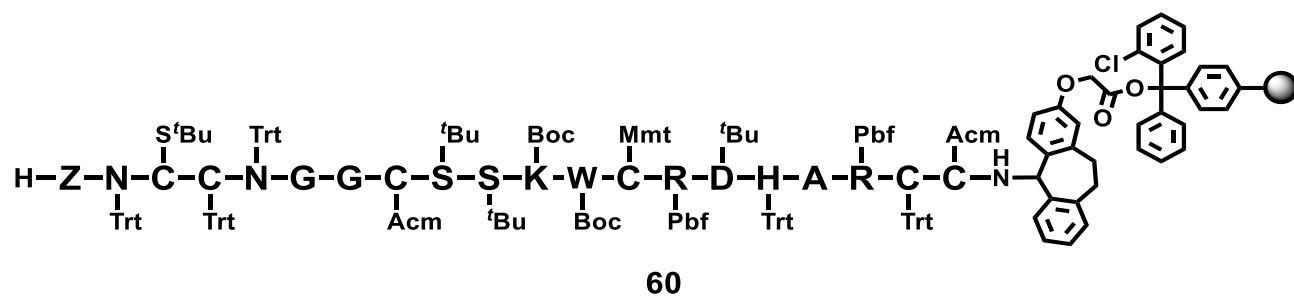

2-Chlorotrityl chloride resin $(0.70 \mathrm{~g}, 0.70 \mathrm{mmol}, 1-1.50 \mathrm{mmol} / \mathrm{g})$ was preloaded with the Ramage linker according to SOP8 and the occupancy $(0.24 \mathrm{mmol} / \mathrm{g})$ was determined following SOP10. The first amino acid Fmoc-Cys(Acm)-OH was coupled to the resin $(416 \mathrm{mg}$, $0.10 \mathrm{mmol}$ ) as described in SOP9 and the peptide sequence was synthesised by automated SPPS following SOP11. The test cleavage of the native $\mu$-SIIIA (102) was carried out with cleavage mixture A as outlined in SOP12, yielding the Acm and $\mathrm{S}^{t} \mathrm{Bu}$ protected peptide.

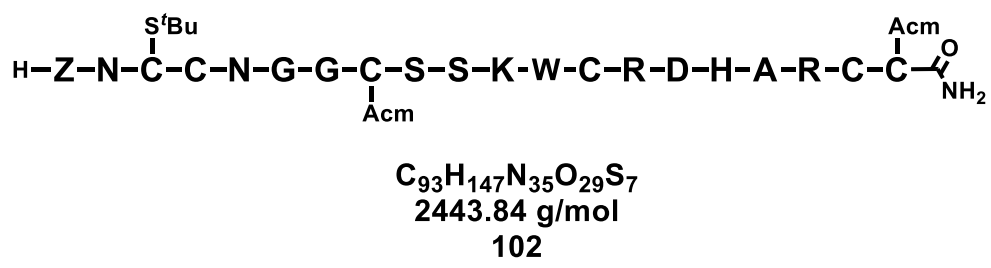

MS (ESI): $\mathbf{m} / \mathbf{z}(\%)=1222.4(22)[\mathrm{M}+2 \mathrm{H}]^{2+}, 815.3(100)[\mathrm{M}+3 \mathrm{H}]^{3+}, 611.7(83)[\mathrm{M}+4 \mathrm{H}]^{4+}$.

HRMS (ESI): calc. for: $\mathrm{C}_{93} \mathrm{H}_{149} \mathrm{~N}_{35} \mathrm{O}_{29} \mathrm{~S}_{7}: 1221.4660$, found.: $1221.4651[\mathrm{M}+2 \mathrm{H}]^{2+}$, calc. for: $\mathrm{C}_{93} \mathrm{H}_{150} \mathrm{~N}_{35} \mathrm{O}_{29} \mathrm{~S}_{7}: 815.3131$, found.: $815.3119[\mathrm{M}+3 \mathrm{H}]^{3+}$, calc. for: $\mathrm{C}_{93} \mathrm{H}_{151} \mathrm{~N}_{35} \mathrm{O}_{29} \mathrm{~S}_{7}$ : 611.7366, found.: $611.7358[\mathrm{M}+4 \mathrm{H}]^{4+}$. 


\section{Synthesis of Native $\mu$-Conotoxin SIIIA: Formation of the Second Disulphide Bridge}

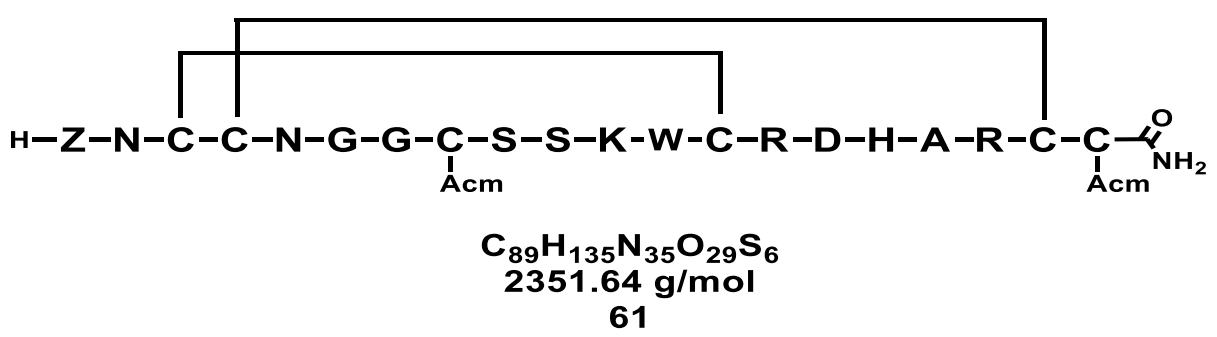

The formation of the first and second disulphide bridge was performed as described in SOP13 and SOP14. The crude peptide was purified by semipreparative HPLC and the product $\mathbf{6 1}$ (8.22 mg, $3.48 \mu \mathrm{mol}$ ) was obtained as a colourless solid.

HPLC: $(250 \cdot 10 \mathrm{~mm}, 5 \mu \mathrm{m}, \mathrm{C} 18$, Gradient $=1-40 \% \mathrm{~B}$ in $30 \mathrm{~min}, \lambda[\mathrm{nm}]: 215,254,280)$ : $t_{\mathrm{R}}=18.7 \mathrm{~min}$.

MS (ESI): $\mathbf{m} / \mathbf{z}(\%)=1176.4(16)[\mathrm{M}+2 \mathrm{H}]^{2+}, 784.6(87)[\mathrm{M}+3 \mathrm{H}]^{3+}, 588.7(100)[\mathrm{M}+4 \mathrm{H}]^{4+}$.

HRMS (ESI): calc. for: $\mathrm{C}_{89} \mathrm{H}_{137} \mathrm{~N}_{35} \mathrm{O}_{29} \mathrm{~S}_{6}: 1176.4333$, found.: $1176.4344[\mathrm{M}+2 \mathrm{H}]^{2+}$, calc. for: $\mathrm{C}_{89} \mathrm{H}_{138} \mathrm{~N}_{35} \mathrm{O}_{29} \mathrm{~S}_{6}: 784.6246$, found.: $784.6266[\mathrm{M}+3 \mathrm{H}]^{3+}$, calc. for: $\mathrm{C}_{89} \mathrm{H}_{139} \mathrm{~N}_{35} \mathrm{O}_{29} \mathrm{~S}_{6}: 588.7203$, found.: $588.7215[\mathrm{M}+4 \mathrm{H}]^{4+}$.

Synthesis of native $\mu$-conotoxin SIIIA: formation of the third disulphide bridge

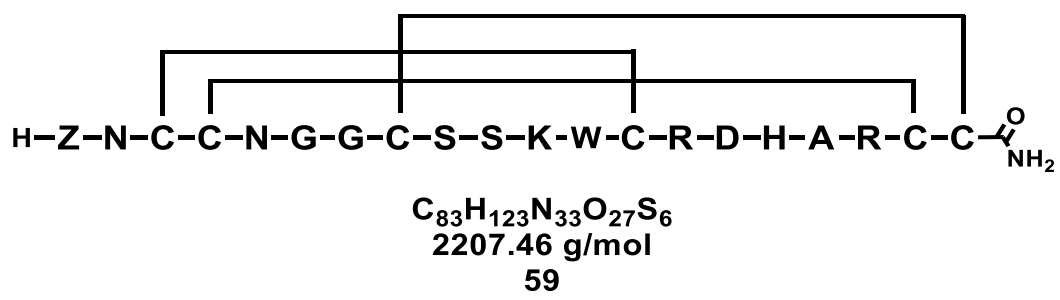

For cleavage of the Acm protecting groups and formation of the third disulphide bridge, 61 (8.22 mg, $3.71 \mu \mathrm{mol}, 1.00 \mathrm{eq})$ and Ac-L-Trp-OMe (9.97 mg, $37.1 \mu \mathrm{mol}, 10.0 \mathrm{eq})$ were dissolved in $\mathrm{AcOH} / \mathrm{H}_{2} \mathrm{O}(1.82 / 0.91, v / v)$. Following, a solution of iodine $(48.5 \mathrm{mg}, 185 \mu \mathrm{mol}$, $50.0 \mathrm{eq})$, dissolved in acetic acid $(1.80 \mathrm{~mL})$, was added and the reaction mixture was stirred for $60 \mathrm{~min}$ at $\mathrm{rt}$. The reaction was quenched by addition of L-ascorbic acid (400 $\mathrm{mg}$ in $1.5 \mathrm{~mL} \mathrm{H}_{2} \mathrm{O}$ ) and the crude peptide was directly purified by semipreparative HPLC. Two isomers of the product 59 were obtained as a colourless solid. 
1.) Isomer 59.2.1 $(0.10 \mu \mathrm{g}, 0.04 \mu \mathrm{mol})$

HPLC: $(250 \cdot 10 \mathrm{~mm}, 5 \mu \mathrm{m}, \mathrm{C} 18$, Gradient $=1-40 \%$ B in $30 \mathrm{~min}, \lambda[\mathrm{nm}]: 215,254,280)$ : $t_{\mathrm{R}}=16.6 \mathrm{~min}$.

MS (ESI): $\mathbf{m} / \mathbf{z}(\%)=1104.4(11)[\mathrm{M}+2 \mathrm{H}]^{2+}, 736.6(100)[\mathrm{M}+3 \mathrm{H}]^{3+}, 552.7(56)[\mathrm{M}+4 \mathrm{H}]^{4+}$.

HRMS (ESI): calc. for: $\mathrm{C}_{83} \mathrm{H}_{125} \mathrm{~N}_{33} \mathrm{O}_{27} \mathrm{~S}_{6}: 1104.3884$, found.: $1104.3880[\mathrm{M}+2 \mathrm{H}]^{2+}$, calc. for: $\mathrm{C}_{83} \mathrm{H}_{126} \mathrm{~N}_{33} \mathrm{O}_{27} \mathrm{~S}_{6}: 736.5947$, found.: $736.5957[\mathrm{M}+3 \mathrm{H}]^{3+}$, calc. for: $\mathrm{C}_{83} \mathrm{H}_{127} \mathrm{~N}_{33} \mathrm{O}_{27} \mathrm{~S}_{6}: 552.6978$, found.: $552.6984[\mathrm{M}+4 \mathrm{H}]^{4+}$.

\section{2.) Isomer 59.2.2 $(0.46 \mu \mathrm{g}, 0.21 \mu \mathrm{mol})$}

HPLC: $(250 \cdot 10 \mathrm{~mm}, 5 \mu \mathrm{m}, \mathrm{C} 18$, Gradient $=1-40 \%$ B in $30 \mathrm{~min}, \lambda[\mathrm{nm}]: 215,254,280)$ : $t_{\mathrm{R}}=17.2 \mathrm{~min}$.

MS (ESI): $\mathbf{m} / \mathbf{z}(\%)=1104.4(15)[\mathrm{M}+2 \mathrm{H}]^{2+}, 736.6(79)[\mathrm{M}+3 \mathrm{H}]^{3+}$.

HRMS (ESI): calc. for: $\mathrm{C}_{83} \mathrm{H}_{125} \mathrm{~N}_{33} \mathrm{O}_{27} \mathrm{~S}_{6}: 1104.3884$, found.: $1104.3883[\mathrm{M}+2 \mathrm{H}]^{2+}$, calc. for: $\mathrm{C}_{83} \mathrm{H}_{126} \mathrm{~N}_{33} \mathrm{O}_{27} \mathrm{~S}_{6}: 736.5947$, found.: $736.5946[\mathrm{M}+3 \mathrm{H}]^{3+}$, calc. for: $\mathrm{C}_{83} \mathrm{H}_{127} \mathrm{~N}_{33} \mathrm{O}_{27} \mathrm{~S}_{6}: 552.6978$, found.: $552.6977[\mathrm{M}+4 \mathrm{H}]^{4+}$.

\section{Synthesis of AlexaFluor 488 modified $\mu$-Conotoxin SIIIA}

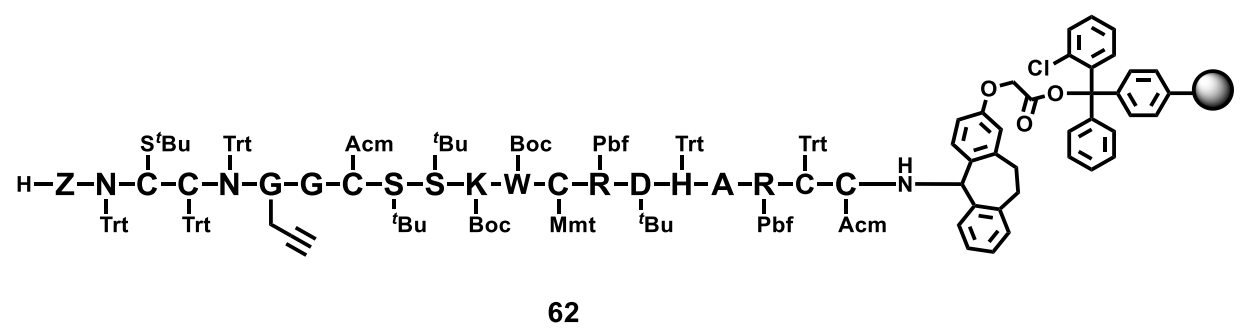

2-Chlorotrityl chloride resin $(0.70 \mathrm{~g}, 0.70 \mathrm{mmol}, 1-1.50 \mathrm{mmol} / \mathrm{g})$ was preloaded with the Ramage linker according to SOP8 and the occupancy $(0.26 \mathrm{mmol} / \mathrm{g})$ was determined following SOP10. The first amino acid Fmoc-Cys(Acm)-OH was coupled to the resin (385 mg, $0.10 \mathrm{mmol}$ ) as described in SOP9 and the peptide sequence was synthesised by automated SPPS following SOP11. The test cleavage of the native $\mu$-SIIIA (103) was carried out with cleavage mixture A as outlined in SOP12, yielding the Acm and $\mathrm{S}^{t} \mathrm{Bu}$ protected peptide. 


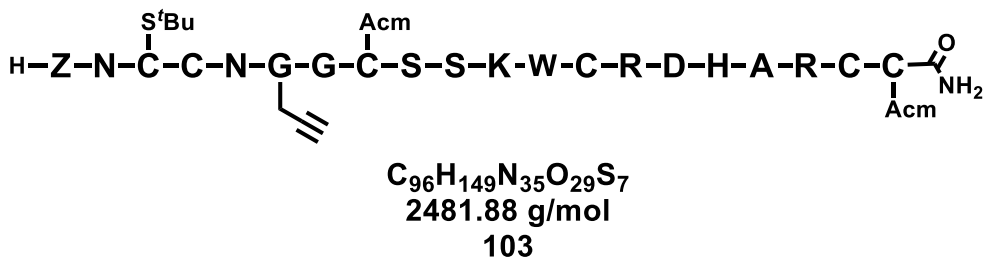

MS (ESI): $\mathbf{m} / \mathbf{z}(\%)=1241.4(21)[\mathrm{M}+2 \mathrm{H}]^{2+}, 828.0(100)[\mathrm{M}+3 \mathrm{H}]^{3+}, 621.2(86)[\mathrm{M}+4 \mathrm{H}]^{4+}$.

HRMS (ESI): calc. for: $\mathrm{C}_{96} \mathrm{H}_{151} \mathrm{~N}_{35} \mathrm{O}_{29} \mathrm{~S}_{7}: 1241.4738$, found.: $1241.4725[\mathrm{M}+2 \mathrm{H}]^{2+}$, calc. for: $\mathrm{C}_{96} \mathrm{H}_{152} \mathrm{~N}_{35} \mathrm{O}_{29} \mathrm{~S}_{7}: 827.9850$, found.: $827.9844[\mathrm{M}+3 \mathrm{H}]^{3+}$, calc. for: $\mathrm{C}_{96} \mathrm{H}_{153} \mathrm{~N}_{35} \mathrm{O}_{29} \mathrm{~S}_{7}$ : 621.2406, found.: $621.2399[\mathrm{M}+4 \mathrm{H}]^{4+}$.

\section{Synthesis of AlexaFluor488 modified $\mu$-Conotoxin SIIIA: Formation of the Third Disulphide Bridge}

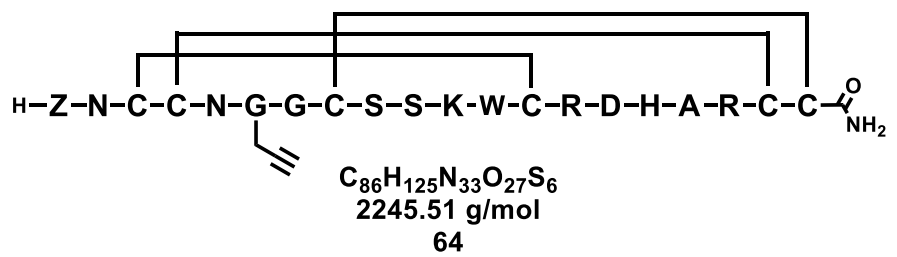

For cleavage of the Acm protecting groups and formation of the third disulphide bridge, $\mathbf{6 3}$ (4.81 mg, $2.14 \mu \mathrm{mol}, 1.00 \mathrm{eq})$ and Ac-L-Trp-OMe (5.23 mg, $21.4 \mu \mathrm{mol}, 10.0 \mathrm{eq})$ were dissolved in $\mathrm{AcOH} / \mathrm{H}_{2} \mathrm{O}(0.91 / 0.46, v / v)$. Following, a solution of iodine $(25.0 \mathrm{mg}, 107 \mu \mathrm{mol}$, $50.0 \mathrm{eq})$, dissolved in acetic acid $(0.91 \mathrm{~mL})$, was added and the reaction mixture was stirred for $15 \mathrm{~min}$ at rt. The reaction was quenched by addition of $\mathrm{L}$-ascorbic acid ( $400 \mathrm{mg}$ in $1.5 \mathrm{~mL} \mathrm{H}_{2} \mathrm{O}$ ) and the crude peptide was directly purified by semipreparative HPLC. The product 64 (0.06 mg,0.03 $\mu \mathrm{mol})$ was obtained as a colourless solid.

HPLC: $(250 \cdot 10 \mathrm{~mm}, 5 \mu \mathrm{m}, \mathrm{C} 18$, Gradient $=20-50 \%$ B in $30 \mathrm{~min}, \lambda[\mathrm{nm}]: 215,254,280)$ : $t_{\mathrm{R}}=14.7 \mathrm{~min}$.

MS (ESI): $\mathbf{~ m / z ~}(\%)=1123.4(21)[\mathrm{M}+2 \mathrm{H}]^{2+}, 749.3(100)[\mathrm{M}+3 \mathrm{H}]^{3+}, 562.2()[\mathrm{M}+4 \mathrm{H}]^{4+}$.

HRMS (ESI): calc. for: $\mathrm{C}_{86} \mathrm{H}_{127} \mathrm{~N}_{33} \mathrm{O}_{27} \mathrm{~S}_{6}: 1123.3962$, found.: $1123.3931[\mathrm{M}+2 \mathrm{H}]^{2+}$, calc. for: $\mathrm{C}_{86} \mathrm{H}_{128} \mathrm{~N}_{33} \mathrm{O}_{27} \mathrm{~S}_{6}: 749.2666$, found: $749.2658[\mathrm{M}+3 \mathrm{H}]^{3+}$, calc. for: $\mathrm{C}_{86} \mathrm{H}_{129} \mathrm{~N}_{33} \mathrm{O}_{27} \mathrm{~S}_{6}: 562.2017$, found: $562.2003[\mathrm{M}+4 \mathrm{H}]^{4+}$. 


\section{Synthesis of AlexaFluor488-labelled $\mu$-Conotoxin SIIIA}

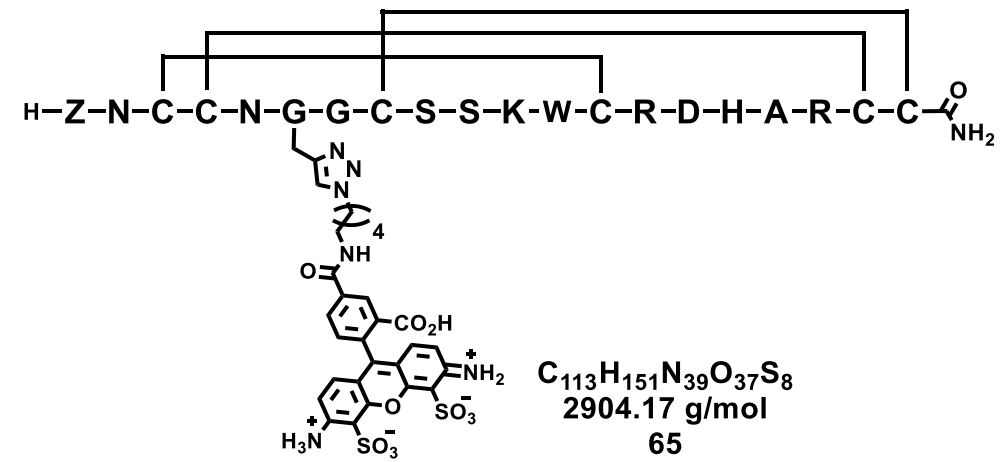

The labelling reaction was performed according to a protocol modified by RINK et al. ${ }^{[241]}$ The alkyne modified $\mu$-SIIA $(64.0 \mu \mathrm{g}, 28.8 \mathrm{nmol}, 1.00 \mathrm{eq})$ and AlexaFluor488 azide (1.64 mg, $1.44 \mu \mathrm{mol}, 50.0 \mathrm{eq})$ were dissolved in degassed MilliQ $(200 \mu \mathrm{L})$ under argon atmosphere. Sodium ascorbate $(10 \mu \mathrm{L}, 100 \mathrm{mM}), \mathrm{CuSO}_{4} / \mathrm{THPTA}(10 \mu \mathrm{L}, 67.0 \mathrm{mM} / 10 \mu \mathrm{L}, 134 \mathrm{mM})$ were mixed in degassed tert-butanol/PBS $(200 \mu \mathrm{L})$ and was transferred to the peptide solution. The reaction mixture was incubated at $37{ }^{\circ} \mathrm{C}$ for $48 \mathrm{~h}$ and was directly purified by analytical HPLC, yielding the product $\mathbf{6 5}(0.04 \mu \mathrm{g}, 13.3 \mathrm{nmol})$ as yellow solid.

HPLC: $(250 \cdot 10 \mathrm{~mm}, 5 \mu \mathrm{m}, \mathrm{C} 18$, Gradient $=20-50 \%$ B in $30 \mathrm{~min}, \lambda[\mathrm{nm}]: 215,280,488)$ : $t_{\mathrm{R}}=14.7 \mathrm{~min}$.

HRMS (ESI): calc. for: $\mathrm{C}_{113} \mathrm{H}_{154} \mathrm{~N}_{39} \mathrm{O}_{37} \mathrm{~S}_{8}$ : 968.6381 , found.: $968.6359[\mathrm{M}+3 \mathrm{H}]^{3+}$.

\section{Synthesis of Allocam Protected Alkyne-modified $\mu$-SIIIA}

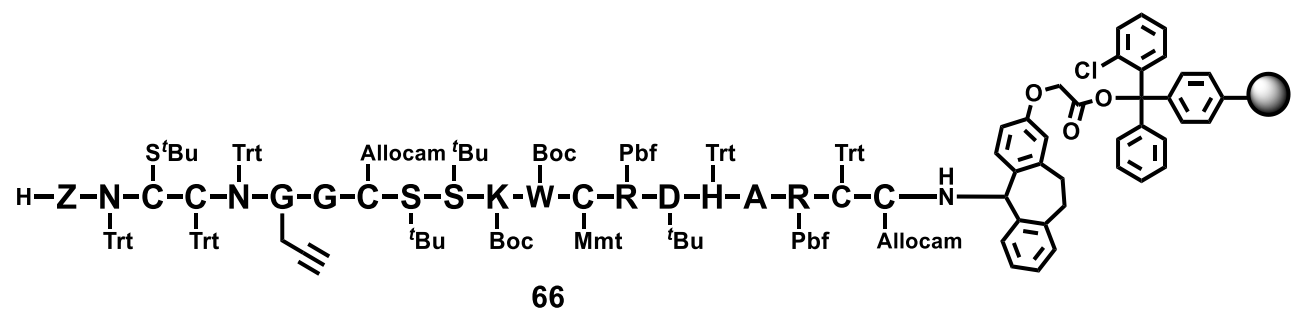

2-Chlorotrityl chloride resin $(0.70 \mathrm{~g}, 0.70 \mathrm{mmol}, 1-1.50 \mathrm{mmol} / \mathrm{g})$ was preloaded with the Ramage linker according to SOP8 and the occupancy $(0.26 \mathrm{mmol} / \mathrm{g})$ was determined following SOP10. The first amino acid Fmoc-Cys(Allocam)-OH was coupled to the resin (385 mg, $0.10 \mathrm{mmol}$ ) as described in SOP9 and the peptide sequence was synthesised by automated SPPS following SOP11. The test cleavage of the alkyne-modified $\mu$-SIIIA (104) was carried out with cleavage mixture A as outlined in SOP12, yielding the Allocam and $\mathrm{S}^{t} \mathrm{Bu}$ protected peptide. 


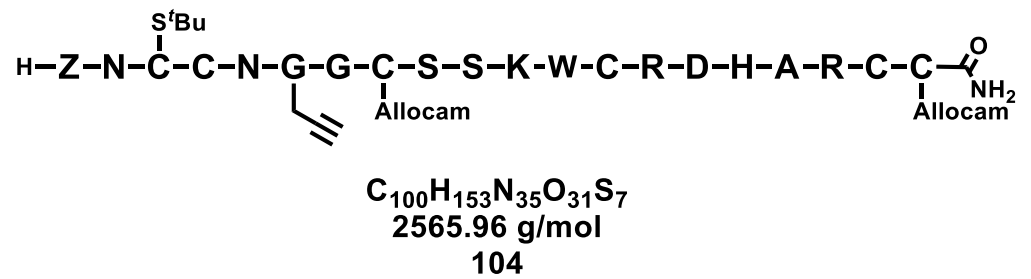

MS (ESI): $\mathbf{m} / \mathbf{z}(\%)=1283.4(4)[\mathrm{M}+2 \mathrm{H}]^{2+}, 856.0(12)[\mathrm{M}+3 \mathrm{H}]^{3+}, 642.2(8)[\mathrm{M}+4 \mathrm{H}]^{4+}$.

HRMS (ESI): calc. for: $\mathrm{C}_{100} \mathrm{H}_{156} \mathrm{~N}_{35} \mathrm{O}_{31} \mathrm{~S}_{7}: 855.9920$, found.: $855.9879[\mathrm{M}+3 \mathrm{H}]^{3+}$, calc. for: $\mathrm{C}_{100} \mathrm{H}_{157} \mathrm{~N}_{35} \mathrm{O}_{31} \mathrm{~S}_{7}$ : 642.2458, found.: $642.2429[\mathrm{M}+4 \mathrm{H}]^{4+}$.

Synthesis of Allocam Protected Alkyne-modified $\mu$-SIIIA: Formation of the First and Second Disulphide Bridge

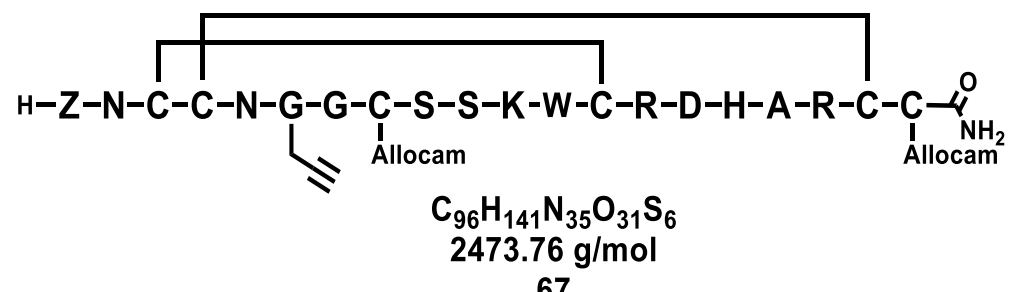

The formation of the first and second disulphide bridge was performed as described in SOP13 and SOP14. The crude peptide was purified by semipreparative HPLC and the product 67 was obtained as a colourless solid.

1.) Isomer $67.1(1.07 \mathrm{mg}, 0.43 \mu \mathrm{mol})$

HPLC: $(250 \cdot 10 \mathrm{~mm}, 5 \mu \mathrm{m}, \mathrm{C} 18$, Gradient $=10-60 \%$ B in $30 \mathrm{~min}, \lambda[\mathrm{nm}]: 215,254,280)$ : $t_{\mathrm{R}}=14.2 \mathrm{~min}$.

MS (ESI): $\mathbf{m} / \mathbf{z}(\%)=1237.5(23)[\mathrm{M}+2 \mathrm{H}]^{2+}, 825.3(100)[\mathrm{M}+3 \mathrm{H}]^{3+}, 619.2(82)[\mathrm{M}+4 \mathrm{H}]^{4+}$.

HRMS (ESI): calc. for: $\mathrm{C}_{96} \mathrm{H}_{143} \mathrm{~N}_{35} \mathrm{O}_{31} \mathrm{~S}_{6}: 1237.4514$, found.: $1237.4505[\mathrm{M}+2 \mathrm{H}]^{2+}$, calc. for: $\mathrm{C}_{96} \mathrm{H}_{144} \mathrm{~N}_{35} \mathrm{O}_{31} \mathrm{~S}_{6}: 825.3034$, found.: $825.3034[\mathrm{M}+3 \mathrm{H}]^{3+}$, calc. for: $\mathrm{C}_{96} \mathrm{H}_{145} \mathrm{~N}_{35} \mathrm{O}_{31} \mathrm{~S}_{6}: 619.2293$, found.: $619.2290[\mathrm{M}+4 \mathrm{H}]^{4+}$.

2.) Isomer $67.2(1.27 \mathrm{mg}, 0.51 \mu \mathrm{mol})$

HPLC: $(250 \cdot 10 \mathrm{~mm}, 5 \mu \mathrm{m}, \mathrm{C} 18$, Gradient $=10-60 \%$ B in $30 \mathrm{~min}, \lambda[\mathrm{nm}]: 215,254,280)$ : $t_{\mathrm{R}}=14.4 \mathrm{~min}$.

MS (ESI): $\mathbf{m} / \mathbf{z}(\%)=1237.5(10)[\mathrm{M}+2 \mathrm{H}]^{2+}, 825.3(70)[\mathrm{M}+3 \mathrm{H}]^{3+}, 619.2(63)[\mathrm{M}+4 \mathrm{H}]^{4+}$. 
HRMS (ESI): calc. for: $\mathrm{C}_{96} \mathrm{H}_{143} \mathrm{~N}_{35} \mathrm{O}_{31} \mathrm{~S}_{6}: 1237.4514$, found.: $1237.4495[\mathrm{M}+2 \mathrm{H}]^{2+}$, calc. for: $\mathrm{C}_{96} \mathrm{H}_{144} \mathrm{~N}_{35} \mathrm{O}_{31} \mathrm{~S}_{6}: 825.3034$, found.: $825.3027[\mathrm{M}+3 \mathrm{H}]^{3+}$, calc. for: $\mathrm{C}_{96} \mathrm{H}_{145} \mathrm{~N}_{35} \mathrm{O}_{31} \mathrm{~S}_{6}: 619.2293$, found.: $619.2288[\mathrm{M}+4 \mathrm{H}]^{4+}$.

\section{Synthesis of ${ }^{t}$ Bu Protected Alkyne-modified $\mu$-SIIIA}

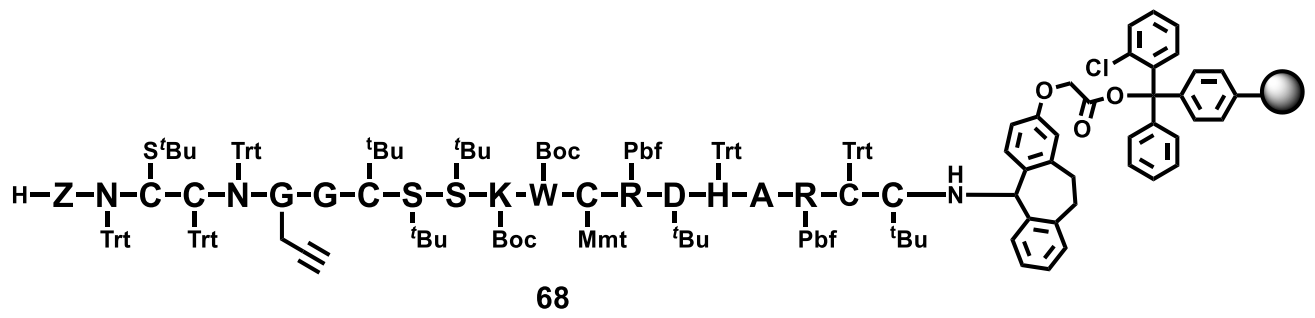

2-Chlorotrityl chloride resin $(0.70 \mathrm{~g}, 0.70 \mathrm{mmol}, 1-1.50 \mathrm{mmol} / \mathrm{g})$ was preloaded with the Ramage linker according to SOP8 and the occupancy $(0.26 \mathrm{mmol} / \mathrm{g})$ was determined following SOP10. The first amino acid Fmoc-Cys $\left({ }^{t} \mathrm{Bu}\right)-\mathrm{OH}$ was coupled to the resin $(385 \mathrm{mg}, 0.10 \mathrm{mmol})$ as described in SOP9 and the peptide sequence was synthesised by automated SPPS following SOP11. The test cleavage of the alkyne-modified $\mu$-SIIIA (105) was carried out with cleavage mixture $\mathrm{A}$ as outlined in $\mathrm{SOP} 12$, yielding the ${ }^{t} \mathrm{Bu}$ and $\mathrm{S}^{t} \mathrm{Bu}$ protected peptide.

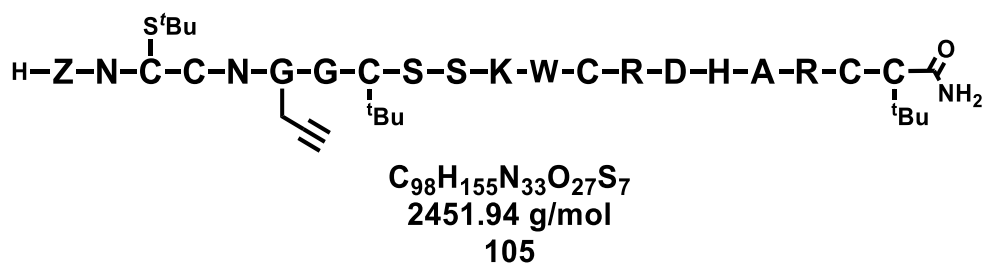

MS (ESI): $\mathbf{m} / \mathbf{z}(\%)=1226.5(18)[\mathrm{M}+2 \mathrm{H}]^{2+}, 818.0(100)[\mathrm{M}+3 \mathrm{H}]^{3+}, 613.7(68)[\mathrm{M}+4 \mathrm{H}]^{4+}$.

HRMS (ESI): calc. for: $\mathrm{C}_{98} \mathrm{H}_{157} \mathrm{~N}_{33} \mathrm{O}_{27} \mathrm{~S}_{7}: 1226.4993$, found.: $1222.4967[\mathrm{M}+2 \mathrm{H}]^{2+}$, calc. for: $\mathrm{C}_{98} \mathrm{H}_{158} \mathrm{~N}_{33} \mathrm{O}_{27} \mathrm{~S}_{7}: 818.0020$, found.: $818.0008[\mathrm{M}+3 \mathrm{H}]^{3+}$, calc. for: $\mathrm{C}_{98} \mathrm{H}_{159} \mathrm{~N}_{33} \mathrm{O}_{27} \mathrm{~S}_{7}: 613.7533$, found.: $613.7522[\mathrm{M}+4 \mathrm{H}]^{4+}$. 
Synthesis of ${ }^{t} \mathrm{Bu}$ Protected Alkyne-modified $\mu$-SIIIA: Formation of the First and Second Disulphide Bridge

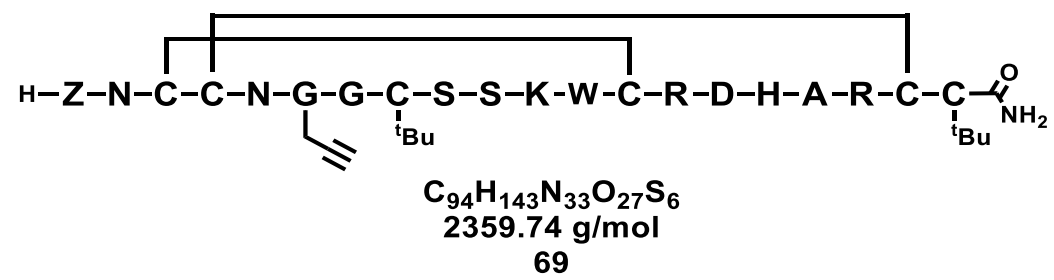

The formation of the first and second disulphide bridge was performed as described in SOP13 and SOP14. The crude peptide was purified by semipreparative HPLC and the product 69 was obtained as a colourless solid.

1.) Isomer $69.1(2.7 \mathrm{mg}, 1.1 \mu \mathrm{mol})$

HPLC: $(250 \cdot 10 \mathrm{~mm}, 5 \mu \mathrm{m}, \mathrm{C} 18$, Gradient $=10-60 \%$ B in $30 \mathrm{~min}, \lambda[\mathrm{nm}]: 215,254,280)$ : $t_{\mathrm{R}}=12.9 \mathrm{~min}$.

MS (ESI): $\mathbf{m} / \mathbf{z}(\%)=1180.4(42)[\mathrm{M}+2 \mathrm{H}]^{2+}, 787.3(100)[\mathrm{M}+3 \mathrm{H}]^{3+}, 590.7(16)[\mathrm{M}+4 \mathrm{H}]^{4+}$.

HRMS (ESI): calc. for: $\mathrm{C}_{94} \mathrm{H}_{145} \mathrm{~N}_{33} \mathrm{O}_{27} \mathrm{~S}_{6}: 1180.4664$, found.: $11180.4657[\mathrm{M}+2 \mathrm{H}]^{2+}$, calc. for: $\mathrm{C}_{94} \mathrm{H}_{146} \mathrm{~N}_{33} \mathrm{O}_{27} \mathrm{~S}_{6}: 787.3133$, found.: $787.3133[\mathrm{M}+3 \mathrm{H}]^{3+}$, calc. for: $\mathrm{C}_{94} \mathrm{H}_{147} \mathrm{~N}_{33} \mathrm{O}_{27} \mathrm{~S}_{6}: 590.7368$, found.: $590.7364[\mathrm{M}+4 \mathrm{H}]^{4+}$.

2.) Isomer $69.2(2.9 \mathrm{mg}, 1.2 \mu \mathrm{mol})$

HPLC: $(250 \cdot 10 \mathrm{~mm}, 5 \mu \mathrm{m}, \mathrm{C} 18$, Gradient $=10-60 \%$ B in $30 \mathrm{~min}, \lambda[\mathrm{nm}]: 215,254,280)$ : $t_{\mathrm{R}}=13.4 \mathrm{~min}$.

MS (ESI): $\mathbf{m} / \mathbf{z}(\%)=1180.5(30)[\mathrm{M}+2 \mathrm{H}]^{2+}, 787.3(100)[\mathrm{M}+3 \mathrm{H}]^{3+}, 590.7(37)[\mathrm{M}+4 \mathrm{H}]^{4+}$.

HRMS (ESI): calc. for: $\mathrm{C}_{94} \mathrm{H}_{145} \mathrm{~N}_{33} \mathrm{O}_{27} \mathrm{~S}_{6}: 1180.4664$, found.: $11180.4667[\mathrm{M}+2 \mathrm{H}]^{2+}$, calc. for: $\mathrm{C}_{94} \mathrm{H}_{146} \mathrm{~N}_{33} \mathrm{O}_{27} \mathrm{~S}_{6}: 787.3133$, found.: $787.3133[\mathrm{M}+3 \mathrm{H}]^{3+}$, calc. for: $\mathrm{C}_{94} \mathrm{H}_{147} \mathrm{~N}_{33} \mathrm{O}_{27} \mathrm{~S}_{6}: 590.7368$, found.: $590.7371[\mathrm{M}+4 \mathrm{H}]^{4+}$. 


\section{Synthesis of MeOBzl Protected Alkyne-modified $\mu$-SIIIA}

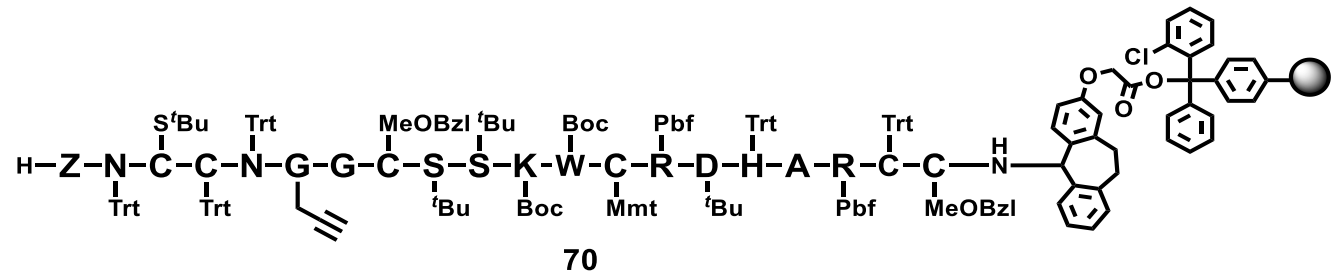

2-Chlorotrityl chloride resin $(0.70 \mathrm{~g}, 0.70 \mathrm{mmol}, 1-1.50 \mathrm{mmol} / \mathrm{g})$ was preloaded with the Ramage linker according to SOP8 and the occupancy $(0.26 \mathrm{mmol} / \mathrm{g})$ was determined following SOP10. The first amino acid Fmoc-Cys(MeOBzl)-OH was coupled to the resin (385 mg, $0.10 \mathrm{mmol}$ ) as described in SOP9 and the peptide sequence was synthesised by automated SPPS following SOP11. The test cleavage of the alkyne-modified $\mu$-SIIIA (106) was carried out with cleavage mixture A as outlined in SOP12, yielding the MeOBzl and $\mathrm{S}^{t} \mathrm{Bu}$ protected peptide.

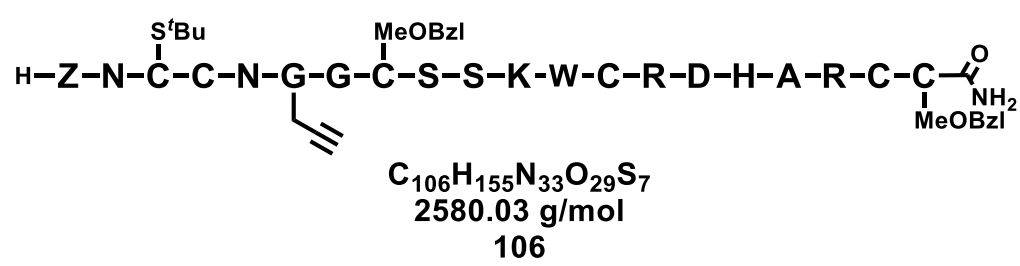

MS (ESI): $\mathbf{m} / \mathbf{z}(\%)=1290.5(5)[\mathrm{M}+2 \mathrm{H}]^{2+}, 860.6(16)[\mathrm{M}+3 \mathrm{H}]^{3+}, 645.7(18)[\mathrm{M}+4 \mathrm{H}]^{4+}$.

HRMS (ESI): calc. for: $\mathrm{C}_{106} \mathrm{H}_{157} \mathrm{~N}_{33} \mathrm{O}_{29} \mathrm{~S}_{7}: 1290.4943$, found.: $1290.4900[\mathrm{M}+2 \mathrm{H}]^{2+}$, calc. for: $\mathrm{C}_{106} \mathrm{H}_{158} \mathrm{~N}_{33} \mathrm{O}_{29} \mathrm{~S}_{7}$ : 860.6653, found.: $860.6635[\mathrm{M}+3 \mathrm{H}]^{3+}$, calc. for: $\mathrm{C}_{106} \mathrm{H}_{159} \mathrm{~N}_{33} \mathrm{O}_{29} \mathrm{~S}_{7}$ : 645.7508, found.: $645.7492[\mathrm{M}+4 \mathrm{H}]^{4+}$.

Synthesis of MeOBzl Protected Alkyne-modified $\mu$-SIIIA: Formation of the First and Second Disulphide Bridge

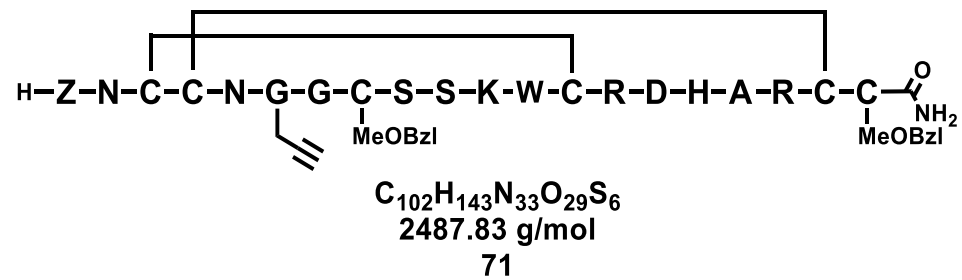


The formation of the first and second disulphide bridge was performed as described in SOP13 and SOP14. The crude peptide was purified by semipreparative HPLC and the product 71 was obtained as a colourless solid.

1.) Isomer $71.1(5.09 \mathrm{mg}, 2.05 \mu \mathrm{mol})$

HPLC: $(250 \cdot 10 \mathrm{~mm}, 5 \mu \mathrm{m}, \mathrm{C} 18$, Gradient $=1-40 \%$ B in $30 \mathrm{~min}, \lambda[\mathrm{nm}]: 215,254,280)$ : $t_{\mathrm{R}}=24.8 \mathrm{~min}$.

MS (ESI): $\mathbf{m} / \mathbf{z}(\%)=1244.5(12)[\mathrm{M}+2 \mathrm{H}]^{2+}, 830.0(100)[\mathrm{M}+3 \mathrm{H}]^{3+}, 622.7(97)[\mathrm{M}+4 \mathrm{H}]^{4+}$.

HRMS (ESI): calc. for: $\mathrm{C}_{102} \mathrm{H}_{145} \mathrm{~N}_{33} \mathrm{O}_{29} \mathrm{~S}_{6}: 1244.4613$, found.: $1244.4607[\mathrm{M}+2 \mathrm{H}]^{2+}$, calc. for: $\mathrm{C}_{102} \mathrm{H}_{146} \mathrm{~N}_{33} \mathrm{O}_{29} \mathrm{~S}_{6}: 829.9766$, found.: $829.9767[\mathrm{M}+3 \mathrm{H}]^{3+}$, calc. for: $\mathrm{C}_{102} \mathrm{H}_{147} \mathrm{~N}_{33} \mathrm{O}_{29} \mathrm{~S}_{6}$ : 622.7343, found.: $622.7342[\mathrm{M}+4 \mathrm{H}]^{4+}$.

2.) Isomer $71.2(2.05 \mathrm{mg}, 0.82 \mu \mathrm{mol})$

HPLC: $(250 \cdot 10 \mathrm{~mm}, 5 \mu \mathrm{m}, \mathrm{C} 18$, Gradient $=1-40 \%$ B in $30 \mathrm{~min}, \lambda[\mathrm{nm}]: 215,254,280)$ : $t_{\mathrm{R}}=25.4 \mathrm{~min}$.

MS (ESI): $\mathbf{m} / \mathbf{z}(\%)=1244.5(17)[\mathrm{M}+2 \mathrm{H}]^{2+}, 830.0(100)[\mathrm{M}+3 \mathrm{H}]^{3+}, 622.7(97)[\mathrm{M}+4 \mathrm{H}]^{4+}$.

HRMS (ESI): calc. for: $\mathrm{C}_{102} \mathrm{H}_{145} \mathrm{~N}_{33} \mathrm{O}_{29} \mathrm{~S}_{6}: 1244.4613$, found.: $1244.4600[\mathrm{M}+2 \mathrm{H}]^{2+}$, calc. for: $\mathrm{C}_{102} \mathrm{H}_{146} \mathrm{~N}_{33} \mathrm{O}_{29} \mathrm{~S}_{6}$ : 829.9766 , found.: $829.9765[\mathrm{M}+3 \mathrm{H}]^{3+}$, calc. for: $\mathrm{C}_{102} \mathrm{H}_{147} \mathrm{~N}_{33} \mathrm{O}_{29} \mathrm{~S}_{6}$ : 622.7343 , found.: $622.7339[\mathrm{M}+4 \mathrm{H}]^{4+}$.

\section{Synthesis of Thiol-modified $\mu$-SIIIA}

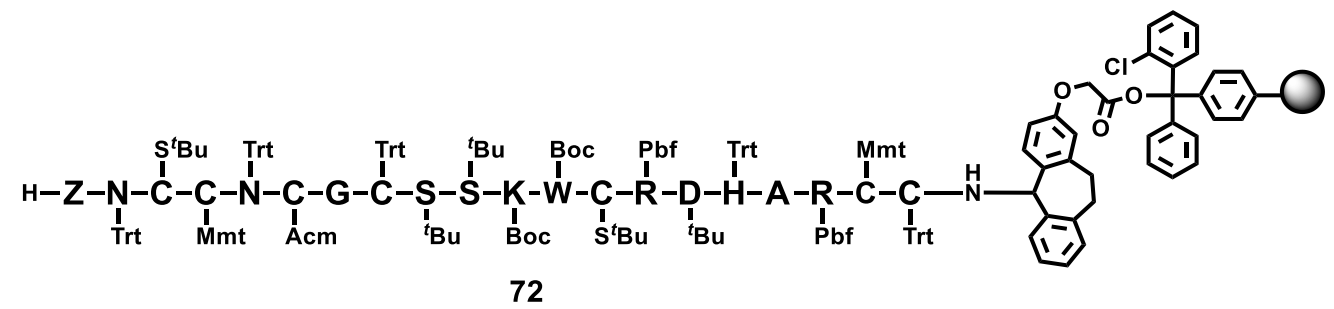

2-Chlorotrityl chloride resin $(0.70 \mathrm{~g}, 0.70 \mathrm{mmol}, 1-1.50 \mathrm{mmol} / \mathrm{g})$ was preloaded with the Ramage linker according to SOP8 and the occupancy $(0.26 \mathrm{mmol} / \mathrm{g})$ was determined following SOP10. The first amino acid Fmoc-Cys(Trt)-OH was coupled to the resin (385 mg, $0.10 \mathrm{mmol}$ ) as described in SOP9 and the peptide sequence was synthesised by automated SPPS following SOP11. The test cleavage of the thiol-modified $\mu$-SIIIA (107) was carried out with cleavage mixture $\mathrm{A}$ as outlined in SOP12, yielding the Acm and $\mathrm{S}^{t} \mathrm{Bu}$ protected peptide. 


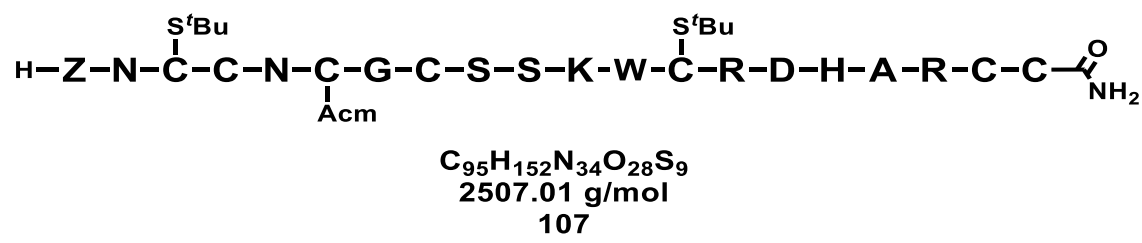

MS (ESI): $\mathbf{m} / \mathbf{z}(\%)=1253.9(16)[\mathrm{M}+2 \mathrm{H}]^{2+}, 836.3(84)[\mathrm{M}+3 \mathrm{H}]^{3+}, 627.7(100)[\mathrm{M}+4 \mathrm{H}]^{4+}$.

HRMS (ESI): calc. for: $\mathrm{C}_{95} \mathrm{H}_{154} \mathrm{~N}_{34} \mathrm{O}_{28} \mathrm{~S}_{9}: 1253.9586$, found.: $1253.9548[\mathrm{M}+2 \mathrm{H}]^{2+}$, calc. for: $\mathrm{C}_{95} \mathrm{H}_{155} \mathrm{~N}_{34} \mathrm{O}_{28} \mathrm{~S}_{9}$ : 836.3082, found.: $836.3071[\mathrm{M}+3 \mathrm{H}]^{3+}$, calc. for: $\mathrm{C}_{95} \mathrm{H}_{156} \mathrm{~N}_{34} \mathrm{O}_{28} \mathrm{~S}_{9}: 627.4830$, found.: $627.4817[\mathrm{M}+4 \mathrm{H}]^{4+}$.

Synthesis of Thiol-modified $\mu$-SIIIA: Formation of the First, Second and Third Disulphide Bridge

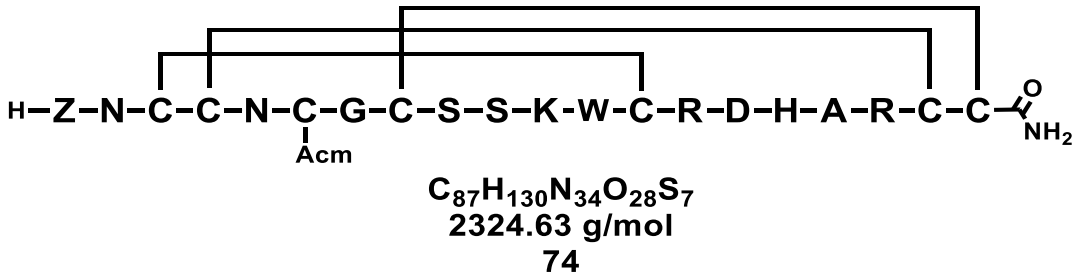

The formation of the first, second and third disulphide bridge was performed following SOP15 and the crude peptide was directly purified by semipreparative HPLC. Two isomers of the product 74 were obtained as a colourless solid.

1.) Isomer $74.1(1.73 \mathrm{mg}, 0.74 \mu \mathrm{mol})$

HPLC: $(250 \cdot 10 \mathrm{~mm}, 5 \mu \mathrm{m}, \mathrm{C} 18$, Gradient $=10-60 \%$ B in $30 \mathrm{~min}, \lambda[\mathrm{nm}]: 215,254,280)$ : $t_{\mathrm{R}}=15.0 \mathrm{~min}$.

MS (ESI): $\mathbf{m} / \mathbf{z}(\%)=1162.9(23)[\mathrm{M}+2 \mathrm{H}]^{2+}, 775.6(100)[\mathrm{M}+3 \mathrm{H}]^{3+}, 581.9(16)[\mathrm{M}+4 \mathrm{H}]^{4+}$.

HRMS (ESI): calc. for: $\mathrm{C}_{87} \mathrm{H}_{132} \mathrm{~N}_{34} \mathrm{O}_{28} \mathrm{~S}_{7}: 1162.9008$, found.: $1162.9001[\mathrm{M}+2 \mathrm{H}]^{2+}$, calc. for: $\mathrm{C}_{87} \mathrm{H}_{133} \mathrm{~N}_{34} \mathrm{O}_{28} \mathrm{~S}_{7}$ : 775.6030, found: $775.6034[\mathrm{M}+3 \mathrm{H}]^{3+}$, calc. for: $\mathrm{C}_{87} \mathrm{H}_{134} \mathrm{~N}_{34} \mathrm{O}_{28} \mathrm{~S}_{7}$ : 581.9540, found: $581.9536[\mathrm{M}+4 \mathrm{H}]^{4+}$. 
2.) Isomer $74.2(1.95 \mathrm{mg}, 0.84 \mu \mathrm{mol})$

HPLC: $(250 \cdot 10 \mathrm{~mm}, 5 \mu \mathrm{m}, \mathrm{C} 18$, Gradient $=10-60 \%$ B in $30 \mathrm{~min}, \lambda[\mathrm{nm}]: 215,254,280)$ : $t_{\mathrm{R}}=15.7 \mathrm{~min}$.

MS (ESI): m/z $(\%)=1162.9(9)[\mathrm{M}+2 \mathrm{H}]^{2+}, 775.6(58)[\mathrm{M}+3 \mathrm{H}]^{3+}, 581.9(21)[\mathrm{M}+4 \mathrm{H}]^{4+}$.

HRMS (ESI): calc. for: $\mathrm{C}_{87} \mathrm{H}_{132} \mathrm{~N}_{34} \mathrm{O}_{28} \mathrm{~S}_{7}: 1162.9008$, found.: $1162.8993[\mathrm{M}+2 \mathrm{H}]^{2+}$, calc. for: $\mathrm{C}_{87} \mathrm{H}_{133} \mathrm{~N}_{34} \mathrm{O}_{28} \mathrm{~S}_{7}$ : 775.6030, found: $775.6019[\mathrm{M}+3 \mathrm{H}]^{3+}$, calc. for: $\mathrm{C}_{87} \mathrm{H}_{134} \mathrm{~N}_{34} \mathrm{O}_{28} \mathrm{~S}_{7}$ : 581.9540, found: $581.9528[\mathrm{M}+4 \mathrm{H}]^{4+}$.

\section{Synthesis of Amine-modified $\mu$-SIIIA}

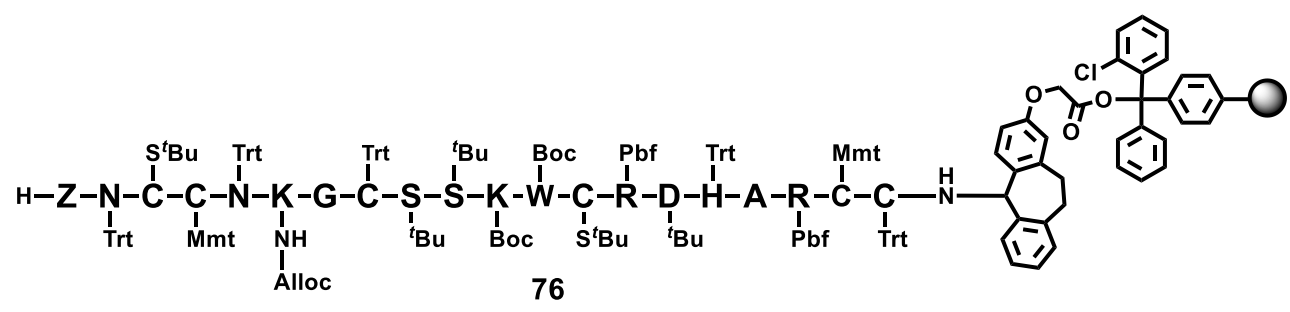

2-Chlorotrityl chloride resin $(0.70 \mathrm{~g}, 0.70 \mathrm{mmol}, 1-1.50 \mathrm{mmol} / \mathrm{g})$ was preloaded with the Ramage linker according to SOP8 and the occupancy $(0.26 \mathrm{mmol} / \mathrm{g})$ was determined following SOP10. The first amino acid Fmoc-Cys(Trt)-OH was coupled to the resin (385 mg, $0.03 \mathrm{mmol}$ ) as described in SOP9 and the peptide sequence was synthesised by automated SPPS following SOP11. The test cleavage of the amine-modified $\mu$-SIIIA (108) was carried out with cleavage mixture A as outlined in SOP12, yielding the Alloc and $\mathrm{S}^{t} \mathrm{Bu}$ protected peptide.

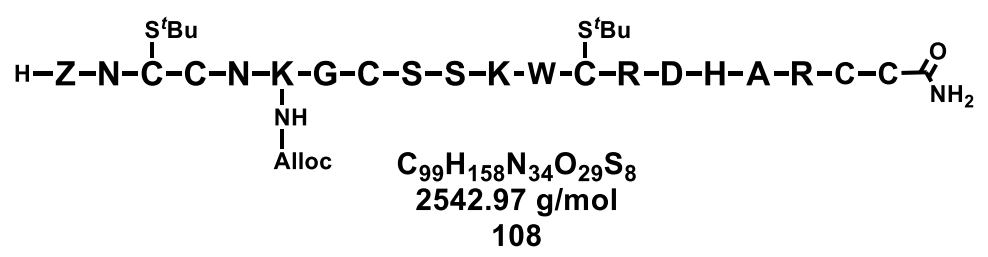

MS (ESI): $\mathbf{m} / \mathbf{z}(\%)=1272.9(24)[\mathrm{M}+2 \mathrm{H}]^{2+}, 848.9(100)[\mathrm{M}+3 \mathrm{H}]^{3+}, 637.0(58)[\mathrm{M}+4 \mathrm{H}]^{4+}$.

HRMS (ESI): calc. for: $\mathrm{C}_{99} \mathrm{H}_{160} \mathrm{~N}_{34} \mathrm{O}_{29} \mathrm{~S}_{8}: 1272.9936$, found.: $1272.9907[\mathrm{M}+2 \mathrm{H}]^{2+}$, calc. for: $\mathrm{C}_{99} \mathrm{H}_{161} \mathrm{~N}_{34} \mathrm{O}_{29} \mathrm{~S}_{8}: 848.9981$, found.: $848.9975[\mathrm{M}+3 \mathrm{H}]^{3+}$, calc. for: $\mathrm{C}_{99} \mathrm{H}_{162} \mathrm{~N}_{34} \mathrm{O}_{29} \mathrm{~S}_{8}: 637.0004$, found.: $636.9993[\mathrm{M}+4 \mathrm{H}]^{4+}$. 
Synthesis of Amine-modified $\mu$-SIIIA: Formation of the First, Second and Third Disulphide Bridge

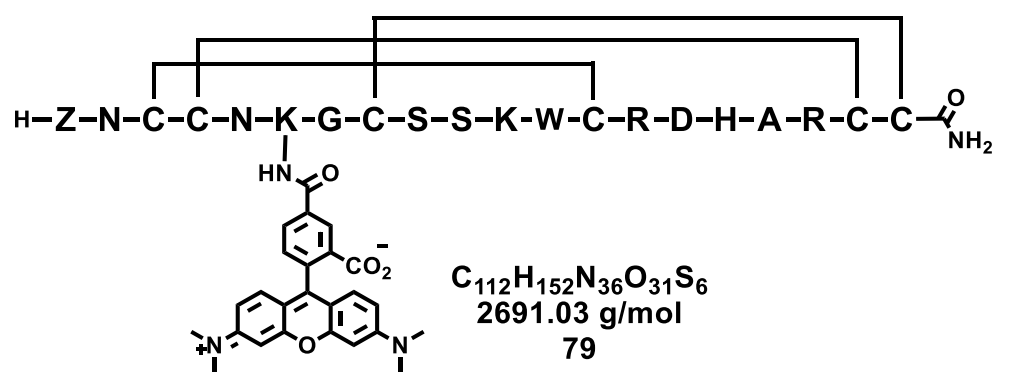

The formation of the first, second and third disulphide bridge was performed following SOP15. After formation of the second disulphide bridge, the Alloc protecting group was cleaved under argon atmosphere with $\mathrm{Pd}\left(\mathrm{PPh}_{3}\right)_{4}(14.4 \mathrm{mg}, 0.01 \mu \mathrm{mol}, 0.50 \mathrm{eq})$ and phenylsilane $(46.2 \mu \mathrm{L}$, $0.38 \mu \mathrm{mol}, 15.0 \mathrm{eq})$ for $12 \mathrm{~h}$ at $\mathrm{rt}$. The $\varepsilon$-amine group of the lysine was labelled with NHSactivated TMR $(65.9 \mathrm{mg}, 0.13 \mu \mathrm{mol}, 5.00 \mathrm{eq})$ in DMF $(400 \mu \mathrm{L})$ and DIPEA $(43.5 \mu \mathrm{L}$, $0.25 \mu \mathrm{mol}, 10.0 \mathrm{eq}$ ) for $12 \mathrm{~h}$ at $\mathrm{rt}$. After labelling the third disulphide bridge was formed, following SOP15 and the crude peptide was directly purified by semipreparative HPLC. Two isomers of the product $\mathbf{7 9}$ were obtained as a colourless solid.

1.) Isomer $79.1(54.3 \mu \mathrm{g}, 20.2 \mathrm{nmol})$

HPLC: $(250 \cdot 10 \mathrm{~mm}, 5 \mu \mathrm{m}, \mathrm{C} 18$, Gradient $=1-40 \%$ B in $30 \mathrm{~min}, \lambda[\mathrm{nm}]: 215,280,540)$ : $t_{\mathrm{R}}=24.0 \mathrm{~min}$.

MS (ESI): $\mathbf{m} / \mathbf{z}(\%)=673.8[\mathrm{M}+4 \mathrm{H}]^{4+}$.

HRMS (ESI): calc. for: $\mathrm{C}_{112} \mathrm{H}_{156} \mathrm{~N}_{36} \mathrm{O}_{31} \mathrm{~S}_{6}: 673.5017$, found.: $673.4994[\mathrm{M}+4 \mathrm{H}]^{4+}$, calc. for: $\mathrm{C}_{112} \mathrm{H}_{157} \mathrm{~N}_{36} \mathrm{O}_{31} \mathrm{~S}_{6}$ : 539.0028 , found.: $539.0008[\mathrm{M}+5 \mathrm{H}]^{5+}$.

2.) Isomer $79.2(115 \mu \mathrm{g}, 42.8 \mathrm{nmol})$

HPLC: $(250 \cdot 10 \mathrm{~mm}, 5 \mu \mathrm{m}, \mathrm{C} 18$, Gradient $=1-40 \%$ B in $30 \mathrm{~min}, \lambda[\mathrm{nm}]: 215,254,540)$ : $t_{\mathrm{R}}=24.6 \mathrm{~min}$.

HRMS (ESI): calc. for: $\mathrm{C}_{112} \mathrm{H}_{156} \mathrm{~N}_{36} \mathrm{O}_{31} \mathrm{~S}_{6}: 673.5017$, found.: $673.5001[\mathrm{M}+4 \mathrm{H}]^{4+}$. 


\section{Synthesis of DEACM modified $\mu$-Conotoxin SIIIA}

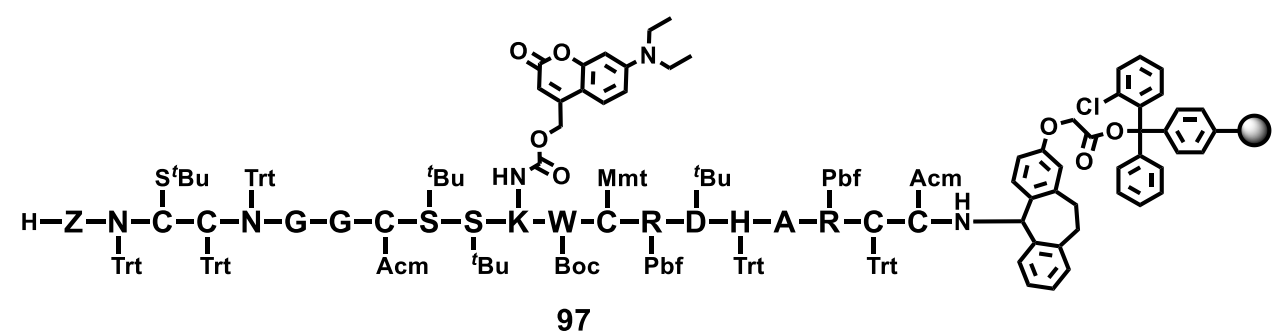

2-Chlorotrityl chloride resin $(0.70 \mathrm{~g}, 0.70 \mathrm{mmol}, 1-1.50 \mathrm{mmol} / \mathrm{g})$ was preloaded with the Ramage linker according to SOP8 and the occupancy $(0.26 \mathrm{mmol} / \mathrm{g})$ was determined following SOP10. The first amino acid Fmoc-Cys(Acm)-OH was coupled to the resin (385 mg, $0.10 \mathrm{mmol}$ ) as described in SOP9 and the peptide sequence was synthesised by automated SPPS following SOP11. The test cleavage of the native $\mu$-SIIIA (109) was carried out with cleavage mixture A as outlined in SOP12, yielding the DEACM, Acm and $S^{t} B u$ protected peptide.

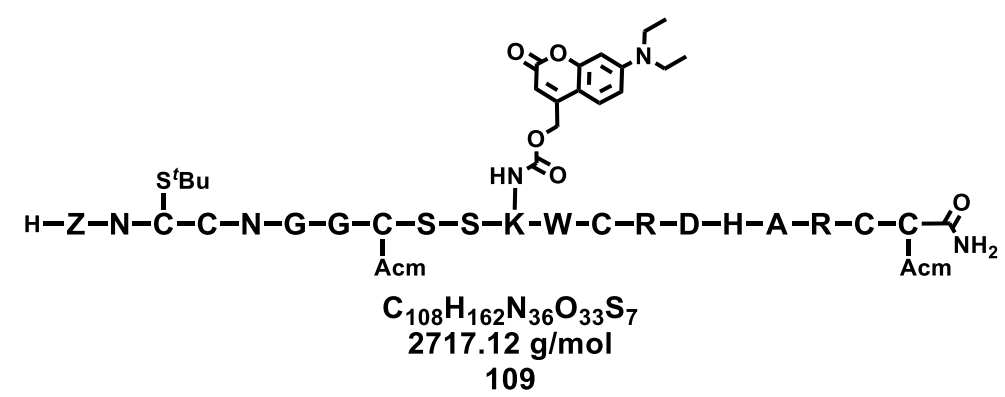

MS (ESI): m/z $(\%)=1359.0(33)[\mathrm{M}+2 \mathrm{H}]^{2+}, 906.3(100)[\mathrm{M}+3 \mathrm{H}]^{3+}$.

HRMS (ESI): calc. for: $\mathrm{C}_{108} \mathrm{H}_{164} \mathrm{~N}_{36} \mathrm{O}_{33} \mathrm{~S}_{7}: 1359.0161$, found.: $1359.0151[\mathrm{M}+2 \mathrm{H}]^{2+}$, calc. for: $\mathrm{C}_{108} \mathrm{H}_{165} \mathrm{~N}_{36} \mathrm{O}_{33} \mathrm{~S}_{7}$ : 906.3465 , found.: $906.3461[\mathrm{M}+3 \mathrm{H}]^{3+}$. 
Synthesis of DEACM modified $\mu$-Conotoxin SIIIA: Formation of the First and Second Disulphide Bridge

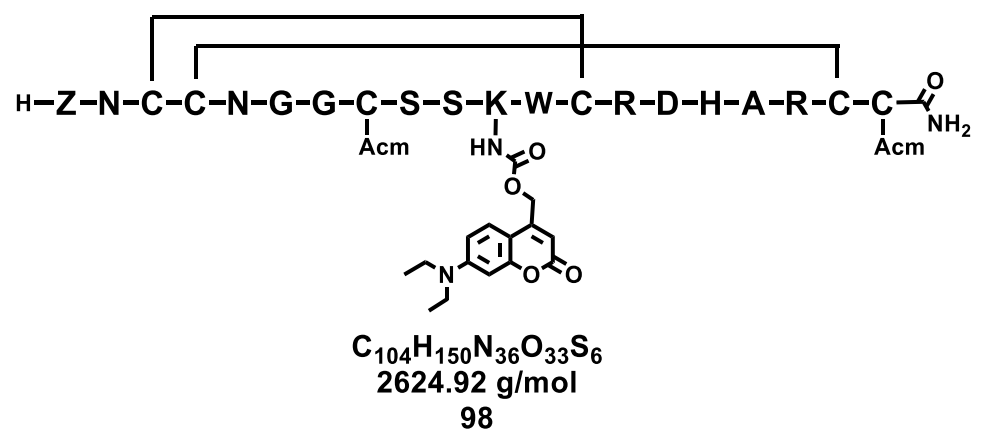

The formation of the first and second disulphide bridge was performed as described in SOP13 and SOP14. The crude peptide was purified by semipreparative HPLC and the product 98 (1.35 mg, $0.54 \mu \mathrm{mol})$ was obtained as a colourless solid.

HPLC: $(250 \cdot 10 \mathrm{~mm}, 5 \mu \mathrm{m}, \mathrm{C} 18$, Gradient $=20-50 \%$ B in $30 \mathrm{~min}, \lambda[\mathrm{nm}]: 215,254,280)$ : $t_{\mathrm{R}}=16.0 \mathrm{~min}$.

MS (ESI): $\mathbf{m} / \mathbf{z}(\%)=1359.0(33)[\mathrm{M}+2 \mathrm{H}]^{2+}, 906.3(100)[\mathrm{M}+3 \mathrm{H}]^{3+}$.

HRMS (ESI): calc. for: $\mathrm{C}_{104} \mathrm{H}_{152} \mathrm{~N}_{36} \mathrm{O}_{33} \mathrm{~S}_{6}: 1312.9831$, found.: $1312.9847[\mathrm{M}+2 \mathrm{H}]^{2+}$, calc. for: $\mathrm{C}_{104} \mathrm{H}_{153} \mathrm{~N}_{36} \mathrm{O}_{33} \mathrm{~S}_{6}: 875.6578$, found.: $875.6592[\mathrm{M}+3 \mathrm{H}]^{3+}$.

Synthesis of DEACM modified $\mu$-Conotoxin SIIIA: Formation of the First and Second Disulphide Bridge

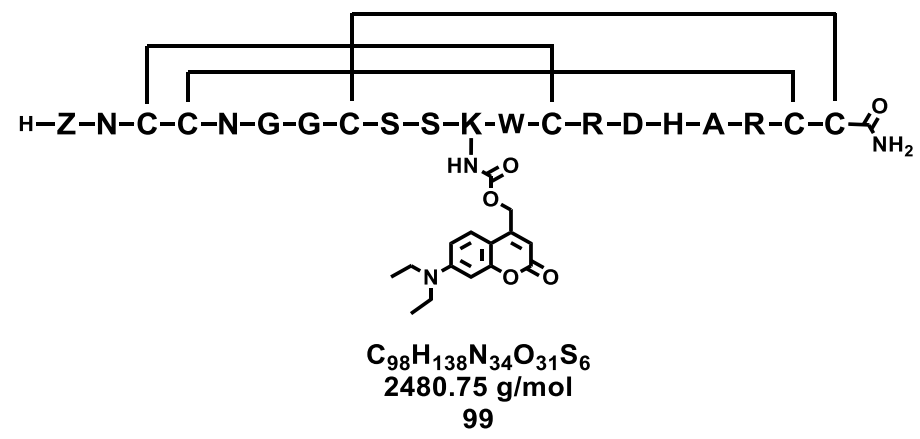

For cleavage of the Acm protecting groups and formation of the third disulphide bridge, 98 $(1.35 \mathrm{mg}, 0.54 \mu \mathrm{mol}, 1.00 \mathrm{eq})$ and Ac-L-Trp-OMe (1.34 mg, $5.44 \mu \mathrm{mol}, 10.0 \mathrm{eq})$ were dissolved in $\mathrm{AcOH} / \mathrm{H}_{2} \mathrm{O}(0.33 / 0.17, v / v)$. Following, a solution of iodine $(6.58 \mathrm{mg}, 27.0 \mu \mathrm{mol}$, 
$50.0 \mathrm{eq})$, dissolved in acetic acid $(0.26 \mathrm{~mL})$, was added and the reaction mixture was stirred for $20 \mathrm{~min}$ at $\mathrm{rt}$. The reaction was quenched by addition of L-ascorbic acid $(100 \mathrm{mg}$ in $0.50 \mathrm{~mL}$ $\mathrm{H}_{2} \mathrm{O}$ ) and the crude peptide was directly purified by semipreparative HPLC. Four isomers of the product 99 were obtained as a colourless solid.

1.) Isomer $99.1(0.25 \mathrm{mg}, 0.10 \mu \mathrm{mol})$

HPLC: $(250 \cdot 10 \mathrm{~mm}, 5 \mu \mathrm{m}, \mathrm{C} 18$, Gradient $=20-50 \%$ B in $30 \mathrm{~min}, \lambda[\mathrm{nm}]: 215,254,280)$ : $t_{\mathrm{R}}=13.5 \mathrm{~min}$.

MS (ESI): $\mathbf{m} / \mathbf{z}(\%)=1240.9(33)[\mathrm{M}+2 \mathrm{H}]^{2+}, 827.6(100)[\mathrm{M}+3 \mathrm{H}]^{3+}$.

HRMS (ESI): calc. for: $\mathrm{C}_{98} \mathrm{H}_{140} \mathrm{~N}_{34} \mathrm{O}_{31} \mathrm{~S}_{6}: 1240.9382$, found.: $1240.9386[\mathrm{M}+2 \mathrm{H}]^{2+}$, calc. for: $\mathrm{C}_{98} \mathrm{H}_{141} \mathrm{~N}_{34} \mathrm{O}_{31} \mathrm{~S}_{6}: 827.6279$, found.: $827.6292[\mathrm{M}+3 \mathrm{H}]^{3+}$.

2.) Isomer $99.2(0.05 \mathrm{mg}, 0.02 \mu \mathrm{mol})$

HPLC: $(250 \cdot 10 \mathrm{~mm}, 5 \mu \mathrm{m}, \mathrm{C} 18$, Gradient $=20-50 \%$ B in $30 \mathrm{~min}, \lambda[\mathrm{nm}]: 215,254,280)$ : $t_{\mathrm{R}}=14.1 \mathrm{~min}$.

MS (ESI): $\mathbf{m} / \mathbf{z}(\%)=1240.9(33)[\mathrm{M}+2 \mathrm{H}]^{2+}, 827.6(100)[\mathrm{M}+3 \mathrm{H}]^{3+}$.

HRMS (ESI): calc. for: $\mathrm{C}_{98} \mathrm{H}_{140} \mathrm{~N}_{34} \mathrm{O}_{31} \mathrm{~S}_{6}: 1240.9382$, found.: $1240.9368[\mathrm{M}+2 \mathrm{H}]^{2+}$, calc. for: $\mathrm{C}_{98} \mathrm{H}_{141} \mathrm{~N}_{34} \mathrm{O}_{31} \mathrm{~S}_{6}: 827.6279$, found.: $827.6290[\mathrm{M}+3 \mathrm{H}]^{3+}$.

3.) Isomer $99.3(0.08 \mathrm{mg}, 0.02 \mu \mathrm{mol})$

HPLC: $(250 \cdot 10 \mathrm{~mm}, 5 \mu \mathrm{m}, \mathrm{C} 18$, Gradient $=20-50 \%$ B in $30 \mathrm{~min}, \lambda[\mathrm{nm}]: 215,254,280)$ : $t_{\mathrm{R}}=14.7 \mathrm{~min}$.

MS (ESI): $\mathbf{m} / \mathbf{z}(\%)=1240.9(33)[\mathrm{M}+2 \mathrm{H}]^{2+}, 827.6(100)[\mathrm{M}+3 \mathrm{H}]^{3+}$.

HRMS (ESI): calc. for: $\mathrm{C}_{98} \mathrm{H}_{140} \mathrm{~N}_{34} \mathrm{O}_{31} \mathrm{~S}_{6}: 1240.9382$, found.: $1240.9389[\mathrm{M}+2 \mathrm{H}]^{2+}$, calc. for: $\mathrm{C}_{98} \mathrm{H}_{141} \mathrm{~N}_{34} \mathrm{O}_{31} \mathrm{~S}_{6}: 827.6279$, found.: $827.6291[\mathrm{M}+3 \mathrm{H}]^{3+}$.

4.) Isomer $99.4(0.12 \mathrm{mg}, 0.05 \mu \mathrm{mol})$

HPLC: $(250 \cdot 10 \mathrm{~mm}, 5 \mu \mathrm{m}, \mathrm{C} 18$, Gradient $=20-50 \%$ B in $30 \mathrm{~min}, \lambda[\mathrm{nm}]: 215,254,280)$ : $t_{\mathrm{R}}=15.5 \mathrm{~min}$.

MS (ESI): $\mathbf{m} / \mathbf{z}(\%)=1240.9(16)[\mathrm{M}+2 \mathrm{H}]^{2+}, 827.6(100)[\mathrm{M}+3 \mathrm{H}]^{3+}$.

HRMS (ESI): calc. for: $\mathrm{C}_{98} \mathrm{H}_{140} \mathrm{~N}_{34} \mathrm{O}_{31} \mathrm{~S}_{6}: 1240.9382$, found.: $1240.9386[\mathrm{M}+2 \mathrm{H}]^{2+}$, calc. for: $\mathrm{C}_{98} \mathrm{H}_{141} \mathrm{~N}_{34} \mathrm{O}_{31} \mathrm{~S}_{6}: 827.6279$, found.: $827.6287[\mathrm{M}+3 \mathrm{H}]^{3+}$. 


\section{Abbreviations}

Standard $\alpha$-amino acids are abbreviated using the three- or one letter code. Nucleobases in aegPNA sequences are abbreviated with small letter $(a, c, g, t)$.

\begin{tabular}{|c|c|}
\hline $\mathrm{Ac}_{2} \mathrm{O}$ & acetic anhydride \\
\hline Acm & acetoamidomethyl \\
\hline $\mathrm{AcOH}$ & acetic acid \\
\hline aeg & $N$-(2-aminoethyl)glycine \\
\hline Alloc & allyloxycarbonyl \\
\hline Allocam & allyloxycarbonyl aminomethyl \\
\hline $\mathrm{AO}$ & acridine orange \\
\hline ATP & adenosine triphosphate \\
\hline AU & absorption unit \\
\hline BAC & 10,10'-bis[3-carboxypropyl]-9,9'biacridinium dinitrate \\
\hline $\mathrm{BD}$ & syringe syringe by Becton Dickinson \\
\hline Bhoc & benzhydryloxycarbonyl protection group \\
\hline Boc & tert-butoxycarbonyl \\
\hline calc & calculated \\
\hline CFP & cyan fluorescent protein \\
\hline CNS & central nervous system \\
\hline COMU & (1-cyano-2-ethoxy-2-oxoethylidenaminooxy)dimethylamino- \\
\hline $\mathrm{CuAAC}$ & copper(I)-catalysed azide-alkyne cycloaddition \\
\hline$\delta$ & chemical shift (NMR) \\
\hline DBU & 1,8-diazabicyclo[5.4.0]undec-7-en \\
\hline DCM & dichlormethane \\
\hline DEACM & (7-(diethylamino)coumarin-4-yl)methyl \\
\hline DIC & $N, N^{\prime}$-diisopropylcarbodiimide \\
\hline DIPEA & $N, N$ '-diisopropylethylamine \\
\hline DLS & dynamic light scattering \\
\hline DMAP & 4-dimethylaminopyridine \\
\hline
\end{tabular}




\begin{tabular}{ll} 
DMF & $N, N$-dimethylformamide \\
DMNPB & 3-(4,5-Dimethoxy-2-nitrophenyl)-2-butyl \\
DMSO & dimethyl sulfoxide \\
DNA & deoxyribonucleic acid \\
DOPC & 1,2-dioleoyl-sn-glycero-3-phosphocholine \\
DOPE & 1,2-dioleoyl-sn-glycero-3-phosphoethanolamine \\
DOPS & 1,2 -dioleoyl-sn-glycero-3-phospho-L-serine \\
$\Delta p H$ & pH gradient \\
DsRed & red fluorescent protein \\
DTT & dithiothreitol \\
$\Delta \mu H^{+}$ & electrochemical gradient \\
$\Delta \Psi$ & membrane potential \\
$\varepsilon$ & extinction coefficient \\
EDC & 1-ethyl-3-(3-dimethylaminopropyl)carbodiimide \\
EDT & 1,2-ethanedithiole \\
EDTA & fluorescence resonance energy transfer \\
eq & FAC fluorescence intensity \\
ESI & equivalents \\
EtOAc & electrospray-ionisation \\
EtOH & ethyl acetate \\
FCCS & fluorescence cross-correlation spectroscopy \\
FDA & Food and Drug Administration (US) \\
G & fluotraacetic acid \\
\hline
\end{tabular}

GABA $\quad \gamma$-aminobutyric acid

GFP green fluorescent protein

HATU O-(7-azabenzotriazol-1-yl)- $N, N, N^{\prime}, N^{\prime}$-tetramethyluronium hexafluorophosphate

HBTU O-(1H-benzotriazol-1-yl)- $N, N, N^{\prime}, N^{\prime}$-tetramethyluronium hexafluorophosphate

HEK human embryonic kidney 


\begin{tabular}{|c|c|}
\hline HOAt & 1-hydroxy-7-azabenzotriazole \\
\hline $\mathrm{HOBt}$ & 1-hydroxybenzotriazole \\
\hline HPLC & high performance liquid chromatography \\
\hline HR-MS & high resolution mass spectrometry \\
\hline$J$ & coupling constant \\
\hline KALP & peptides of general sequence: GKK(LA) n LKKA \\
\hline$K_{\mathrm{D}}$ & dissociation constant \\
\hline $\mathrm{k}_{\mathrm{obs}}$ & observed association rate \\
\hline $\mathrm{k}_{\mathrm{off}}$ & dissociation rate \\
\hline $\mathrm{k}_{\mathrm{on}}$ & association rate \\
\hline$K_{\mathrm{SV}}$ & STERN-VOLLMER quenching constant \\
\hline LC-MS & liquid chromatography mass spectrometry \\
\hline LUV & large unilamellar vesicle \\
\hline $\mathrm{m} / \mathrm{z}$ & mass-to-charge ratio \\
\hline $\mathrm{MeCN}$ & acetonitrile \\
\hline MeIm & methylimidazole \\
\hline $\mathrm{MeOH}$ & methanol \\
\hline MEQ & 6-methoxy- $N$-ethylquinolinium iodide \\
\hline & morpholino-carbenium hexafluorophosphate \\
\hline MQAE & $N$-(ethoxycarbonylmethyl)-6-methoxyquninolinium bromide \\
\hline MS & mass spectrometry \\
\hline MSNT & 1-(mesitylene-2-sulfonyl)-3-nitro-1,2,4-triazole \\
\hline $\mathrm{Nav}$ & voltage gated sodium channels \\
\hline NBD & 7-nitrobenz-2-oxa-1,3-diazol-4-yl \\
\hline NHS-ester & $N$-hydroxysuccinimide ester \\
\hline NMR & nuclear magnetic resonance \\
\hline $\mathrm{nt}$ & nucleotides \\
\hline NTA & Nitrilotriacetic acid \\
\hline NVOC & 6-nitroveratryloxycarbonyl \\
\hline Oxyma & ethyl cyanohydroxyiminoacetate \\
\hline PAGE & polyacrylamide gel electrophoresis \\
\hline
\end{tabular}


$\mathrm{Pbf}$

$\mathrm{Pd}\left(\mathrm{PPh}_{3}\right)_{4}$

PG

pGlu

PNA

PNS

PPG

Ppm

PTMs

PyAOP

$\mathrm{R}$

RNA

Rpm

rrp

$\mathrm{rt}$

SLC17

SNARE

SOP

SPPS

SPQ

SPyr

$\mathrm{S}^{t} \mathrm{Bu}$

STX

SUV

SV

${ }^{t} \mathrm{Bu}$

Tert

TES

TEV

TFA 2,2,4,6,7-pentamethyldihydrofurane-5-sulphonyl

Tetrakis(triphenylphosphine)palladium

protecting group

pyroglutamate

peptide nucleic acid

peripheral nervous system

photoremovable protecting group

parts per million

post-translational modifications

7-azabenzotriazol-1-yloxy)tripyrrolidinophosphonium hexafluorophosphate

AlexaFluor 647 fluorescence intensity

ribonucleic acid

rotations per minute

readily releasable pool

room temperature

solute carrier transmembrane transporters

soluble $N$-ethylmaleimide-sensitive factor attachment protein receptor

standard operating procedure

solid phase peptide synthesis

6-methoxy- $N$-(3-sulfopropyl)quinolinium

2-pyridinesulphenyl

tert-butylthio

saxitoxin

small unilamellar vesicle

synaptic vesicles

tert-butyl

tertiary

triethylsilane

tobacco etch virus

trifluoroacetic acid 


\begin{tabular}{|c|c|}
\hline TFE & trifluoroethanol \\
\hline TFMSA & trifluoromethanesulfonic acid \\
\hline THPTA & $\operatorname{tris}((1-$ hydroxy-propyl-1H-1,2,3-triazol-4-yl)methyl)amine \\
\hline TIRF & total internal reflection fluorescence \\
\hline TIS & triisopropylsilan \\
\hline TLC & thin layer chromatography \\
\hline TMD & transmembrane domain \\
\hline TMR & tetramethylrhodamine \\
\hline TMS & trimethylsilan \\
\hline $\mathrm{TMSN}_{3}$ & trimethylsilyl azide \\
\hline$t_{\mathrm{R}}$ & retention time \\
\hline TR & Texas Red \\
\hline Trt & trityl \\
\hline TTX & tetrodotoxin \\
\hline $\mathrm{U}$ & units \\
\hline U-HPLC & ultra-high performance liquid chromatography \\
\hline UV & ultraviolet \\
\hline $\mathrm{v} / \mathrm{v}$ & volume to volume \\
\hline VAchT & vesicular acetylcholine transporter \\
\hline V-ATPase & vacuolar-type $\mathrm{H}^{+}$-ATPase \\
\hline VGLUT & vesicular glutamate transporter \\
\hline VGSC & voltage-gated sodium channel \\
\hline VIAAT & vesicular inhibitory amino acid transporter \\
\hline VIS & visual \\
\hline VMATs & vesicular monoamine transporters \\
\hline YFP & yellow fluorescent protein \\
\hline
\end{tabular}




\section{Bibliography}

[1] T. C. Südhof, Annu. Rev. Neurosci. 2004, 27, 509-547.

[2] S. Takamori, M. Holt, K. Stenius, E. A. Lemke, M. Grønborg, D. Riedel, H. Urlaub, S. Schenck, B. Brügger, P. Ringler, et al., Cell 2006, 127, 831-846.

[3] J. Preobraschenski, J. F. Zander, T. Suzuki, G. Ahnert-Hilger, R. Jahn, Neuron 2014, 84, 12871301.

[4] S. Schenck, S. M. Wojcik, N. Brose, S. Takamori, Nat. Neurosci. 2009, 12, 156-162.

[5] S. Saha, V. Prakash, S. Halder, K. Chakraborty, Y. Krishnan, Nat. Nanotechnol. 2015, 10, 645651.

[6] V. Prakash, S. Saha, K. Chakraborty, Y. Krishnan, Chem. Sci. 2016, 7, 1946-1953.

[7] N. D. Sonawane, J. R. Thiagarajah, A. S. Verkman, J. Biol. Chem. 2002, 277, 5506-5513.

[8] M. Zajac, K. Chakraborty, S. Saha, V. Mahadevan, D. T. Infield, A. Accardi, Z. Qiu, Y. Krishnan, J. Cell Sci. 2020, 133, 1-13.

[9] M. De Lera Ruiz, R. L. Kraus, J. Med. Chem. 2015, 58, 7093-7118.

[10] S. D. Dib-Hajj, Y. Yang, J. A. Black, S. G. Waxman, Nat. Rev. Neurosci. 2013, 14, 49-62.

[11] S. J. Sanders, M. T. Murtha, A. R. Gupta, J. D. Murdoch, M. J. Raubeson, A. J. Willsey, A. G. Ercan-Sencicek, N. M. Di Lullo, N. N. Parikshak, J. L. Stein, et al., Nature 2012, 485, 237-241.

[12] G. Bulaj, P. J. West, J. E. Garrett, M. Marsh, M. M. Zhang, R. S. Norton, B. J. Smith, D. Yoshikami, B. M. Olivera, Biochemistry 2005, 44, 7259-7265.

[13] C. I. Schroeder, J. Ekberg, K. J. Nielsen, D. Adams, M. L. Loughnan, L. Thomas, D. J. Adams, P. F. Alewood, R. J. Lewis, J. Biol. Chem. 2008, 283, 21621-21628.

[14] K. B. Akondi, R. J. Lewis, P. F. Alewood, Biopolymers 2014, 101, 347-354.

[15] L. Qu, Y. Akbergenova, Y. Hu, T. Schikorski, J. Comp. Neurol. 2009, 514, 343-352.

[16] J. S. Bonifacino, B. S. Glick, Cell 2004, 116, 153-166.

[17] T. C. Südhof, R. Jahn, Neuron 1991, 6, 665-677.

[18] C. Rosenmund, C. F. Stevens, Neuron 1996, 16, 1197-1207.

[19] P. S. Kaeser, W. G. Regehr, Curr. Opin. Neurobiol. 2017, 43, 63-70.

[20] N. L. Chanaday, M. A. Cousin, I. Milosevic, S. Watanabe, J. R. Morgan, J. Neurosci. 2019, 39, 
8209-8216.

[21] B. Katz, Science 1971, 173, 123-126.

[22] R. H. Edwards, Neuron 2007, 55, 835-858.

[23] Z. Farsi, J. Preobraschenski, G. Van Den Bogaart, D. Riedel, R. Jahn, A. Woehler, Science 2016, $351,981-984$.

[24] M. Beinlich, Mechanisms Underlying Biphasic Synaptic Vesicle Acidification in Glutamatergic Synapses, Dissertation, Heinrich-Heine Universität Düsseldorf, 2018.

[25] H. Fei, A. Grygoruk, E. S. Brooks, A. Chen, D. E. Krantz, Traffic 2008, 9, 1425-1436.

[26] D. Peter, J. Jimenez, Y. Liu, J. Kim, R. H. Edwards, J. Biol. Chem. 1994, 269, 7231-7237.

[27] J. D. Erickson, M. K. H. Schäfer, T. I. Bonner, L. E. Eiden, E. Weihe, Proc. Natl. Acad. Sci. U. S. A. 1996, 93, 5166-5171.

[28] M. L. Nguyen, G. D. Cox, S. M. Parsons, Biochemistry 1998, 37, 13400-13410.

[29] R. G. Johnson, Physiol. Rev. 1988, 68, 232-307.

[30] J. D. Erickson, H. Varoqui, M. K. H. Schafer, W. Modi, M. F. Diebler, E. Weihe, J. Rand, L. E. Eiden, T. I. Bonner, T. B. Usdin, J. Biol. Chem. 1994, 269, 21929-21932.

[31] S. L. McIntire, R. J. Reimer, K. Schuske, R. H. Edwards, E. M. Jorgensen, Nature 1997, 389, 870-876.

[32] N. Juge, A. Muroyama, M. Hiasa, H. Omote, Y. Moriyama, J. Biol. Chem. 2009, 284, 3507335078 .

[33] P. R. Maycox, T. Deckwerth, J. W. Hell, R. Jahn, J. Biol. Chem. 1988, 263, 15423-15428.

[34] E. E. Bellocchio, R. J. Reimer, J. Fremeau, R. H. Edwards, Science 2000, 289, 957-960.

[35] S. Takamori, J. S. Rhec, C. Rosenmund, R. Jahn, Nature 2000, 407, 189-194.

[36] R. T. Fremeau, S. Voglmaier, R. P. Seal, R. H. Edwards, Trends Neurosci. 2004, 27, 98-103.

[37] S. M. Wojcik, J. S. Rhee, E. Herzog, A. Sigler, R. Jahn, S. Takamori, N. Brose, C. Rosenmund, Proc. Natl. Acad. Sci. U. S. A. 2004, 101, 7158-7163.

[38] Å. Wallén-Mackenzie, H. Gezelius, M. Thoby-Brisson, A. Nygård, A. Enjin, F. Fujiyama, G. Fortin, K. Kullander, J. Neurosci. 2006, 26, 12294-12307.

[39] D. Balschun, D. Moechars, Z. Callaerts-Vegh, B. Vermaercke, N. Van Acker, L. Andries, R. D’Hooge, Cereb. Cortex 2010, 20, 684-693. 
[40] R. M. Tordera, S. Totterdell, S. M. Wojcik, N. Brose, N. Elizalde, B. Lasheras, J. Del Rio, Eur. J. Neurosci. 2007, 25, 281-290.

[41] E. E. Benarroch, Neurology 2018, 91, 125-132.

[42] A. Kashani, È. Lepicard, O. Poirel, C. Videau, J. P. David, C. Fallet-Bianco, A. Simon, A. Delacourte, B. Giros, J. Epelbaum, et al., Neurobiol. Aging 2008, 29, 1619-1630.

[43] W. S. Van Der Hel, S. A. M. W. Verlinde, D. H. M. Meijer, M. De Wit, M. G. Rensen, K. L. I. Van Gassen, P. C. Van Rijen, C. W. M. Van Veelen, P. N. E. De Graan, Epilepsia 2009, 50, 1717-1728.

[44] N. Pietrancosta, M. Djibo, S. Daumas, S. El Mestikawy, J. D. Erickson, Mol. Neurobiol. 2020, $57,3118-3142$.

[45] K. Disbrow, J. Gershten, J. A. Ruth, Biochem. Biophys. Res. Commun. 1982, 108, $1221-1227$.

[46] P. R. Maycox, J. W. Hell, R. Jahn, Trends Neurosci. 1990, 13, 83-87.

[47] M. Martineau, R. E. Guzman, C. Fahlke, J. Klingauf, Nat. Commun. 2017, 8, 1-13.

[48] S. Takamori, J. S. Rhee, C. Rosenmund, R. Jahn, J. Neurosci. 2001, 21, 1-6.

[49] S. Takamori, P. Malherbe, C. Broger, R. Jahn, EMBO Rep. 2002, 3, 798-803.

[50] S. El Mestikawy, ̊̊. Wallén-Mackenzie, G. M. Fortin, L. Descarries, L. E. Trudeau, Nat. Rev. Neurosci. 2011, 12, 204-216.

[51] S. M. Voglmaier, K. Kam, H. Yang, D. L. Fortin, Z. Hua, R. A. Nicoll, R. H. Edwards, Neuron 2006, 51, 71-84.

[52] S. De Gois, E. Jeanclos, M. Morris, S. Grewal, H. Varoqui, J. D. Erickson, Cell. Mol. Neurobiol. 2006, 26, 679-693.

[53] T. S. Hnasko, N. Chuhma, H. Zhang, G. Y. Goh, D. Sulzer, R. D. Palmiter, S. Rayport, R. H. Edwards, Neuron 2010, 65, 643-656.

[54] C. Gras, B. Amilhon, È. M. Lepicard, O. Poirel, J. Vinatier, M. Herbin, S. Dumas, E. T. Tzavara, M. R. Wade, G. G. Nomikos, et al., Nat. Neurosci. 2008, 11, 292-300.

[55] F. Li, J. Eriksen, J. Finer-Moore, R. Chang, P. Nguyen, A. Bowen, A. Myasnikov, Z. Yu, D. Bulkley, Y. Cheng, et al., Science 2020, 368, 893-897.

[56] J. Almqvist, Y. Huang, A. Laaksonen, D.-N. Wang, S. Hovmöller, Protein Sci. 2007, 16, 18191829.

[57] J. B. Leano, S. Batarni, J. Eriksen, N. Juge, J. E. Pak, T. Kimura-Someya, Y. Robles-Colmenares, 
Y. Moriyama, R. M. Stroud, R. H. Edwards, PLoS Biol. 2019, 17, 1-25.

[58] H. H. Füldner, H. Stadler, Eur. J. Biochem. 1982, 121, 519-524.

[59] H. Wolosker, D. O. De Souza, L. De Meis, J. Biol. Chem. 1996, 271, 11726-11731.

[60] J. S. Tabb, P. E. Kish, R. Van Dyke, T. Ueda, J. Biol. Chem. 1992, 267, 15412-15416.

[61] J. Preobraschenski, C. Cheret, M. Ganzella, J. F. Zander, K. Richter, S. Schenck, R. Jahn, G. Ahnert-Hilger, Cell Rep. 2018, 23, 535-545.

[62] G. Y. Goh, H. Huang, J. Ullman, L. Borre, T. S. Hnasko, L. O. Trussell, R. H. Edwards, Nat. Neurosci. 2011, 14, 1285-1292.

[63] J. Eriksen, R. Chang, M. McGregor, K. Silm, T. Suzuki, R. H. Edwards, Neuron 2016, 90, 768 780.

[64] S. Naito, T. Ueda, J. Neurochem. 1985, 44, 99-109.

[65] J. Hartinger, R. Jahn, J. Biol. Chem. 1993, 268, 23122-23127.

[66] G. Ahnert-Hilger, M. Höltje, I. Pahner, S. Winter, I. Brunk, Rev. Physiol. Biochem. Pharmacol. 2003, 150, 140-160.

[67] S. M. Stobrawa, T. Breiderhoff, S. Takamori, D. Engel, M. Schweizer, A. A. Zdebik, M. R. Bösl, K. Ruether, H. Jahn, A. Draguhn, et al., Neuron 2001, 29, 185-196.

[68] T. Maritzen, D. J. Keating, I. Neagoe, A. A. Zdebik, T. J. Jentsch, J. Neurosci. 2008, 28, 1058710598.

[69] X. Li, T. Wang, Z. Zhao, S. A. Weinman, Am. J. Physiol. - Cell Physiol. 2002, 282, 1483-1491.

[70] M. Hara-Chikuma, B. Yang, N. D. Sonawane, S. Sasaki, S. Uchida, A. S. Verkman, J. Biol. Chem. 2005, 280, 1241-1247.

[71] R. E. Guzman, M. Grieschat, C. Fahlke, A. K. Alekov, ACS Chem. Neurosci. 2013, 4, 994-1003.

[72] R. E. Guzman, A. K. Alekov, M. Filippov, J. Hegermann, C. Fahlke, Front. Cell. Neurosci. 2014, $8,1-9$.

[73] M. Yoshikawa, S. Uchida, J. Ezaki, T. Rai, A. Hayama, K. Kobayashi, Y. Kida, M. Noda, M. Koike, Y. Uchiyama, et al., Genes to Cells 2002, 7, 597-605.

[74] R. Chang, J. Eriksen, R. H. Edwards, Elife 2018, 7, 1-16.

[75] J. Eriksen, F. Li, R. H. Edwards, BBA - Biomembr. 2020, 104743.

[76] T. Y. Chen, T. C. Hwang, Physiol. Rev. 2008, 88, 351-387. 
[77] U. F. Aslanova, T. Morimoto, E. I. Farajov, N. Kumagai, M. Nishino, N. Sugawara, A. Ohsaga, Y. Maruyama, S. Tsuchiya, S. Takahashi, et al., Tohoku J. Exp. Med. 2006, 210, 291-300.

[78] T. Stauber, T. J. Jentsch, Annu. Rev. Physiol. 2013, 75, 453-477.

[79] A. Di, M. E. Brown, L. V. Deriy, C. Li, F. L. Szeto, Y. Chen, P. Huang, J. Tong, A. P. Naren, V. Bindokas, et al., Nat. Cell Biol. 2006, 8, 933-944.

[80] T. O. Neild, R. C. Thomas, J. Physiol. 1974, 242, 453-470.

[81] R. A. Harris, A. M. Allan, Science 1985, 228, 1108-1110.

[82] D. Arosio, G. M. Ratto, Front. Cell. Neurosci. 2014, 8, 1-12.

[83] R. M. Wachter, S. J. Remington, Curr. Biol. 1999, 9, 628-629.

[84] L. J. V. Galietta, P. M. Haggie, A. S. Verkman, FEBS Lett. 2001, 499, 220-224.

[85] S. Jayaraman, P. Haggie, R. M. Wachter, S. J. Remington, A. S. Verkman, J. Biol. Chem. 2000, $275,6047-6050$.

[86] O. Markova, M. Mukhtarov, E. Real, Y. Jacob, P. Bregestovski, J. Neurosci. Methods 2008, 170, $67-76$.

[87] T. Kuner, G. J. Augustine, Neuron 2000, 27, 447-459.

[88] D. Arosio, F. Ricci, L. Marchetti, R. Gualdani, L. Albertazzi, F. Beltram, Nat. Methods 2010, 7, $516-518$.

[89] D. Arosio, G. Garau, F. Ricci, L. Marchetti, R. Bizzarri, R. Nifosì, F. Beltram, Biophys. J. 2007, 93, 232-244.

[90] T. Wasiluk, M. Roueinfar, K. Hiryak, M. Torsiello, A. Miner, J. Lee, M. Venditto, W. Terzaghi, D. Lucent, A. L. VanWert, Biol. Open 2019, 8, 1-8.

[91] C. D. Geddes, Meas. Sci. Technol. 2001, 12, 53-88.

[92] S. Saha, V. Prakash, S. Halder, K. Chakraborty, Y. Krishnan, Nat. Nanotechnol. 2015, 10, 645651.

[93] R. Y. Tsien, Biochemistry 1980, 19, 2396-2404.

[94] A. Minta, J. P. Y. Kao, R. Y. Tsien, J. Biol. Chem. 1989, 264, 8171-8178.

[95] A. S. Verkman, Am. J. Physiol. - Cell Physiol. 1990, 259, 375-388.

[96] T. D. Ashton, K. A. Jolliffe, F. M. Pfeffer, Chem. Soc. Rev. 2015, 44, 4547-4595.

[97] N. P. Illsley, A. S. Verkman, Biochemistry 1987, 26, 1215-1219. 
[98] A. S. Verkman, M. C. Sellers, A. C. Chao, T. Leung, R. Ketcham, Anal. Biochem. 1989, 178, $355-361$.

[99] L. J. MacVinish, T. Reancharoen, A. W. Cuthbert, Br. J. Pharmacol. 1993, 108, 469-478.

[100] N. Chub, G. Z. Mentis, M. J. O’Donovan, J. Neurophysiol. 2006, 95, 323-330.

[101] M. K. Mansoura, J. Biwersi, M. A. Ashlock, A. S. Verkman, Hum. Gene Ther. 1999, 10, 861875.

[102] R. G. Painter, G. Wang, Anal. Chem. 2006, 78, 3133-3137.

[103] Y. Şener, G. Tosun, F. Kahvecioğlu, A. Gökalp, H. Koç, Eur. J. Dent. 2007, 01, 21-24.

[104] H. A. Olszowy, J. Rossiter, J. Hegarty, P. Geoghegan, M. Haswell-Elkins, J. Anal. Toxicol. 1998, $22,225-230$.

[105] A. M. Gbadebo, C. O. Nwufoh, J. Geochemical Explor. 2010, 107, 169-174.

[106] N. D. Sonawane, A. S. Verkman, J. Cell Biol. 2003, 160, 1129-1138.

[107] S. Modi, M. G. Swetha, D. Goswami, G. D. Gupta, S. Mayor, Y. Krishnan, Nat. Nanotechnol. 2009, 4, 325-330.

[108] S. Modi, C. Nizak, S. Surana, S. Halder, Y. Krishnan, Nat. Nanotechnol. 2013, 8, 459-467.

[109] S. Surana, J. M. Bhat, S. P. Koushika, Y. Krishnan, Nat. Commun. 2011, 2, 1-7.

[110] J. R. Lakowicz, Principles of Fluorescence Spectroscopy, Springer $3^{\text {rd }}$ Edition, 2006.

[111] A. M. Hansen, G. Bonke, W. F. J. Hogendorf, F. Björkling, J. Nielsen, K. T. Kongstad, D. Zabicka, M. Tomczak, M. Urbas, P. E. Nielsen, et al., Eur. J. Med. Chem. 2019, 168, 134-145.

[112] P. E. Nielsen, M. Egholm, R. H. Berg, O. Buchardt, Science 1991, 254, 1497-1500.

[113] G. T. Hwang, Y. J. Seo, B. H. Kim, J. Am. Chem. Soc. 2004, 126, 6528-6529.

[114] P. E. Nielsen, Curr. Opin. Struct. Biol. 1999, 9, 353-357.

[115] J. C. Wu, Q. C. Meng, H. M. Ren, H. T. Wang, J. Wu, Q. Wang, Acta Pharmacol. Sin. 2017, 38, 798-805.

[116] P. E. Nielsen, Appl. Biochem. Biotechnol. - Part B Mol. Biotechnol. 2004, 26, 233-248.

[117] S. Siddiquee, Adv. Tech. Biol. Med. 2015, 03, 1-10.

[118] U. Giesen, W. Kleider, C. Berding, A. Geiger, H. Ørum, P. E. Nielsen, Nucleic Acids Res. 1998, 26, 5004-5006.

[119] J. Tailhades, H. Takizawa, M. J. Gait, D. A. Wellings, J. D. Wade, Y. Aoki, F. Shabanpoor, 
Front. Chem. 2017, 5, 1-7.

[120] S. Middel, C. H. Panse, S. Nawratil, U. Diederichsen, ChemBioChem 2017, 18, 2328-2332.

[121] B. E. Hubrich, P. M. Menzel, B. Kugler, U. Diederichsen, Pept. Nucleic Acids Methods Protoc. Springer, $3^{\text {rd }}$ Edition, 2020.

[122] K. R. Wilson, S. Sedberry, R. Pescatore, D. Vinton, B. Love, S. Ballard, B. C. Wham, S. K. Hutchison, E. J. Williamson, J. Pept. Sci. 2016, 22, 622-627.

[123] U. Rost, C. Steinem, U. Diederichsen, Chem. Sci. 2016, 7, 5900-5907.

[124] D. Pahlke, Synthesis, characterisation and sensor-functionalisation of transmembrane $\beta$ peptides, Dissertation, Georg-August Universität Göttingen, 2018.

[125] E. I. Vrettos, N. Sayyad, E. M. Mavrogiannaki, E. Stylos, A. D. Kostagianni, S. Papas, T. Mavromoustakos, V. Theodorou, A. G. Tzakos, RSC Adv. 2017, 7, 50519-50526.

[126] G. A. Höger, M. Wiegand, B. Worbs, U. Diederichsen, ChemBioChem 2020, 21, 2599-2603.

[127] G. Höger, Self-Organization of $\beta$-Peptide Nucleic Acid Helices for Membrane Scaffolding, Dissertation, Georg-August-Universität Göttingen, 2019.

[128] Q. Sun, S. Cai, B. R. Peterson, Org. Lett. 2009, 11, 567-570.

[129] R. Ramage, S. Irving, C. McInnes, Tetrahedron Lett. 1993, 354, 6599-6602.

[130] P. Linning, U. Haussmann, I. Beyer, S. Weidlich, H. Schieb, J. Wiltfang, H. W. Klafki, H. J. Knölker, Org. Biomol. Chem. 2012, 10, 8216-8235.

[131] C. S. Bennett, C. H. Wong, Chem. Soc. Rev. 2007, 36, 1227-1238.

[132] T. J. Tolbert, D. Franke, C. H. Wong, Bioorganic Med. Chem. 2005, 13, 909-915.

[133] H. Nam, B. J. Hwang, D. Y. Choi, S. Shin, M. Choi, FEBS Open Bio 2020, 10, 619-626.

[134] David S. Waugh, Protein Expr. Purif. 2011, 80, 283-293.

[135] J. Zerweck, B. S. Venkata, J. M. Pittman, A. K. Srivastava, P. C. Moore, J. R. Sachleben, G. Thinakaran, S. C. Meredith, Protein Expr. Purif. 2019, 162, 72-82.

[136] G. M. Dubowchik, R. A. Firestone, L. Padilla, D. Willner, S. J. Hofstead, K. Mosure, J. O. Knipe, S. J. Lasch, P. A. Trail, Bioconjug. Chem. 2002, 13, 855-869.

[137] M. K. Shim, Y. Moon, S. Yang, J. Kim, H. Cho, S. Lim, H. Y. Yoon, J. K. Seong, K. Kim, Biomaterials 2020, 261, 1-14.

[138] J. D. Bargh, A. Isidro-Llobet, J. S. Parker, D. R. Spring, Chem. Soc. Rev. 2019, 48, 4361-4374. 
[139] J. D. Wehland, A. S. Lygina, P. Kumar, S. Guha, B. E. Hubrich, R. Jahn, U. Diederichsen, Mol. Biosyst. 2016, 12, 2770-2776.

[140] K. Halbmair, J. Wegner, U. Diederichsen, M. Bennati, Biophys. J. 2016, 111, 2345-2348.

[141] A. Holt, J. A. Killian, Eur. Biophys. J. 2010, 39, 609-621.

[142] V. Vasic, Reconstitution of retrotranslocation by the Hrdl ubiquitin ligase with purified components, Dissertation, Georg-August Universität Göttingen, 2019.

[143] W. van den Nest, S. Yuval, F. Albericio, J. Pept. Sci. 2001, 120, 115-120.

[144] N. Hartrampf, A. Saebi, M. Poskus, Z. P. Gates, A. J. Callahan, A. E. Cowfer, S. Hanna, S. Antilla, C. K. Schissel, A. J. Quartararo, et al., Science 2020, 368, 980-987.

[145] S. Lata, A. Reichel, R. Brock, R. Tampé, J. Piehler, J. Am. Chem. Soc. 2005, 127, 10205-10215.

[146] R. Wieneke, N. Labòria, M. Rajan, A. Kollmannsperger, F. Natale, M. C. Cardoso, R. Tampé, J. Am. Chem. Soc. 2014, 136, 13975-13978.

[147] J. L. Douglas, C. A. Trieber, M. Afara, H. S. Young, Protein Expr. Purif. 2005, 40, 118-125.

[148] P. Bannas, J. Hambach, F. Koch-Nolte, Front. Immunol. 2017, 8, 1-13.

[149] M. M. Harmsen, H. J. De Haard, Appl. Microbiol. Biotechnol. 2007, 77, 13-22.

[150] H. Götzke, M. Kilisch, M. Martínez-Carranza, S. Sograte-Idrissi, A. Rajavel, T. Schlichthaerle, N. Engels, R. Jungmann, P. Stenmark, F. Opazo, et al., Nat. Commun. 2019, 10, 1-12.

[151] M. Petukhov, Y. Tatsu, K. Tamaki, S. Murase, H. Uekawa, S. Yoshikawa, L. Serrano, N. Yumoto, J. Pept. Sci. 2009, 15, 359-365.

[152] G. V. Los, L. P. Encell, M. G. McDougall, D. D. Hartzell, N. Karassina, C. Zimprich, M. G. Wood, R. Learish, R. F. Ohana, M. Urh, et al., ACS Chem. Biol. 2008, 3, 373-382.

[153] R. S. Erdmann, S. W. Baguley, J. H. Richens, R. F. Wissner, Z. Xi, E. S. Allgeyer, S. Zhong, A. D. Thompson, N. Lowe, R. Butler, et al., Cell Chem. Biol. 2019, 26, 584-592.e6.

[154] E. I. Vrettos, G. Mező, A. G. Tzakos, Beilstein J. Org. Chem. 2018, 14, 930-954.

[155] J. R. McCombs, S. C. Owen, AAPS J. 2015, 17, 339-351.

[156] K. Tsuchikama, Z. An, Protein Cell 2018, 9, 33-46.

[157] M. J. Roberts, M. D. Bentley, J. M. Harris, Adv. Drug Deliv. Rev. 2002, 54, 459-476.

[158] H. Duclohier, BBA - Biomembr. 2009, 1788, 2374-2379.

[159] W. A. Catterall, J. Physiol. 2012, 590, 2577-2589. 
[160] A. L. Hodgkin, A. F. Huxley, J. Physiol. 1952, 116, 473-496.

[161] A. L. Hodgkin, A. F. Huxley, J. Physiol. 1952, 116, 449-472.

[162] D. A. Beneski, W. A. Catterall, J. Supramol. Cell. Biochem. 1980, 12, 639-643.

[163] R. P. Hartshorne, W. A. Catterall, Proc. Natl. Acad. Sci. U. S. A. 1981, 78, 4620-4624.

[164] E. C. McCusker, C. Bagnéris, C. E. Naylor, A. R. Cole, N. D’Avanzo, C. G. Nichols, B. A. Wallace, Nat. Commun. 2012, 3, 1-8.

[165] J. Payandeh, T. Scheuer, N. Zheng, W. A. Catterall, Nature 2012, 475, 353-358.

[166] W. A. Catterall, Exp Physiol. 2014, 99, 1-7.

[167] D. L. Bennett, X. A. J. Clark, J. Huang, S. G. Waxman, S. D. Dib-Hajj, Physiol. Rev. 2019, 99, 1079-1151.

[168] S. A. Pless, F. D. Elstone, A. P. Niciforovic, J. D. Galpin, R. Yang, H. T. Kurata, C. A. Ahern, J. Gen. Physiol. 2014, 143, 645-656.

[169] W. A. Catterall, A. L. Goldin, S. G. Waxman, Pharmacol. Rev. 2005, 57, 397-409.

[170] W. A. Catterall, Neuron 2000, 26, 13-25.

[171] M. D. L. Ruiz, R. L. Kraus, J. Med. Chem. 2015, 58, 7093-7118.

[172] T. Dudev, C. Lim, Acc. Chem. Res. 2014, 47, 3580-3587.

[173] S. H. Heinemann, H. Terlau, W. Stuhmer, K. Imoto, S. Numa, Nature 1992, 356, 441-443.

[174] W. A. Catterall, A. L. Goldin, S. G. Waxman, Pharmacol. Rev. 2005, 57, 397-409.

[175] K. Jurkat-Rott, B. Holzherr, M. Fauler, F. Lehmann-Horn, Pflugers Arch. Eur. J. Physiol. 2010, $460,239-248$.

[176] C. Antzelevitch, V. Nesterenko, J. Shyrock, S. Rajamani, Y. Song, L. Belardinelli, Handb. Exp. Pharmacol. 2014, 221, 137-168.

[177] A. Moreau, P. Gosselin-Badaroudine, M. Chahine, Front. Pharmacol. 2014, 5, 1-19.

[178] A. Nardi, N. Damann, T. Hertrampf, A. Kless, ChemMedChem 2012, 7, 1712-1740.

[179] K. Nakamura, M. Kato, H. Osaka, S. Yamashita, E. Nakagawa, K. Haginoya, J. Tohyama, M. Okuda, T. Wada, S. Shimakawa, et al., Neurology 2013, 81, 992-998.

[180] J. W. Theile, T. R. Cummins, Front. Pharmacol. 2011, 2, 1-14.

[181] K. S. Walia, E. A. Khan, D. H. Ko, S. S. Raza, Y. N. Khan, Pain Pract. 2004, 4, 194-203. 
[182] J. P. Meeks, S. Mennerick, J. Neurophysiol. 2007, 97, 3460-3472.

[183] D. A. Wollner, W. A. Catterall, Proc. Natl. Acad. Sci. 1986, 83, 8424-8428.

[184] C. M. Colbert, E. Pan, Nat. Neurosci. 2002, 5, 533-538.

[185] T. A. Stanly, M. Fritzsche, S. Banerji, E. Garcia, J. B. De La Serna, D. G. Jackson, C. Eggeling, Biol. Open 2016, 5, 1343-1350.

[186] E. G. Moczydlowski, Toxicon 2013, 63, 165-183.

[187] R. Chen, S. H. Chung, Biochem. Biophys. Res. Commun. 2014, 446, 370-374.

[188] H. M. Garraffo, T. F. Spande, Heterocycles 2009, 79, 195-205.

[189] J. Wang, V. Yarov-Yarovoy, R. Kahn, D. Gordon, M. Gurevitz, T. Scheuer, W. A. Catterall, Proc. Natl. Acad. Sci U.S.A. 2011, 108, 15426-15431.

[190] J. K. Klint, S. Senff, D. B. Rupasinghe, S. Y. Er, V. Herzig, G. M. Nicholson, G. F. King, Toxicon 2012, 60, 478-491.

[191] B. G. Livett, K. R. Gayler, Z. Khalil, Curr. Med. Chem. 2004, 11, 1715-1723.

[192] K. B. Akondi, M. Muttenthaler, S. Dutertre, Q. Kaas, D. J. Craik, R. J. Lewis, P. F. Alewood, Chem. Rev. 2014, 114, 5815-5847.

[193] I. Vetter, R. J. Lewis, Curr. Top. Med. Chem. 2012, 12, 1546-1552.

[194] K. B. Akondi, M. Muttenthaler, S. Dutertre, Q. Kaas, D. J. Craik, R. J. Lewis, P. F. Alewood, Chem. Rev. 2014, 114, 5815-5847.

[195] J. Davis, A. Jones, R. J. Lewis, Peptides 2009, 30, 1222-1227.

[196] B. Gao, C. Peng, B. Lin, Q. Chen, J. Zhang, Q. Shi, Toxins (Basel). 2017, 9, 1-10.

[197] Q. Kaas, J. C. Westermann, D. J. Craik, Toxicon 2010, 55, 1491-1509.

[198] S. D. Robinson, R. S. Norton, Mar. Drugs 2014, 12, 6058-6101.

[199] F. Y. Dao, H. Yang, Z. D. Su, W. Yang, Y. Wu, H. Ding, W. Chen, H. Tang, H. Lin, Molecules 2017, 22, 1-20.

[200] J. E. Pope, T. R. Deer, Expert Opin. Pharmacother. 2013, 14, 957-966.

[201] J. G. McGivern, Neuropsychiatr. Dis. Treat. 2007, 3, 69-85.

[202] E. Leipold, R. Markgraf, A. Miloslavina, M. Kijas, J. Schirmeyer, D. Imhof, S. H. Heinemann, Neuropharmacology 2011, 61, 105-111.

[203] B. R. Green, B. M. Olivera, Curr. Top. Membr. 2016, 78, 65-86. 
[204] E. Tosti, R. Boni, A. Gallo, Mar. Drugs 2017, 15, 1-16.

[205] R. S. Norton, Molecules 2010, 15, 2825-2844.

[206] R. Chen, A. Robinson, S. H. Chung, PLoS One 2014, 9, 1-8.

[207] B. R. Green, G. Bulaj, R. S. Norton, Futur. Med. Chem. 2014, 6, 1677-1698.

[208] S. Yao, M. M. Zhang, D. Yoshikami, L. Azam, B. M. Olivera, G. Bulaj, R. S. Norton, Biochemistry 2008, 47, 10940-10949.

[209] C. Z. Wang, H. Zhang, H. Jiang, W. Lu, Z. Q. Zhao, C. W. Chi, Toxicon 2006, 47, 122-132.

[210] B. R. Green, P. Catlin, M. M. Zhang, B. Fiedler, W. Bayudan, A. Morrison, R. S. S. Norton, B. J. Smith, D. Yoshikami, B. M. Olivera, et al., Chem. Biol. 2007, 14, 399-407.

[211] D. Chelius, K. Jing, A. Lueras, D. S. Rehder, T. M. Dillon, A. Vizel, R. S. Rajan, T. Li, M. J. Treuheit, P. V. Bondarenko, Anal. Chem. 2006, 78, 2370-2376.

[212] E. F. Pettersen, T. D. Goddard, C. C. Huang, G. S. Couch, D. M. Greenblatt, E. C. Meng, T. E. Ferrin, J. Comput. Chem. 2004, 25, 1605-1612.

[213] A. M. Steiner, K. J. Woycechowsky, B. M. Olivera, G. Bulaj, Angew. Chemie - Int. Ed. 2012, $51,5580-5584$.

[214] A. Walewska, M. M. Zhang, J. J. Skalicky, D. Yoshikami, B. M. Olivera, G. Bulaj, Angew. Chemie - Int. Ed. 2009, 48, 2221-2224.

[215] K. K. Khoo, M. J. Wilson, B. J. Smith, M.-M. Zhang, J. Gulyas, D. Yoshikami, J. E. Rivier, G. Bulaj, R. S. Norton, J. Med. Chem. 2011, 54, 7558-7566.

[216] C. Pi, J. Liu, L. Wang, X. Jiang, Y. Liu, C. Peng, S. Chen, A. Xu, J. Biotechnol. 2007, 128, 184 193.

[217] A. H. Jin, M. Muttenthaler, S. Dutertre, S. W. A. Himaya, Q. Kaas, D. J. Craik, R. J. Lewis, P. F. Alewood, Conotoxins: Chemistry and Biology, 2019.

[218] A. M. Steiner, G. Bulaj, J. Pept. Sci. 2011, 17, 1-7.

[219] A. A. Miloslavina, E. Leipold, M. Kijas, A. Stark, S. H. Heinemann, D. Imhof, J. Pept. Sci. 2009, $15,72-77$.

[220] H. A. Radzey, Synthesis of fluorescent toxin and nucleotide derivatives to specifically address membrane proteins, Dissertation, Georg-August Universität Göttingen, 2015.

[221] L. Moroder, H. J. Musiol, M. Götz, C. Renner, Biopolym. - Pept. Sci. Sect. 2004, 80, 85-97.

[222] G. Bulaj, B. M. Olivera, Antioxid. Redox Signal. 2008, 10, 141-155. 
[223] C. Boulègue, H. J. Musiol, V. Prasad, L. Moroder, Chim. Oggi 2006, 24, 24-36.

[224] T. M. Postma, F. Albericio, European J. Org. Chem. 2014, 2014, 3519-3530.

[225] A. Cuthbertson, B. Indrevoll, Org. Lett. 2003, 5, 2955-2957.

[226] A. I. Kuzmenkov, A. A. Vassilevski, Neurosci. Lett. 2018, 679, 15-23.

[227] A. J. Hone, M. Scadden, J. Gajewiak, S. Christensen, J. Lindstrom, J. M. Mcintosh, 2012, 82, 972-982.

[228] D. S. Kudryavtsev, I. V. Shelukhina, L. V. Son, L. O. Ojomoko, E. V. Kryukova, E. N. Lyukmanova, M. N. Zhmak, D. A. Dolgikh, I. A. Ivanov, I. E. Kasheverov, et al., J. Biol. Chem. 2015, 290, 22747-22758.

[229] Z. Zhang, D. Yomo, C. Gradinaru, BBA - Biomembr. 2017, 1859, 1242-1253.

[230] P. Heimer, A. A. Tietze, C. A. Bäuml, A. Resemann, F. J. Mayer, D. Suckau, O. Ohlenschläger, D. Tietze, D. Imhof, Anal. Chem. 2018, 90, 3321-3327.

[231] M. Ieronymaki, M. E. len. Androutsou, A. Pantelia, I. Friligou, M. Crisp, K. High, K. Penkman, D. Gatos, T. Tselios, Biopolymers 2015, 104, 506-514.

[232] J. P. Tam, C. R. Wu, W. Liu, J. W. Zhang, J. Am. Chem. Soc. 1991, 113, 6657-6662.

[233] B. Kamber, A. Hartmann, K. Eisler, B. Riniker, H. Rink, P. Sieber, W. Rittel, Helv. Chim. Acta 1980, 63, 899-915.

[234] M. J. Espiritu, Disulfide Bond and Topological Isomerization of the Conopeptide PNID: Disulfide Bonds with a Twist, Dissertation, University of Hawaii, 2017.

[235] Y. Shimonishi, Y. Hidaka, M. Koizumi, M. Hane, S. Aimoto, T. Takeda, T. Miwatani, Y. Takeda, FEBS Lett. 1987, 215, 165-170.

[236] M. Okumura, S. Shimamoto, Y. Hidaka, FEBS J. 2012, 279, 2283-2295.

[237] M. J. Wilson, D. Yoshikami, L. Azam, J. Gajewiak, B. M. Olivera, G. Bulaj, M. M. Zhang, Proc. Natl. Acad. Sci. U. S. A. 2011, 108, 10302-10307.

[238] L. D. Hughes, R. J. Rawle, S. G. Boxer, PLoS One 2014, 9, 1-8.

[239] P. Thirumurugan, D. Matosiuk, K. Jozwiak, Chem. Rev. 2013, 113, 4905-4979.

[240] F. Saito, H. Noda, J. W. Bode, ACS Chem. Biol. 2015, 10, 1026-1033.

[241] W. M. Rink, F. Thomas, Org. Lett. 2018, 20, 7493-7497.

[242] M. Empting, O. Avrutina, R. Meusinger, S. Fabritz, M. Reinwarth, M. Biesalski, S. Voigt, G. 
Buntkowsky, H. Kolmar, Angew. Chemie - Int. Ed. 2011, 50, 5207-5211.

[243] T. D. Kondasinghe, H. Y. Saraha, S. B. Odeesho, J. L. Stockdill, Org. Biomol. Chem. 2017, 15, 2914-2918.

[244] T. D. Kondasinghe, H. Y. Saraha, S. T. Jackowski, J. L. Stockdill, Tetrahedron Lett. 2019, 60, 23-28.

[245] F. Wu, J. P. Mayer, V. M. Gelfanov, F. Liu, R. D. Dimarchi, J. Org. Chem. 2017, 82, 35063512.

[246] F. Czerny, Development of Zinc-Finger-Based Artificial Restriction Endonucleases and Fluorescent Peptidyl Metal Sensors, Dissertation, Georg-August Universität Göttingen, 2016.

[247] E. J. Ste.Marie, R. J. Hondal, J. Pept. Sci. 2018, 24, 1-8.

[248] A. Otaka, T. Koide, A. Shide, N. Fujii, Tet 1991, 32, 1223-1226.

[249] A. Schulz, E. Klüver, S. Schulz-Maronde, K. Adermann, Biopolym. - Pept. Sci. Sect. 2005, 80, 34-49.

[250] B. H. Northrop, S. H. Frayne, U. Choudhary, Polym. Chem. 2015, 6, 3415-3430.

[251] J. S. Nanda, J. R. Lorsch, Methods Enzymol. 2014, 536, 87-94.

[252] C. González-Cabrera, R. Meza, L. Ulloa, P. Merino-Sepúlveda, V. Luco, A. Sanhueza, A. OnatePonce, J. P. Bolam, P. Henry, J. Comp. Neurol. 2017, 1-37.

[253] H. Yu, J. Li, D. Wu, Z. Qiu, Y. Zhang, Chem. Soc. Rev. 2010, 39, 464-473.

[254] H.-M. Lee, D. R. Larson, D. S. Lawrence, ACS Chem. Biol. 2009, 4, 409-427.

[255] M. J. Hansen, W. A. Velema, M. M. Lerch, W. Szymanski, B. L. Feringa, Chem. Soc. Rev. 2015, 44, 3358-3377.

[256] W. H. So, C. T. T. Wong, J. Xia, Chinese Chem. Lett. 2018, 29, 1058-1062.

[257] P. Klán, T. Šolomek, C. G. Bochet, A. Blanc, R. Givens, M. Rubina, V. Popik, A. Kostikov, J. Wirz, Chem. Rev. 2013, 113, 119-191.

[258] Y. V. Il’ichev, M. A. Schwörer, J. Wirz, J. Am. Chem. Soc. 2004, 126, 4581-4595.

[259] N. Fomina, C. Mcfearin, M. Sermsakdi, O. Edigin, A. Almutairi, J. Am. Chem. Soc. 2010, 132, $9540-9542$.

[260] M. Quast, Synthesis and Analyis of Modified SNARE Proteins with Respect to Assembly and Disassembly of the SNARE Complex, Dissertation, Georg-August Universität Göttingen, 2016. 
[261] S. Zhou, O. Mozziconacci, B. A. Kerwin, C. Schöneich, Pharm. Res. 2013, 30, 1291-1299.

[262] S. Bourgault, M. Létourneau, A. Fournier, Peptides 2007, 28, 1074-1082.

[263] A. Specht, J. S. Thomann, K. Alarcon, W. Wittayanan, D. Ogden, T. Furuta, Y. Kurakawa, M. Goeldner, ChemBioChem 2006, 7, 1690-1695.

[264] S. B. Cambridge, D. Geissler, S. Keller, B. Cürten, Angew. Chemie - Int. Ed. 2006, 45, 2229 2231.

[265] V. Hagen, J. Bendig, S. Frings, T. Eckardt, S. Helm, D. Reuter, B. Kaupp, Angew. Chem. Int. Ed. 2001, 40, 1046-1048.

[266] L. Fan, R. W. Lewis, G. P. Hess, B. Ganem, Bioorg. Med. Chem. Lett 2010, 19, 3932-3933.

[267] D. Geißler, W. Kresse, B. Wiesner, J. Bendig, H. Kettenmann, V. Hagen, ChemBioChem 2003, $4,162-170$.

[268] R. O. Schönleber, J. Bendig, V. Hagen, B. Giese, Bioorganic Med. Chem. 2002, 10, 97-101.

[269] N. Kotzur, B. Briand, M. Beyermann, V. Hagen, J. Am. Chem. Soc. 2009, 131, 16927-16931.

[270] J. Gonzales, P. Demetrio De Souza Franca, Y. Jiang, G. Pirovano, S. Kossatz, N. Guru, D. Yarilin, A. J. Agwa, C. I. Schroeder, S. G. Patel, et al., Bioconjug. Chem. 2019, 30, 2879-2888.

[271] A. E. Ondrus, H. L. D. Lee, S. Iwanaga, W. H. Parsons, B. M. Andresen, W. E. Moerner, J. Du Bois, Chem. Biol. 2012, 19, 902-912.

[272] M. H. Litt, J. Kim, J. M. Rodriguezparada, J. Polym. Sci. Polym. Chem. Ed. 1985, 23, 13071319.

[273] E. Masson, Y. M. Shaker, J. Masson, M. E. Kordesch, Org. Lett. 2011, 13, 3872-3875.

[274] C. De Gracia Lux, J. Olejniczak, N. Fomina, M. L. Viger, A. Almutairi, J. Polym. Sci. Part A Polym. Chem. 2013, 51, 3783-3790.

[275] A. Schirmacher, Modification of transmembrane peptides to probe SNARE-induced membrane fusion and cross-presentation membrane-buried epitopes. Dissertation, Georg-AugustUniversität Göttingen, 2020.

[276] X. Zhang, W. Xi, C. Wang, M. Podgórski, C. N. Bowman, ACS Macro Lett. 2016, 5, 229-233. 


\section{List of Figures}

Figure 1.1: Cycling of Neurotransmitters and Synaptic vesicles. ..................................................... 4

Figure 1.2: Proton-dependent neurotransmitter transport into vesicles.................................................. 5

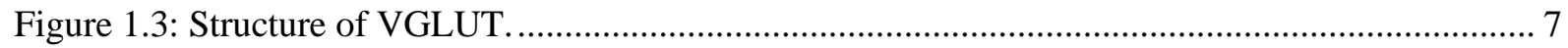

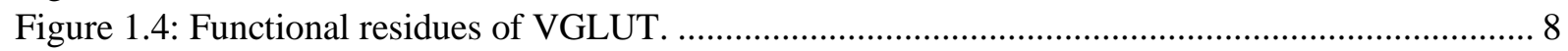

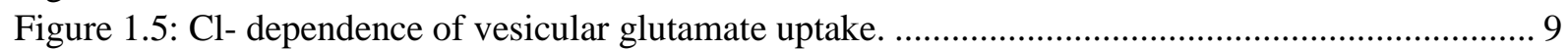

Figure 1.6: Proteoliposome model for $\mathrm{Cl}^{-}$transport mechanism....................................................... 10

Figure 1.7: Proposed mechanism of glutamate uptake in the synaptic vesicle. ................................. 12

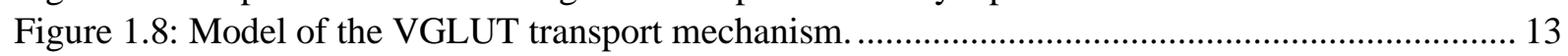

Figure 1.9: Elevated model of the VGLUT transport mechanism. .................................................... 14

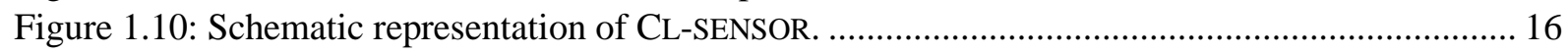

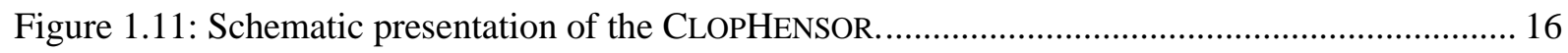

Figure 1.12: Examples for the first-generation $\mathrm{Cl}^{-}$-sensitive small molecules.................................... 18

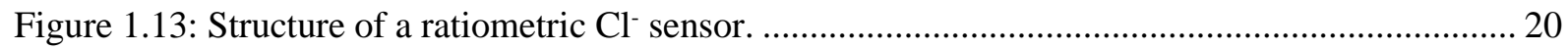

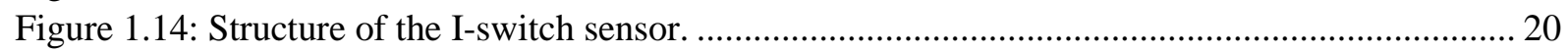

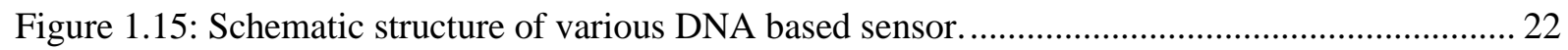

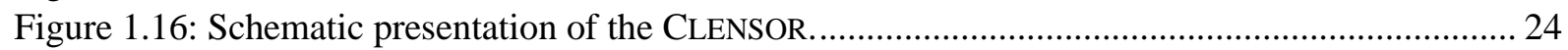

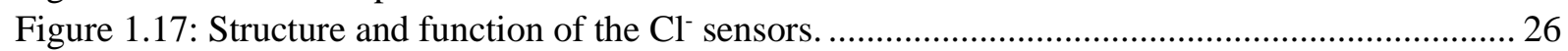

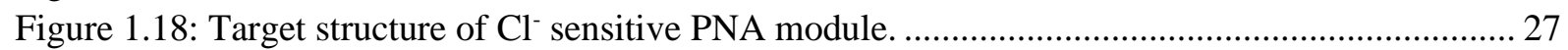

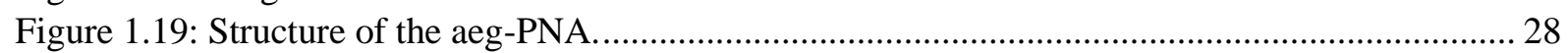

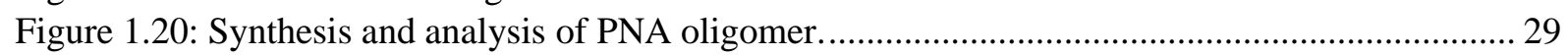

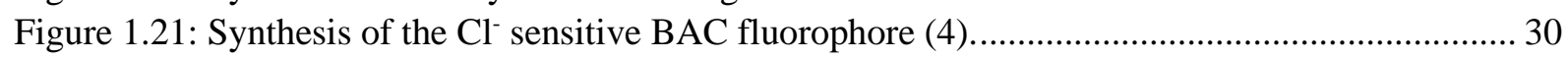

Figure 1.22: Synthesis and Analysis of BAC labelled PNA oligomer............................................. 31

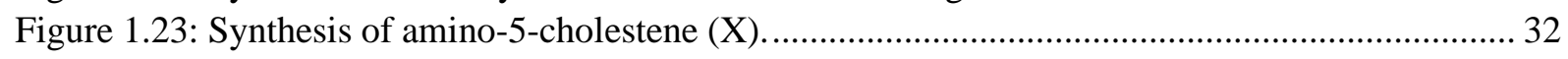

Figure 1.24: Synthesis approach of cholesterol modified peptide-PNA oligomers. ............................. 33

Figure 1.25: Synthesis approach of cholesterol modified peptide-PNA oligomer in solution.............. 33

Figure 1.26: Synthesis of Fmoc-Asp(palmitoyl)-OH (X) over two steps. .......................................... 34

Figure 1.27: Synthesis and analysis of palmitoyl and BAC modified PNA oligomer. ......................... 35

Figure 1.28: Synthesis of palmitoyl, TEV and BAC modified peptide-PNA oligomer........................ 36

Figure 1.29: TEV-protease cleavage experiments of the palmitoyl modified PNA sensor.................... 37

Figure 1.30: TEV-cleavage experiments of different test peptides................................................... 38

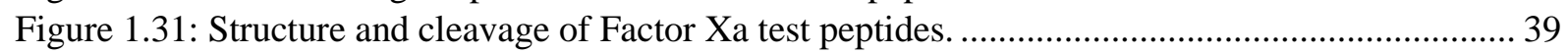

Figure 1.32: Synthesis and Factor Xa cleavage of palmitoyl-modified PNA sensors........................... 40

Figure 1.33: Synthesis and cathepsin B cleavage of palmitoyl-modified PNA sensors....................... 41

Figure 1.34: Separation of incorporated sensors by density-gradient centrifugation.......................... 42

Figure 1.35: Incorporation of the palmitoyl-modified PNA sensor in liposomes. ............................... 42

Figure 1.36: Synthesis and Cathepsin B cleavage of TMD-modified PNA sensors. ........................... 44

Figure 1.37: Incorporation assay of the TMD-modified PNA sensor in liposomes............................. 45

Figure 1.38: Quenching experiments in fluorophore labelled TMD peptides...................................... 45

Figure 1.39: Synthesis and incorporation assay of NBD labelled TMD peptides............................... 46

Figure 1.40: Gel mobility shift assay for visualisation of the $\mathrm{Cl}^{-}$sensor formation............................47

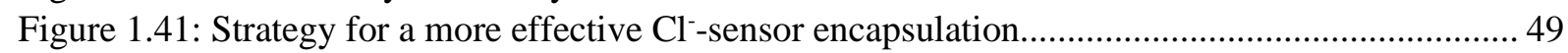

Figure 1.42: Epimerization of Fmoc-His(PG)-OH during SPPS activation......................................... 50

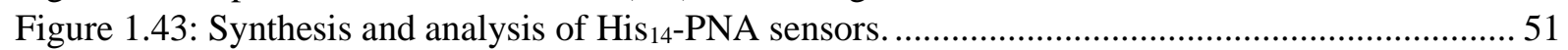

Figure 1.44: Gel mobility assay visualizing the $\mathrm{His}_{14}$ Clensor formation............................................ 52

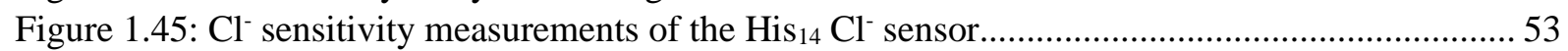

Figure 1.46: Integrity analysis of $\mathrm{His}_{14} \mathrm{Cl}^{-}$sensor by gel mobility shift assay.................................... 54

Figure 1.47: Encapsulation and cleavage of $\mathrm{His}_{14} \mathrm{Cl}^{-}$sensor in $\mathrm{Ni}^{2+}$ modified liposomes. .................. 55

Figure 1.48: Purification and $\mathrm{Cl}^{-}$measurements of encapsulated $\mathrm{His}_{14}$ sensors in liposomes. ............. 56 
Figure 1.49: Activity measurements of encapsulated VGLUT $\mathrm{TF}_{0} \mathrm{~F}_{1} \mathrm{His}_{14}$ in liposomes. ...................58

Figure 1.50: Time-dependent BAC fluorescence intensity of $\mathrm{His}_{14}-\mathrm{Cl}^{-}$-sensor. .....................................5 59

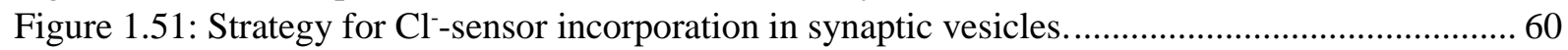

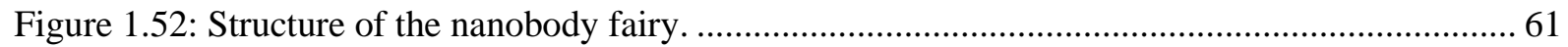

Figure 1.53: Synthesis and analysis of ALFA-tag modified PNA sensor.......................................... 62

Figure 1.54: Gel mobility shift assay of the ALFA-Cl-sensor formation with the ALFA-nanobody. . 63

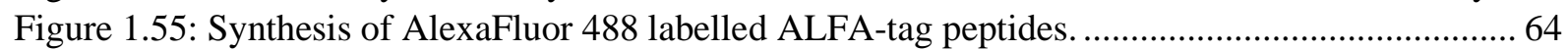

Figure 1.56: Gel mobility shift assay visualizing the AFLA-peptide nanobody formation................... 64

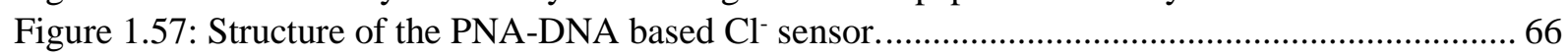

Figure 1.58: Overview of the results of the TMD and Cathepsin B modified PNA sensor................. 68

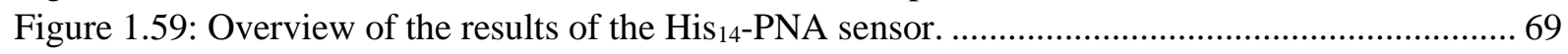

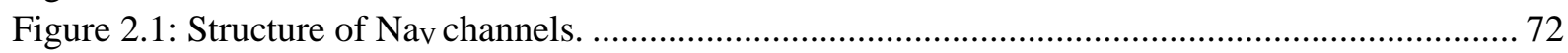

Figure 2.2: Tissue expression of the nine different voltage gated sodium channel subtypes. ${ }^{[158]} \ldots \ldots \ldots . .73$

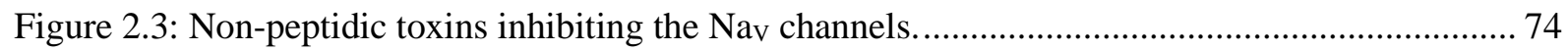

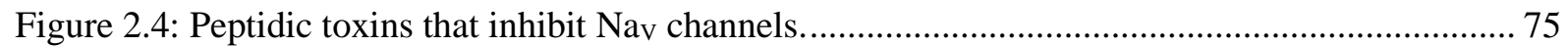

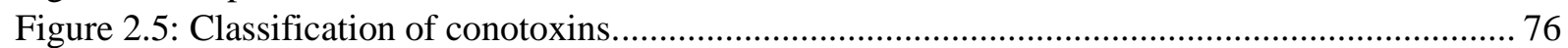

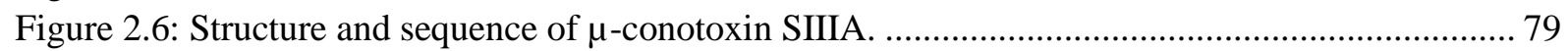

Figure 2.7: Formation possibilities in a peptide containing two-disulphide bonds............................. 80

Figure 2.8: Comparison of oxidative one-step folding and regioselective synthesis strategy............... 81

Figure 2.9: 3D Structure of the $\mu$-SIIIA modified with AlexaFluor 488 ............................................ 84

Figure 2.10: Schematic overview of the $\mu$-SIIIA synthesis into a fluorescent labelled toxin............... 85

Figure 2.11: Formation of the first disulphide bridge of the native $\mu$-SIIIA....................................... 86

Figure 2.12: Formation of the second disulphide bridge of the native $\mu$-SIIIA................................. 87

Figure 2.13: Formation of the third disulphide bridge of the $\mu$-SIIIA. ............................................. 88

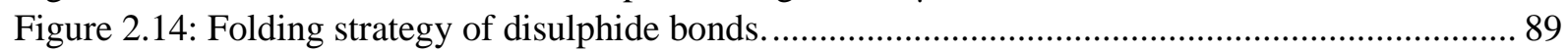

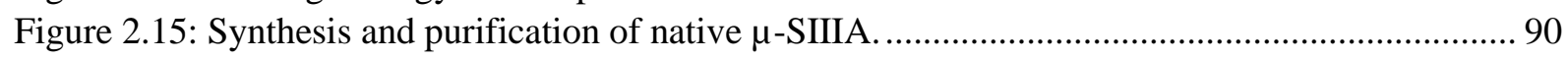

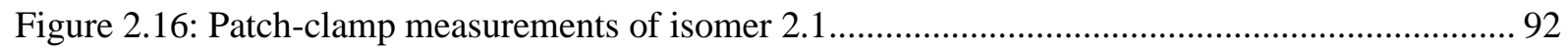

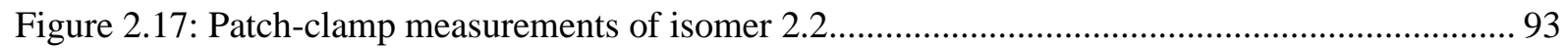

Figure 2.18: Synthesis and purification of alkyne modified $\mu$-SIIIA................................................. 95

Figure 2.19: Synthesis and purification of AlexaFluor 488 labelled $\mu$-SIIIA. ..................................... 96

Figure 2.20: Synthesis strategy for the Allocam-protected alkyne-modified $\mu$-SIIIA......................... 98

Figure 2.21: Synthesis strategy of tert-butyl-protected and alkyne modified $\mu$-SIIIA......................... 99

Figure 2.22: Synthesis strategy of MeOBzl-protected and alkyne modified $\mu$-SIIIA......................... 100

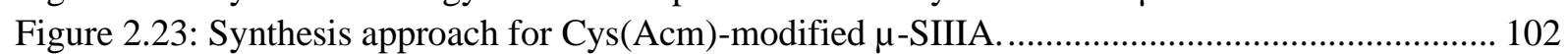

Figure 2.24: Synthesis and purification of TMR labelled $\mu$-SIIIA. ................................................. 104

Figure 2.25: Overview of the synthesis and application of caged neurotoxins................................. 105

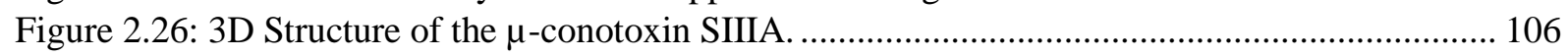

Figure 2.27: Synthesis of the caged Fmoc-Lys(Nvoc)-OH building block for SPPS over two steps. 107

Figure 2.28: Structure and uncaging experiments of Nvoc modified peptides. .................................. 108

Figure 2.29: Synthesis of the caged Fmoc-Asp(DMNPB)-OH building block for SPPS. .................. 109

Figure 2.30: Structure and uncaging experiments of DMNPB modified peptides.............................. 110

Figure 2.31: Three step synthesis of Fmoc-Lys(DEACM)-OH building block for SPPS................... 111

Figure 2.32: Structure and uncaging experiments of DEACM modified peptides............................. 111

Figure 2.33: Synthesis and purification of the DECAM caged $\mu$-SIIIA. ........................................... 113

Figure 2.34: Photocleavage experiments of DEACM caged $\mu$-SIIIA................................................ 114

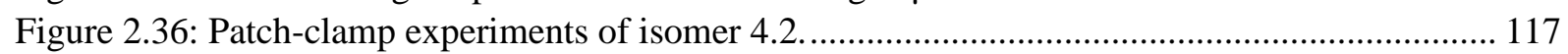

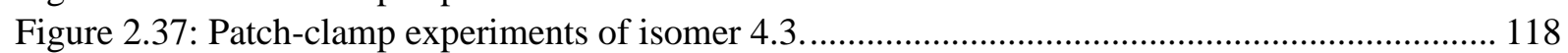

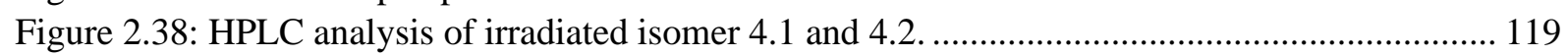

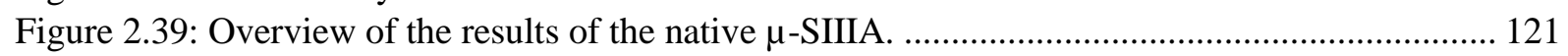

Figure 2.40: Overview of the results of the AlexaFluor 488 labelled $\mu$-SIIIA. ................................. 121

Figure 2.41: Overview of the results of the PPG modified $\mu$-SIIIA. .............................................. 123 


\section{List of Tables}

Table 1.1: Overview of the fluorescent $\mathrm{Cl}^{-}$-sensitive small molecule dyes........................................ 18

Table 1.2: Overview of the photophysical characteristics of different BAC-labelled constructs. ........ 23

Table 1.3: Overview of the different test peptides with the TEV recognition sequence........................ 38

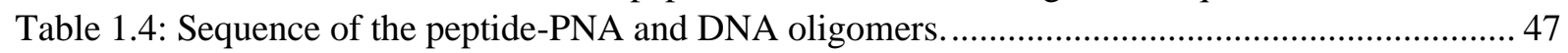

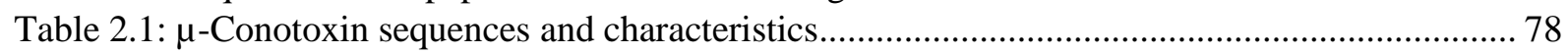

Table 2.2: Overview of frequently used thiol protecting groups for SPPS . ....................................... 82

Table 3.1: The extinction coefficients used for concentration estimations. ...................................... 128

Table 3.2: Overview of coupling and deprotection conditions depending on the synthesis scale..... 131 


\section{Acknowledgements}

Mein besonderer Dank gilt Prof. Dr. Ulf Diederichsen für die Möglickeit meine Dissertation bei Ihnen im Arbeitskreis anfertigen zu dürfen, sowie für die sehr gute Betreeung, ständige Diskussionsbereitschaft und die wissenschaftliche Freiheit. Ich bin sehr dankbar für das Vertrauen und die enge Zusammenarbeit nicht nur in wissenschaftlichen Fragen, sondern auch in der Betreung von Bachelorstudenten und in der Organisation von Praktika.

Meiner Zweitgutachterin Prof. Dr. Claudia Steinem möchte ich danken für die Übernahme des Koreferats, sowie für die jährlichen Treffen und die daraus resultierenden Diskussionen und hilfreichen Vorschläge für mein Projekt.

Darüber hinaus danke ich Prof. Dr. Reinhard Jahn, Prof. Dr. Silvio Rizzoli, Dr. Stefan Glöggler und Dr. Holm Frauendorf für die Bereitschaft meiner Prüfungskommsission anzugehören.

Mein besonderer Dank gilt Rashi Goel und Jennifer Struck für die intensive Kooperiation, die Diskussions- und Hilfsbereitschaft bei biologischen und physikalischen Fragestellungen, sowie für die enge und gute Zusammenarbeit auf dem Chlorid-Sensor Thema. Zusätzlich möchte ich mich bei Benjamin Ide und Frederike Maßß für die Durchführung von Experimenten bedanken.

Bei Vivien Kistmacher und Prof. Dr. Enrico Leipold möchte ich mich für die Messungen der Conotoxin Proben sowie für ausführlichen Diskussionen und Erklärungen zur den Toxin Eigenschaften bedanken.

Dr. Michael John und dem Team der NMR Abteilung danke für die Anfertigung der Kernresonanzspektren. Bei Dr. Holm Frauendorf und seinen Mitarbeitern aus der Massenspektrometrie Abteilung möchte ich mich ganz besonders bedanken, für die schnelle und gewissenhafte Bearbeitung meiner unzähligen Proben.

Ganz besonders möchte ich mich bei Angela Heinemann bedanken: für die ständige Unterstützung in allen Situationen, dein offenes Ohr und dafür, dass du dir immer Zeit für meine kleinen und großen Probleme genommen hast. Besonders dankbar bin ich, dass du mir das Verlegen von Rechnungen oder das Chaos im Labor nie übel genommen hast.

Allen aktuellen und ehemaligen Mitgliedern des Arbeitskreises Diederichsen danke ich für die vielen schönen Stunden, beim Mittagessen, der Kaffeepause oder auf den AK-Fahrten.

Meinen Laborkollegen Dr. Matthias Krull und Ilze Lace danke ich für die schöne und lustige Zeit im Labor 106. 
Bei Dr. Denis Pahlke, Dr. Geralin Höger, Dr. Anastasiya Schirmacher, Mathis Rink und Benedikt Kugler möchte ich mich für die vielen hilfreichen wissenschaftlichen Diskussionen und Ratschläge bedanken.

Ein unendlich großer Dank gehört den von mir betreuten Bachelorstudenten Lina Kopp, Lucas Hensen, Manuel Müller und Katharina Overhoff für die großartige Unterstützung im Labor, die vielen schönen Stunden und euren Beitrag zu meinen Projekten .

Für das Korrekturlesen meiner Arbeit möchte ich mich ganz besonders bei Iriyna Portnova, Benedikt Kugler, Tobias Schmidt, Lina Kopp, Florian Grimm, Dr. Julia Menzel und Katharina Ewers bedanken. Vielen Dank für eure hilfreichen Korrekturvorschläge

Ein besonderes großer Dank geht an Florian Grimm für all die Laufrunden am Donnerstagabend im Regen und bei Schneefall. Zusätzlich danke ich dir für alle Mittagspausen am Freitag, deine Hilfsbeitschaft in allen Lebenslagen und deine Freundschaft.

Den Handballern vom NSC danke ich für die unzähligen schönen Stunden in der Halle und auf dem Sportplatz, sowie für die Ablenkung und den Ausgleich zum Laboralltag.

Meinen „Schwiegereltern“ in spe möchte ich danken für all die gemeinsame Zeit sowie eure Unterstützung und Hilfe während der letzten Jahre. Auch bei Maximilian und Alexander möchte ich mich bedanken für eurer Hilfsbereitschaft und die vielen lustigen Studen. Ganz besonderer Dank geht an Constantin, für die beste WG aller Zeiten, fürs gemeinsame Handball spielen, Football schauen und für all die schönen Stunden.

Bei Julia und Marcel möchte ich mich bedanken für die unfassbar schöne Zeit, sowie für eure Unterstützung und Hilfe in allen Lebenslagen. Danke für all die Grillabende, Laufrunden, für das Anfeuern bei Heimspielen, Spieleabende und Urlaube.

Ein unendlich großer Dank geht an meine Eltern. Danke für die Ermöglichung des Studiums, sowie eure bedingungslose Unterstützung und euren Rückhalt, die mich so weit gebracht haben.

Mein größter Dank geht an Katharina, für all die Unterstützung, Hilfsbereitsschaft, gute Laune in den letzten Jahren, in allen guten und schlechten Augenblicken. Vielen Dank für deine Begeisterung für all unsere Abenteuer und dafür, dass du immer an meiner Seite bist und warst. 


\section{Curriculum Vitae}

Patrick Matthias Menzel

Born July 29, 1992 in Burgwedel, Germany

Scientific Education

$01 / 2017-11 / 2020$

$10 / 2014-11 / 2016$

06/2014-10/2014

$10 / 2011-06 / 2014$

2011
PhD thesis in the working group of Prof. Dr. Ulf Diederichsen at the Institute of Organic and Biomolecular Chemistry (IOBC), Georg-August University Göttingen

Synthesis of Modified Biomolecules to Investigate Neuronal Processes

M. Sc. Chemistry Philipps University Marburg

Overall grade: very good (1.1)

Master Thesis (04-11/2016) in the working group of Prof. Dr. Martin Schlitzer at the Institute of Pharmaceutical Chemistry

Synthesis and Investigation of Sulfonamide-Derivates as Inhibitors of the eIF4F Complex

IRTG Research student at the IOBC in the working group of Prof. Dr. Ulf Diederichsen

Synthesis and Modification of Zinc Finger Zif268

B.Sc. Biochemistry Georg-August-University Göttingen

Overall grade: good (1.7)

Bachelor Thesis (04-06/2014) in the working group of Prof. Dr. Ulf Diederichsen at the IOBC

Synthesis and Investigation of Modified $\beta$-Peptides

Abitur Gymnasium Großburgwedel 
Scientific Contributions

2020

Oral Presentation, $12^{\text {th }}$ Göttinger Chemie Forum, Synthesis of Fluorescent Neurotoxins to Investigate Neuronal Processes

2019

Poster Presentation, $14^{\text {th }}$ German Peptide Symposium, Cologne, Germany, Modified Biomolecules to Address Membrane Proteins and to Quantify Ion Concentrations

2018

Poster Presentation, $8^{\text {th }}$ Peptide Engineering Meeting, Berlin, Germany, Peptide Nucleic Acid (PNA) as a Reaction Template and Recognition Motif in Bioorganic Chemistry

Selected Teaching Experience

$01 / 2017-11 / 2020$

Supervision of four bachelor theses and one student internship

$01 / 2017-11 / 2020$

Senior assistant: practical course Biological Chemistry

$01 / 2017-11 / 2020$

Senior assistant: practical course Enzyme catalysis and biological chemistry

Awards

2020

Travel Grand, $12^{\text {th }}$ International Peptide Symposium, Barcelona, Spain

2018

Poster Award, $8^{\text {th }}$ Peptide Engineering Meeting, Berlin, Germany

Publications

B. E. Hubrich, P. M. Menzel, B. Kugler, U. Diederichsen, Peptide Nucleic Acids: Methods and Protocols, P. Nielsen (Ed.), $3^{\text {rd }}$ Edition, Springer Verlag, Wiesbaden, 2020. 